Analysis of GEM-3 Data from the Advanced UXO Detection/Discrimination Technology Demonstration - U.S. Army Jefferson Proving Ground, Madison, Indiana

Ricky A. Goodson, Hollis H. (Jay) Bennett, Jr., Tere' A. DeMoss, Diane M. Cargile, John C. Morgan, and Morris P. Fields
US Army Corps of Engineers ${ }_{\circledast}$ Engineer Research and Development Center 
The contents of this report are not to be used for advertising, publication, or promotional purposes. Citation of trade names does not constitute an official endorsement or approval of the use of such commercial products.

The findings of this report are not to be construed as an official Department of the Army position, unless so designated by other authorized documents. 


\section{Analysis of GEM-3 Data from the Advanced UXO Detection/Discrimination Technology Demonstration - U.S. Army Jefferson Proving Ground, Madison, Indiana}

by Ricky A. Goodson, Hollis H. (Jay) Bennett, Jr., Tere' A. DeMoss, Diane M. Cargile Environmental Laboratory

U.S. Army Engineer Research and Development Center 3909 Halls Ferry Road

Vicksburg, MS 39180-6199

John C. Morgan, Morris P. Fields

Illinois Institute of Technology Research Institute 10 W. $35^{\text {th }}$ Street

Chicago, IL 60616-3717

Final report

Approved for public release; distribution is unlimited 


\section{Contents}

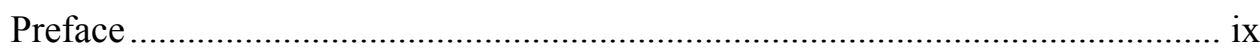

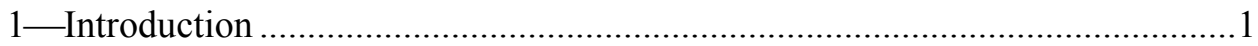

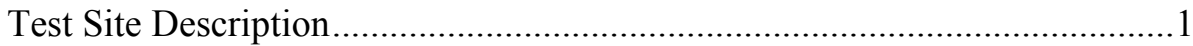

Description of Data Collection Equipment...................................................

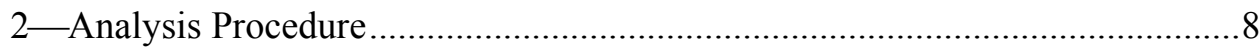

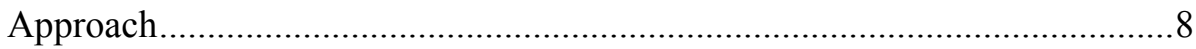

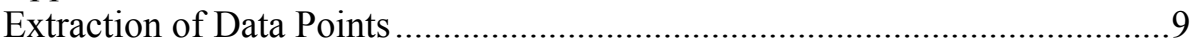

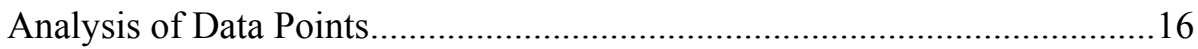

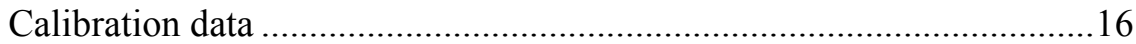

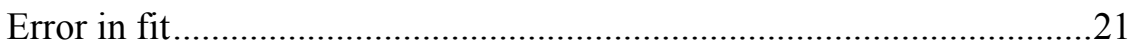

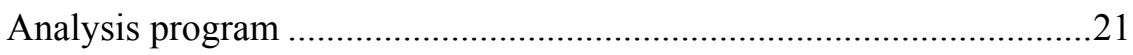

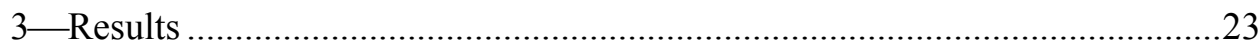

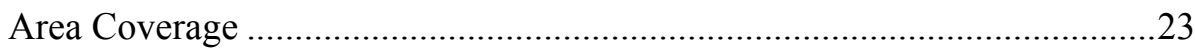

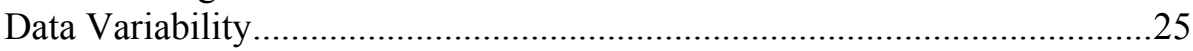

Comparison of Data Points with Calibration Data........................................34

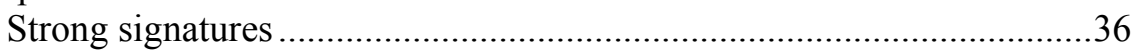

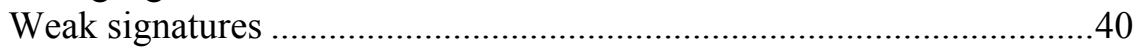

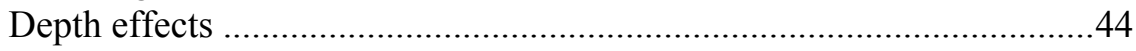

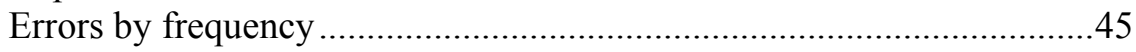

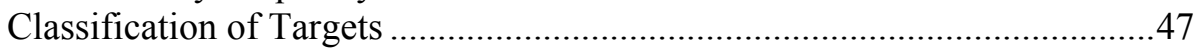

Classification of All Detected Anomalies.....................................................50

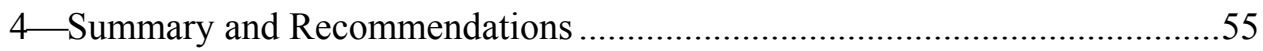

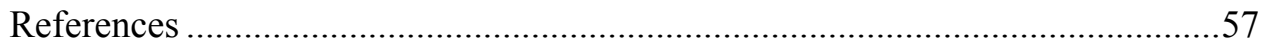

Appendix A: Area Coverage Maps with Histograms ......................................A1

Appendix B: Plots of Data Point Locations and Comparison with

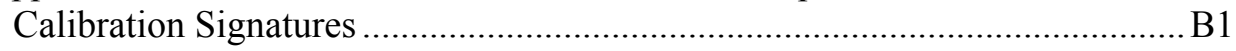

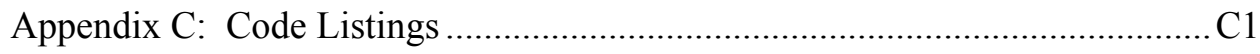

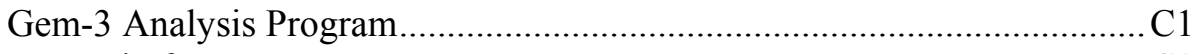

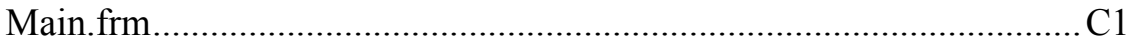

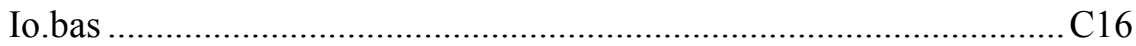

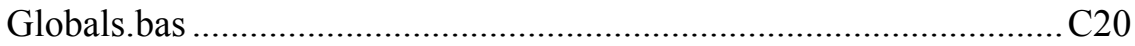

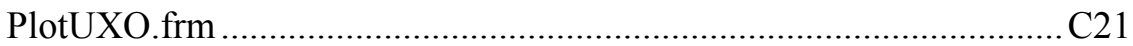


GridScan Program $\mathrm{C} 23$

GridTool Program

SF 298

\section{List of Figures}

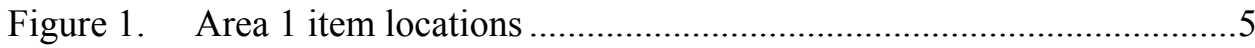

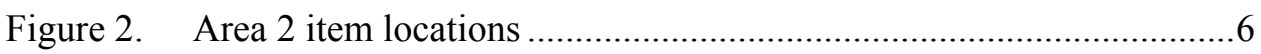

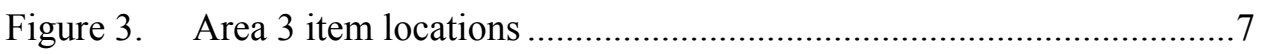

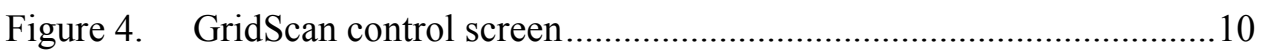

Figure 5. GridScan control screen showing the area selection pulldown menu ...................................................................... 11

Figure 6. GridScan control screen showing the target/item selection pulldown menu ....................................................................... 11

Figure 7. GridScan control screen showing the file action pulldown menu ....12

Figure 8. Histogram representation of background for target 3-74 at

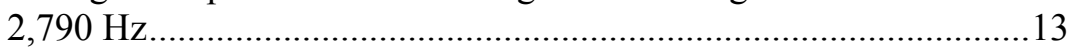

Figure 9. Histogram representation of background for target 3-100

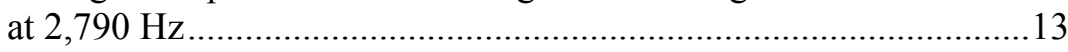

Figure 10. Histogram of background for target 1-123 at frequency $90 \mathrm{~Hz}$........14

Figure 11. Histogram of background for target 1-123 at frequency $150 \mathrm{~Hz}$...... 14

Figure 12. Histogram of background for target 1-123 at frequency $330 \mathrm{~Hz}$...... 14

Figure 13. Histogram of background for target 1-123 at frequency $930 \mathrm{~Hz}$......15

Figure 14. Histogram of background for target 1-123 at frequency $2,790 \mathrm{~Hz}$ .15

Figure 15. Histogram of background for target 1-123 at frequency $8,190 \mathrm{~Hz}$ 15

Figure 16. Histogram of background for target 1-123 at frequency $20,010 \mathrm{~Hz}$ .15

Figure 17. Acquisition of calibration data with the GEM-3. 16 
Figure 18. Calibration data for 20-mm projectile.

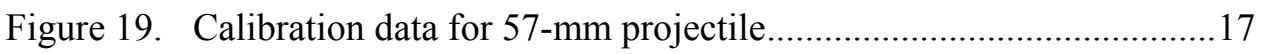

Figure 20. Calibration data for $60-\mathrm{mm}$ mortar ............................................... 18

Figure 21. Calibration data for 2.75-in. rocket............................................ 18

Figure 22. Calibration data for 81-mm mortar ............................................19

Figure 23. Calibration data for 4.2-in. mortar ................................................... 19

Figure 24. Calibration data for $152-\mathrm{mm}$ projectile..........................................20

Figure 25. Calibration data for $155-\mathrm{mm}$ projectile.........................................20

Figure 26. GEM-3 Analysis Program screen dump ......................................22

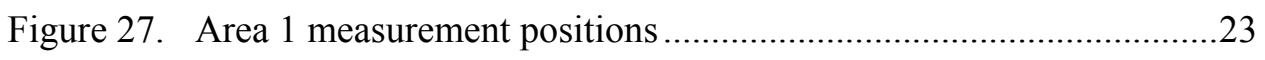

Figure 28. Area 2 measurement positions …................................................24

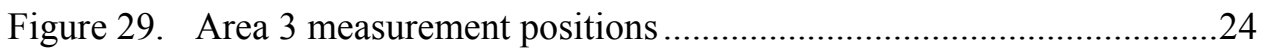

Figure 30. In-phase and quadrature histograms for background at $90 \mathrm{~Hz}$........26

Figure 31. In-phase and quadrature histograms for background at $150 \mathrm{~Hz}$.......26

Figure 32. In-phase and quadrature histograms for background at $330 \mathrm{~Hz}$.......26

Figure 33. In-phase and quadrature histograms for background at $930 \mathrm{~Hz}$.......27

Figure 34. In-phase and quadrature histograms for background at 2,790 Hz.

Figure 35. In-phase and quadrature histograms for background at $8,190 \mathrm{~Hz}$. .27

Figure 36. In-phase and quadrature histograms for background at 20,010 Hz. .28

Figure 37. In-phase and quadrature histograms for $20-\mathrm{mm}$ round at $90 \mathrm{~Hz} \ldots . . .28$

Figure 38. In-phase and quadrature histograms for $20-\mathrm{mm}$ round at $150 \mathrm{~Hz} \ldots .28$

Figure 39. In-phase and quadrature histograms for $20-\mathrm{mm}$ round at $330 \mathrm{~Hz} \ldots .29$

Figure 40. In-phase and quadrature histograms for $20-\mathrm{mm}$ round at $930 \mathrm{~Hz}$...29

Figure 41. In-phase and quadrature histograms for 20-mm round at 2,790 Hz. .29 
Figure 42. In-phase and quadrature histograms for $20-\mathrm{mm}$ round at $8,190 \mathrm{~Hz}$.

Figure 43. In-phase and quadrature histograms for $20-\mathrm{mm}$ round at $20,010 \mathrm{~Hz}$. 30

Figure 44. In-phase and quadrature histograms for $57-\mathrm{mm}$ mortar at $90 \mathrm{~Hz}$.....30

Figure 45. In-phase and quadrature histograms for $57-\mathrm{mm}$ mortar at $150 \mathrm{~Hz} \ldots 31$

Figure 46. In-phase and quadrature histograms for $57-\mathrm{mm}$ mortar at $330 \mathrm{~Hz} . .31$

Figure 47. In-phase and quadrature histograms for $57-\mathrm{mm}$ mortar at $930 \mathrm{~Hz} . .31$

Figure 48. In-phase and quadrature histograms for 57-mm mortar at $2,790 \mathrm{~Hz}$.

Figure 49. In-phase and quadrature histograms for 57-mm mortar at $8,190 \mathrm{~Hz}$ .32

Figure 50. In-phase and quadrature histograms for 57-mm mortar at $20,010 \mathrm{~Hz}$. .32

Figure 51. Best matching point for target 2-158 (20-mm projectile). 36

Figure 52. Best matching point for target 3-96 (57-mm mortar) 36

Figure 53. Best matching point for target 3-74 (60-mm mortar) .37

Figure 54. Best matching point for target 1-149 (2.75-in. rocket) .37

Figure 55. Best matching point for target 1-96 (81-mm mortar) ......................38

Figure 56. Best matching point for target 2-134 (4.2-in. mortar) ......................38

Figure 57. Best matching point for target 1-119 (152-mm projectile) ...............39

Figure 58. Best matching point for target 1-121 (155-mm projectile) ...............39

Figure 59. Best matching point for target 1-126 (57-mm mortar) …...............40

Figure 60. Best matching point for target 2-118 (60-mm mortar) ...................41

Figure 61. Best matching point for target 2-166 (2.75-in. rocket) ....................41

Figure 62. Best matching point for target 3-84 (81-mm mortar) .....................42

Figure 63. Best matching point for target 3-100 (152-mm projectile) ...............42

Figure 64. Best matching point for target 2-142 (152-mm projectile) ...............43 
Figure 65. Best matching point for target 3-102 (155-mm projectile)

Figure 66. Average magnitude vs. depth for selected ordnance types

Figure 67. Calibration match with all frequencies for target 1-132

(57-mm mortar).

Figure 68. Calibration match without $90 \mathrm{~Hz}$ for target 1-132

(57-mm mortar).

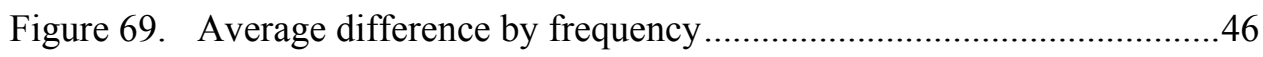

Figure 70. Calibration data with targets at 0 deg inclination ...........................47

Figure 71. Calibration data with targets at $90 \mathrm{deg}$ inclination (noseup) ...........48

Figure 72. Calibration data with targets at -90 deg inclination (nosedown) ......48

\section{List of Tables}

Table 1. JPG - Area 1 Target Items .............................................................2

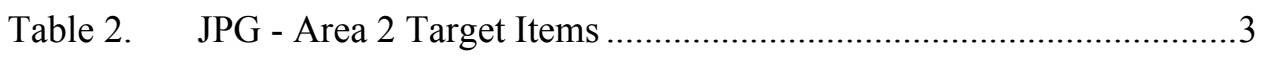

Table 3. JPG - Area 3 Target Items ..........................................................

Table 4. Geophex Classification Matrix for Areas 1, 2, and 3 .......................

Table 5. Geophex Classification Matrix for Areas 1, 2, and 3 Aggregated

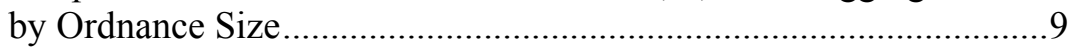

Table 6. Proximity of GEM-3 Data Points to Targets at JPG.......................25

Table 7. Statistical Information on Fixed Target Variation Sample Size

$\mathrm{N}=15,480$

Table 8. Comparison of Data Points with Calibration Signatures in Area 1 ..34

Table 9. Comparison of Data Points with Calibration Signatures in Area 2 ..35

Table 10. Comparison of Data Points with Calibration Signatures in Area 3 ..35

Table 11. Classification Matrix Using All Frequencies .................................49

Table 12. Classification Matrix Using All Frequencies Aggregated

by Ordnance Size. 
Table 13. Classification Matrix without 90-Hz Data.

Table 14. Classification Matrix without 90-Hz Data Aggregated

by Ordnance Size. 50

Table 15. Classification Matrix without 90-Hz and 150-Hz Data 51

Table 16. Classification Matrix without $90-\mathrm{Hz}$ and $150-\mathrm{Hz}$ Data

Aggregated by Ordnance Size . .51

Table 17. Classification Matrix with Threshold of $0.05 \ldots \ldots \ldots \ldots \ldots \ldots \ldots \ldots \ldots \ldots \ldots . . . .52$

Table 18. Classification Matrix Aggregated by Ordnance Size

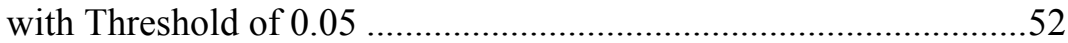

Table 19. Classification Matrix with Threshold of 0.1 ....................................53

Table 20. Classification Matrix Aggregated by Ordnance Size

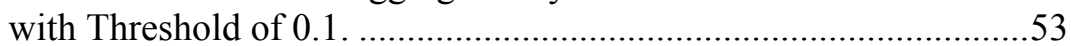

Table 21. Classification Matrix with Threshold of 0.15 ..................................54

Table 22. Classification Matrix Aggregated by Ordnance Size with Threshold of 0.15 54 


\section{Preface}

The research documented in this report was performed at the Environmental Laboratory (EL), U.S. Army Engineer Research and Development Center (ERDC), Vicksburg, MS, during January through June 2001. The work involved was a part of the 62720A/AF25 program "301U-Subsurface UXO Detection and Discrimination" for Project "UXO03-Advanced Sensor Data Analysis Techniques for Improved Buried Target Detection." This research was conducted under the direct supervision of Dr. Ernesto Cespedes, Environmental Sensing Branch, EL, in support of Project UXO03.

The research was conducted and the report was compiled by Messrs. Ricky Goodson, Jay Bennett, Mses. Tere' DeMoss and Diane Cargile, Environmental Sensing Branch, and Messrs. John C.Morgan and Morris P. Fields, Illinois Institute of Technology Research Institute. Messrs. Morgan and Fields performed the analysis of the background data. The statistical analysis was performed by Ms. DeMoss. Initial Jefferson Proving Ground data analysis, tabulation, and map generation were conducted by Ms. Cargile. The discrimination analysis was performed by Messrs. Goodson and Bennett.

Dr. Ernesto Cespedes was the UXO Characterization Team Leader. Mr. Harold W. West was Chief, Environmental Systems Branch. Dr. Dave Tazik was Chief, Ecosystem Evaluation and Engineering Division, and Dr. Edwin A. Theriot was Director, EL.

At the time of publication of this report, Dr. James Houston was Director of ERDC, and COL John W. Morris III, EN, was Commander and Executive Director.

The contents of this report are not to be used for advertising, publication, or promotional purposes. Citation of trade names does not constitute an official endorsement or approval of the use of such commercial products. 


\section{Introduction}

This report documents the analysis of the GEM-3 data collected for the Advanced UXO Detection/Discrimination Technology Demonstration at the U.S. Army Jefferson Proving Ground (JPG), Madison, IN. The analysis is funded through the 62720A/AF25 Program "301U-Subsurface UXO Detection and Discrimination" for Project "UXO03-Advance Sensor Data Analysis Techniques for Improved Buried Target Detection." This postdemonstration analysis focuses on the evaluation of the stability of the data collected, improvements in target detection/discrimination, and positioning errors of the system.

The stability of the system is evaluated through histograms and statistical measurements of data collected during the technology demonstration. Based on findings of the characteristics of the collected data and initial work performed on target detection/discrimination (Miller et al. 2001), target detection/ discrimination techniques are applied and evaluated.

\section{Test Site Description}

The JPG test site consisted of three areas referred to as Areas 1, 2, and 3 (Cespedes 2001). Each area is 1 hectare in size and contains known targets and clutter items. The naming convention for items in the test site is the area number followed by a hyphen and the item number (i.e., item number 43 in area 3 is 343, item number 48 in area 2 is 2-48). The target items for Areas 1, 2, and 3 are given in Tables 1, 2, and 3, respectively. The tables list the item number followed by the location of the item in UTM zone 16 NAD 83 coordinate system (Welch and Homsey 1996). The depth of the item is measured from the top of the item to the ground surface. The UXO type, length, width, thickness, weight, azimuth, and inclination are recorded for each item. The inclination is oriented with respect to $90 \mathrm{deg}$ being a nose-up position and the nose-down position is indicated by $-90 \mathrm{deg}$. The item locations for each area are shown in Figures 1, 2, and 3.

The maps showing the item locations for Areas 1, 2, and 3 are shown in Figures 1, 2, and 3, respectively. 


\begin{tabular}{|c|c|c|c|c|c|c|c|c|c|c|}
\hline \multicolumn{11}{|c|}{$\begin{array}{l}\text { Table } 1 \\
\text { JPG - Area } 1 \text { Target Items }\end{array}$} \\
\hline Item \# & $\begin{array}{c}\text { Northing } \\
\mathrm{m}\end{array}$ & $\begin{array}{c}\text { Easting } \\
\mathrm{m}\end{array}$ & $\begin{array}{c}\text { Depth } \\
\text { m }\end{array}$ & UXO Type & $\begin{array}{c}\text { Length } \\
\text { m }\end{array}$ & $\begin{array}{c}\text { Width } \\
\text { m }\end{array}$ & $\begin{array}{c}\text { Thick } \\
\text { m }\end{array}$ & $\begin{array}{l}\text { Weight } \\
\text { Kg }\end{array}$ & $\begin{array}{l}\text { Azimuth } \\
\text { deg }\end{array}$ & $\begin{array}{l}\text { Incl. } \\
\text { deg }\end{array}$ \\
\hline $1-86$ & 4309700.512 & 641416.750 & 0.2 & 4.2-in. Mortar & 0.52 & 0.105 & 0.105 & 21.9 & 120 & 45 \\
\hline $1-88$ & 4309684.035 & 641361.390 & 0.35 & 60-mm Mortar & 0.182 & 0.058 & 0.008 & 1.05 & 0 & 0 \\
\hline $1-90$ & 4309679.388 & 641352.247 & 0.35 & 4.2-in. Mortar & 0.52 & 0.105 & 0.105 & 22.1 & 270 & 0 \\
\hline $1-92$ & 4309668.548 & 641348.945 & 0.2 & 81-mm Mortar & 0.276 & 0.08 & 0.008 & 2.95 & 30 & 20 \\
\hline $1-94$ & 4309670.663 & 641365.981 & 0.25 & 81-mm Mortar & 0.276 & 0.08 & 0.008 & 2.95 & 75 & 55 \\
\hline $1-96$ & 4309663.286 & 641345.020 & 0.15 & 81-mm Mortar & 0.276 & 0.08 & 0.008 & 2.85 & 330 & 35 \\
\hline $1-98$ & 4309667.056 & 641369.619 & 0.1 & 60-mm Mortar & 0.182 & 0.058 & 0.008 & 1.05 & 275 & 20 \\
\hline $1-100$ & 4309681.176 & 641375.100 & 0.2 & 60-mm Mortar & 0.182 & 0.058 & 0.008 & 1.1 & 195 & 30 \\
\hline $1-102$ & 4309656.291 & 641370.045 & 0.25 & 81-mm Mortar & 0.276 & 0.08 & 0.008 & 2.85 & 210 & 45 \\
\hline $1-104$ & 4309659.265 & 641378.327 & 0.35 & 81-mm Mortar & 0.276 & 0.08 & 0.008 & 2.85 & 180 & 0 \\
\hline $1-106$ & 4309650.172 & 641372.159 & 0.25 & 60-mm Mortar & 0.182 & 0.058 & 0.008 & 1.05 & 75 & 35 \\
\hline $1-108$ & 4309638.289 & 641383.795 & 0.2 & 60-mm Mortar & 0.182 & 0.058 & 0.008 & 1.05 & 100 & 45 \\
\hline $1-112$ & 4309670.570 & 641356.944 & 0.1 & 20-mm Projectile & 0.074 & 0.018 & 0.018 & $100 \mathrm{~g}$ & 30 & 10 \\
\hline $1-113$ & 4309695.860 & 641344.701 & 0.5 & 105-mm Projectile & 0.374 & 0.106 & 0.004 & 28.4 & 120 & 30 \\
\hline $1-114$ & 4309666.352 & 641379.460 & 0.15 & 20-mm Projectile & 0.074 & 0.018 & 0.018 & $100 \mathrm{~g}$ & 175 & 20 \\
\hline $1-115$ & 4309686.931 & 641393.476 & 0.25 & 76-mm Projectile & 0.498 & 0.076 & 0.006 & 7.6 & 270 & 30 \\
\hline $1-116$ & 4309660.003 & 641370.325 & 0.15 & 20-mm Projectile & 0.074 & 0.018 & 0.018 & $100 \mathrm{~g}$ & 0 & 0 \\
\hline $1-117$ & 4309687.658 & 641422.319 & 0.9 & 152-mm Projectile & 0.478 & 0.152 & 0.152 & 42.6 & 0 & 45 \\
\hline $1-118$ & 4309665.079 & 641410.099 & 0.45 & 5-in. Projectile & 0.63 & 0.118 & 0.118 & 71 & 120 & 30 \\
\hline $1-119$ & 4309634.012 & 641351.264 & 0.4 & 152-mm Projectile & 0.478 & 0.152 & 0.152 & 42.7 & 270 & 30 \\
\hline $1-120$ & 4309629.459 & 641360.225 & 0.25 & 76-mm Projectile & 0.498 & 0.076 & 0.006 & 7.6 & 0 & 20 \\
\hline $1-121$ & 4309627.556 & 641387.439 & 0.5 & 155-mm Projectile & 0.602 & 0.15 & 0.008 & 99.1 & 200 & 0 \\
\hline $1-122$ & 4309647.428 & 641403.828 & 0.91 & 5-in. Projectile & 0.63 & 0.118 & 0.118 & 70.7 & 270 & 55 \\
\hline $1-123$ & 4309647.987 & 641416.776 & 0 & 20-mm Projectile & 0.074 & 0.018 & 0.018 & $100 \mathrm{~g}$ & - & 90 \\
\hline $1-124$ & 4309670.579 & 641337.765 & 0 & 20-mm Projectile & 0.074 & 0.018 & 0.018 & $100 \mathrm{~g}$ & - & -90 \\
\hline $1-126$ & 4309671.696 & 641343.041 & 0.2 & 57-mm Projectile & 0.118 & 0.054 & 0.004 & 0.8 & 120 & 30 \\
\hline $1-128$ & 4309662.646 & 641354.238 & 0.1 & 20-mm Projectile & 0.074 & 0.018 & 0.018 & $100 \mathrm{~g}$ & 0 & 0 \\
\hline $1-130$ & 4309657.656 & 641350.291 & 0.7 & 5-in. Projectile & 0.63 & 0.118 & 0.118 & 32.25 & 180 & 30 \\
\hline $1-132$ & 4309657.497 & 641359.337 & 0.25 & 57-mm Projectile & 0.118 & 0.054 & 0.004 & 0.8 & 180 & 0 \\
\hline $1-134$ & 4309665.044 & 641374.342 & 0.05 & 20-mm Projectile & 0.074 & 0.018 & 0.018 & $100 \mathrm{~g}$ & 120 & 30 \\
\hline $1-136$ & 4309671.569 & 641380.922 & 0.5 & 155-mm Projectile & 0.602 & 0.15 & 0.008 & 45.05 & 90 & 75 \\
\hline $1-138$ & 4309661.180 & 641385.835 & 0.15 & 57-mm Projectile & 0.118 & 0.054 & 0.004 & 0.8 & 95 & 45 \\
\hline $1-140$ & 4309656.690 & 641384.660 & 0.05 & 20-mm Projectile & 0.074 & 0.018 & 0.018 & $100 \mathrm{~g}$ & 275 & 15 \\
\hline $1-142$ & 4309654.752 & 641392.730 & 0.15 & 57-mm Projectile & 0.118 & 0.018 & 0.004 & 0.8 & 265 & 45 \\
\hline $1-144$ & 4309681.026 & 641399.341 & 0.5 & 105-mm Projectile & 0.374 & 0.106 & 0.004 & 13 & 180 & 0 \\
\hline $1-146$ & 4309657.423 & 641400.847 & 0.05 & 20-mm Projectile & 0.074 & 0.018 & 0.018 & $100 \mathrm{~g}$ & 270 & 0 \\
\hline $1-147$ & 4309613.119 & 641435.288 & 0.25 & 57-mm Projectile & 0.118 & 0.018 & 0.004 & 0.8 & 0 & 0 \\
\hline $1-148$ & 4309619.590 & 641374.925 & 0.1 & 20-mm Projectile & 0.074 & 0.018 & 0.018 & $100 \mathrm{~g}$ & 235 & 45 \\
\hline $1-149$ & 4309704.619 & 641371.539 & 0.5 & 2.75-in. Rocket & 0.406 & 0.072 & 0.008 & 4.2 & 30 & 55 \\
\hline $1-150$ & 4309678.038 & 641341.944 & 0.7 & 2.75-in. Rocket & 0.406 & 0.072 & 0.008 & 4.2 & 275 & 45 \\
\hline $1-151$ & 4309708.045 & 641432.206 & 0.25 & 76-mm Projectile & 0.496 & 0.076 & 0.076 & 3.542 & 150 & 45 \\
\hline $1-152$ & 4309651.123 & 641345.675 & 0.15 & 2.75-in. Rocket & 0.406 & 0.072 & 0.008 & 4.2 & 30 & 0 \\
\hline $1-153$ & 4309632.829 & 641426.973 & 0.76 & 2.75-in. Rocket & 0.406 & 0.072 & 0.008 & 4.3 & 0 & 90 \\
\hline
\end{tabular}




\begin{tabular}{|c|c|c|c|c|c|c|c|c|c|c|}
\hline \multicolumn{11}{|c|}{$\begin{array}{l}\text { Table } 2 \\
\text { JPG - Area } 2 \text { Target Items }\end{array}$} \\
\hline Item \# & $\begin{array}{c}\text { Northing } \\
\mathrm{m}\end{array}$ & $\begin{array}{c}\text { Easting } \\
\mathbf{m}\end{array}$ & $\begin{array}{c}\text { Depth } \\
\mathrm{m}\end{array}$ & UXO Type & $\begin{array}{c}\text { Length } \\
\mathrm{m}\end{array}$ & $\begin{array}{c}\text { Width } \\
\mathrm{m}\end{array}$ & $\begin{array}{c}\text { Thick } \\
\mathrm{m} \\
\end{array}$ & $\begin{array}{c}\text { Weight } \\
\text { Kg }\end{array}$ & \begin{tabular}{|c|}
$\begin{array}{c}\text { Azimuth } \\
\text { deg }\end{array}$ \\
\end{tabular} & $\begin{array}{l}\text { Incl. } \\
\text { deg }\end{array}$ \\
\hline $2-112$ & 4309743.578 & 641696.258 & 0.1 & 81-mm Mortar & 0.28 & 0.08 & 0.08 & 2.85 & -- & 90 \\
\hline $2-114$ & 4309719.108 & 641679.737 & 0.2 & 81-mm Mortar & 0.28 & 0.08 & 0.08 & 2.9 & -- & -90 \\
\hline $2-116$ & 4309707.453 & 641707.489 & 0.3 & 81-mm Mortar & 0.275 & 0.08 & 0.08 & 2.85 & 45 & 0 \\
\hline $2-118$ & 4309700.220 & 641704.486 & 0.35 & 60-mm Mortar & 0.18 & 0.06 & 0.06 & 1.19 & 0 & 45 \\
\hline $2-120$ & 4309688.298 & 641704.687 & 0.3 & 60-mm Mortar & 0.18 & 0.06 & 0.06 & 1.15 & 270 & 0 \\
\hline $2-122$ & 4309692.113 & 641624.184 & 0.1 & 60-mm Mortar & 0.18 & 0.06 & 0.06 & 1.16 & 180 & 30 \\
\hline $2-124$ & 4309683.303 & 641622.452 & 0.2 & 60-mm Mortar & 0.18 & 0.06 & 0.06 & 1.12 & 330 & 10 \\
\hline $2-126$ & 4309674.004 & 641675.583 & 0.35 & 81-mm Mortar & 0.27 & 0.08 & 0.08 & 2.85 & 45 & 45 \\
\hline $2-128$ & 4309687.368 & 641691.822 & 0.1 & 60-mm Mortar & 0.182 & 0.06 & 0.08 & 1.13 & 95 & 20 \\
\hline $2-130$ & 4309671.657 & 641700.823 & 0.2 & 81-mm Mortar & 0.274 & 0.08 & 0.08 & 2.8 & & 0 \\
\hline $2-131$ & 4309733.931 & 641654.484 & 0.25 & 81-mm Mortar & 0.274 & 0.08 & 0.08 & 2.95 & 0 & 0 \\
\hline $2-132$ & 4309733.468 & 641687.011 & 0.91 & 5-in. Projectile & 0.758 & 0.12 & & 31.55 & 90 & 0 \\
\hline $2-134$ & 4309731.276 & 641702.487 & 0.4 & 4.2-in. Mortar & & & & & 0 & 0 \\
\hline $2-136$ & 4309707.296 & 641622.792 & 0.05 & 20-mm Projectile & 0.074 & 0.02 & 0.02 & 0.1 & 120 & 10 \\
\hline $2-138$ & 4309708.488 & 641660.344 & 0.05 & 20-mm Projectile & 0.074 & 0.02 & 0.02 & 0.1 & 275 & 15 \\
\hline $2-140$ & 4309704.836 & 641684.996 & 0.7 & 105-mm Projectile & 0.262 & 0.105 & 0.105 & 13 & 330 & 10 \\
\hline $2-142$ & 4309697.175 & 641693.249 & 0.91 & 152-mm Projectile & 0.484 & 0.152 & 0.152 & 19.3 & 300 & 45 \\
\hline $2-144$ & 4309686.347 & 641637.892 & 0.45 & 152-mm Projectile & 0.484 & 0.152 & 0.152 & 19.35 & 50 & 30 \\
\hline $2-146$ & 4309683.272 & 641663.370 & flush & 20-mm Projectile & 0.074 & 0.02 & 0.02 & 0.098 & -- & 90 \\
\hline $2-148$ & 4309669.271 & 641656.726 & flush & 20-mm Projectile & 0.074 & 0.02 & 0.02 & 0.098 & -- & -90 \\
\hline $2-150$ & 4309679.845 & 641676.348 & 0.1 & 20-mm Projectile & 0.074 & 0.02 & 0.02 & 0.1 & 0 & 0 \\
\hline $2-152$ & 4309680.435 & 641693.918 & 0.25 & 57-mm Projectile & 0.117 & 0.057 & 0.057 & 0.826 & 90 & 0 \\
\hline $2-154$ & 4309667.086 & 641689.383 & 0.2 & 57-mm Projectile & 0.118 & 0.054 & 0.054 & 0.826 & 180 & 45 \\
\hline $2-156$ & 4309654.624 & 641626.571 & 102 & 155-mm Projectile & 0.6 & 0.155 & 0.155 & 42.25 & -- & 90 \\
\hline $2-158$ & 4309653.883 & 641647.917 & 0.1 & 20-mm Projectile & 0.074 & 0.02 & 0.02 & 0.1 & 220 & 20 \\
\hline $2-160$ & 4309651.428 & 641691.019 & 0.35 & 57-mm Projectile & 0.117 & 0.056 & 0.056 & 0.826 & 270 & 40 \\
\hline $2-161$ & 4309741.089 & 641627.124 & 0.75 & 155-mm Projectile & 0.6 & 0.155 & 0.155 & 42.2 & 270 & 30 \\
\hline $2-162$ & 4309730.194 & 641669.085 & 0.5 & 76-mm Projectile & 0.5 & 0.076 & 0.076 & 3.55 & 0 & 0 \\
\hline $2-164$ & 4309725.318 & 641622.007 & 0.6 & 2.75-in. Rocket & 0.4 & 0.08 & 0.08 & 4.2 & 90 & 10 \\
\hline $2-166$ & 4309675.298 & 641640.848 & 0.75 & 2.75-in. Rocket & 0.4 & 0.4 & 0.8 & 4.2 & 180 & 20 \\
\hline
\end{tabular}

\section{Description of Data Collection Equipment}

The GEM-3 is a multifrequency frequency domain electromagnetic (FDEM) system (Won et al. 1997) (Geophex 1998). The collection of multifrequency FDEM data allows for Electromagnetic Induction Spectroscopy (EMIS) of the targets and background materials (Won, Keiswetter, and Novikova 1998). The EMIS signatures are characteristic of the objects geometry and material composition and consist of complex (in-phase and quadrature) frequency responses. These EMIS signatures can provide a method to discriminate targets from natural and manmade clutter background materials. The frequencies used during the data collection were $90 ; 150 ; 330 ; 930 ; 2,790 ; 8,190$; and 20;010 Hz. The system has been developed by Geophex Ltd. with improvements funded by the Army's SBIR Phase II program (Contract DACA39-99-C-0001) and was operated by Geophex during the JPG technology demonstration. Geophex performed the initial target detection. The initial target discrimination was performed by AETC Inc. 


\begin{tabular}{|c|c|c|c|c|c|c|c|c|c|c|}
\hline \multicolumn{11}{|c|}{$\begin{array}{l}\text { Table } 3 \\
\text { JPG - Area } 3 \text { Target Items }\end{array}$} \\
\hline Item \# & $\begin{array}{l}\text { Northing } \\
\mathrm{m}\end{array}$ & $\begin{array}{c}\text { Easting } \\
\mathrm{m}\end{array}$ & $\begin{array}{c}\text { Depth } \\
\text { m }\end{array}$ & UXO Type & $\begin{array}{l}\text { Length } \\
\mathrm{m}\end{array}$ & $\begin{array}{c}\text { Width } \\
\text { m }\end{array}$ & $\begin{array}{c}\text { Thick } \\
\text { m }\end{array}$ & $\begin{array}{l}\text { Weight } \\
\text { Kg }\end{array}$ & $\begin{array}{l}\text { Azimuth } \\
\text { deg }\end{array}$ & $\begin{array}{l}\text { Incl. } \\
\text { deg }\end{array}$ \\
\hline $3-68$ & 4309868.372 & 641625.085 & 0.2 & 60-mm Mortar & 0.182 & 0.058 & 0.008 & 2.5 & $F$ & 90 \\
\hline $3-70$ & 4309858.666 & 641651.432 & 0.25 & 81-mm Mortar & 0.276 & 0.08 & 0.008 & 6.4 & 0 & 0 \\
\hline $3-72$ & 4309843.426 & 641632.303 & 0.25 & 60-mm Mortar & 0.182 & 0.058 & 0.008 & 2.5 & 45 & 30 \\
\hline $3-74$ & 4309848.102 & 641650.780 & 0.3 & 60-mm Mortar & 0.182 & 0.058 & 0.008 & 2.7 & 225 & 45 \\
\hline $3-76$ & 4309843.472 & 641664.147 & 0.2 & 81-mm Mortar & 0.276 & 0.08 & 0.008 & 6.4 & - & -90 \\
\hline $3-78$ & 4309830.166 & 641650.755 & 0.35 & 60-mm Mortar & 0.182 & 0.058 & 0.008 & 2.5 & 330 & 40 \\
\hline $3-80$ & 4309817.969 & 641633.484 & 0.25 & 81-mm Mortar & 0.276 & 0.08 & 0.008 & 6.4 & 180 & 0 \\
\hline $3-82$ & 4309814.256 & 641657.245 & 0.2 & 60-mm Mortar & 0.182 & 0.058 & 0.008 & 2.7 & 270 & 15 \\
\hline $3-84$ & 4309805.611 & 641679.788 & 0.25 & 81-mm Mortar & 0.276 & 0.08 & 0.008 & 6.4 & 0 & 0 \\
\hline $3-86$ & 4309885.192 & 641665.607 & 0.01 & 20-mm Projectile & 0.074 & 0.018 & 0.018 & $100 \mathrm{~g}$ & - & 90 \\
\hline $3-88$ & 4309854.961 & 641639.131 & 0.01 & 20-mm Projectile & 0.074 & 0.018 & 0.018 & $100 \mathrm{~g}$ & 0 & 0 \\
\hline $3-90$ & 4309846.260 & 641635.938 & 0.15 & 20-mm Projectile & 0.074 & 0.018 & 0.018 & $100 \mathrm{~g}$ & 90 & 0 \\
\hline $3-92$ & 4309835.209 & 641642.572 & 0.15 & 20-mm Projectile & 0.074 & 0.018 & 0.018 & $100 \mathrm{~g}$ & 120 & 30 \\
\hline $3-94$ & 4309841.825 & 641651.824 & 0.35 & 57-mm Projectile & 0.118 & 0.054 & 0.004 & 0.8 & 330 & 0 \\
\hline $3-96$ & 4309808.212 & 641616.158 & 0.25 & 57-mm Projectile & 0.118 & 0.054 & 0.004 & 0.8 & 30 & 20 \\
\hline $3-98$ & 4309873.375 & 641597.294 & 0.5 & 105-mm Projectile & 0.374 & 0.106 & 0.004 & 13 & 0 & -45 \\
\hline $3-100$ & 4309869.507 & 641658.196 & 0.91 & 152-mm Projectile & 0.478 & 0.152 & 0.152 & 42.7 & 90 & 35 \\
\hline |3-102 & 4309828.646 & 641617.038 & 1.2 & 155-mm Projectile & 0.602 & 0.015 & 0.008 & 45.05 & 30 & 20 \\
\hline 3-104 & 4309849.677 & 641644.479 & 0.76 & 76-mm Projectile & 0.488 & 0.076 & 0.076 & 3.486 & 0 & 0 \\
\hline $3-106$ & 4309853.860 & 641597.683 & 0.5 & 2.75 in. Rocket & 0.406 & 0.072 & 0.008 & 9.3 & 120 & 30 \\
\hline
\end{tabular}




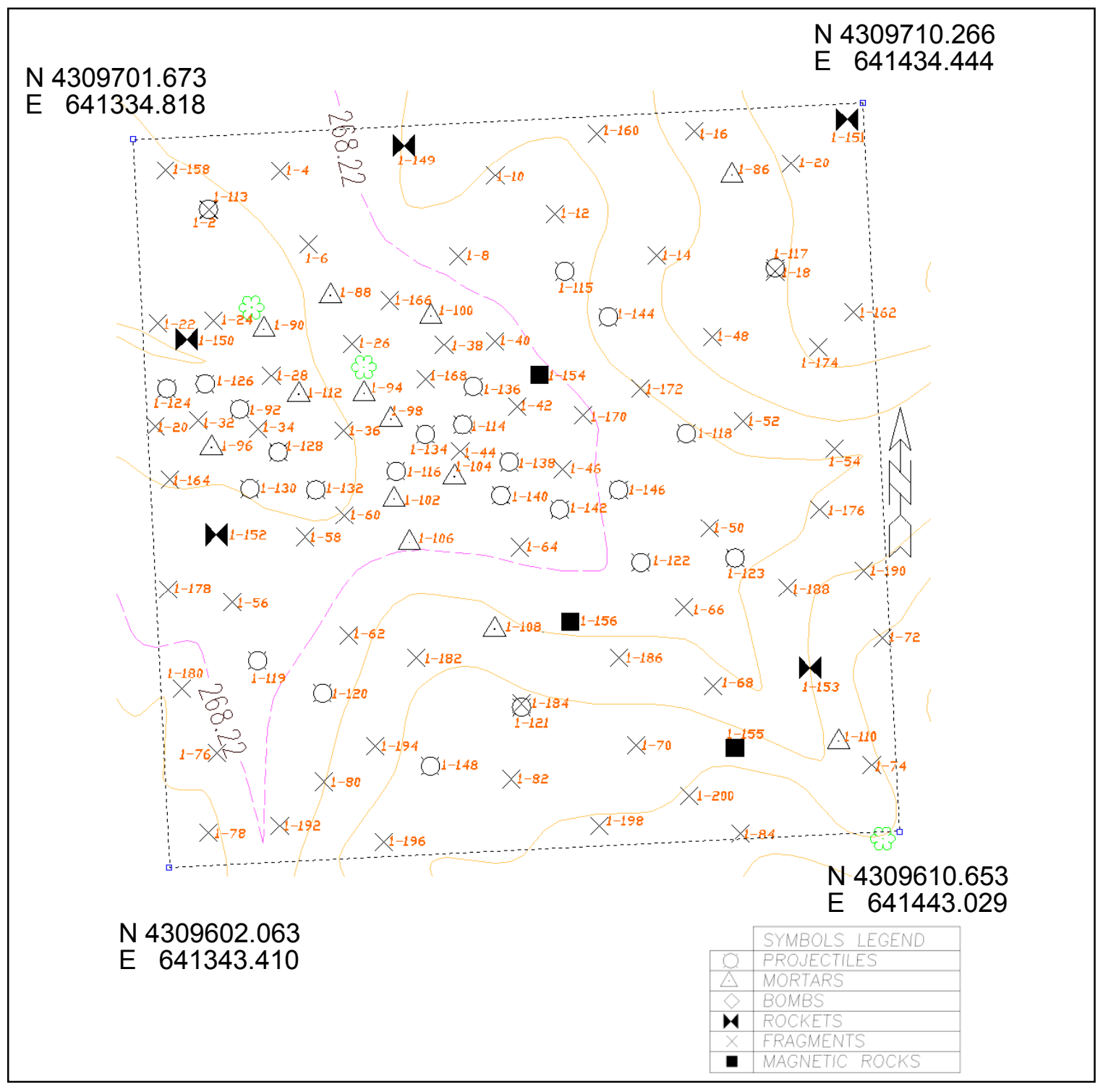

Figure 1. Area 1 item locations 


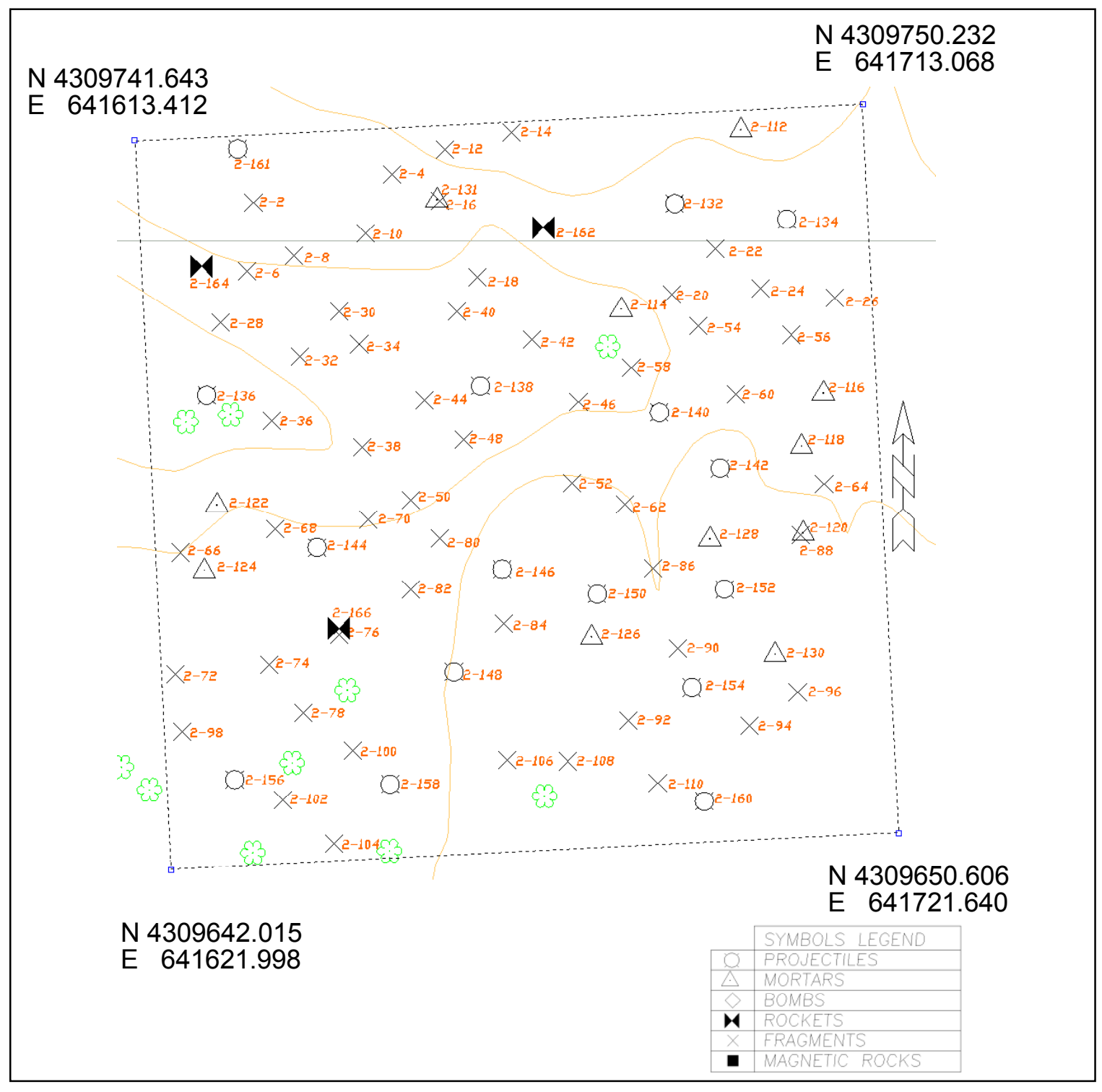

Figure 2. Area 2 item locations 


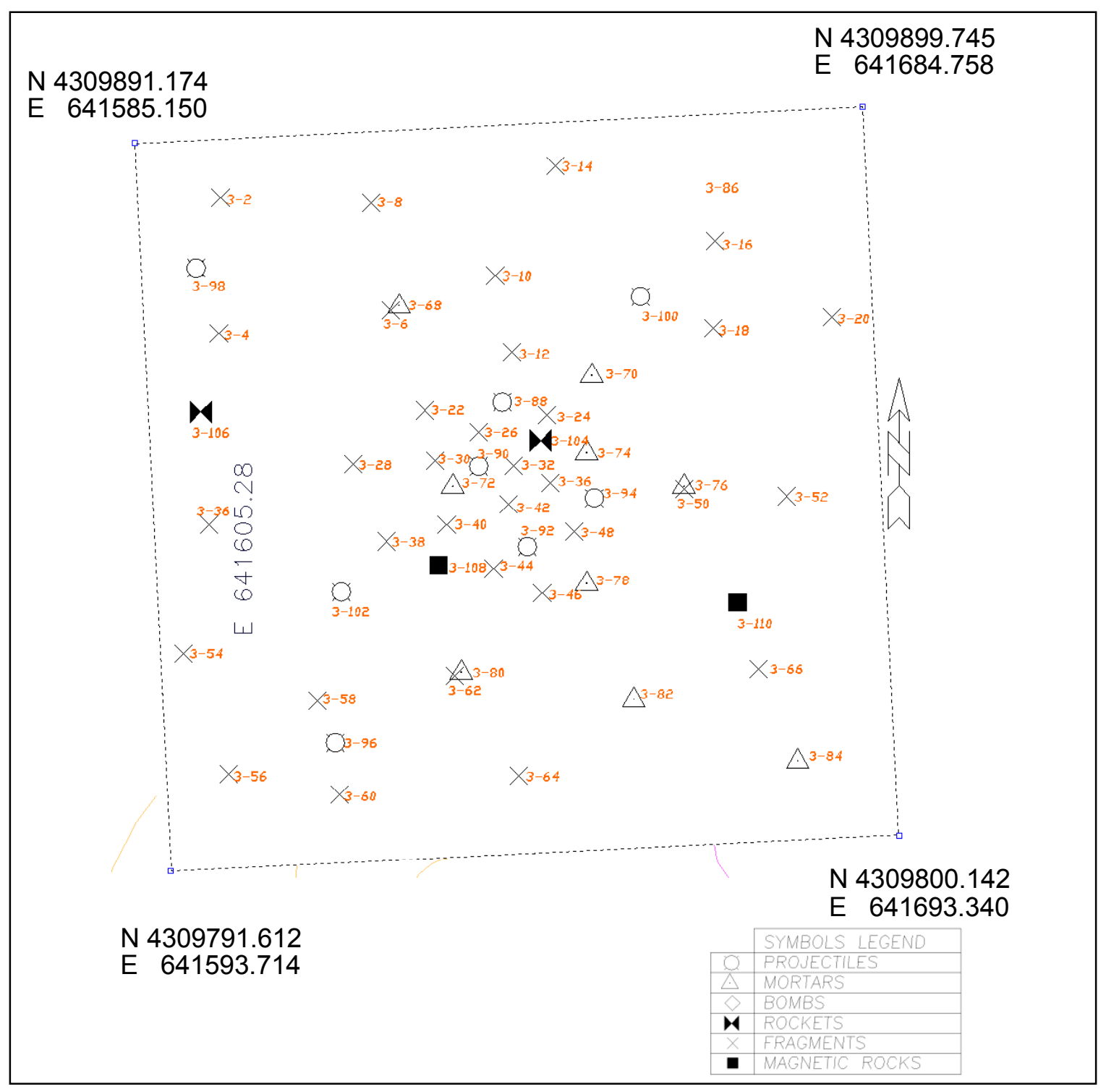

Figure 3. Area 3 item locations 


\section{Analysis Procedure}

The target classification procedure performed on the JPG data by AETC for Geophex is based on estimating dipole model parameters from a number of GEM-3 measurements around a suspected target location (Cespedes 2001). While this classification procedure had worked well during previous demonstrations (Miller et al. 2001), it did not work very well at JPG. The target classification results achieved by Geophex for each ordnance type included in the JPG demonstration are shown in Table 4 . Geophex correctly classified 39.39 percent $(13 / 33)$ of the mortars, 20.75 percent $(11 / 53)$ of the projectiles, and 0 percent $(0 / 7)$ of the rockets for a total of 25.8 percent $(24 / 93)$ of the UXO items in the area. The results for projectiles were actually worse than it appears, because only 2 out of 33 of the larger projectiles were properly classified. The other nine properly classified projectiles were $20-\mathrm{mm}$ projectiles, the classification of which generated a large number of false alarms as well. Over one-half (48/93) of the targets were classified as non-ordnance clutter. The Geophex Classification results aggregated by ordnance size are shown in Table 5. The GEM-3 data from the JPG demonstration were analyzed by ERDC personnel to determine whether classification results better than those achieved by Geophex were possible and to identify possible problems or limitations of the GEM-3.

\section{Approach}

The approach taken in the U.S. Army Engineer Research and Develoment Center (ERDC), Vicksburg, MS, analysis of the performance of the GEM-3 at JPG was to extract data points collected near each of the actual target locations and compare them to the calibration data acquired with known targets at the beginning of the demonstration. Throughout this report, the term "data point" refers to a GEM-3 measurement consisting of both the in-phase and quadrature measurements for each of the seven frequencies for which data were collected during the JPG demonstration. The seven frequencies used were 90; 150; 330; 930; 2,790; 8,190; and 20,010 Hz. A computer program was developed to extract the data points and analyze the background signature for the set of data points near each target. A second computer program was developed to compare the extracted data points with calibration signatures. This was done to determine how well the data collected near each actual target matched the calibration signatures for the same ordnance type and the extent to which the data could be differentiated from other ordnance types. In addition, data points near all the objects declared by Geophex were compared with the calibration signatures to 


\begin{tabular}{|c|c|c|c|c|c|c|c|c|c|c|c|c|}
\hline \multicolumn{13}{|l|}{ Table 4} \\
\hline \multirow[b]{2}{*}{$\begin{array}{l}\text { Geophex } \\
\text { Classification }\end{array}$} & \multirow[b]{2}{*}{$\begin{array}{l}20- \\
\mathrm{mm}\end{array}$} & \multirow[b]{2}{*}{$\begin{array}{l}57- \\
\mathrm{mm}\end{array}$} & \multirow[b]{2}{*}{$\begin{array}{l}76- \\
\mathrm{mm}\end{array}$} & \multirow[b]{2}{*}{$\begin{array}{l}105 \\
-\mathrm{mm} \\
\end{array}$} & \multicolumn{3}{|c|}{ Ordnance Type } & \multirow[b]{2}{*}{$\begin{array}{l}60- \\
\mathrm{mm}\end{array}$} & \multirow[b]{2}{*}{$\begin{array}{l}81- \\
\mathrm{mm}\end{array}$} & \multirow[b]{2}{*}{$\begin{array}{l}4.2- \\
\text { in. }\end{array}$} & \multirow[b]{2}{*}{$\begin{array}{l}2.75- \\
\text { in. }\end{array}$} & \multirow[b]{2}{*}{\begin{tabular}{|l|} 
False \\
Alarms
\end{tabular}} \\
\hline & & & & & \begin{tabular}{|l}
$5-$ \\
in.
\end{tabular} & $\begin{array}{l}152- \\
\mathrm{mm}\end{array}$ & \begin{tabular}{|c|}
155 \\
$-\mathrm{mm}$ \\
\end{tabular} & & & & & \\
\hline Projectile 20-mm & 9 & 0 & 1 & 0 & 0 & 0 & 0 & 0 & 0 & 0 & 0 & 152 \\
\hline Projectile 57-mm & 0 & 1 & 0 & 0 & 0 & 0 & 0 & 1 & 0 & 0 & 0 & 6 \\
\hline Projectile 76-mm & 0 & 0 & 0 & 0 & 0 & 1 & 0 & 2 & 0 & 0 & 4 & 15 \\
\hline Projectile 105-mm & 0 & 0 & 0 & 0 & 0 & 0 & 0 & 0 & 1 & 0 & 0 & 1 \\
\hline Projectile 5-in. & 0 & 0 & 0 & 0 & 0 & 0 & 0 & 0 & 0 & 0 & 0 & 4 \\
\hline Projectile 152-mm & 0 & 0 & 0 & 0 & 1 & 1 & 1 & 0 & 0 & 0 & 0 & 4 \\
\hline Projectile 155-mm & 0 & 0 & 0 & 0 & 0 & 0 & 0 & 0 & 0 & 0 & 0 & 1 \\
\hline Mortar 60-mm & 0 & 2 & 0 & 1 & 0 & 0 & 0 & 4 & 0 & 0 & 0 & 56 \\
\hline Mortar 81-mm & 0 & 0 & 0 & 1 & 0 & 1 & 0 & 0 & 7 & 0 & 0 & 10 \\
\hline Mortar 4.2-in. & 0 & 0 & 0 & 0 & 2 & 0 & 0 & 0 & 0 & 2 & 0 & 1 \\
\hline Rocket 2.75-in. & 0 & 0 & 1 & 0 & 0 & 0 & 0 & 0 & 1 & 0 & 0 & 5 \\
\hline \begin{tabular}{|l} 
Nonordnance \\
Low/Med
\end{tabular} & 10 & 6 & 2 & 0 & 1 & 1 & 1 & 6 & 1 & 0 & 3 & 181 \\
\hline \multirow[t]{2}{*}{\begin{tabular}{|l|} 
Nonordnance High \\
\end{tabular}} & 1 & 1 & 1 & 2 & 0 & 1 & 3 & 2 & 5 & 1 & 0 & 65 \\
\hline & & & & & & & & & & & & 0 \\
\hline Total & 20 & 10 & 5 & 4 & 4 & 5 & 5 & 15 & 15 & 3 & 7 & 501 \\
\hline$\%$ classified & 45.0 & 10.0 & 0.0 & 0.0 & 0.0 & 20.0 & 0.0 & 26.7 & 46.7 & 66.7 & 0.0 & \\
\hline
\end{tabular}

\begin{tabular}{|c|c|c|c|c|}
\hline $\begin{array}{l}\text { Table } 5 \\
\text { Geophex } \\
\text { Ordnance }\end{array}$ & ificatic & x for $A$ & , 2, and 3 & yregated by \\
\hline Geophex & & & ance Type & \\
\hline Classification & $20-\mathrm{mm}$ & 57-81-mm & 105-155-mm & False Alarm \\
\hline 20-mm & 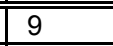 & $\overline{c 1}$ & 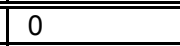 & 152 \\
\hline 57-81-mm & 0 & 23 & 4 & 92 \\
\hline 105-155-mm & 0 & 1 & 7 & 11 \\
\hline Nonordnance & 11 & 27 & 10 & 246 \\
\hline Total & 20 & 52 & 21 & 501 \\
\hline$\%$ classified & 45.0 & 44.2 & 33.3 & 49.1 \\
\hline
\end{tabular}

determine the degree of confusion caused by nonordnance clutter. In addition to analysis of the JPG data, new measurements were made with the GEM-3 for several minutes over a target area to determine the statistical variability of the instrument.

\section{Extraction of Data Points}

Because of the size of these data sets (in excess of 20 megabytes each) that were acquired for each demonstration area at JPG, it was necessary to develop a way to extract the relevant data for analysis. A graphical user interface program, GridScan, was written to allow the user to extract the data near a given by Global 
Positioning System (GPS) coordinate for analysis. GridScan reads in a list of targets and their location and extracts these data within a user-defined box about the item for analysis. The list of points consists of either the GPS locations given by Geophex or in the ground truth. In addition, GridScan generates histograms of a user-defined square annulus around the target and outputs these data for use in background determination. A screen shot of GridScan can be seen in Figure 4. This program was written first in an effort to determine if there was anything wrong with the locations chosen for analysis or the data itself.

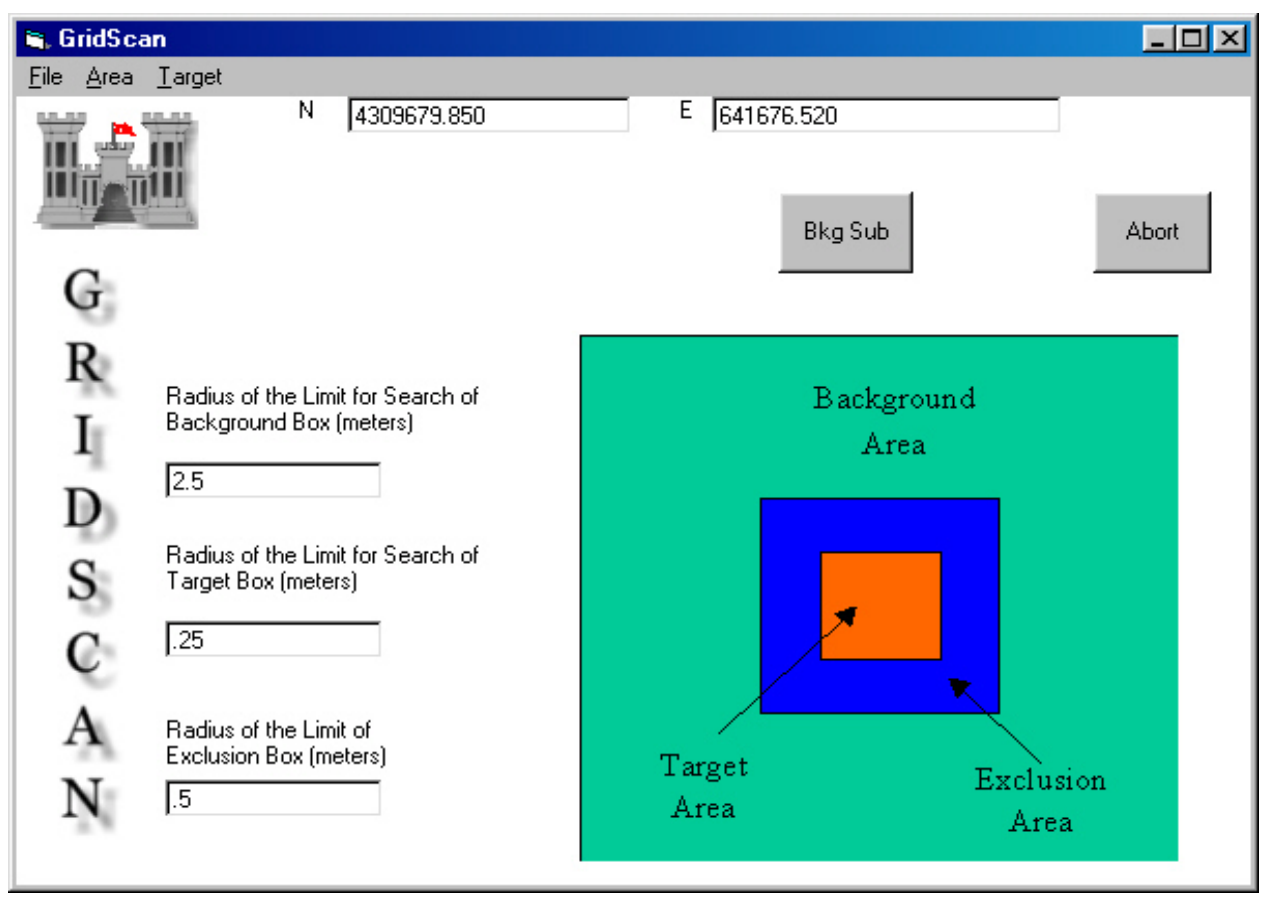

Figure 4. GridScan control screen

The procedure used by ERDC personnel to analyze the JPG GEM-3 data began with first selecting one of the three test plot areas to be searched by checking the appropriate choice in the pull down menu (Figure 5). This selects the correct file that contains all of the raw data from that area. Next the user would select to search for either target items, fragments (clutter items), or for both target and fragment items (Figure 6). The choice of options will determine which file containing item locations is to be used. If "targets" is selected, then the file containing the target GPS locations is used and searches these data one at a time. The last option was to allow for the use of the Geophex chosen locations file to be used.

The user then determined the area of the search by selecting the area around the point to include in the search area, the area to exclude around that point, and then the area to be included as background. The exclusion area was used to remove the influence of any large target from the background to better determine the actual background signal in the area of the target. This selection of the background area size must be done carefully. If the area chosen is too large, it may include other targets or clutter items. 


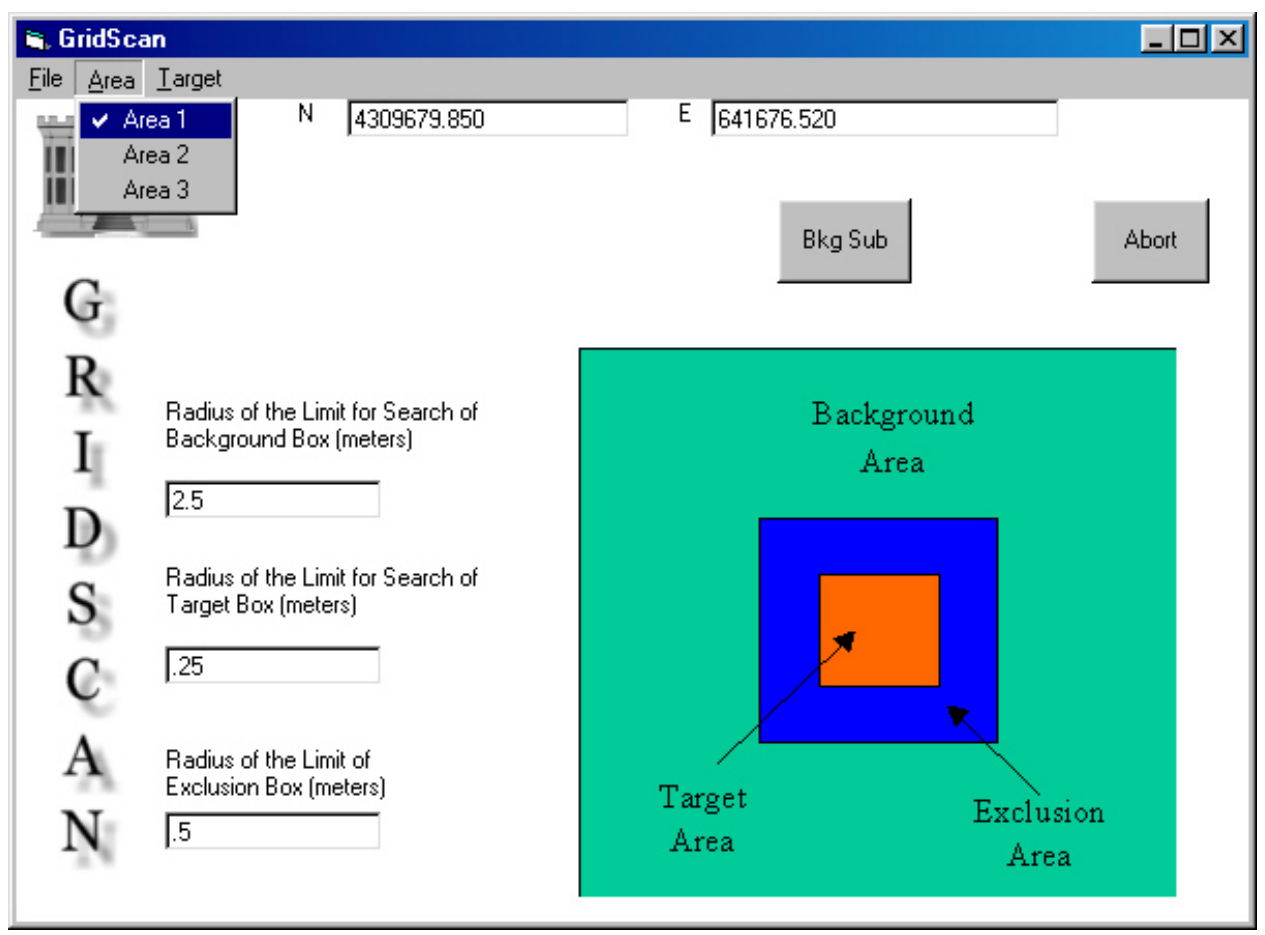

Figure 5. GridScan control screen showing the area selection pulldown menu

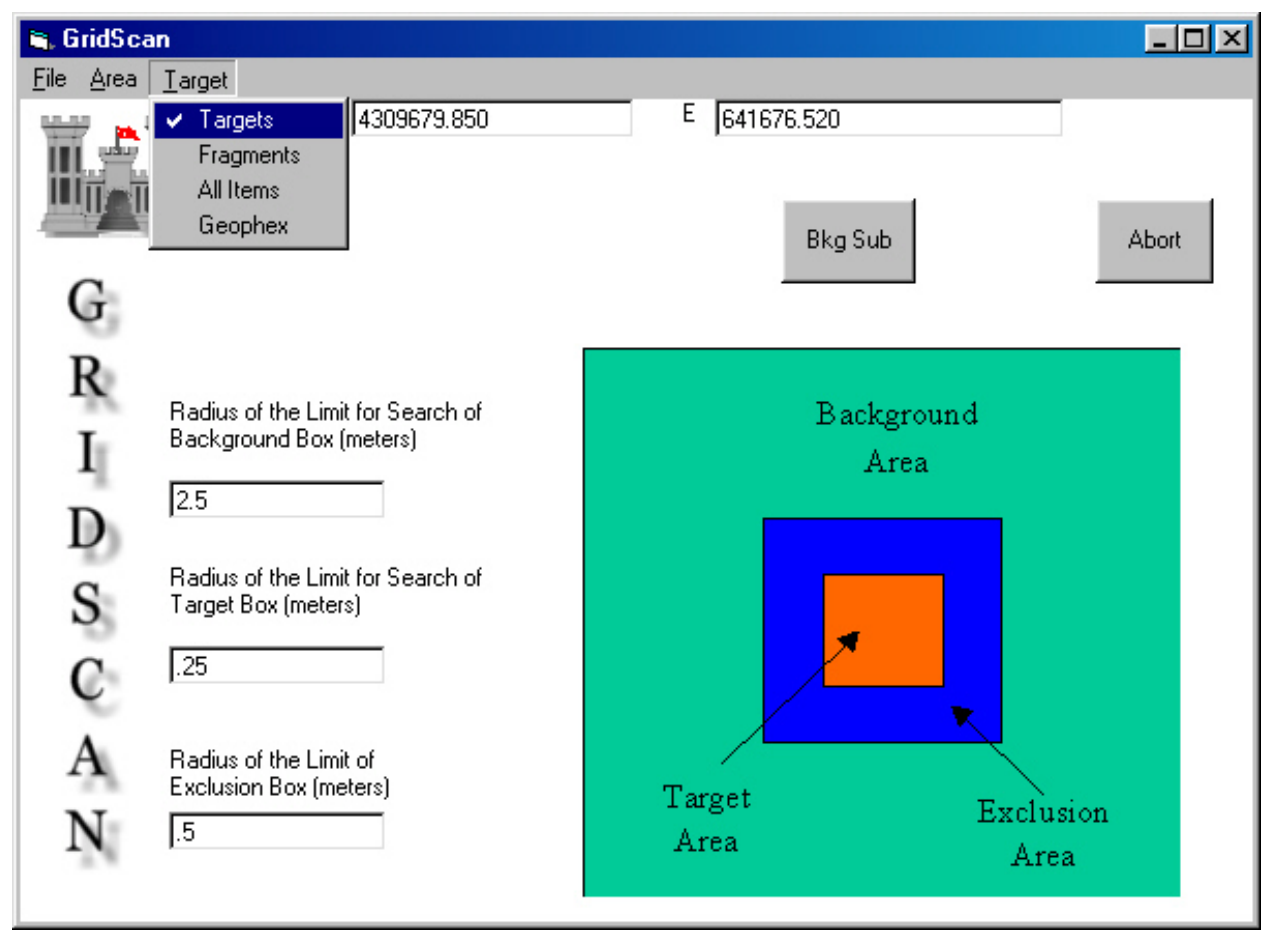

Figure 6. GridScan control screen showing the target/item selection pulldown menu 
Once all of the parameters have been decided, the user runs the program by selecting File $>$ Process Data (Figure 7). Now the data will be searched for the points of interest. When this is finished running, the user will implement the Background Subtract. This will use an average of the local background around the item as the background to be subtracted for each item. All of this information is saved in an ASCII file that has the item number, GPS location, and all the measured data associated with that point for more indepth analysis.

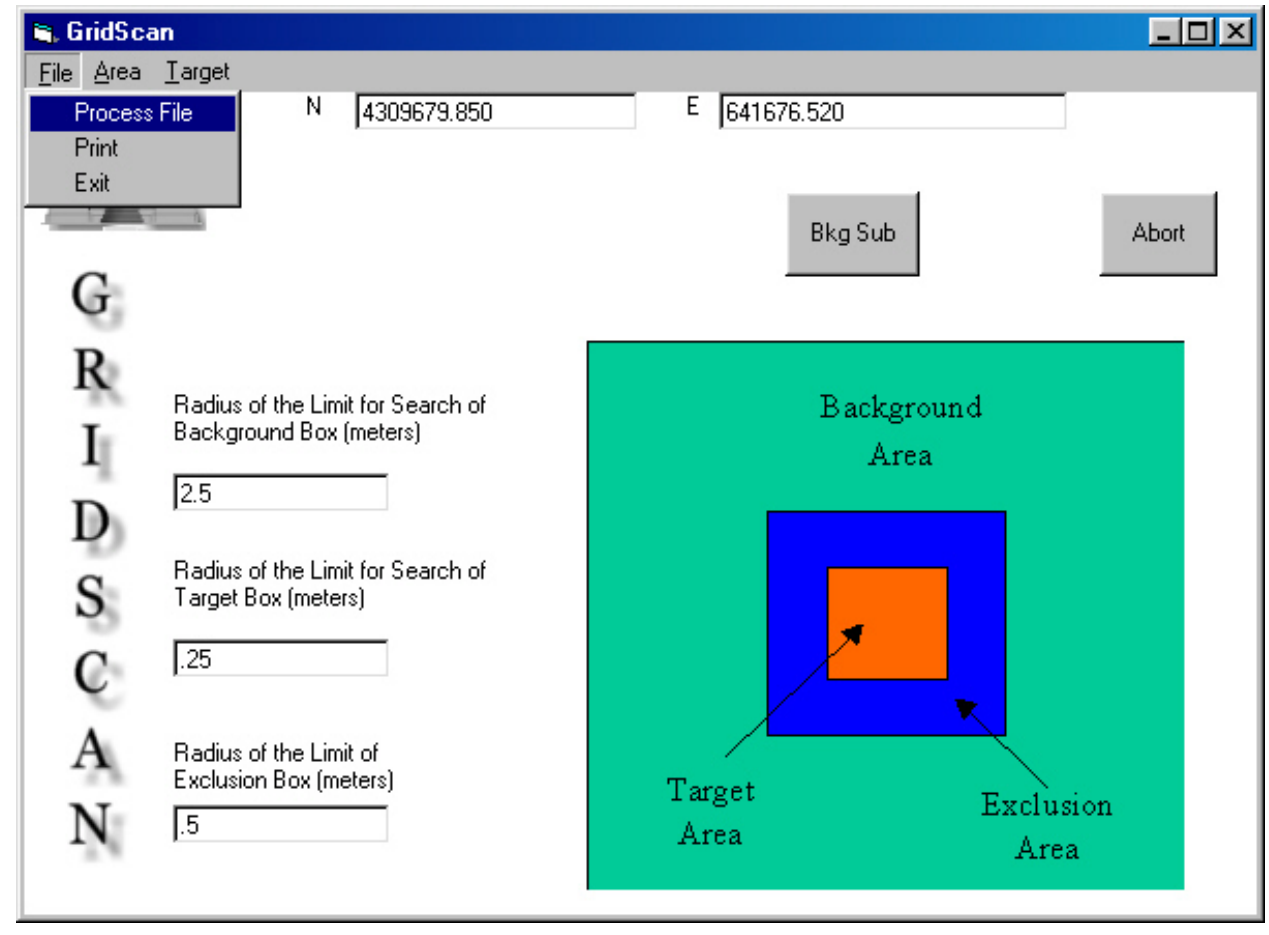

Figure 7. GridScan control screen showing the file action pulldown menu

An additional feature was implemented into GridScan for help in background calculation. The routine creates a histogram of the data that were selected for use as the background. If these data create a single well-defined peak, this indicates that the background is fairly homogenous and isotropic. Dual peaks or skewed distributions indicate that a different method of determining the background should be used. This could be caused by a detector shift and should be handled by treating the shifted areas separately. The background will be the most prominent feature and should have distributions around the average background signal strength. Also, smaller peaks will be seen. These peaks will represent the various items buried in the area.

Background variability in the data set adds uncertainty or error to the target signature and this degrades the viability of classification algorithms. To better understand the background contribution to the uncertainty in the data set, it is necessary to characterize the background so that it could be removed from the target data set. For the purpose of this investigation, the target data were defined as the points within a 1-m box centered on each target, and these data were extracted for classification analysis. The background data were defined as the 
data within a 5-m box about the target excluding the 1-m target data set. To determine the best background value to subtract from the target, a histogram of the values in the background was created. Then, the centroid of the largest peak in that set was chosen as the background value.

From Figure 8, it is apparent that the majority of the measured values around item 3-74 at frequency $2,790 \mathrm{~Hz}$ have a value of approximately 2.5 . This welldefined peak is what would be expected from a measurement over a fairly homogeneous medium. In this case, the centroid is a good measure of the background value.

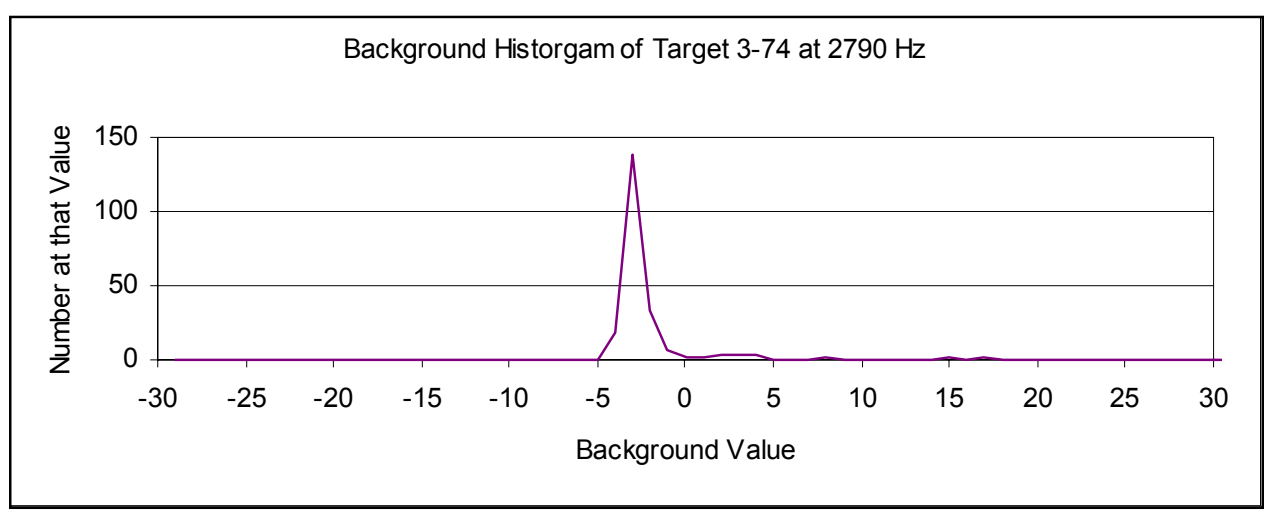

Figure 8. Histogram representation of background for target $3-74$ at $2,790 \mathrm{~Hz}$

Figure 9 shows an upward shift, but it also displays a double peak. Simply picking the largest peak will not work for this case. It is obvious from the multiple peak structure here that the background is not homogeneous over the region that was extracted. For the purpose of this analysis, background was chosen as the center of mass of this distribution. When analyzing targets with this sort of background, the analyst can consider the error introduced by the background to determine if the measurement has sufficient accuracy to make a classification.

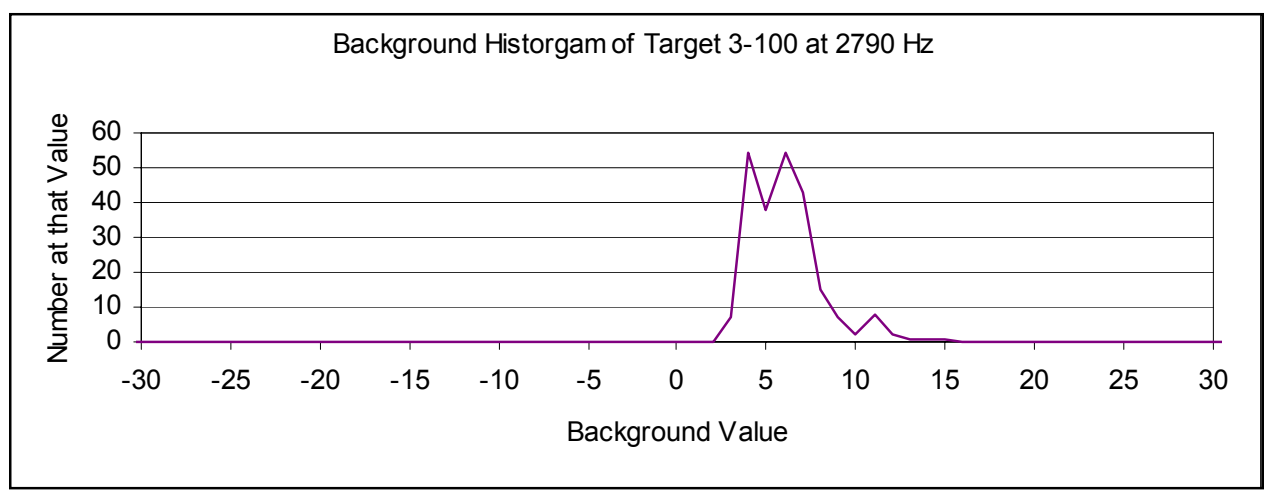

Figure 9. Histogram representation of background for target $3-100$ at $2,790 \mathrm{~Hz}$ 
Figures 10 through 16 show a typical set of in-phase measurements and are presented so the variation in the distribution as a function of frequency can be seen.

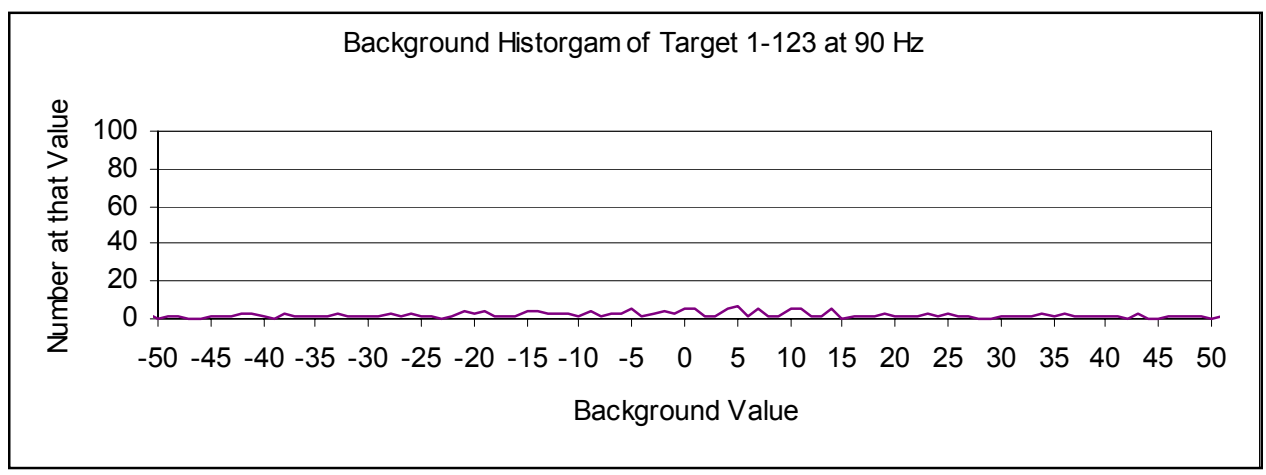

Figure 10. Histogram of background for target $1-123$ at frequency $90 \mathrm{~Hz}$

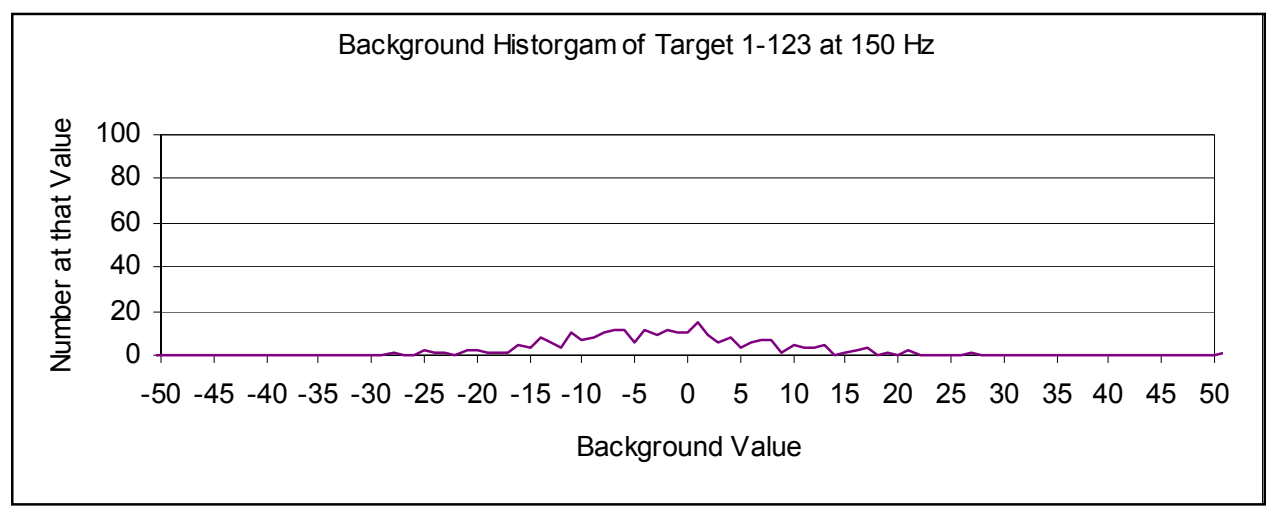

Figure 11. Histogram of background for target 1-123 at frequency $150 \mathrm{~Hz}$

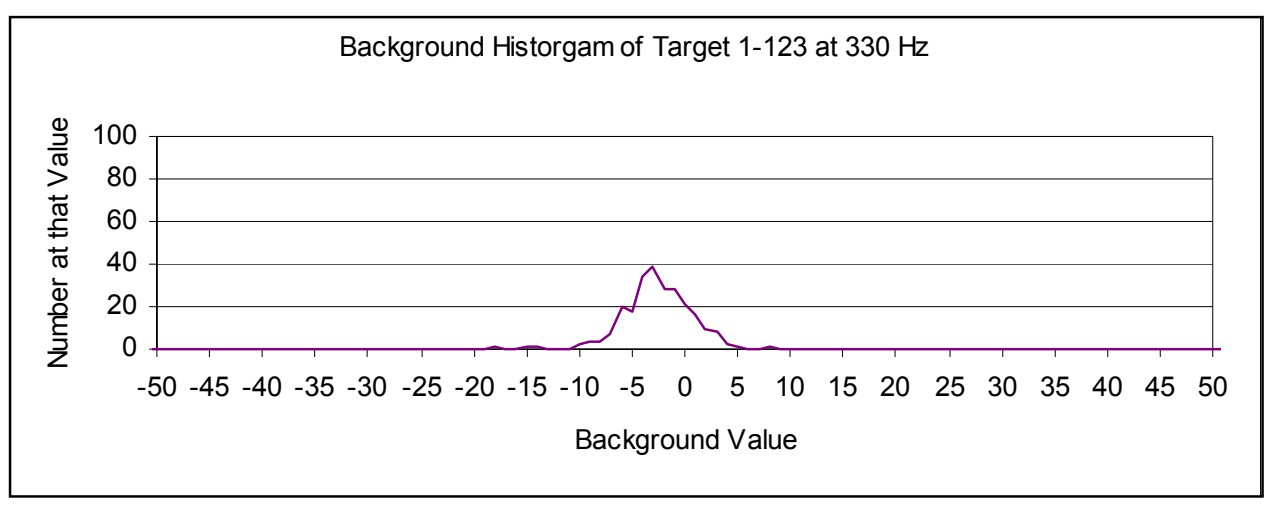

Figure 12. Histogram of background for target $1-123$ at frequency $330 \mathrm{~Hz}$ 


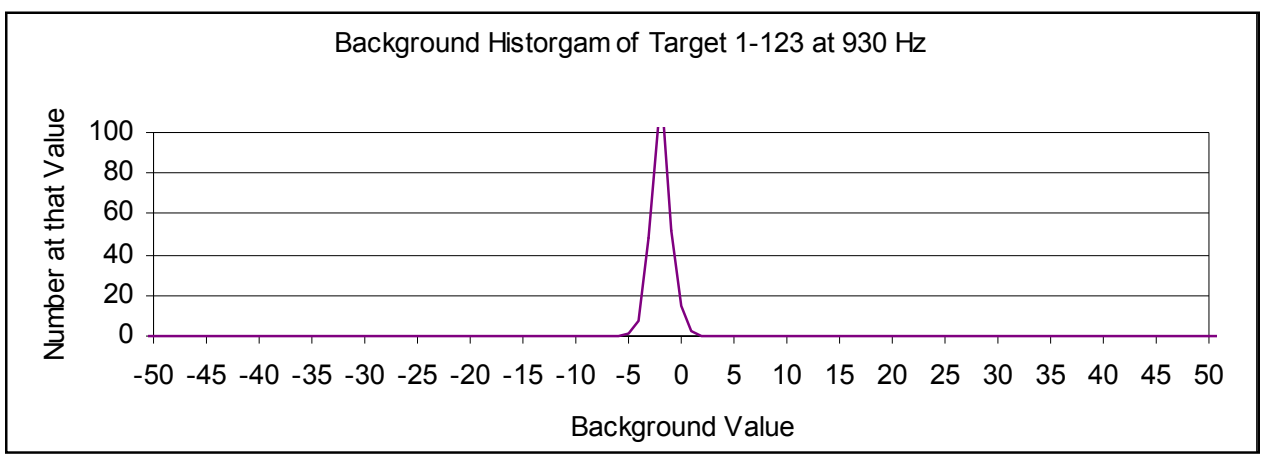

Figure 13. Histogram of background for target 1-123 at frequency $930 \mathrm{~Hz}$

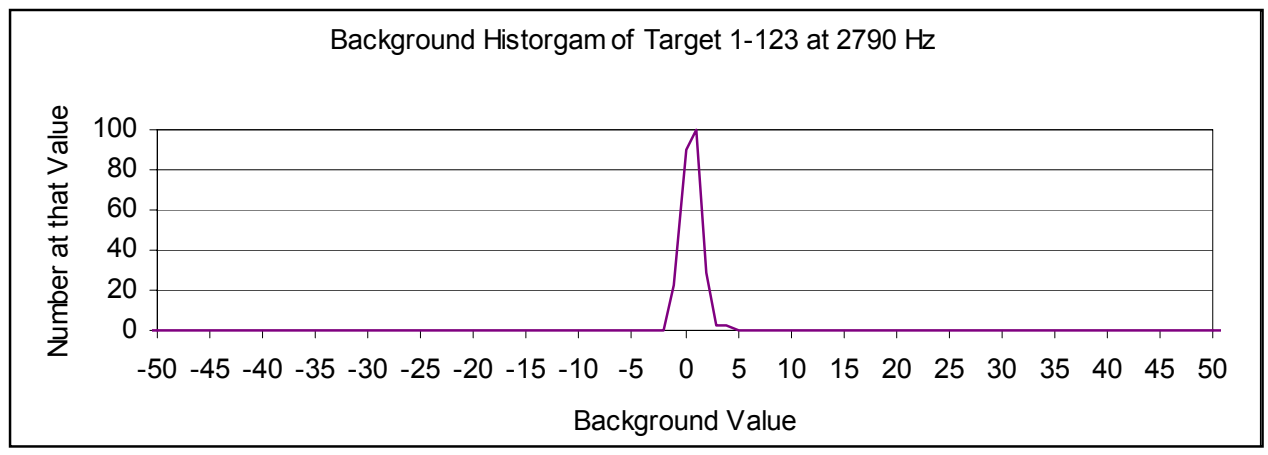

Figure 14. Histogram of background for target $1-123$ at frequency $2,790 \mathrm{~Hz}$

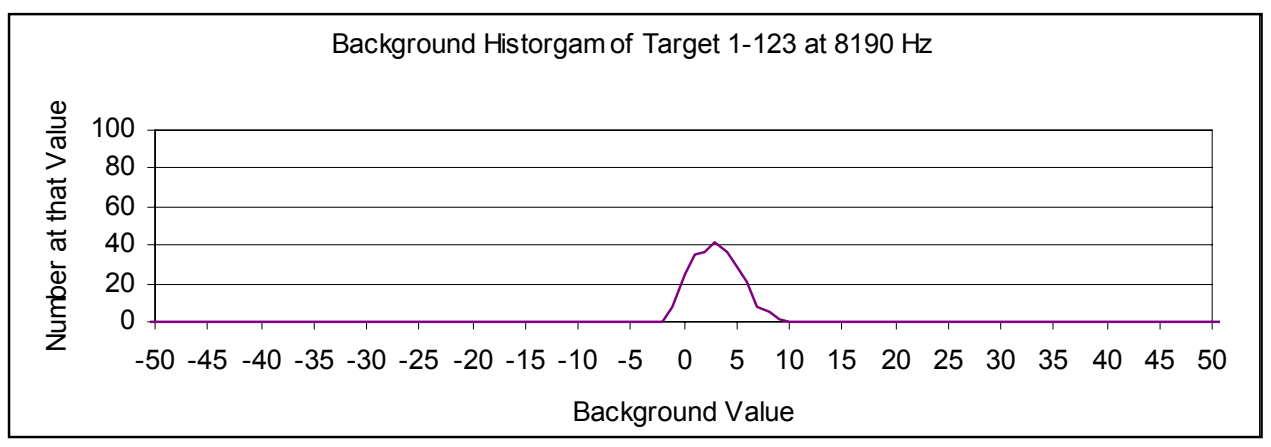

Figure 15. Histogram of background for target $1-123$ at frequency $8,190 \mathrm{~Hz}$

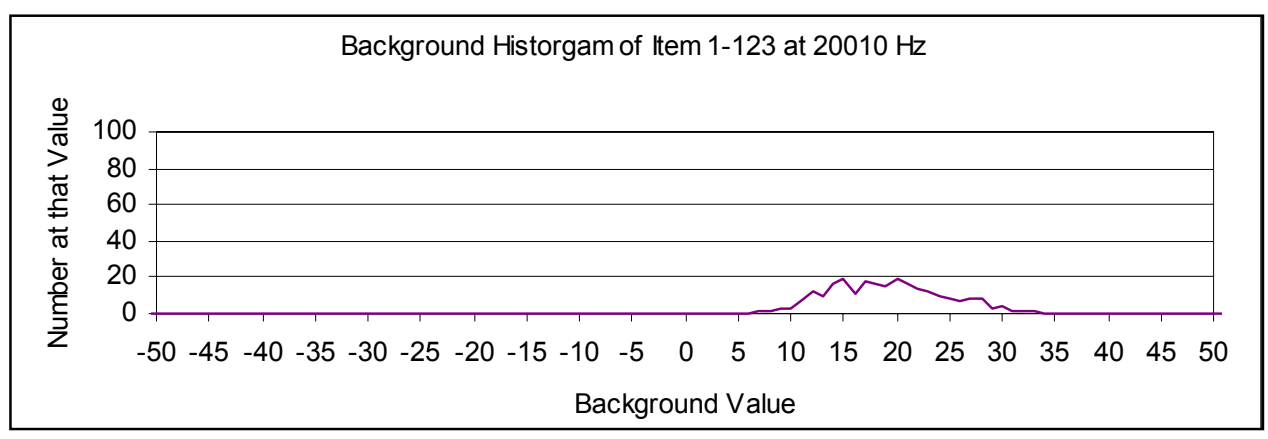

Figure 16. Histogram of background for target $1-123$ at frequency $20,010 \mathrm{~Hz}$ 
Typically, the lower frequencies produce a broader distribution in the measurements narrowing at frequencies 930 and 2,790 Hz while broadening back out for 8,190 and $20,010 \mathrm{~Hz}$. With most scientific instruments, it is common to discuss the accuracy of the instrument. In this case, that is not a straightforward thing; the uncertainty in the measurement is a function of the background homogeneity as well as frequency.

\section{Analysis of Data Points}

\section{Calibration data}

While at the site, calibration data were acquired by passing the GEM-3 over each type of ordnance placed in an open trench, as shown in Figure 17. Because of sensor problems at the site, the available calibration data were very limited. No calibration data were available for the 76- $\mathrm{mm}, 105-\mathrm{mm}$, and $127 \mathrm{~mm}$ (5-in.) projectiles. For the other ordnance types, data were collected with inclination angles of 0, 90 (nose up), and -90 (nose down) deg. Because the actual targets were buried with many different inclination angles and the inclination angle has a significant influence on the signature of the target, data were interpolated between these three measurements at 5-deg increments. Data were collected at multiple depths for some ordnance types but not for others. Because depth appears to affect only the magnitude of the data and not the shape of the curves, the data at the shallowest depth for each ordnance type were selected for analysis to simplify the process. The calibration data at 0,90 , and -90 deg are shown for each of the available ordnance types in Figures 18 through 25.

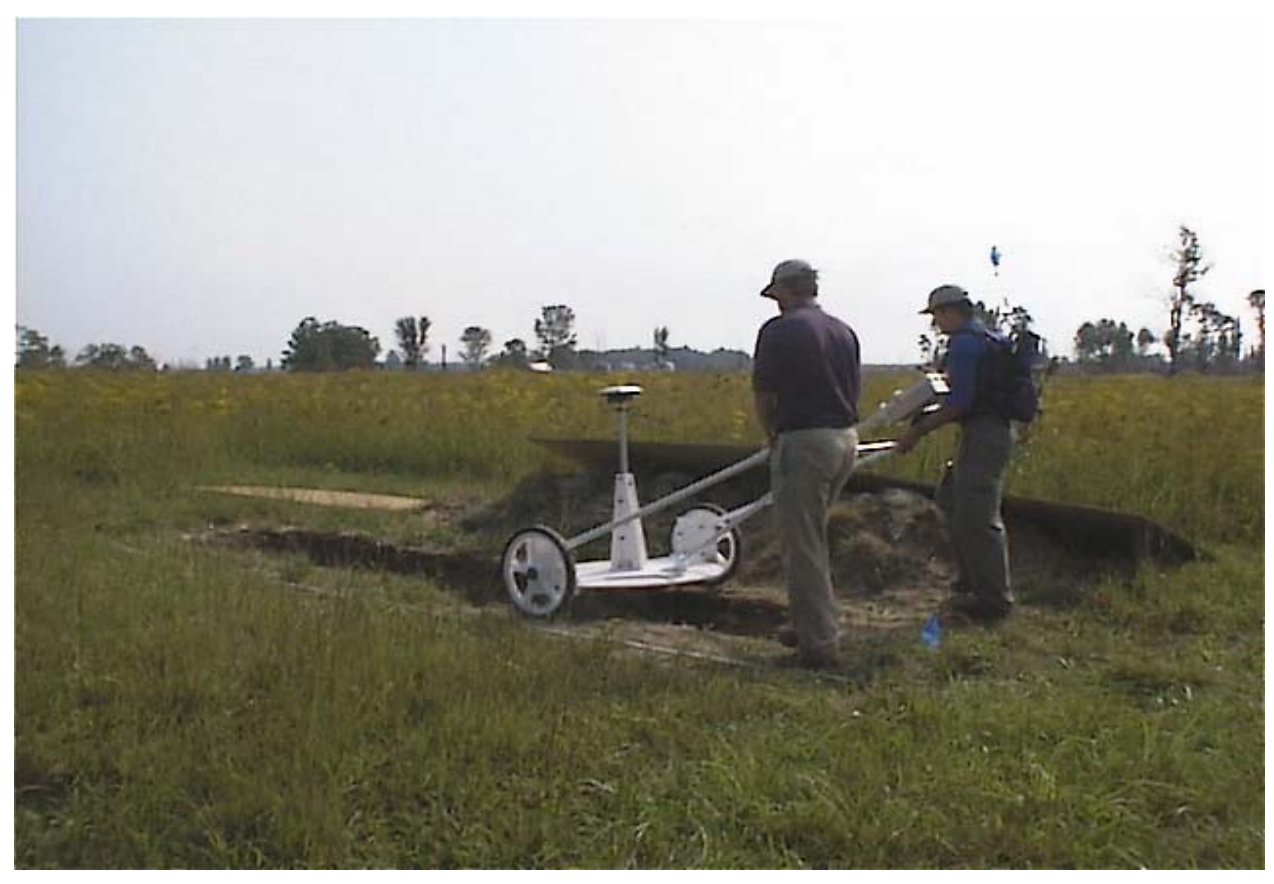

Figure 17. Acquisition of calibration data with the GEM-3 


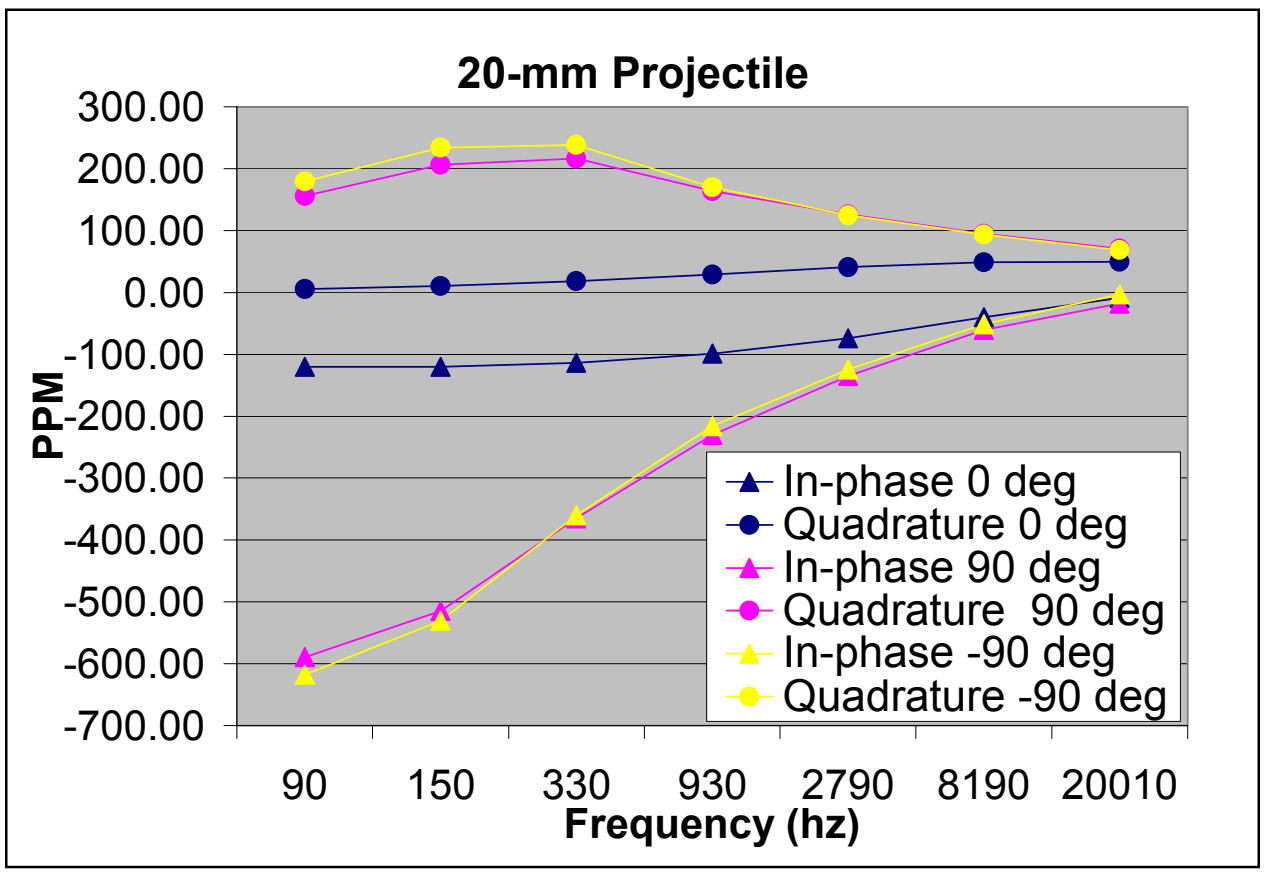

Figure 18. Calibration data for $20-\mathrm{mm}$ projectile

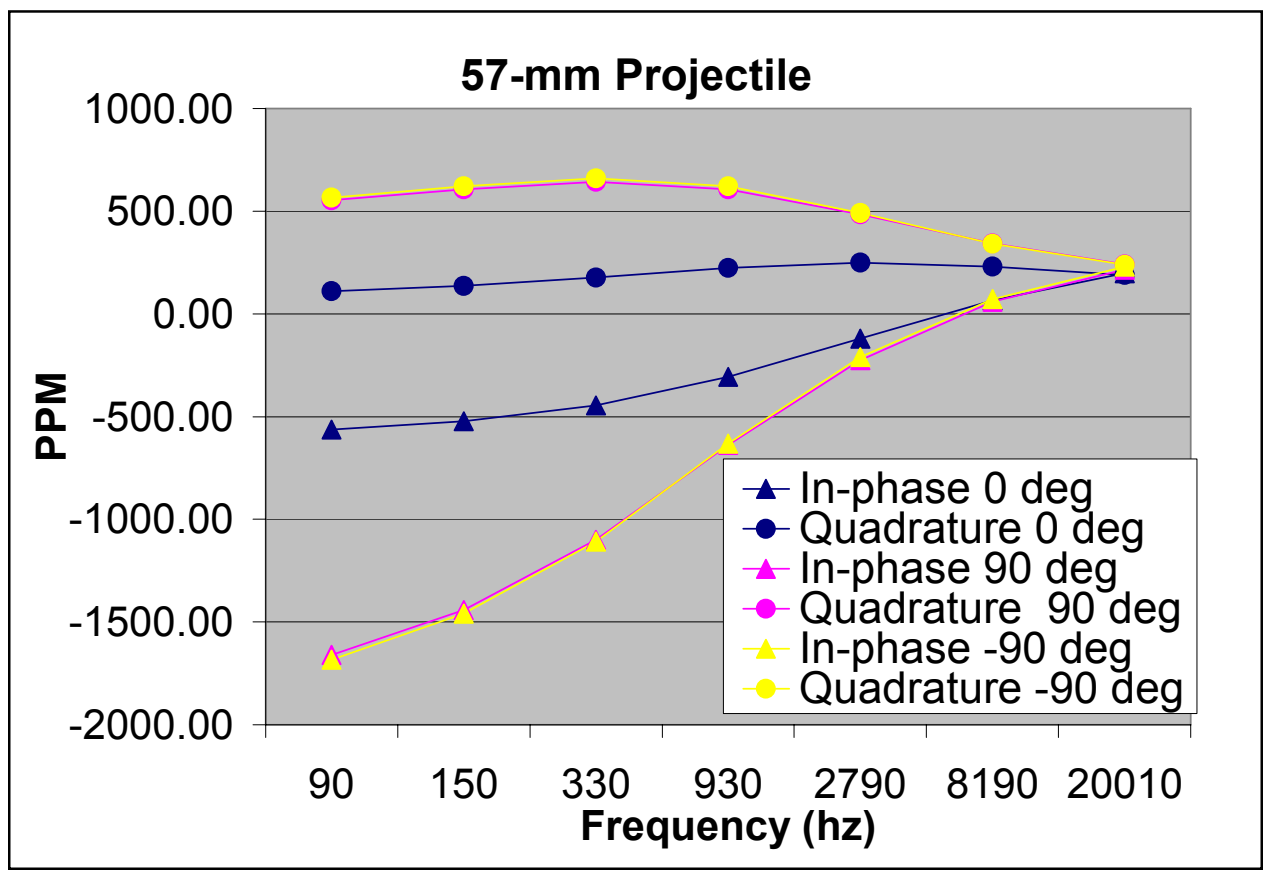

Figure 19. Calibration data for $57-\mathrm{mm}$ projectile 


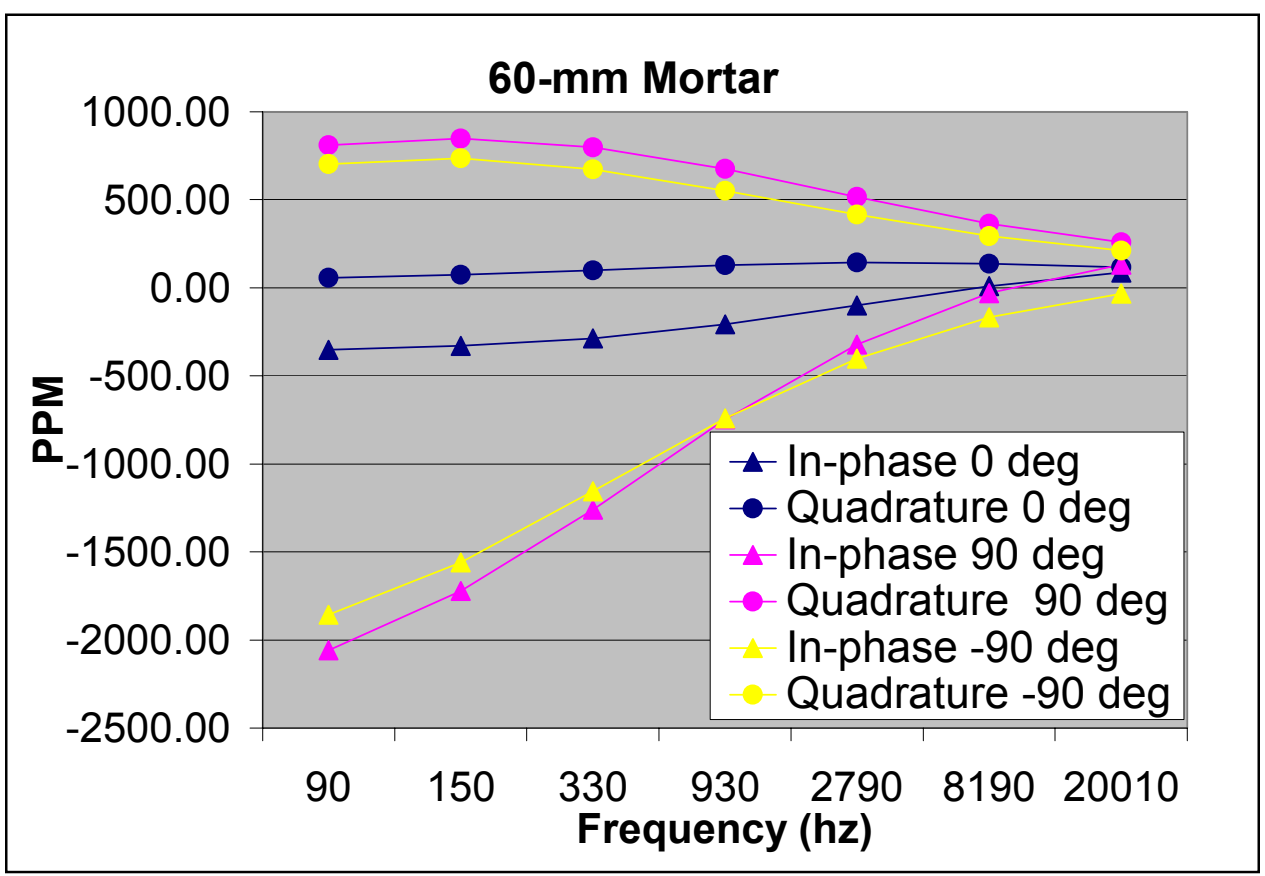

Figure 20. Calibration data for $60-\mathrm{mm}$ mortar

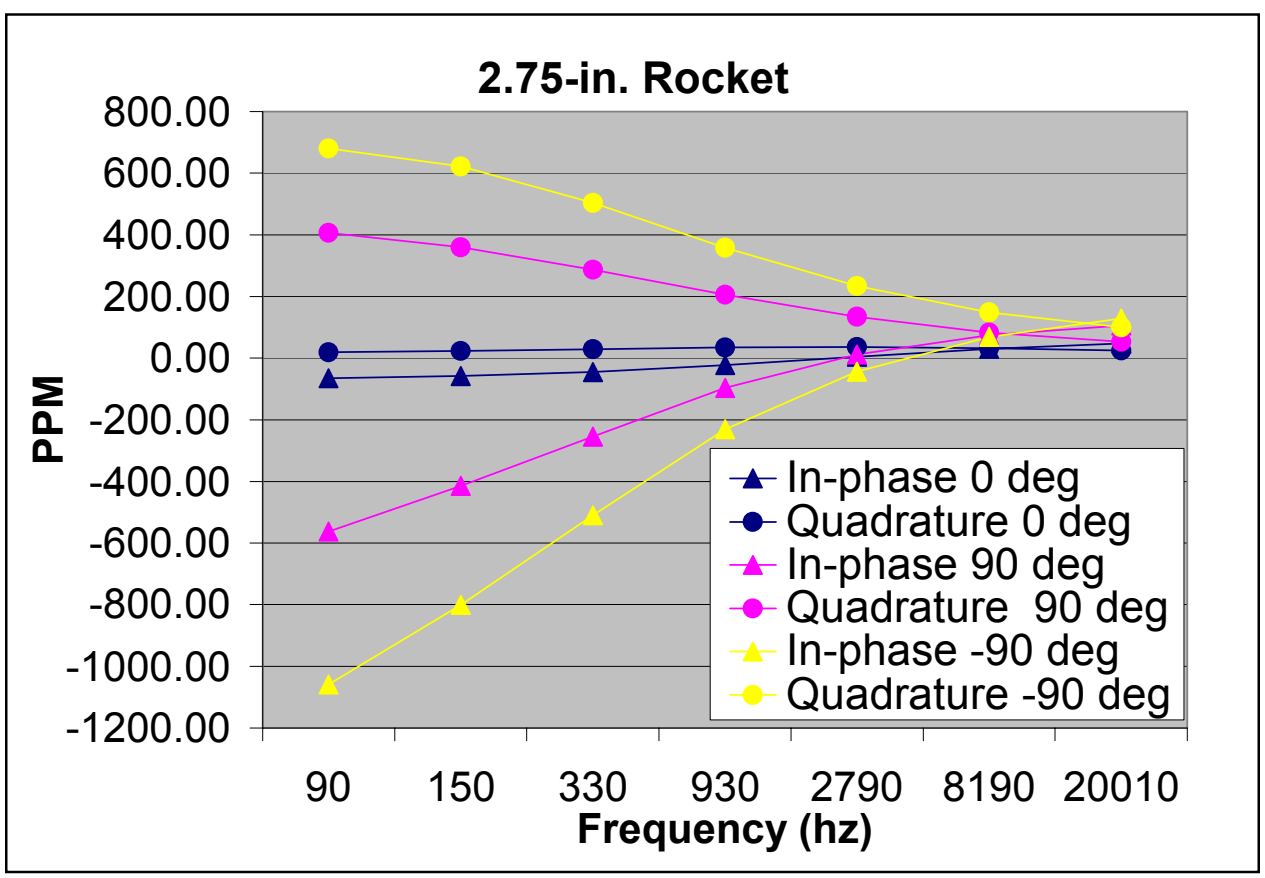

Figure 21. Calibration data for 2.75-in. rocket (To convert inches to millimeters, multiply by 25.4 ) 


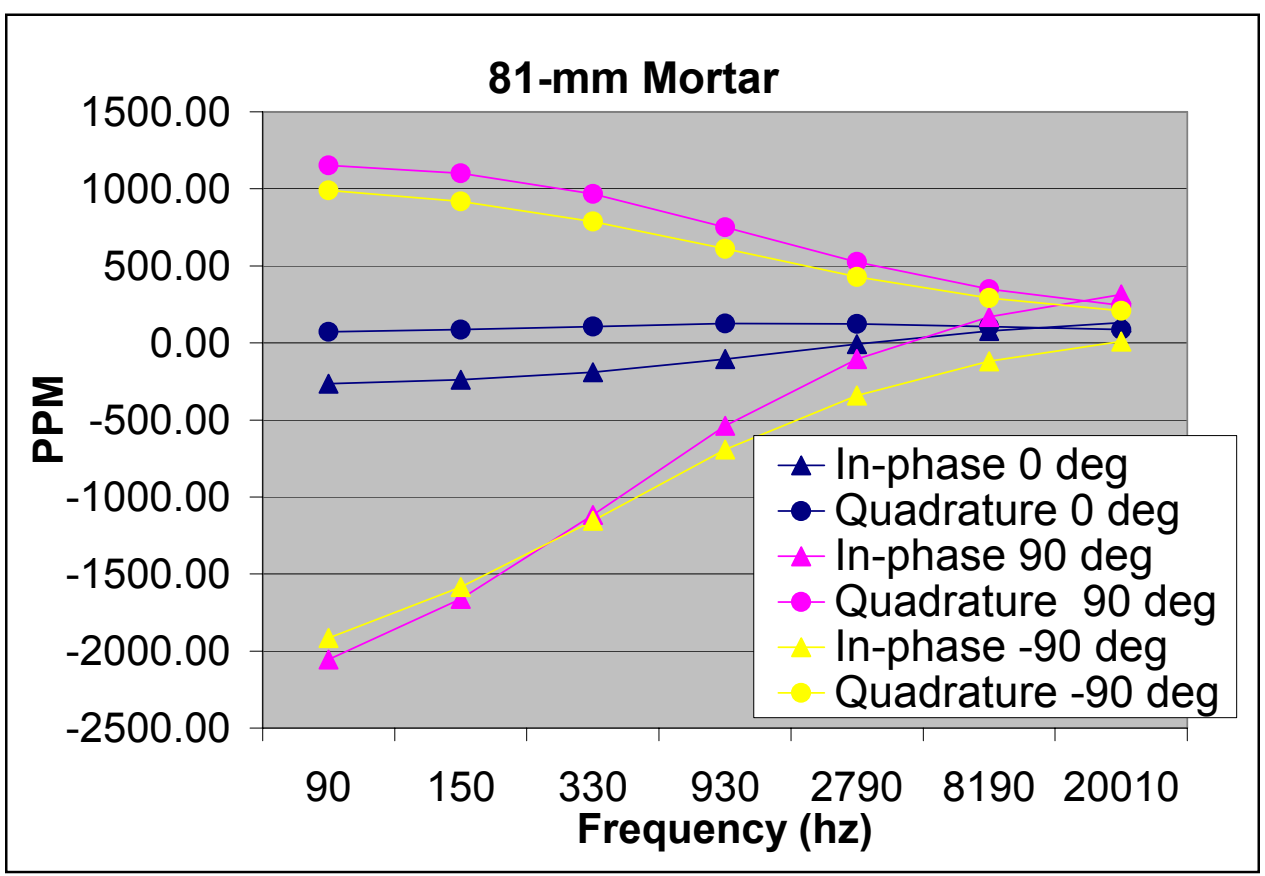

Figure 22. Calibration data for 81-mm mortar

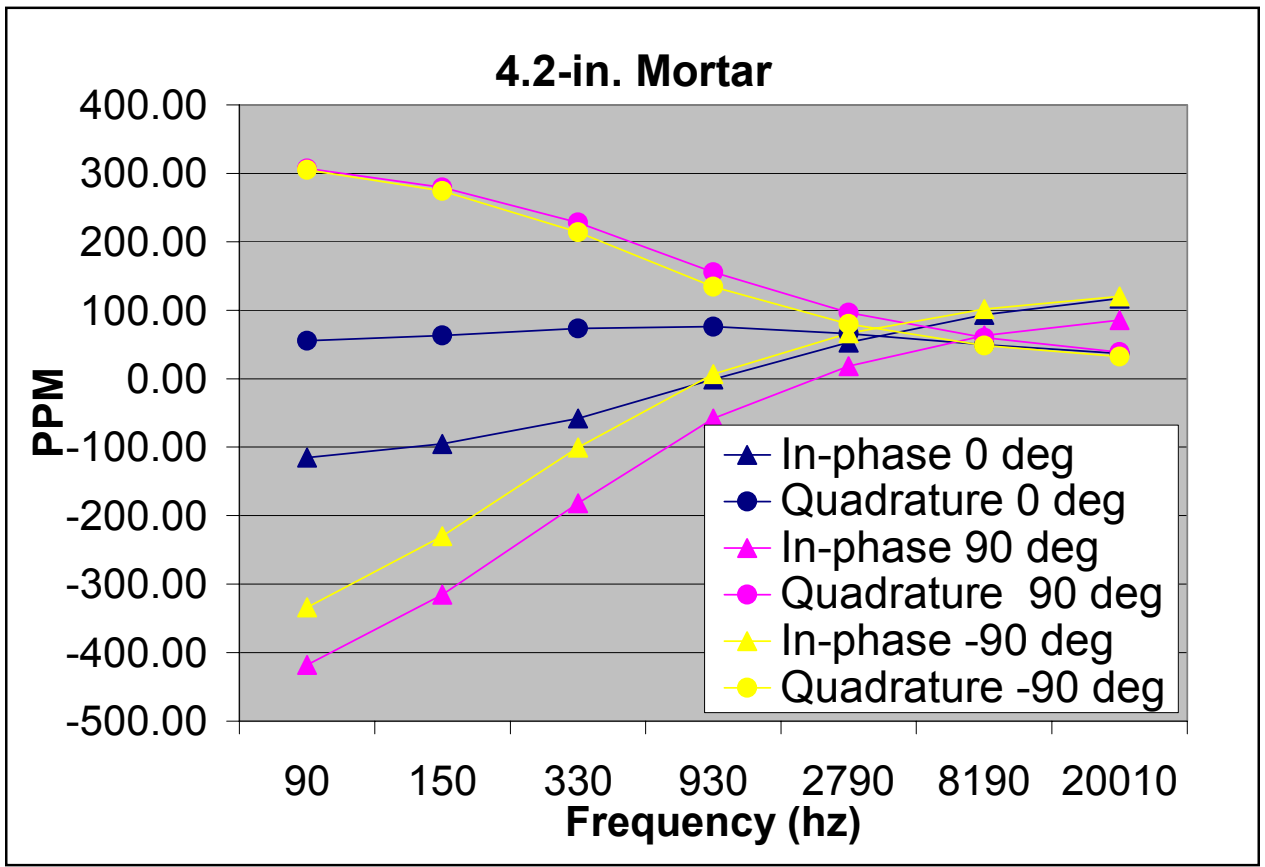

Figure 23. Calibration data for 4.2-in. mortar (To convert inches to millimeters, multiply by 25.4) 


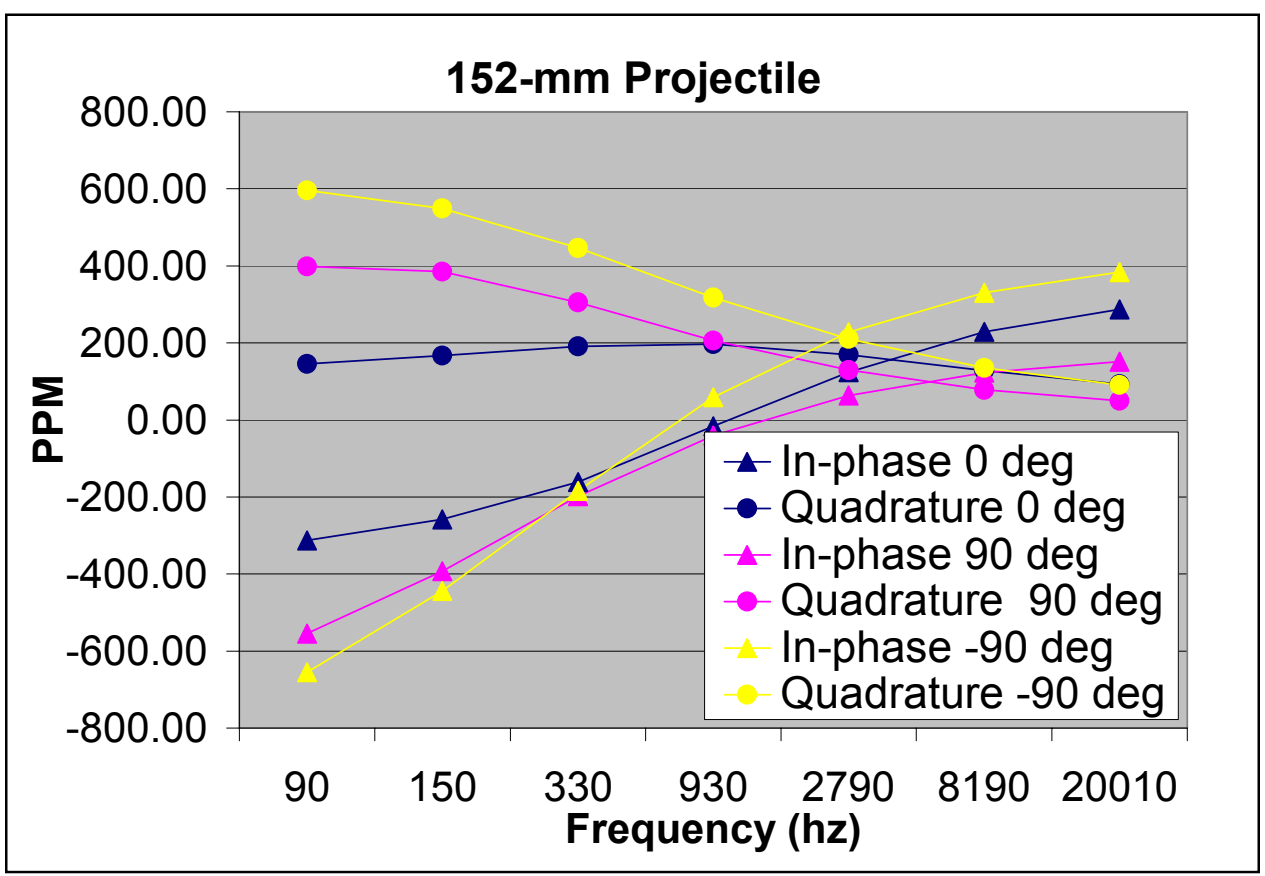

Figure 24. Calibration data for $152-\mathrm{mm}$ projectile

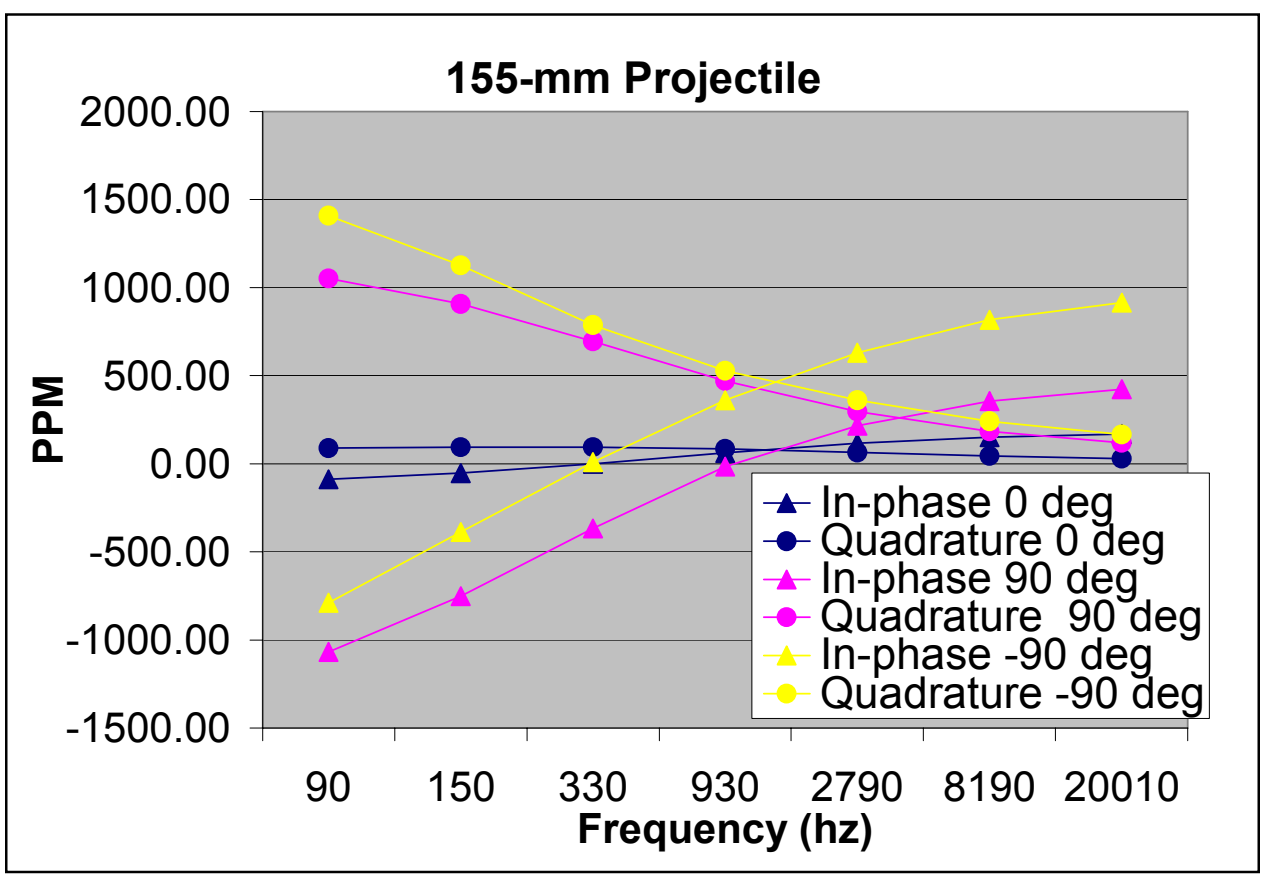

Figure 25. Calibration data for $155-\mathrm{mm}$ projectile 


\section{Error in fit}

A simple measure of the error in fit between a data point and a calibration signature was used. The error in fit was calculated separately for the in-phase and quadrature and then summed to get a single measure. When comparing a data point to a calibration signature, the frequency measurements for the data point were normalized to make the total in-phase and quadrature responses for the data point equal to the total in-phase and quadrature responses, respectively, of the calibration signature. Once the magnitudes were equalized, the error in fit, E, was calculated by

$$
E=\frac{\sqrt{\sum_{k=1}^{k=7}\left(C I_{k}-P I_{k}\right)^{2}}}{\sum_{k=1}^{k=7}\left|C I_{k}\right|}+\frac{\sqrt{\sum_{k=1}^{k=7}\left(C Q_{k}-P Q_{k}\right)^{2}}}{\sum_{k=1}^{k=7}\left|C Q_{k}\right|}
$$

where $C I_{k}=$ calibration in-phase response

$$
\begin{gathered}
P I_{k}=\text { data point in-phase response at frequency } \mathrm{k} \\
C Q_{k} \text { and } P Q_{k}=\text { calibration and data point quadrature responses, } \\
\text { respectively, at frequency } \mathrm{k}
\end{gathered}
$$

\section{Analysis program}

A graphical user interface computer program was developed to automate the process of comparing data points with calibration signatures under various conditions. A view of the program's user interface is shown in Figure 26. The two text boxes at the top of the screen are used to select objects of interest. The "Actual Targets" box lists the Target IDs and ground truth information for all of the targets that were emplaced in one of the three areas at JPG. The area currently visible is selected by radio buttons at the bottom of the screen. The "Detected Objects" box lists the IDs for all objects declared as targets by Geophex in the currently selected area. If the object corresponds to an actual target, the Target ID is listed. If not, the Geophex ID for the object is shown. Selecting an ID in either of these text boxes will cause the other text boxes and graphics windows to be updated reflecting the selection. The center textbox, labeled "Best Matches to Calibration Data," consists of one line for each data point near the selected object that lists the ID, the total in phase and quadrature magnitude, the distance from the target if the object is a known target, and information about the three closest matches of the point with calibration data. The information for each match consists of the ordnance type, depth, and inclination and the error between the data point and the calibration signature. The left graphics window shows the in-phase and quadrature data for the selected data point and for the calibration data. The calibration data displayed is for either the best matching ordnance or the actual ordnance corresponding to the current Target ID, depending on the status of the "Show Best Match" check box. The right graphics box shows the 


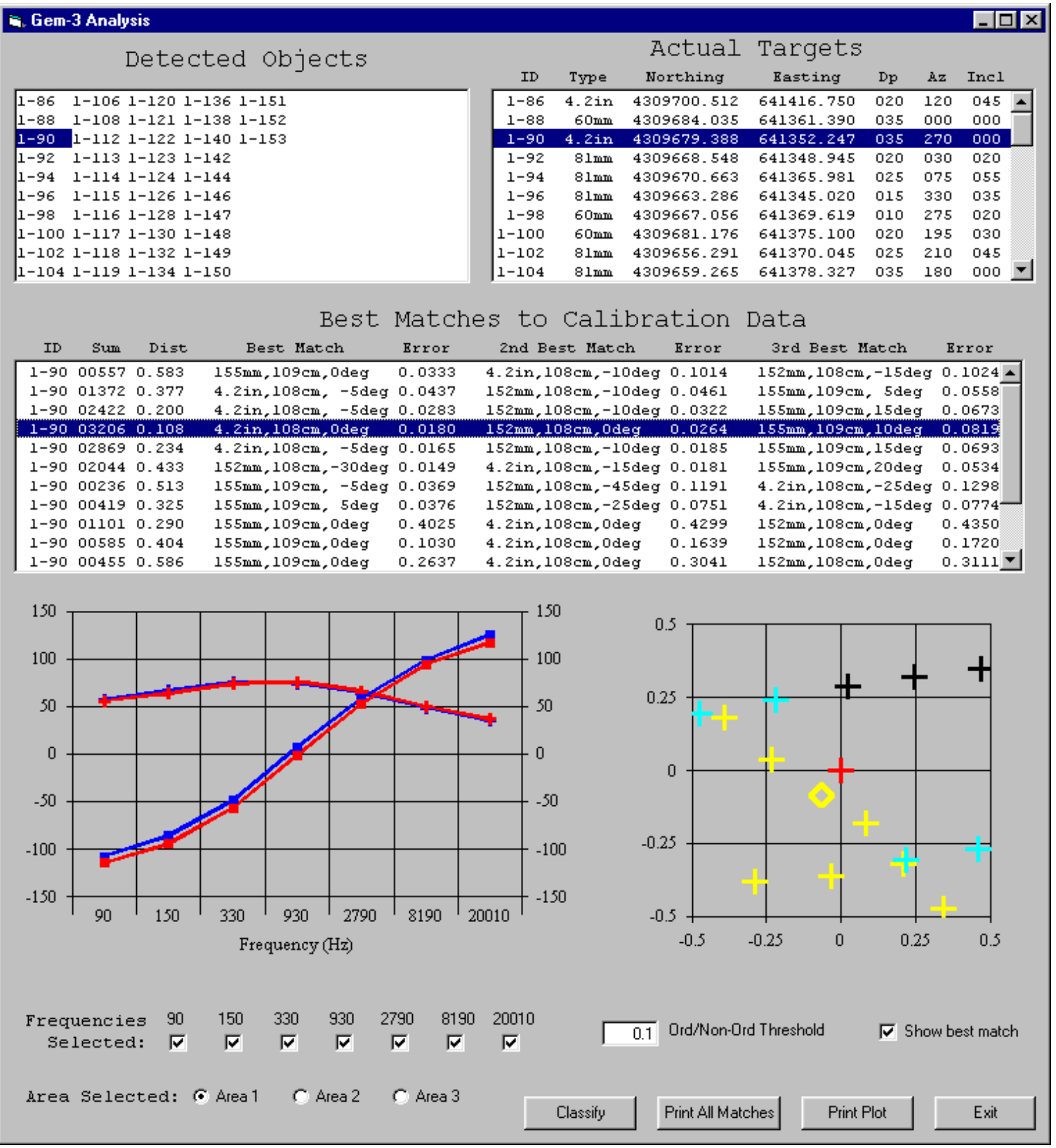

Figure 26. GEM-3 Analysis Program screen dump

positions of the data points around the actual target location shown at the origin in red. Points with match errors greater than the current ordnance/nonordnance threshold are shown in black. Points with errors less than the threshold, but greater than two-thirds of the threshold, are shown in dark blue. Points with errors between one-third and two-thirds of the threshold are shown in cyan. Points with errors below one-third of the threshold are displayed in yellow. The currently displayed data point is shown as a diamond rather than a plus. A row of check boxes at the bottom of the screen allows individual frequencies to be excluded from calculations. An operator can also select individual frequencies to be excluded from calculations through the graphical user interface. 


\section{Results}

\section{Area Coverage}

Complete area coverage is essential for determining the performance of a UXO detection system. A rendering of the positions where measurements were made in Areas 1, 2, and 3 can be seen in Figures 27, 28, and 29, respectively. Coverage maps showing the distribution of quadrature and in-phase values for each frequency are included as Appendix A. From the graphics it can be seen that there were some failures in coverage, but fortunately these glitches did not occur over any of the target locations.

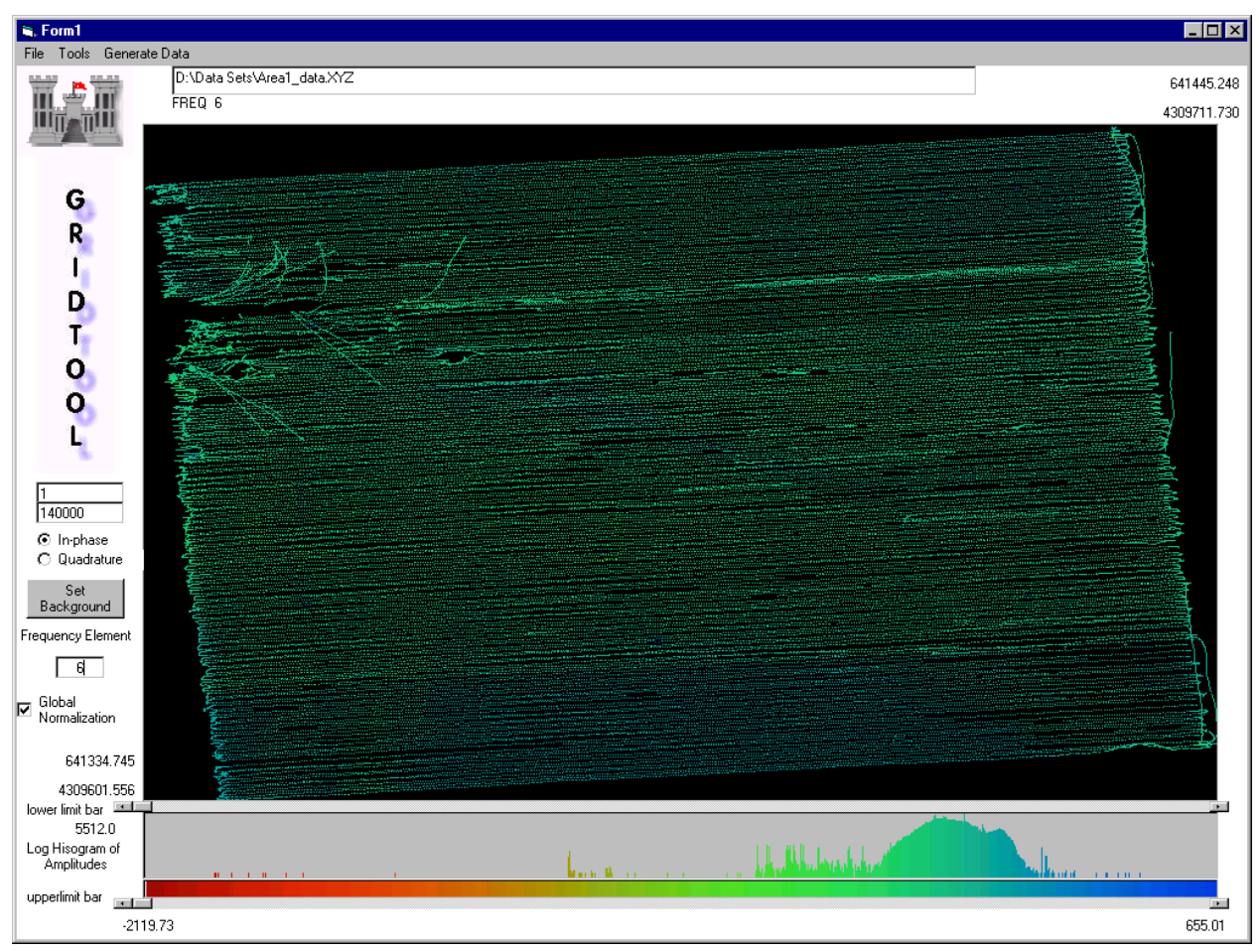

Figure 27. Area 1 measurement positions 


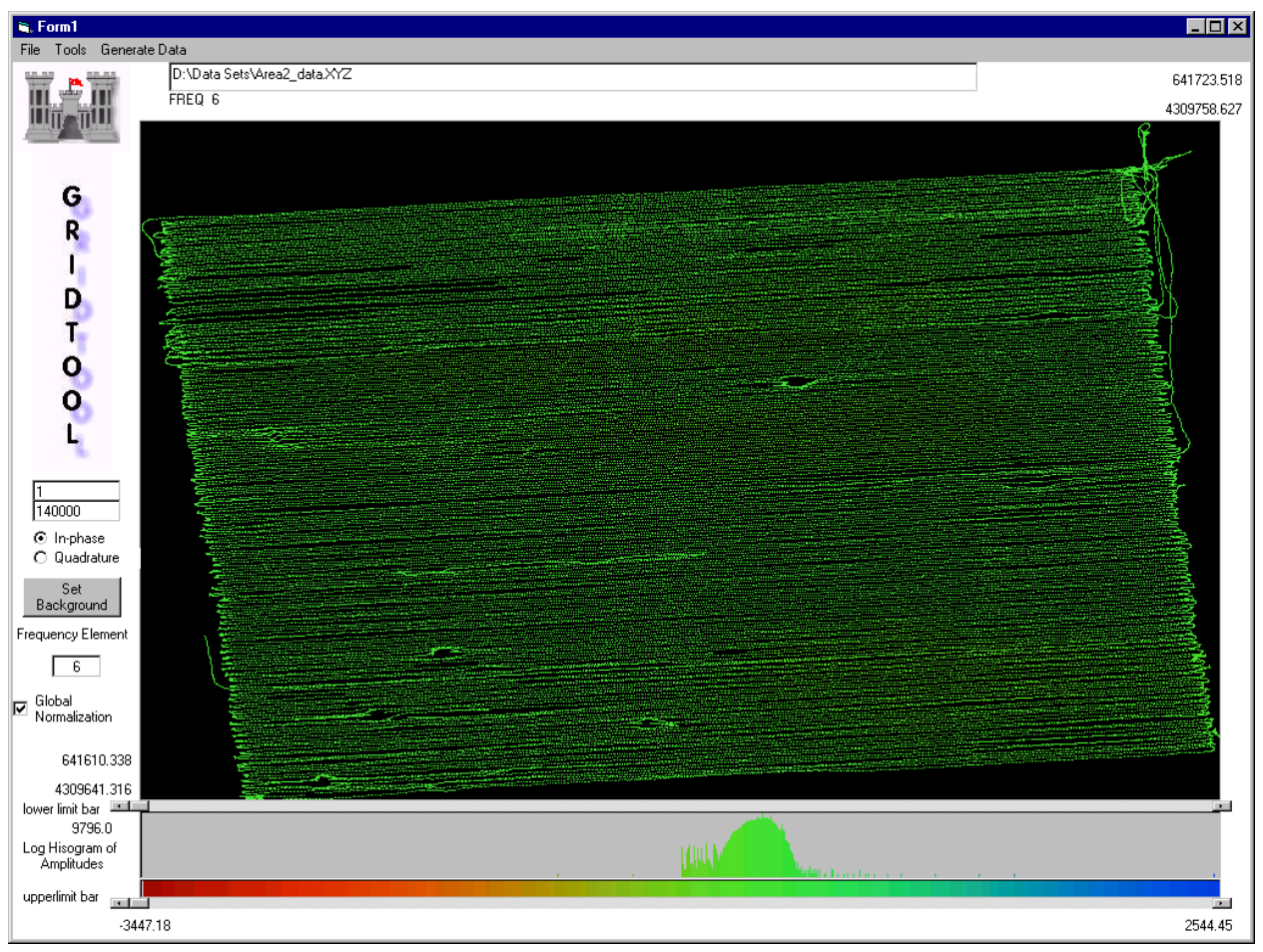

Figure 28. Area 2 measurement positions

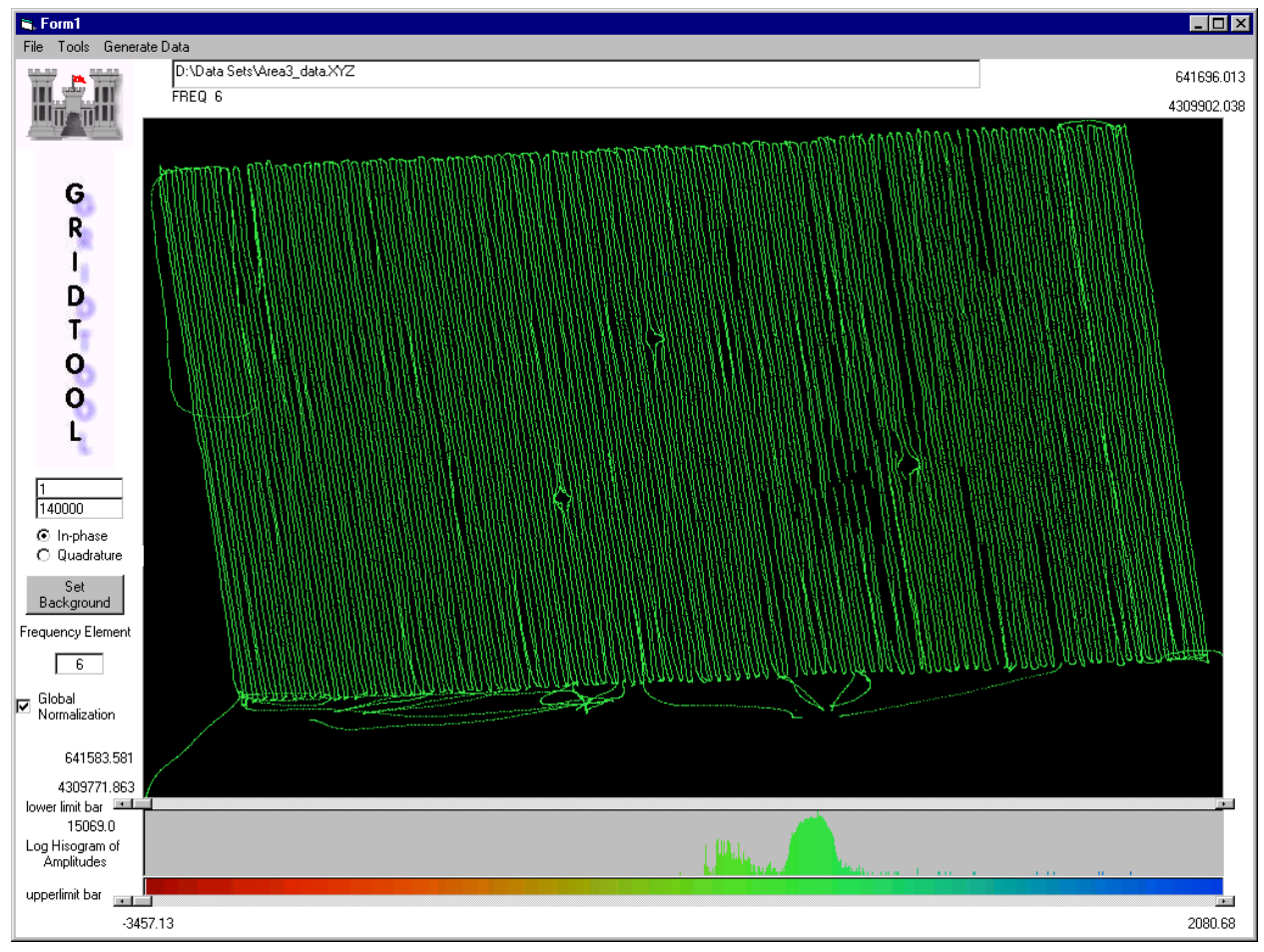

Figure 29. Area 3 measurement positions 
Table 6 shows the number of data points within a 1-m box centered on each target and the distance from the target of the closest point. No target is identified for which measurements were not taken within $1 / 2 \mathrm{~m}$.

\begin{tabular}{|c|c|c|c|c|c|c|c|c|}
\hline \multicolumn{9}{|c|}{\begin{tabular}{|l} 
Table 6 \\
Proximity of GEM-3 Data Points to Targets at JPG
\end{tabular}} \\
\hline Target ID & $\begin{array}{l}\text { Number } \\
\text { of Points }\end{array}$ & $\begin{array}{l}\text { Closest } \\
\text { Point } m\end{array}$ & Target ID & $\begin{array}{l}\begin{array}{l}\text { Number } \\
\text { of Points }\end{array} \\
\end{array}$ & $\begin{array}{l}\text { Closest } \\
\text { Point m }\end{array}$ & Target ID & $\begin{array}{l}\begin{array}{l}\text { Number } \\
\text { of Points }\end{array} \\
\end{array}$ & $\begin{array}{l}\text { Closest } \\
\text { Point m }\end{array}$ \\
\hline $1-86$ & 8 & 0.1845 & $1-138$ & 9 & 0.2583 & $2-148$ & 8 & 0.2552 \\
\hline $1-88$ & 12 & 0.1163 & $1-140$ & 9 & 0.2118 & $2-150$ & 11 & 0.078 \\
\hline $1-90$ & 15 & 0.1084 & $1-142$ & 8 & 0.1012 & $2-152$ & 10 & 0.1217 \\
\hline $1-92$ & 11 & 0.2047 & 1-144 & 8 & 0.0221 & 2-154 & 11 & 0.1662 \\
\hline $1-94$ & 5 & 0.3789 & $1-146$ & 14 & 0.1985 & $2-156$ & 10 & 0.094 \\
\hline $1-96$ & 10 & 0.254 & $1-147$ & 6 & 0.1779 & $2-158$ & 9 & 0.1112 \\
\hline $1-98$ & 8 & 0.0524 & $1-148$ & 8 & 0.1818 & $2-160$ & 9 & 0.1 \\
\hline $1-100$ & 7 & 0.1802 & $1-149$ & 8 & 0.159 & $2-161$ & 7 & 0.1206 \\
\hline $1-102$ & 7 & 0.037 & $1-150$ & 40 & 0.0716 & $2-162$ & 10 & 0.3109 \\
\hline $1-104$ & 7 & 0.0226 & $1-151$ & 9 & 0.1703 & $2-164$ & 4 & 0.0481 \\
\hline $1-106$ & 8 & 0.2547 & $1-152$ & 9 & 0.2302 & $2-166$ & 4 & 0.1448 \\
\hline $1-108$ & 8 & 0.1743 & $1-153$ & 9 & 0.0804 & $3-68$ & 10 & 0.099 \\
\hline $1-112$ & 9 & 0.2053 & $2-112$ & 3 & 0.3122 & 3-70 & 9 & 0.312 \\
\hline $1-113$ & 8 & 0.1205 & 2-114 & 8 & 0.3113 & 3-72 & 10 & 0.1537 \\
\hline $1-114$ & 11 & 0.1056 & $2-116$ & 8 & 0.2823 & 3-74 & 10 & 0.1845 \\
\hline \begin{tabular}{|l|}
$1-115$ \\
\end{tabular} & 8 & 0.2459 & $2-118$ & 9 & 0.1788 & 3-76 & 4 & 0.2053 \\
\hline $1-116$ & 6 & 0.3548 & $2-120$ & 8 & 0.2594 & 3-78 & 9 & 0.2821 \\
\hline $1-117$ & 18 & 0.1302 & $2-122$ & 10 & 0.1062 & $3-80$ & 13 & 0.1604 \\
\hline $1-118$ & 10 & 0.0977 & $2-124$ & 8 & 0.063 & $3-82$ & 7 & 0.2288 \\
\hline $1-119$ & 12 & 0.0546 & $2-126$ & 7 & 0.2474 & $3-84$ & 9 & 0.126 \\
\hline $1-120$ & 7 & 0.2299 & $2-128$ & 8 & 0.2001 & $3-86$ & 8 & 0.3152 \\
\hline $1-121$ & 9 & 0.0663 & $2-130$ & 10 & 0.3674 & $3-88$ & 5 & 0.0757 \\
\hline $1-122$ & 5 & 0.0944 & $2-131$ & 7 & 0.2354 & $3-90$ & 9 & 0.1502 \\
\hline $1-123$ & 10 & 0.1379 & $2-132$ & 5 & 0.1081 & 3-92 & 6 & 0.0715 \\
\hline $1-124$ & 31 & 0.0136 & $2-134$ & 8 & 0.0648 & 3-94 & 8 & 0.2611 \\
\hline $1-126$ & 10 & 0.1342 & $2-136$ & 8 & 0.1592 & $3-96$ & 8 & 0.2901 \\
\hline $1-128$ & 11 & 0.0224 & $2-138$ & 7 & 0.4204 & $3-98$ & 11 & 0.0272 \\
\hline $1-130$ & 8 & 0.0774 & $2-140$ & 8 & 0.1918 & $3-100$ & 10 & 0.058 \\
\hline $1-132$ & 9 & 0.305 & $2-142$ & 11 & 0.1674 & $3-102$ & 8 & 0.1774 \\
\hline $1-134$ & 10 & 0.0854 & $2-144$ & 7 & 0.3494 & 3-104 & 12 & 0.124 \\
\hline $1-136$ & 5 & 0.144 & $2-146$ & 18 & 0.1793 & $3-106$ & 11 & 0.1329 \\
\hline
\end{tabular}

\section{Data Variability}

To determine the precision of the instrument, an experiment was set up to collect a statistically significant number of measurements for a fixed source. The Gem-3 was placed in a wooden rack $10 \mathrm{~cm}$ above the ground, and first data were collected in remote mode using a $233 \mathrm{MHz}$ Pentium II computer. The Geophex data display program was used to collect the data, but anomalies in the data were observed.

At irregular intervals, obviously corrupted data were plotted to the screen. A initial examination of the collected data showed that there were shifts in the data stream. Geophex advised that a $500 \mathrm{MHz}$ class machine was required to make use of this software, so more data were collected in "survey mode" and the following is a discussion of these data. 
Figures 30 through 50 show approximately $45 \mathrm{~min}$ of data that were collected to make histograms for three separate cases, background, a $20-\mathrm{mm}$ projectile, and a 57-mm mortar. Table 7 shows the statistical results of this analysis.

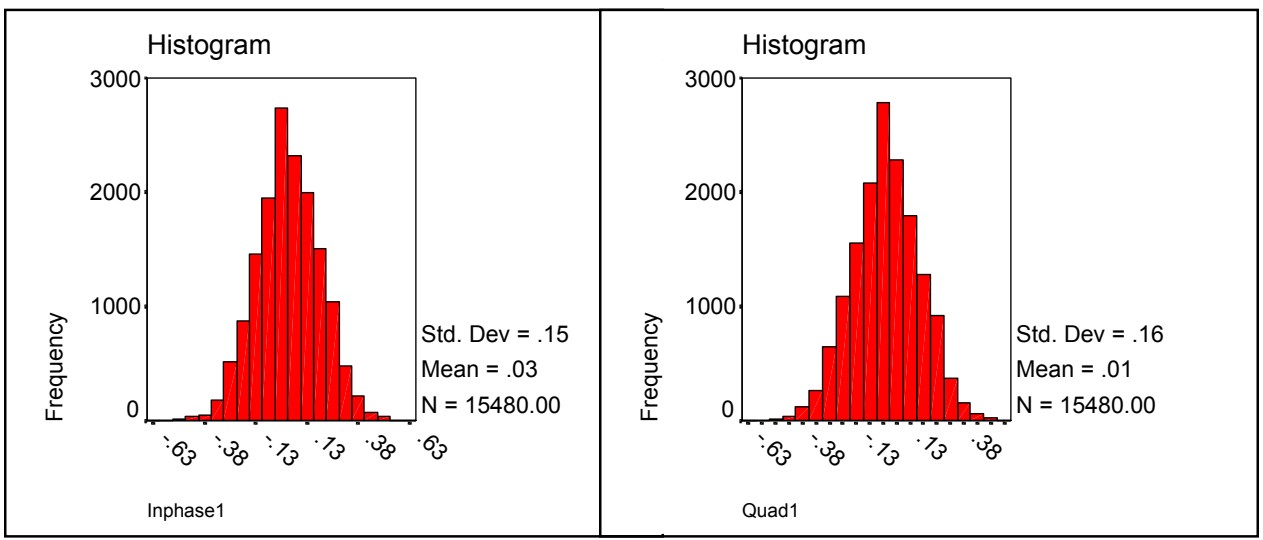

Figure 30. In-phase and quadrature histograms for background at $90 \mathrm{~Hz}$

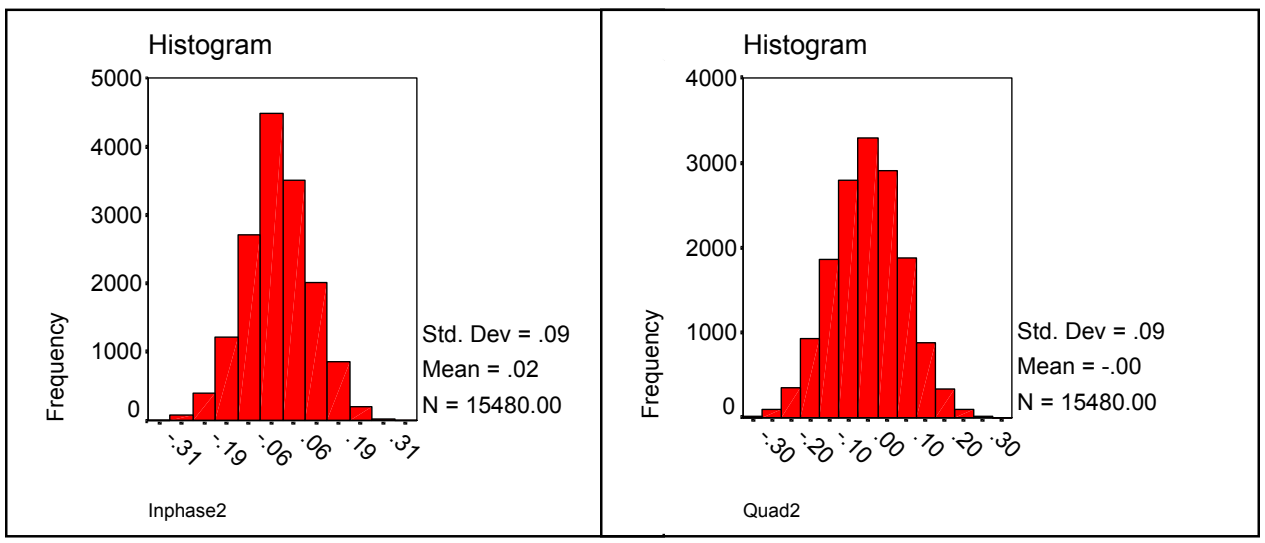

Figure 31. In-phase and quadrature histograms for background at $150 \mathrm{~Hz}$

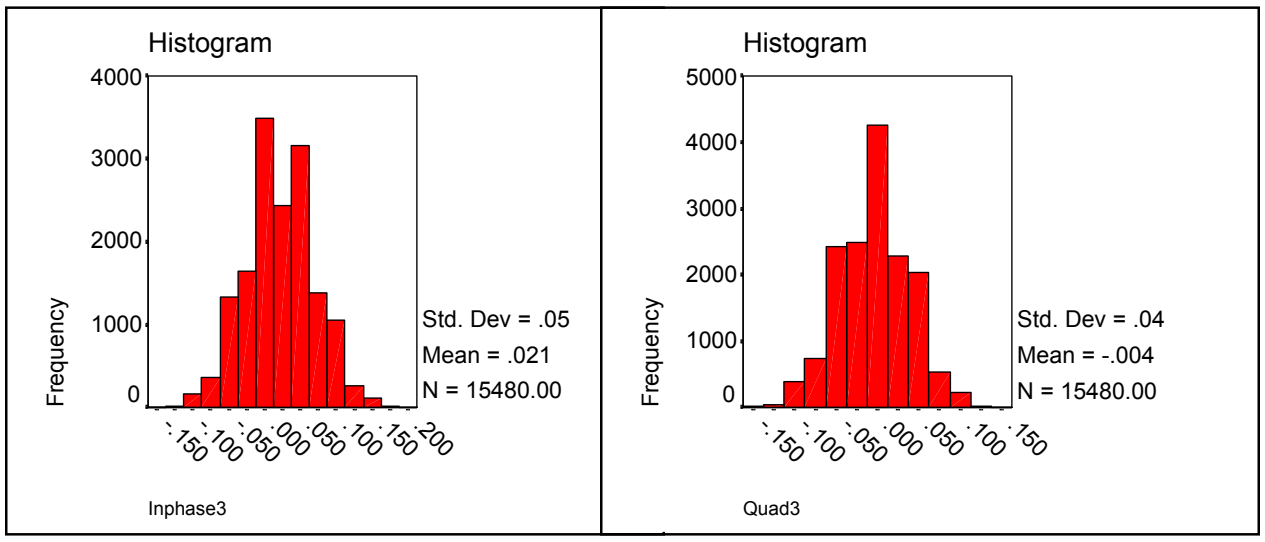

Figure 32. In-phase and quadrature histograms for background at $330 \mathrm{~Hz}$ 


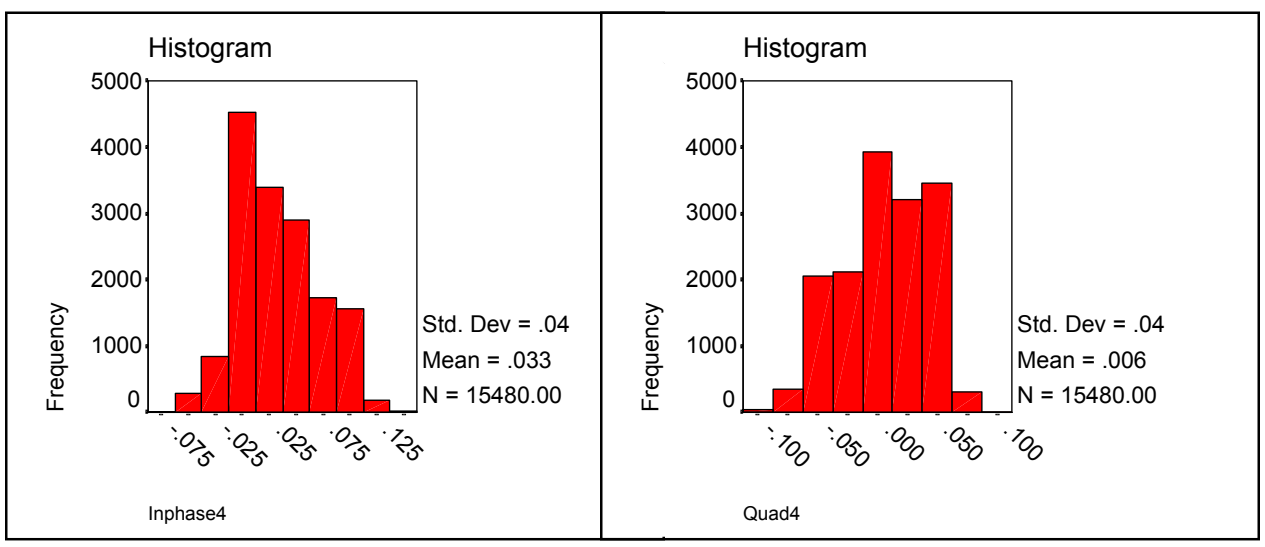

Figure 33. In-phase and quadrature histograms for background at $930 \mathrm{~Hz}$

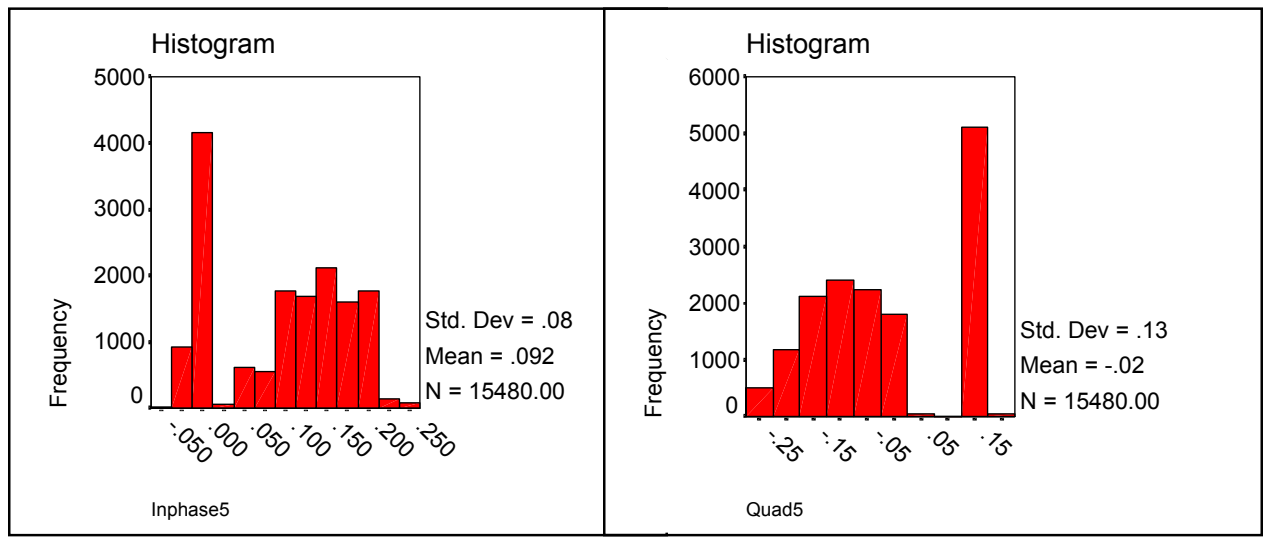

Figure 34. In-phase and quadrature histograms for background at $2,790 \mathrm{~Hz}$

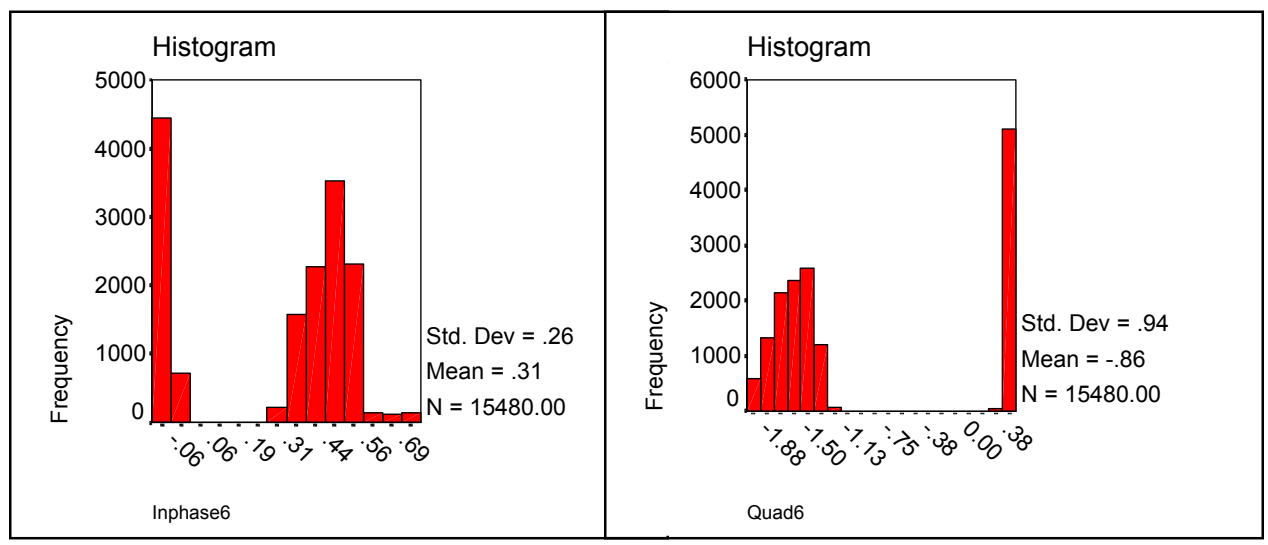

Figure 35. In-phase and quadrature histograms for background at $8,190 \mathrm{~Hz}$ 


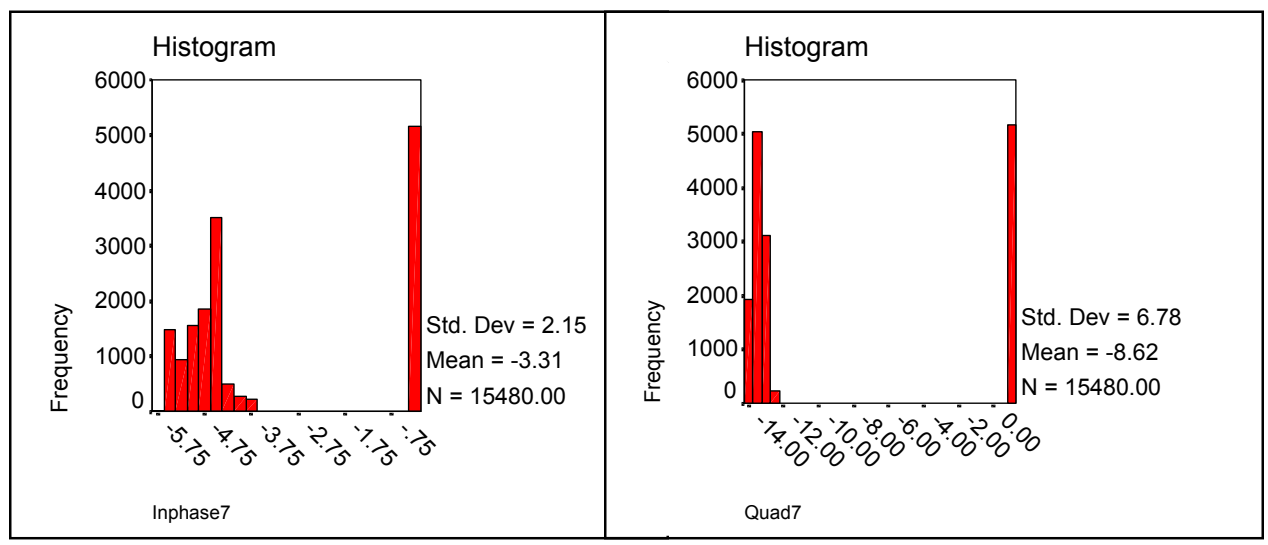

Figure 36. In-phase and quadrature histograms for background at $20,010 \mathrm{~Hz}$

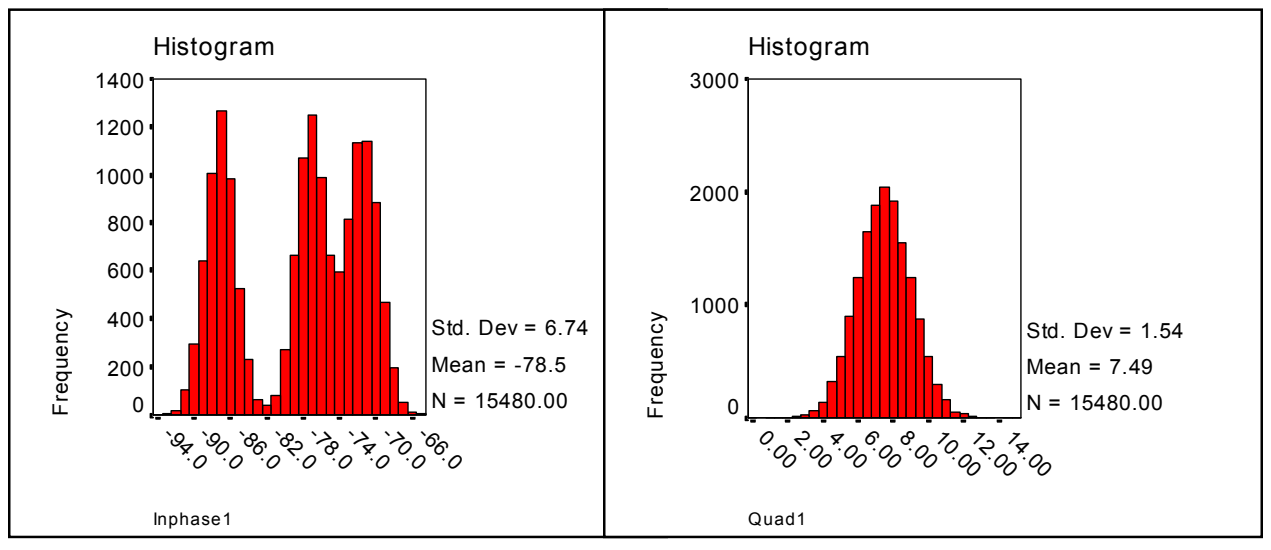

Figure 37 . In-phase and quadrature histograms for $20-\mathrm{mm}$ round at $90 \mathrm{~Hz}$

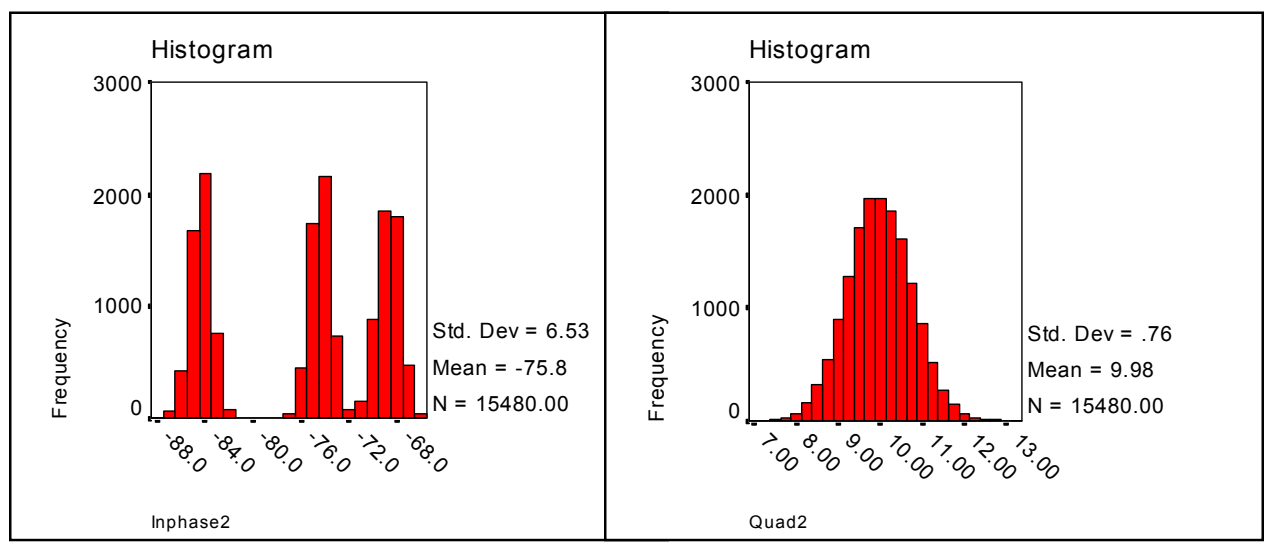

Figure 38. In-phase and quadrature histograms for $20-\mathrm{mm}$ round at $150 \mathrm{~Hz}$ 


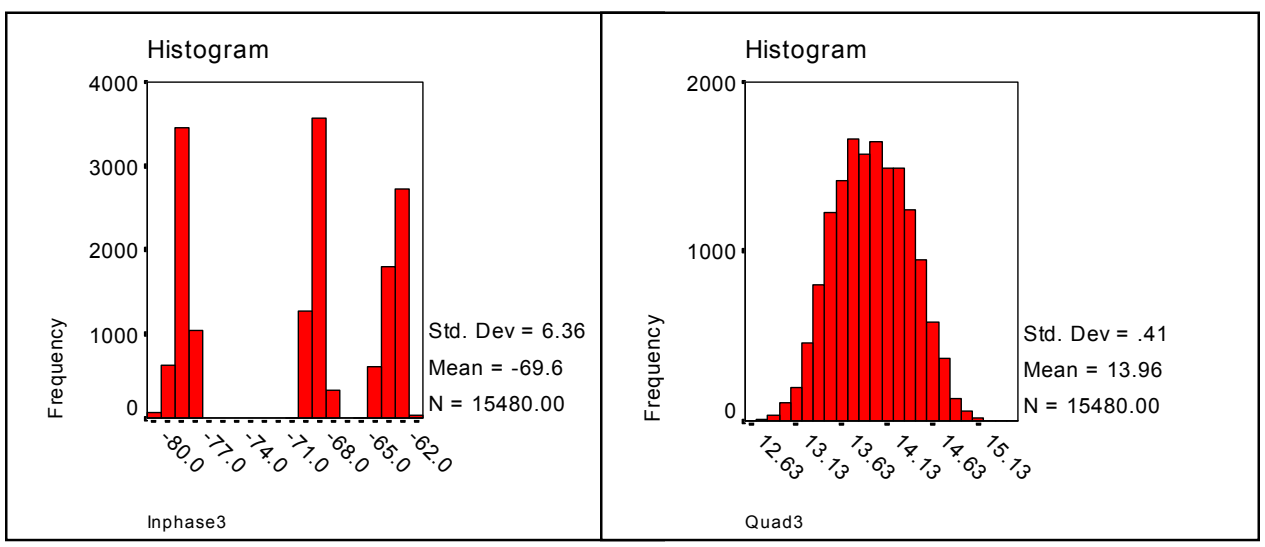

Figure 39. In-phase and quadrature histograms for $20-\mathrm{mm}$ round at $330 \mathrm{~Hz}$

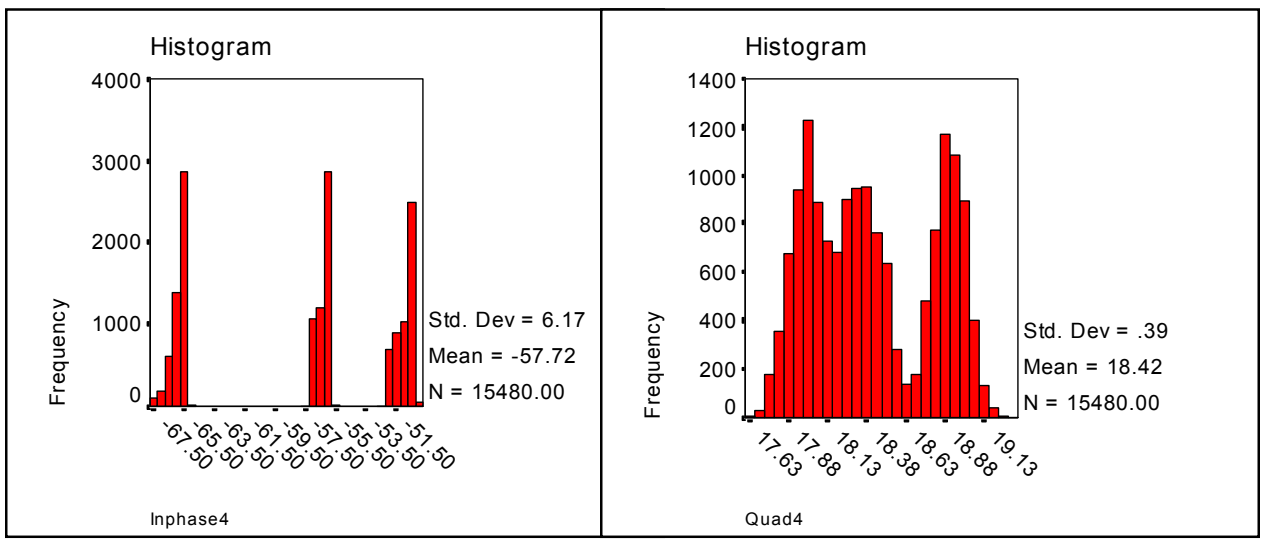

Figure 40 . In-phase and quadrature histograms for $20-\mathrm{mm}$ round at $930 \mathrm{~Hz}$

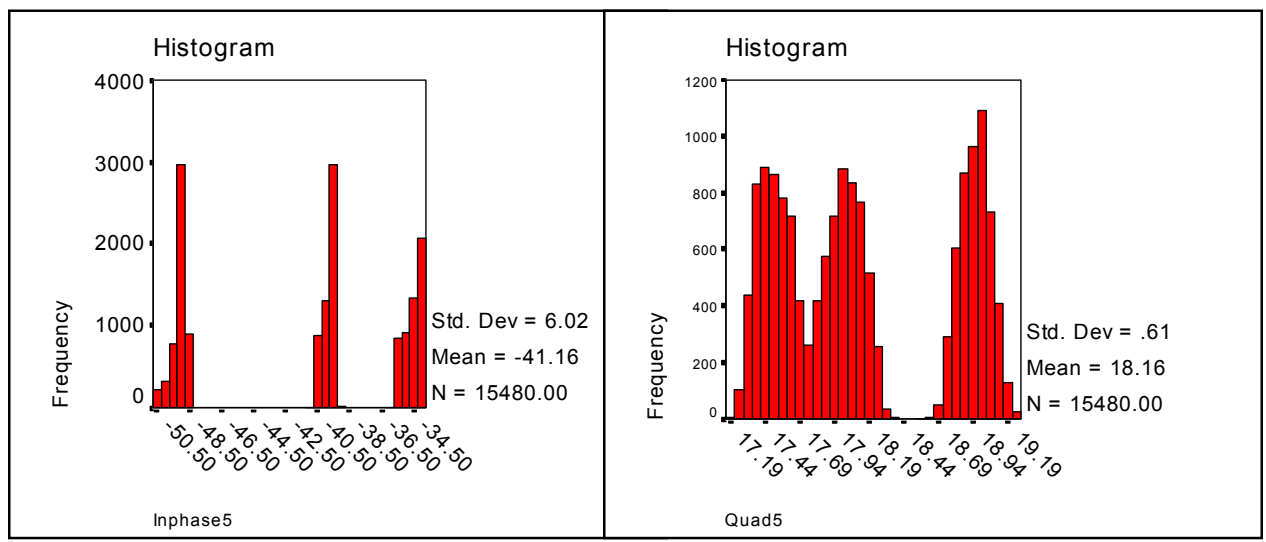

Figure 41 . In-phase and quadrature histograms for $20-\mathrm{mm}$ round at $2,790 \mathrm{~Hz}$ 


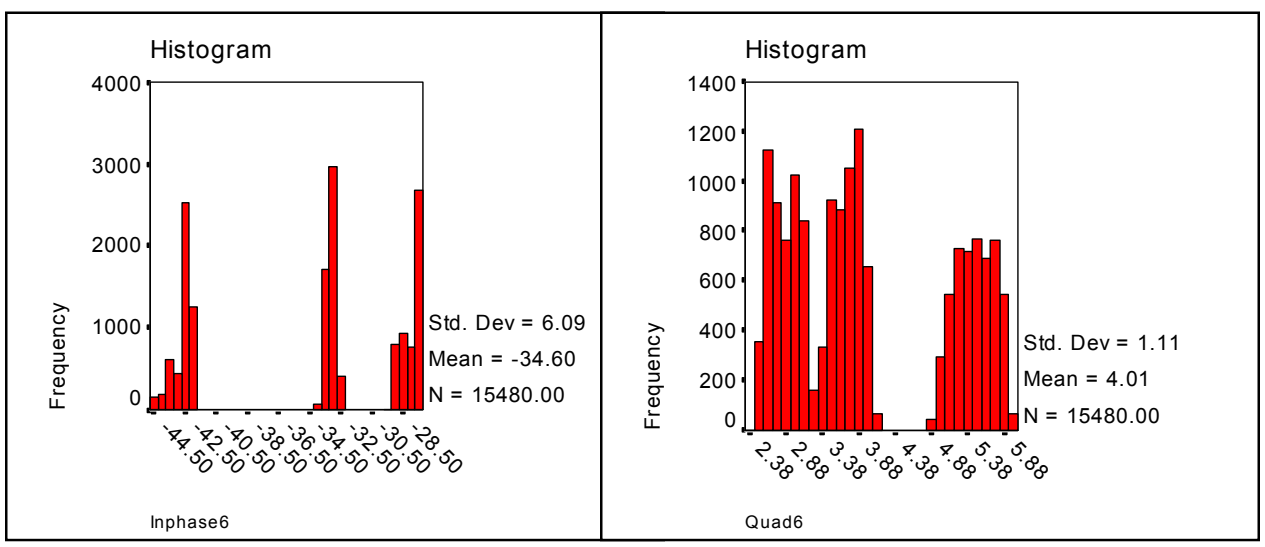

Figure 42. In-phase and quadrature histograms for $20-\mathrm{mm}$ round at $8,190 \mathrm{~Hz}$

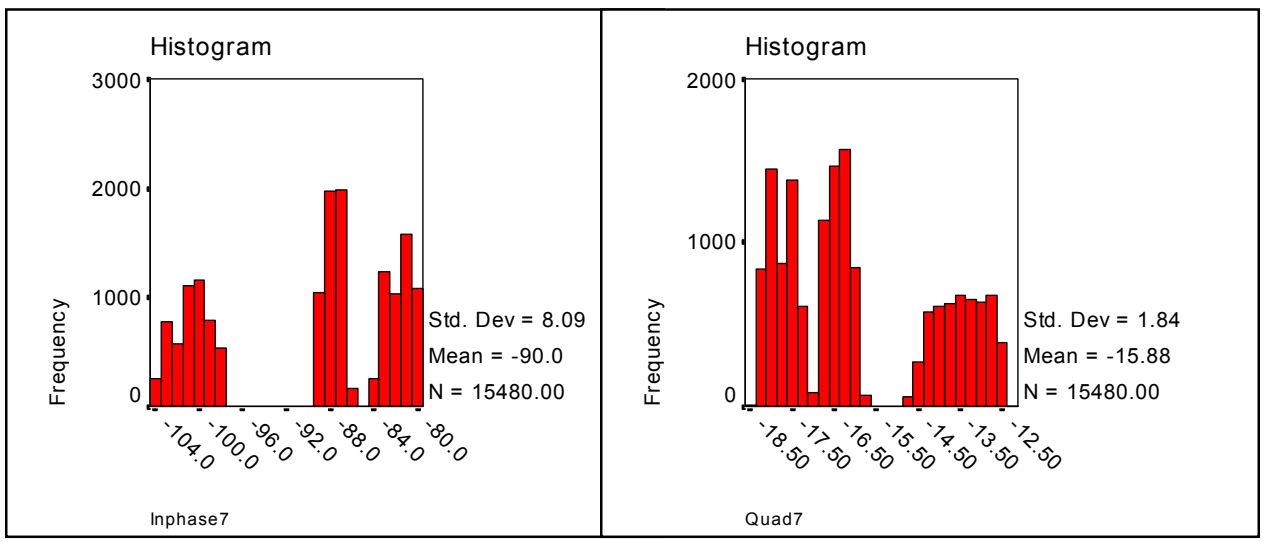

Figure 43. In-phase and quadrature histograms for $20-\mathrm{mm}$ round at $20,010 \mathrm{~Hz}$

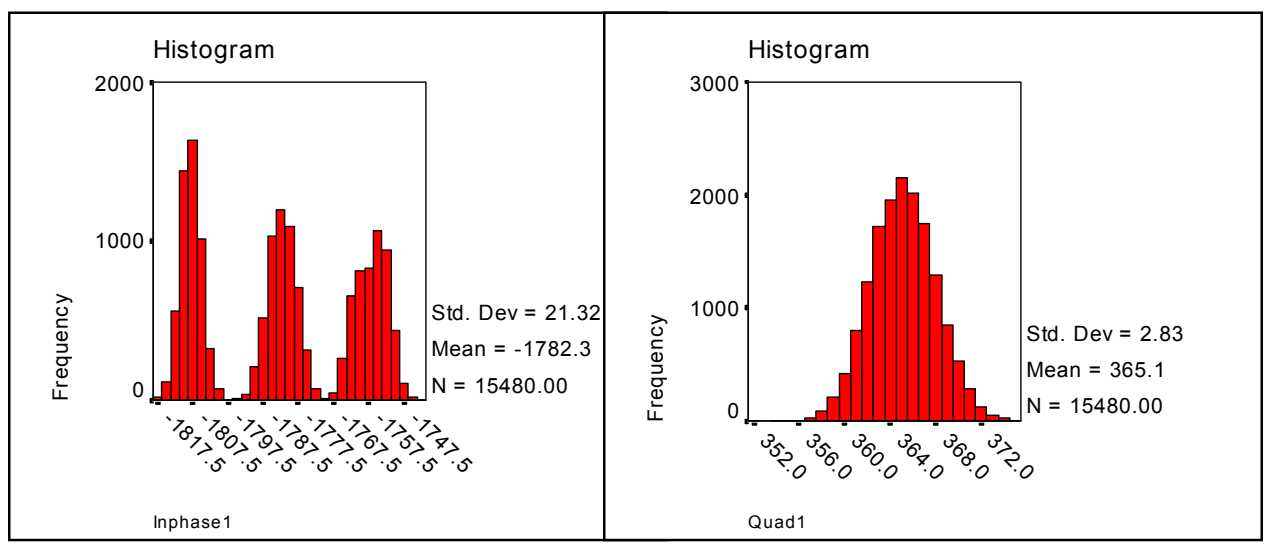

Figure 44. In-phase and quadrature histograms for $57-\mathrm{mm}$ mortar at $90 \mathrm{~Hz}$ 


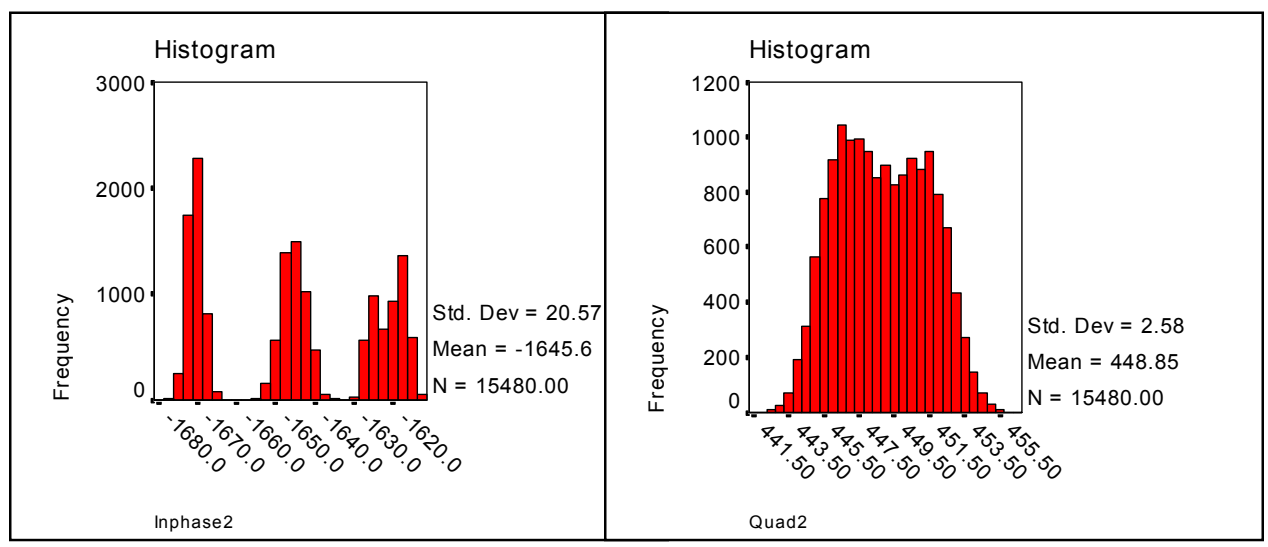

Figure 45. In-phase and quadrature histograms for $57-\mathrm{mm}$ mortar at $150 \mathrm{~Hz}$

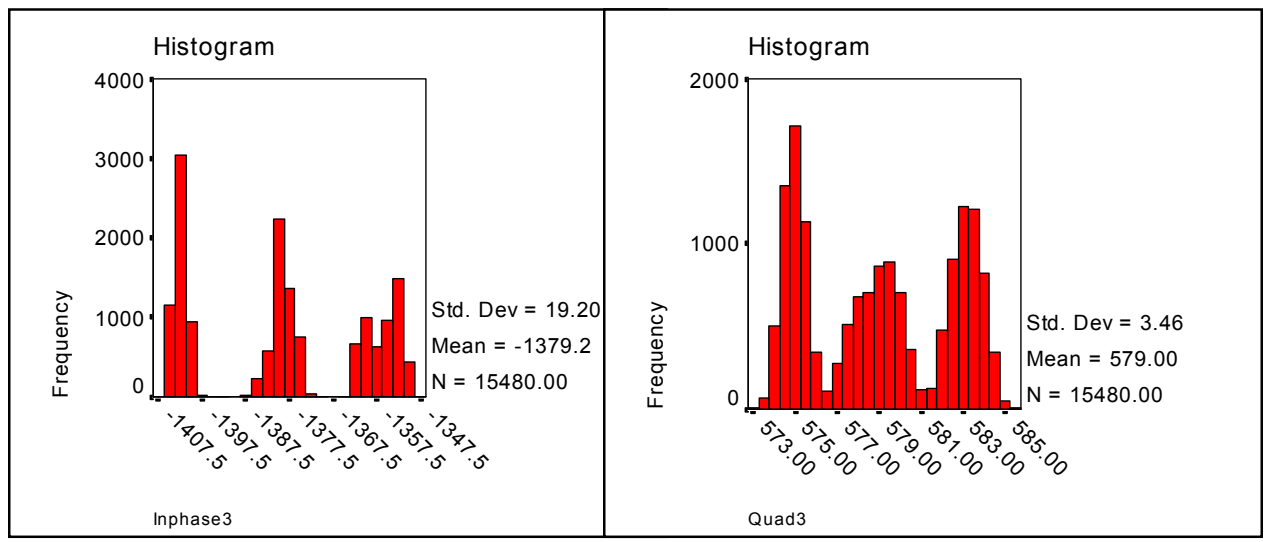

Figure 46. In-phase and quadrature histograms for $57-\mathrm{mm}$ mortar at $330 \mathrm{~Hz}$

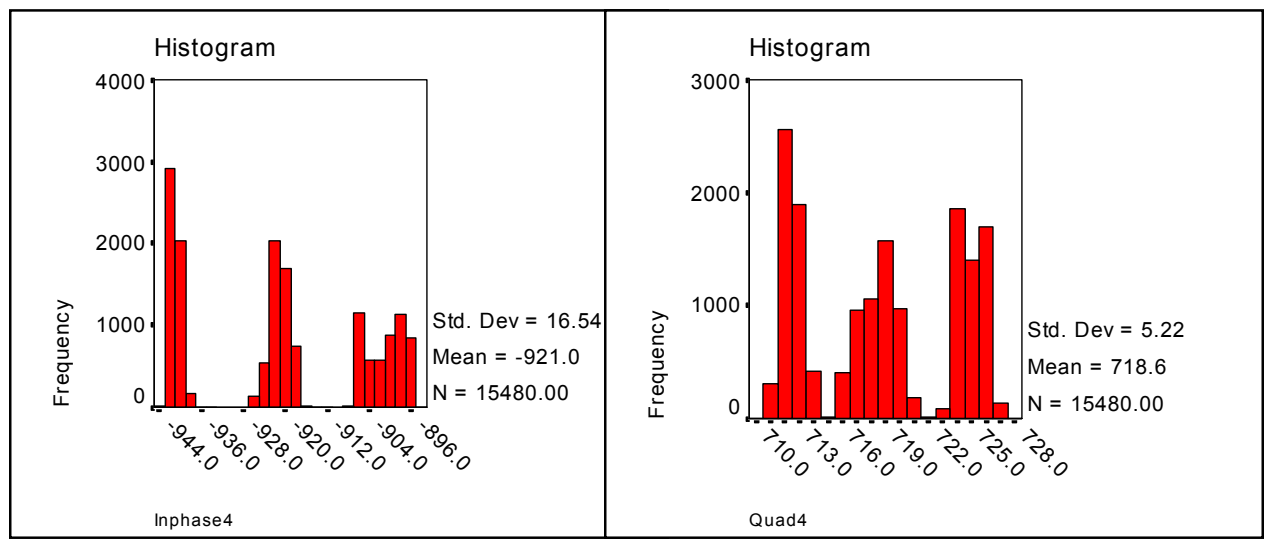

Figure 47. In-phase and quadrature histograms for $57-\mathrm{mm}$ mortar at $930 \mathrm{~Hz}$ 


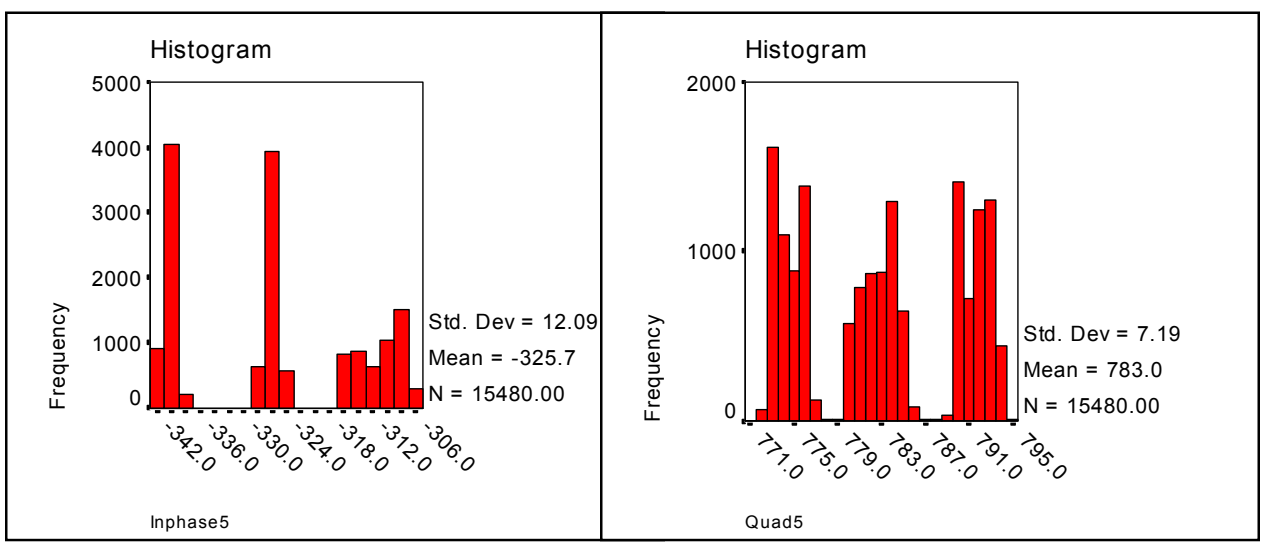

Figure 48. In-phase and quadrature histograms for $57-\mathrm{mm}$ mortar at $2,790 \mathrm{~Hz}$

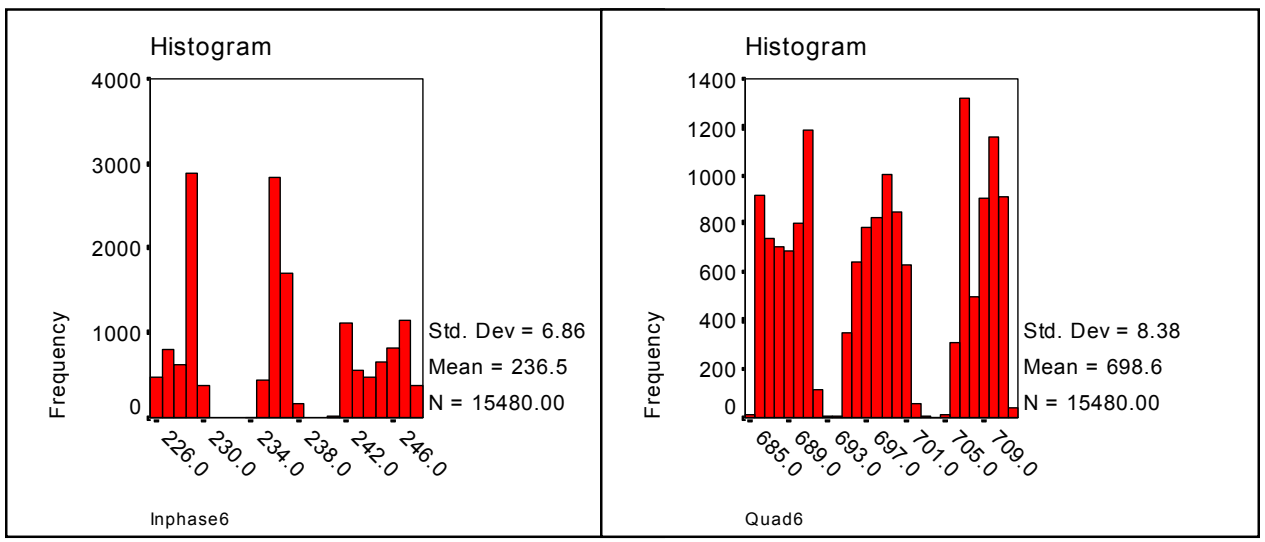

Figure 49. In-phase and quadrature histograms for $57-\mathrm{mm}$ mortar at $8,190 \mathrm{~Hz}$

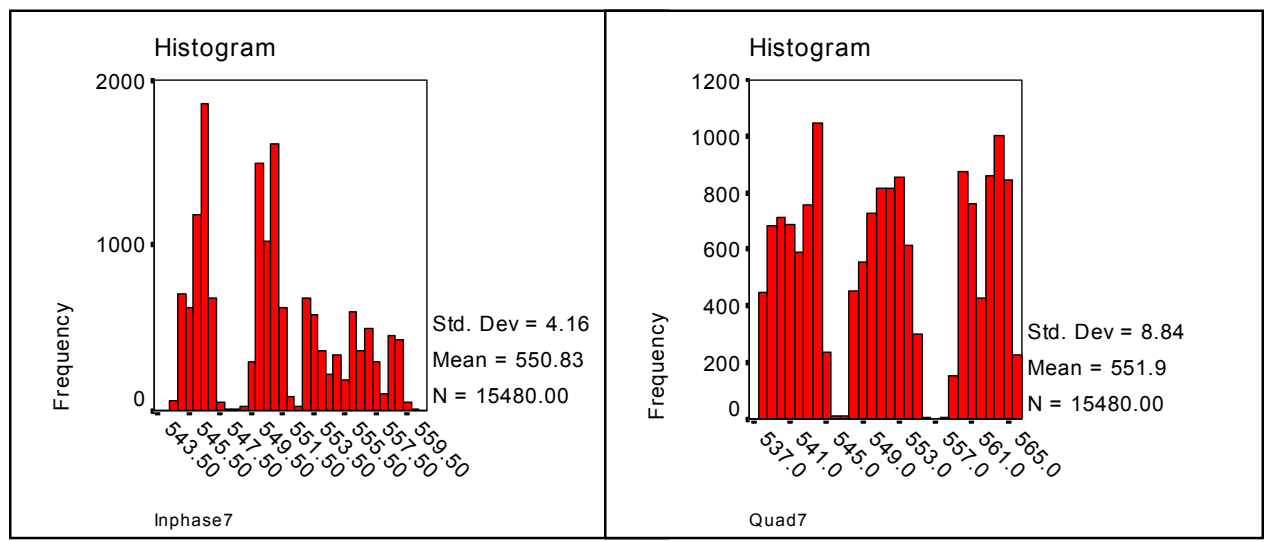

Figure 50 . In-phase and quadrature histograms for $57-\mathrm{mm}$ mortar at $20,010 \mathrm{~Hz}$ 


\begin{tabular}{|c|c|c|c|c|}
\hline \multicolumn{5}{|c|}{$\begin{array}{l}\text { Table } 7 \\
\text { Statistical Information on Fixed Target Variation } \\
\text { Sample Size } N=15,480\end{array}$} \\
\hline Description & Component & Frequency & Mean & Std. Deviation \\
\hline \multirow[t]{14}{*}{ Background } & In-phase1 & 90 & 0.0342 & 0.15307 \\
\hline & In-phase2 & 150 & 0.0179 & 0.091938 \\
\hline & In-phase3 & 330 & 0.0213 & 0.04845 \\
\hline & In-phase4 & 930 & 0.0331 & 0.03764 \\
\hline & In-phase5 & 2,790 & 0.0922 & 0.07864 \\
\hline & In-phase6 & 8,190 & 0.3074 & 0.25669 \\
\hline & In-phase7 & 20,010 & -3.3078 & 2.14604 \\
\hline & Quad1 & 90 & 0.0137 & 0.15560 \\
\hline & Quad2 & 150 & -.0000155 & 0.09292 \\
\hline & Quad3 & 330 & -.00406 & 0.04263 \\
\hline & Quad4 & 930 & 0.0057 & 0.03581 \\
\hline & Quad5 & 2,790 & -0.0158 & 0.13205 \\
\hline & Quad6 & 8,190 & -0.8627 & 0.94256 \\
\hline & Quad7 & 20,010 & -8.6170 & 6.78427 \\
\hline \multirow[t]{14}{*}{ 20-mm } & In-phase1 & 90 & -78.5324 & 6.7391 \\
\hline & In-phase2 & 150 & -75.8057 & 6.5315 \\
\hline & In-phase3 & 330 & -69.5563 & 6.3570 \\
\hline & In-phase4 & 930 & -57.7184 & 6.1737 \\
\hline & In-phase5 & 2,790 & -41.1624 & 6.0193 \\
\hline & In-phase6 & 8,190 & -34.5964 & 6.0938 \\
\hline & In-phase7 & 20,010 & -90.0381 & 8.0931 \\
\hline & Quad1 & 90 & 7.4923 & 1.5388 \\
\hline & Quad2 & 150 & 9.9834 & 0.7586 \\
\hline & Quad3 & 330 & 13.9623 & 0.4118 \\
\hline & Quad4 & 930 & 18.4170 & 0.3942 \\
\hline & Quad5 & 2,790 & 18.1551 & 0.6143 \\
\hline & Quad6 & 8,190 & 4.0144 & 1.1135 \\
\hline & Quad7 & 20,010 & -15.8804 & 1.8402 \\
\hline \multirow[t]{14}{*}{ 57-mm } & In-phase1 & 90 & -1782.262 & 21.3220 \\
\hline & In-phase2 & 150 & -1645.632 & 20.5687 \\
\hline & In-phase3 & 330 & -1379.235 & 19.2048 \\
\hline & In-phase4 & 930 & -920.9837 & 16.5396 \\
\hline & In-phase5 & 2,790 & -325.7171 & 12.0936 \\
\hline & In-phase6 & 8,190 & 23.4787 & 6.8598 \\
\hline & In-phase7 & 20,010 & 550.8331 & 4.1577 \\
\hline & Quad1 & 90 & 365.1280 & 2.8266 \\
\hline & Quad2 & 150 & 448.8472 & 2.5750 \\
\hline & Quad3 & 330 & 578.9956 & 3.4596 \\
\hline & Quad4 & 930 & 718.6377 & 5.2193 \\
\hline & Quad5 & 2,790 & 782.9586 & 7.1893 \\
\hline & Quad6 & 8,190 & 698.6334 & 8.3765 \\
\hline & Quad7 & 20,010 & 551.8812 & 8.8383 \\
\hline
\end{tabular}




\section{Comparison of Data Points with Calibration Data}

To determine how closely the data points acquired near targets during the demonstration match the corresponding calibration signatures, the data points within a 1-m box centered on each target were extracted. They were compared to the calibration signature for the same ordnance type as the target at all inclination angles. The best matching angle was selected. The exact inclination angle of the target was not used because the position of the sensor relative to the target during the demonstration varied from point to point, unlike the calibration data where the sensor was always directly above the target. Tables 8, 9, and 10 summarize the results of this comparison. Targets for which no calibration data were available are not included. Plots showing the comparison between each data point and the calibration signature, as well as the locations of the points relative to the target, are shown for every target in Appendix B. Code listings for the programs are given in Appendix C.

\begin{tabular}{|c|c|c|c|c|c|c|c|c|}
\hline \multicolumn{9}{|c|}{ Table 8} \\
\hline \multirow{2}{*}{\begin{tabular}{|l} 
Target ID \\
$1-86$
\end{tabular}} & Type & $\begin{array}{c}\text { Depth } \\
\text { cm }\end{array}$ & $\begin{array}{l}\text { Inclination } \\
\text { angle, deg }\end{array}$ & \begin{tabular}{|c|}
$\begin{array}{c}\text { Number } \\
\text { of Points }\end{array}$ \\
\end{tabular} & $\begin{array}{c}\text { Minimum } \\
\text { Error }\end{array}$ & $\begin{array}{c}\begin{array}{c}\text { Maximum } \\
\text { Error }\end{array} \\
\end{array}$ & Mean Error & $\begin{array}{l}\begin{array}{l}\text { Std Dev } \\
\text { of Error }\end{array} \\
\end{array}$ \\
\hline & 4.2-in. & 20 & 45 & 8 & 0.0100 & 0.1095 & 0.0491 & 0.0361 \\
\hline $1-88$ & $60-\mathrm{mm}$ & 35 & 0 & 12 & 0.0235 & 0.6212 & 0.1954 & 0.1850 \\
\hline $1-90$ & 4.2-in. & 35 & 0 & 15 & 0.0165 & 0.4299 & 0.0968 & 0.1209 \\
\hline $1-92$ & $81-\mathrm{mm}$ & 20 & 20 & 11 & 0.0425 & 0.2417 & 0.0873 & 0.0592 \\
\hline $1-94$ & $81-\mathrm{mm}$ & 25 & 55 & 5 & 0.0890 & 0.3049 & 0.1846 & 0.0785 \\
\hline $1-96$ & $81-\mathrm{mm}$ & 15 & 35 & 10 & 0.0095 & 0.3622 & 0.0803 & 0.1099 \\
\hline $1-98$ & 60-mm & 10 & 20 & 8 & 0.0097 & 0.1492 & 0.0574 & 0.0483 \\
\hline $1-100$ & $60-\mathrm{mm}$ & 20 & 30 & 7 & 0.0239 & 0.4182 & 0.2251 & 0.1611 \\
\hline $1-102$ & $81-\mathrm{mm}$ & 25 & 45 & 7 & 0.0084 & 0.2707 & 0.0865 & 0.0902 \\
\hline $1-104$ & $81-\mathrm{mm}$ & 35 & 0 & 7 & 0.0299 & 0.1377 & 0.0709 & 0.0404 \\
\hline $1-106$ & $60-\mathrm{mm}$ & 25 & 35 & 8 & 0.0513 & 0.2855 & 0.1362 & 0.0702 \\
\hline $1-108$ & 60-mm & 20 & 45 & 8 & 0.0258 & 0.5122 & 0.2734 & 0.1695 \\
\hline $1-112$ & 20-mm & 10 & 10 & 9 & 0.1090 & 0.3726 & 0.2028 & 0.0860 \\
\hline 1-114 & 20-mm & 15 & 20 & 11 & 0.2824 & 0.5345 & 0.3857 & 0.0841 \\
\hline 1-116 & $20-\mathrm{mm}$ & 15 & 0 & 6 & 0.2400 & 0.5572 & 0.4014 & 0.1324 \\
\hline $1-117$ & 152-mm & 90 & 45 & 18 & 0.0760 & 0.6827 & 0.2678 & 0.1644 \\
\hline $1-119$ & 152-mm & 40 & 30 & 12 & 0.0224 & 0.1922 & 0.0996 & 0.0508 \\
\hline $1-121$ & $155-\mathrm{mm}$ & 50 & 0 & 9 & 0.0119 & 0.1403 & 0.0391 & 0.0390 \\
\hline $1-123$ & $20-\mathrm{mm}$ & 0 & 90 & 10 & 0.1027 & 0.4538 & 0.2964 & 0.1240 \\
\hline $1-124$ & $20-\mathrm{mm}$ & 0 & -90 & 31 & 0.2523 & 0.6976 & 0.3938 & 0.1128 \\
\hline $1-126$ & $57-\mathrm{mm}$ & 20 & 30 & 10 & 0.2184 & 0.6329 & 0.5026 & 0.1241 \\
\hline $1-128$ & $20-\mathrm{mm}$ & 10 & 0 & 11 & 0.0438 & 0.2624 & 0.1512 & 0.0799 \\
\hline $1-132$ & 57-mm & 25 & 0 & 9 & 0.0247 & 0.4117 & 0.1392 & 0.1157 \\
\hline $1-134$ & 20-mm & 5 & 30 & 10 & 0.0758 & 0.6445 & 0.4085 & 0.1999 \\
\hline $1-136$ & $155-\mathrm{mm}$ & 50 & 75 & 5 & 0.0267 & 0.0686 & 0.0487 & 0.0158 \\
\hline $1-138$ & $57-\mathrm{mm}$ & 15 & 45 & 9 & 0.0302 & 0.2422 & 0.1038 & 0.0813 \\
\hline $1-140$ & $20-\mathrm{mm}$ & 5 & 15 & 9 & 0.1186 & 0.467 & 0.2615 & 0.1275 \\
\hline $1-142$ & $57-\mathrm{mm}$ & 15 & 45 & 8 & 0.0058 & 0.038 & 0.0235 & 0.0119 \\
\hline $1-146$ & 20-mm & 5 & 0 & 14 & 0.1084 & 0.5391 & 0.3326 & 0.1517 \\
\hline $1-147$ & $57-\mathrm{mm}$ & 25 & 0 & 6 & 0.0114 & 0.4985 & 0.2442 & 0.2105 \\
\hline $1-148$ & $20-\mathrm{mm}$ & \begin{tabular}{|l|}
10 \\
\end{tabular} & 45 & 8 & 0.1830 & 0.6268 & 0.3457 & 0.1805 \\
\hline $1-149$ & 2.75-in. & 50 & 55 & 8 & 0.0160 & 0.2399 & 0.1055 & 0.0726 \\
\hline $1-150$ & 2.75-in. & 70 & 45 & 40 & 0.0357 & 0.5201 & 0.1944 & 0.1025 \\
\hline 1-152 & 2.75-in. & 15 & 0 & 9 & 0.0458 & 0.109 & 0.0772 & 0.0243 \\
\hline $1-153$ & 2.75-in. & 76 & 90 & 9 & 0.0767 & 0.6012 & 0.4352 & 0.1697 \\
\hline
\end{tabular}




\begin{tabular}{|c|c|c|c|c|c|c|c|c|}
\hline \multicolumn{9}{|c|}{ Table 9} \\
\hline Target ID & Type & $\begin{array}{r}\text { Depth } \\
\text { (cm) }\end{array}$ & $\begin{array}{c}\begin{array}{c}\text { Inclination } \\
\text { angle }\end{array} \\
\end{array}$ & \begin{tabular}{|c|}
$\begin{array}{c}\text { Number } \\
\text { of Points }\end{array}$ \\
\end{tabular} & $\begin{array}{c}\text { Minimum } \\
\text { Error }\end{array}$ & $\begin{array}{c}\begin{array}{c}\text { Maximum } \\
\text { Error }\end{array} \\
\end{array}$ & Mean Error & $\begin{array}{l}\begin{array}{l}\text { Std Dev } \\
\text { of Error }\end{array} \\
\end{array}$ \\
\hline $2-112$ & $81-\mathrm{mm}$ & 10 & 90 & 3 & 0.0096 & 0.0643 & 0.0289 & 0.0307 \\
\hline $2-114$ & $81-\mathrm{mm}$ & 20 & -90 & 8 & 0.0070 & 0.2249 & 0.0690 & 0.0684 \\
\hline $2-116$ & $81-\mathrm{mm}$ & 30 & 0 & 8 & 0.0122 & 0.1463 & 0.0606 & 0.0463 \\
\hline $2-118$ & $60-\mathrm{mm}$ & 35 & 45 & 9 & 0.1200 & 0.5099 & 0.2611 & 0.1142 \\
\hline $2-120$ & 60-mm & 30 & 0 & 8 & 0.0424 & 0.3254 & 0.1130 & 0.0932 \\
\hline $2-122$ & 60-mm & 10 & 30 & 10 & 0.0232 & 0.5161 & 0.1351 & 0.1592 \\
\hline $2-124$ & $60-\mathrm{mm}$ & 20 & 10 & 8 & 0.0188 & 0.6151 & 0.1506 & 0.2004 \\
\hline $2-126$ & $81-\mathrm{mm}$ & 35 & 45 & 7 & 0.0274 & 0.1084 & 0.0570 & 0.0323 \\
\hline $2-128$ & $60-\mathrm{mm}$ & 10 & 20 & 8 & 0.0046 & 0.0888 & 0.0364 & 0.0279 \\
\hline $2-130$ & $81-\mathrm{mm}$ & 70 & 0 & 10 & 0.0159 & 0.3097 & 0.0759 & 0.0882 \\
\hline $2-131$ & $81-\mathrm{mm}$ & 25 & 0 & 7 & 0.0057 & 0.0379 & 0.0200 & 0.0115 \\
\hline $2-134$ & 4.2-in. & 40 & 0 & 8 & 0.0118 & 0.0377 & 0.0257 & 0.0112 \\
\hline $2-136$ & $20-\mathrm{mm}$ & 5 & 10 & 8 & 0.2054 & 0.6724 & 0.4012 & 0.1689 \\
\hline $2-138$ & $20-\mathrm{mm}$ & 5 & 15 & 7 & 0.2121 & 0.5602 & 0.3455 & 0.1183 \\
\hline $2-142$ & 152-mm & 91 & 45 & 11 & 0.1341 & 0.3596 & 0.2438 & 0.0717 \\
\hline $2-144$ & 152-mm & 45 & 30 & 7 & 0.0642 & 0.3177 & 0.1472 & 0.1082 \\
\hline $2-146$ & $20-\mathrm{mm}$ & 0 & 90 & 18 & 0.0610 & 0.6574 & 0.3535 & 0.1625 \\
\hline $2-148$ & $20-\mathrm{mm}$ & 0 & -90 & 8 & 0.1893 & 0.6583 & 0.4192 & 0.1874 \\
\hline $2-150$ & $20-\mathrm{mm}$ & 10 & 0 & 11 & 0.0284 & 0.6097 & 0.3680 & 0.1845 \\
\hline $2-152$ & $57-\mathrm{mm}$ & 25 & 0 & 10 & 0.0224 & 0.2997 & 0.1039 & 0.0993 \\
\hline $2-154$ & $57-\mathrm{mm}$ & 20 & 45 & 11 & 0.0252 & 0.2989 & 0.1201 & 0.0891 \\
\hline $2-156$ & 155-mm & 102 & 90 & 10 & 0.0843 & 0.5366 & 0.1896 & 0.1412 \\
\hline $2-158$ & $20-\mathrm{mm}$ & 10 & 20 & 9 & 0.0276 & 0.5122 & 0.1775 & 0.1465 \\
\hline $2-160$ & $57-\mathrm{mm}$ & 35 & 40 & 9 & 0.0285 & 0.5212 & 0.2257 & 0.1471 \\
\hline $2-161$ & $155-\mathrm{mm}$ & 75 & 30 & 7 & 0.0215 & 0.1410 & 0.0649 & 0.0430 \\
\hline $2-164$ & 2.75-in. & 60 & 10 & 4 & 0.0660 & 0.1433 & 0.1047 & 0.0316 \\
\hline $2-166$ & 2.75 -in. & 75 & 20 & 4 & 0.2687 & 0.5892 & 0.4617 & 0.1464 \\
\hline
\end{tabular}

\begin{tabular}{|c|c|c|c|c|c|c|c|c|}
\hline \multicolumn{9}{|c|}{ Table 10} \\
\hline Target ID & Type & \begin{tabular}{|r|} 
Depth \\
(cm)
\end{tabular} & $\begin{array}{c}\text { Inclination } \\
\text { angle }\end{array}$ & \begin{tabular}{|c|}
$\begin{array}{c}\text { Number } \\
\text { of Points }\end{array}$ \\
\end{tabular} & $\begin{array}{c}\text { Minimum } \\
\text { Error }\end{array}$ & $\begin{array}{c}\text { Maximum } \\
\text { Error }\end{array}$ & Mean Error & \begin{tabular}{|l|} 
Std Dev \\
of Error
\end{tabular} \\
\hline $3-68$ & 60-mm & 20 & 90 & 10 & 0.0149 & 0.3716 & 0.1308 & 0.1373 \\
\hline $3-70$ & 81-mm & 25 & 0 & 9 & 0.0158 & 0.0521 & 0.0301 & 0.0138 \\
\hline $3-72$ & 60-mm & 25 & 30 & 10 & 0.0202 & 0.4133 & 0.2271 & 0.1573 \\
\hline $3-74$ & 60-mm & 30 & 45 & 10 & 0.0152 & 0.0884 & 0.0390 & 0.0231 \\
\hline $3-76$ & $81-\mathrm{mm}$ & 20 & -90 & 4 & 0.0065 & 0.0454 & 0.0261 & 0.0185 \\
\hline $3-78$ & 60-mm & 35 & 40 & 9 & 0.0213 & 0.4177 & 0.1242 & 0.1205 \\
\hline $3-80$ & $81-\mathrm{mm}$ & 25 & 0 & 11 & 0.0111 & 0.1795 & 0.0350 & 0.0503 \\
\hline $3-82$ & 60-mm & 20 & 15 & 7 & 0.0181 & 0.3424 & 0.1001 & 0.1114 \\
\hline $3-84$ & $81-m m$ & 25 & 0 & 9 & 0.1225 & 0.1694 & 0.1452 & 0.0137 \\
\hline $3-86$ & 20-mm & 1 & 90 & 8 & 0.1028 & 0.3071 & 0.1919 & 0.0804 \\
\hline $3-88$ & 20-mm & 1 & 0 & 5 & 0.0545 & 0.4379 & 0.1992 & 0.1542 \\
\hline $3-90$ & 20-mm & 15 & 0 & 9 & 0.0732 & 0.5454 & 0.2431 & 0.1550 \\
\hline $3-92$ & 20-mm & 15 & 30 & 6 & 0.0622 & 0.5500 & 0.2650 & 0.1949 \\
\hline $3-94$ & 57-mm & 35 & 0 & 8 & 0.0462 & 0.2992 & 0.1548 & 0.0933 \\
\hline $3-96$ & $57-\mathrm{mm}$ & 25 & 20 & 8 & 0.0185 & 0.3410 & 0.0764 & 0.1088 \\
\hline $3-100$ & 152-mm & 91 & 35 & 10 & 0.1582 & 0.4296 & 0.2549 & 0.0835 \\
\hline 3-102 & 155-mm & 120 & 20 & 8 & 0.2182 & 0.5247 & 0.3507 & 0.1185 \\
\hline 3-106 & 2.75 -in. & 50 & 30 & 11 & 0.0562 & 0.4900 & 0.2228 & 0.1468 \\
\hline
\end{tabular}




\section{Strong signatures}

The minimum error column of Tables 8,9 , and 10 shows the error in fit of the data point near the target that most closely matches the calibration signature for the ordnance type of the target. Figures 51 through 58 show examples of data points near targets of each of the different ordnance types that closely match the corresponding calibration signatures.

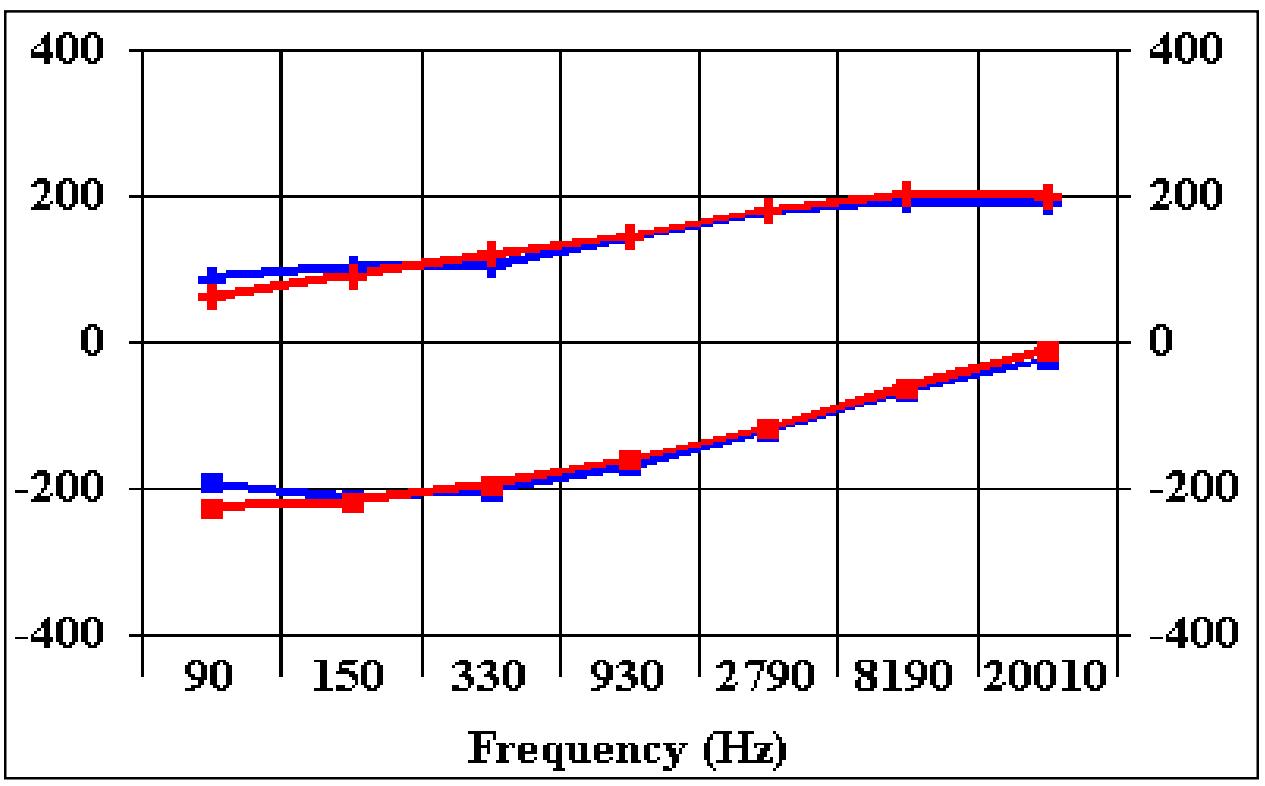

Figure 51. Best matching point for target 2-158 (20-mm projectile)

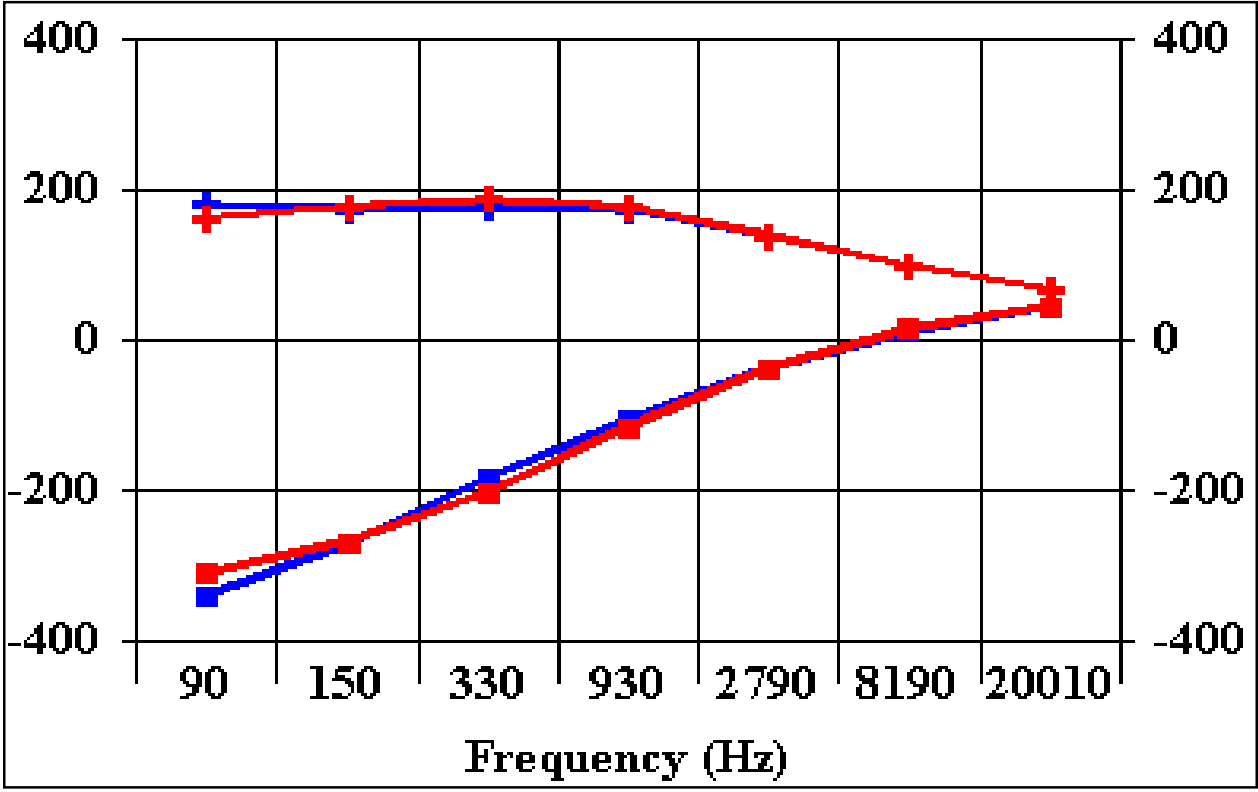

Figure 52. Best matching point for target 3-96 (57-mm mortar) 


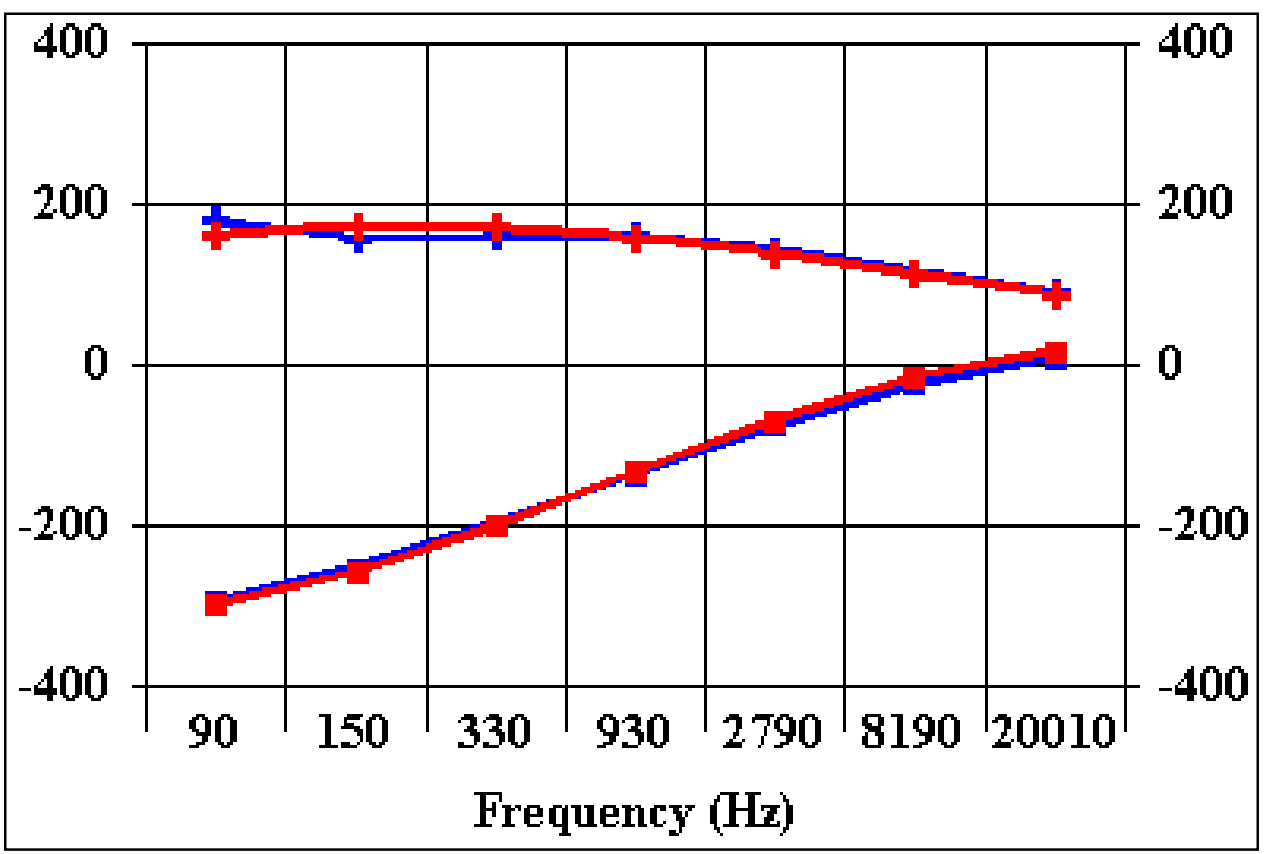

Figure 53. Best matching point for target 3-74 (60-mm mortar)

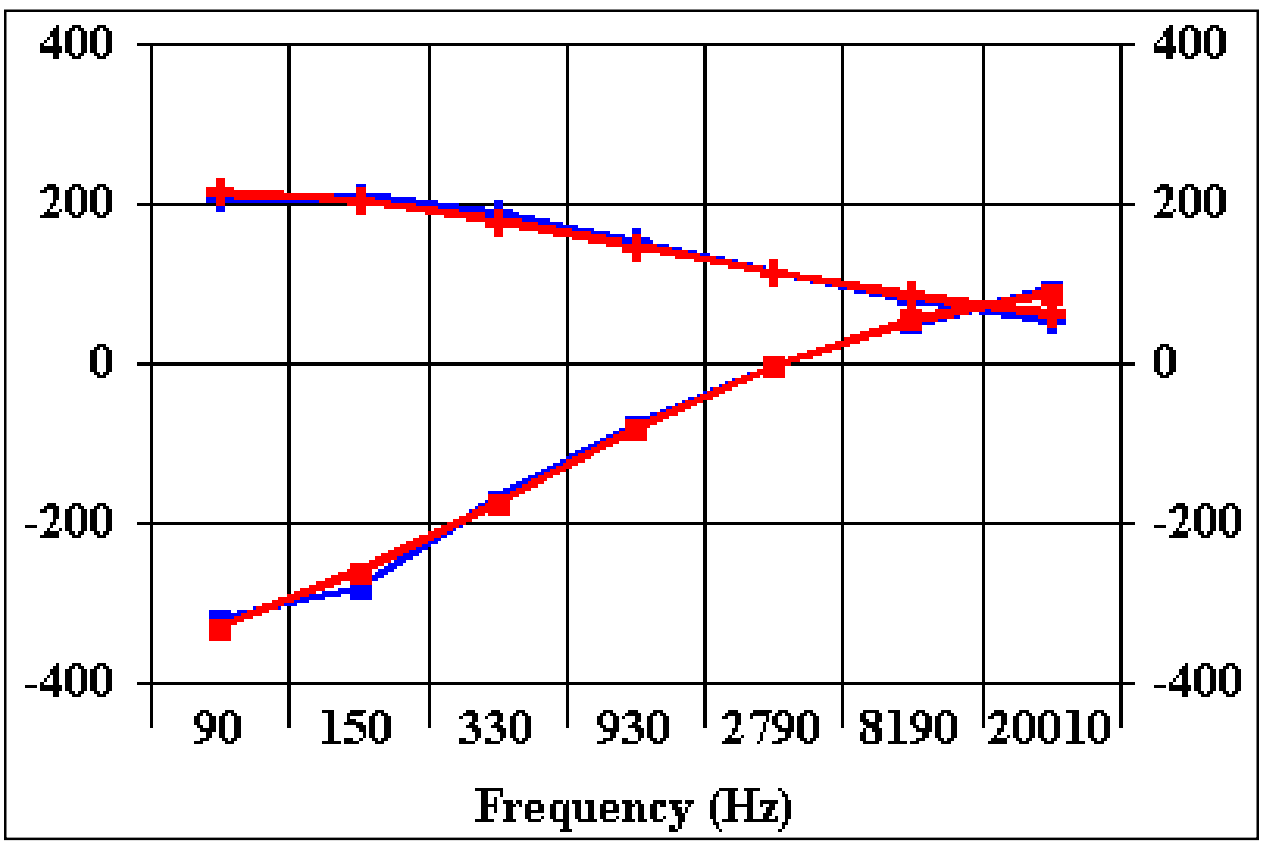

Figure 54. Best matching point for target 1-149 (2.75-in. rocket) (To convert inches to meters, multiply by 0.0254 ) 


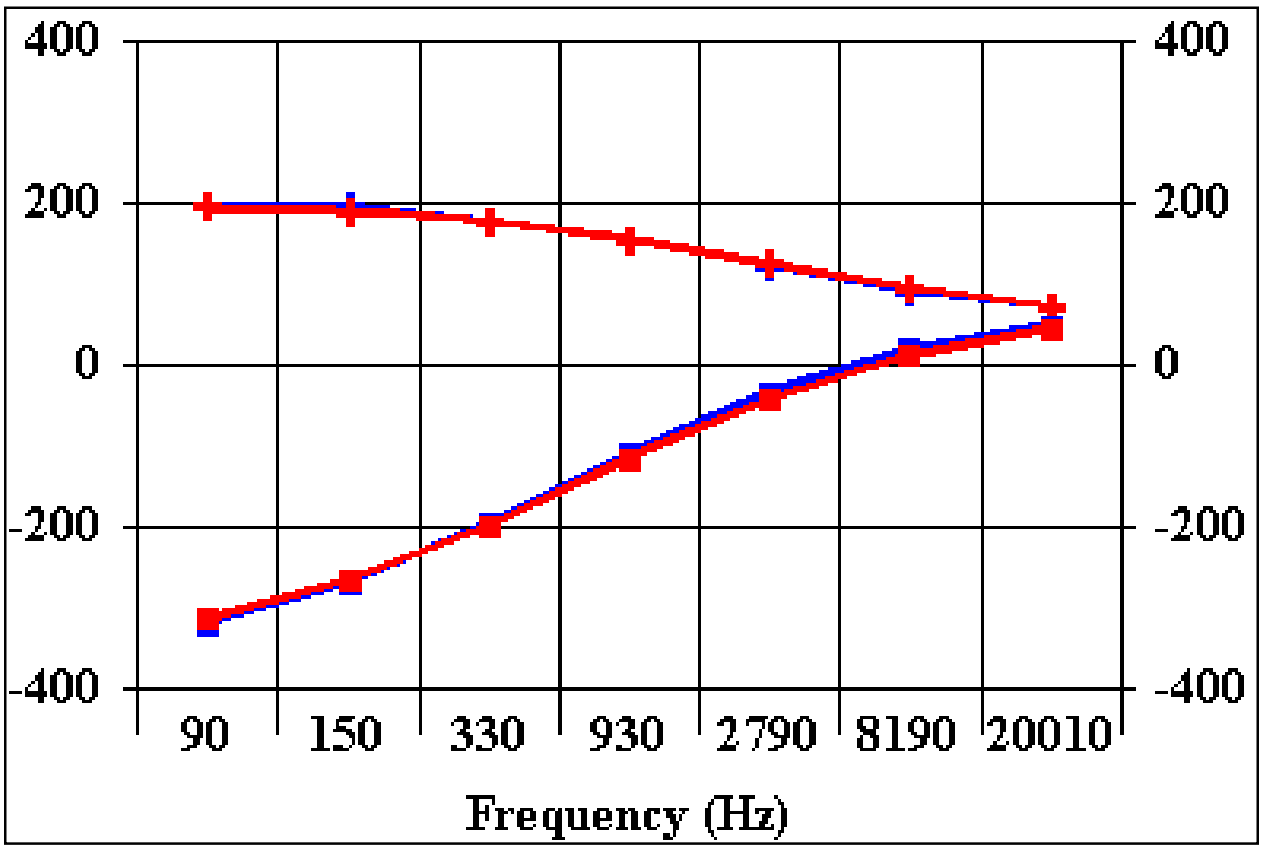

Figure 55. Best matching point for target 1-96 (81-mm mortar)

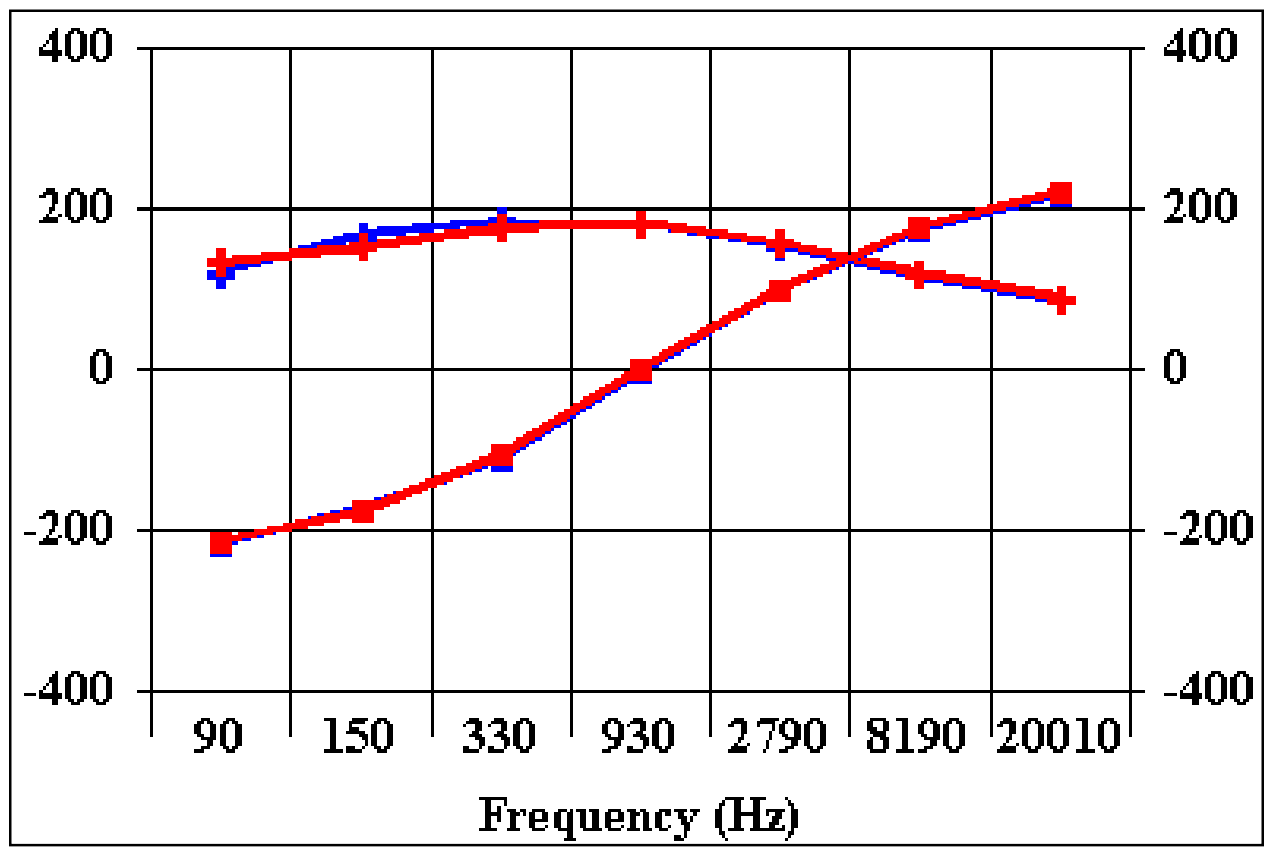

Figure 56. Best matching point for target 2-134 (4.2-in. mortar) (To convert inches to meters, multiply by 0.0254 ) 


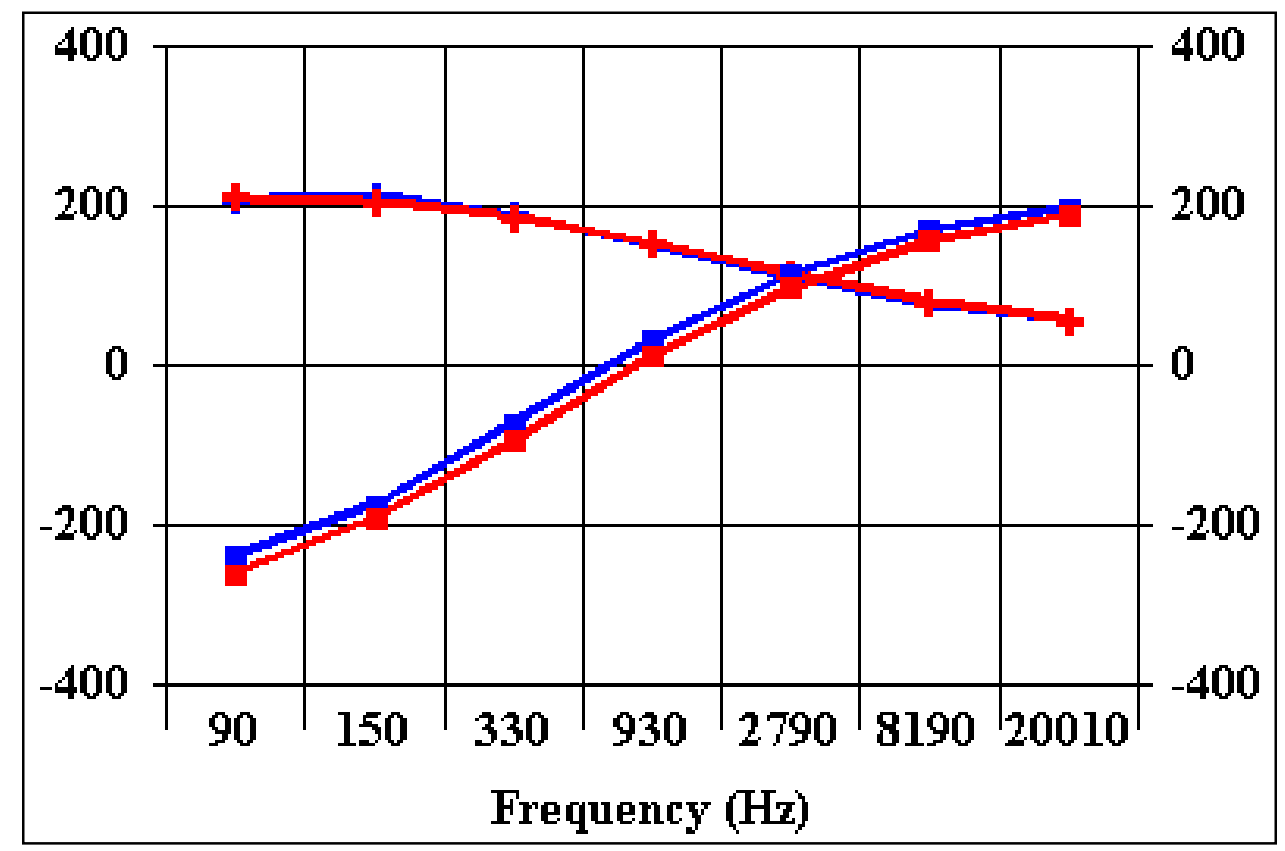

Figure 57. Best matching point for target 1-119 (152-mm projectile)

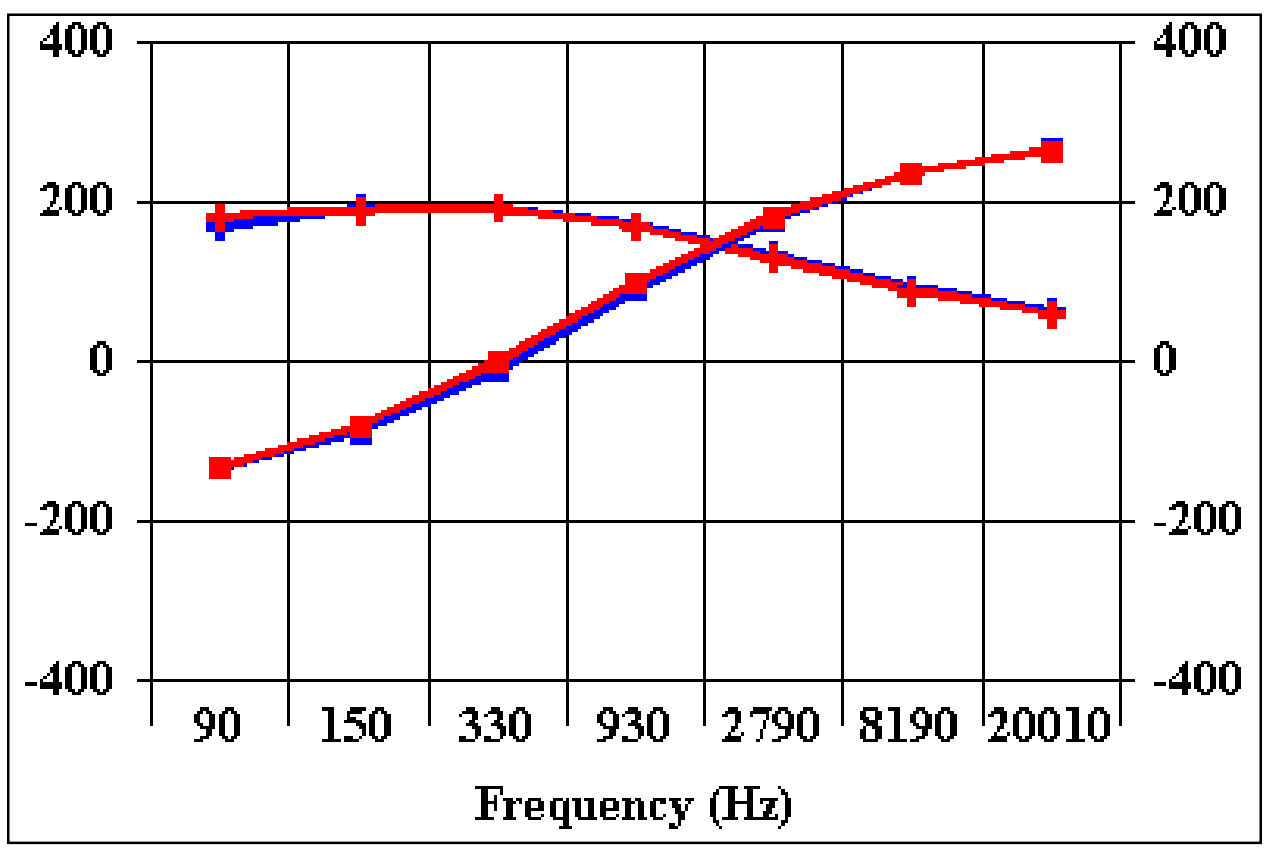

Figure 58. Best matching point for target 1-121 (155-mm projectile) 


\section{Weak signatures}

A total of 19 targets had no points near them with an error in fit less than 0.1 . Twelve of these targets were $20-\mathrm{mm}$ projectiles and the weak signatures were likely a result of the small size of the target. The few data points with good signatures near $20-\mathrm{mm}$ projectiles were very close to the target (within $0.25 \mathrm{~m}$ ). Targets 2-150 and 2-158 are examples of 20-mm targets that had data points with good signatures very near the target. In some cases, the sensor did not get that close to a target. However, even when the sensor did get very close to a $20-\mathrm{mm}$ projectile, the signature was not necessarily strong enough to classify it correctly. Target 1-124, with a total of 12 data points within $0.25 \mathrm{~m}$, but none with a good signature, is a good example of this.

Figures 59 through 65 show the best matching data points for the seven larger targets that had no points with error in fit less than 0.1. All but two of these targets, 3-84 and 1-126, were among the deepest targets of their type emplaced at JPG, and their weak signatures are probably the result of their depth. The in-phase data for target 3-84 consists of large magnitude values of a nearly constant value for every frequency, possibly because of a recording error of some kind. Figure 59 shows that the quadrature data for the target matches the calibration data very well. The data points for target 1-126, a 57-mm mortar at a depth of $20 \mathrm{~cm}$, had an average magnitude of only 62, a much weaker signal than for similar targets at similar depths. The total magnitude of a data point is the sum of the absolute values of the in-phase and quadrature measurements for all frequencies.

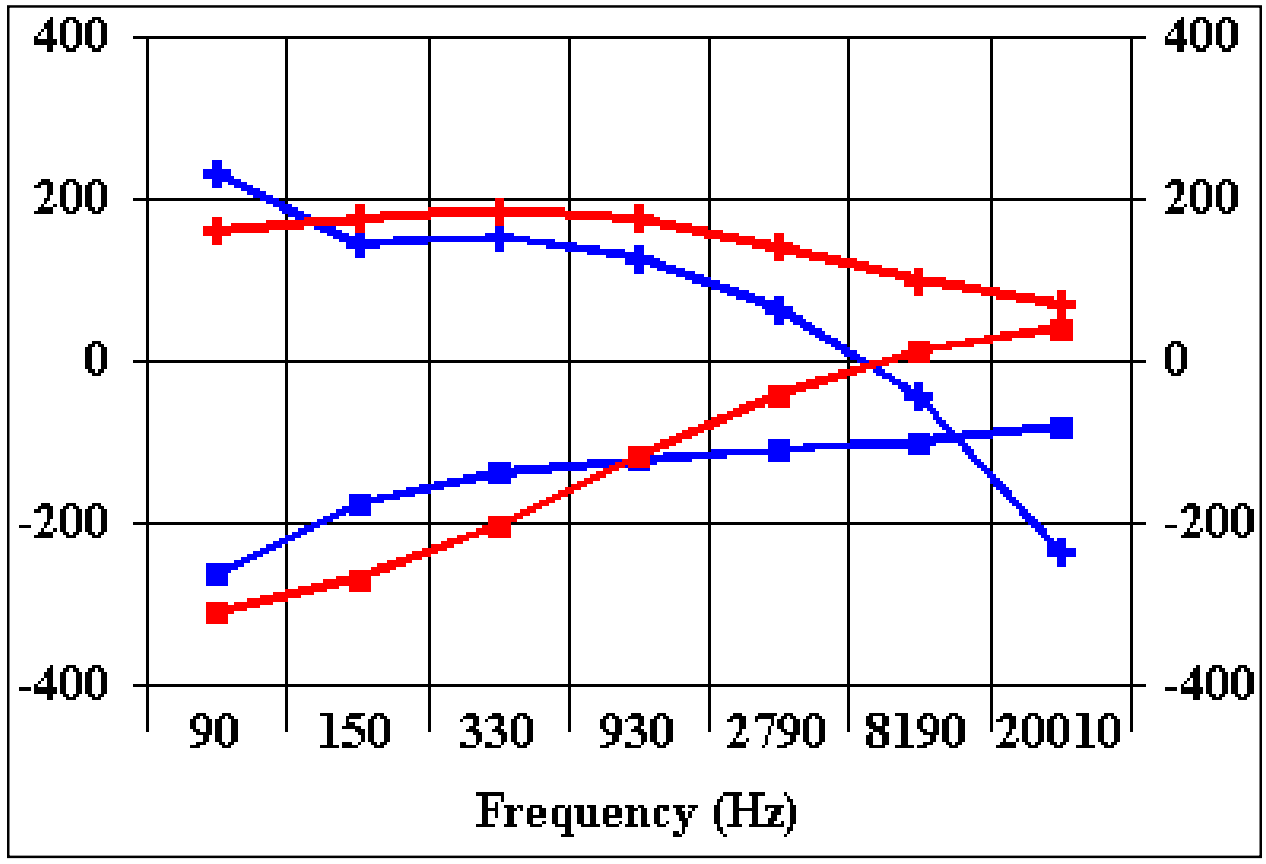

Figure 59. Best matching point for target 1-126 (57-mm mortar) 


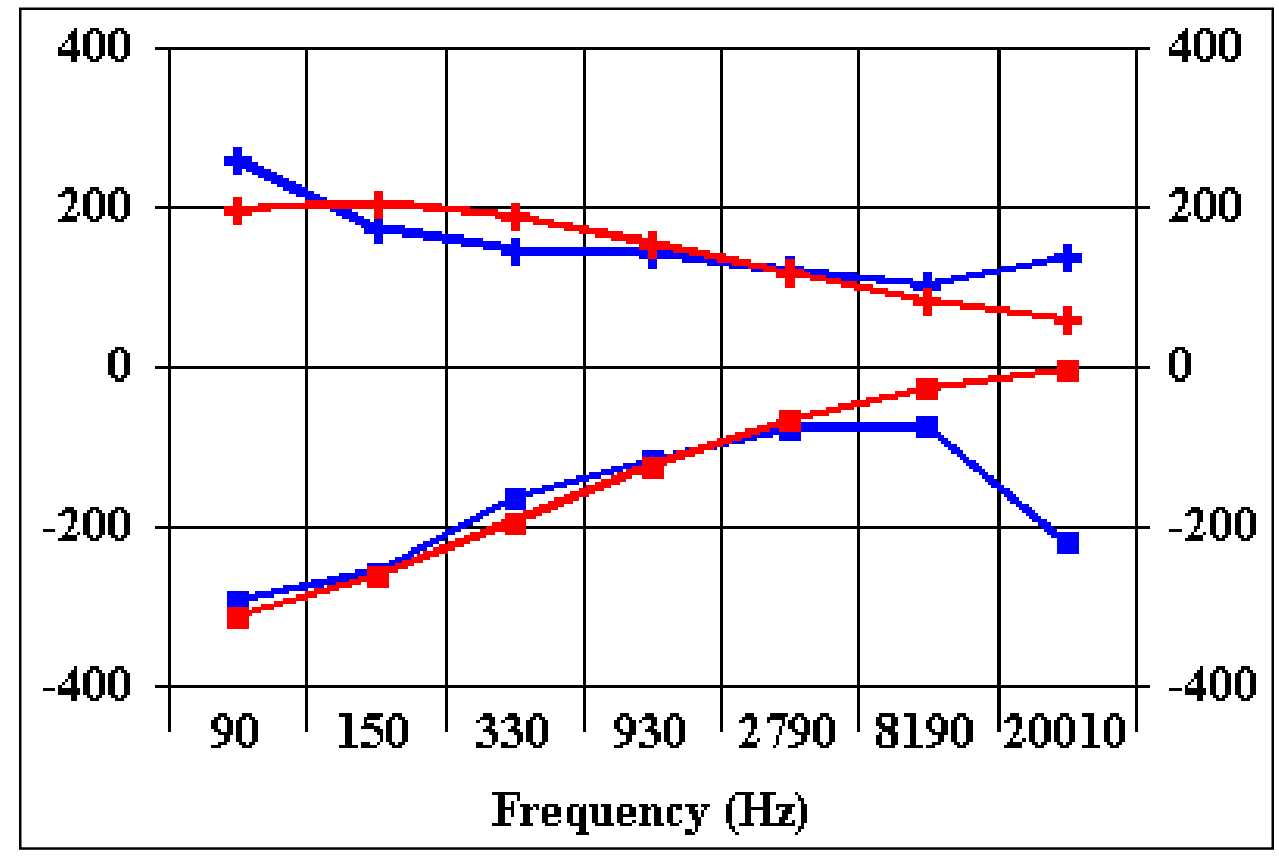

Figure 60. Best matching point for target 2-118 (60-mm mortar)

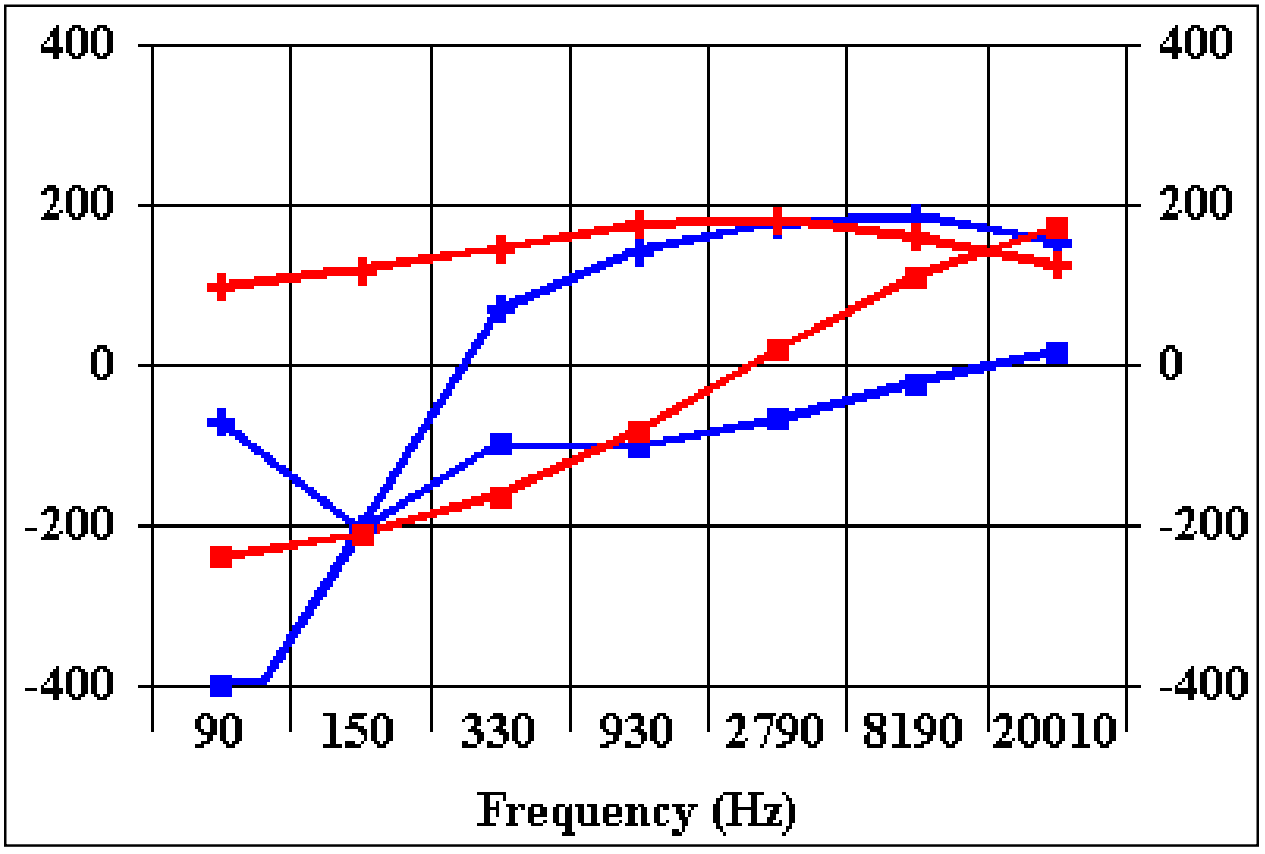

Figure 61. Best matching point for target 2-166 (2.75-in. rocket) (To convert inches to meters, multiply by 0.0254 ) 


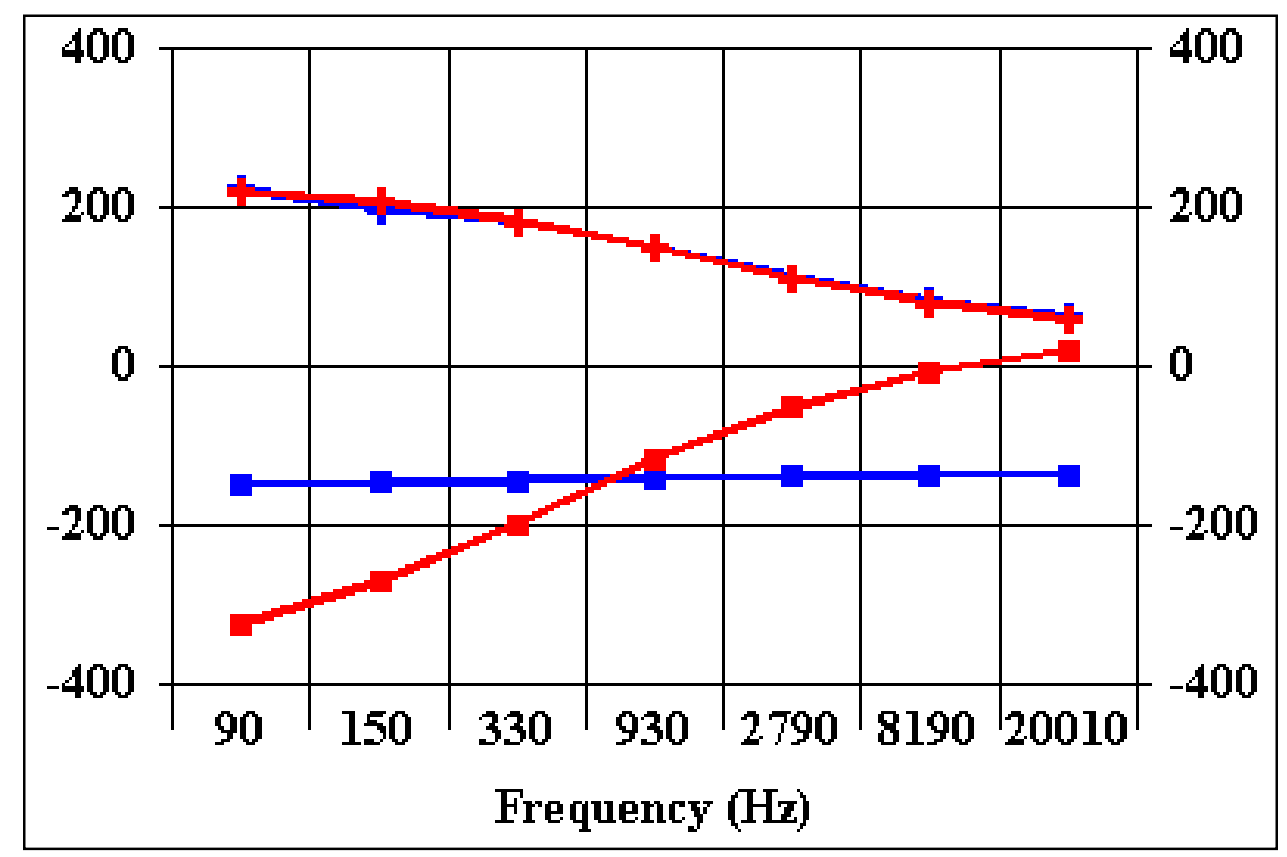

Figure 62. Best matching point for target 3-84 (81-mm mortar)

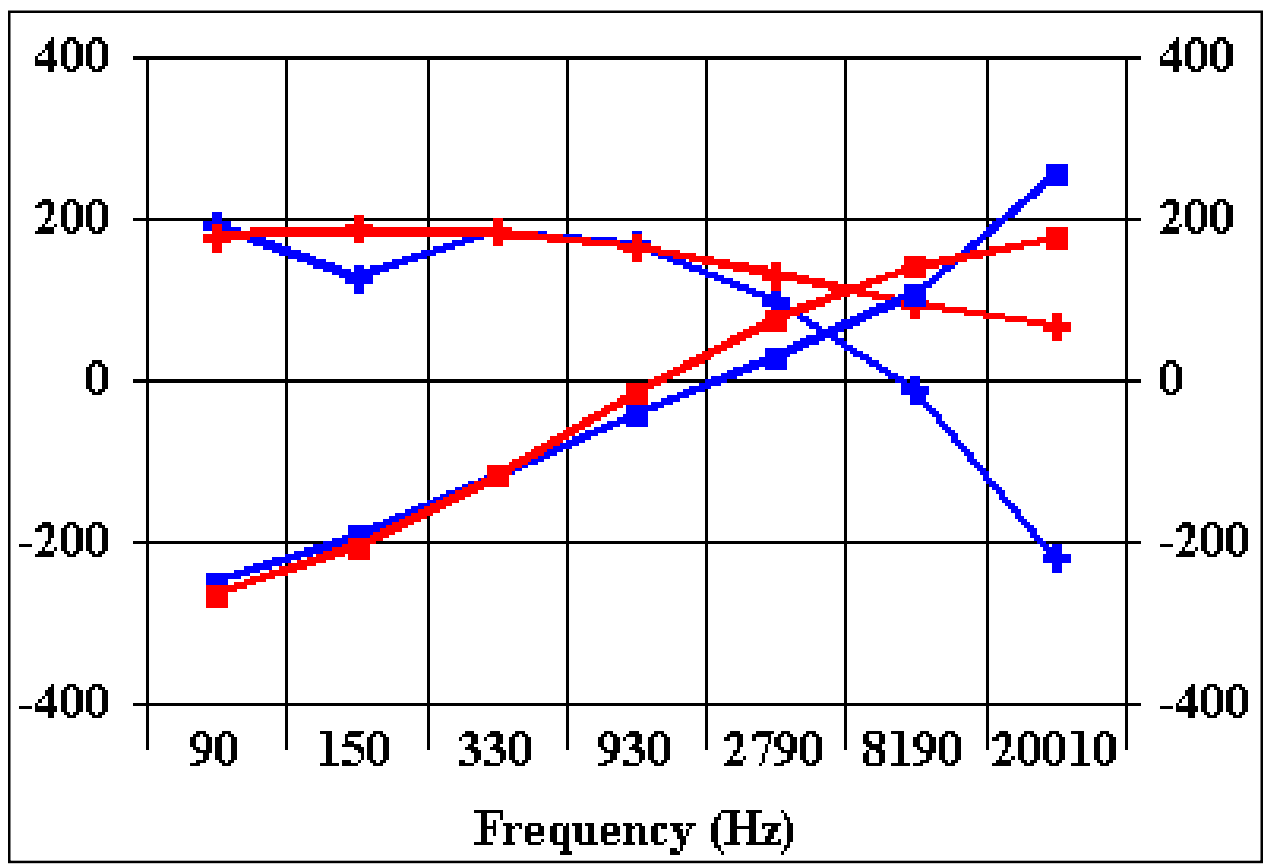

Figure 63. Best matching point for target 3-100 (152-mm projectile) 


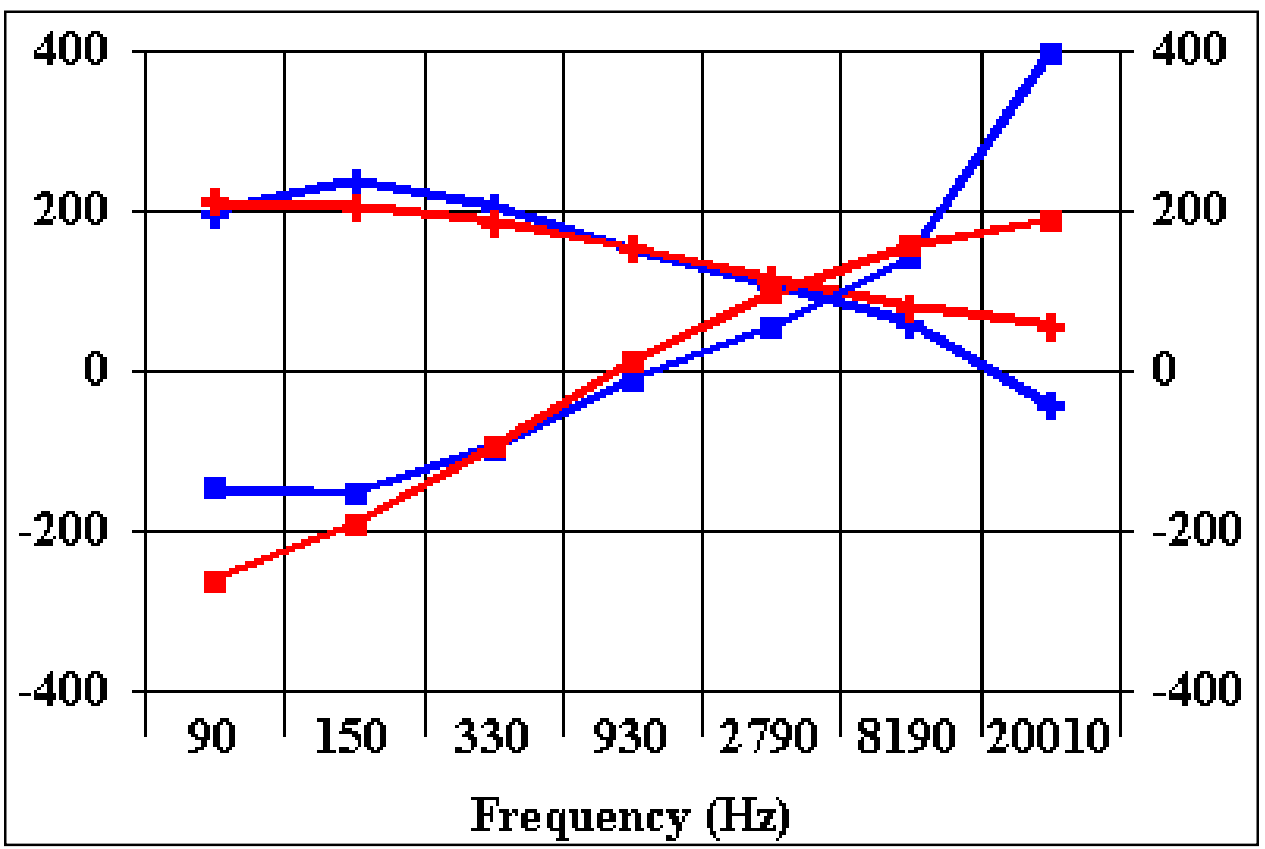

Figure 64. Best matching point for target 2-142 (152-mm projectile)

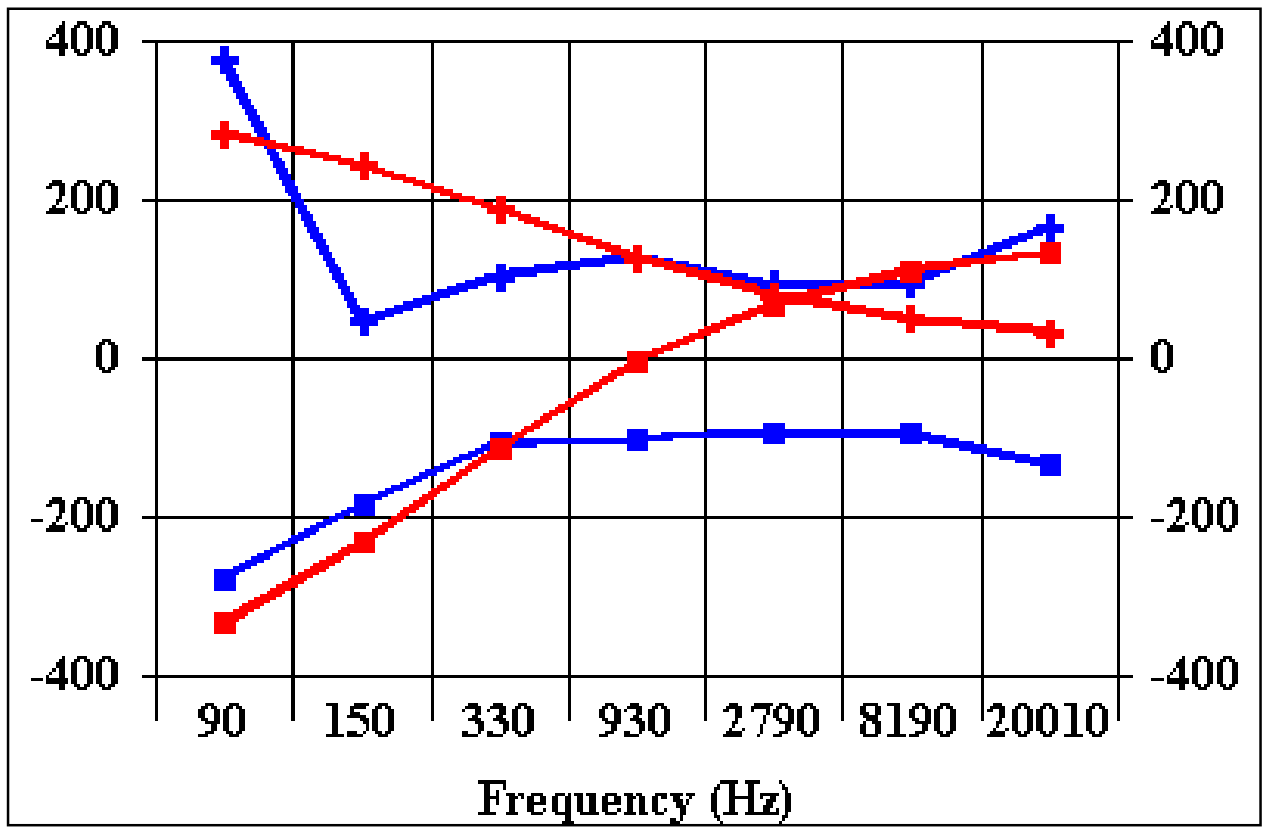

Figure 65. Best matching point for target 3-102 (155-mm projectile) 


\section{Depth effects}

The effect of depth on the signal strength of the GEM-3 is apparent in Error!

Reference source not found., which shows the average total magnitude of data points within a $1-\mathrm{m}$ box centered on the target vs. target depth for the $155-\mathrm{mm}$ projectiles, 81-mm mortars, and 60-mm mortars emplaced at JPG. The total magnitude of a data point is the sum of the absolute values of the in-phase and quadrature measurements for all frequesncies. For all three ordnance types, the signal strength decreases sharply with increasing depth. The effect of target depth on the classification of targets can best be illustrated by examining the data for the $155-\mathrm{mm}$ projectiles, which were the largest targets in the demonstration. A total of five 155-mm projectiles were included in the demonstration at depths of $50,75,102$, and $120 \mathrm{~cm}$. The data points near the two $155-\mathrm{mm}$ projectiles buried at $50 \mathrm{~cm}$, targets 1-121 and 1-136, had an average total magnitude of over 2,000 , and nearly all of them matched the calibration signatures quite well. The points near target 2-161, buried at $75 \mathrm{~cm}$, have an average magnitude of 396 and also match the calibration signatures well. The data points near the two deepest $155-\mathrm{mm}$ projectiles, targets 2-156 and 3-102, have an average magnitude of less than 200 . The deepest, target 3-102, has no points with a recognizable signature. Target 2-156 has three points that match the calibration signature for a $155-\mathrm{mm}$ projectile with an error just under 0.1, although they match other ordnance types slightly better. From those data, it appears that reliable classification of $155-\mathrm{mm}$ projectiles at depths greater than $1 \mathrm{~m}$ is unlikely. Classification of smaller ordnance types will suffer from weak signal at shallower depths. Other targets that produced weak signatures because of depth include three $152-\mathrm{mm}$ projectiles, targets $1-117,2-142$, and 3-100, all at depths of approximately $90 \mathrm{~cm}$ and two $0.07-\mathrm{m}(2.75-\mathrm{in}$.) rockets, targets $1-153$ and 2-166, both at depths of $76 \mathrm{~cm}$. Three targets, [2-132 $(0.13 \mathrm{~m}(5$-in. $)$ projectile at a depth of $91 \mathrm{~cm})$, 2-140 (105-mm projectile at a depth of 70 $\mathrm{cm})$, and 3-104 $(76-\mathrm{mm}$ projectile at a depth of $76 \mathrm{~cm}$ )], for which there were no calibration data also appeared to be too deep for classification. Targets of these types at shallower depths were classified as targets of similar size for which calibration data were available.

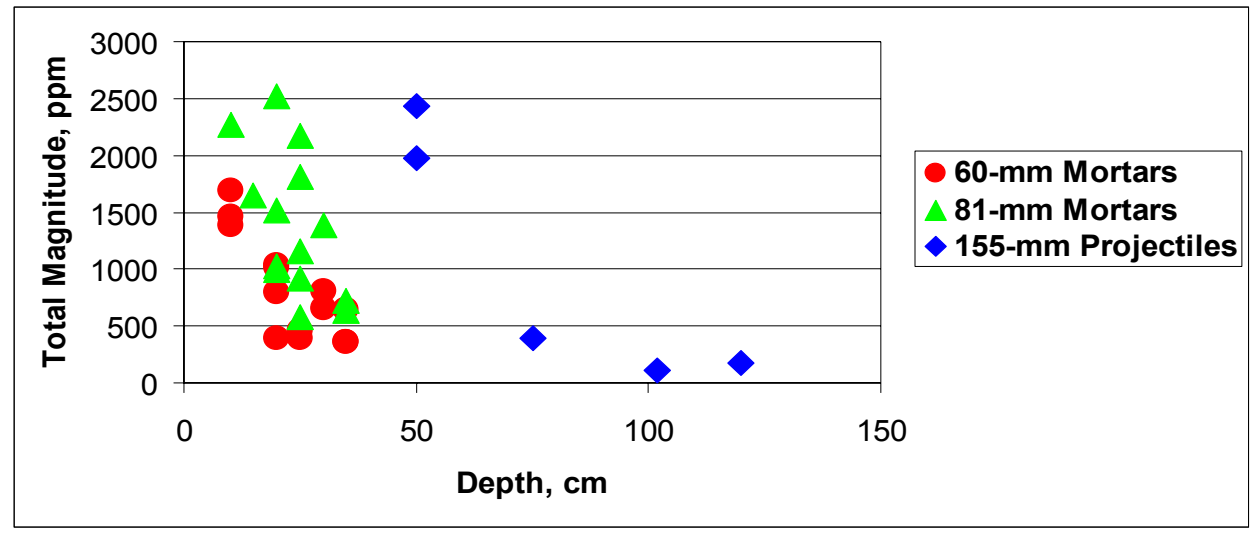

Figure 66. Average magnitude vs. depth for selected ordnance types 


\section{Errors by frequency}

For many data points, the error in fit with the calibration data was dominated by the measurement for a single frequency. In such cases, excluding that frequency from the calculations produced a much closer fit of the point with the calibration data. An example of this is shown in Figures 67 and 68. The $90-\mathrm{Hz}$ data are included in the graph in Figure 68 for reference but not included in the magnitude normalization or error calculations. Using all frequencies, as shown in Figure 67, the error in fit was calculated to be 0.1369 , while excluding the 90 $\mathrm{Hz}$ data reduced the error to only 0.0645 .

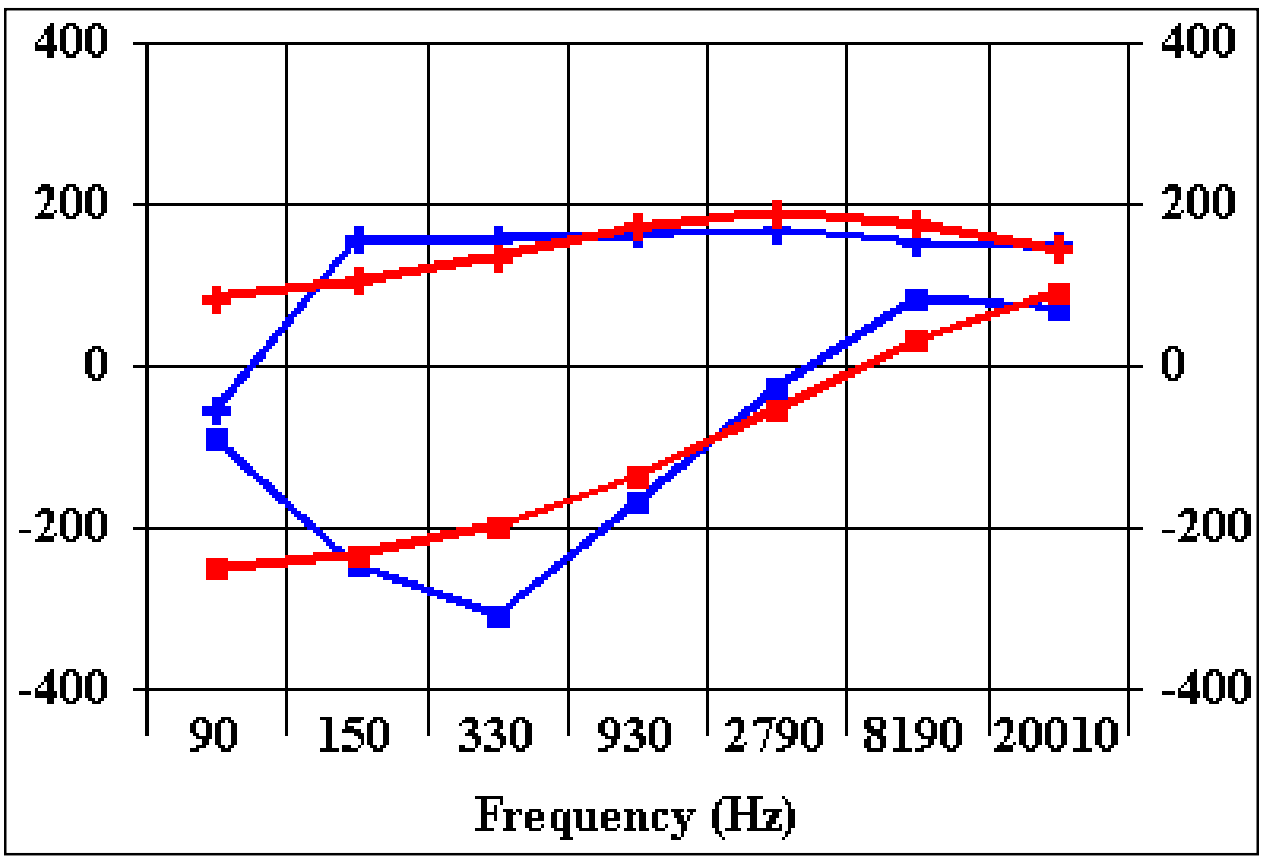

Figure 67. Calibration match with all frequencies for target 1-132 (57-mm mortar)

Although $90 \mathrm{~Hz}$ was the most likely frequency to cause problems, it was not the only one that did. The error in fit of the data points shown in a previous section for targets 2-118, 2-142, and 3-100 are dominated by the $20,010 \mathrm{~Hz}$ measurement. Excluding that frequency brings the error in fit for all three data points to less than 0.08 . In almost all cases, data points with large errors in one frequency were relatively low in magnitude.

To determine the overall contributions of particular frequencies to the error between data points and calibration signatures throughout the data set, the differences between all the data points and corresponding calibration signatures for each frequency were averaged. The results are shown in Figure 69. The lowest frequency, $90 \mathrm{~Hz}$, has a substantially larger average difference than the other frequencies. 


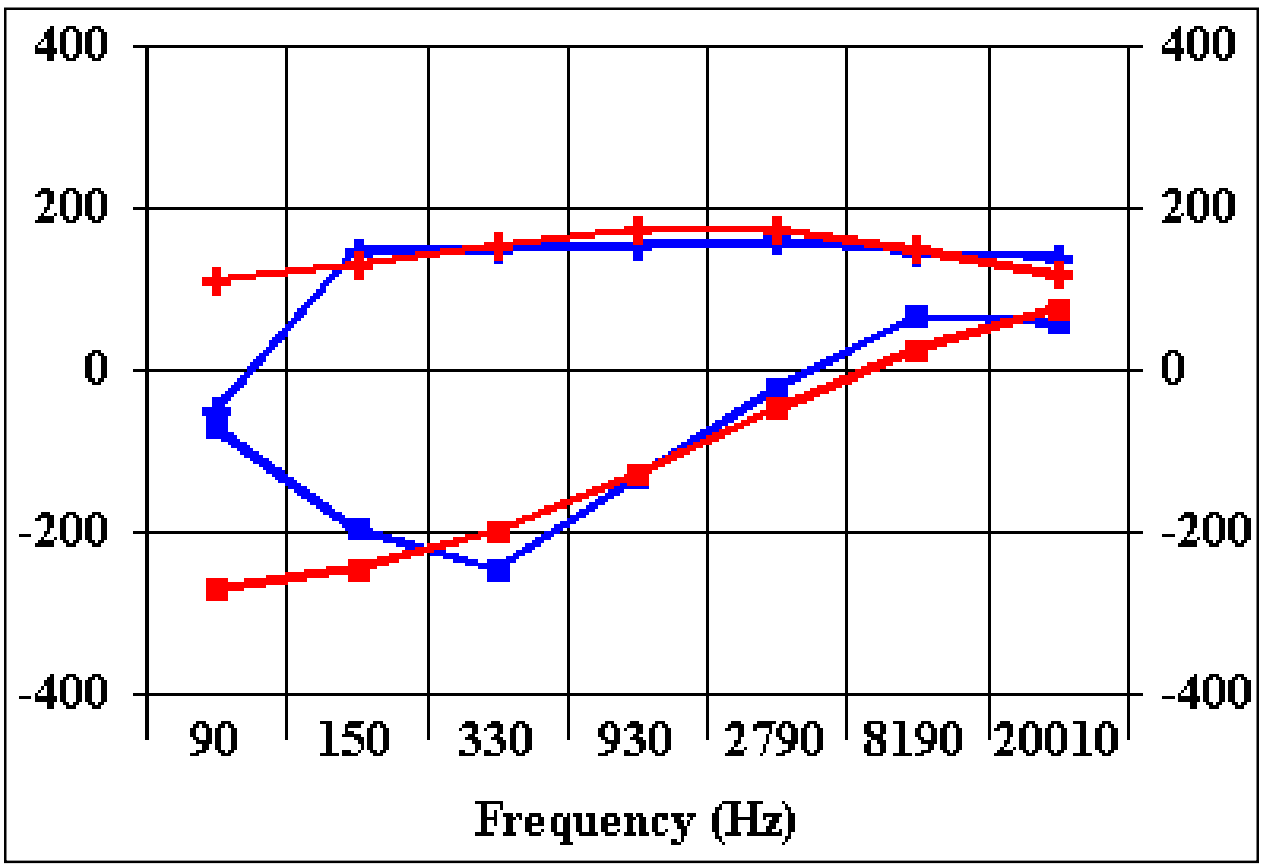

Figure 68. Calibration match without $90 \mathrm{~Hz}$ for target 1-132 (57-mm mortar)

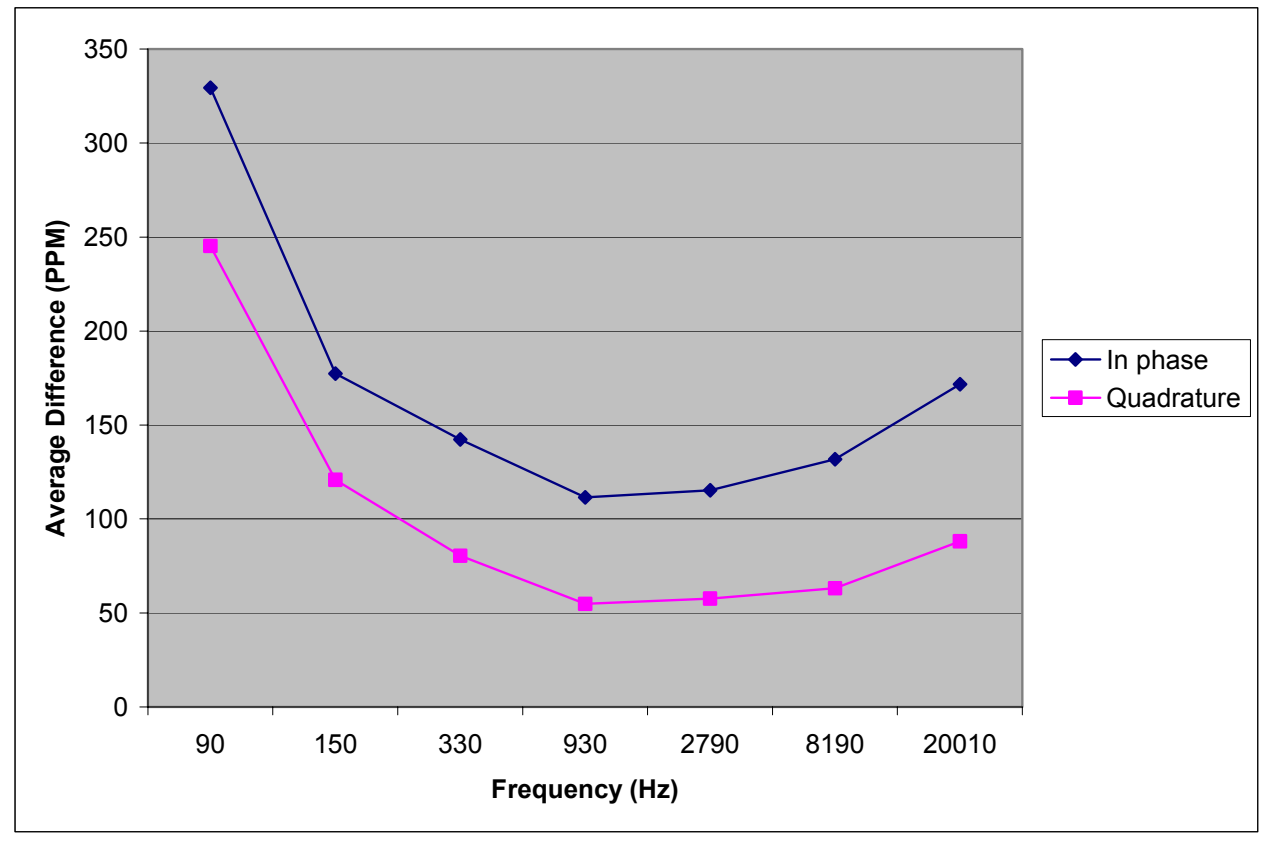

Figure 69. Average difference by frequency 


\section{Classification of Targets}

Figure 70 through 72 show the calibration data for all the different ordnance types at 0,90 , and $-90 \mathrm{deg}$, respectively. These data have been normalized to the same total quadrature and the same total in-phase responses so that the relative responses of each ordnance type at each of the seven frequencies can be compared. Some of the ordnance types have very similar signatures to each other. A simple classification procedure was applied to the JPG data to examine the separability of the different ordnance types from each other. Each target was classified using the single data point with the greatest magnitude of the points near the target. The error in fit between the data point and each ordnance type at each inclination angle was calculated. The target was classified as the ordnance type that had the lowest error in fit at any inclination angle. The results are shown in Table 11. Overall, 41.9 percent (39/93) of the targets were classified correctly. Excluding the three ordnance types for which no calibration data were available, 48.8 percent $(39 / 80)$ of the targets were correctly classified, including 60.1 percent (20/33) of the mortars, 40.0 percent (16/40) of the projectiles, and 42.9 percent $(3 / 7)$ of the rockets. Because of the very similar signatures of some of the targets, a more informative way to look at the classification matrix would be to group the targets by size as shown in Table 12 . With aggregation by size, 71.0 percent (66/93) of the targets are classified in the correct group, including 78.1 percent $(56 / 73)$ of the medium and large targets.

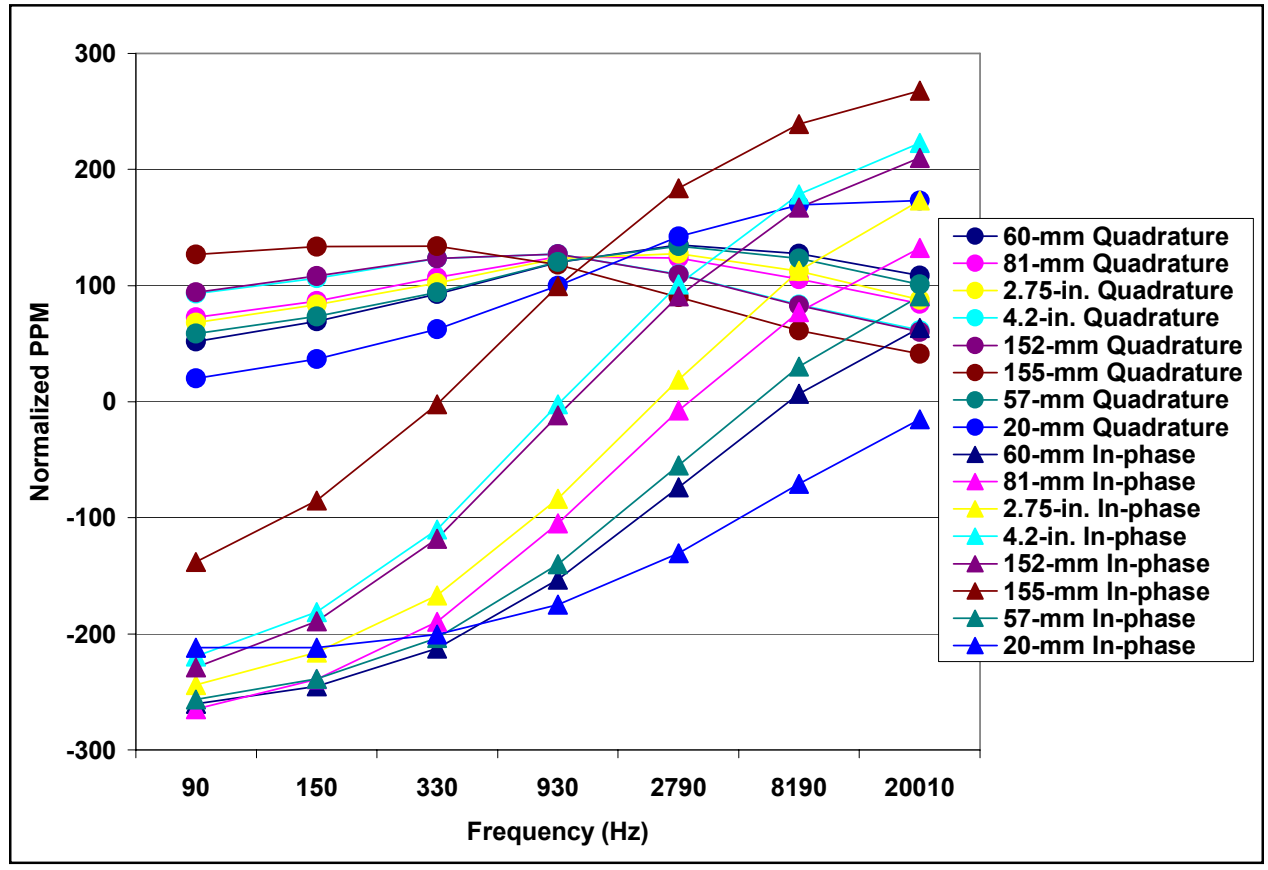

Figure 70. Calibration data with targets at 0-deg inclination 


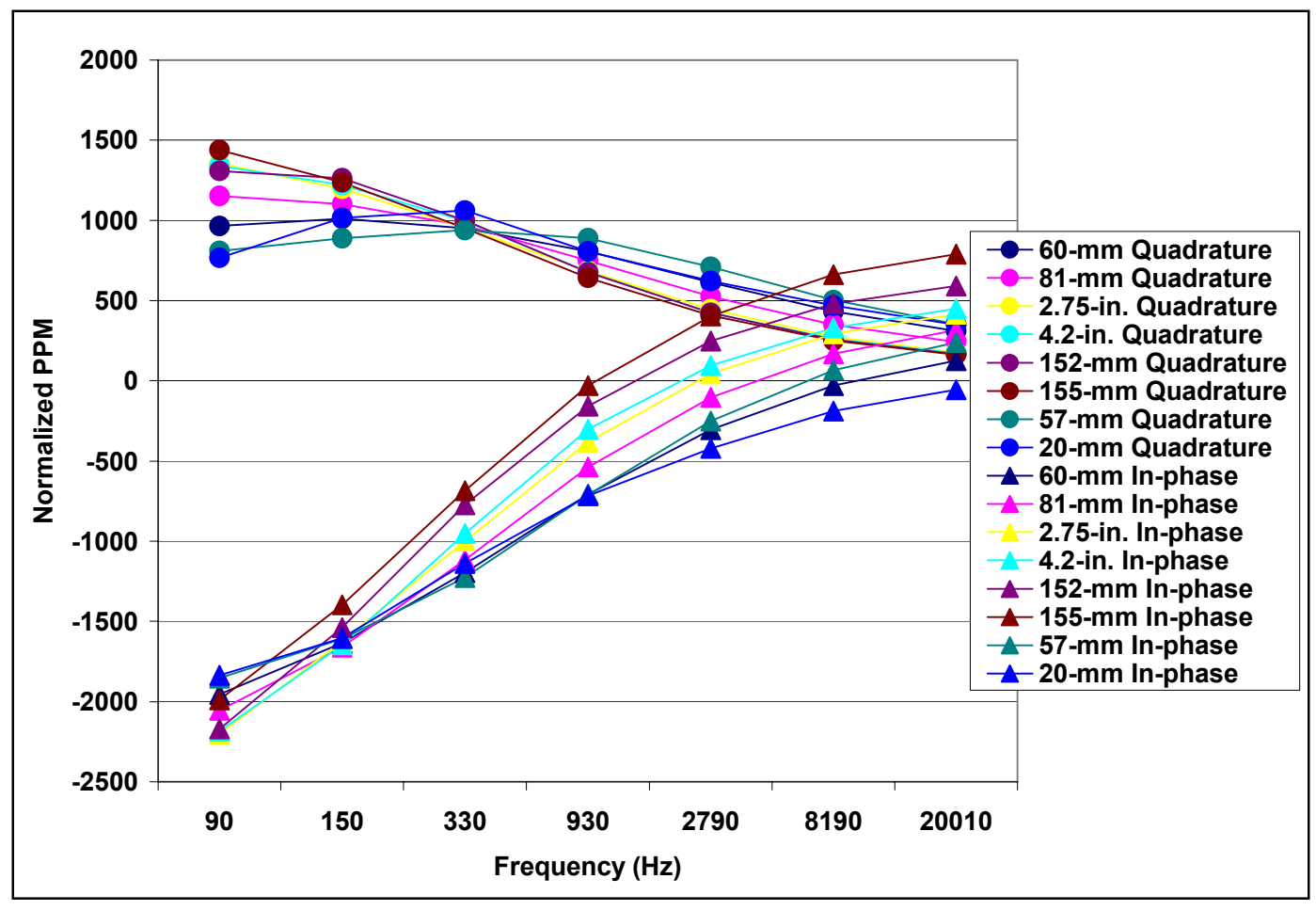

Figure 71. Calibration data with targets at 90 deg inclination (noseup)

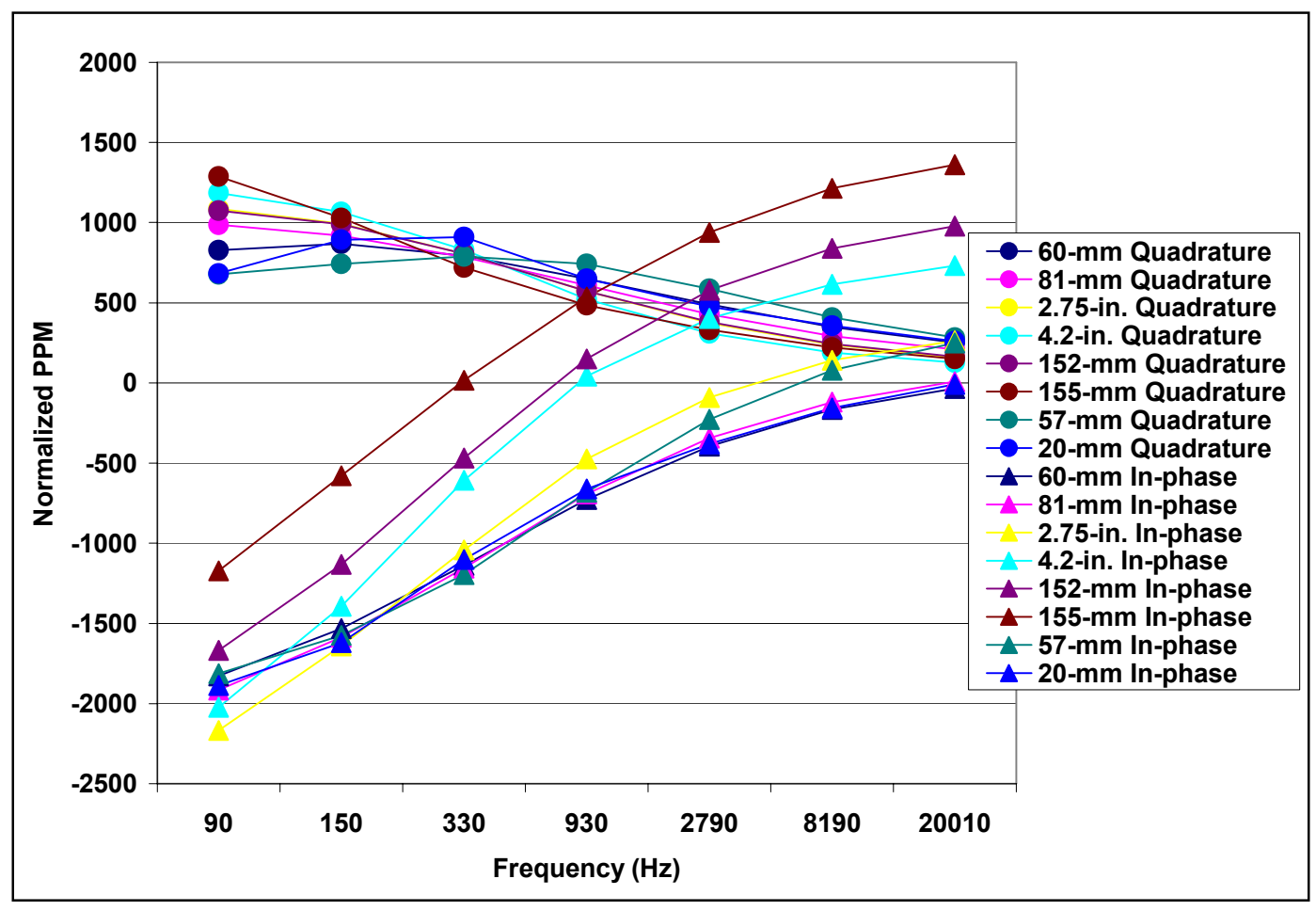

Figure 72. Calibration data with targets at -90 deg inclination (nosedown) 


\begin{tabular}{|c|c|c|c|c|c|c|c|c|c|c|c|c|c|}
\hline \multicolumn{14}{|c|}{$\begin{array}{l}\text { Table } 11 \\
\text { Classification Matrix Using All Frequencies }\end{array}$} \\
\hline \multirow[b]{2}{*}{ Classification } & \multicolumn{13}{|c|}{ "Ordnance Type } \\
\hline & $\begin{array}{l}20- \\
\mathrm{mm}\end{array}$ & 57-mm & $60-\mathrm{mm}$ & $\begin{array}{l}2.75- \\
\text { in. } \\
\end{array}$ & $76-\mathrm{mm}$ & $81-\mathrm{mm}$ & $105-\mathrm{mm}$ & 4.2-in. & \begin{tabular}{|l|}
$5.0-$ \\
in.
\end{tabular} & $\begin{array}{l}152- \\
\mathrm{mm}\end{array}$ & $\begin{array}{l}155- \\
\mathrm{mm}\end{array}$ & FA & Total \\
\hline 20-mm & 9 & 1 & 3 & 2 & 0 & 0 & 0 & 0 & 0 & 0 & 0 & 0 & 15 \\
\hline $57-\mathrm{mm}$ & 1 & 4 & 2 & 0 & 0 & 3 & 0 & 0 & 0 & 0 & 0 & 0 & 10 \\
\hline $60-\mathrm{mm}$ & 3 & 3 & 8 & 2 & 0 & 1 & 0 & 0 & 0 & 0 & 1 & 0 & 18 \\
\hline 2.75 -in & 4 & 0 & 0 & 3 & 1 & 2 & 0 & 0 & 0 & 1 & 1 & 0 & 12 \\
\hline $76-\mathrm{mm}$ & 0 & 0 & 0 & 0 & 0 & 0 & 0 & 0 & 0 & 0 & 0 & 0 & 0 \\
\hline $81-\mathrm{mm}$ & 0 & 1 & 1 & 0 & 1 & 9 & 1 & 0 & 0 & 1 & 0 & 0 & 14 \\
\hline $105-\mathrm{mm}$ & 0 & 0 & 0 & 0 & 0 & 0 & 0 & 0 & 0 & 0 & 0 & 0 & 0 \\
\hline 4.2 -in & 0 & 0 & 0 & 0 & 1 & 0 & 0 & 3 & 0 & 0 & 0 & 0 & 4 \\
\hline 5.0 -in & 0 & 0 & 0 & 0 & 0 & 0 & 0 & 0 & To & 0 & 0 & 0 & 0 \\
\hline $152-\mathrm{mm}$ & 0 & 1 & 0 & 0 & 0 & 0 & 0 & 0 & 1 & 0 & 0 & 0 & 2 \\
\hline $155-\mathrm{mm}$ & 3 & 0 & 1 & 0 & 2 & 0 & 3 & 0 & 3 & 3 & 3 & 0 & 18 \\
\hline Nonord & 0 & 0 & 0 & 0 & 0 & 0 & 0 & 0 & 0 & 0 & 0 & 0 & 0 \\
\hline \multirow{2}{*}{ Total } & 20 & 10 & 15 & 7 & 5 & 15 & 4 & 3 & 4 & 5 & 5 & 0 & 93 \\
\hline & & & & & & & & & & & & & \\
\hline $\begin{array}{l}\text { \%classified } \\
\end{array}$ & 45.0 & 40.0 & 53.3 & 42.9 & 0.0 & 60.0 & 0.0 & 100.0 & 0.0 & 0.0 & 60.0 & & \\
\hline
\end{tabular}

\begin{tabular}{|c|c|c|c|}
\hline $\begin{array}{l}\text { Table } 12 \\
\text { Classification } \\
\text { Ordnance Size }\end{array}$ & atrix Using & I Frequencies & ggregated by \\
\hline & & Ordnan & уре \\
\hline Classification & 20-mm & 57 to $81-\mathrm{mm}$ & 105 to $155-\mathrm{mm}$ \\
\hline 20-mm & $\overline{99}$ & $\bar{~} 6$ & 0 \\
\hline 57- to $81-\mathrm{mm}$ & 8 & 41 & 5 \\
\hline $105-$ to $155-\mathrm{mm}$ & 3 & 5 & 16 \\
\hline Total & 20 & 52 & 21 \\
\hline$\%$ classified & 45.0 & 78.8 & 76.2 \\
\hline
\end{tabular}

Because of the larger average difference between the data points and the calibration data for the $90 \mathrm{~Hz}$ data, that frequency was excluded from the calculations to see if the results would improve. Tables 13 and 14 show the complete and aggregated results of this classification, respectively. Also, because of reports from Geophex that both the $90 \mathrm{~Hz}$ and $150 \mathrm{~Hz}$ data were noisy, a classification was performed excluding both of those frequencies. The complete and aggregated classification matrices excluding 90 and $150 \mathrm{~Hz}$ are shown in and Tables 15 and 16, respectively. The results did not change significantly in either of these classifications compared with the classification using all frequencies. A total of 40 targets were classified correctly in each of them, one more than were classified correctly using all frequencies. Several targets changed classifications when the $90 \mathrm{~Hz}$ data were excluded, but the gains and losses essentially canceled each other out. Viewing the data for individual targets indicates that excluding the $90 \mathrm{~Hz}$ data reduces the errors of data points 


\begin{tabular}{|c|c|c|c|c|c|c|c|c|c|c|c|c|c|}
\hline \multicolumn{14}{|c|}{\begin{tabular}{|l} 
Table 13 \\
Classification Matrix without $90 \mathrm{~Hz}$ Data
\end{tabular}} \\
\hline \multirow[b]{2}{*}{ Classification } & \multicolumn{13}{|c|}{ "Ordnance Type } \\
\hline & $\begin{array}{l}20- \\
\mathrm{mm}\end{array}$ & $57-\mathrm{mm}$ & $60-\mathrm{mm}$ & $\begin{array}{l}2.75- \\
\text { in. }\end{array}$ & 76-mm & $81-\mathrm{mm}$ & 105-mm & 4.2-in. & $\begin{array}{l}5.0- \\
\text { in. } \\
\end{array}$ & $152-\mathrm{mm}$ & $155-\mathrm{mm}$ & FA & Total \\
\hline 20-mm & 9 & 1 & 3 & 3 & 0 & 2 & 1 & 0 & 0 & 2 & 1 & 0 & 22 \\
\hline $57-\mathrm{mm}$ & 3 & 4 & 2 & 0 & 0 & 3 & 0 & 0 & 0 & 0 & 0 & 0 & 12 \\
\hline $60-\mathrm{mm}$ & 5 & 3 & 9 & 1 & 0 & 1 & 0 & 0 & 0 & 0 & 0 & 0 & 19 \\
\hline 2.75-in. & 0 & 0 & 1 & 2 & 0 & 0 & 0 & 0 & 0 & 0 & 0 & 0 & 3 \\
\hline 76-mm & 0 & 0 & 0 & 0 & 0 & 0 & 0 & 0 & 0 & 0 & 0 & 0 & 0 \\
\hline 81-mm & 1 & 1 & 0 & 1 & 1 & 9 & 0 & 0 & 1 & 0 & 0 & 0 & 14 \\
\hline $105-\mathrm{mm}$ & 0 & 0 & 0 & 0 & 0 & 0 & 0 & 0 & 0 & 0 & 0 & 0 & 0 \\
\hline 4.2-in. & 0 & 1 & 0 & 0 & 1 & 0 & 0 & 2 & 0 & 0 & 0 & 0 & 4 \\
\hline 5.0-in. & 0 & 0 & 0 & 0 & 0 & 0 & 0 & 0 & 0 & 0 & 0 & 0 & 0 \\
\hline 152-mm & 0 & 0 & 0 & 0 & 1 & 0 & 0 & 1 & 1 & 1 & 0 & 0 & 4 \\
\hline 155-mm & 2 & 0 & 0 & 0 & 2 & 0 & 3 & 0 & 2 & 2 & 4 & 0 & 15 \\
\hline Nonord & 0 & 0 & 0 & 0 & 0 & 0 & 0 & 0 & 0 & 0 & 0 & 0 & 0 \\
\hline Total & 20 & 10 & 15 & 7 & 5 & 15 & 4 & 3 & 4 & 5 & 5 & 0 & 93 \\
\hline & & & & & & & & & & & & & \\
\hline \%classified & 45.0 & 40.0 & 60.0 & 28.6 & 0.0 & 60.0 & 0.0 & 66.7 & 0.0 & 20.0 & 80.0 & & \\
\hline
\end{tabular}

\begin{tabular}{|c|c|c|c|}
\hline \begin{tabular}{|l} 
Table 14 \\
Classificatior \\
Size
\end{tabular} & trix with & 0-Hz Data A & regated by Ordnance \\
\hline & & Ordnan & Type \\
\hline Classification & 20-mm & 57 to $81-\mathrm{mm}$ & 105 to $155-\mathrm{mm}$ \\
\hline $20-\mathrm{mm}$ & 9 & 9 & 4 \\
\hline 57 - to $81-\mathrm{mm}$ & 9 & 38 & 1 \\
\hline 105- to $155-\mathrm{mm}$ & 2 & 5 & 16 \\
\hline Total & 20 & 52 & 21 \\
\hline \% classified & 45.0 & 73.1 & 76.2 \\
\hline
\end{tabular}

relative to the calibration data, particular for data points with weak signals. This is as expected, given the greater noise level of the data acquired at that frequency. However, excluding some frequencies increases the potential for confusion between similar ordnance types.

\section{Classification of All Detected Anomalies}

The classifications in the preceding section involved only data points near actual targets; therefore, it demonstrates only the capability to separate different types of ordnance from each other given the presence of a target. However, in a realistic search scenario, real targets must be separated from clutter. To examine this aspect of classification with the GEM-3, data points near all objects declared by Geophex were extracted and the classification procedure repeated. An 


\begin{tabular}{|c|c|c|c|c|c|c|c|c|c|c|c|c|c|}
\hline \multicolumn{14}{|c|}{\begin{tabular}{|l} 
Table 15 \\
Classification Matrix without $90-\mathrm{Hz}$ and $150-\mathrm{Hz}$ Data
\end{tabular}} \\
\hline \multirow[b]{2}{*}{ Classification } & \multicolumn{13}{|c|}{ "Ordnance Type } \\
\hline & \begin{tabular}{|l|}
$20-$ \\
$\mathrm{mm}$
\end{tabular} & $57-\mathrm{mm}$ & $60-\mathrm{mm}$ & $\begin{array}{l}2.75- \\
\text { in. }\end{array}$ & 76-mm & $81-\mathrm{mm}$ & 105-mm & 4.2-in. & $\begin{array}{l}5.0- \\
\text { in. } \\
\end{array}$ & $152-\mathrm{mm}$ & $155-\mathrm{mm}$ & FA & Total \\
\hline 20-mm & 11 & $\begin{array}{ll}1 \\
\end{array}$ & 3 & 3 & \begin{tabular}{l|l}
0 \\
\end{tabular} & 2 & 1 & 0 & 0 & 2 & 1 & 0 & 24 \\
\hline $57-\mathrm{mm}$ & 1 & 4 & 2 & 0 & 0 & 3 & 0 & 0 & 0 & 0 & 0 & 0 & 10 \\
\hline $60-\mathrm{mm}$ & 4 & 3 & 8 & 1 & 0 & 1 & 0 & 0 & 0 & 0 & 0 & 0 & 17 \\
\hline 2.75 -in. & 0 & 0 & 1 & 1 & 0 & 0 & 0 & 0 & 0 & 0 & 0 & 0 & 2 \\
\hline 76-mm & 0 & 0 & 0 & 0 & 0 & 0 & 0 & 0 & 0 & 0 & 0 & 0 & 0 \\
\hline $81-m m$ & 2 & 1 & 1 & 1 & 1 & 9 & 0 & 0 & 0 & 0 & 0 & 0 & 15 \\
\hline $105-\mathrm{mm}$ & 0 & 0 & 0 & 0 & 0 & 0 & 0 & 0 & 0 & 0 & 0 & 0 & 0 \\
\hline 4.2-in. & 0 & 0 & 0 & 0 & 1 & 0 & 0 & 3 & 0 & 1 & 0 & 0 & 5 \\
\hline 5.0-in. & 0 & 0 & 0 & 0 & 0 & 0 & 0 & 0 & 0 & 0 & 0 & 0 & 0 \\
\hline 152-mm & 0 & 0 & 0 & 0 & 1 & 0 & 0 & 0 & 1 & 0 & 0 & 0 & 2 \\
\hline $155-\mathrm{mm}$ & 2 & 1 & 0 & 1 & 2 & 0 & 3 & 0 & 3 & 2 & 4 & 0 & 18 \\
\hline \multirow[t]{2}{*}{ Nonord } & 0 & 0 & 0 & 0 & 0 & 0 & 0 & 0 & 0 & 0 & 0 & 0 & 0 \\
\hline & & & & & & & & & & & & & \\
\hline \multirow[t]{2}{*}{ Total } & 20 & 10 & 15 & 7 & 5 & 15 & 4 & 3 & 4 & 5 & 5 & 0 & 93 \\
\hline & & & & & & & & & & & & & \\
\hline \%classified & 55.0 & 40.0 & 53.3 & 14.3 & 0.0 & 60.0 & 0.0 & 100.0 & 0.0 & 0.0 & 80.0 & & \\
\hline
\end{tabular}

\begin{tabular}{|c|c|c|c|}
\hline $\begin{array}{l}\text { Table } 16 \\
\text { Classification } \\
\text { Ordnance Siz }\end{array}$ & atrix with & $90-\mathrm{Hz}$ and 15 & Az Data Aggregated by \\
\hline & & Ordnan & Type \\
\hline Classification & 20-mm & 57 to $81-\mathrm{mm}$ & 105 to $155-\mathrm{mm}$ \\
\hline $20-\mathrm{mm}$ & 11 & 9 & 4 \\
\hline $57-$ to $81-\mathrm{mm}$ & 7 & 37 & 0 \\
\hline 105- to $155-\mathrm{mm}$ & 2 & 6 & 17 \\
\hline Total & 20 & 52 & 21 \\
\hline$\%$ classified & 55.0 & 71.2 & 81.0 \\
\hline
\end{tabular}

ordnance/nonordnance threshold was specified to separate targets from clutter. The results of this classification using all frequencies at three different threshold levels are given in Tables 17 through 22. At the lowest threshold value used, $0.05,23$ of the 80 targets with calibration data were properly classified with 74 false alarms classified as ordnance. At a threshold of $0.1,31$ of the 80 targets are classified correctly with 155 false alarms classified as ordnance. At a threshold of $0.15,38$ of the 80 targets were correctly classified with 212 false alarms classified as ordnance. The biggest gains in the number of correctly classified targets as the threshold was raised were in the smaller-ordnance categories, especially the $20-\mathrm{mm}$ projectiles where the number correct went from 1 at the lowest threshold to 8 at the highest. 


\begin{tabular}{|c|c|c|c|c|c|c|c|c|c|c|c|c|c|}
\hline \multicolumn{14}{|c|}{\begin{tabular}{|l} 
Table 17 \\
Classification Matrix with Threshold of 0.05
\end{tabular}} \\
\hline \multirow[b]{2}{*}{ Classification } & \multicolumn{13}{|c|}{ Ordnance Type } \\
\hline & \begin{tabular}{|l|}
$20-$ \\
$\mathrm{mm}$
\end{tabular} & 57-mm & 60-mm & \begin{tabular}{|l|}
$2.75-$ \\
in.
\end{tabular} & 76-mm & 81-mm & $105-\mathrm{mm}$ & 4.2-in. & $\begin{array}{l}5.0- \\
\text { in. } \\
\end{array}$ & $\begin{array}{l}152- \\
\mathrm{mm}\end{array}$ & $\begin{array}{l}155- \\
\mathrm{mm}\end{array}$ & FA & Total \\
\hline $20-\mathrm{mm}$ & 1 & 0 & 1 & 0 & \begin{tabular}{|l|l|}
0 \\
\end{tabular} & \begin{tabular}{|l|}
0 \\
\end{tabular} & 0 & 0 & 0 & 0 & 0 & 18 & 20 \\
\hline $57-\mathrm{mm}$ & 0 & 4 & 2 & 0 & 0 & 2 & 0 & 0 & 0 & 0 & 0 & 5 & 13 \\
\hline $60-\mathrm{mm}$ & 0 & 2 & 5 & 1 & 0 & 0 & 0 & 0 & 0 & 0 & 0 & 12 & 20 \\
\hline $2.75 \mathrm{in}$ & 1 & 0 & 0 & 0 & 1 & 0 & 0 & 0 & 0 & 0 & 0 & 12 & 14 \\
\hline 76-mm & 0 & 0 & 0 & 0 & 0 & 0 & 0 & 0 & 0 & 0 & 0 & 0 & 0 \\
\hline $81-\mathrm{mm}$ & 0 & 1 & 1 & 0 & 1 & 8 & 0 & 0 & 0 & 0 & 0 & 13 & 24 \\
\hline $105-\mathrm{mm}$ & 0 & 0 & 0 & 0 & 0 & 0 & 0 & 0 & 0 & 0 & 0 & 0 & 0 \\
\hline 4.2-in. & 0 & 0 & 0 & 0 & 0 & 0 & 0 & 3 & 0 & 0 & 0 & 7 & 10 \\
\hline 5.0-in. & 0 & 0 & 0 & 0 & 0 & 0 & 0 & 0 & 0 & 0 & 0 & 0 & 0 \\
\hline 152-mm & 0 & 0 & 0 & 0 & 1 & 0 & 0 & 0 & 1 & 0 & 0 & 5 & 7 \\
\hline 155-mm & 0 & 0 & 0 & 0 & 0 & 0 & 1 & 0 & 0 & 0 & 2 & 2 & 5 \\
\hline Nonord & 17 & 3 & 6 & 6 & 2 & 4 & 3 & 0 & 3 & 5 & 3 & 450 & 502 \\
\hline Total & 19 & 10 & 15 & 7 & 5 & 14 & 4 & 3 & 4 & 5 & 5 & 524 & 615 \\
\hline & & & & & & & & & & & & & \\
\hline \%classified & 5.3 & 40.0 & 33.3 & 0.0 & 0.0 & 57.1 & 0.0 & 100.0 & 0.0 & 0.0 & 40.0 & & \\
\hline
\end{tabular}

\begin{tabular}{|c|c|c|c|c|}
\hline $\begin{array}{l}\text { Table } 18 \\
\text { Classification } \\
\text { of } 0.05\end{array}$ & Matrix & ated by 0 & ance Size u & Threshold \\
\hline & & & ince Type & \\
\hline Classification & 20-mm & 57 to $81-\mathrm{mm}$ & 105 to $155-\mathrm{mm}$ & False Alarm \\
\hline 20-mm & 1 & 1 & 0 & 18 \\
\hline $57-$ to $81-\mathrm{mm}$ & 1 & 28 & 0 & 42 \\
\hline $105-$ to $155-\mathrm{mm}$ & 0 & 1 & 7 & 14 \\
\hline \begin{tabular}{|l|} 
Nonordnance \\
\end{tabular} & 17 & 21 & 14 & 450 \\
\hline Total & 19 & 51 & 21 & 524 \\
\hline$\%$ classified & 5.3 & 54.9 & 33.3 & 85.9 \\
\hline
\end{tabular}




\begin{tabular}{|c|c|c|c|c|c|c|c|c|c|c|c|c|c|}
\hline \multicolumn{14}{|c|}{\begin{tabular}{|ll} 
Table 19 \\
Classification Matrix with Threshold of 0.1
\end{tabular}} \\
\hline \multicolumn{14}{|c|}{ "Ordnance Type } \\
\hline Classification & 20-mm & $57-\mathrm{mm}$ & 60-mm & \begin{tabular}{|l}
$2.75-$ \\
in. \\
\end{tabular} & 76-mm & 81-mm & 105-mm & 4.2-in. & \begin{tabular}{|l}
$5.0-$ \\
in.
\end{tabular} & $\begin{array}{l}152- \\
\mathrm{mm}\end{array}$ & $\begin{array}{l}155- \\
\mathrm{mm}\end{array}$ & FA & Total \\
\hline 20-mm & 3 & 0 & $\bar{~} 1$ & 0 & 0 & 0 & 1 & 0 & 0 & 0 & 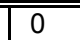 & 60 & 65 \\
\hline $57-\mathrm{mm}$ & 0 & 4 & 2 & 0 & 0 & 2 & 0 & 0 & 0 & 0 & 0 & 13 & 21 \\
\hline $60-\mathrm{mm}$ & 0 & 3 & 7 & 1 & 0 & 0 & 0 & 0 & 1 & 0 & 0 & 18 & 30 \\
\hline $2.75 \mathrm{in}$ & 2 & 0 & 0 & 1 & 1 & 0 & 0 & 0 & 0 & 0 & 0 & 20 & 24 \\
\hline 76-mm & 0 & 0 & 0 & 0 & 0 & 0 & 0 & 0 & 0 & 0 & 0 & 0 & 0 \\
\hline $81-\mathrm{mm}$ & 0 & 2 & 1 & 0 & 1 & 10 & 0 & 0 & 0 & 0 & 0 & 17 & 31 \\
\hline $105-\mathrm{mm}$ & 0 & 0 & 0 & 0 & 0 & 0 & 0 & 0 & 0 & 0 & 0 & 0 & 0 \\
\hline 4.2-in. & 0 & 0 & 0 & 1 & 0 & 0 & 0 & 3 & 0 & 1 & 0 & 11 & 16 \\
\hline 5.0-in. & 0 & 0 & 0 & 0 & 0 & 0 & 0 & 0 & 0 & 0 & 0 & 0 & 0 \\
\hline 152-mm & 0 & 0 & 0 & 0 & 1 & 0 & 0 & 0 & 1 & 0 & 0 & 6 & 8 \\
\hline 155-mm & 0 & 0 & 0 & 0 & 0 & 0 & 2 & 0 & 0 & 2 & 3 & 10 & 17 \\
\hline Nonord & 14 & 1 & 4 & 4 & 2 & 2 & 1 & 0 & 2 & 2 & 2 & 369 & 403 \\
\hline Total & 19 & 10 & 15 & 7 & 5 & 14 & 4 & 3 & 4 & 5 & 5 & 524 & 615 \\
\hline & & & & & & & & & & & & & \\
\hline \%classified & 15.8 & 40.0 & 46.7 & 14.3 & 0.0 & 71.4 & 0.0 & 100.0 & 0.0 & 0.0 & 60.0 & & \\
\hline
\end{tabular}

\begin{tabular}{|c|c|c|c|c|}
\hline \multicolumn{5}{|c|}{\begin{tabular}{|l} 
Table 20 \\
Classification Matrix Aggregated by Ordnance Size with Threshold \\
of 0.1
\end{tabular}} \\
\hline \multirow[b]{2}{*}{ Classification } & \multicolumn{4}{|c|}{$\begin{array}{ll}\text { Ordnance Type } \\
\end{array}$} \\
\hline & $20-\mathrm{mm}$ & 57- to 81-mm & 105- to $155-\mathrm{mm}$ & False Alarm \\
\hline 20-mm & 3 & 1 & 1 & 60 \\
\hline 57- to 81-mm & 2 & 35 & 1 & 68 \\
\hline 105 - to $155-\mathrm{mm}$ & 0 & 2 & 12 & 27 \\
\hline Nonordnance & 14 & 13 & 7 & 369 \\
\hline Total & 19 & 51 & 21 & 524 \\
\hline$\%$ classified & 15.8 & 68.6 & 57.1 & 70.4 \\
\hline
\end{tabular}




\begin{tabular}{|c|c|c|c|c|c|c|c|c|c|c|c|c|c|}
\hline \begin{tabular}{|l} 
Table 21 \\
Classifica \\
\end{tabular} & ion & atrix & with TI & hres & old of & 0.15 & & & & & & & \\
\hline & Ordn & ance Typ & & & & & & & & & & & \\
\hline Classification & $\begin{array}{l}20- \\
\mathrm{mm}\end{array}$ & 57-mm & 60-mm & \begin{tabular}{|l}
$2.75-$ \\
in.
\end{tabular} & 76-mm & 81-mm & $105-\mathrm{mm}$ & 4.2-in. & $\begin{array}{l}5.0- \\
\text { in. }\end{array}$ & $\begin{array}{l}152- \\
\mathrm{mm}\end{array}$ & $\begin{array}{l}155- \\
\mathrm{mm}\end{array}$ & FA & Total \\
\hline $20-\mathrm{mm}$ & 8 & 0 & 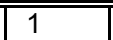 & 0 & 0 & 0 & 1 & 0 & 0 & 0 & 0 & 90 & 100 \\
\hline $57-\mathrm{mm}$ & 1 & 4 & 2 & 0 & 0 & 2 & 0 & 0 & 0 & 0 & 0 & 13 & 22 \\
\hline 60-mm & 1 & 3 & 8 & 1 & 0 & 1 & 0 & 0 & 1 & 0 & 0 & 21 & 36 \\
\hline $2.75 \mathrm{in}$ & 2 & 1 & 0 & 2 & 1 & 1 & 0 & 0 & 0 & 0 & 0 & 24 & 31 \\
\hline $76-\mathrm{mm}$ & 0 & 0 & 0 & 0 & 0 & 0 & 0 & 0 & 0 & 0 & 0 & 0 & 0 \\
\hline $81-\mathrm{mm}$ & 0 & 2 & 1 & 0 & 1 & 10 & 0 & 0 & 0 & 1 & 0 & 20 & 35 \\
\hline 105-mm & 0 & 0 & 0 & 0 & 0 & 0 & 0 & 0 & 0 & 0 & 0 & 0 & 0 \\
\hline 4.2-in. & 0 & 0 & 1 & 1 & 0 & 0 & 0 & 3 & 0 & 1 & 0 & 13 & 19 \\
\hline 5.0-in. & 0 & 0 & 0 & 0 & 0 & 0 & 0 & 0 & 0 & 0 & 0 & 0 & 0 \\
\hline 152-mm & 0 & 0 & 0 & 0 & 1 & 0 & 0 & 0 & 1 & 0 & 0 & 7 & 9 \\
\hline 155-mm & 0 & 0 & 0 & 0 & 1 & 0 & 2 & 0 & 1 & 2 & 3 & 24 & 33 \\
\hline Nonord & 7 & 0 & 2 & 3 & 1 & 0 & 1 & 0 & 1 & 1 & 2 & 312 & 330 \\
\hline & & & & & & & & & & & & & \\
\hline Total & 19 & 10 & 15 & 7 & 5 & 14 & 4 & 3 & 4 & 5 & 5 & 524 & 615 \\
\hline \begin{tabular}{|l}
$\%$ classified \\
\end{tabular} & 421 & 400 & 533 & 286 & 00 & 714 & 00 & 1000 & 00 & 00 & 600 & & \\
\hline
\end{tabular}

\begin{tabular}{|c|c|c|c|c|}
\hline $\begin{array}{l}\text { Table } 22 \\
\text { Classificatior } \\
\text { of } 0.15\end{array}$ & Matrix & ated by $\mathrm{O}$ & ance Size w & h Threshold \\
\hline & & Orc & Ince Type & \\
\hline Classification & $20-\mathrm{mm}$ & 57- to 81-mm & 105- to $155-\mathrm{mm}$ & False Alarm \\
\hline 20-mm & 8 & 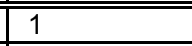 & 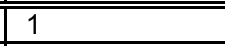 & 90 \\
\hline $57-$ to $81-\mathrm{mm}$ & 4 & 40 & 2 & 78 \\
\hline 105- to $155-\mathrm{mm}$ & 0 & 4 & 13 & 44 \\
\hline \begin{tabular}{|l} 
Nonordnance \\
\end{tabular} & 7 & 6 & 5 & 312 \\
\hline Total & 19 & 51 & 21 & 524 \\
\hline$\%$ classified & 42.1 & 78.4 & 61.9 & 59.5 \\
\hline
\end{tabular}




\section{Summary and Recommendations}

The GEM-3 data collected as part of the Advanced UXO Detection/ Discrimination Technology Demonstration at JPG have been analyzed to determine the UXO classification capabilities of the GEM-3. Although the GEM-3 performed well in detecting anomalies at the site, the classification results achieved by Geophex at JPG were somewhat disappointing, especially for the larger ordnance types. The results presented here indicate that, while sensor constraints and test parameters contributed to some misclassifications, the results could have been significantly better than they were.

Sensor coverage of the target areas at a spacing of $0.5 \mathrm{~m}$ appears to be adequate. Several data points were acquired near each target. Tighter spacing of measurements would likely improve performance against very small targets like the 20 -mm projectiles. Good signatures for these targets were obtained only when the sensor was nearly centered on the target. It is also possible that the density of measurements near some targets was not sufficient to support the classification scheme used by Geophex.

The data points acquired near each target were individually compared with calibration data for the target's ordnance type so that the variability of the data could be examined. Even allowing for differences that result from the differing orientation of the target relative to the sensor, the comparisons showed a high degree of variability among the data points near many targets. While at least one point near almost every target closely matched the signature of the correct ordnance type, there were other points near most targets that did not match the signature for any ordnance favorably. The degree of uncertainty in a given measurement makes classification unreliable, especially for small or deep targets. This report also presents statistical analysis of data of fixed targets over a period of time acquired by ERDC personnel with the GEM-3 in a preliminary attempt to determine the precision of the instrument.

The effect of the size and depth of the targets on classification was examined. The smallest ordnance in the demonstration, $20-\mathrm{mm}$ projectiles, was the only ordnance that was difficult to detect regardless of depth. All the other ordnance types produced strong responses in the sensor at shallower depths. However, the deepest targets of several ordnance types resulted in signal strength on the order of the sensor fluctuations, making reliable classification unlikely. 
To quantify the classification capabilities of the GEM-3, the targets were classified using a template-matching algorithm that compared the data point having the greatest magnitude near each object to calibration signatures for the different ordnance types and assigned the object to the closest matching ordnance type. This procedure resulted in an exact classification match for nearly half of the targets for more than two-thirds of the medium and large targets. This classification, based on a single selected data point for each detected object, is intended to serve as a baseline. In theory, more sophisticated algorithms that use all of the available data points near each object should perform better. However, because of the variability among data points near the same target, this may not be the case.

The results presented here indicate that the GEM-3, has outstanding potential to detect and classify UXO. While sensor limitations and test parameters contributed to some misclassification of objects from the JPG demonstration, the primary problem identified in this analysis is the variability in sensor response throughout the demonstration. The data taken at JPG and ERDC indicate that both drift and abrupt shifts in the level of sensor response occurred in the GEM-3 system. A careful look into the extent and cause of these sensor variations is necessary to determine if they can be removed or characterized such that they may be minimized and not affect the results of the data analysis. 


\section{References}

Cespedes, E. R. (2001). “Advanced UXO detection/discrimination technology demonstration - U.S. Army Jefferson Proving Ground, Madison, Indiana," Technical Report ERDC/EL TR-01-20, U. S. Army Engineer Research and Development Center, Vicksburg, MS.

Geophex, Ltd. (1998). GEM-3 instruction manual, Geophex, Ltd., Raleigh, NC.

Miller, J., Bell, T., Keiswetter, D., and Wright, D. (2001). "Feature-based characterization of UXO-like targets using broadband electromagnetic induction," UXO Forum 2001 Proceedings. UXO Forum 2001, New Orleans, LA.

Welch, R., and Homsey, A. (1996). "Datum shifts for UTM coordinates," Photogrammetric Engineering \& Remote Sensing 63(4), 371-375.

Won, I. J., Keiswetter, D. A., Hanson, D. R., Novikova, E., and Hall, T. M. (1997). "GEM-3: A monostatic broadband electromagnetic induction sensor," Journal of Environmental and Engineering Geophysics 2(1), 53-64.

Won, I. J., Keiswetter, D., and Novikova, E. (1998). "Electromagnetic induction spectroscopy," Journal of Environmental and Engineering Geophysics 3(1), $27-40$. 


\section{Appendix A Area Coverage Maps with Histograms}

In an effort to better understand what the data look like, it was essential to view the data in an easily understandable way. To visualize the data, it was necessary to create a separate map for each frequency measured for both the inphase and quadrature components. The pixel, representing that coordinate, is assigned a color based on a scale generated to cover the dynamic range of the data for all of the frequencies for a given component. Using the histogram that was produced earlier, the background values may be removed using the slider bars on the histogram panel, thus leaving only the "target" items visible on the plot. This can be done for either the entire area or a smaller section of the data.

Background values may be "removed" from the plots by selecting a threshold range for each frequency for both in-phase and quadrature; this allows the user to interactively select values to define background from the histogram by viewing the area map with those values removed or blacked out. The result of this process is a significantly enhanced view of the anomalies in the area map. The user may step through the different frequencies interactively removing the background from the in-phase and quadrature measurements to visually locate the anomalies in the area.

Once the plots have been rendered, the user may click on a suspected target and get the GPS coordinates and the full spectra of frequencies for both in-phase and quadrature components.

An investigation into the variation of the measurements throughout each site produces some interesting results. Figures A1-A42 show renderings of the values that were found in all the areas for both the quadrature and the in-phase measurements. These mappings were normalized globally so that the scale is the same frequency for a given area. In-phase and quadrature values are scaled to use the color map underneath the histograms at the bottom of each screen. The histograms at the bottom of the page are plotted on a vertical log scale in order to increase the vertical dynamic range that is displayed. Consequently, this will enhance the anomalies, which have fewer points and are well away from the background peak(s). 
These histograms show the distribution of all the measurements made of that site for a given frequency. An ideal site would have a large peak, which would represent the background value, and other much smaller peaks that would be indicative of targets. The less homogeneous the background, the broader the background peak should be.

Since the background peak was not sharply defined, a method to determine a more accurate background for the target was developed. The technique was to select an area around the anomaly but exclude the local area about the anomaly, and calculate a histogram for that area. It was expected that these areas would have a single well-defined peak. The location of the peak would be a good representation of the background value in that area. This was not always true and the exceptions to this are discussed further in the analysis. 


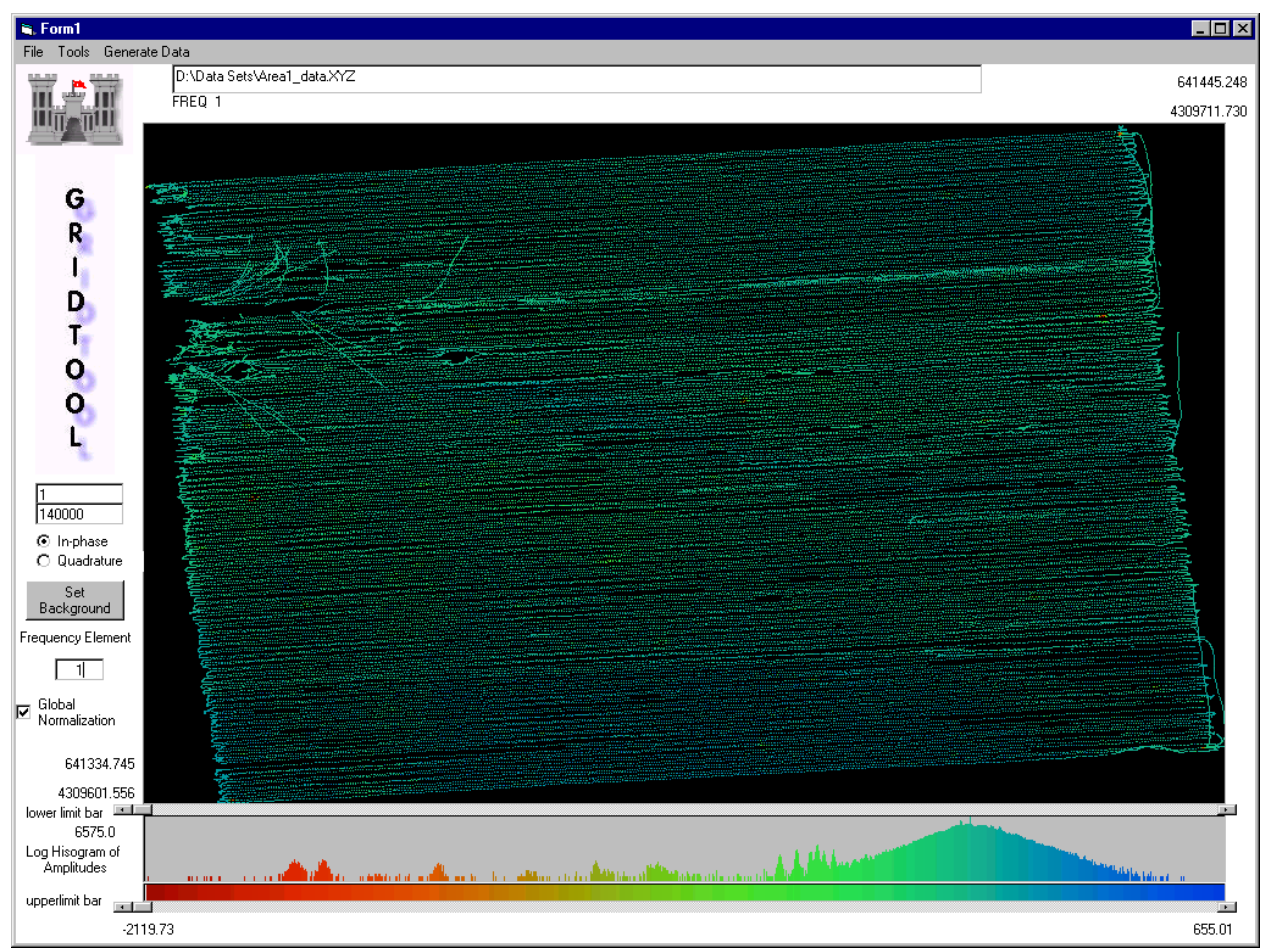

Figure A1. Area 1 in-phase coverage map for $90 \mathrm{~Hz}$

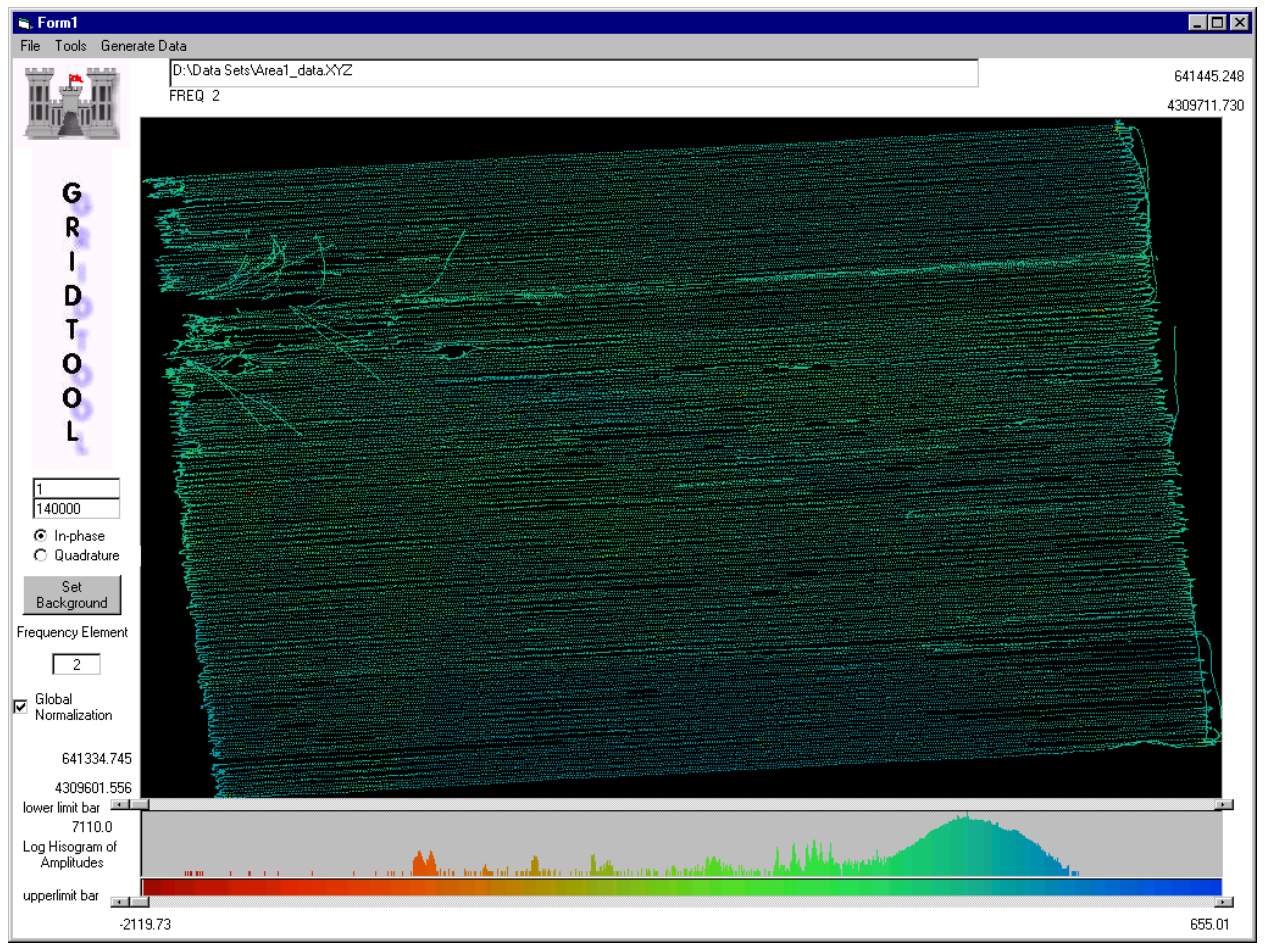

Figure A2. Area 1 in-phase coverage map for $150 \mathrm{~Hz}$ 


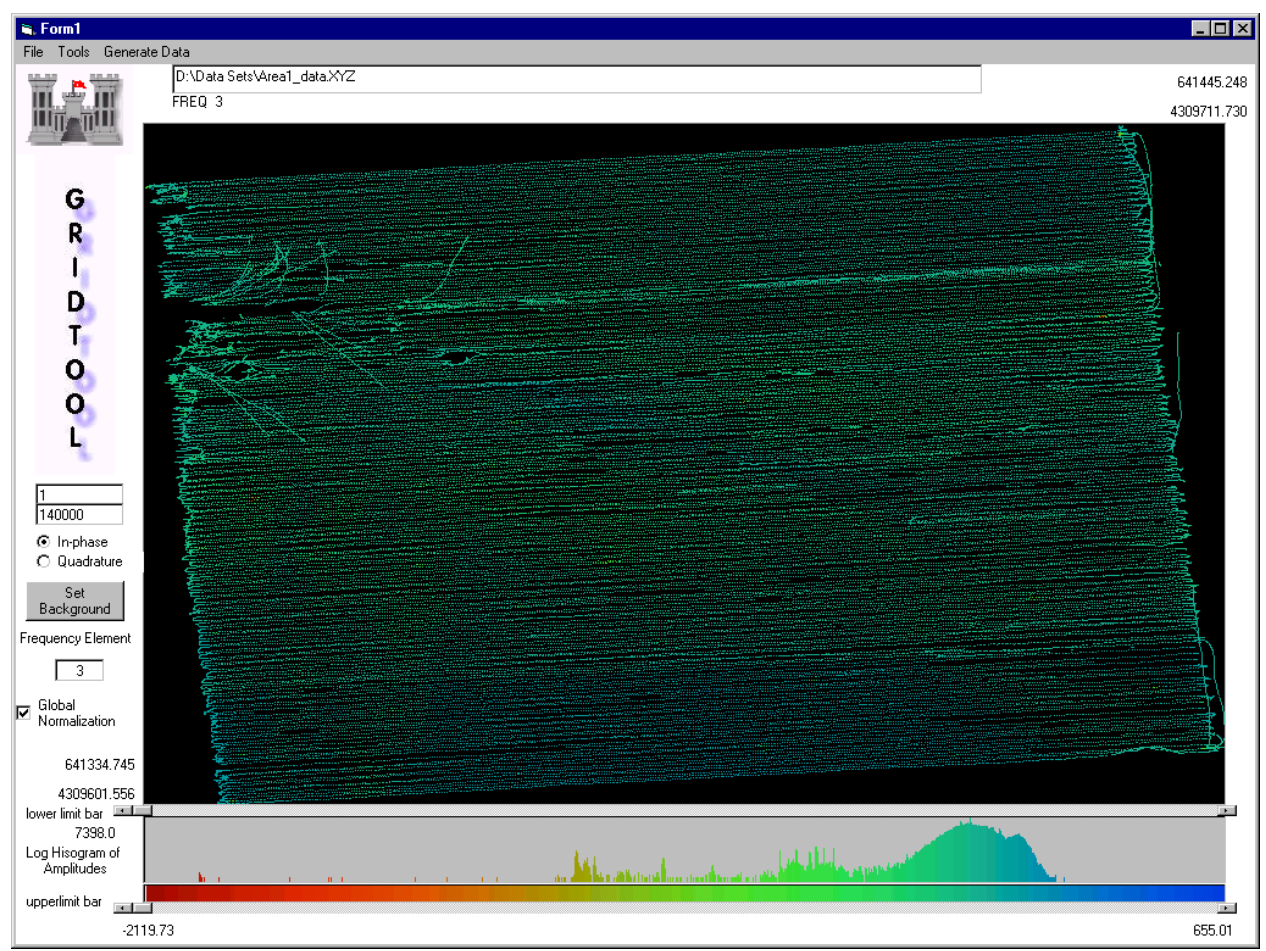

Figure A3. Area 1 in-phase coverage map for $330 \mathrm{~Hz}$

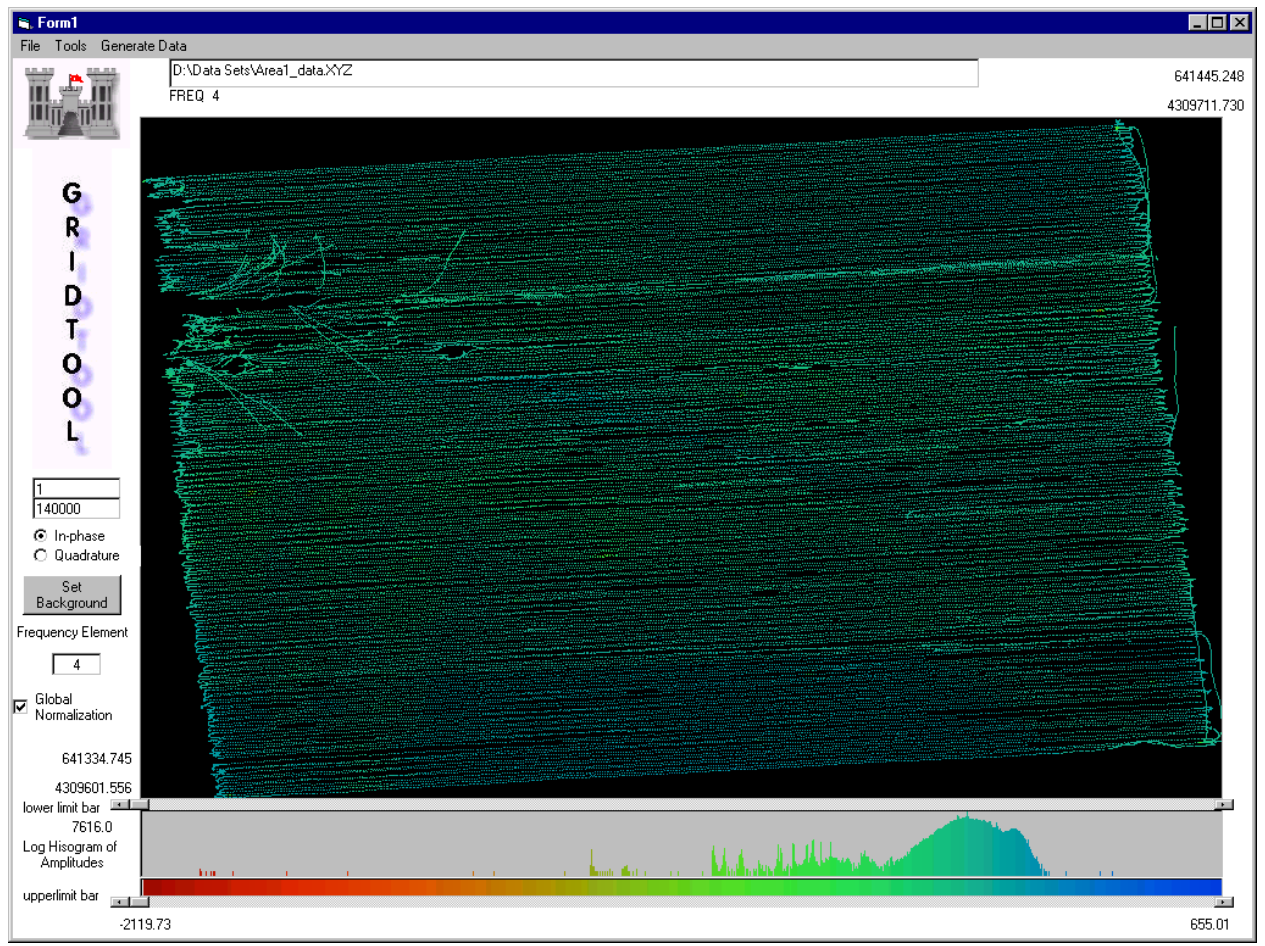

Figure A4. Area 1 in-phase coverage map for $930 \mathrm{~Hz}$ 


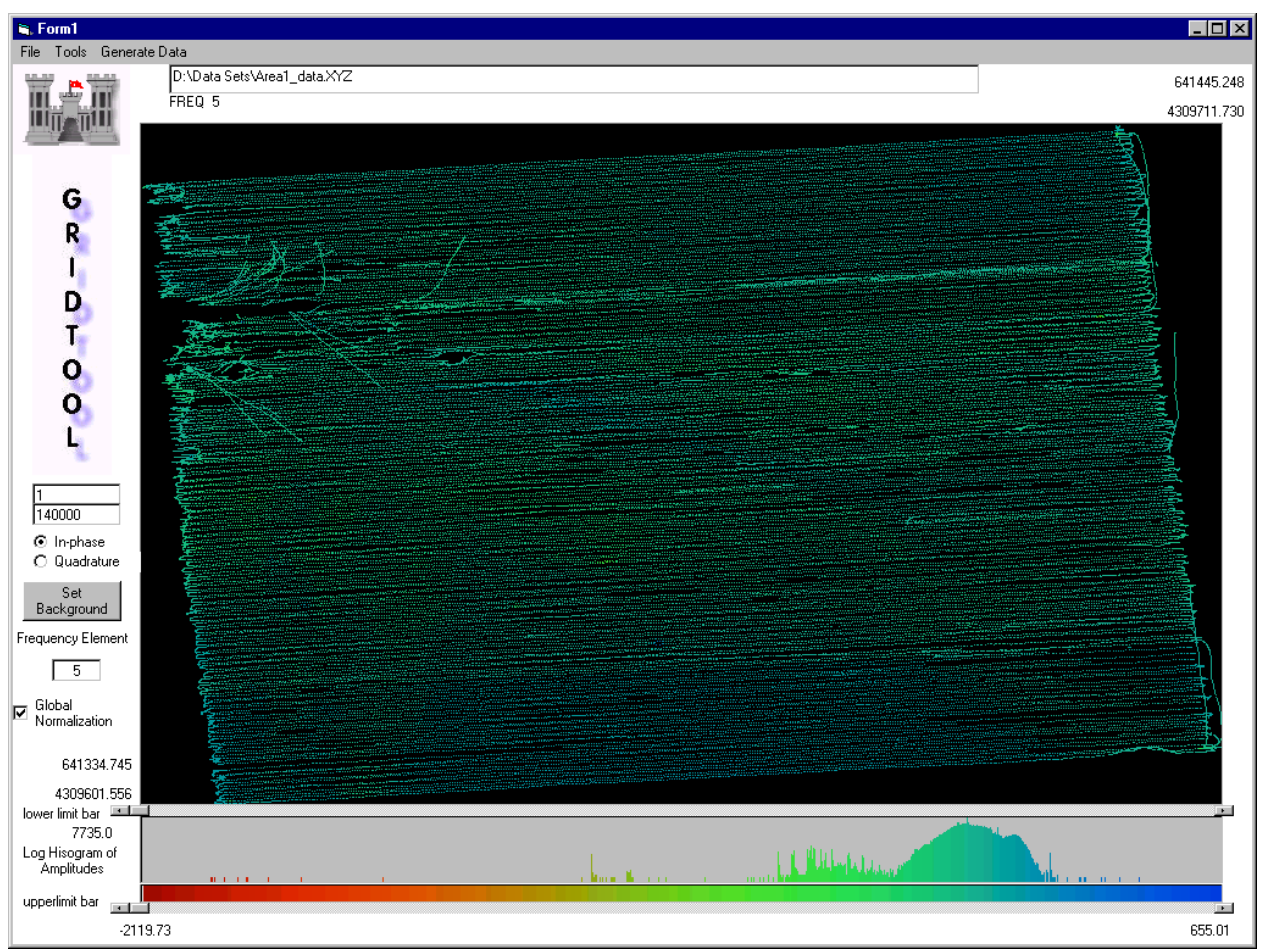

Figure A5. Area 1 in-phase coverage map for $2,790 \mathrm{~Hz}$

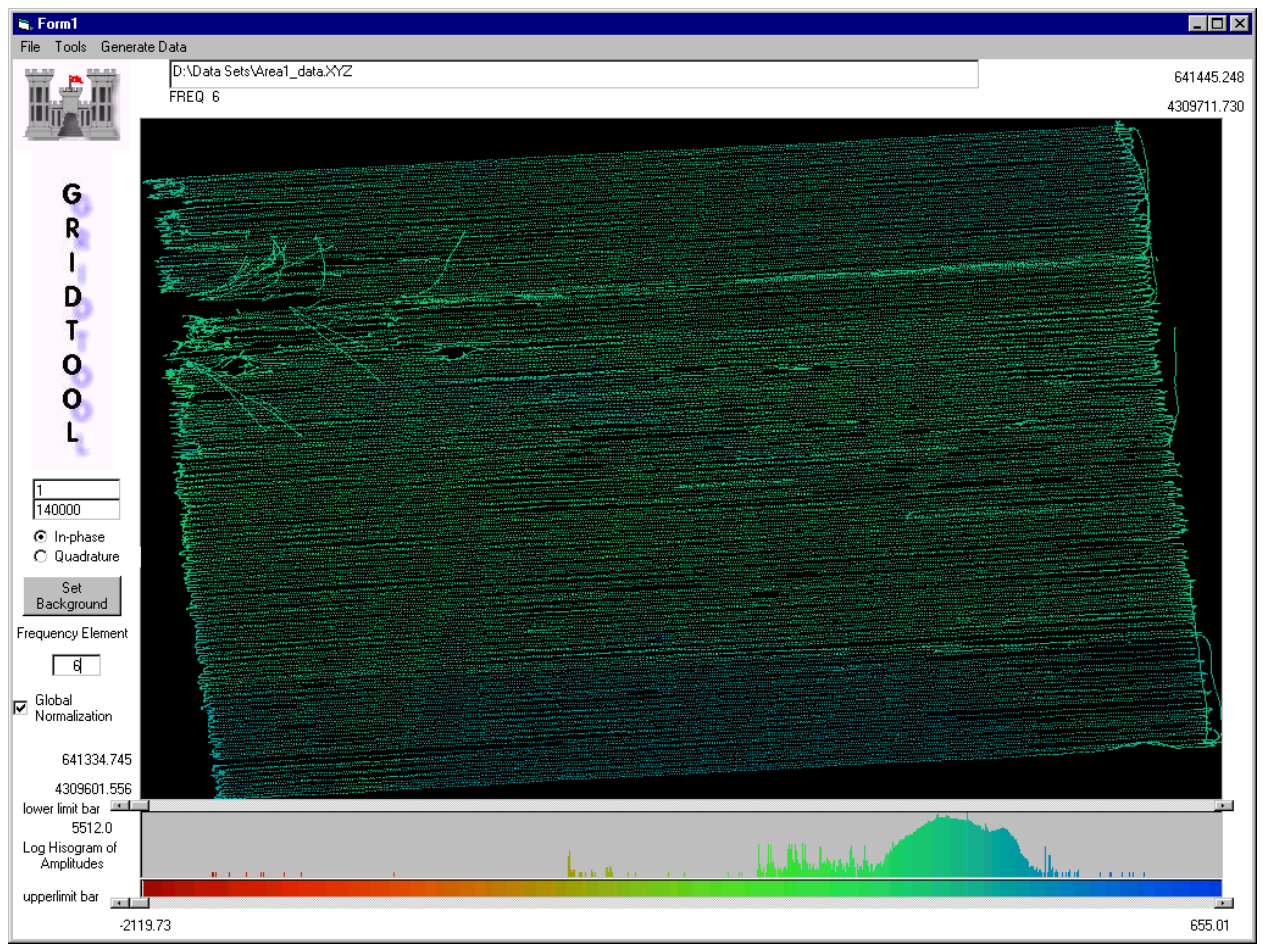

Figure A6. Area 1 in-phase coverage map for $8,190 \mathrm{~Hz}$ 


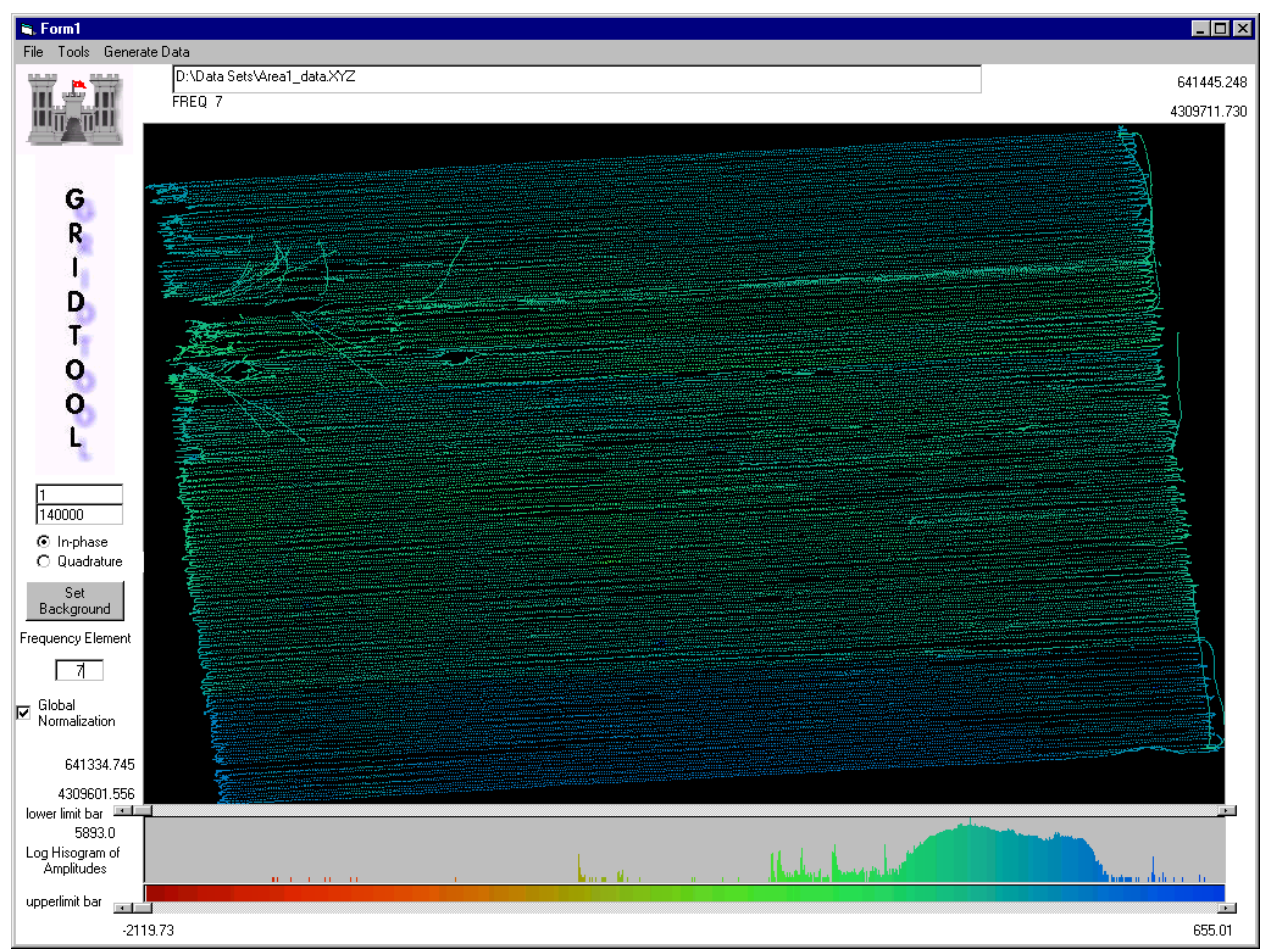

Figure A7. Area 1 in-phase coverage map for $20,010 \mathrm{~Hz}$

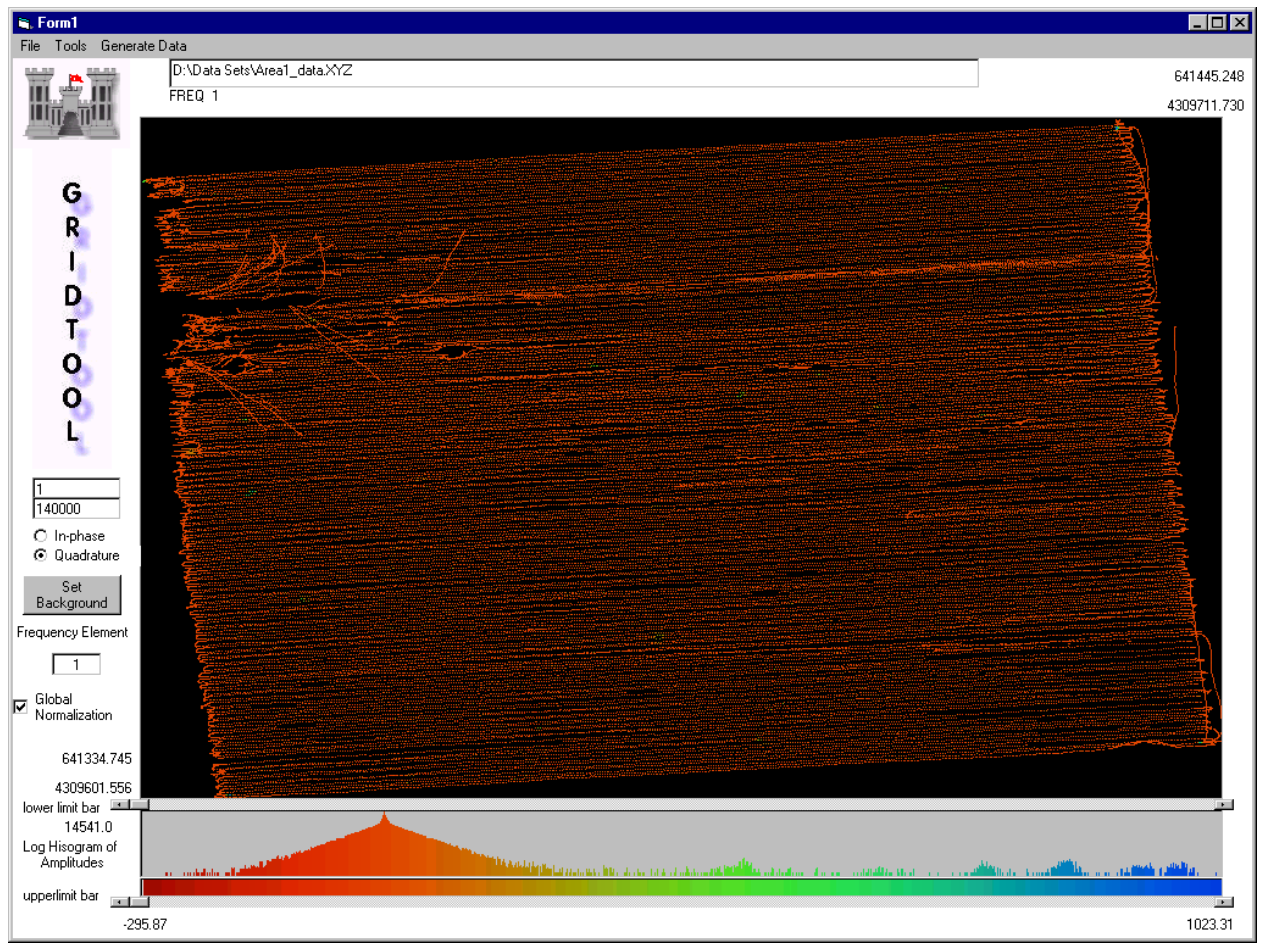

Figure A8. Area 1 quadrature coverage map for $90 \mathrm{~Hz}$ 


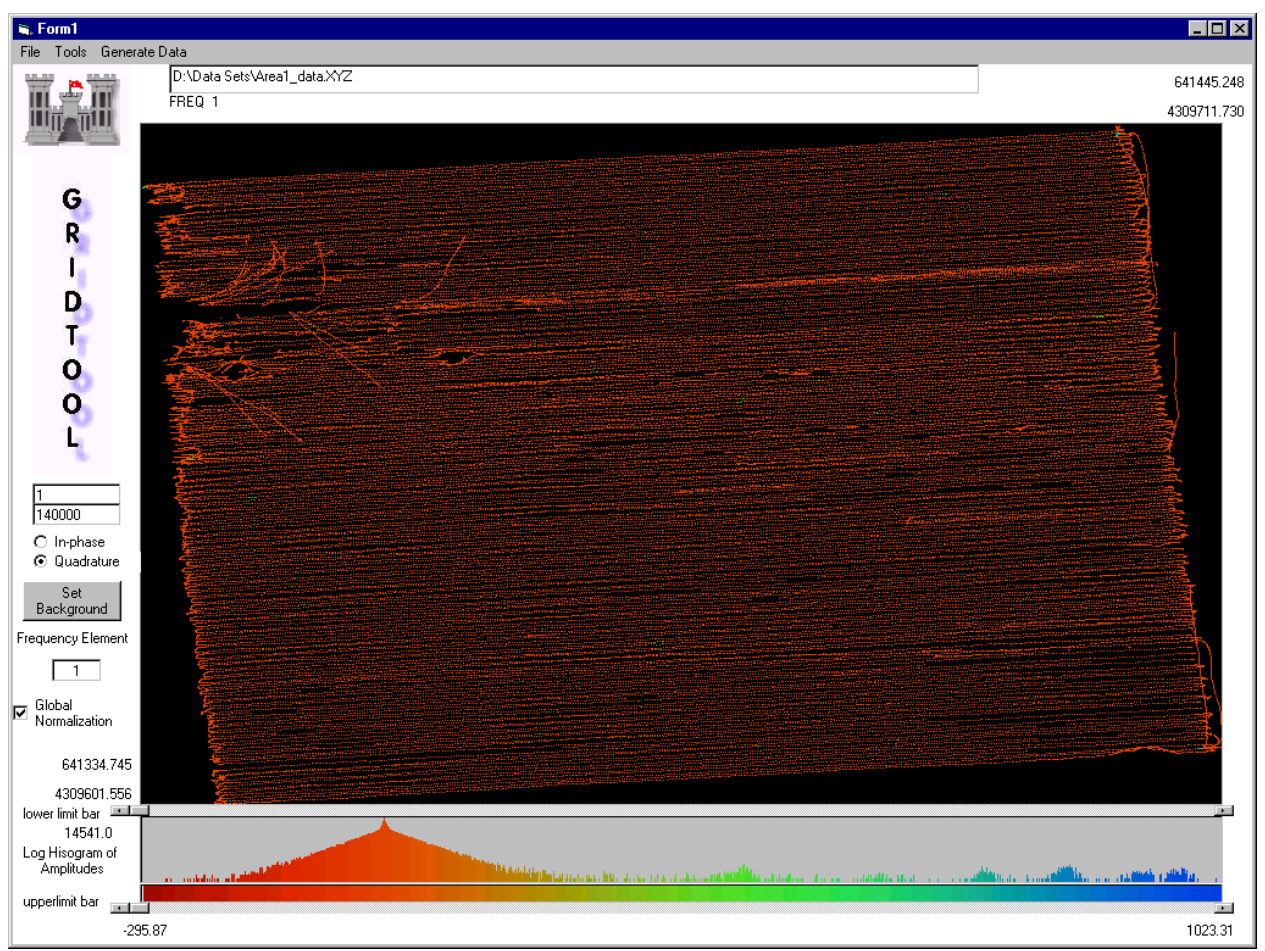

Figure A9. Area 1 quadrature coverage map for $150 \mathrm{~Hz}$

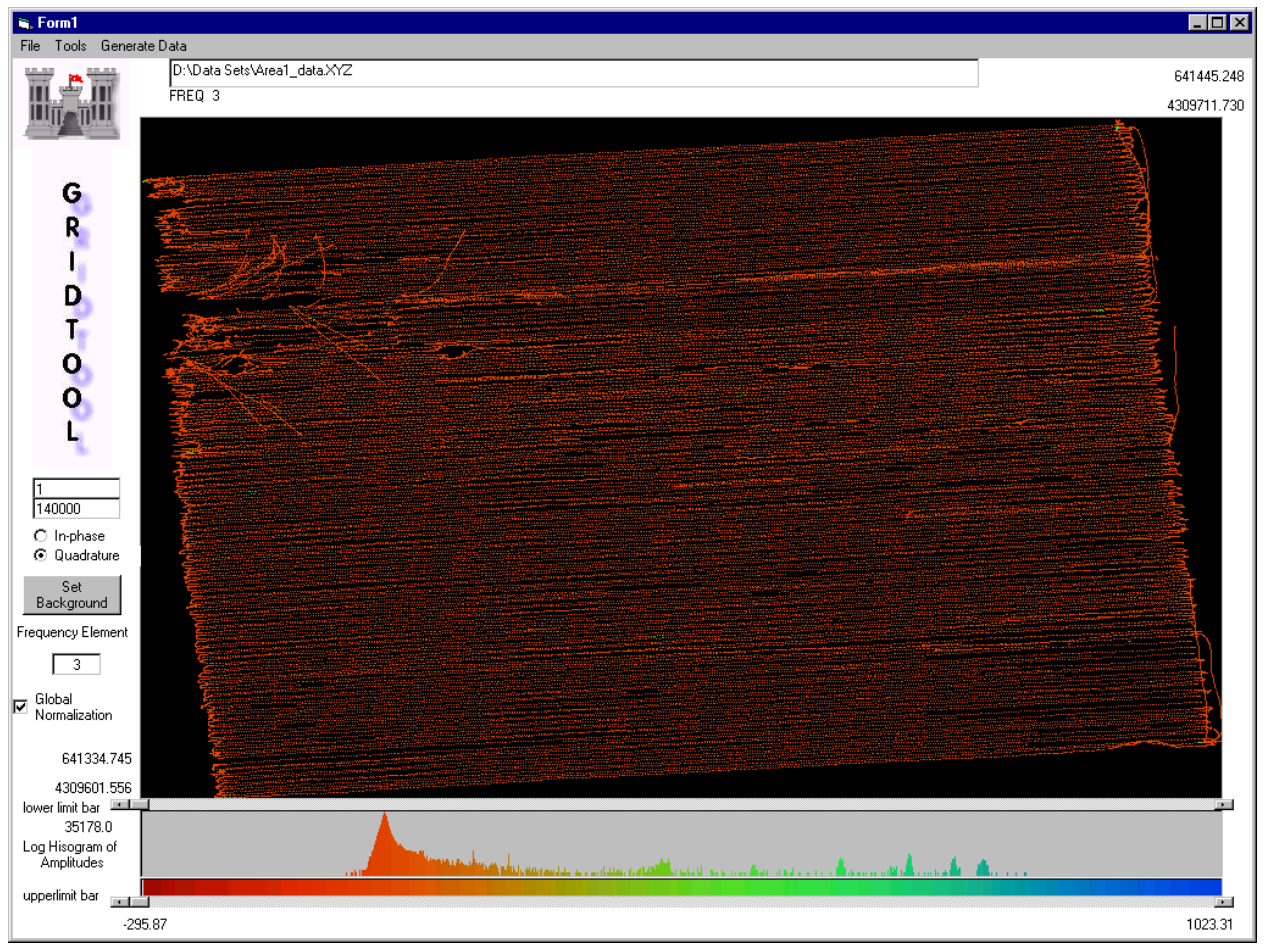

Figure A10. Area 1 quadrature coverage map for $330 \mathrm{~Hz}$ 


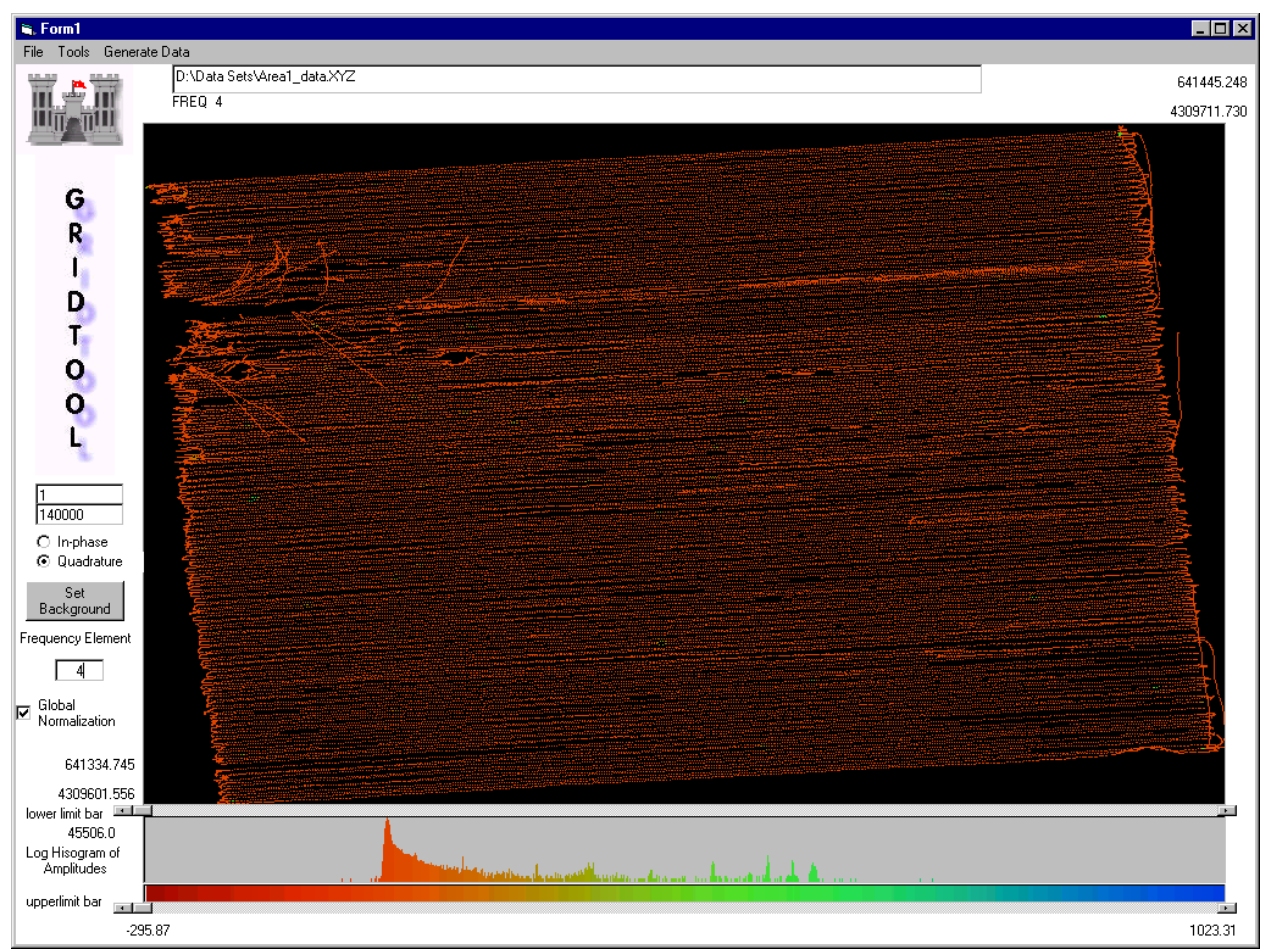

Figure A11. Area 1 quadrature coverage map for $930 \mathrm{~Hz}$

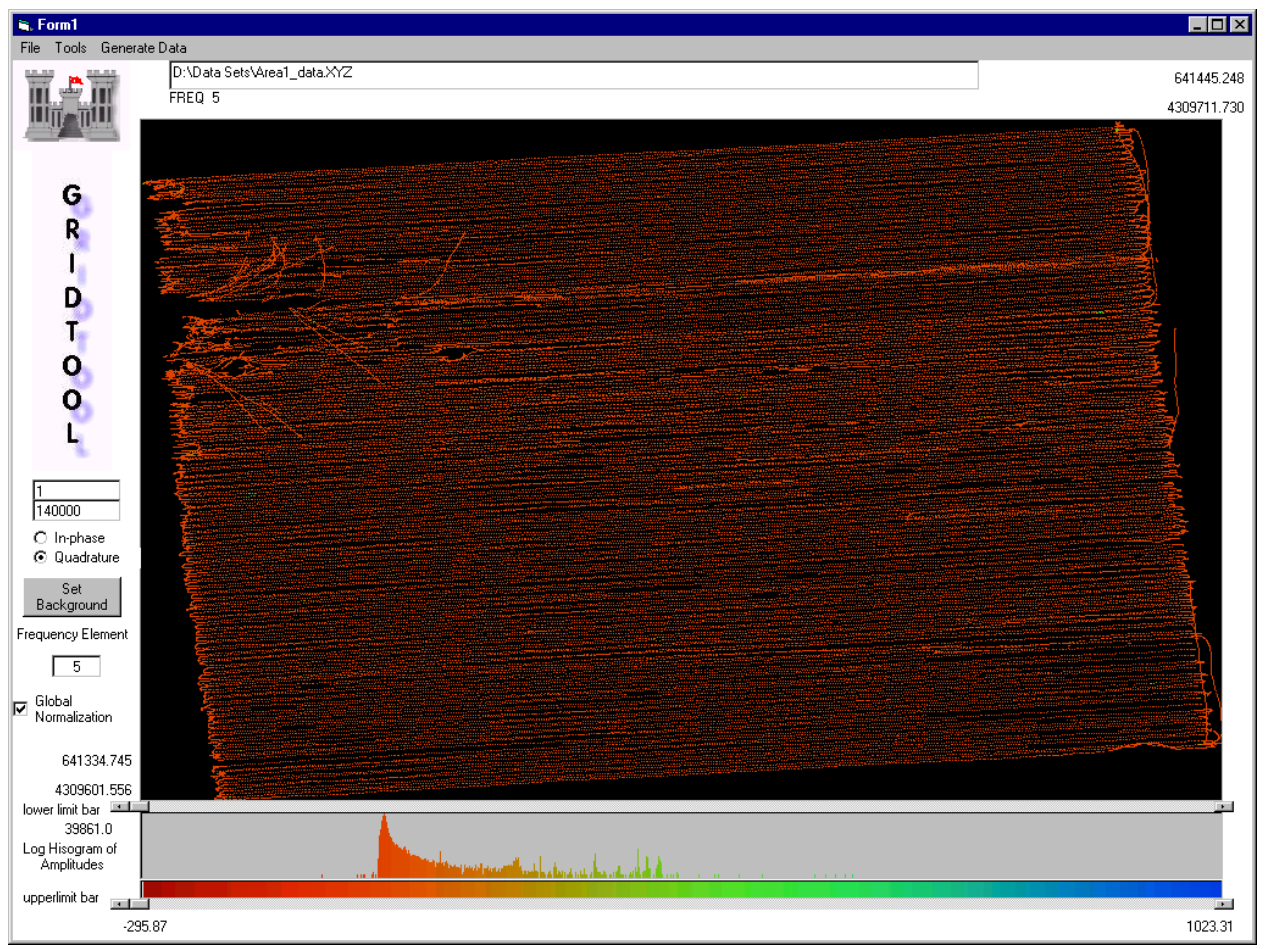

Figure A12. Area 1 quadrature coverage map for $2,790 \mathrm{~Hz}$ 


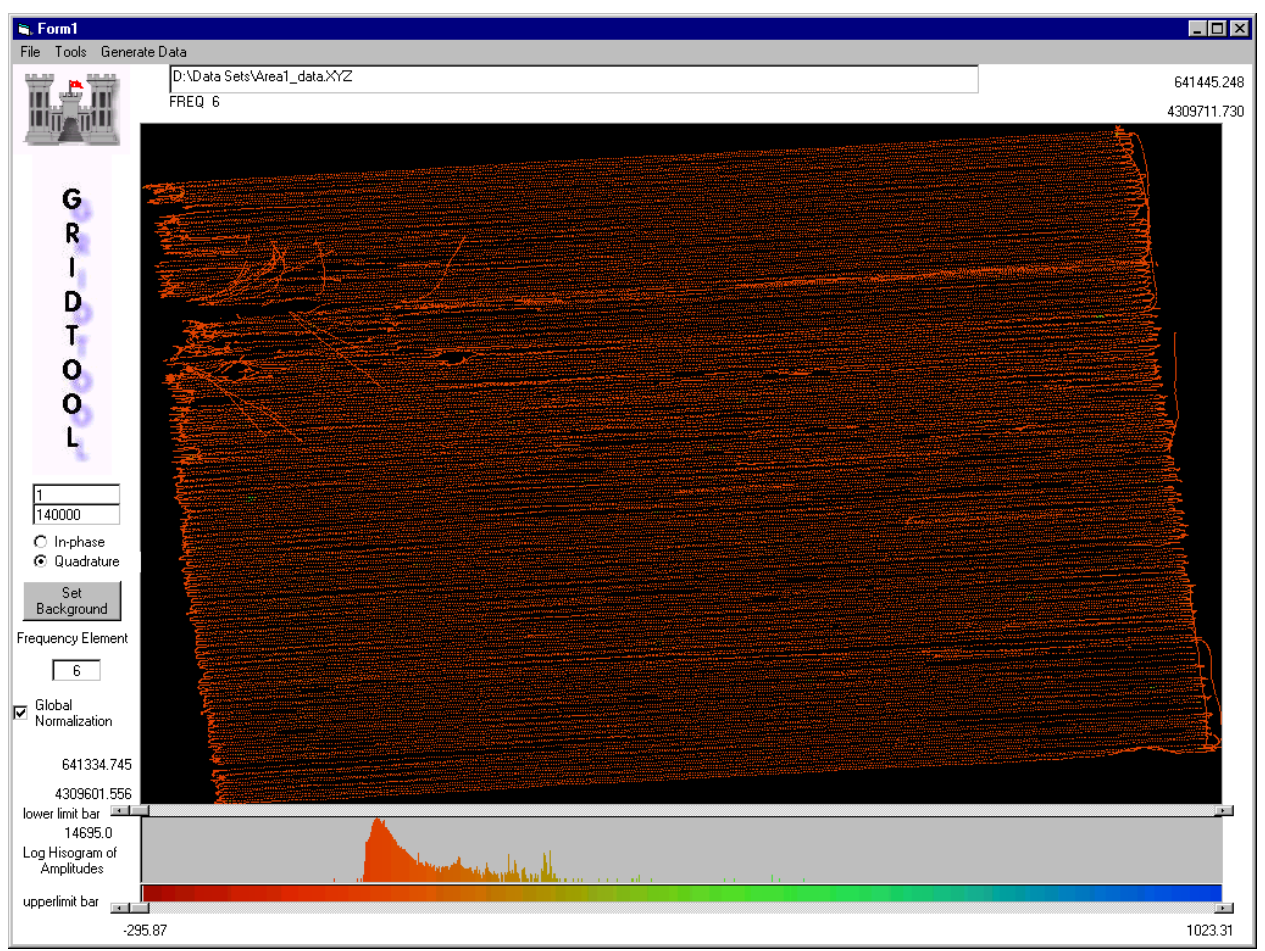

Figure A13. Area 1 quadrature coverage map for $8,190 \mathrm{~Hz}$

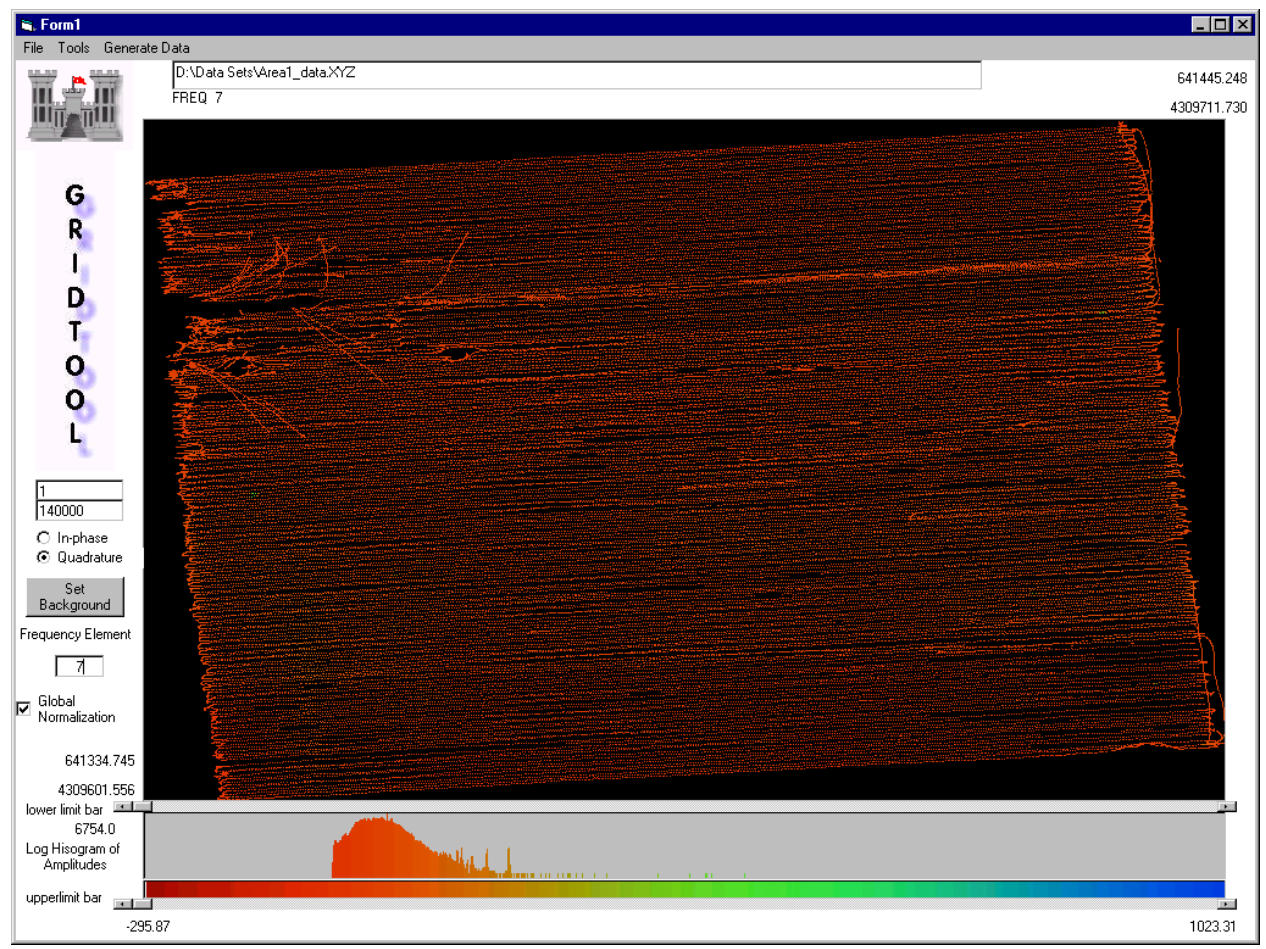

Figure A14. Area 1 quadrature coverage map for $20,010 \mathrm{~Hz}$ 


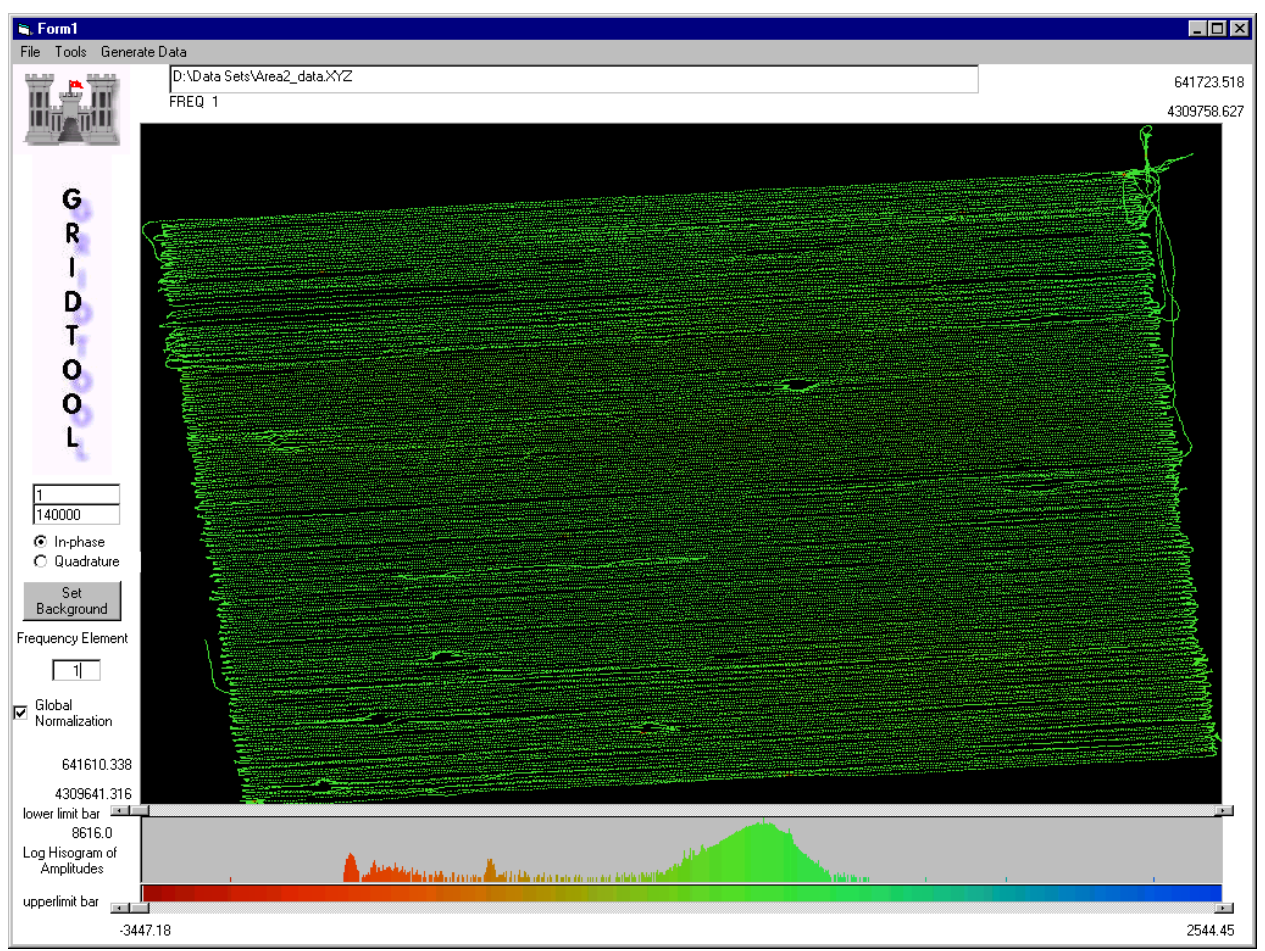

Figure A15. Area 2 in-phase coverage map for $90 \mathrm{~Hz}$

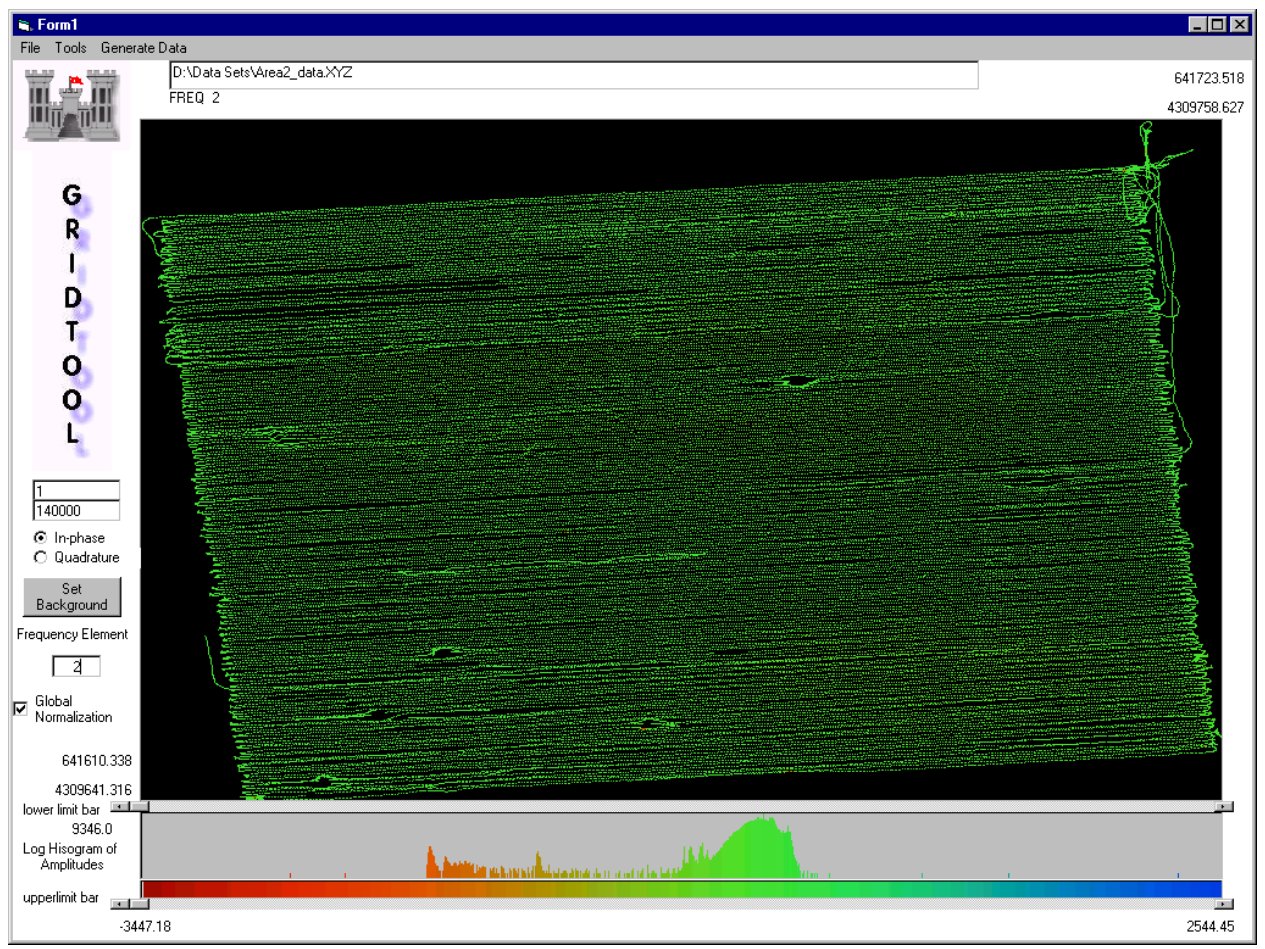

Figure A16. Area 2 in-phase coverage map for $150 \mathrm{~Hz}$ 


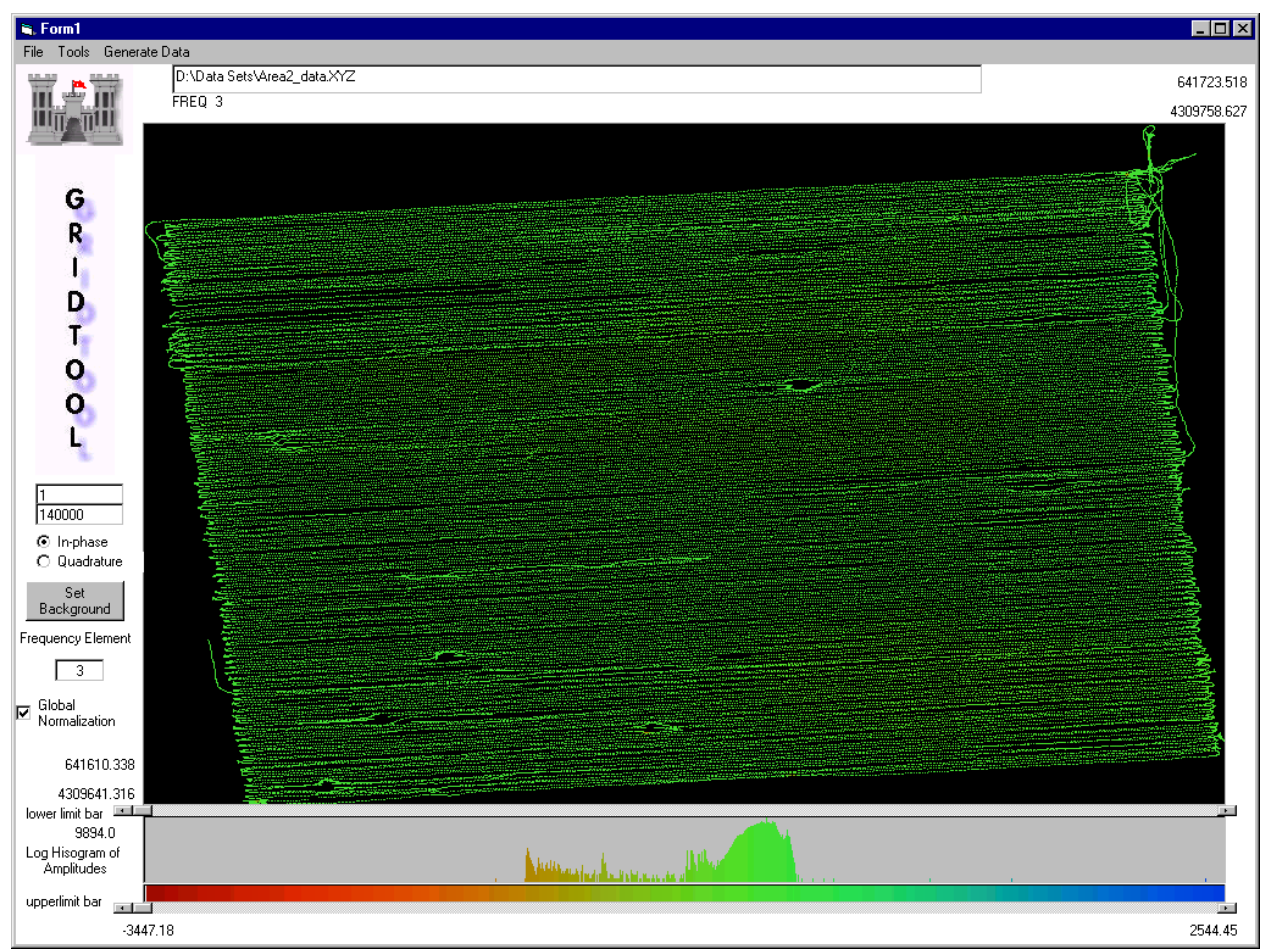

Figure A17. Area 2 in-phase coverage map for $330 \mathrm{~Hz}$

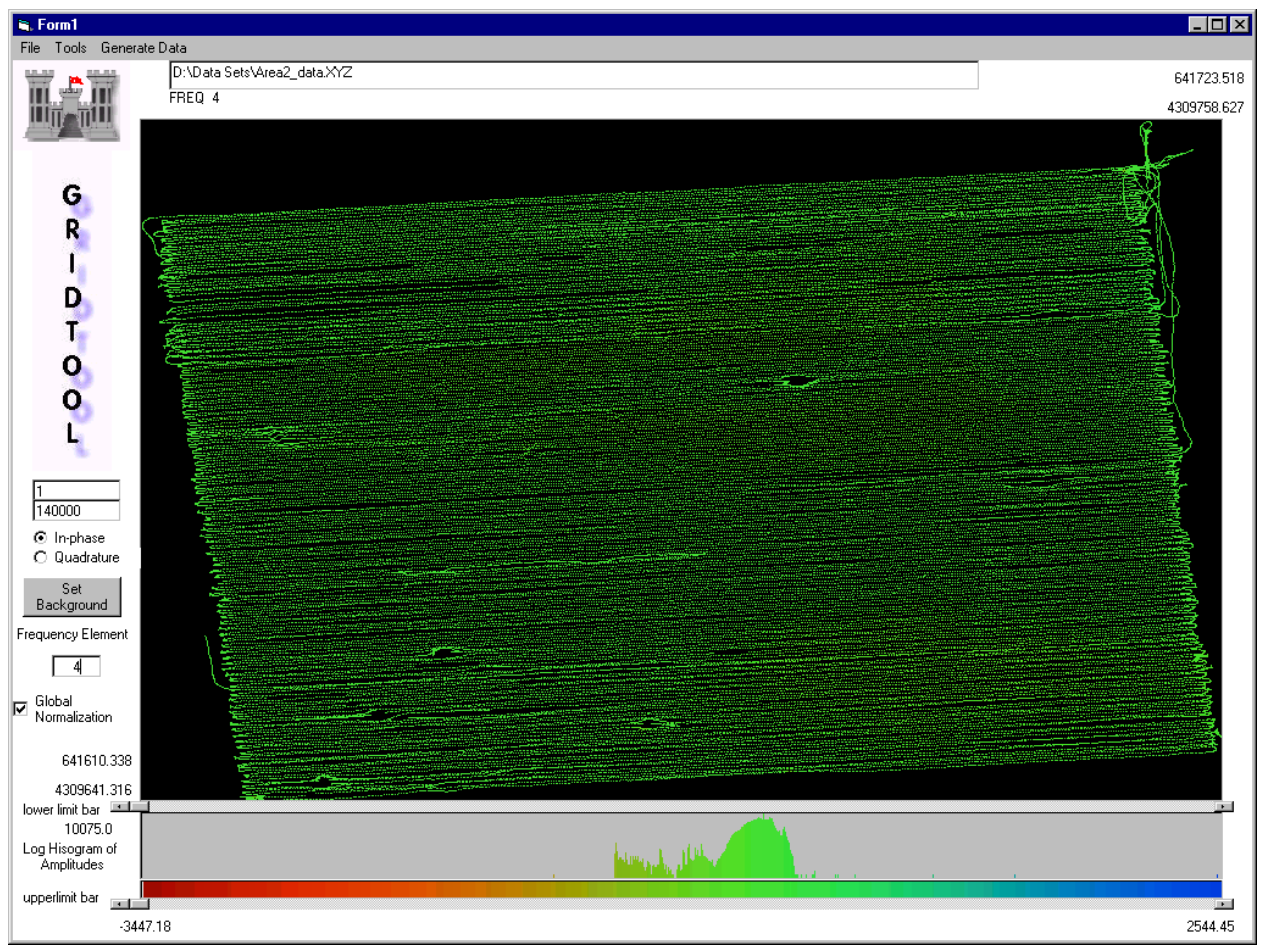

Figure A18. Area 2 in-phase coverage map for $930 \mathrm{~Hz}$ 


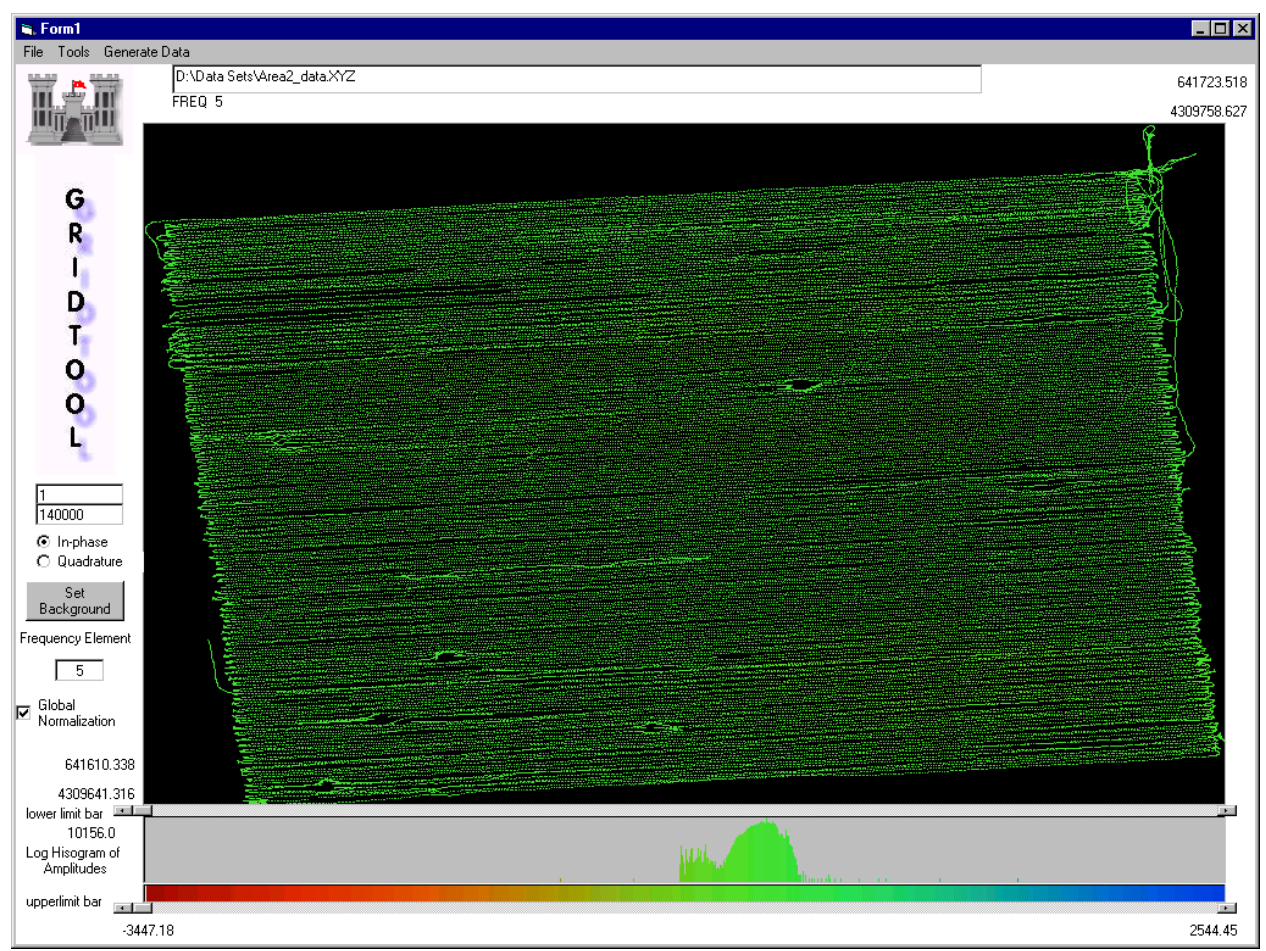

Figure A19. Area 2 in-phase coverage map for $2,790 \mathrm{~Hz}$

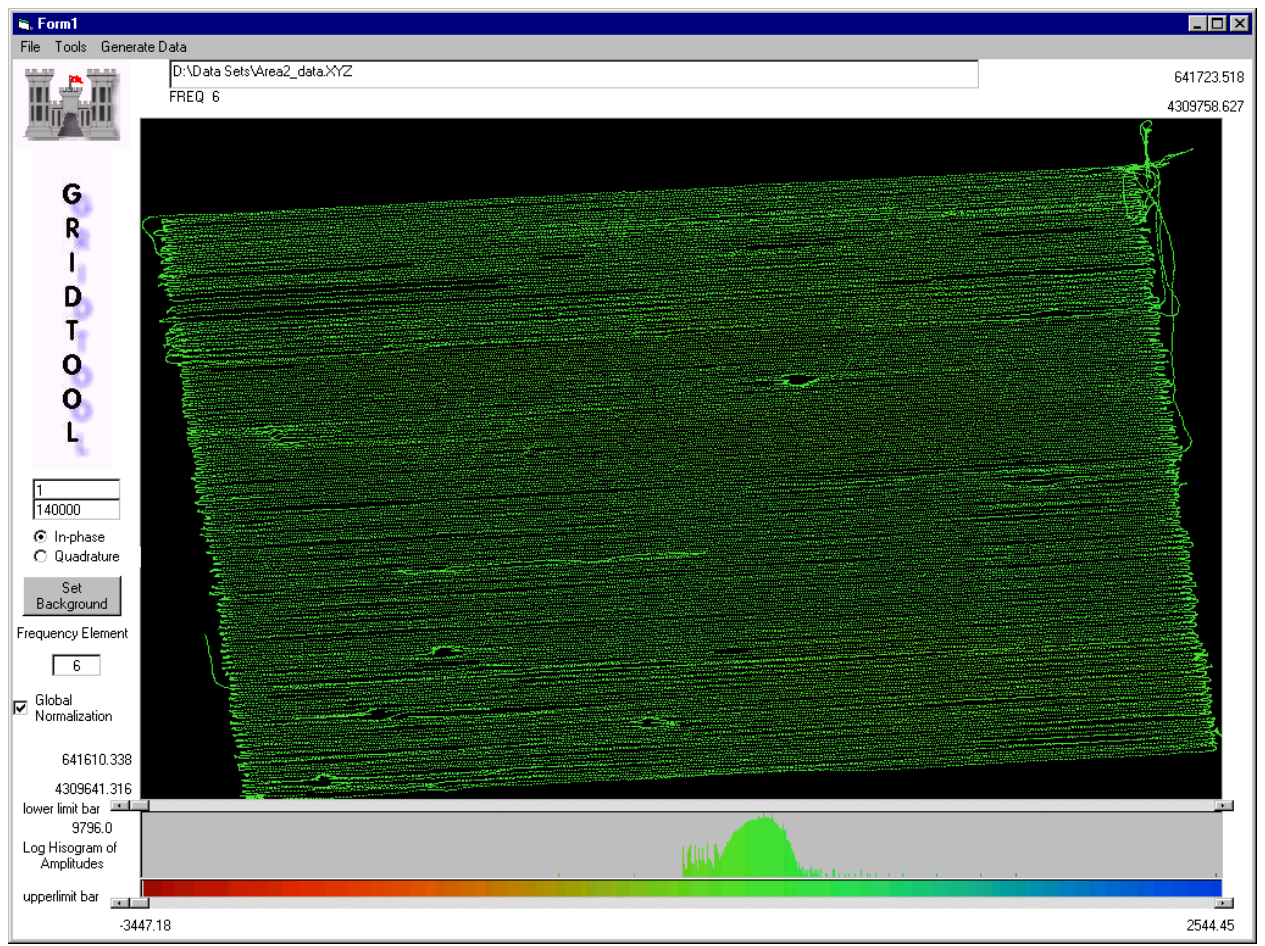

Figure A20. Area 2 in-phase coverage map for $8,190 \mathrm{~Hz}$ 


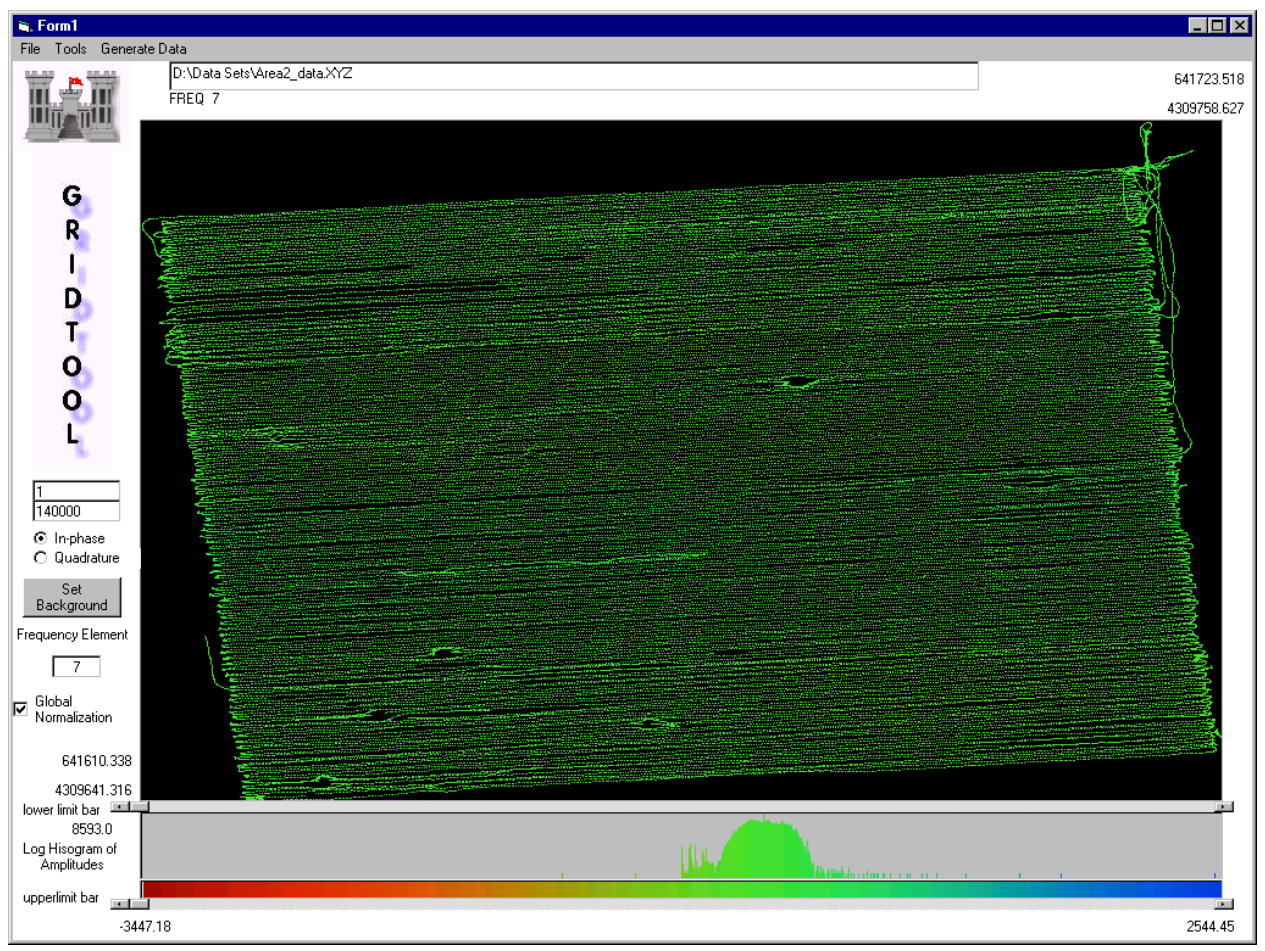

Figure A21. Area 2 in-phase coverage map for $20,010 \mathrm{~Hz}$

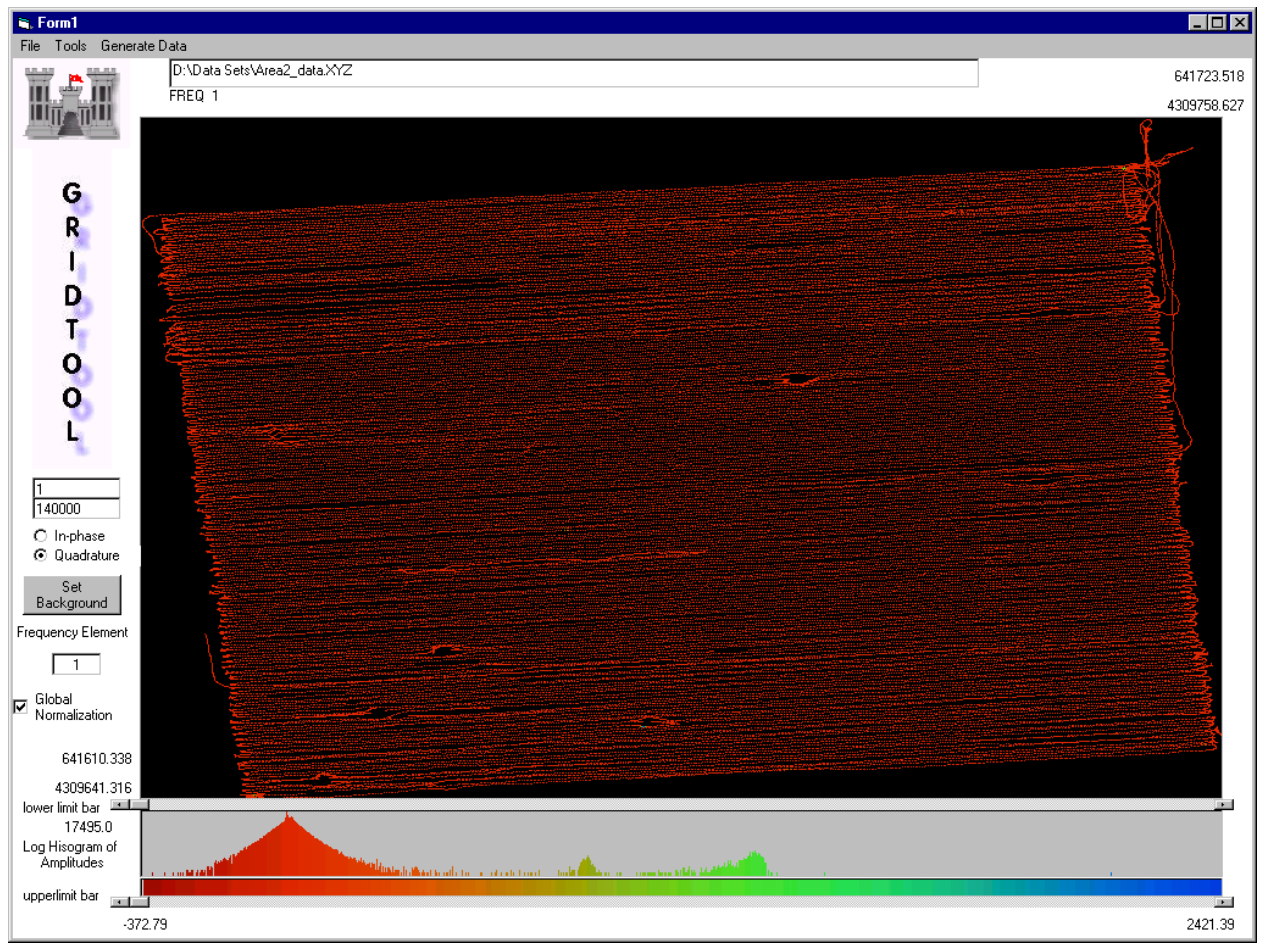

Figure A22. Area 2 quadrature coverage map for $90 \mathrm{~Hz}$ 


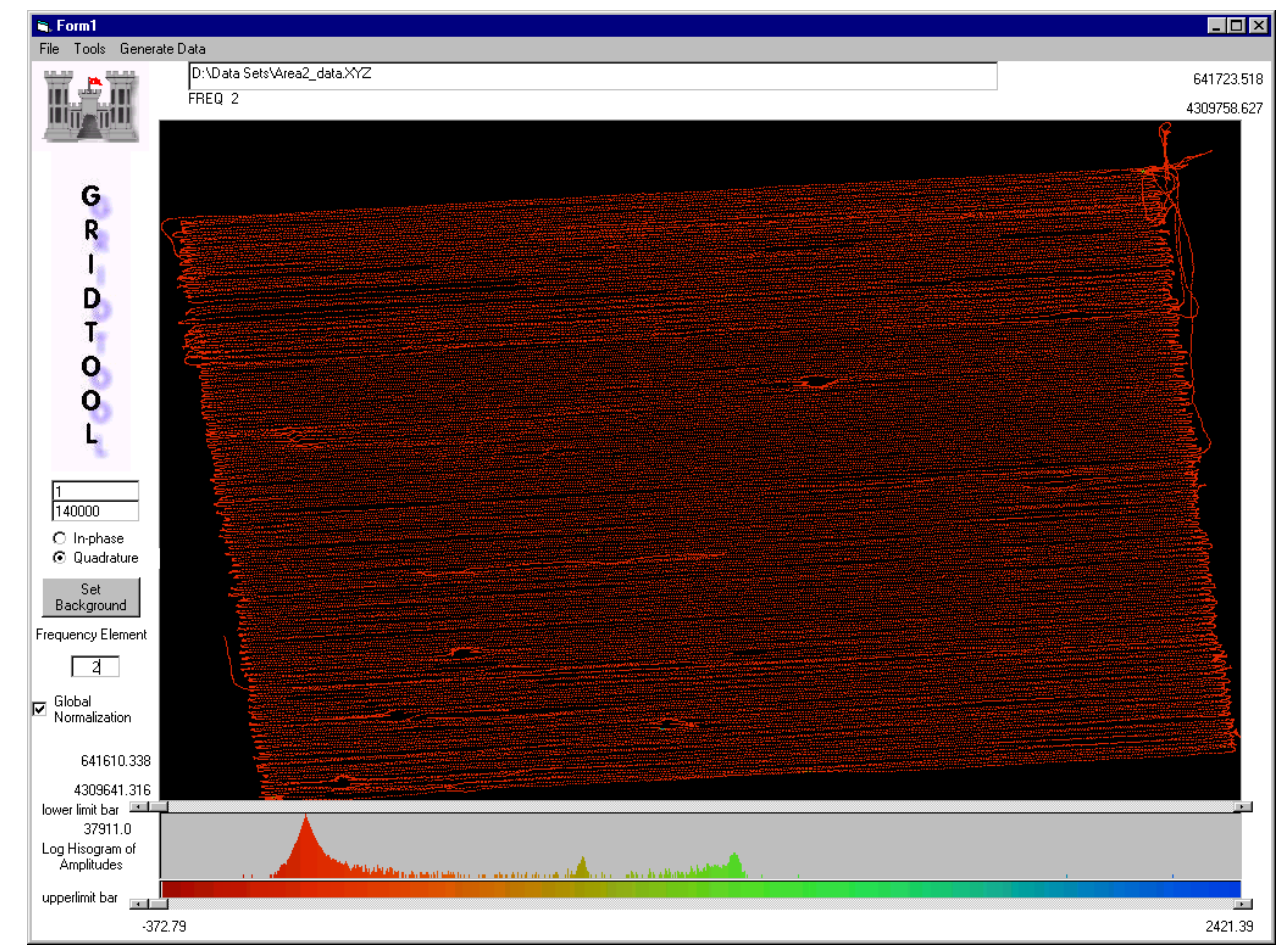

Figure A23. Area 2 quadrature coverage map for $150 \mathrm{~Hz}$

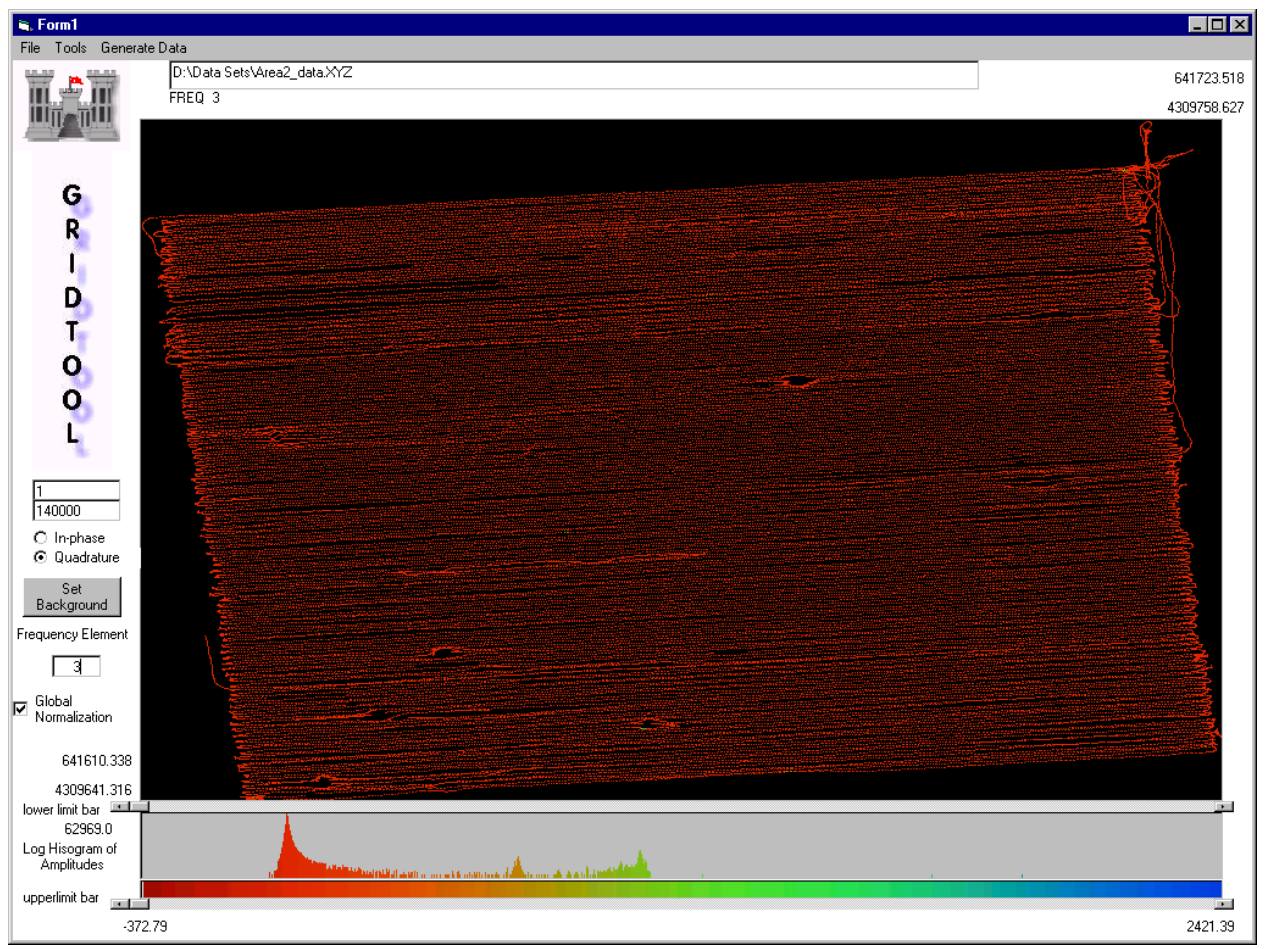

Figure A24. Area 2 quadrature coverage map for $330 \mathrm{~Hz}$ 


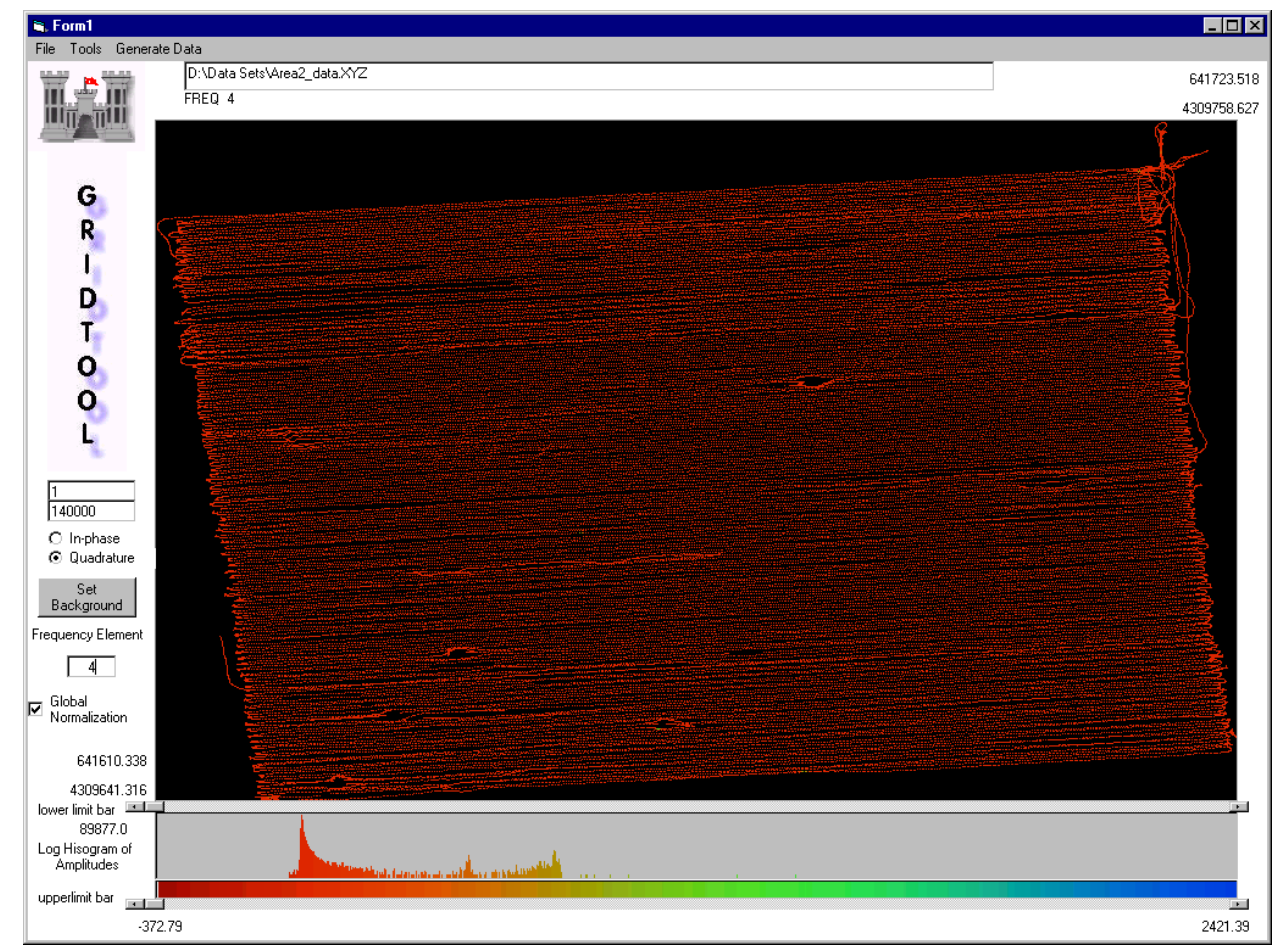

Figure A25. Area 2 quadrature coverage map for $930 \mathrm{~Hz}$

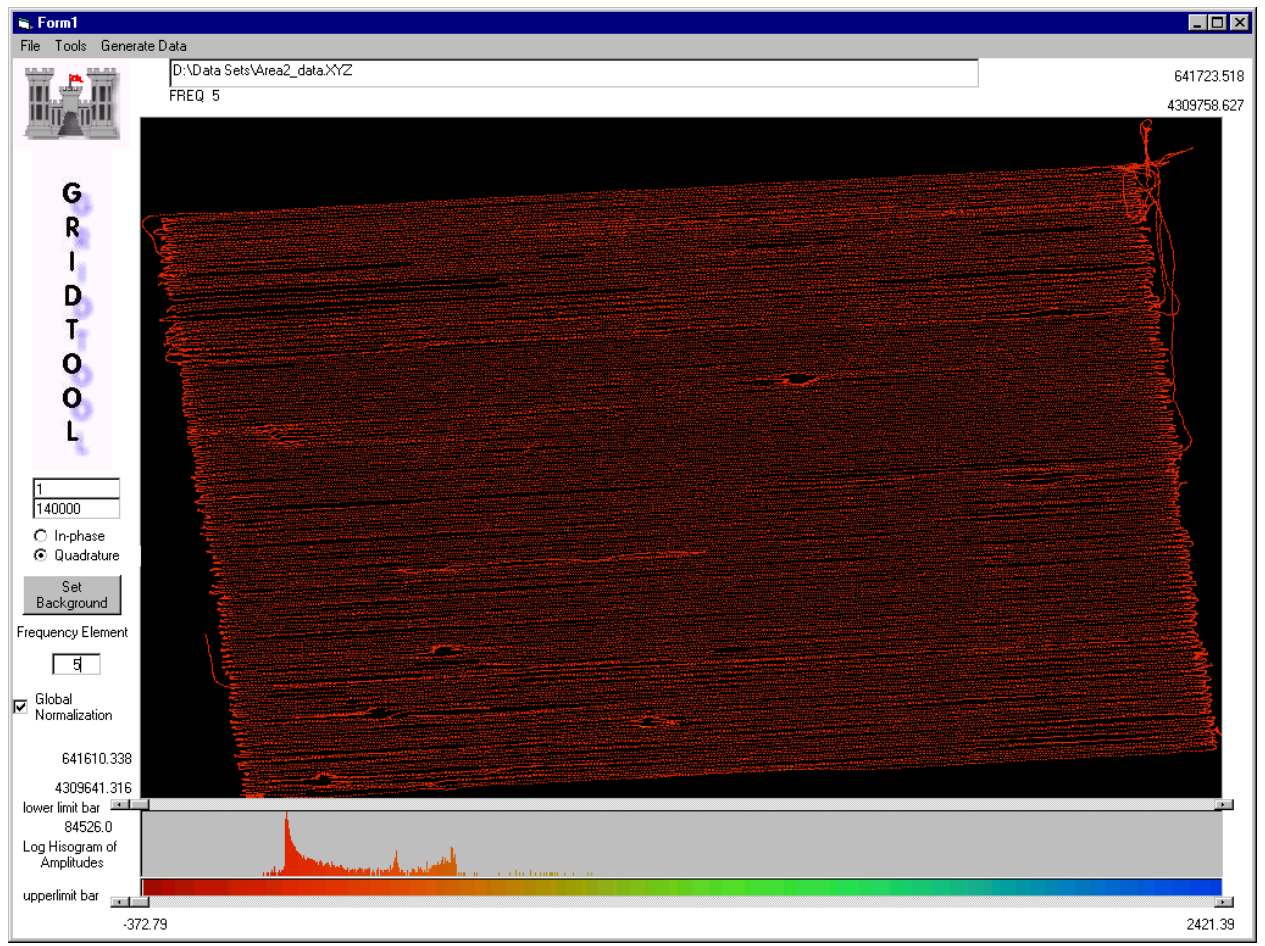

Figure A26. Area 2 quadrature coverage map for $2,790 \mathrm{~Hz}$ 


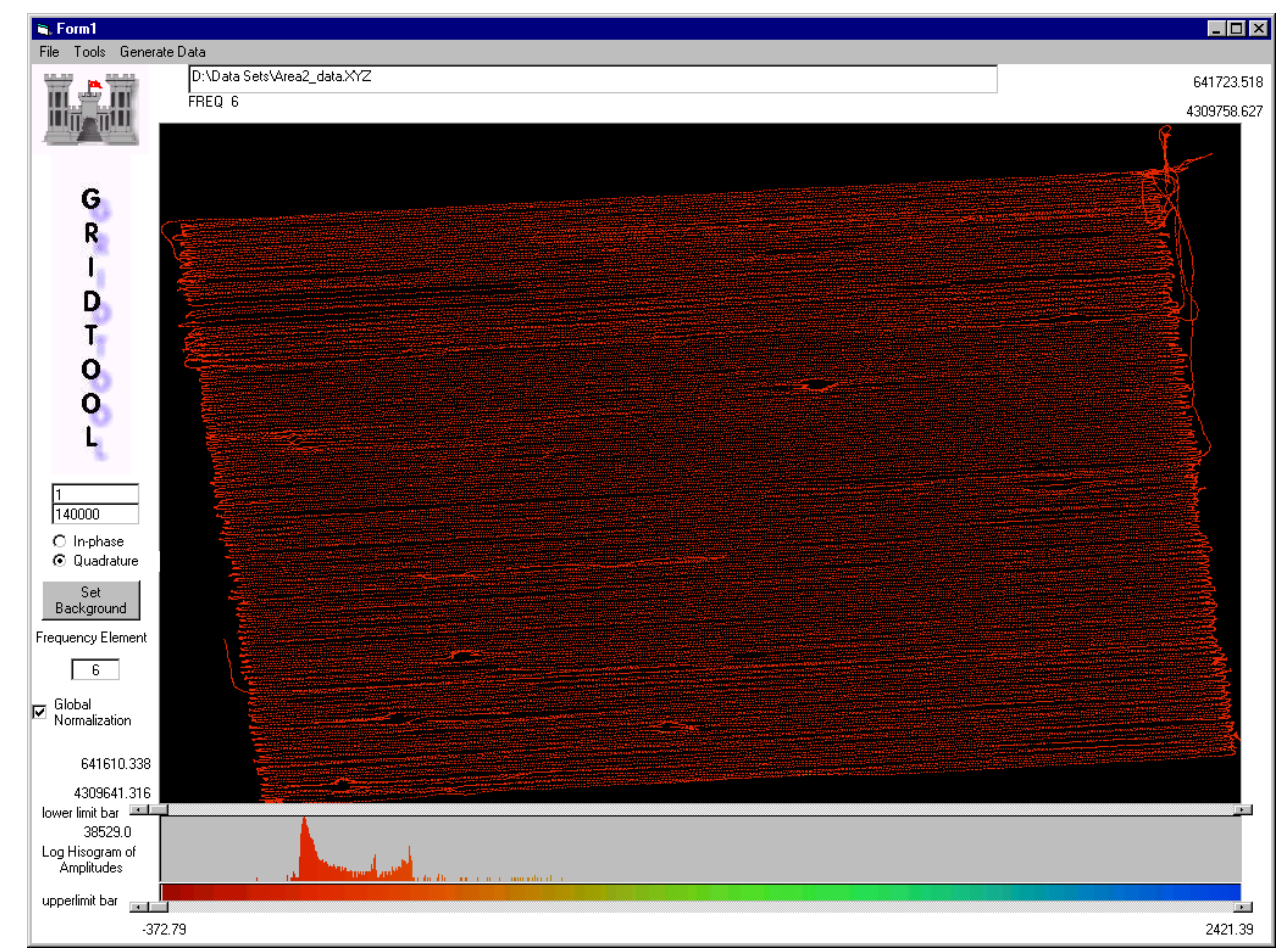

Figure A27. Area 2 quadrature coverage map for $8,190 \mathrm{~Hz}$

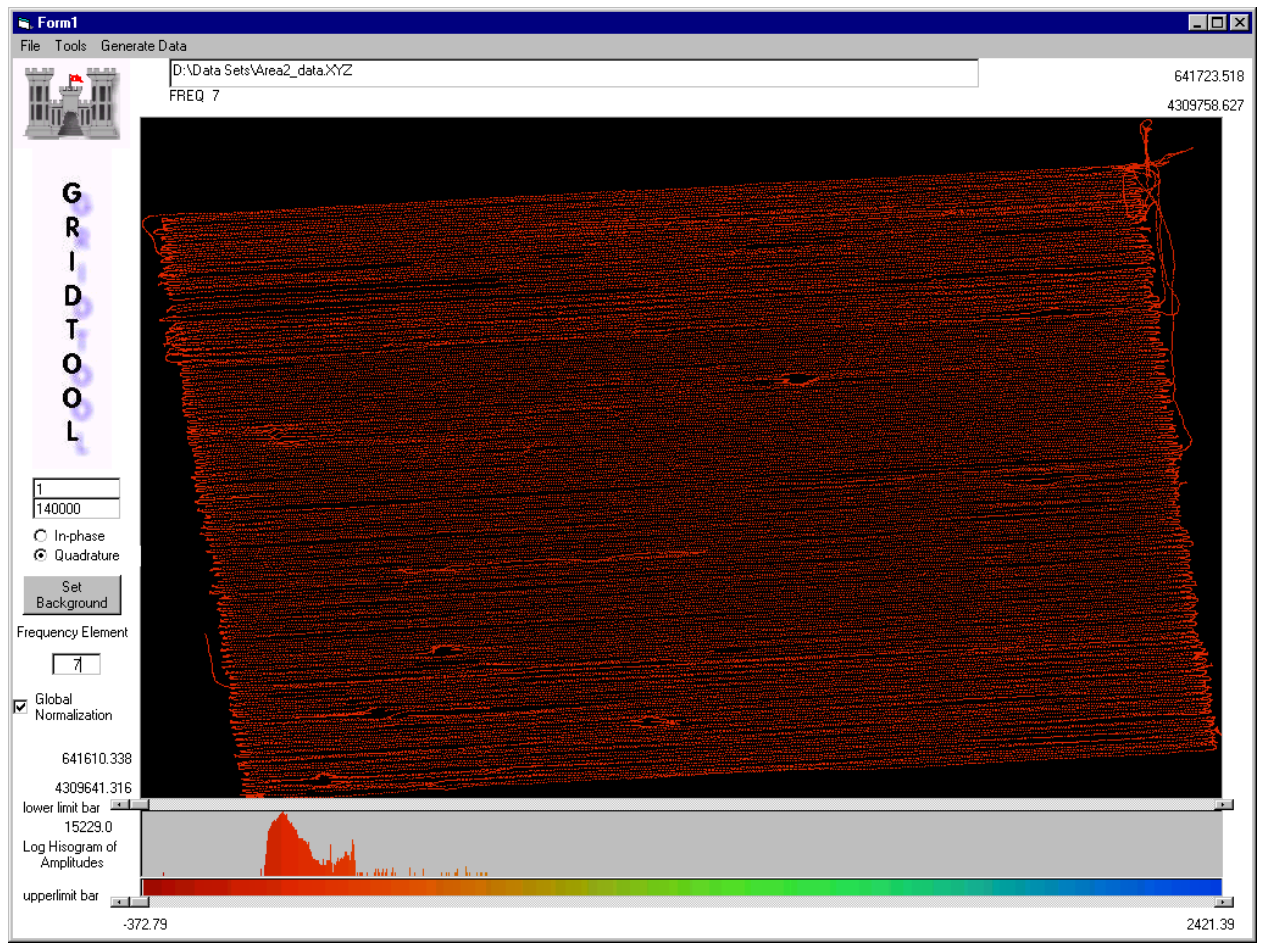

Figure A28. Area 2 quadrature coverage map for $20,010 \mathrm{~Hz}$ 


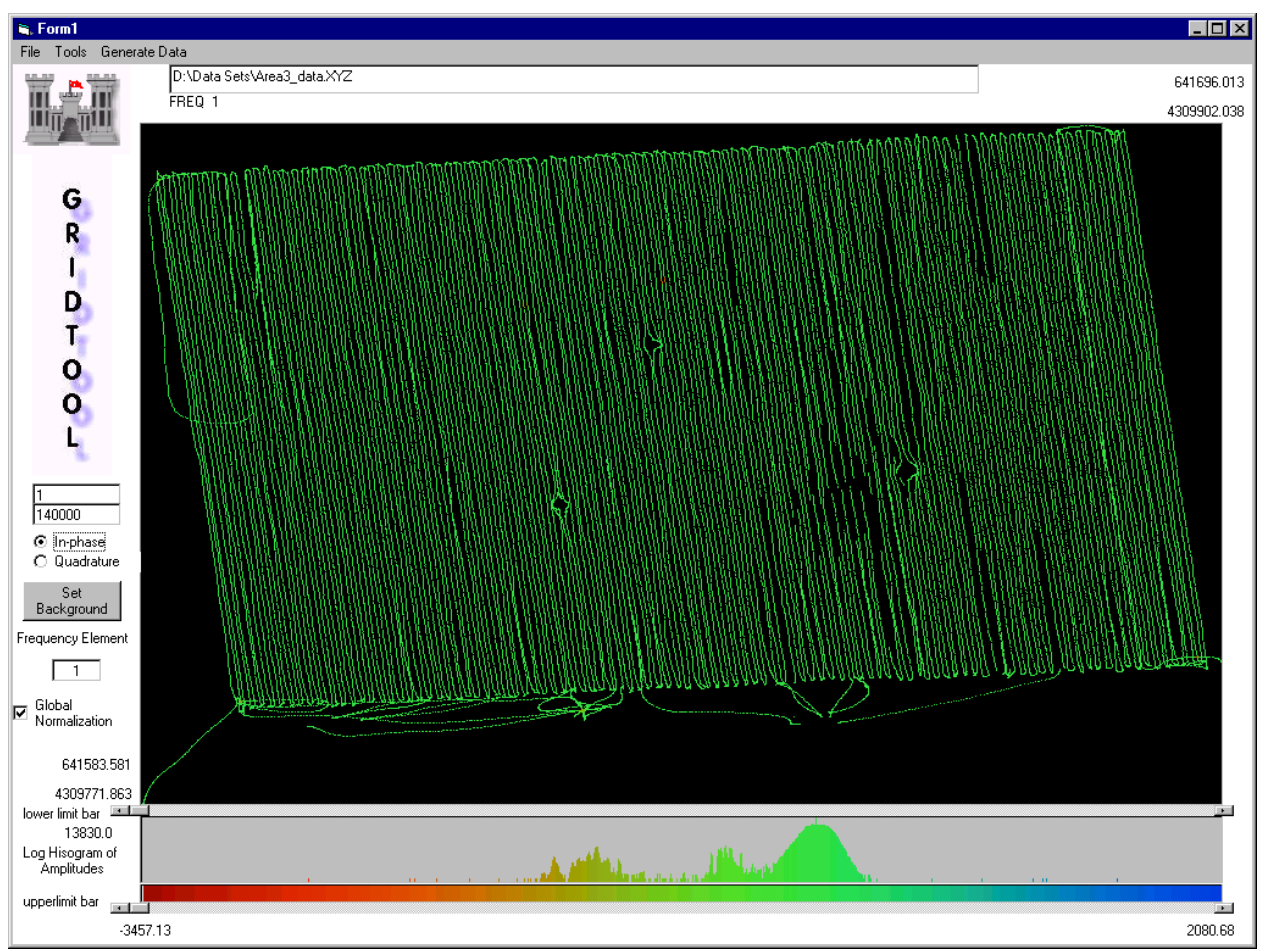

Figure A29. Area 3 in-phase coverage map for $90 \mathrm{~Hz}$

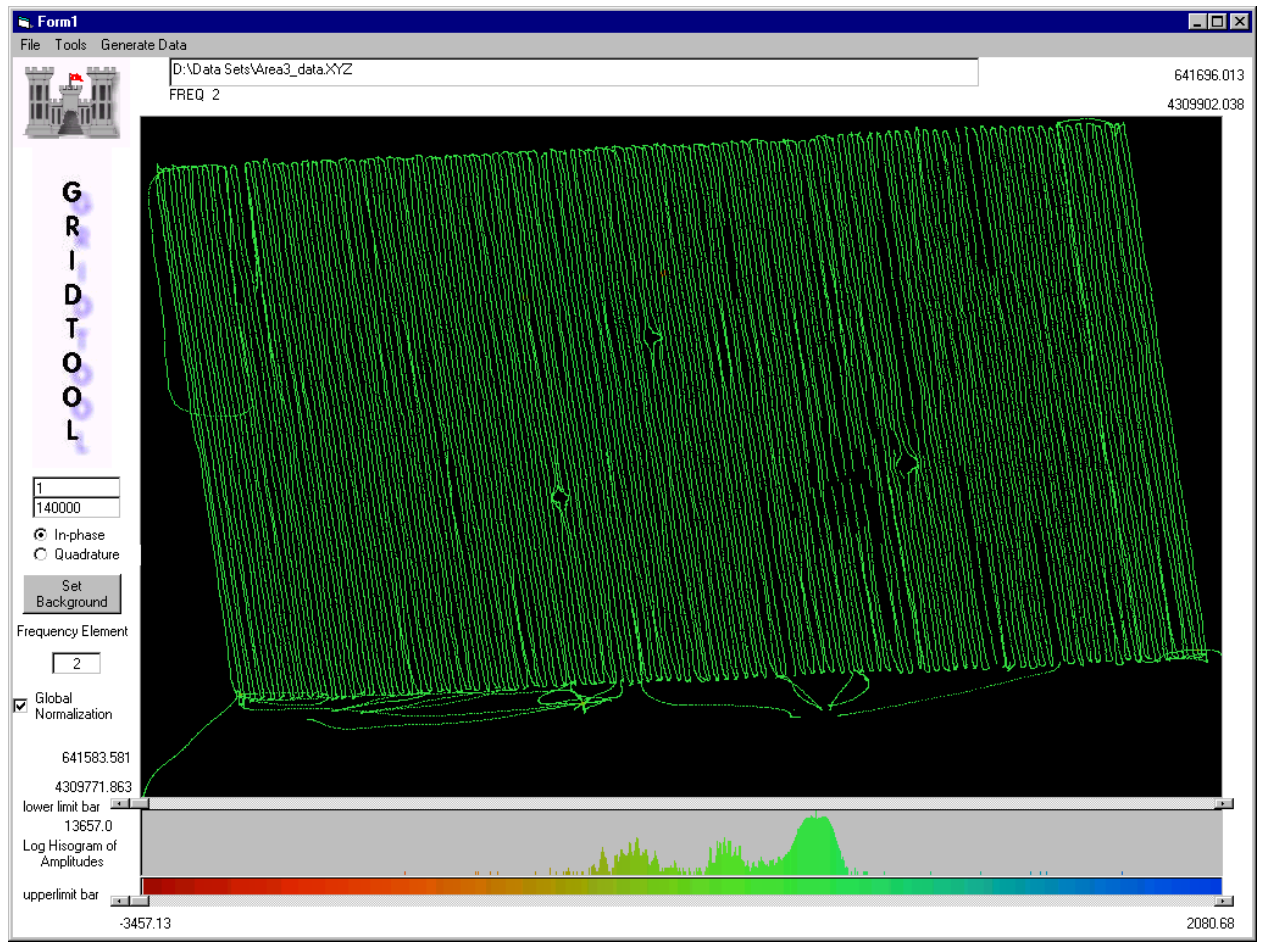

Figure A30. Area 3 in-phase coverage map for $150 \mathrm{~Hz}$ 


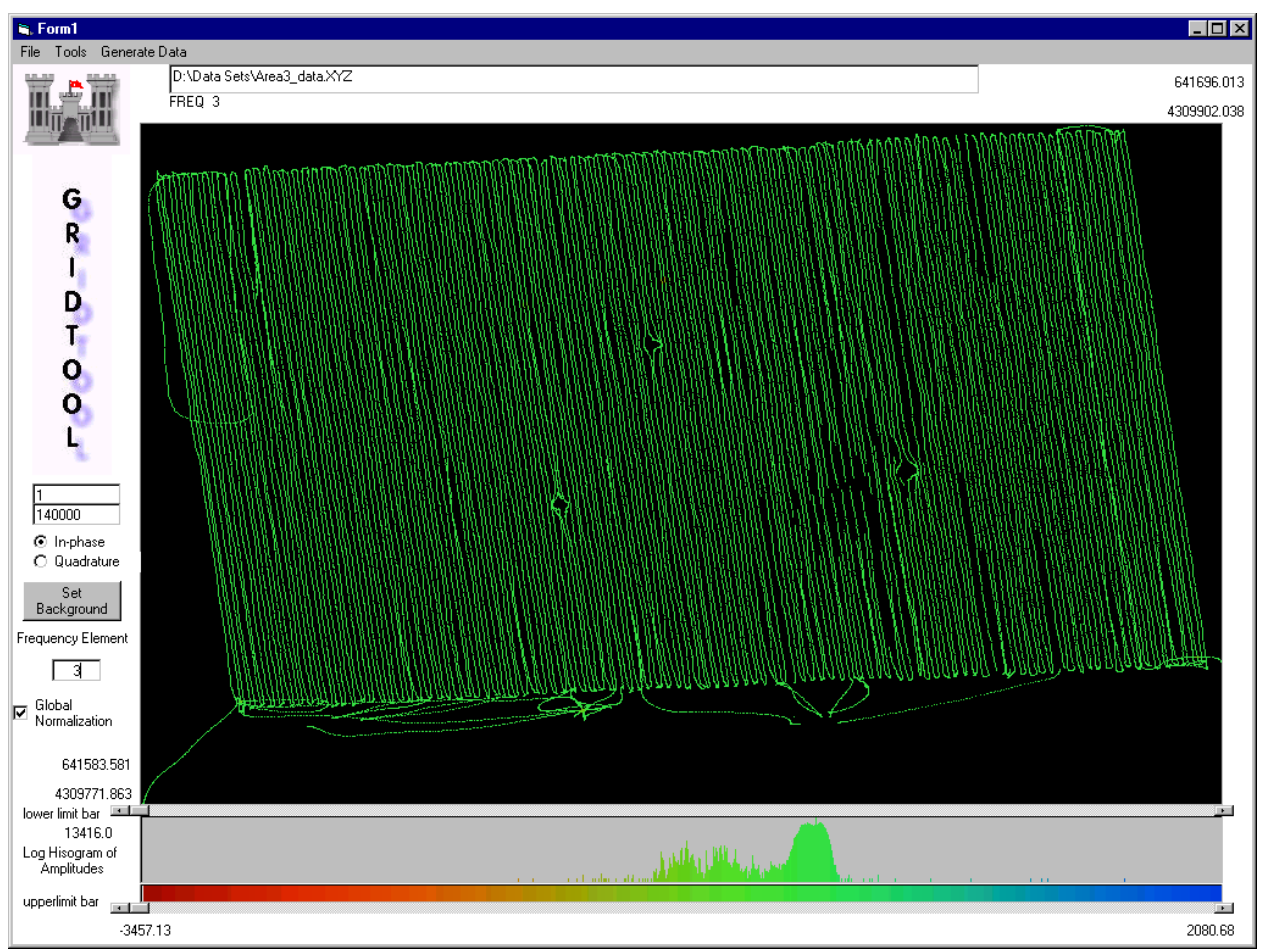

Figure A31. Area 3 in-phase coverage map for $330 \mathrm{~Hz}$

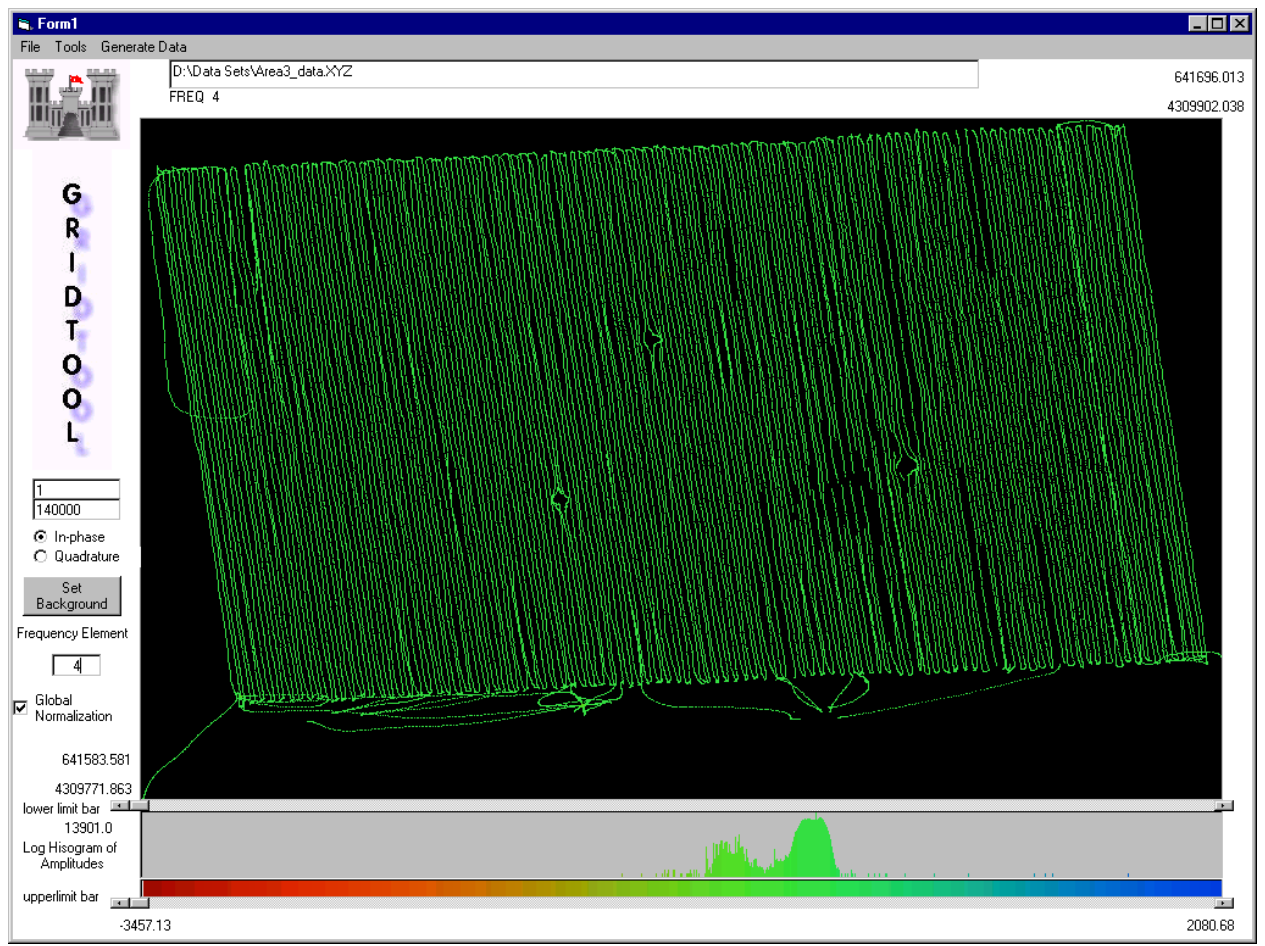

Figure A32. Area 3 in-phase coverage map for $930 \mathrm{~Hz}$ 


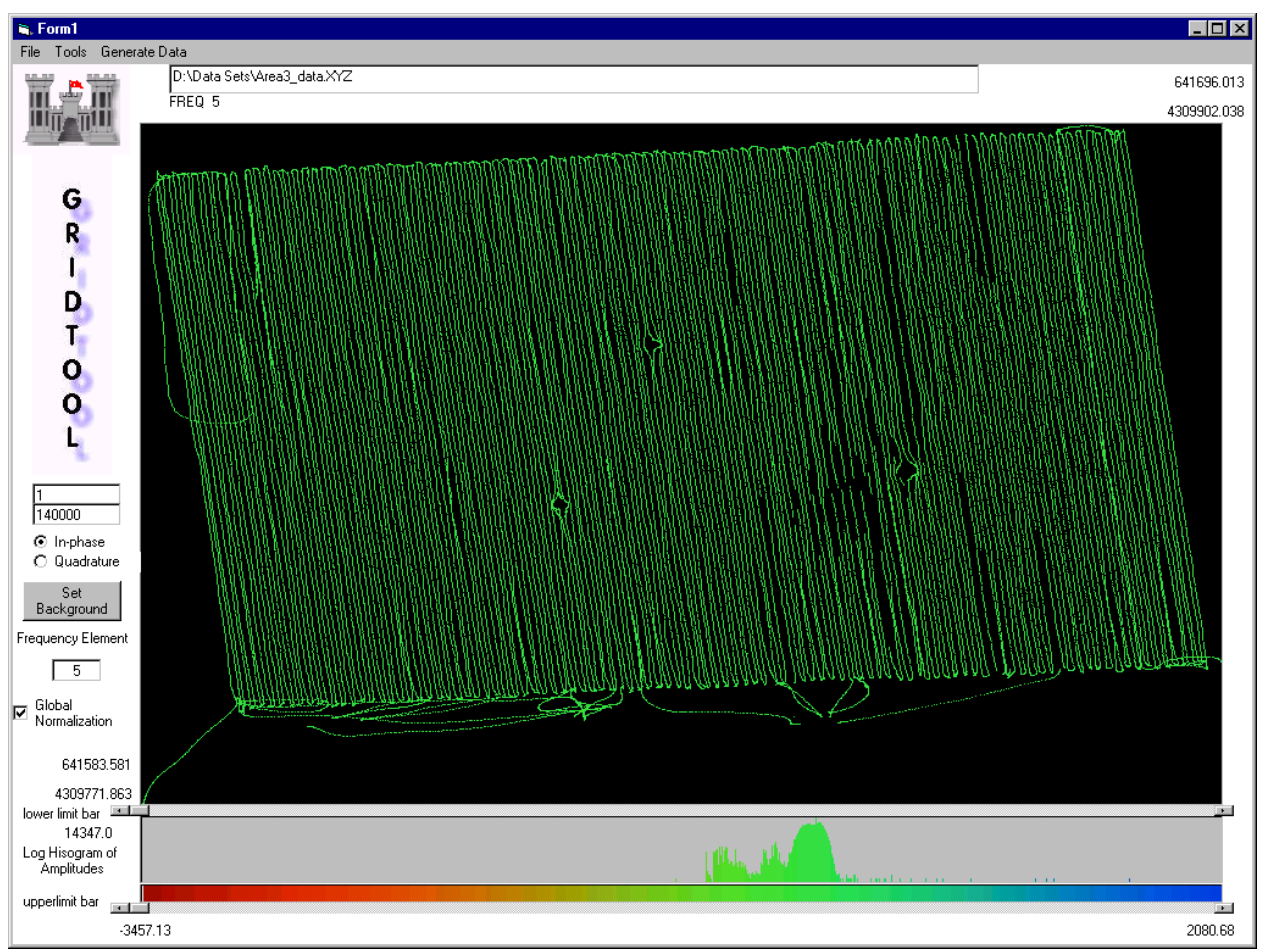

Figure A33. Area 3 in-phase coverage map for $2,790 \mathrm{~Hz}$

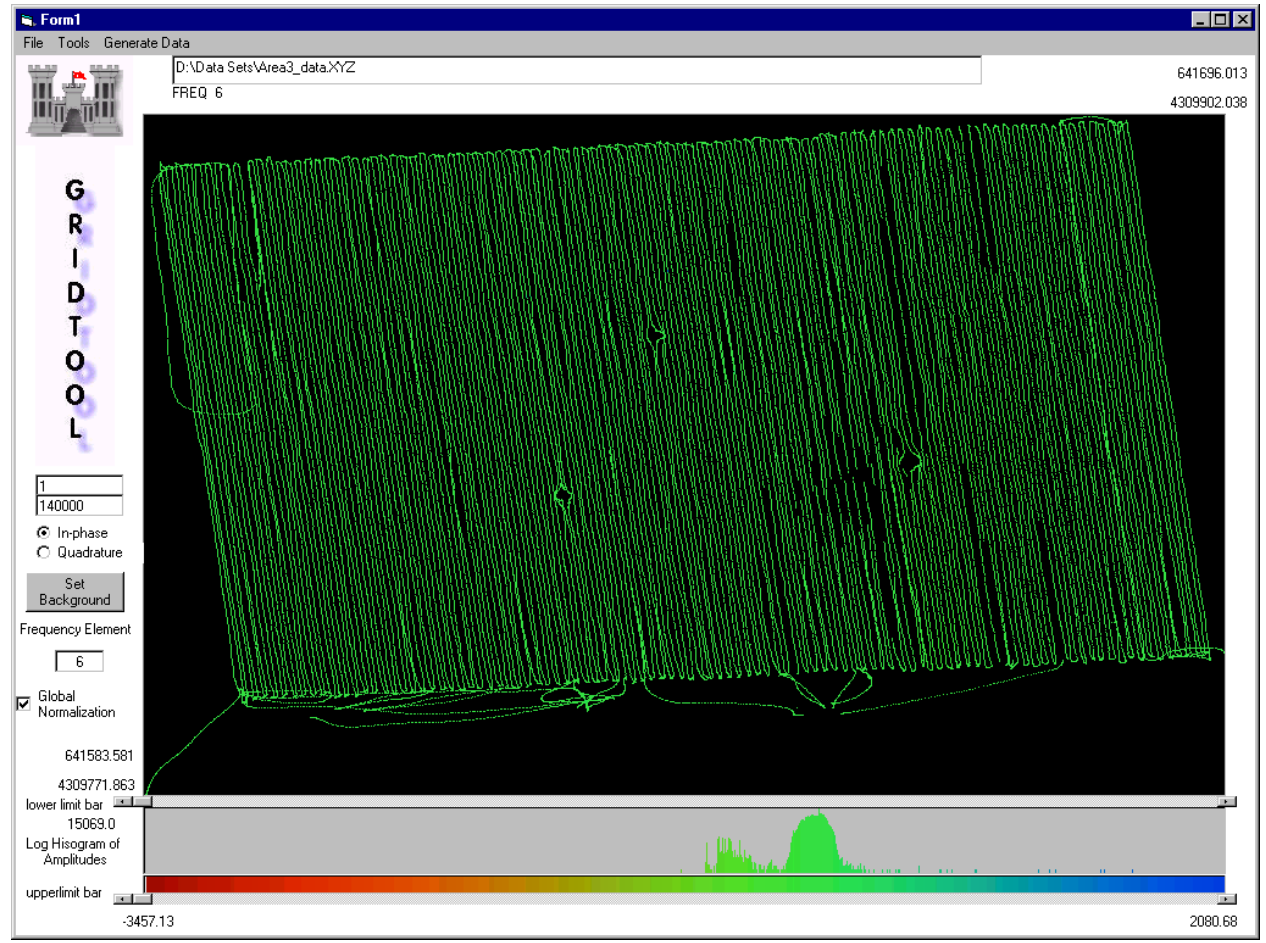

Figure A34. Area 3 in-phase coverage map for $8,190 \mathrm{~Hz}$ 


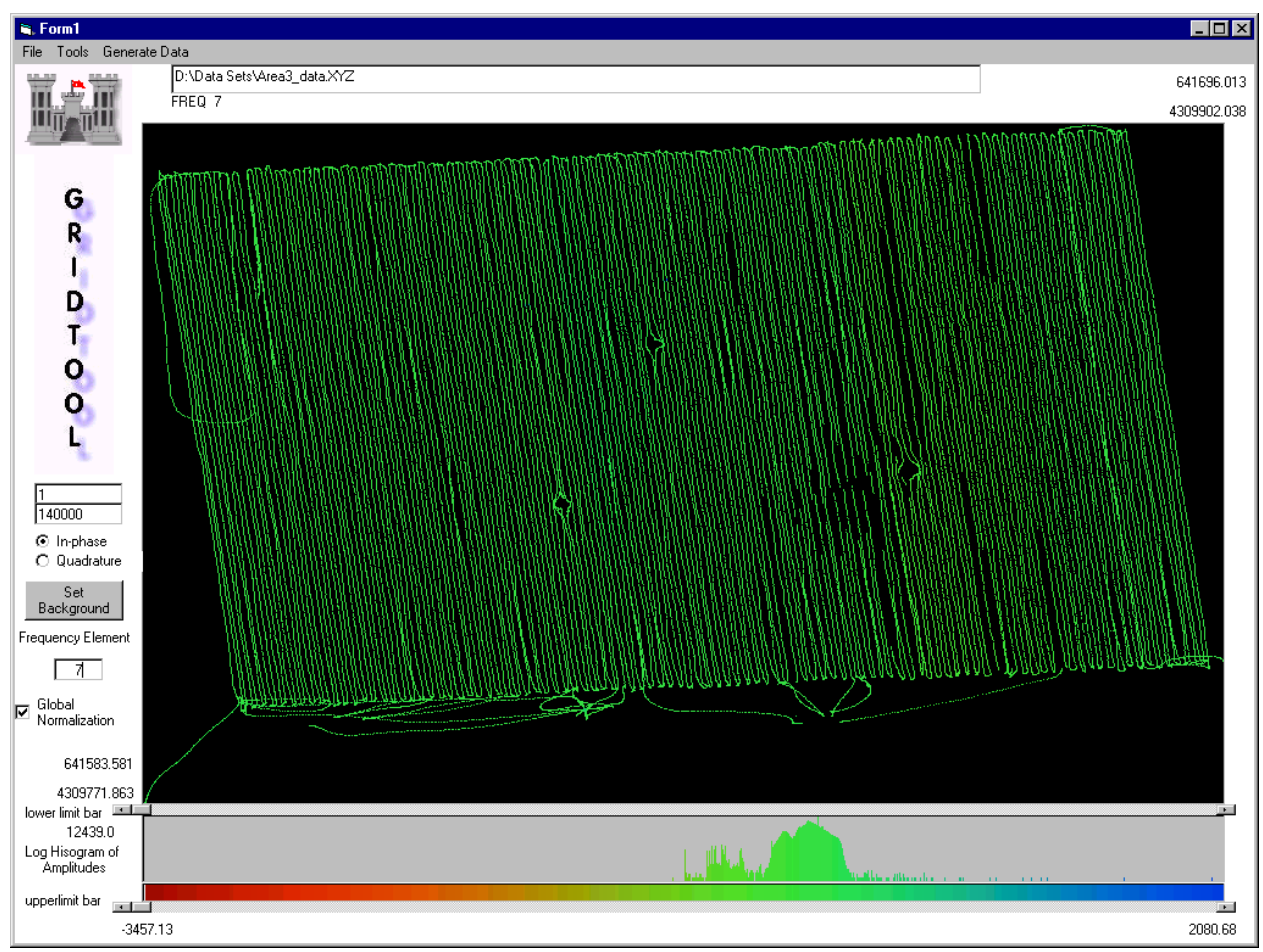

Figure A35. Area 3 in-phase coverage map for $20,010 \mathrm{~Hz}$

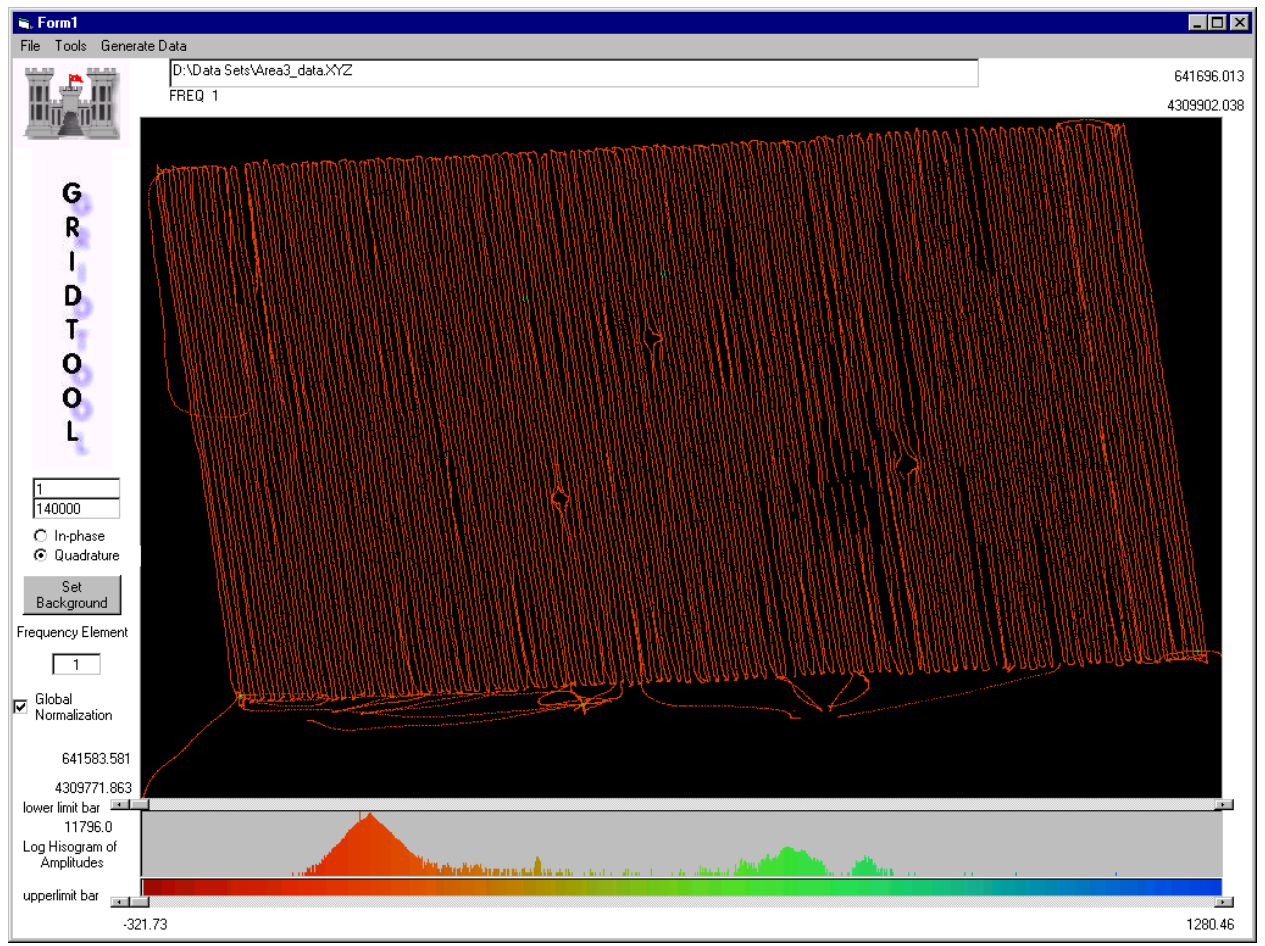

Figure A36. Area 3 quadrature coverage map for $90 \mathrm{~Hz}$ 


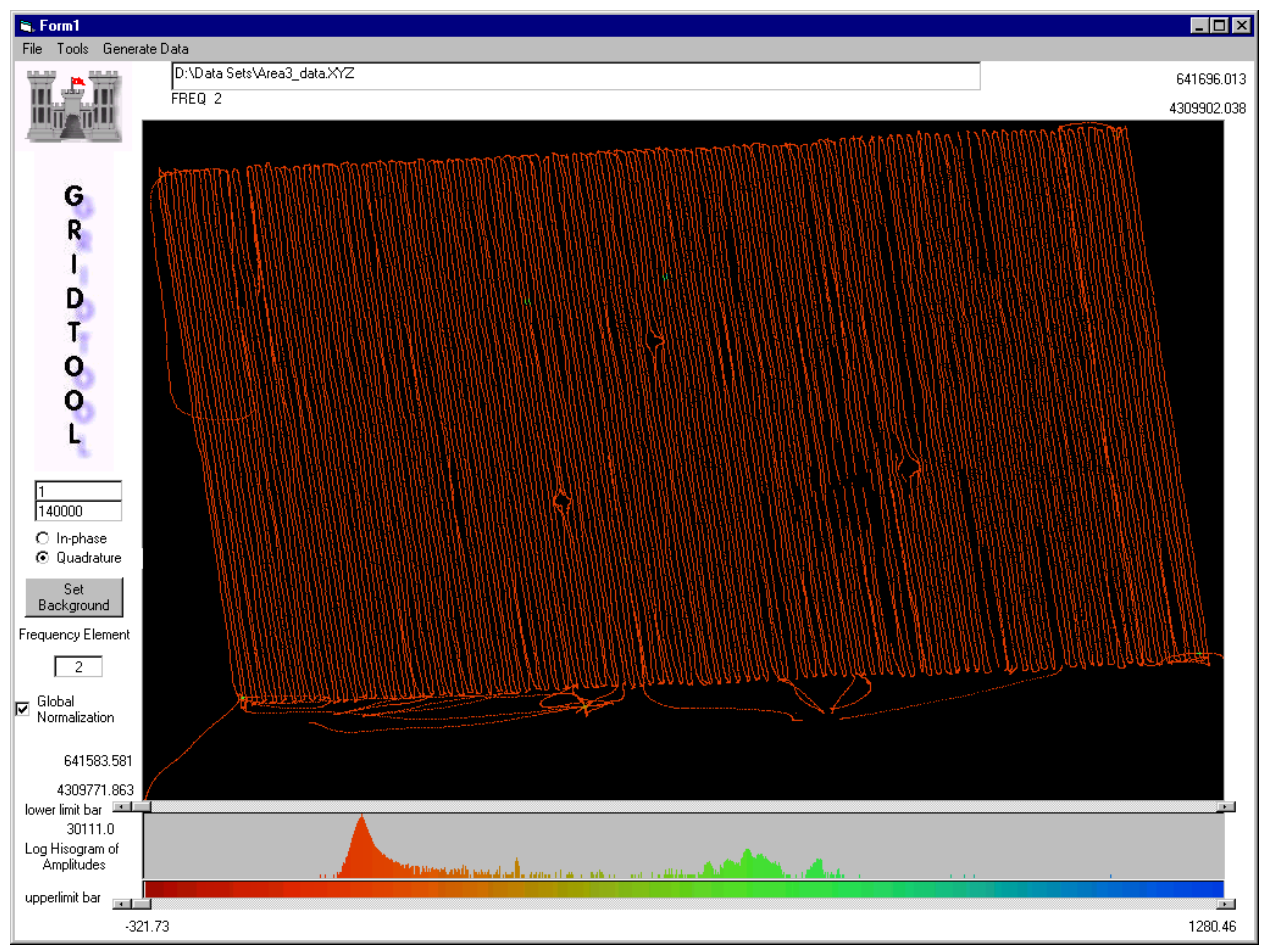

Figure A37. Area 3 quadrature coverage map for $150 \mathrm{~Hz}$

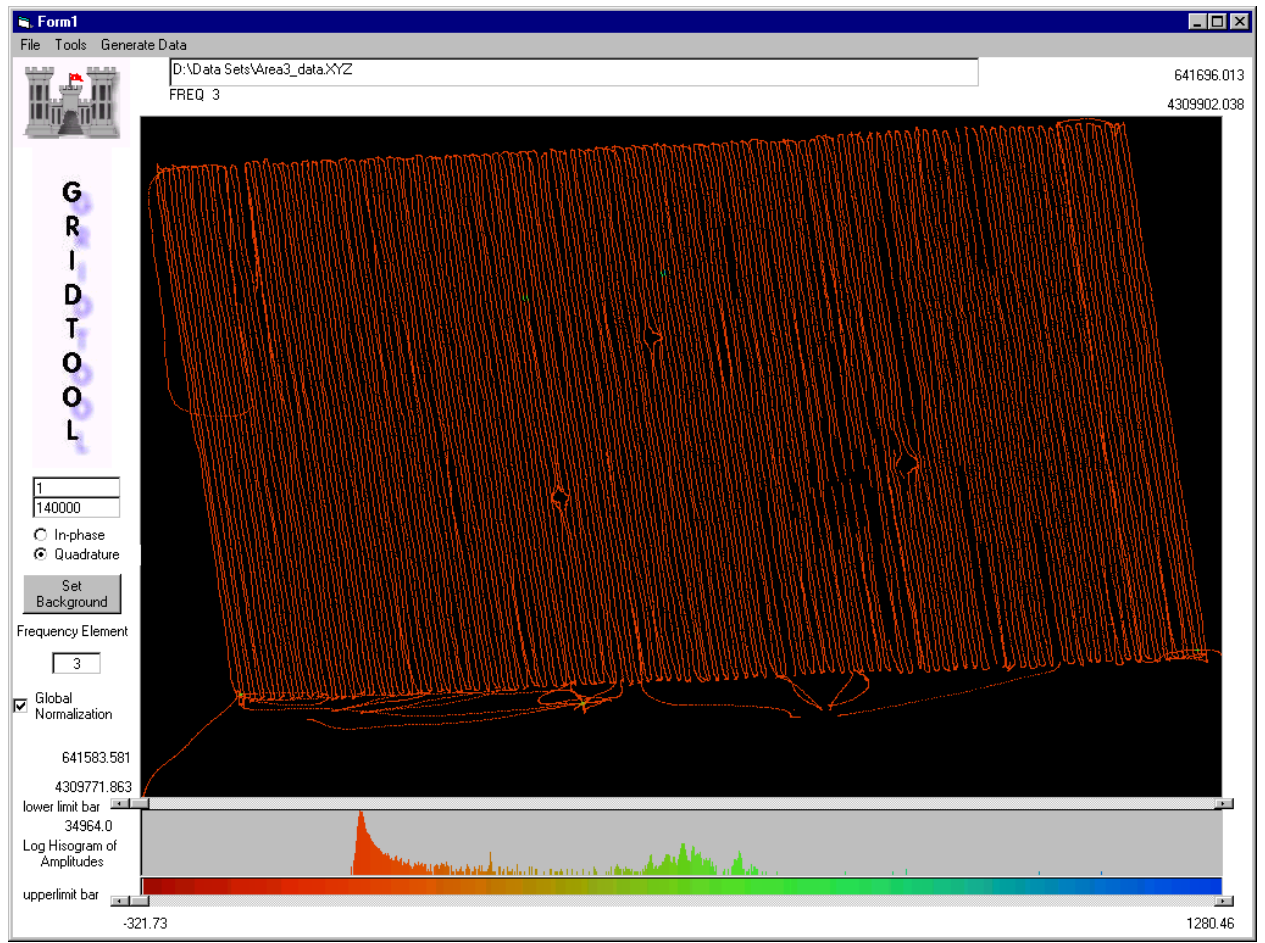

Figure A38. Area 3 quadrature coverage map for $330 \mathrm{~Hz}$ 


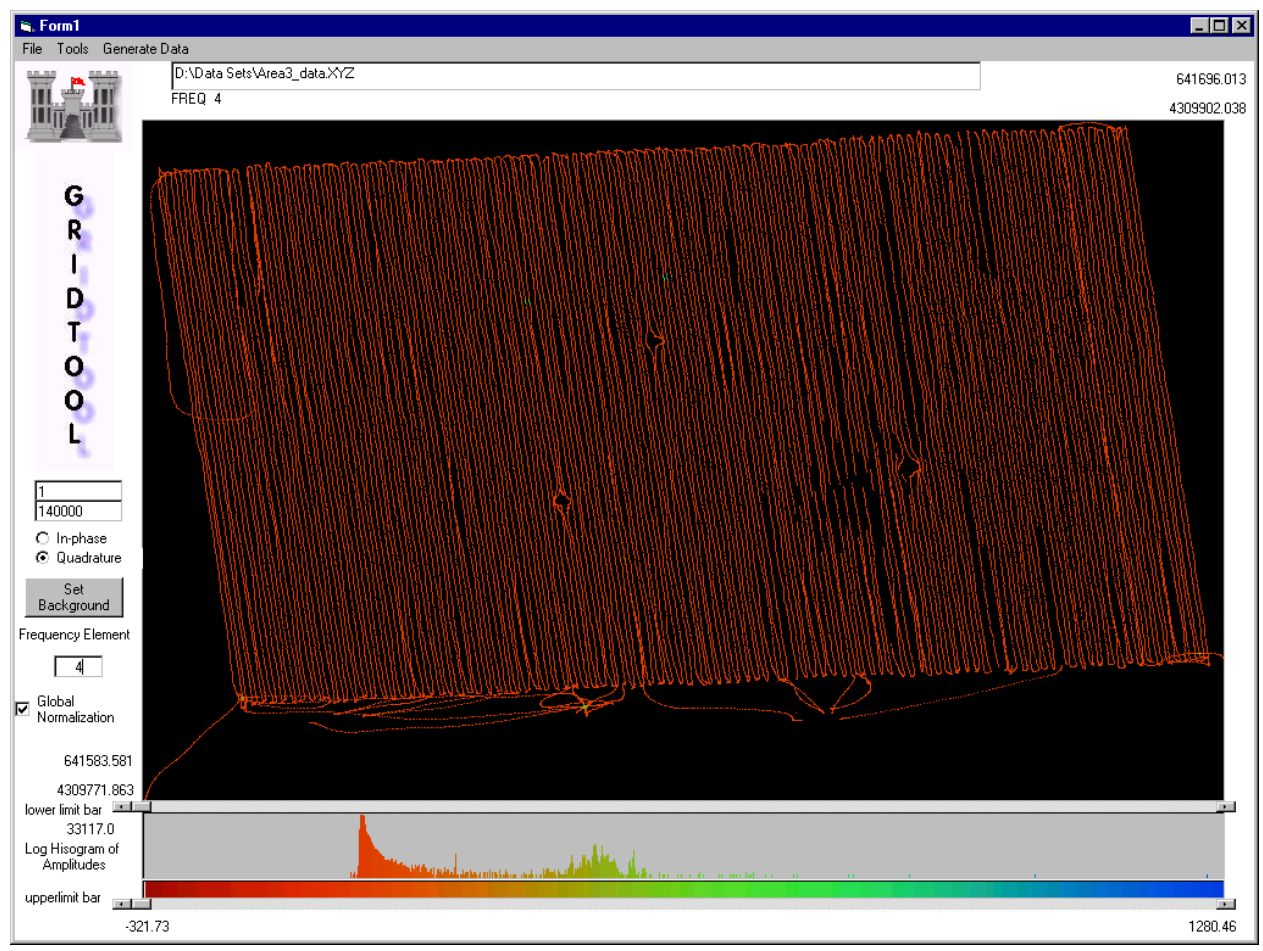

Figure A39. Area 3 quadrature coverage map for $930 \mathrm{~Hz}$

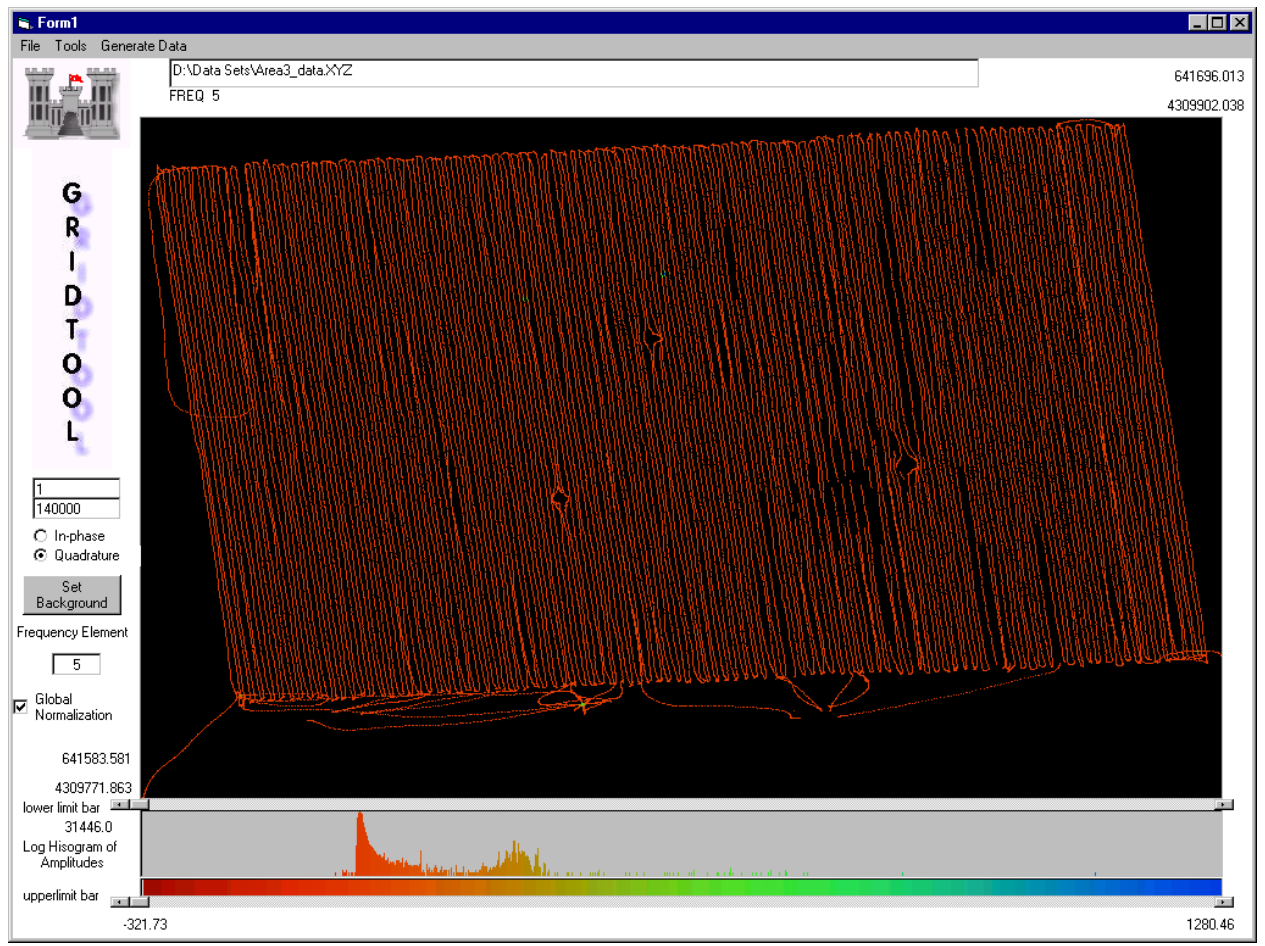

Figure A40. Area 3 quadrature coverage map for $2,790 \mathrm{~Hz}$ 


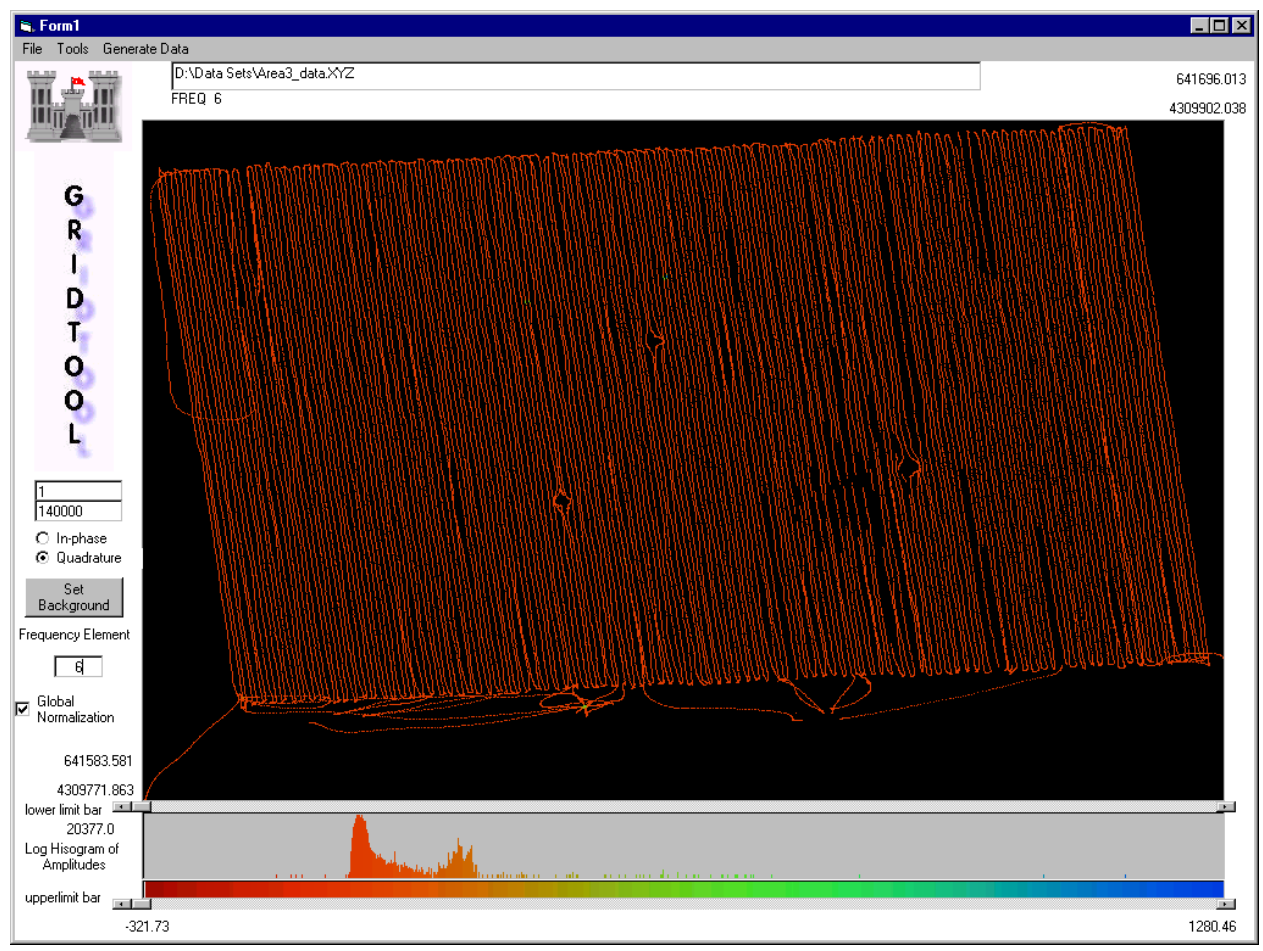

Figure A41. Area 3 quadrature coverage map for $8,190 \mathrm{~Hz}$

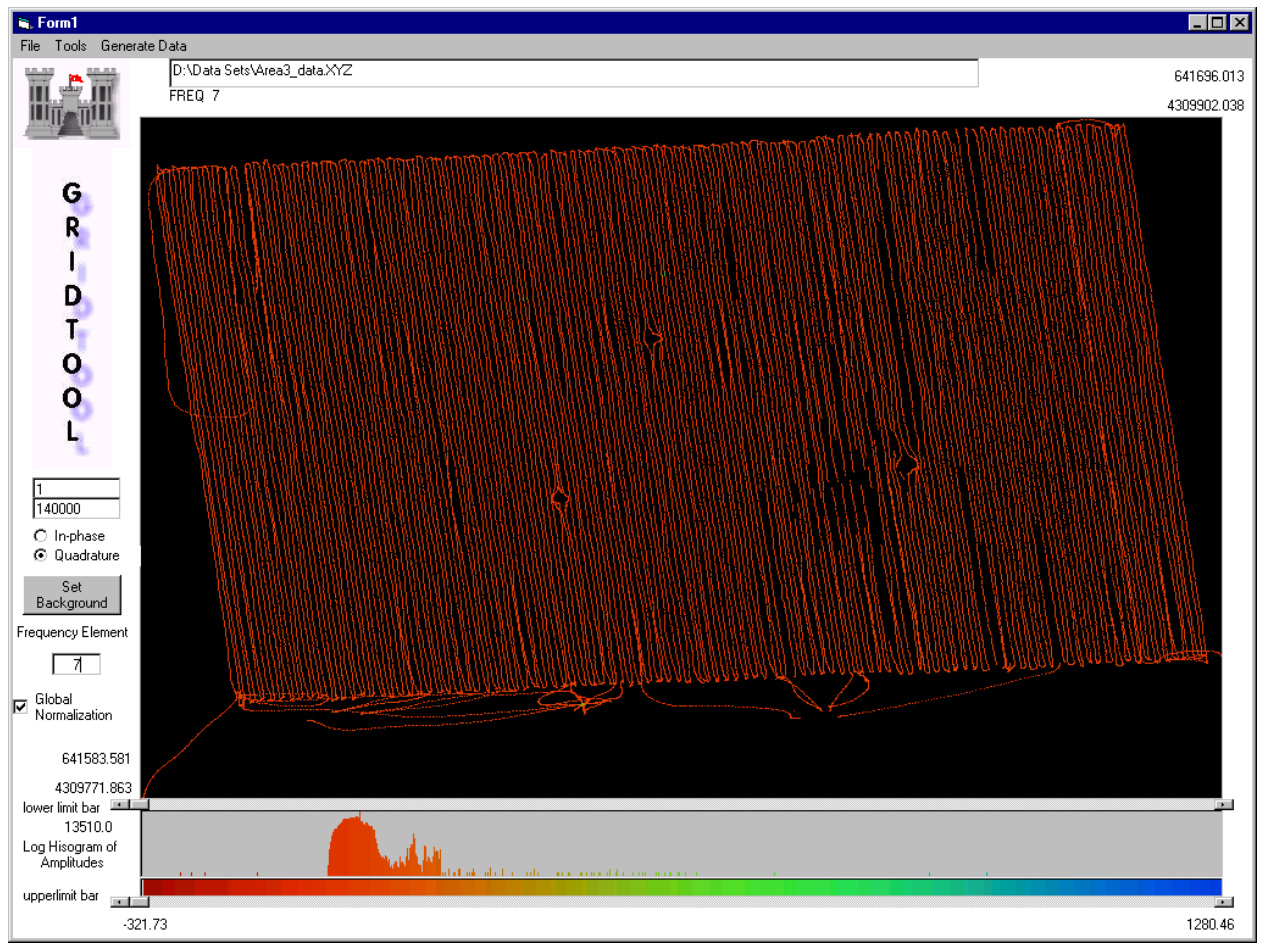

Figure A42. Area 3 quadrature coverage map for $20,010 \mathrm{~Hz}$ 


\section{Appendix B Plots of Data Point Locations and Comparison with Calibration Signatures}

This appendix contains a series of plots for each target in the JPG test. The first plot shows the locations of all data points in a $1-\mathrm{m}$ box centered on the target. The points are represented by plus markers that are color-coded to indicate the closeness of fit of the point to the calibration signature for the target's ordnance type. If no calibration data were available for the ordnance type of the target, all plots are based on the best matching ordnance type for each data point. Yellow indicates a fit error of $\leq 0.0333$. Cyan indicates a fit error of $>0.0333$ and $\leq 0.0666$. Dark blue represents values $>0.0666$ and $\leq 0.1$. The red marker in the center represents the target. Black represents fit error values of $>0.1$.

Each remaining plot shows the in-phase and quadrature values for a data point and the calibration signature at the seven frequencies used in the test. The red line represents the calibration data, and the blue line represents the data point. The seven frequencies on the x-axis are $90 \mathrm{~Hz}, 150 \mathrm{~Hz}, 330 \mathrm{~Hz}, 930 \mathrm{~Hz}$, $2,790 \mathrm{~Hz}, 8,190 \mathrm{~Hz}$, and 20,010 Hz. The y-axis shows normalized PPM. 
Target $\mathbf{1 - 8 6}$

Diameter : 4.2in

Depth : 20

Azimuth : 120

Inclination : 45
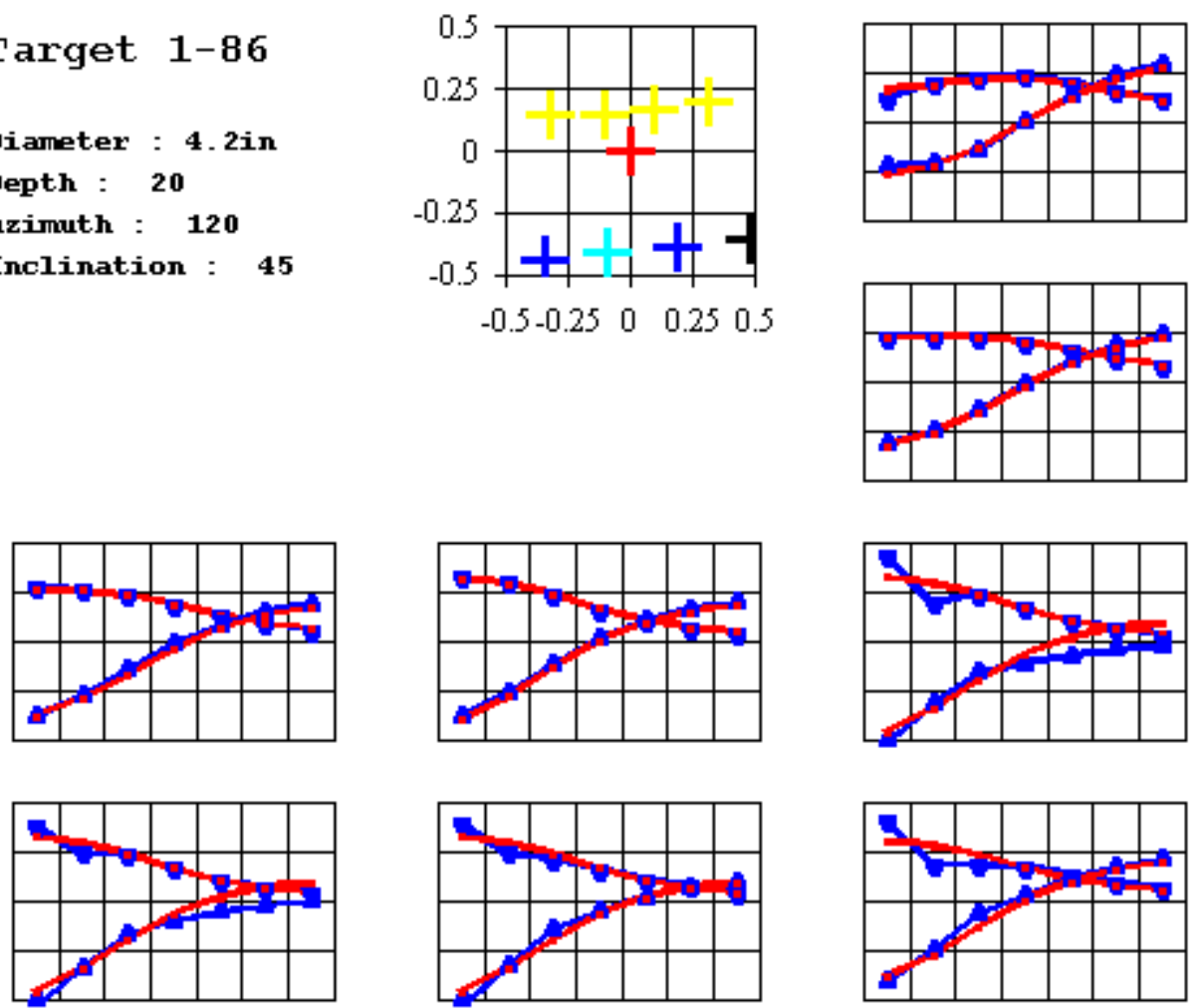
Target $\mathbf{1 - 8 8}$
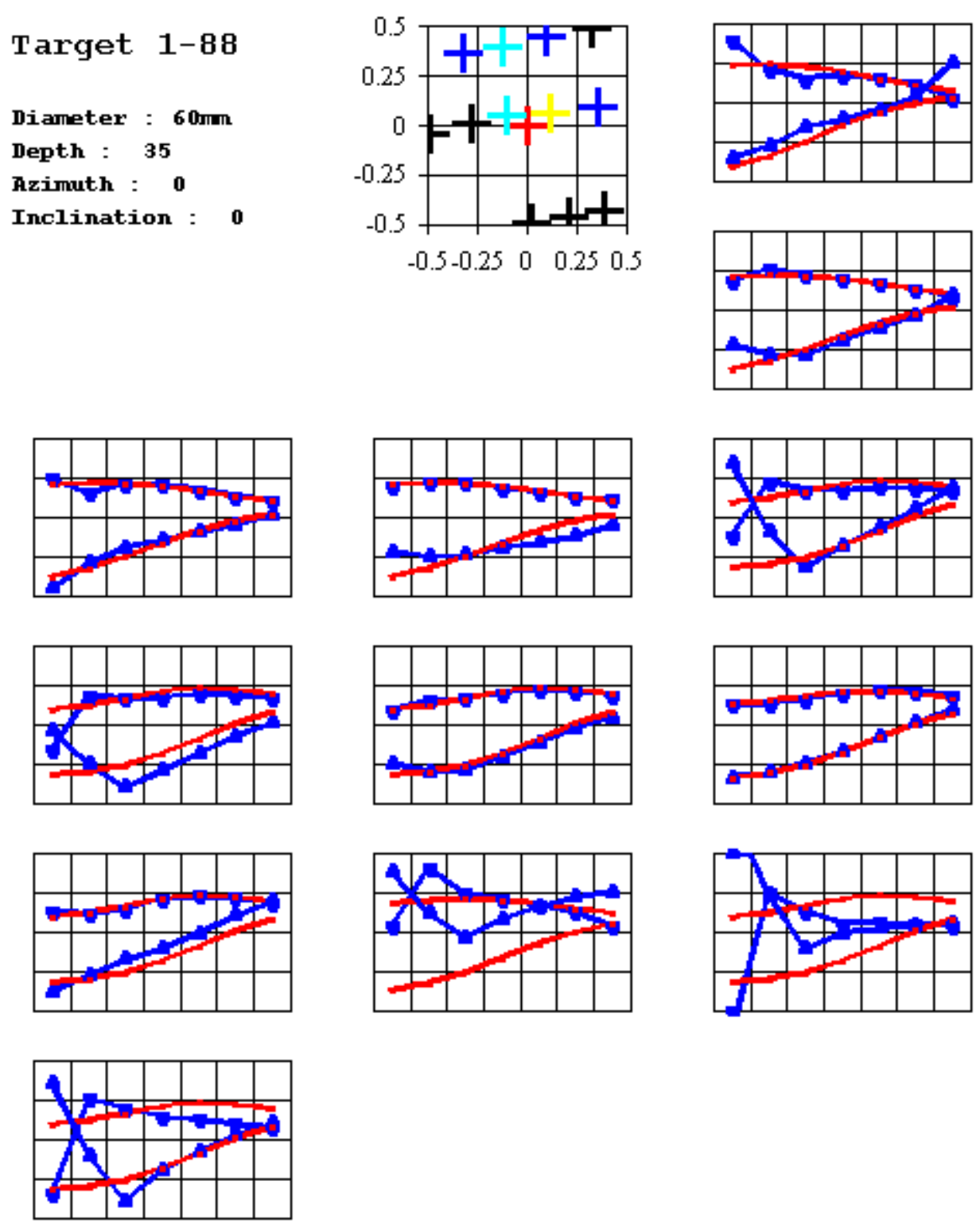
Target $1-90$

Diameter : 4.2in

Depth : 35

Azimuth : 270

Inclination : 0
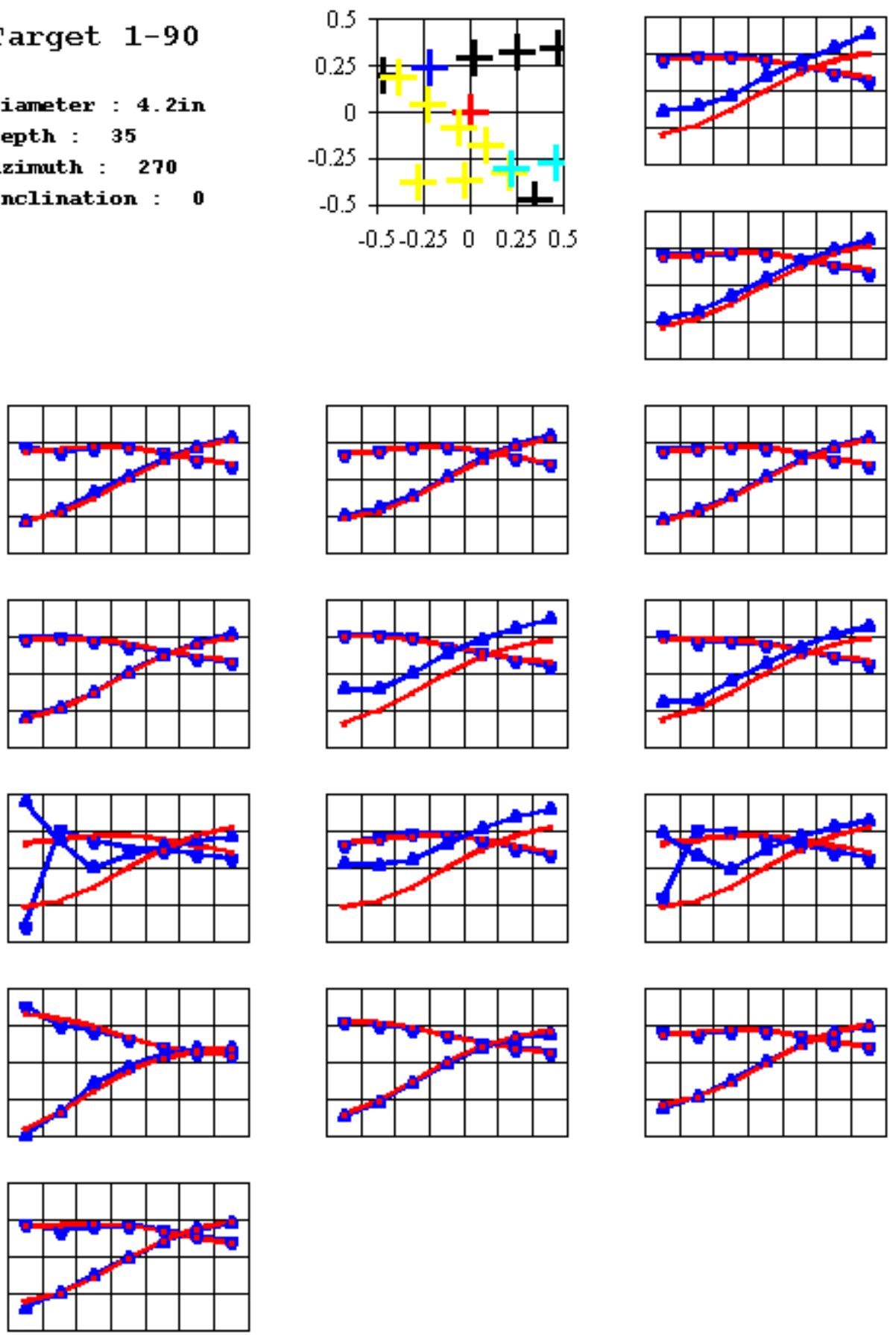

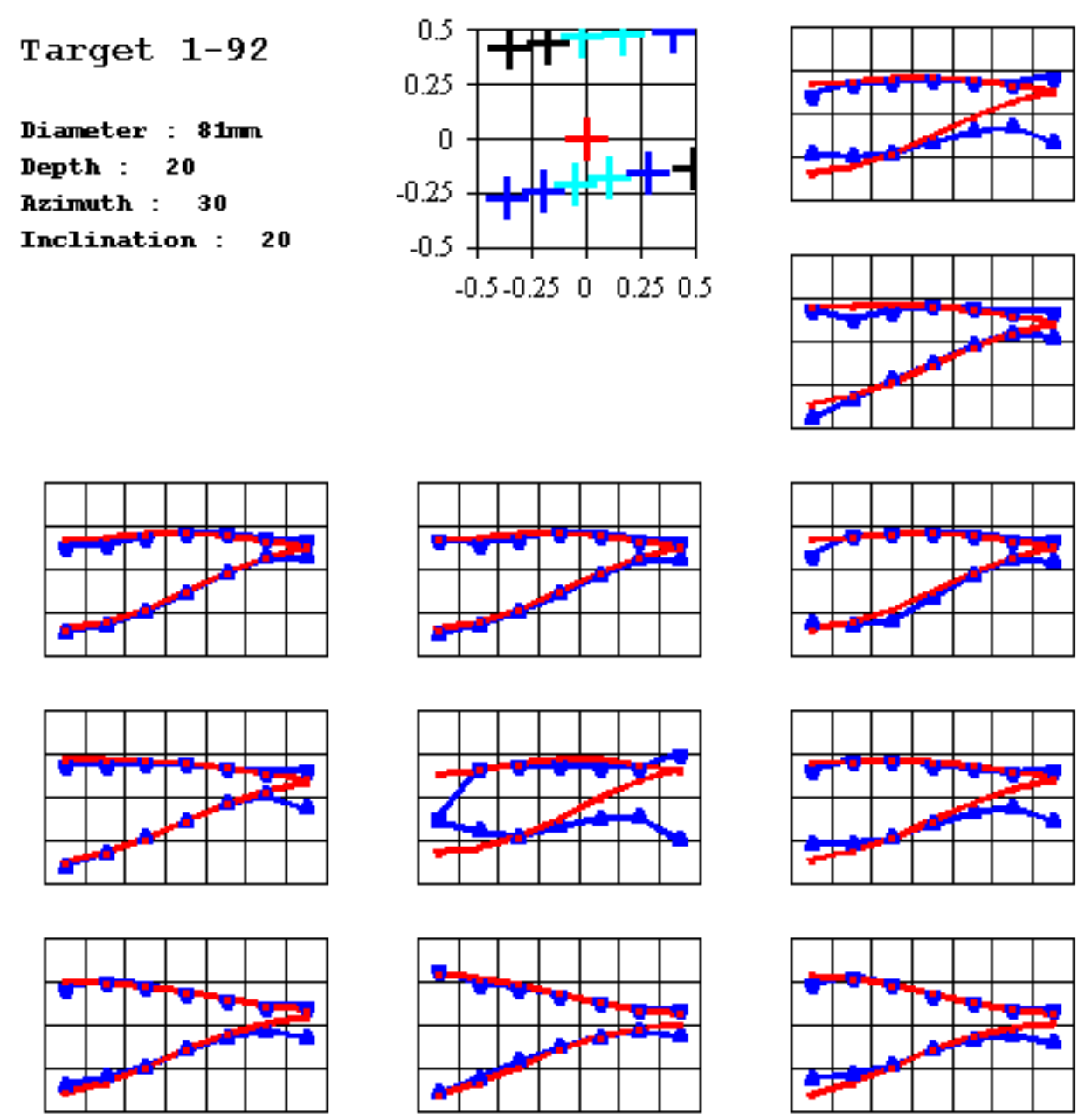

Target 1-94
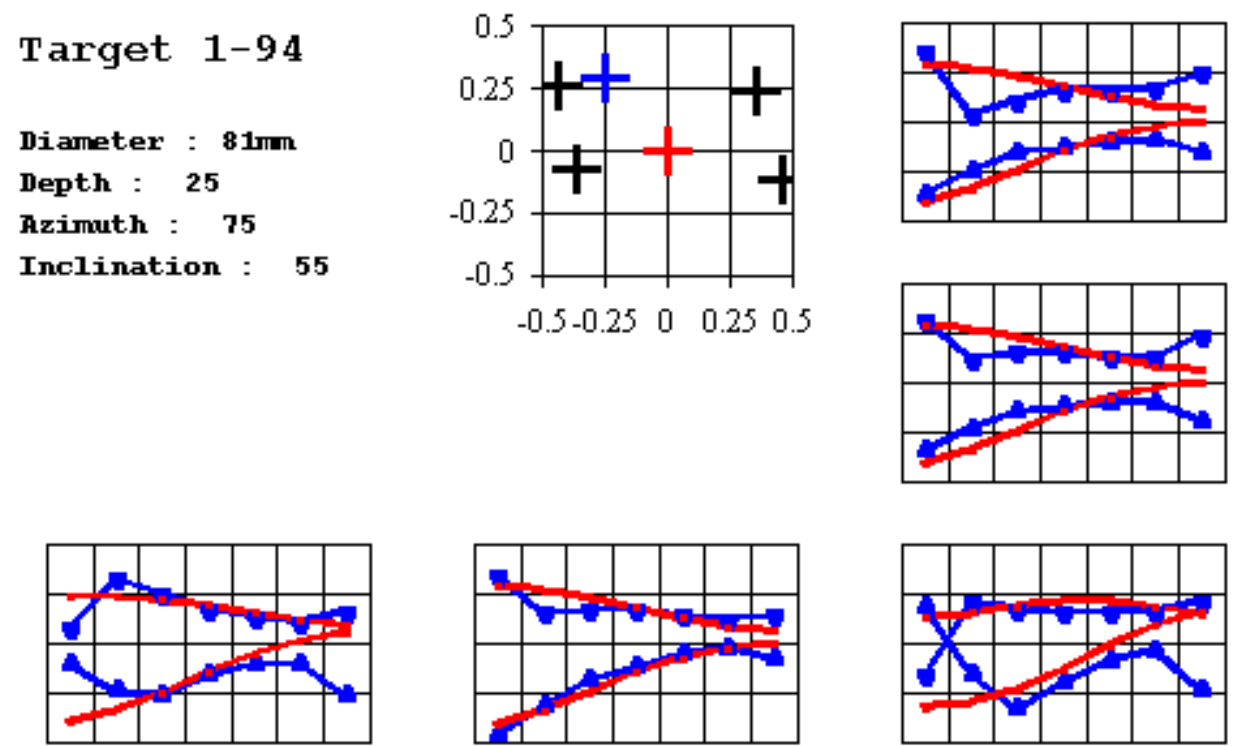
Target $1-96$

Diameter : $\mathbf{8 1 m m}$

Depth : 15

Azimuth : $\mathbf{3 3 0}$

Inclination : 35
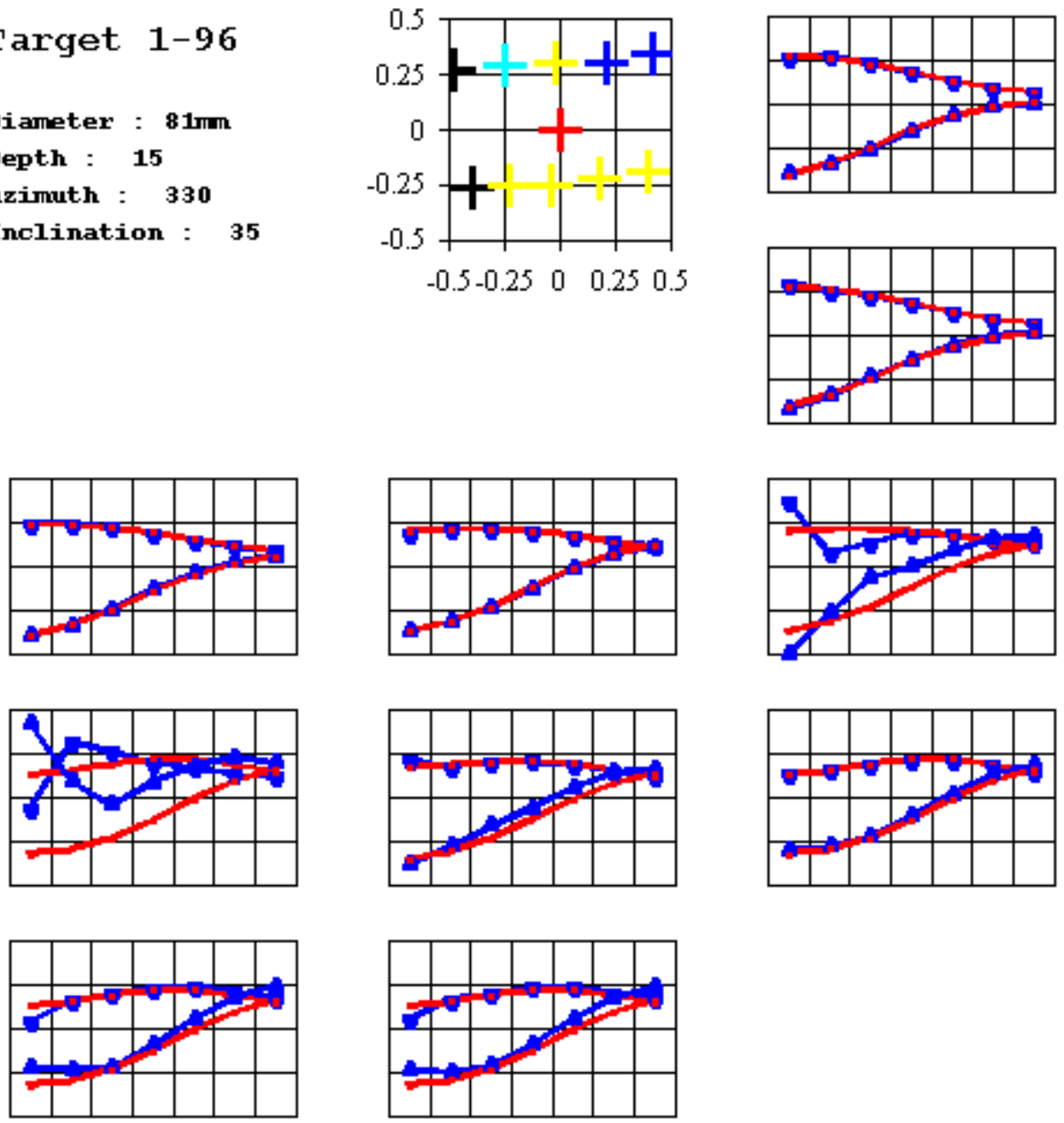
Target 1-98

Diameter : 60mm

Depth : 10

Azimuth : 275

Inclination : 20
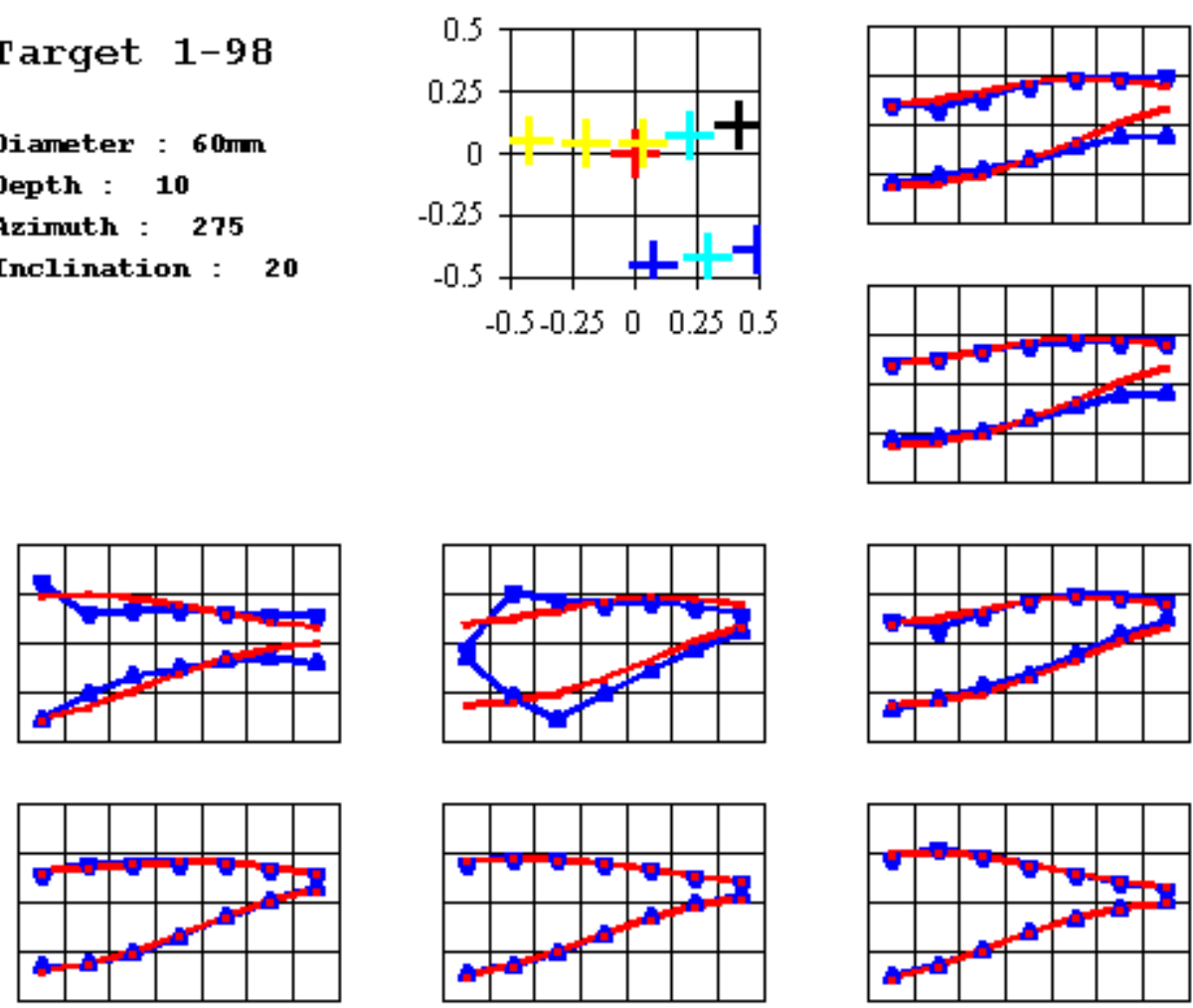

Target 1-100

Diameter : $60 \mathrm{~mm}$

Depth : 20

Azimuth : 195

Inclination : $\mathbf{3 0}$
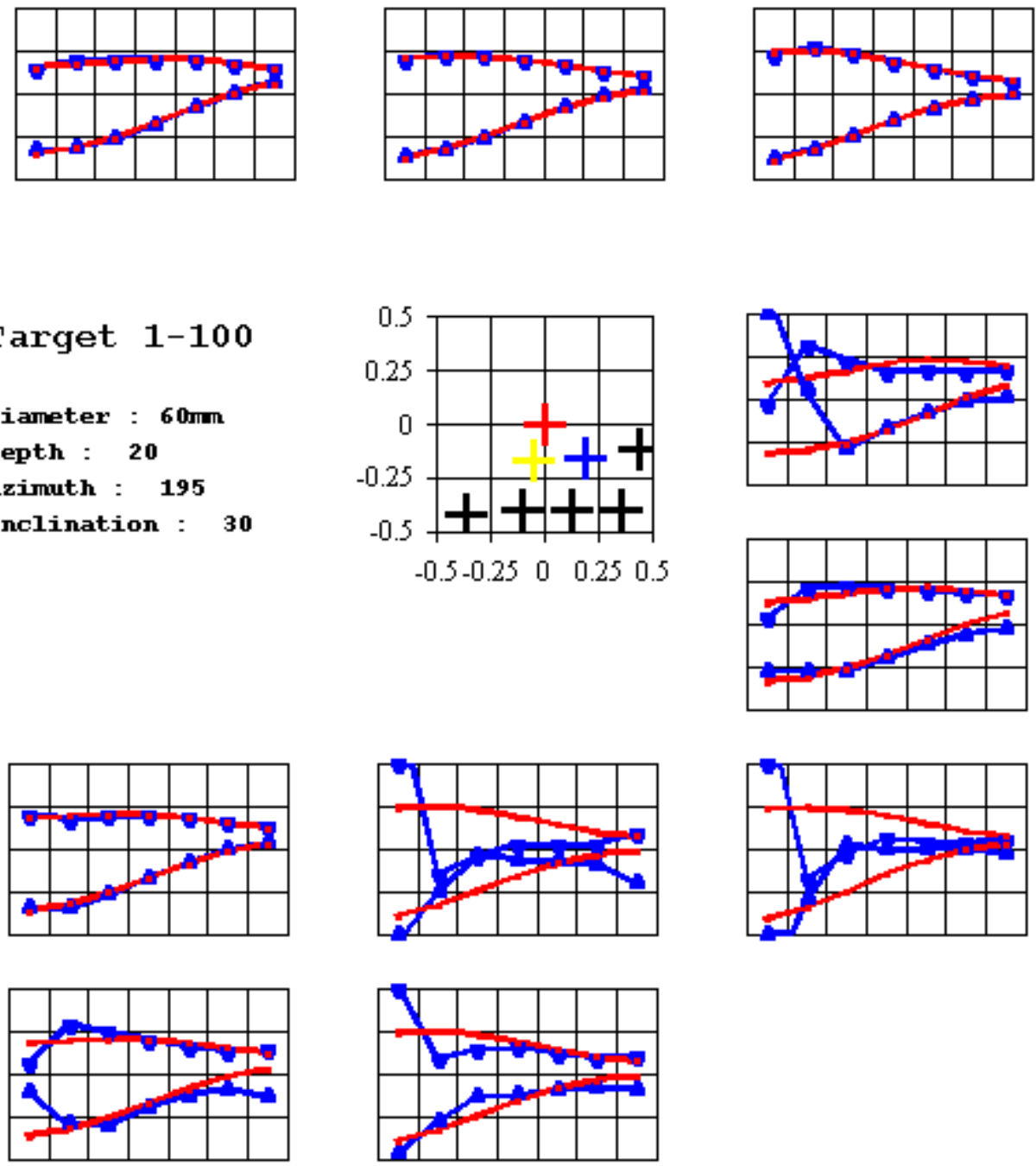
Target 1-102

Diameter : $81 \mathrm{~mm}$

Depth : 25

Fzimuth : $\mathbf{2 1 0}$

Inclination : 45
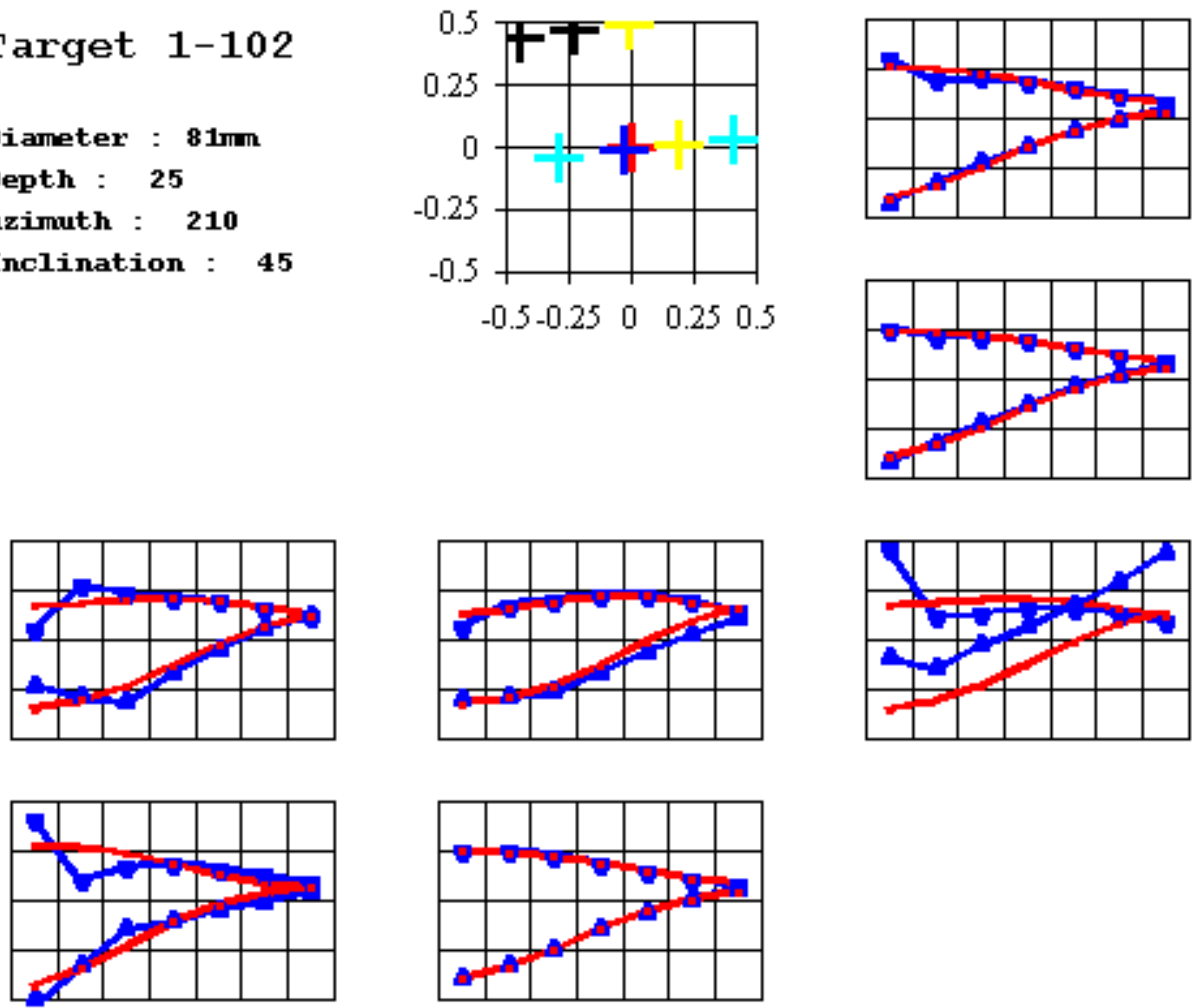
Target 1-104

Diameter : $81 \mathrm{~mm}$

Depth : 35

Azimuth : 180

Inclination : 0
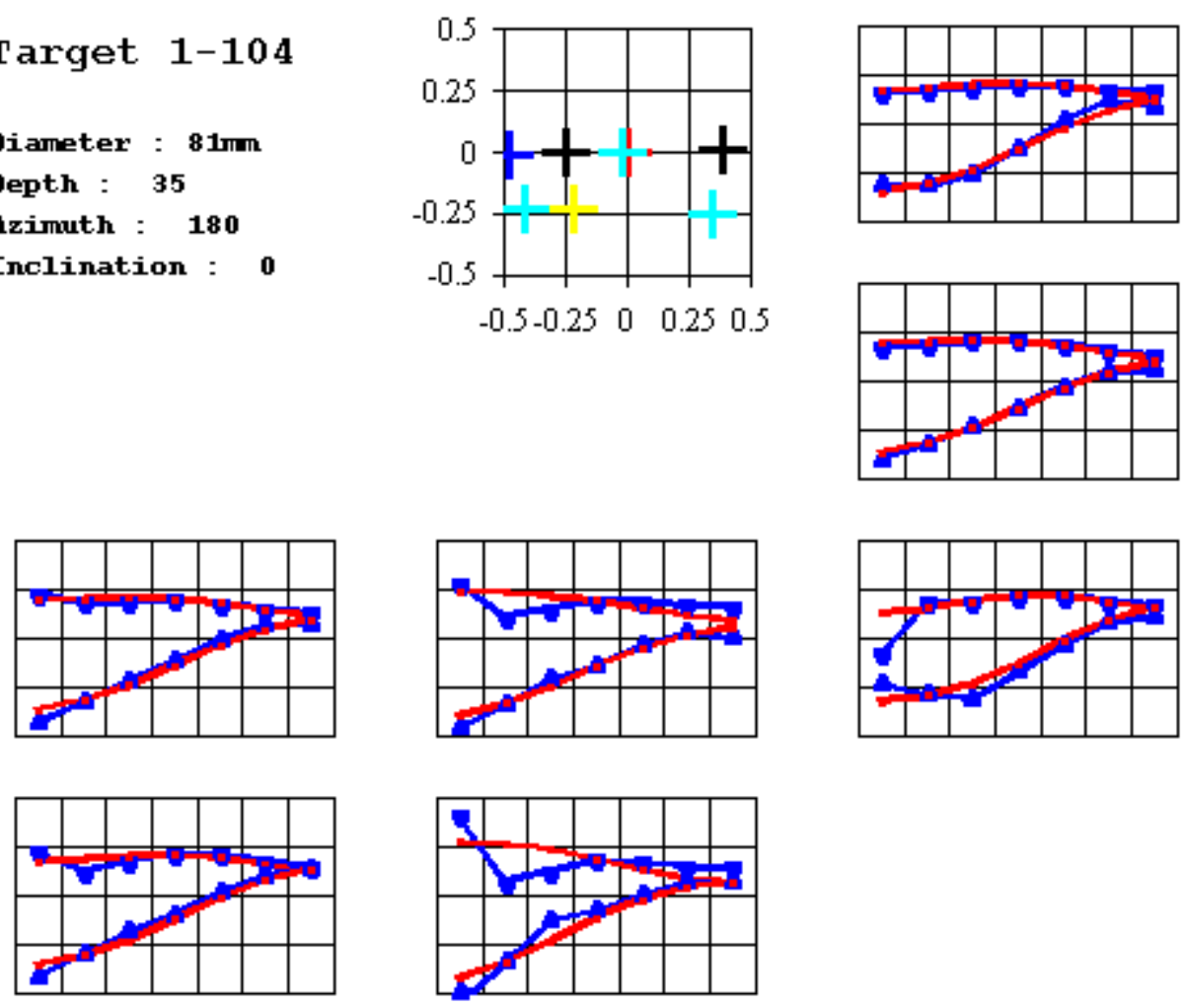

Target 1-106

Diameter : $60 \mathrm{~mm}$

Depth : 25

Azimuth : 75

Inclination : 35
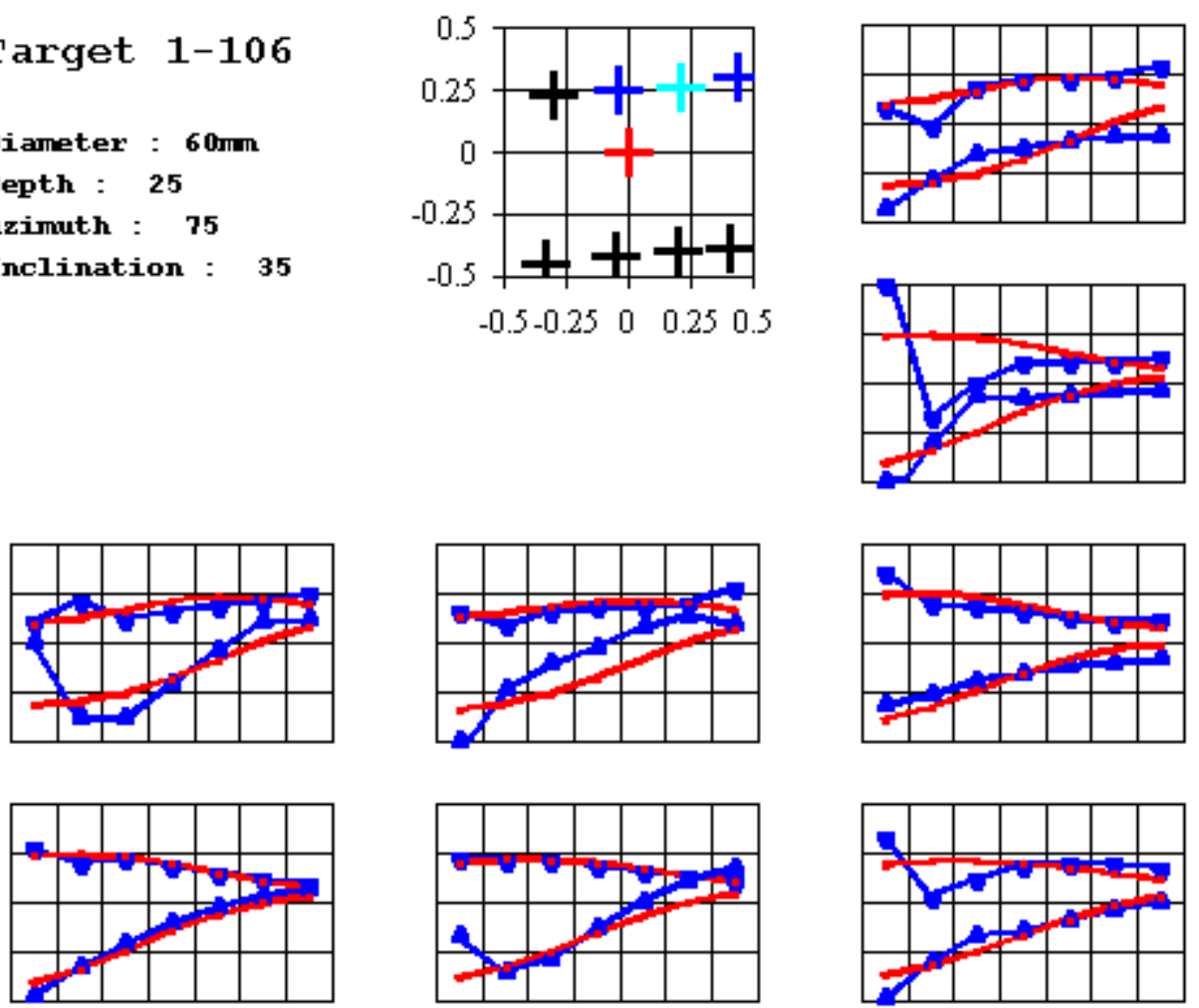
Target 1-108

Diameter : $60 \mathrm{~mm}$

Depth : 20

Azimuth : 100

Inclination : 45
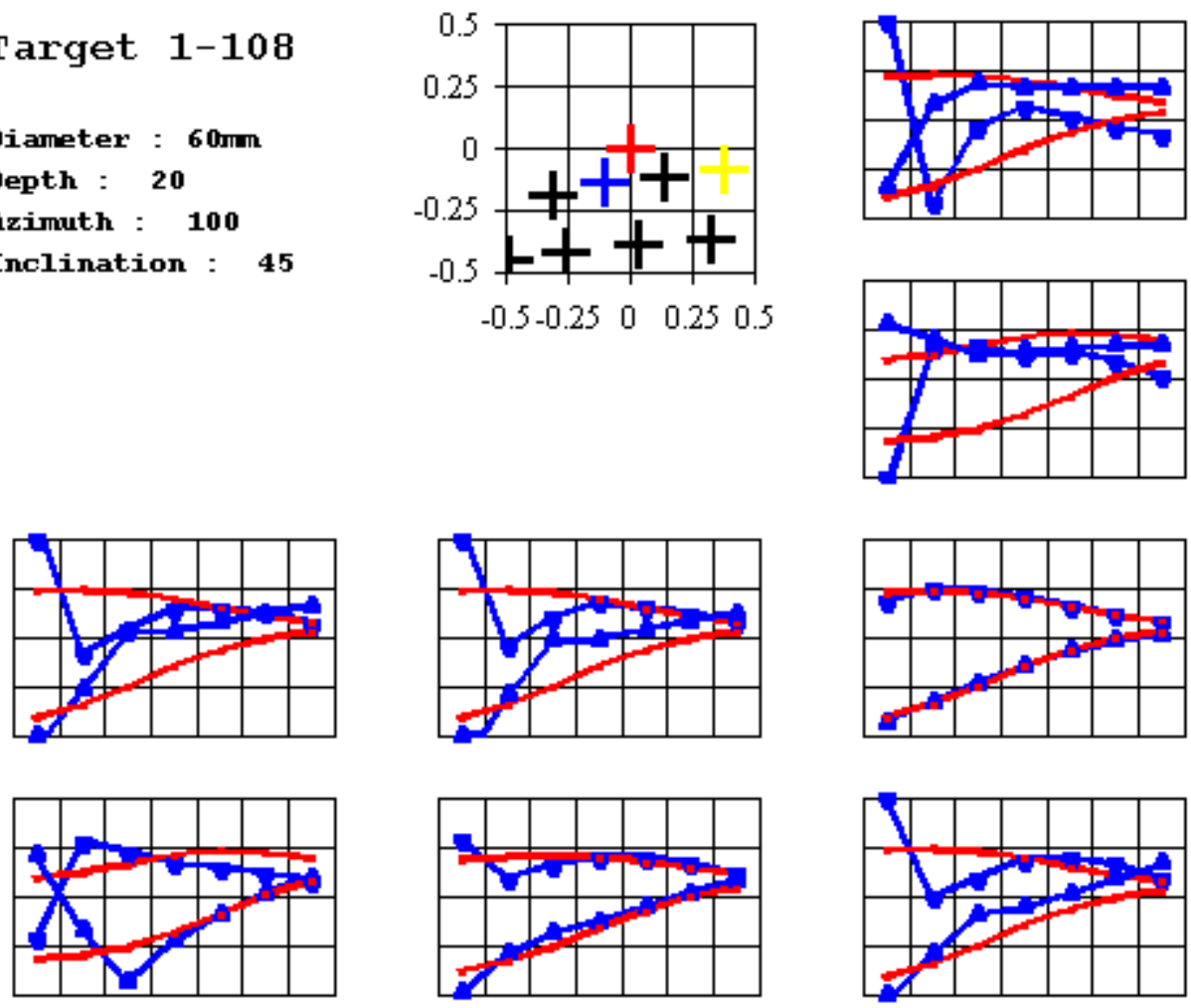
Target 1-112

Diameter : 20mm

Depth : 10

Azimuth : $\mathbf{3 0}$

Inclination : 10
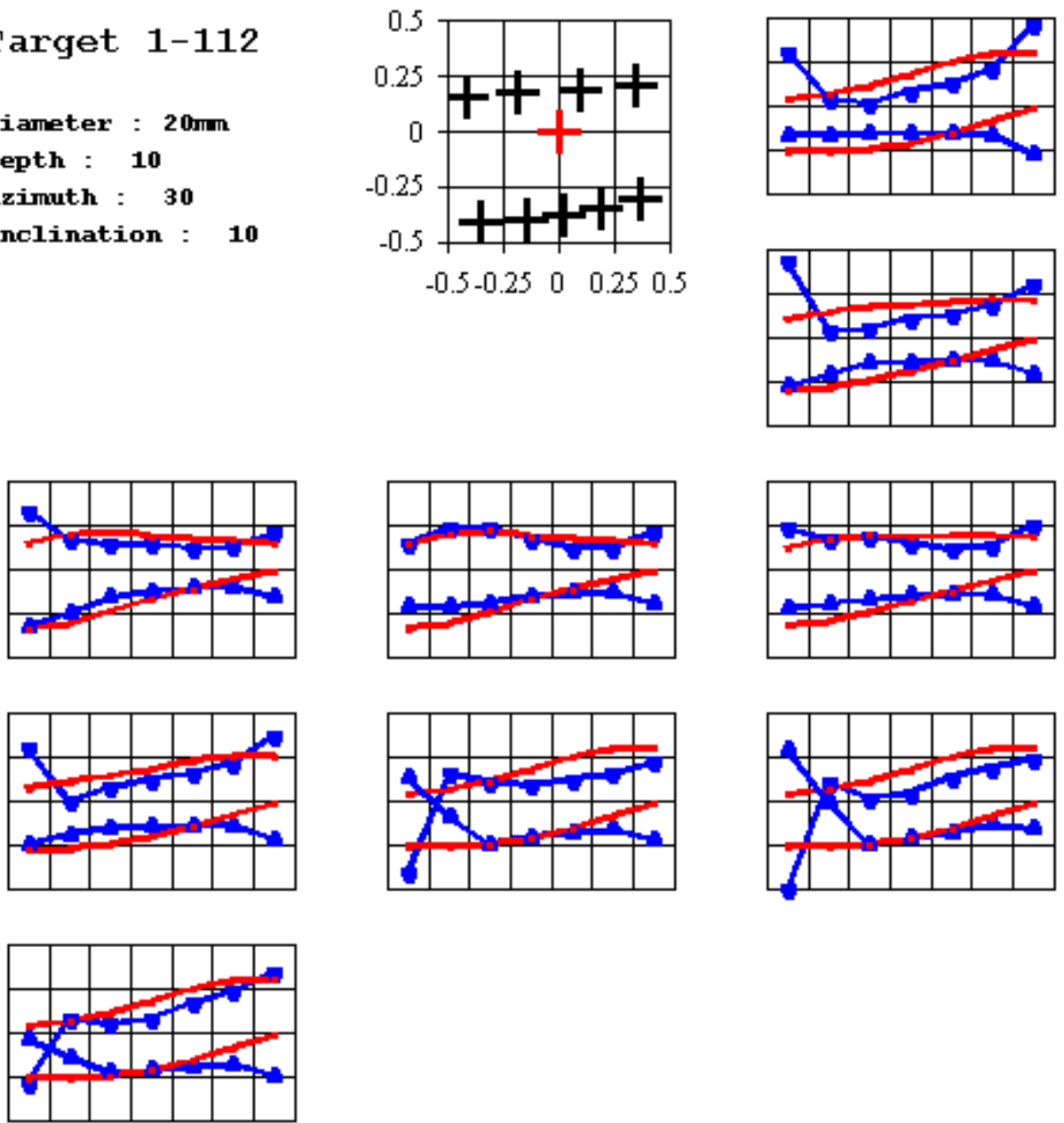
Target 1-113

Diameter : $105 \mathrm{~mm}$

Depth : 50

Azimuth : 120

Inclination : $\mathbf{3 0}$
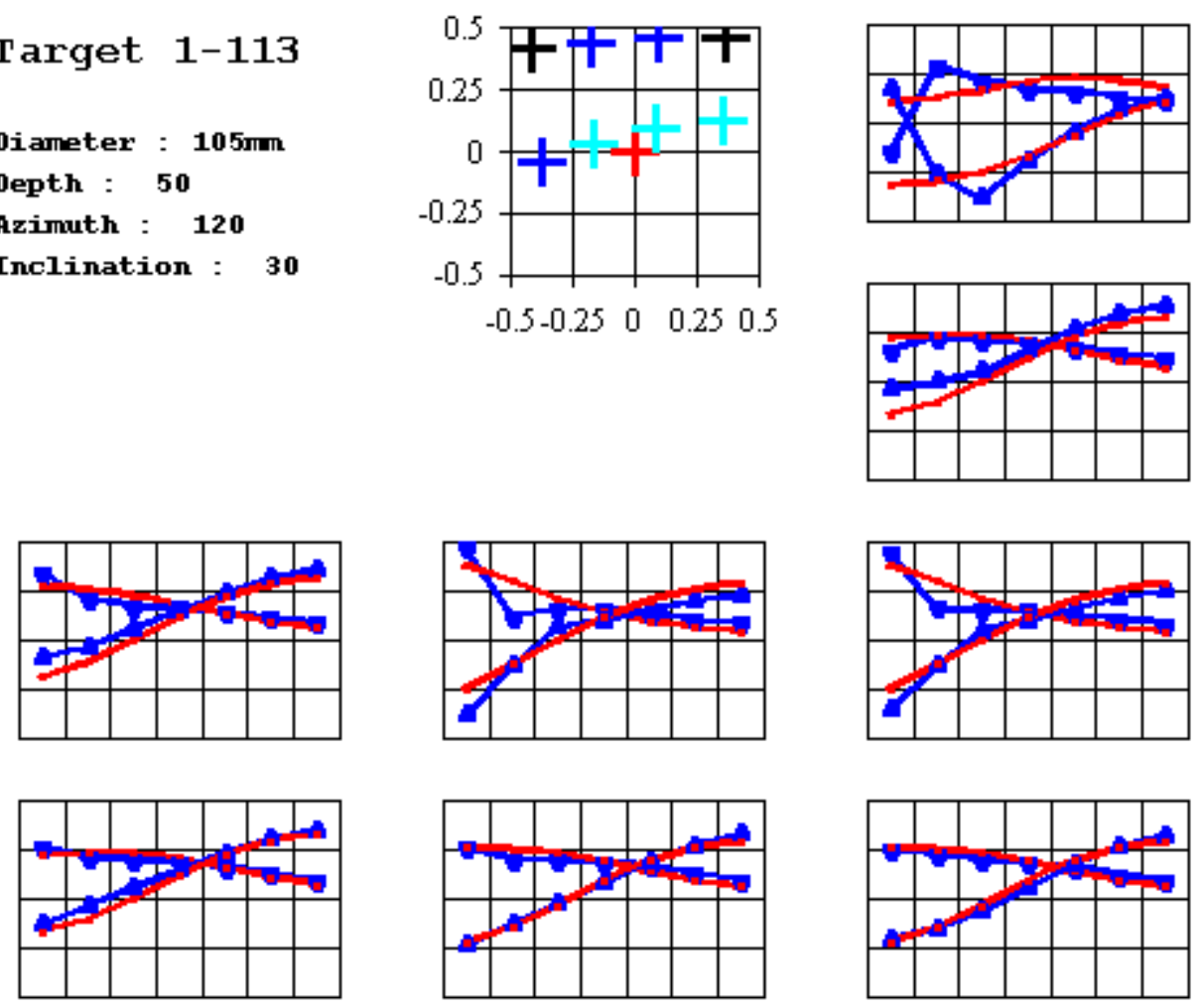
Target 1-114

Diameter : $20 \mathrm{~mm}$

Depth : 15

Azimuth : 175

Inclination : 20
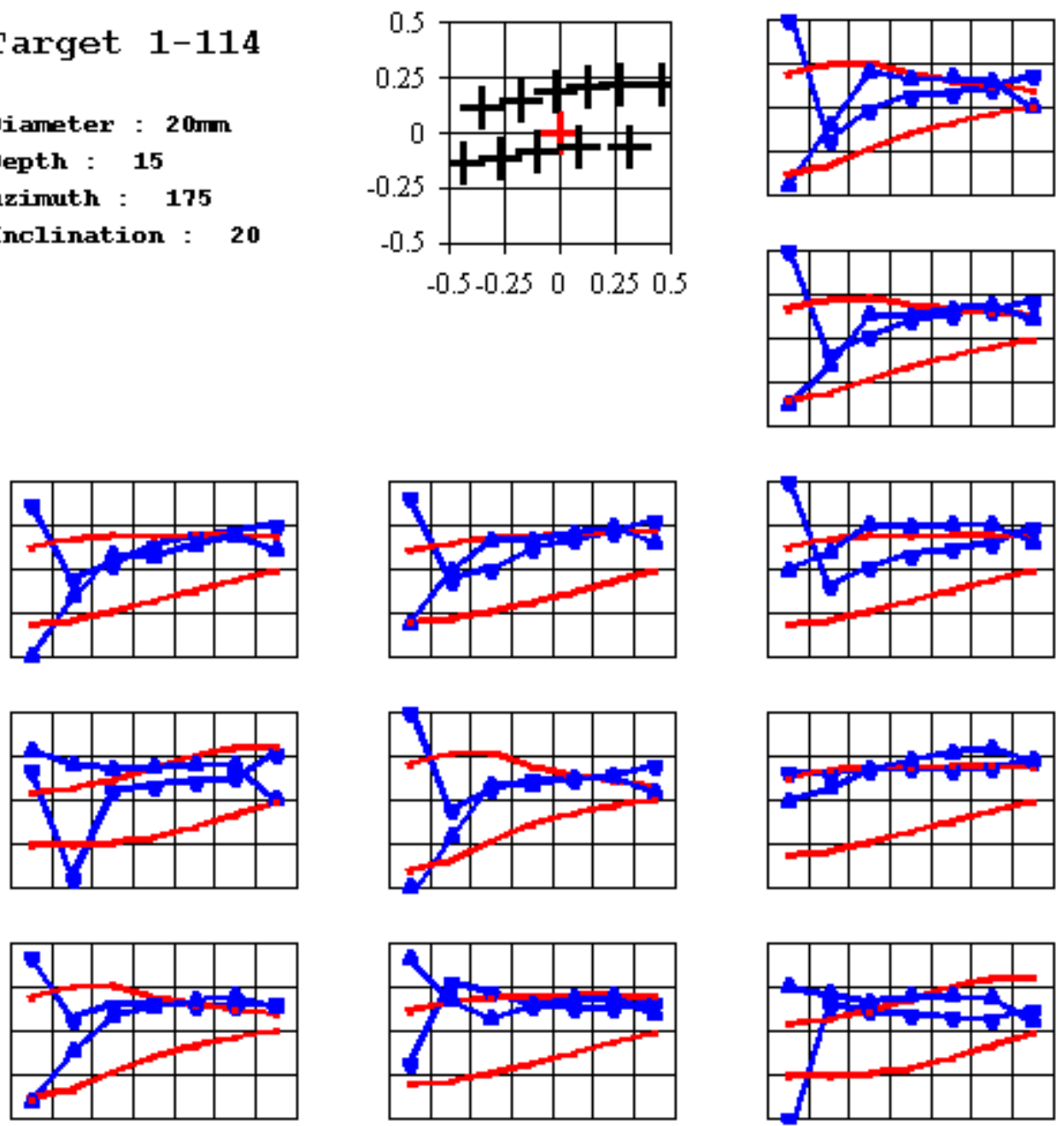
Target 1-115

Diameter : $76 \mathrm{~mm}$

Depth : 25

Azimuth : 270

Inclination : $\mathbf{3 0}$
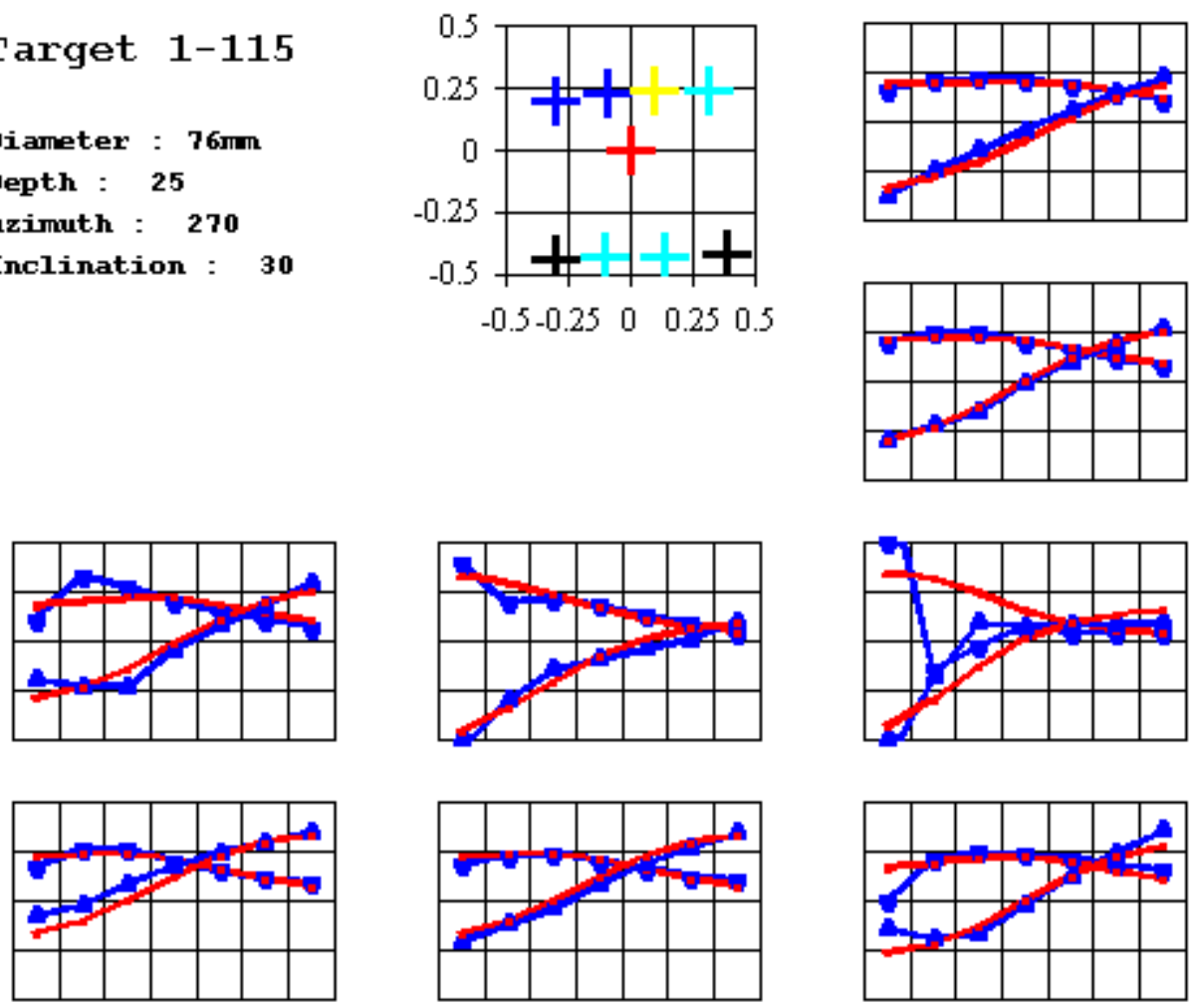

Target 1-116
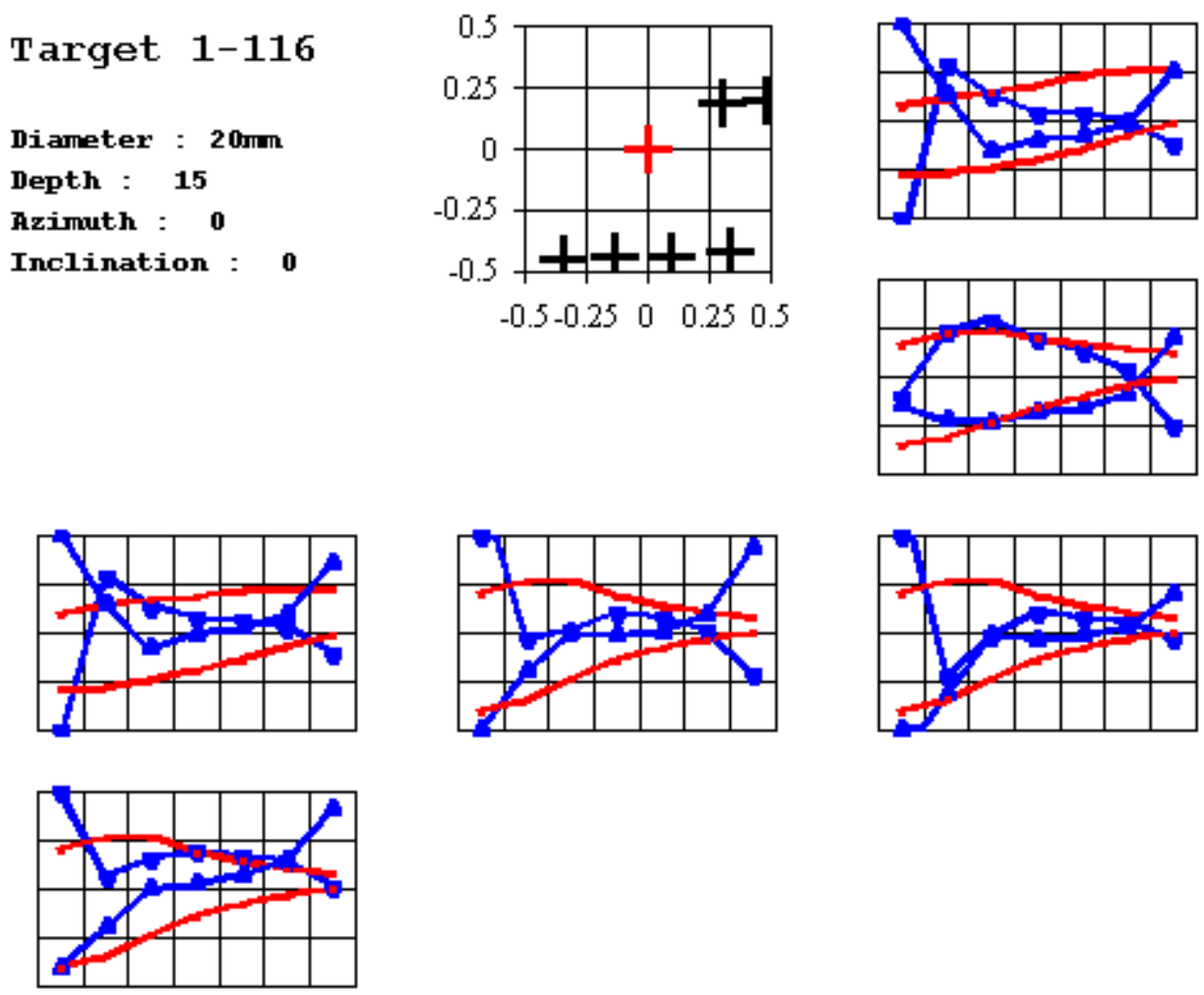
Target $1-117$

Diameter : $152 m m$

Depth : 90

Azimuth : 0

Inclination : $\mathbf{4 5}$
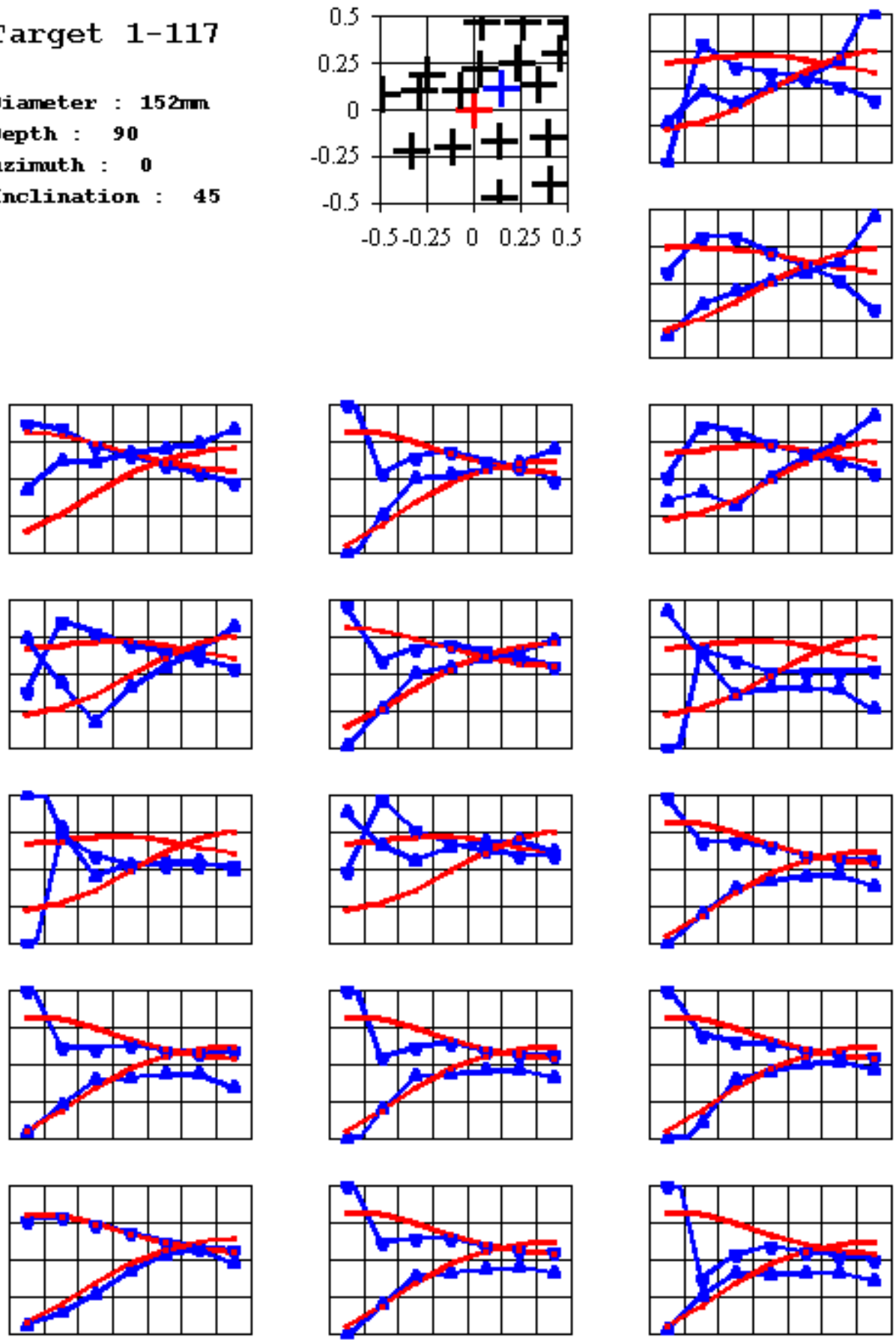
Target 1-118

Diameter : $5.0 i n$

Depth : $\mathbf{4 5}$

Azimuth : 120

Inclination : $\mathbf{3 0}$
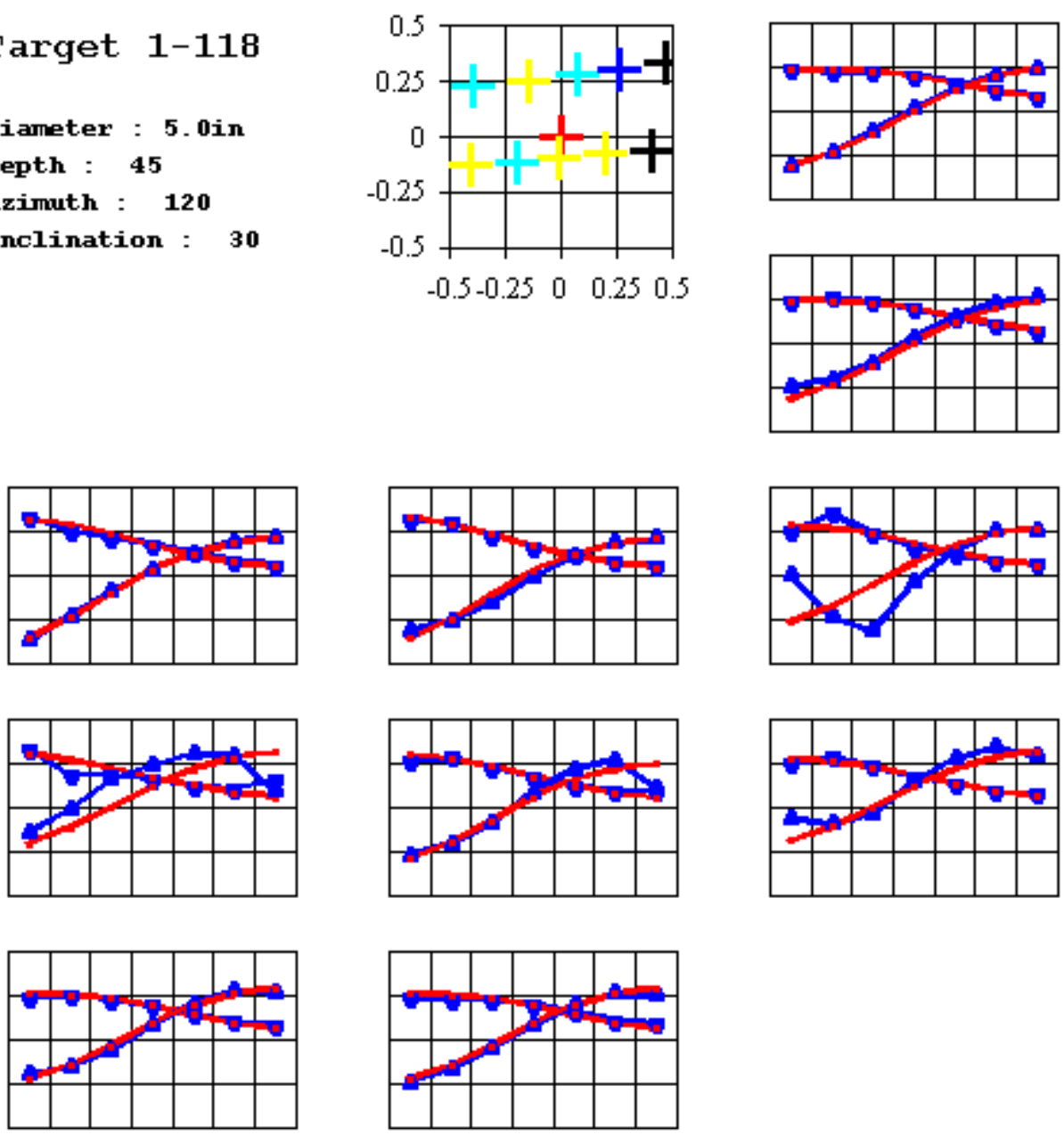
Target 1-119

Diameter : $152 \mathrm{~mm}$

Depth : 40

Fzimuth : $\mathbf{2 7 0}$

Inclination : $\mathbf{3 0}$
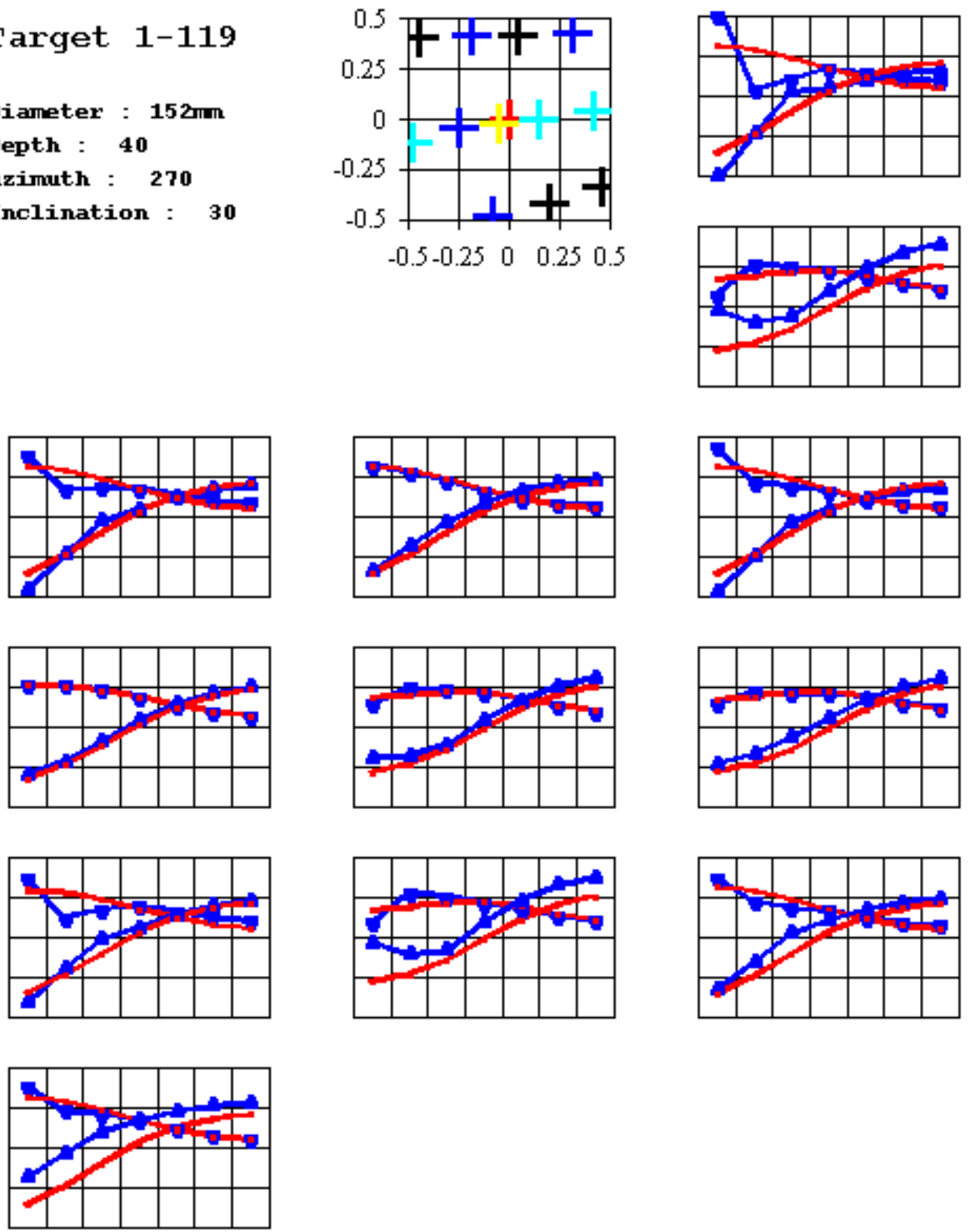
Target $1-120$

Diameter : $76 \mathrm{~mm}$

Depth : 25

Azimuth : 0

Inclination : $\mathbf{2 0}$
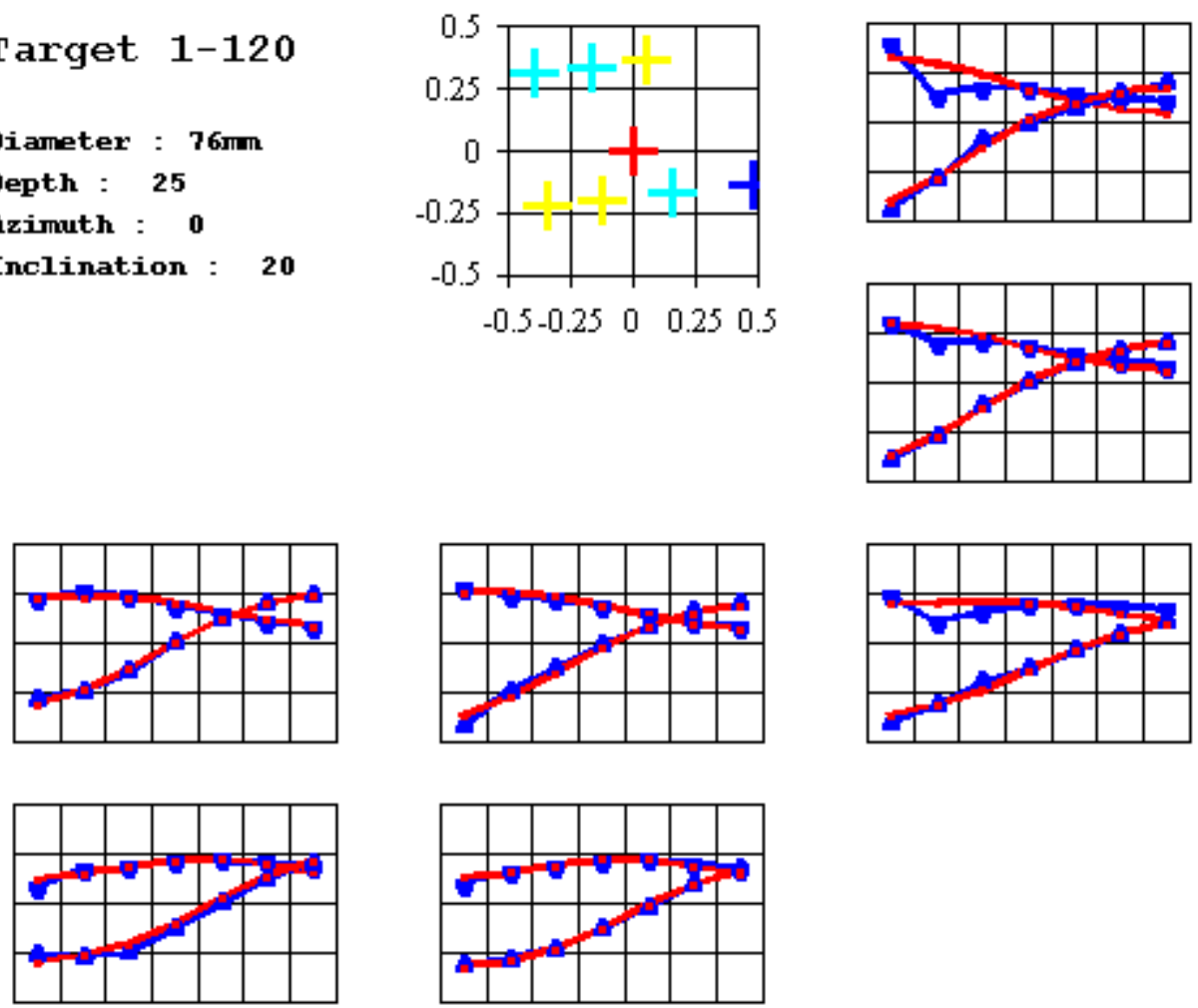
Target 1-121

Diameter : $155 \mathrm{~mm}$

Depth : 50

Fzimuth : $\mathbf{2 0 0}$

Inclination : 0
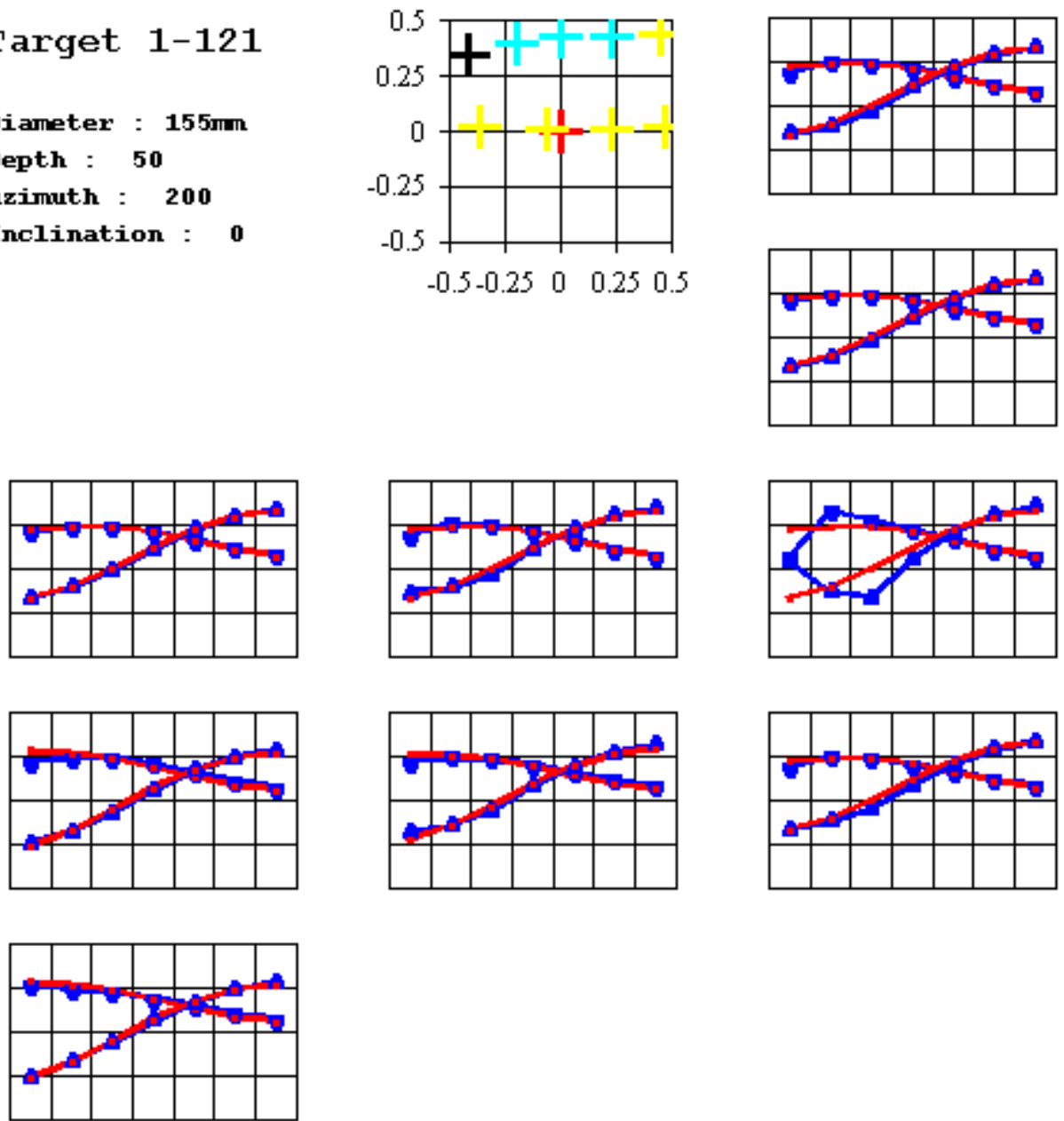
Target $1-123$

Diameter : $20 \mathrm{~mm}$

Depth : 0

Azimuth : 0

Inclination : 90
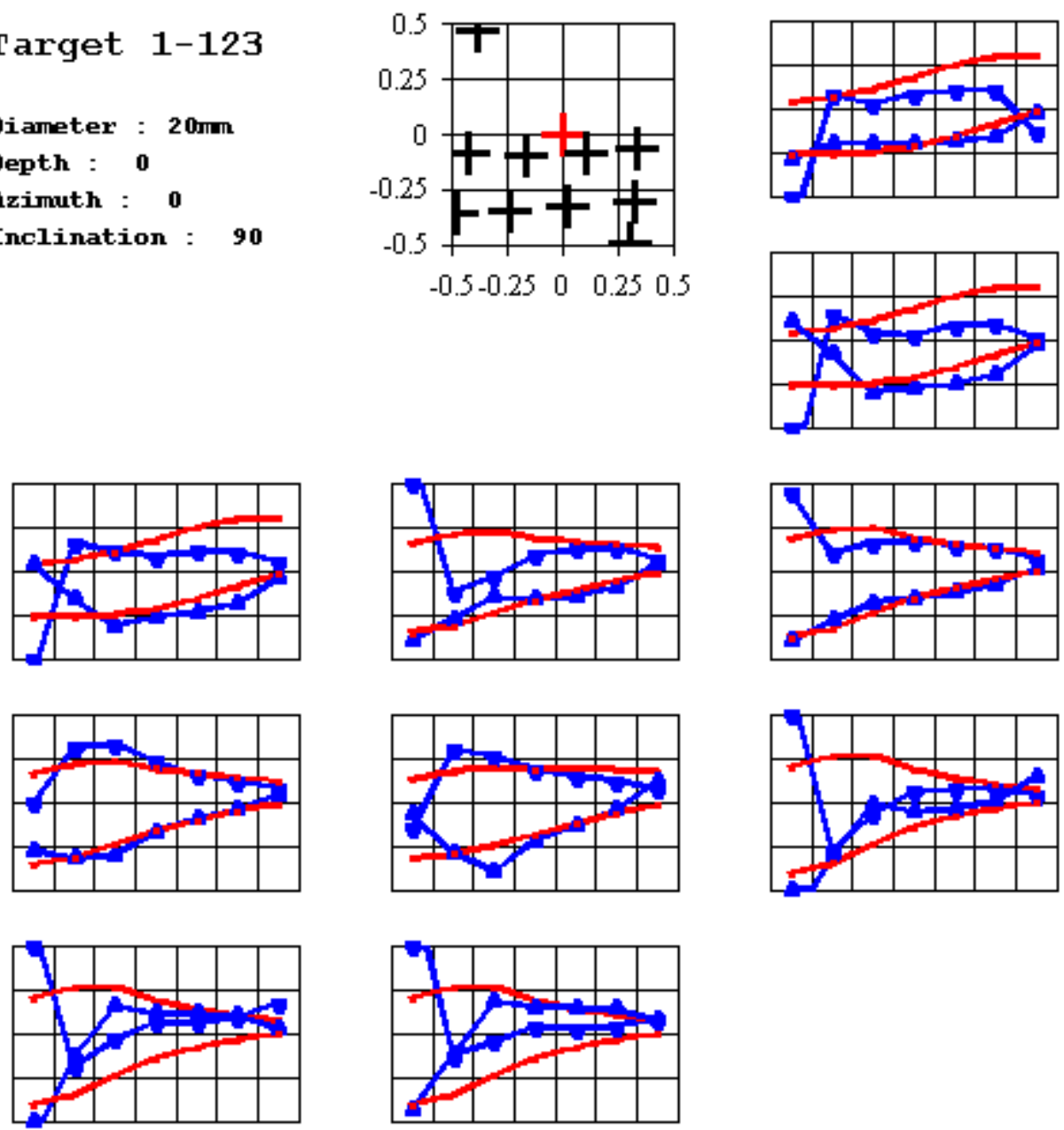
Target 1-124

Diameter : 20mm

Depth : 0

Azimuth : 0

Inclination : -90
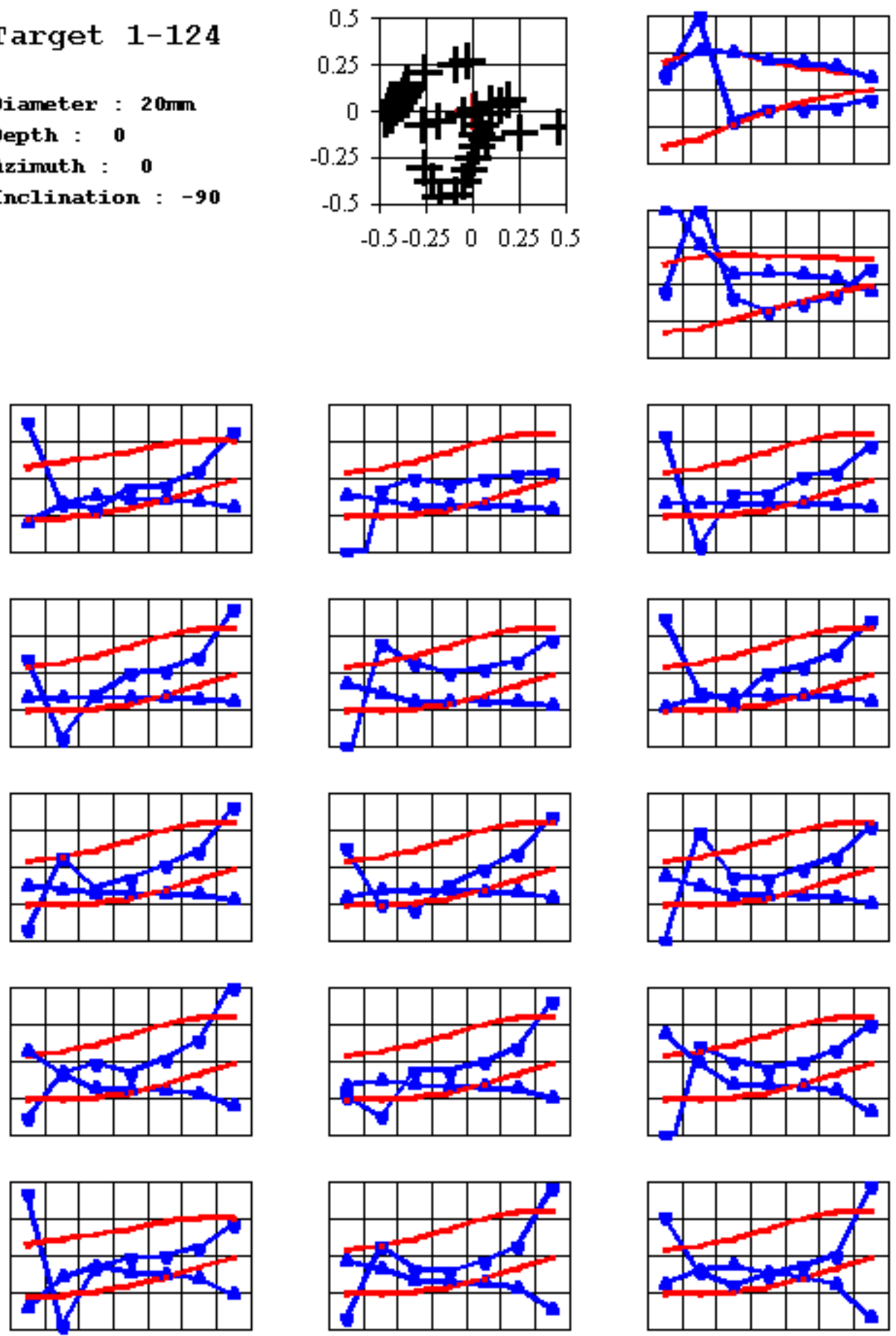
Target $1-126$

Diameter : $5 \mathrm{7mm}$

Depth : 20

Azimuth : 120

Inclination : $\mathbf{3 0}$
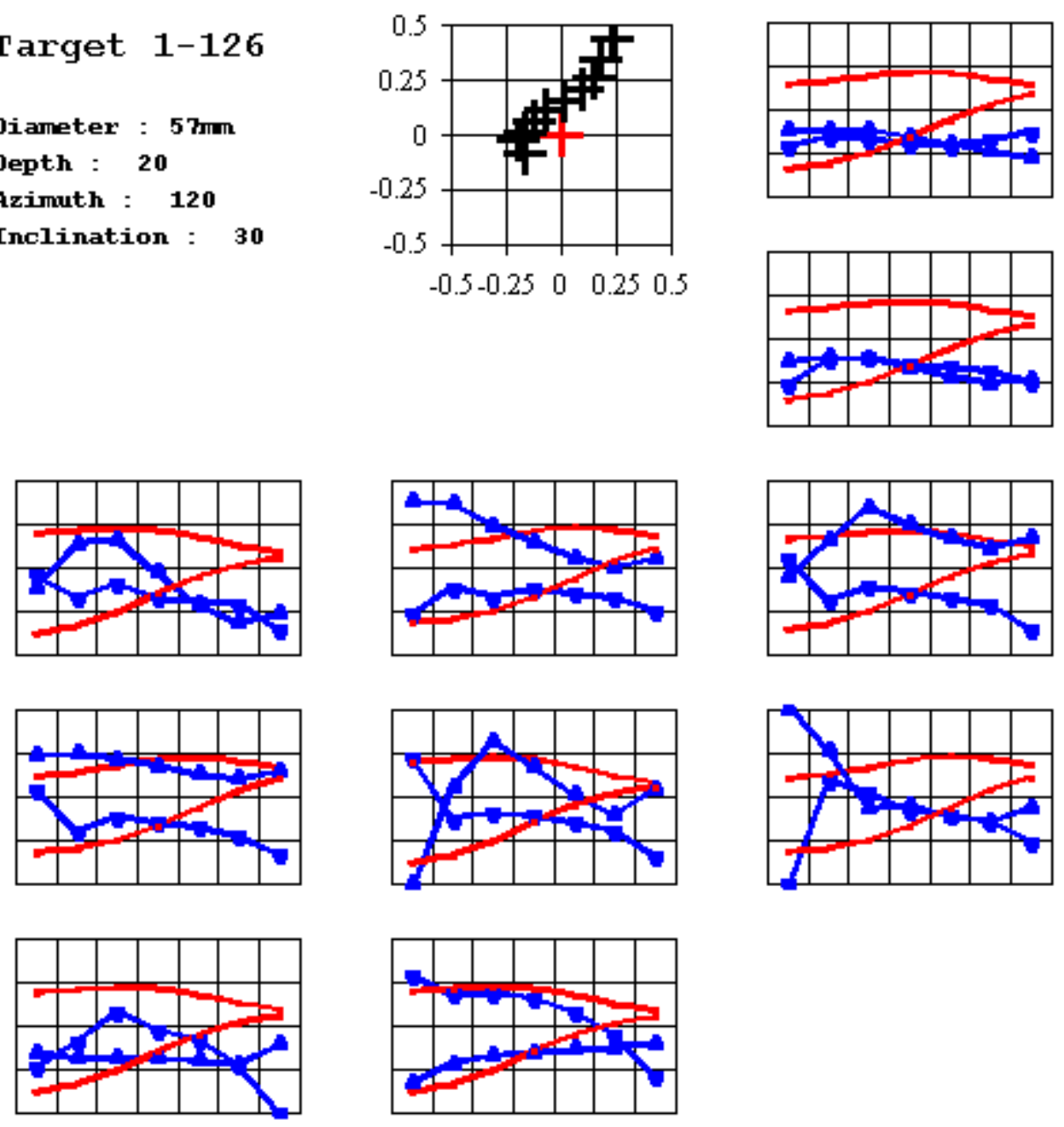
Target 1-128

Diameter : $20 \mathrm{~mm}$

Depth : 10

Azimuth : 0

Inclination : 0
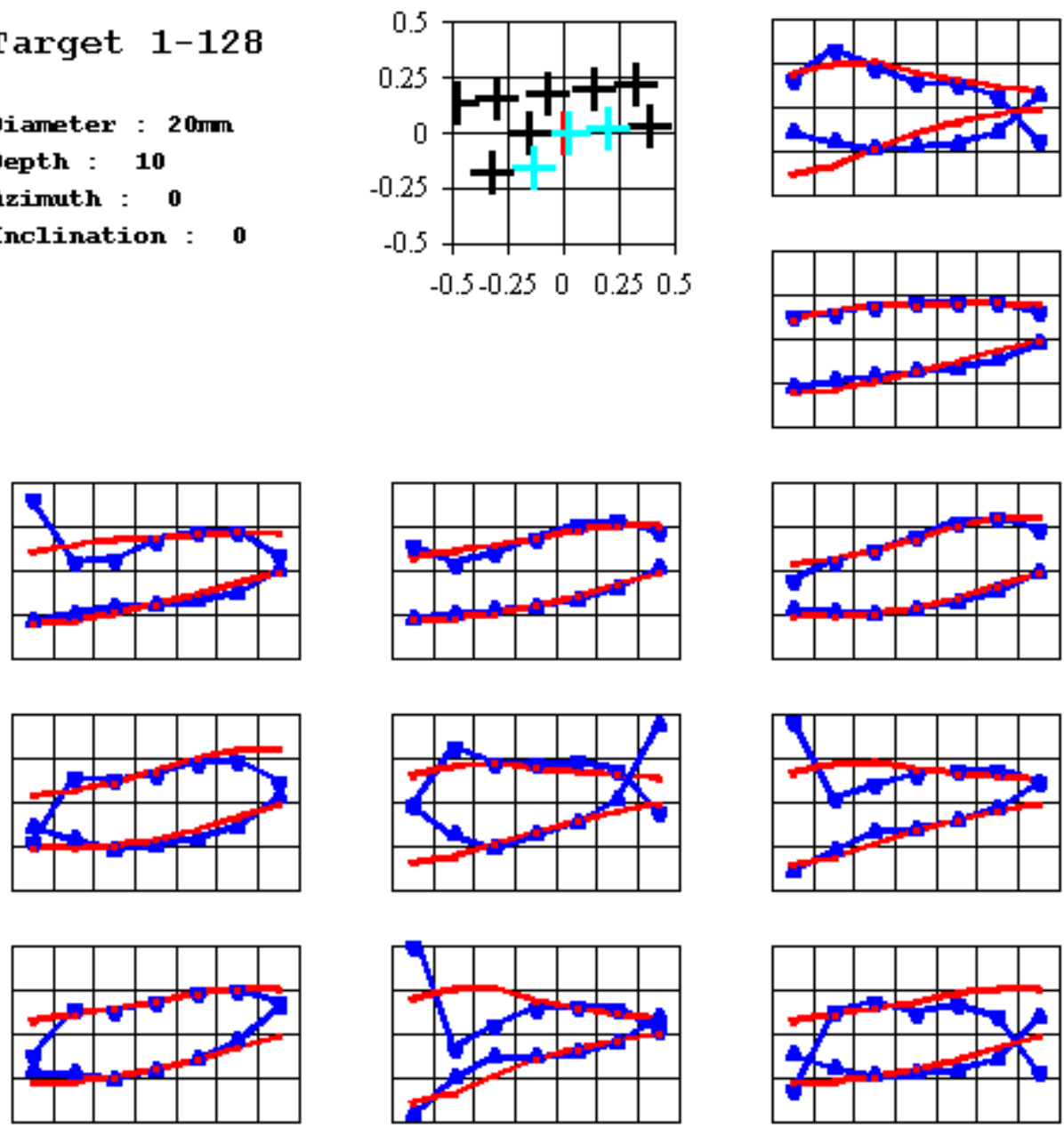
Target $1-130$

Diameter : $5.0 i n$

Depth : 70

Azimuth : 180

Inclination : $\mathbf{3 0}$
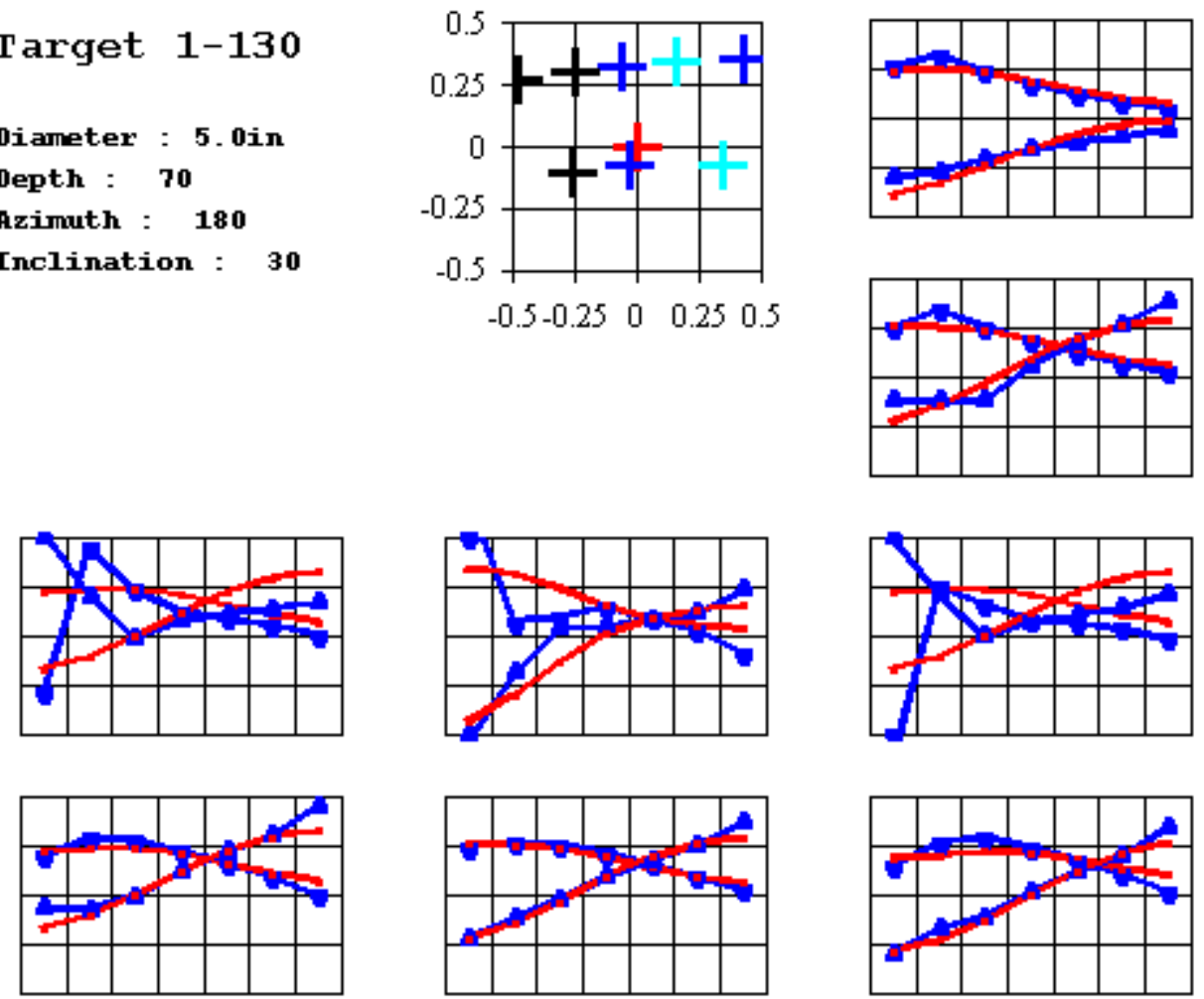
Target 1-132

Diameter : $57 \mathrm{~mm}$

Depth : 25

Azimuth : 180

Inclination : 0
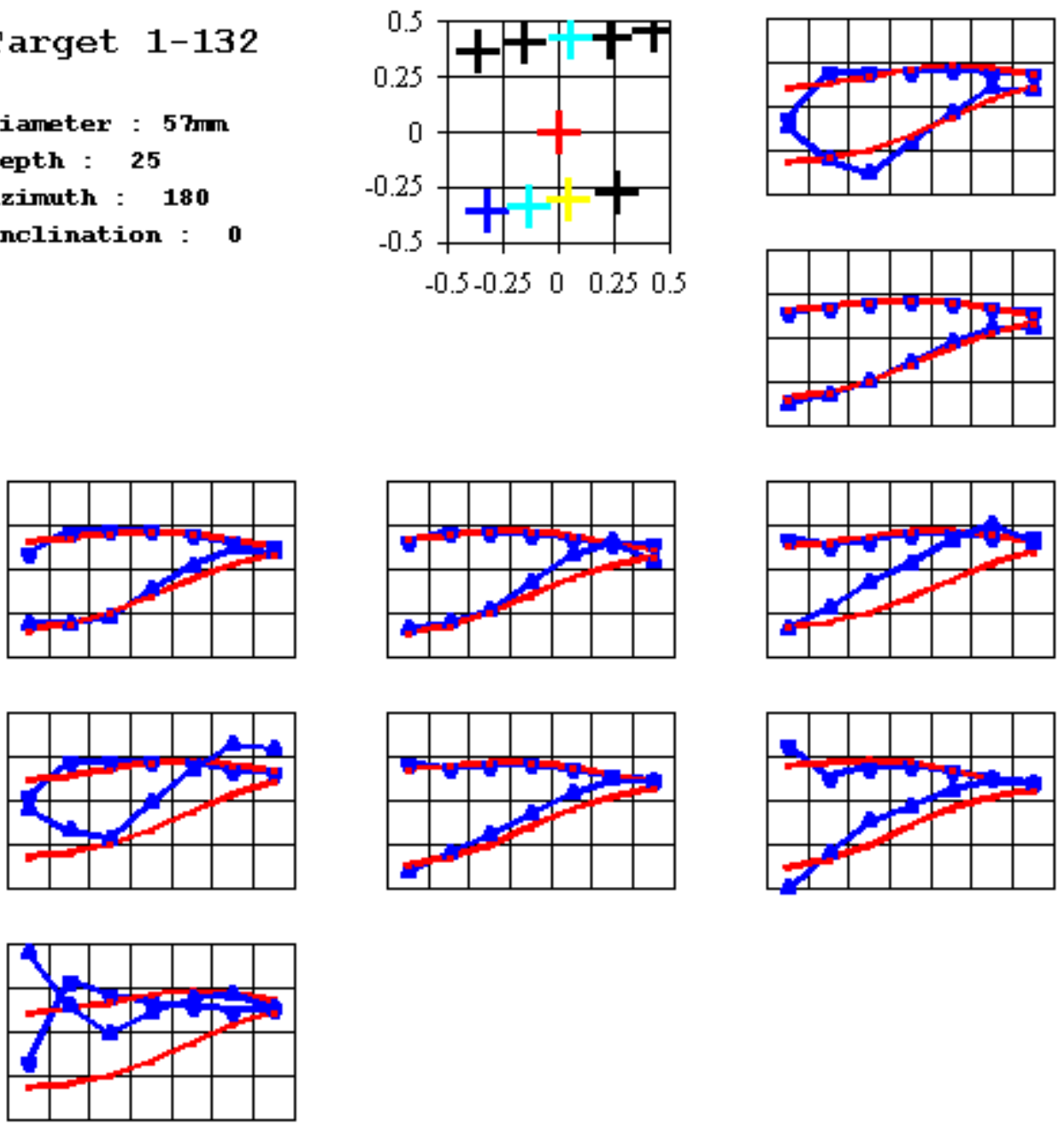

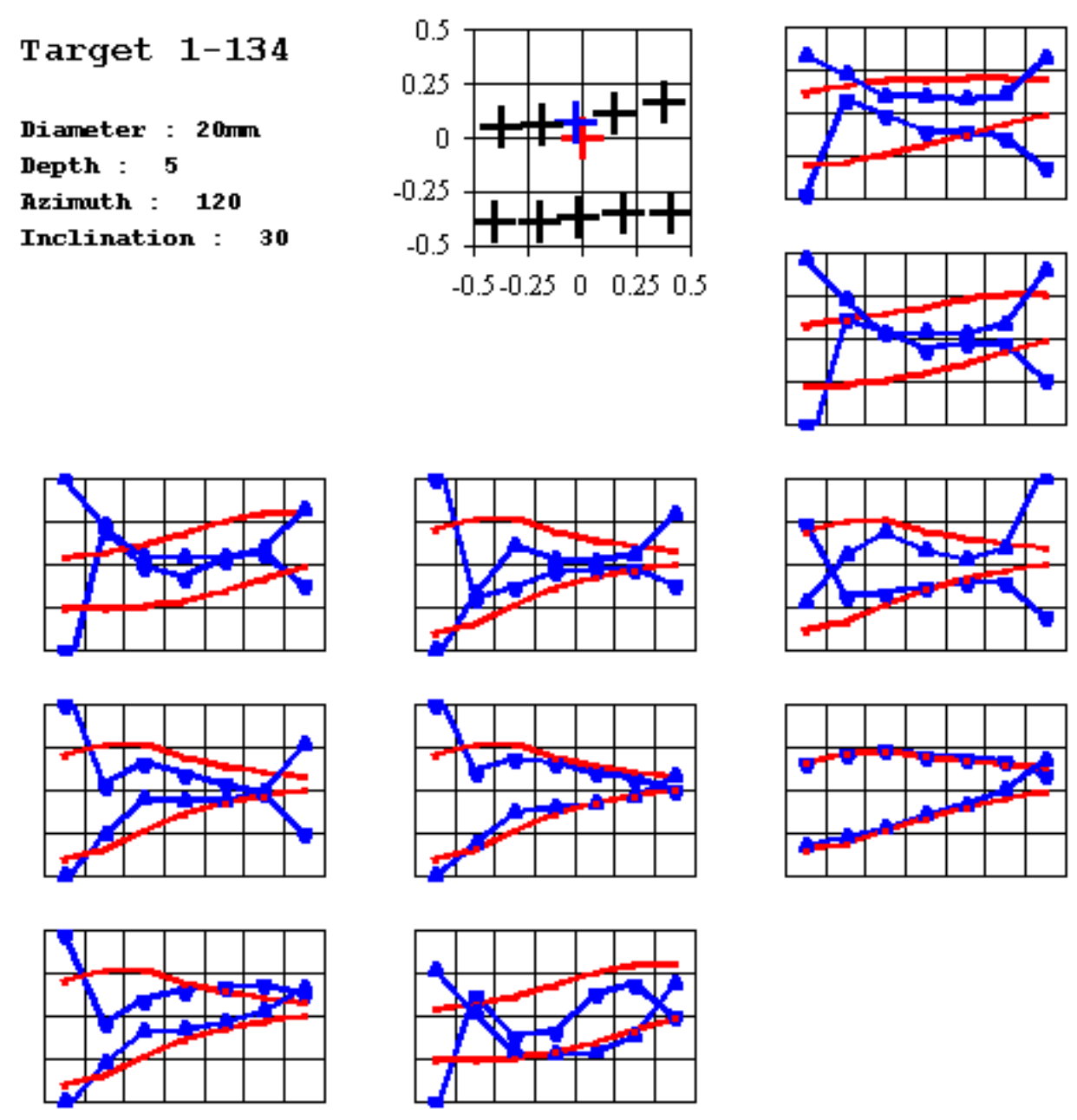

Target 1-136

Diameter : $155 \mathrm{~mm}$

Depth : 50

Azimuth : 90

Inclination : 75
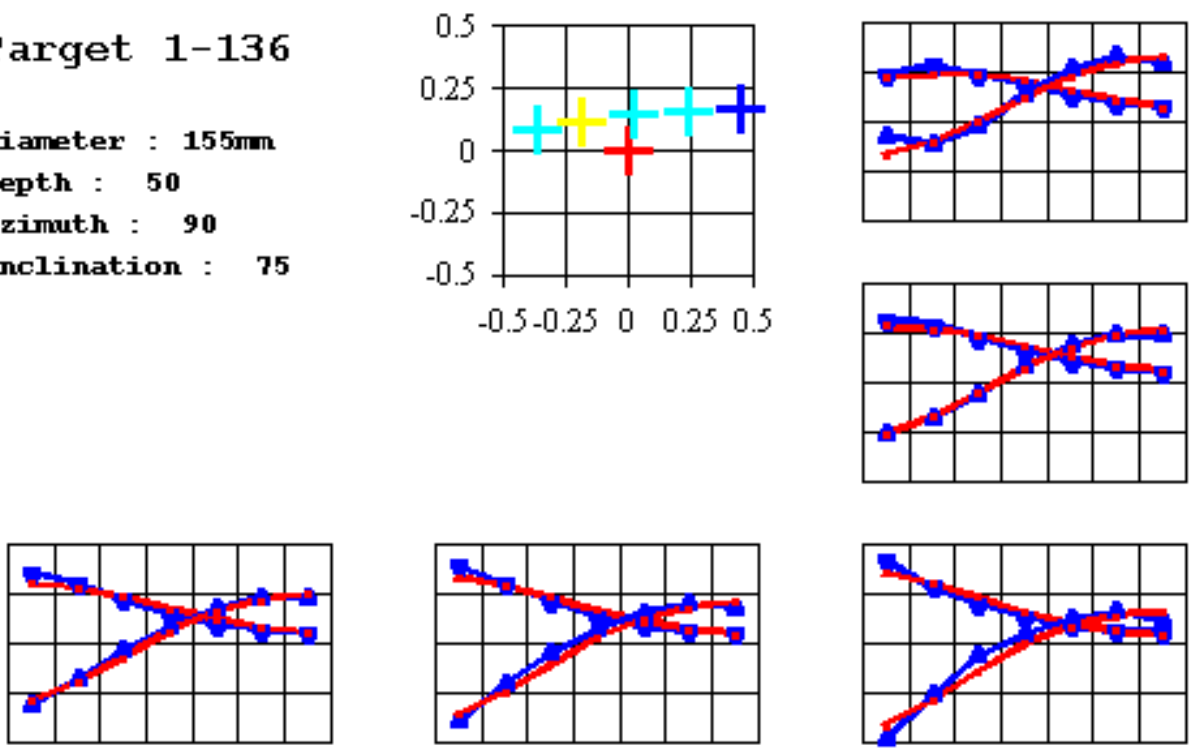
Target 1-138

Diameter : $57 \mathrm{~mm}$

Depth : 15

Azimuth : 95

Inclination : 45
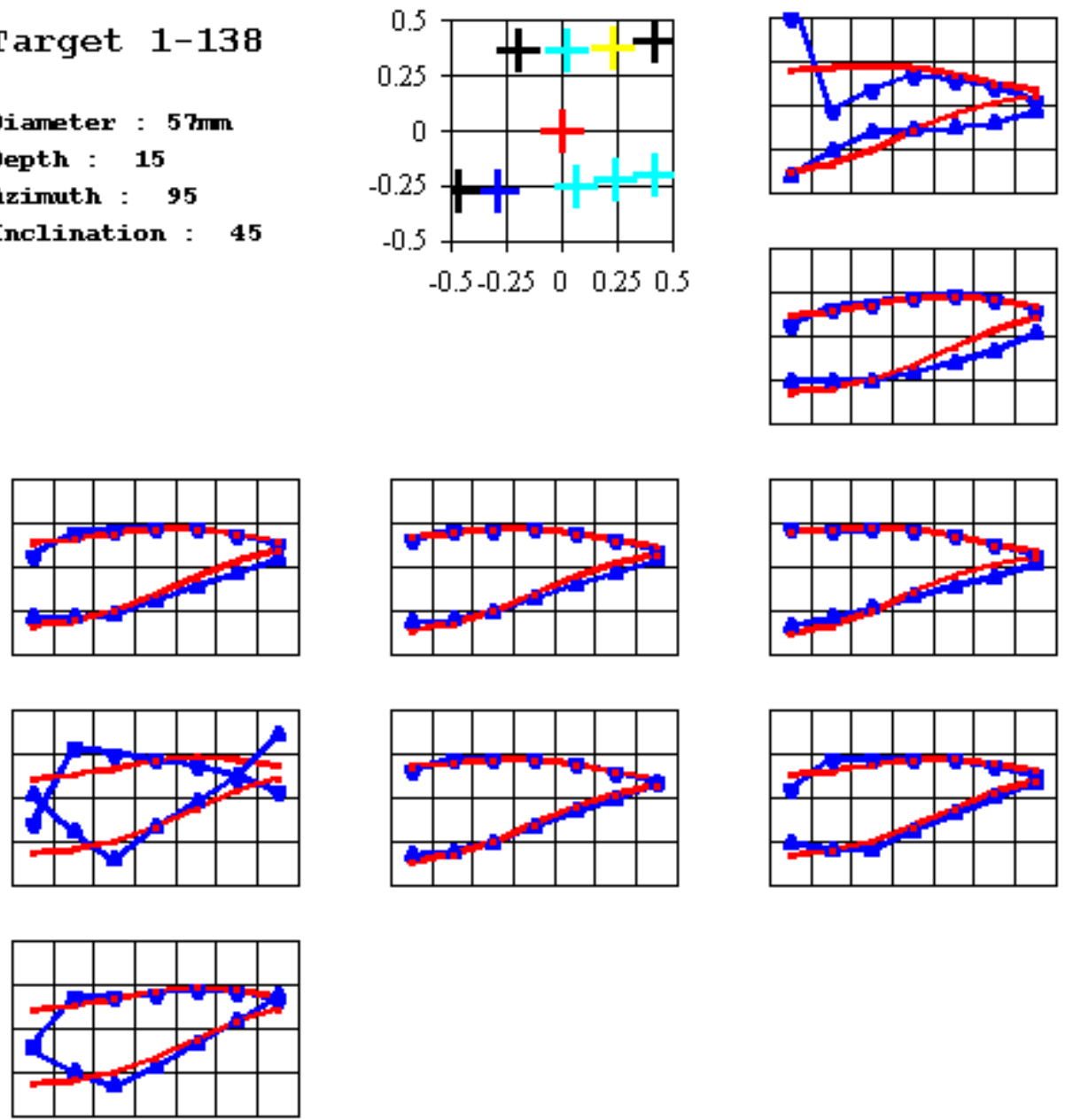
Target $1-140$

Diameter : $20 \mathrm{~mm}$

Depth : 5

Azimuth : 275

Inclination : 15
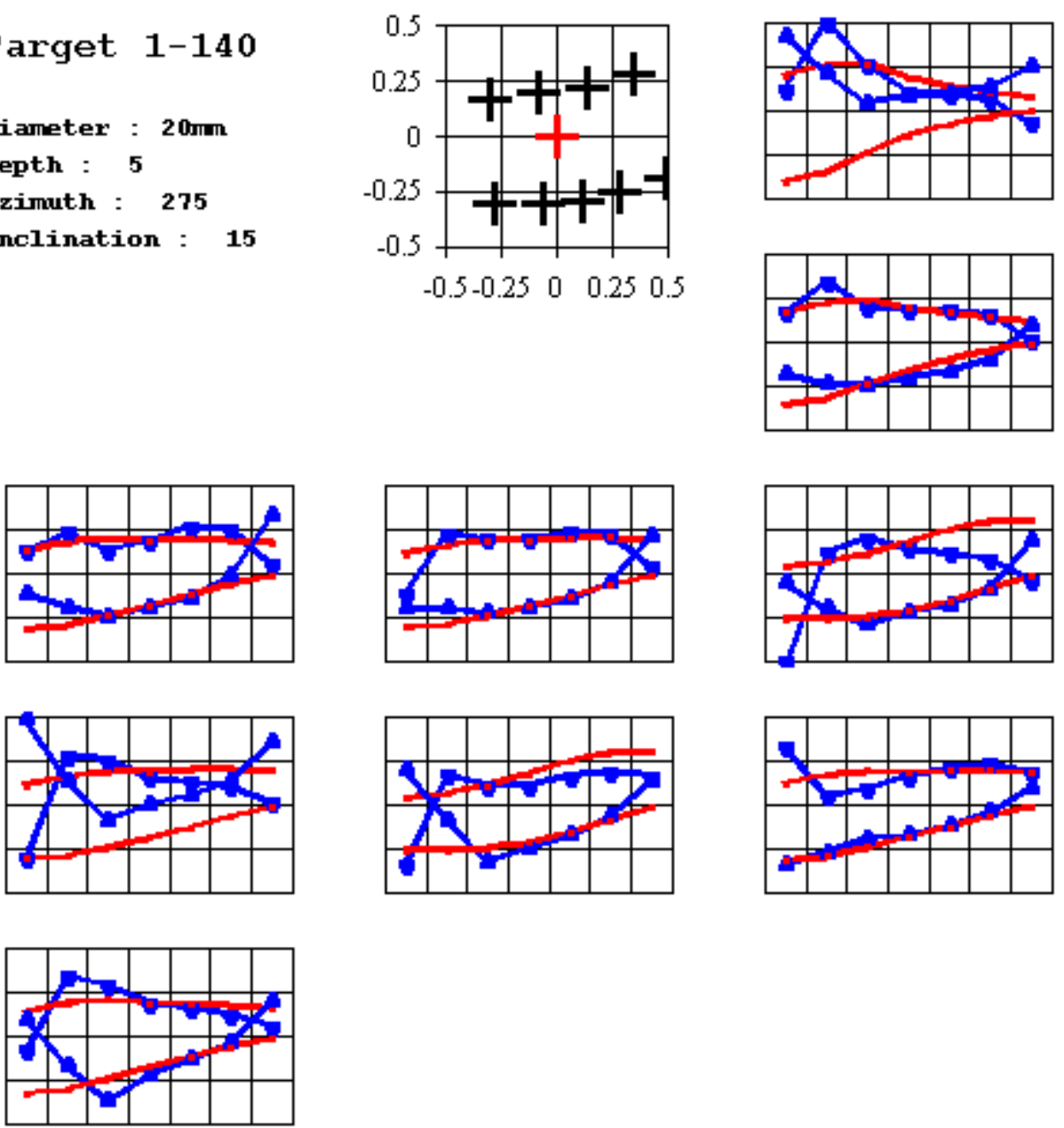

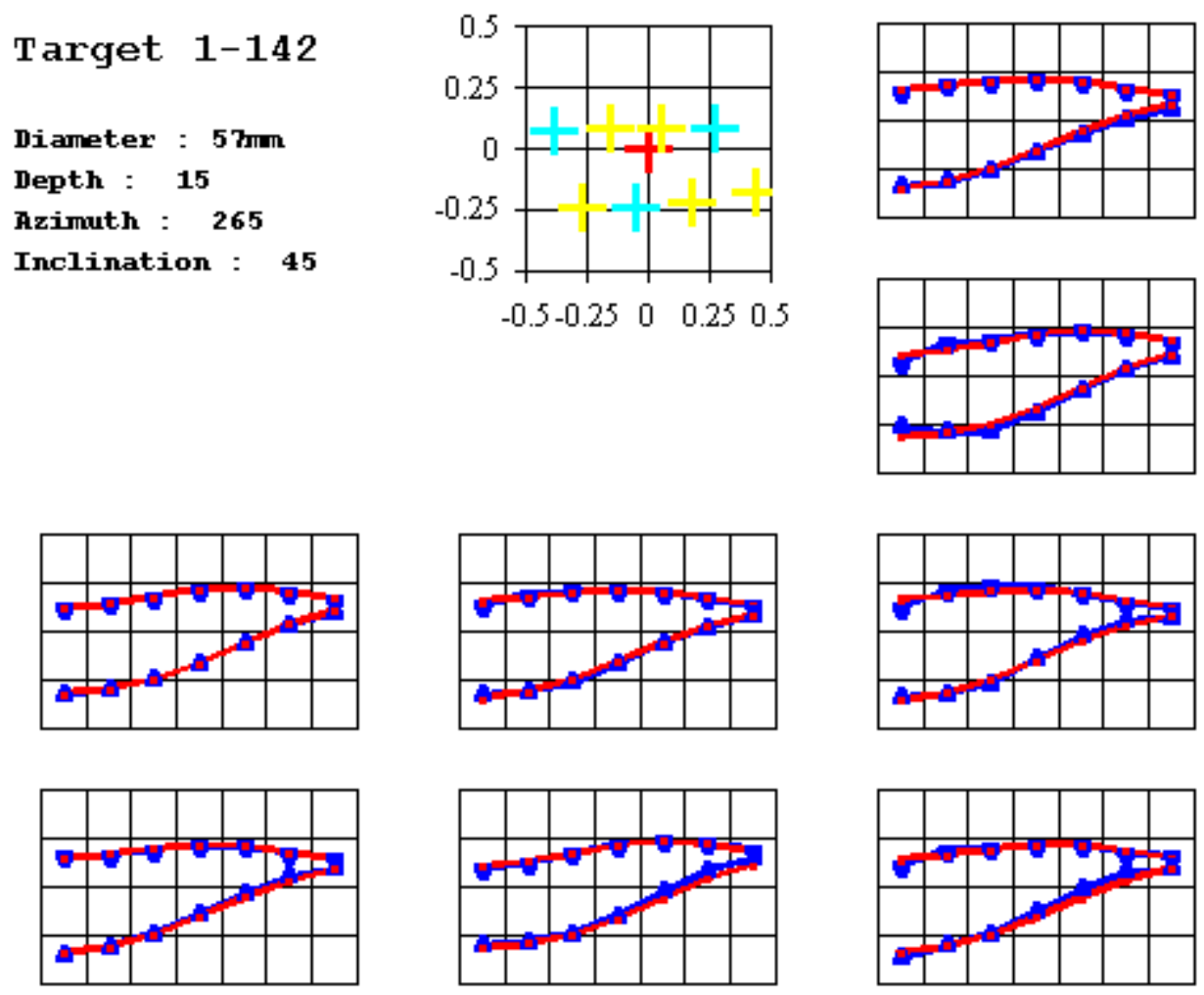

Target 1-144
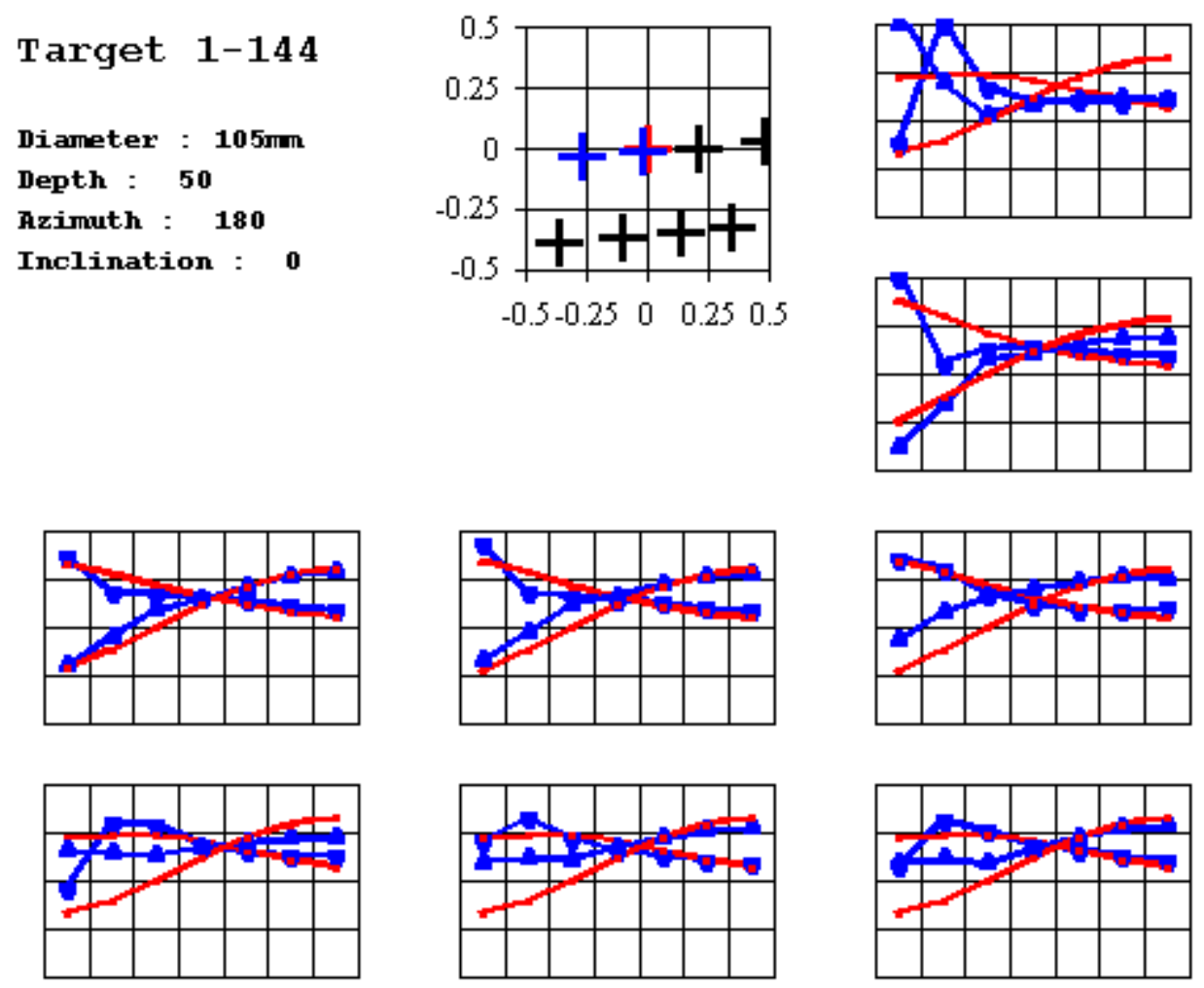
Target 1-146

Diameter : $20 \mathrm{~mm}$

Depth : 5

Azimuth : $\mathbf{2 7 0}$

Inclination : 0
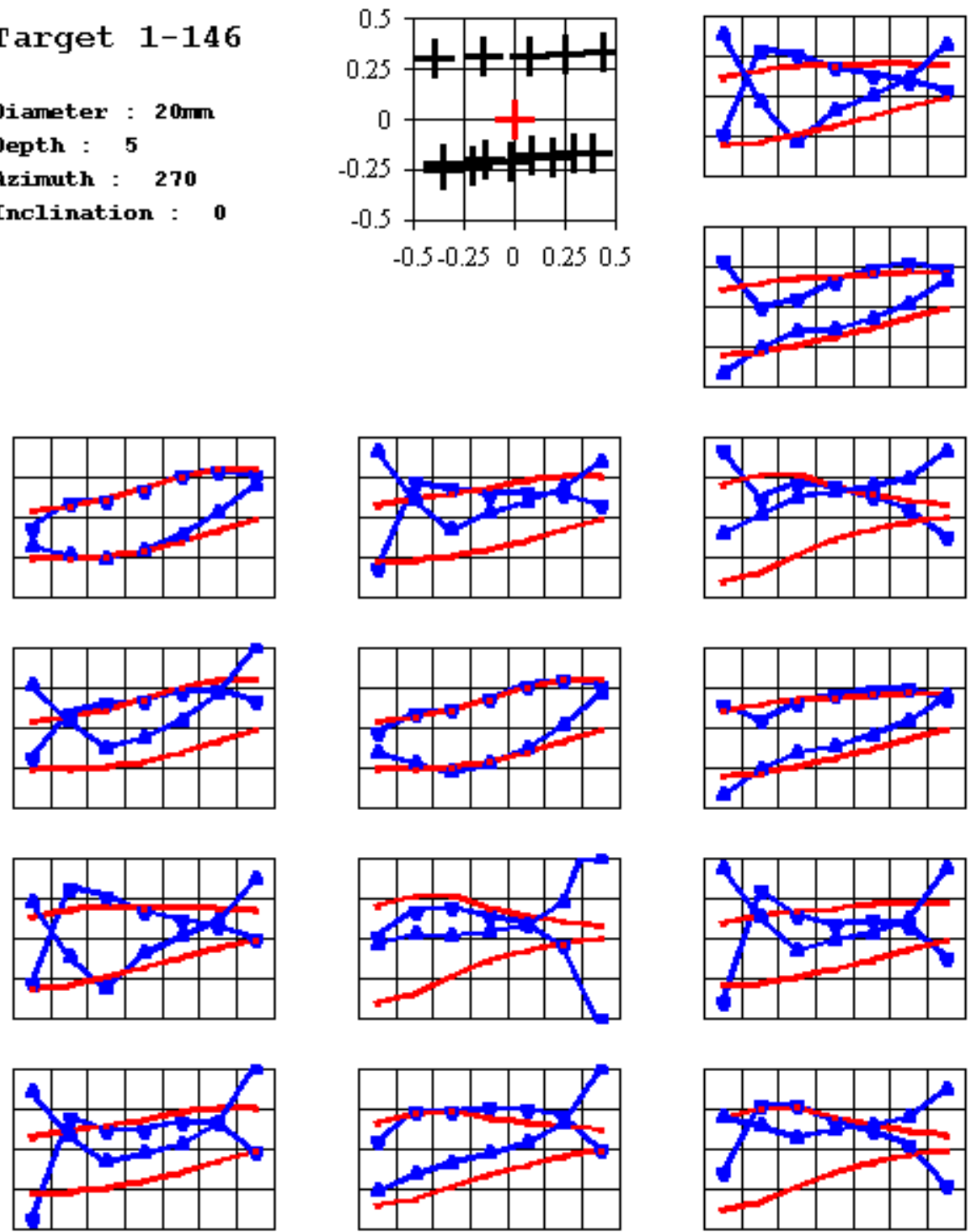
Target 1-147

Diameter : $5 \mathrm{kmm}$

Depth : 25

Azimuth : 0

Inclination : 0
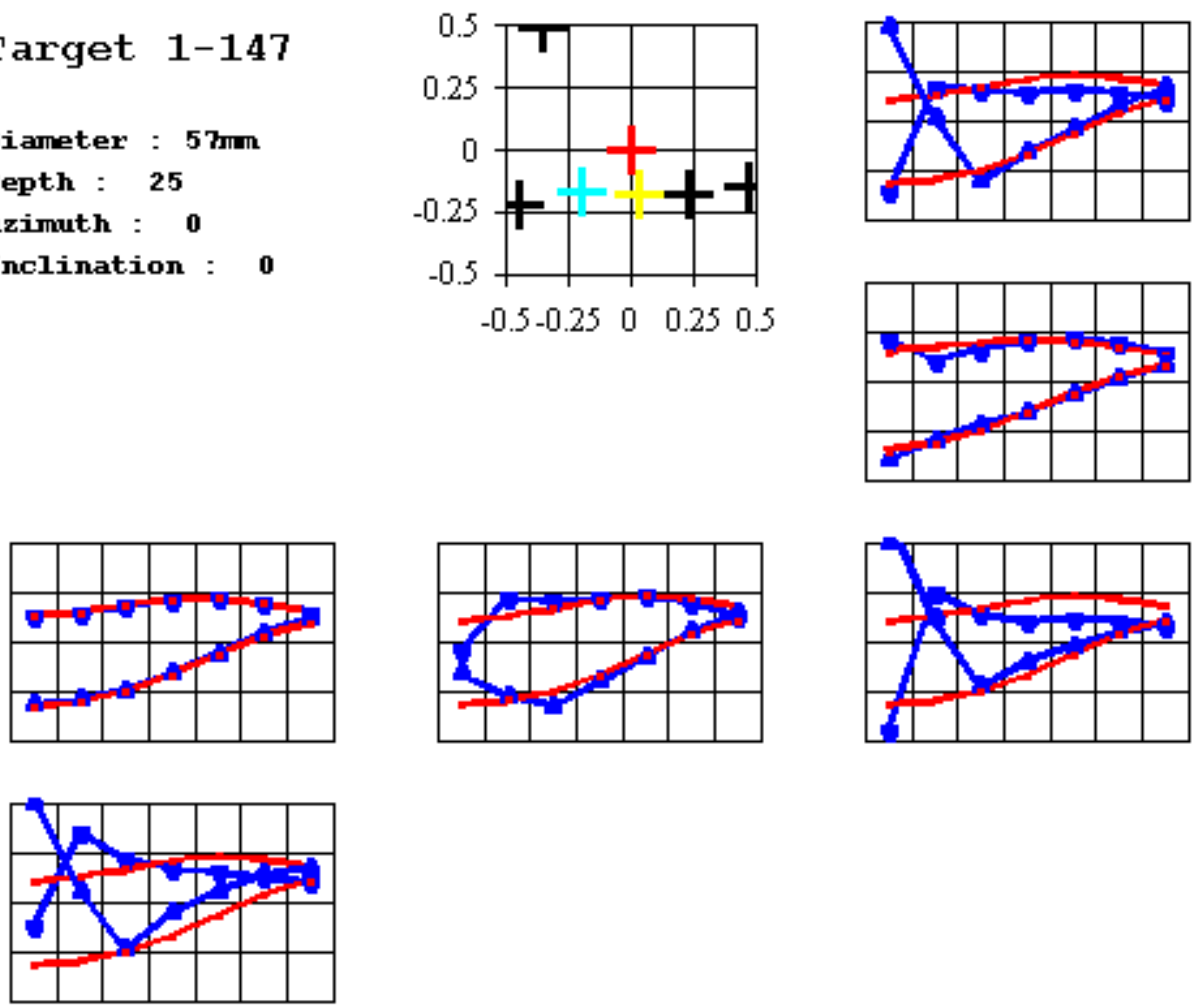

Target 1-148

Diameter : $20 \mathrm{~mm}$

Depth : 10

Azimuth : 235

Inclination : 45
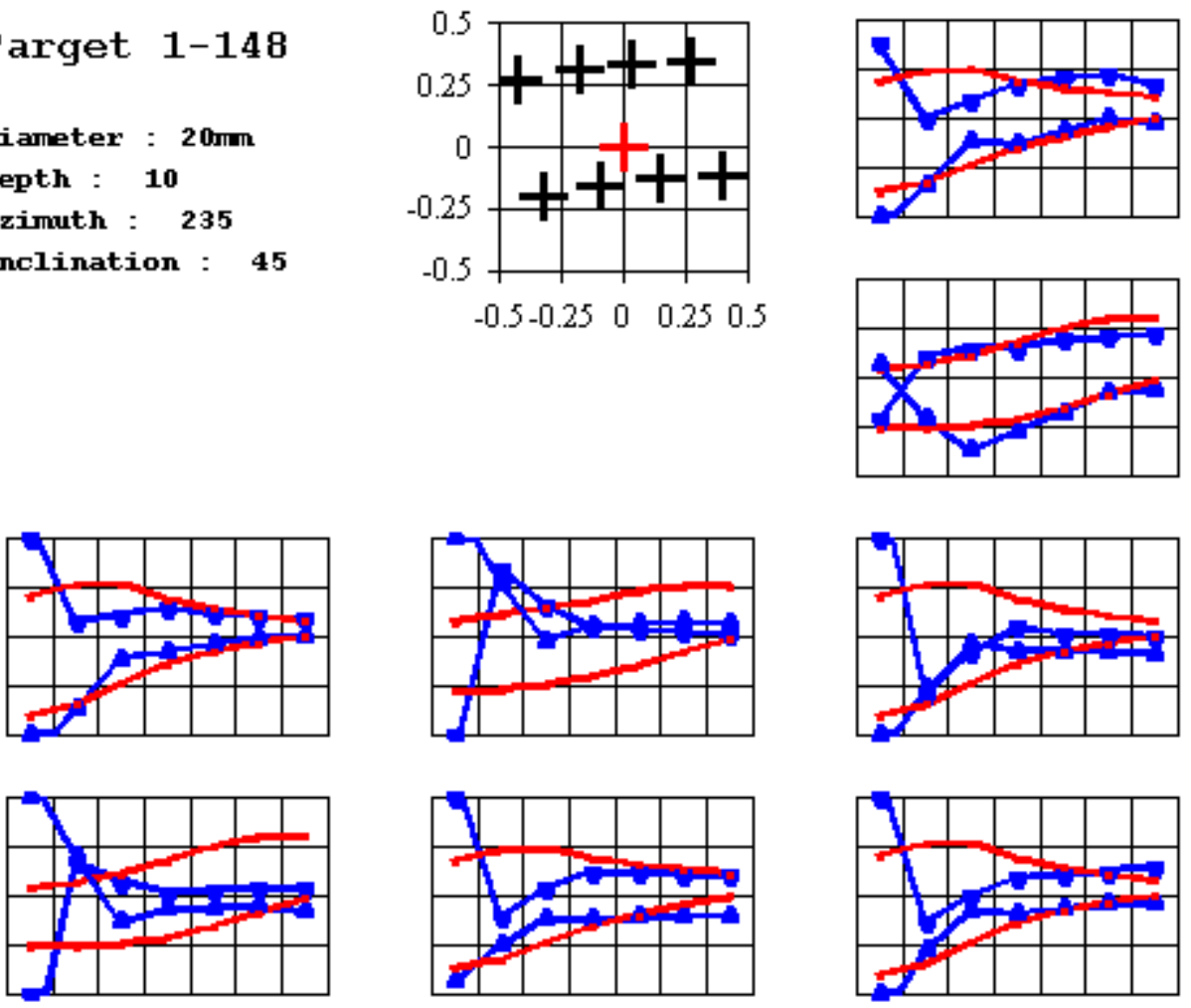
Target 1-149

Diameter : 2.75in

Depth : 50

Azimuth : $\mathbf{3 0}$

Inclination : 55
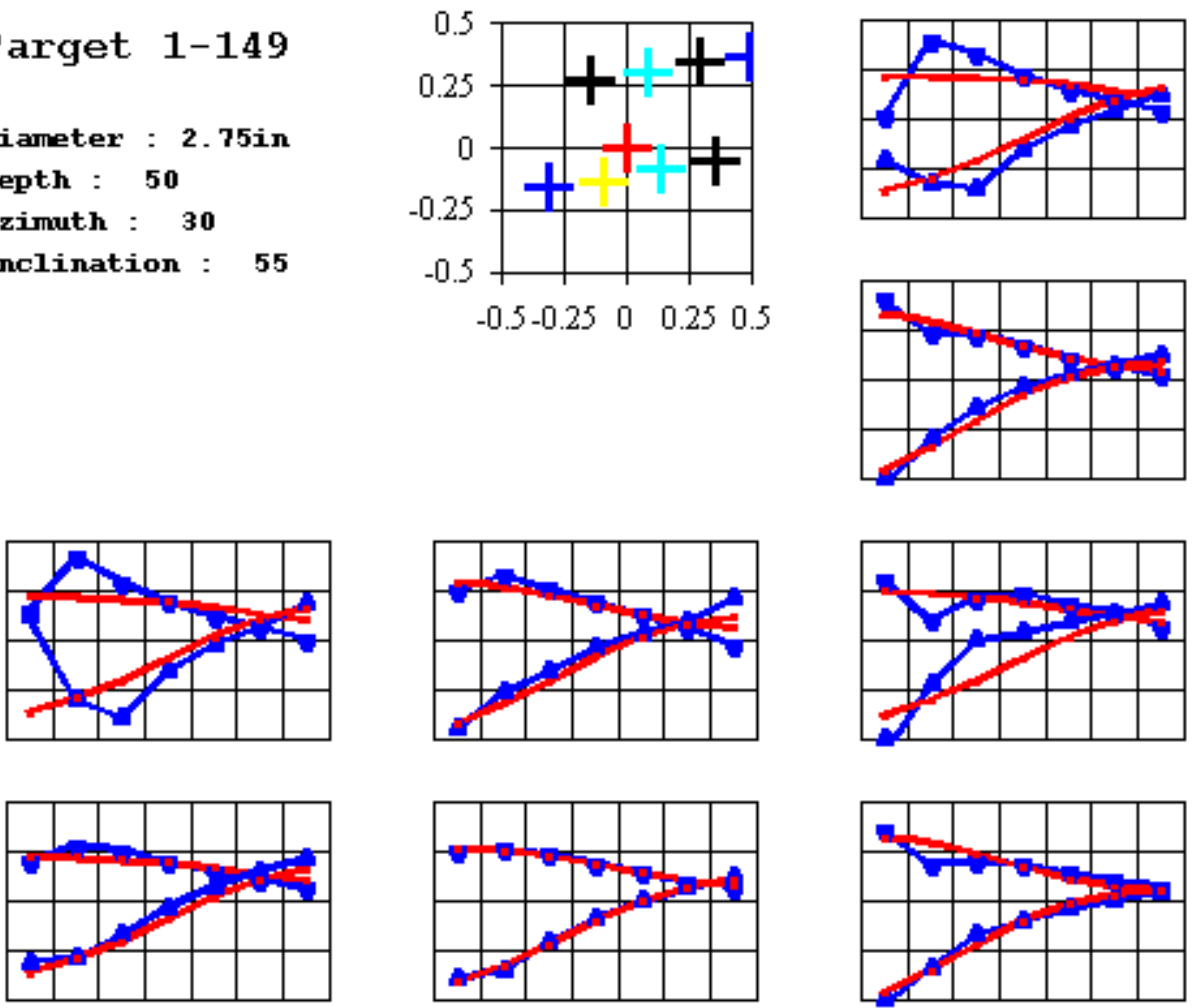
Target $1-150$

Diameter : 2.75in

Depth : 70

Azimuth : 275

Inclination : $\mathbf{4 5}$
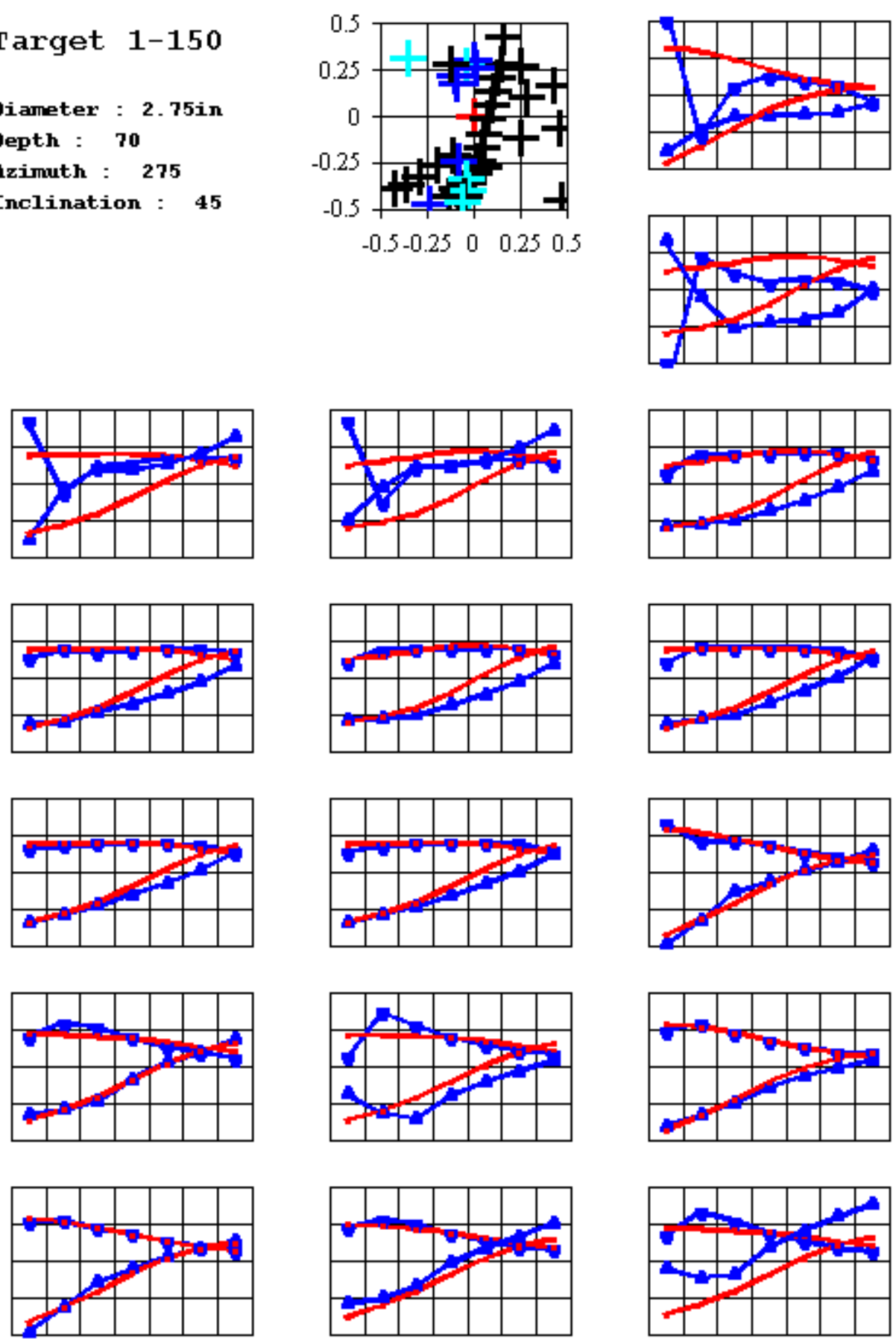
Target 1-151

Diameter : $76 \mathrm{~mm}$

Depth : 25

Azimuth : $\mathbf{1 5 0}$

Inclination : 45
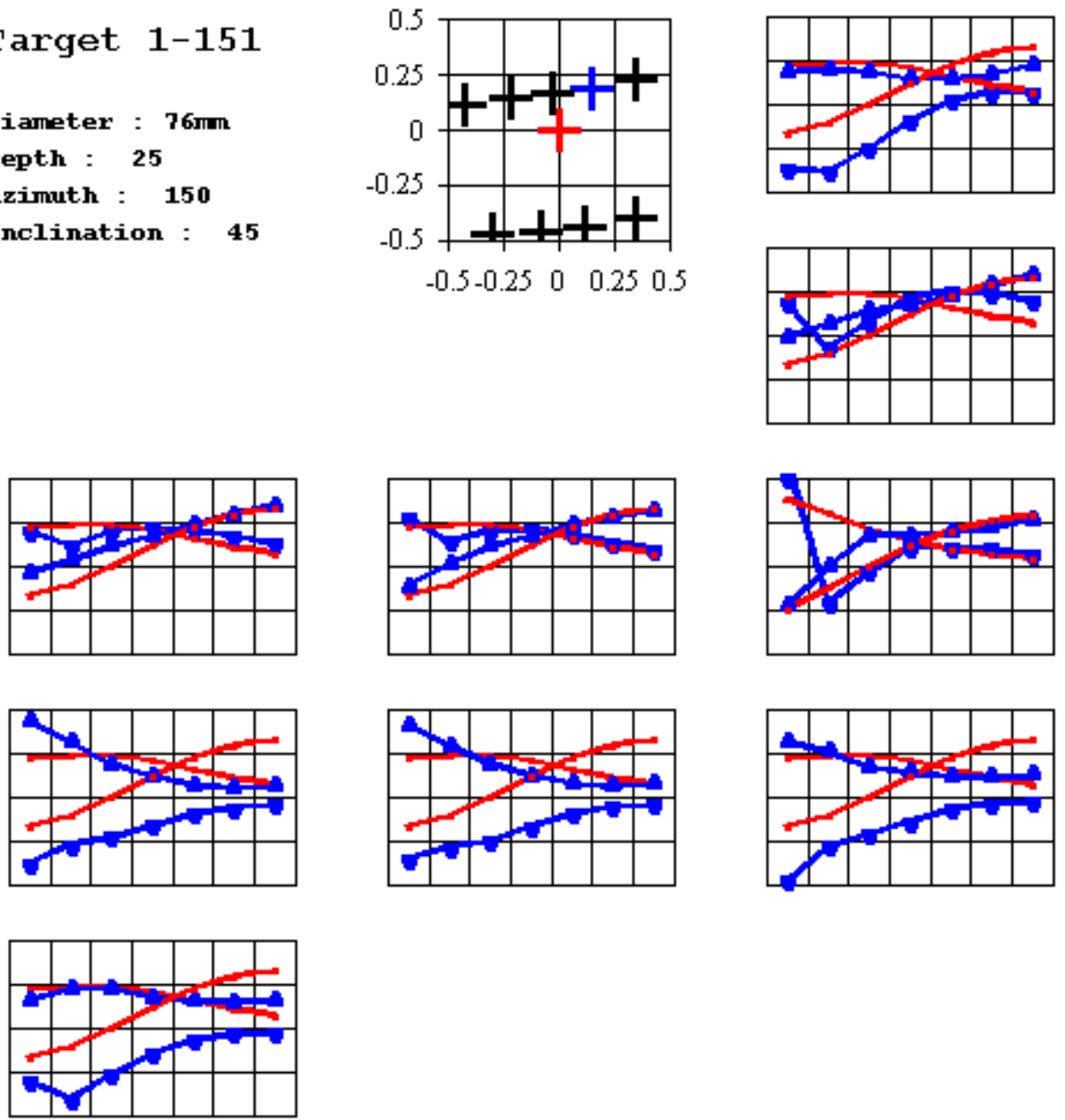
Target 1-152

Diameter : $2.75 i n$

Depth : 15

Azimuth : $\mathbf{3 0}$

Inclination : 0
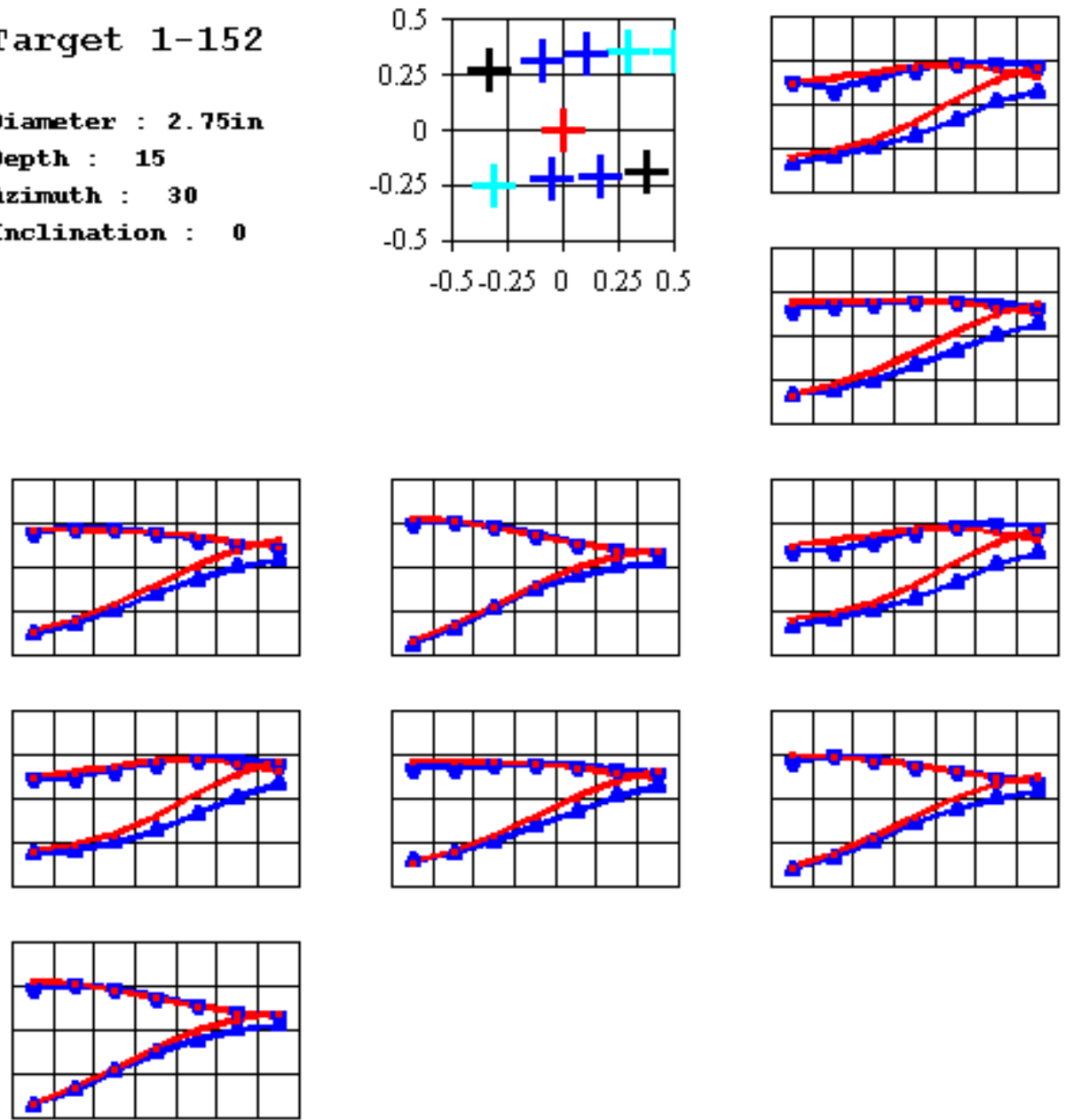
Target 1-153

Diameter : 2.75in

Depth : 76

Azimuth : 0

Inclination : 90
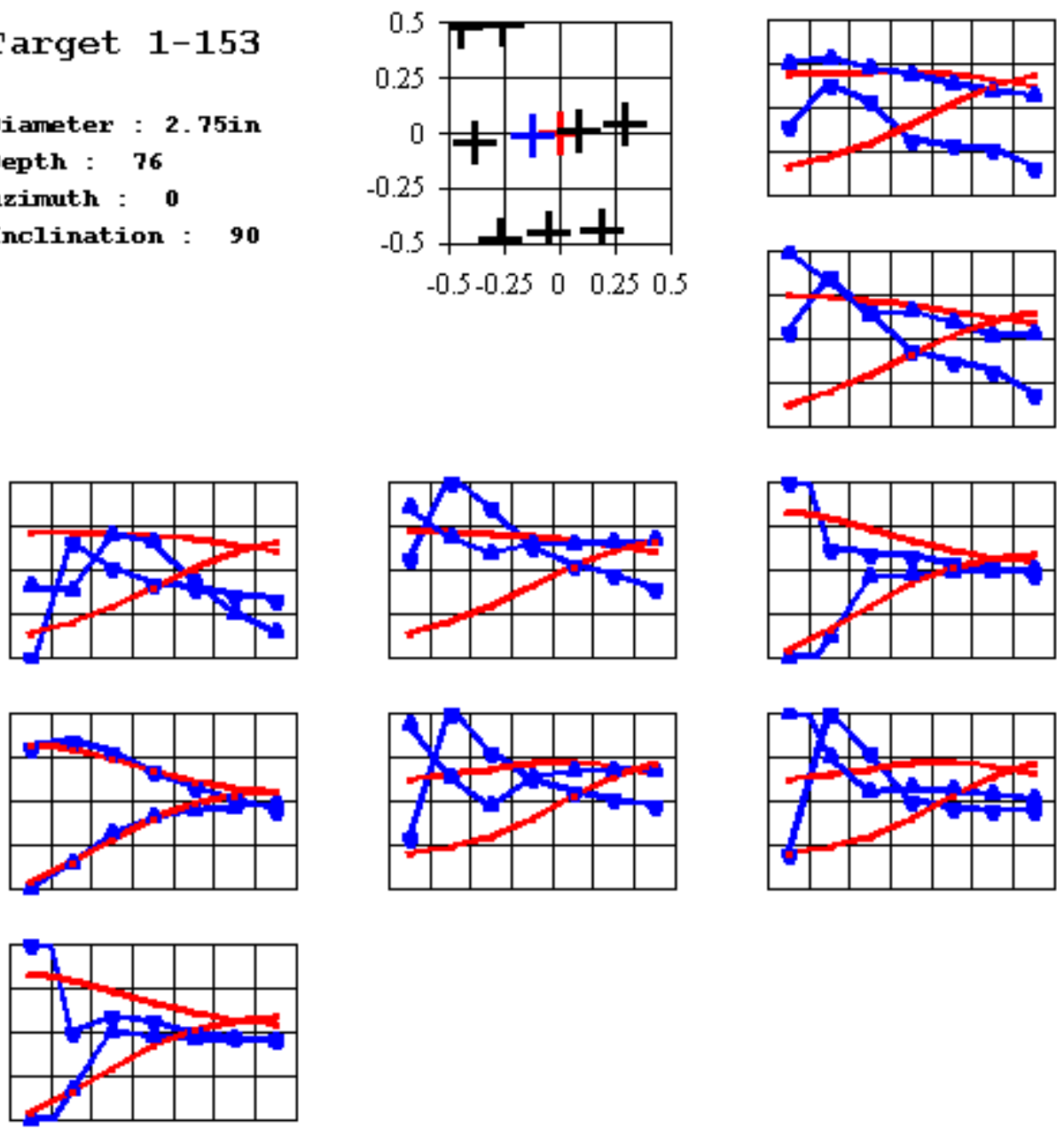

Target $2-112$

Diameter : $81 \mathrm{~mm}$

Depth : 10

Azimuth : 0

Inclination : 90
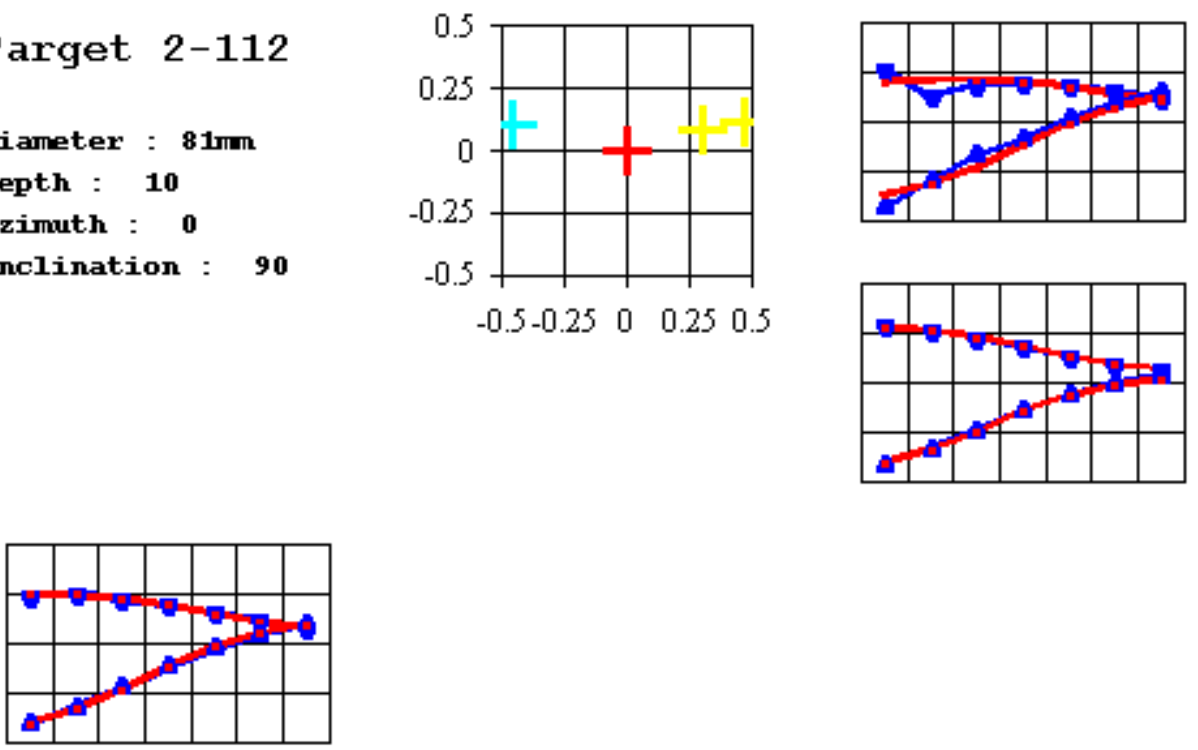

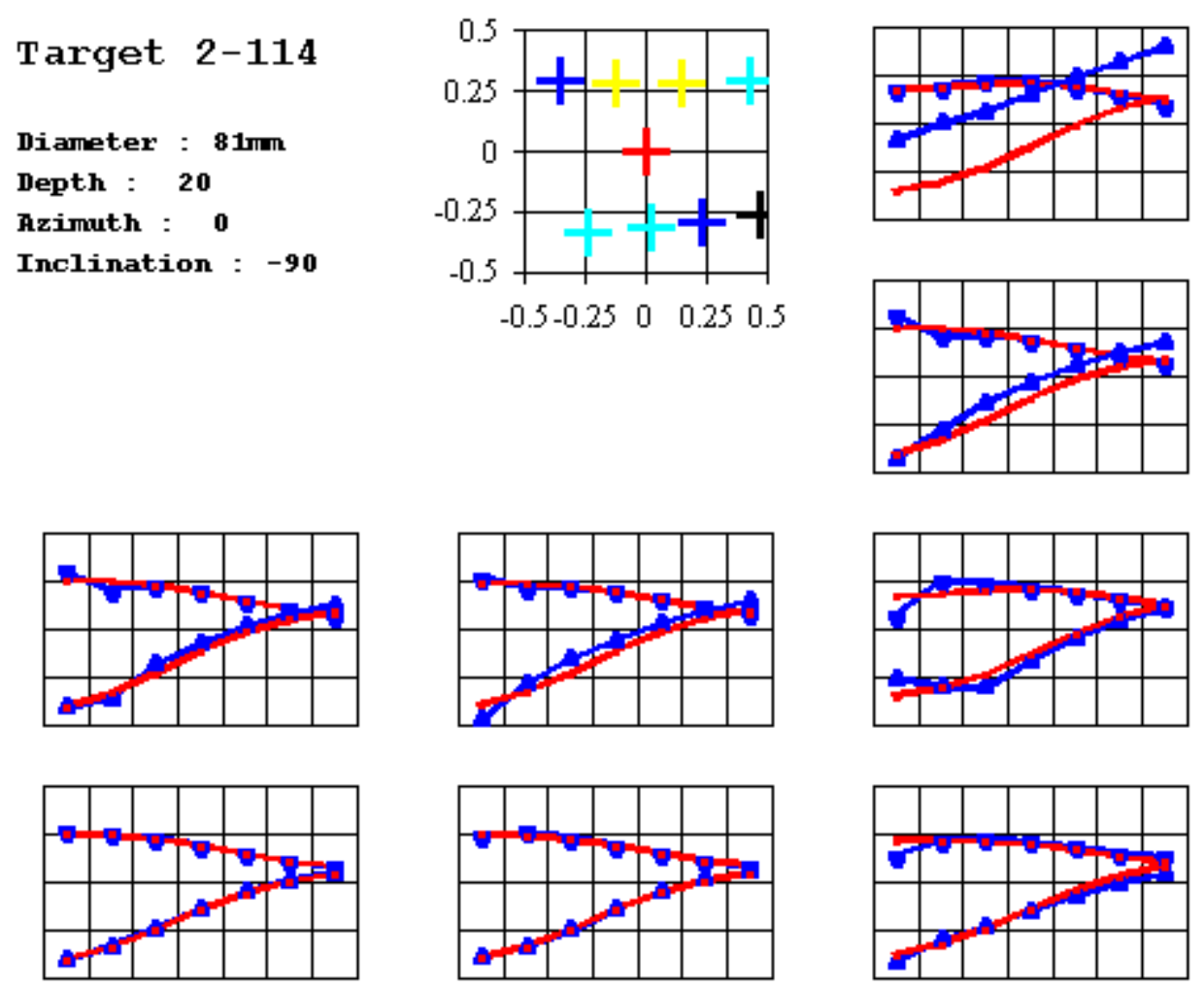
Target 2-116

Diameter : $81 \mathrm{~mm}$

Depth : $\mathbf{3 0}$

Azimuth : $\mathbf{4 5}$

Inclination : 0
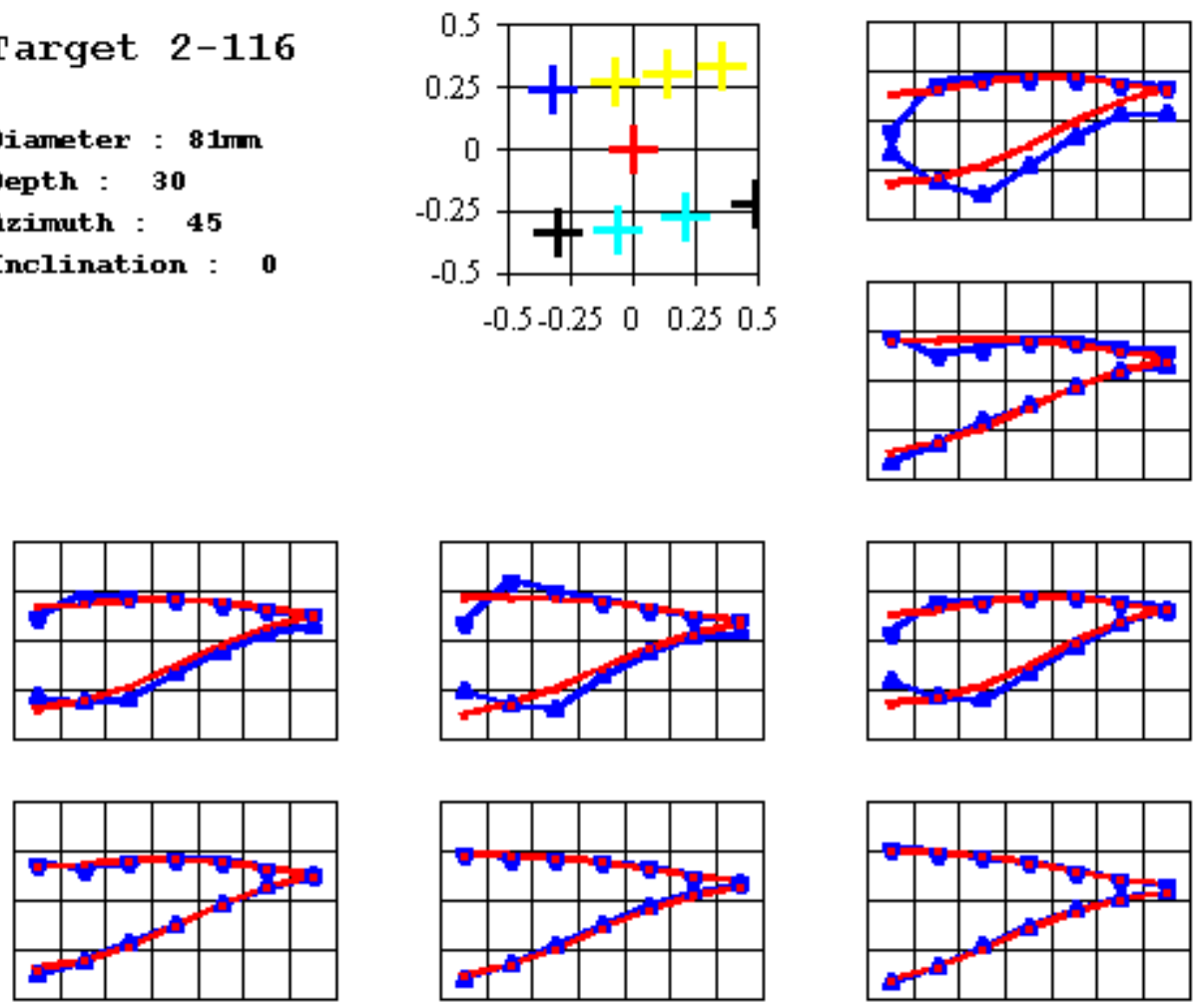
Target 2-118

Diameter : $60 \mathrm{~mm}$

Depth : $\mathbf{3 5}$

Azimuth : 0

Inclination : 45
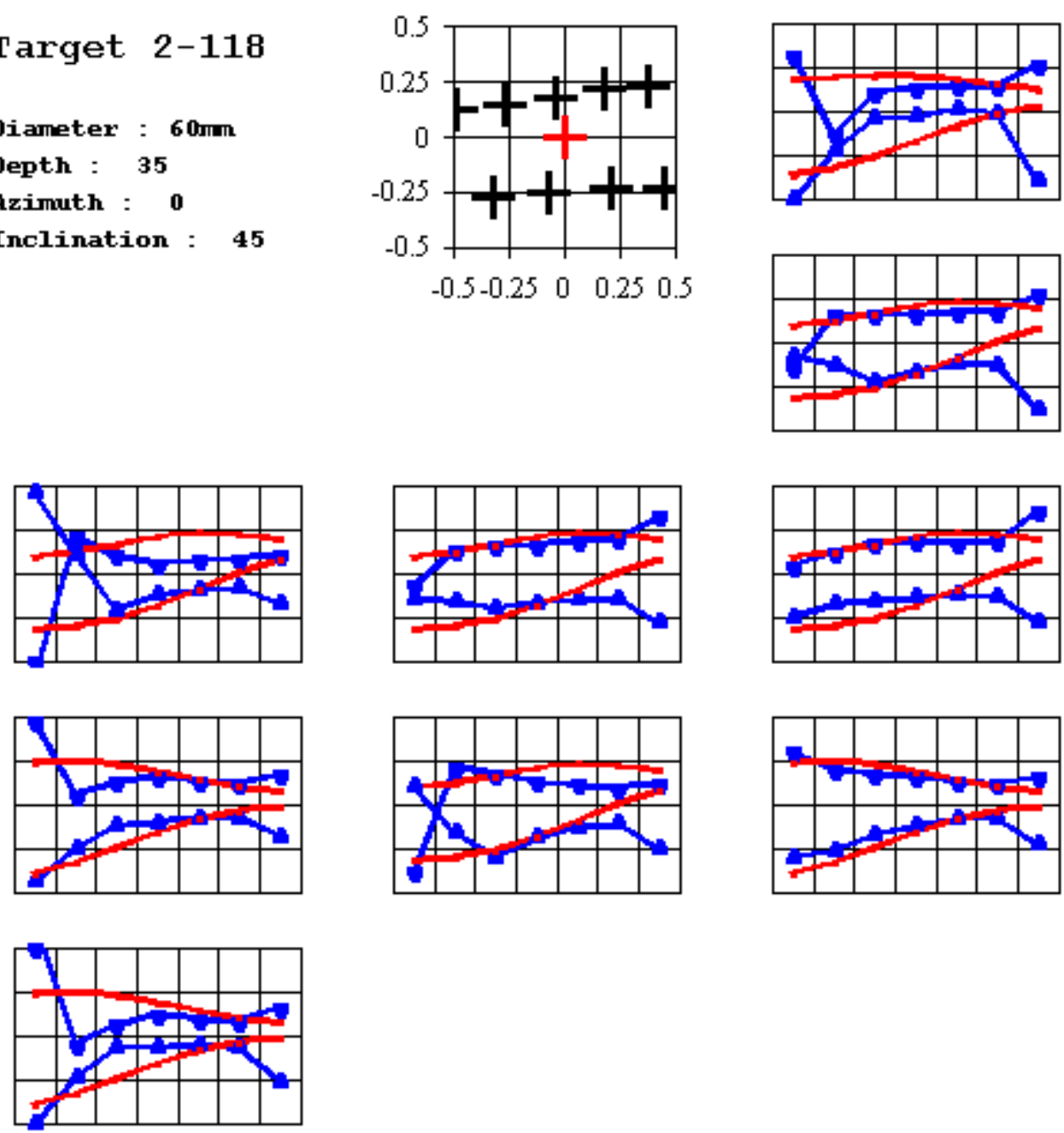
Target 2-120

Diameter : $60 \mathrm{~mm}$

Depth : $\mathbf{3 0}$

fzimuth : 270

Inclination : 0
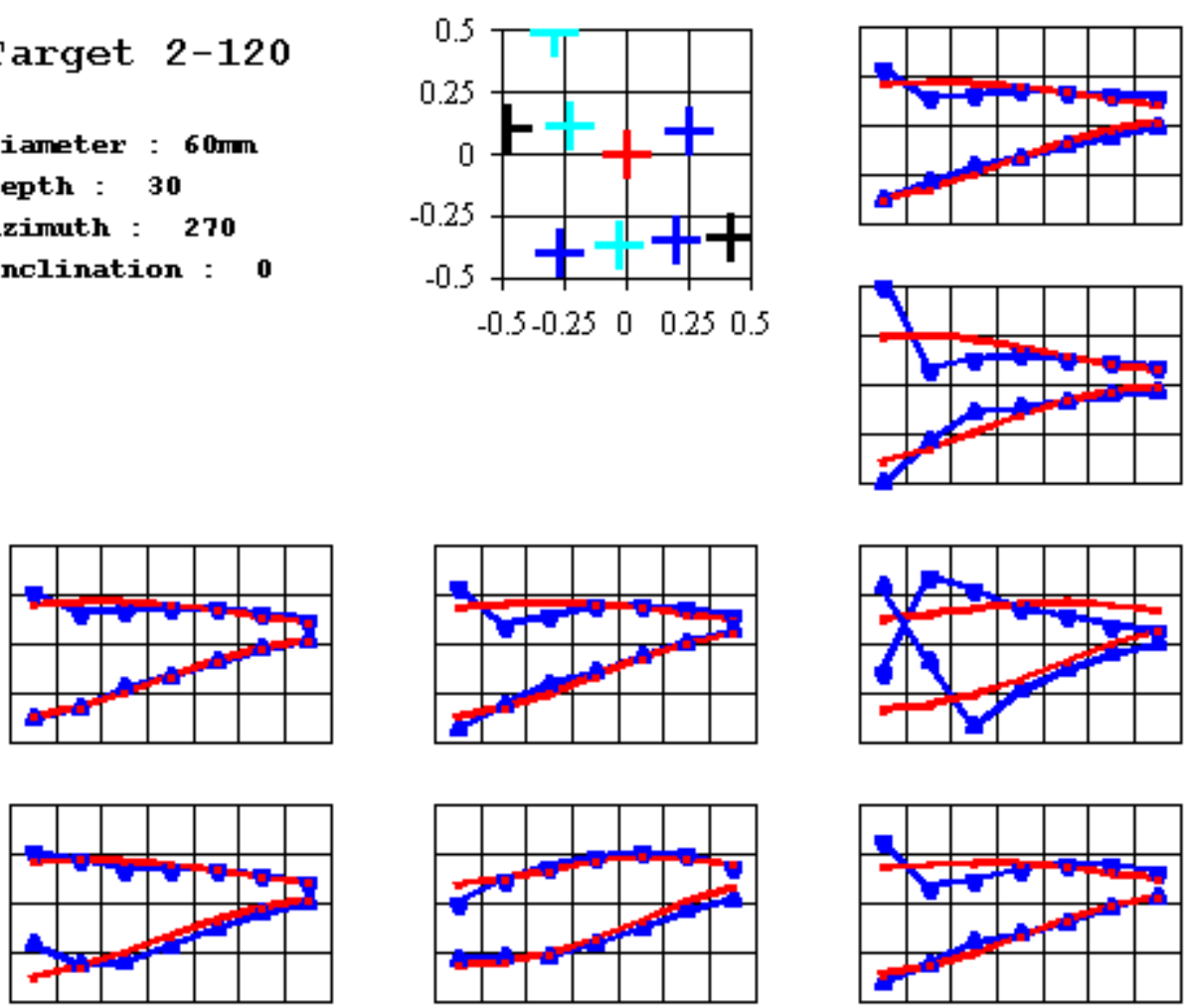

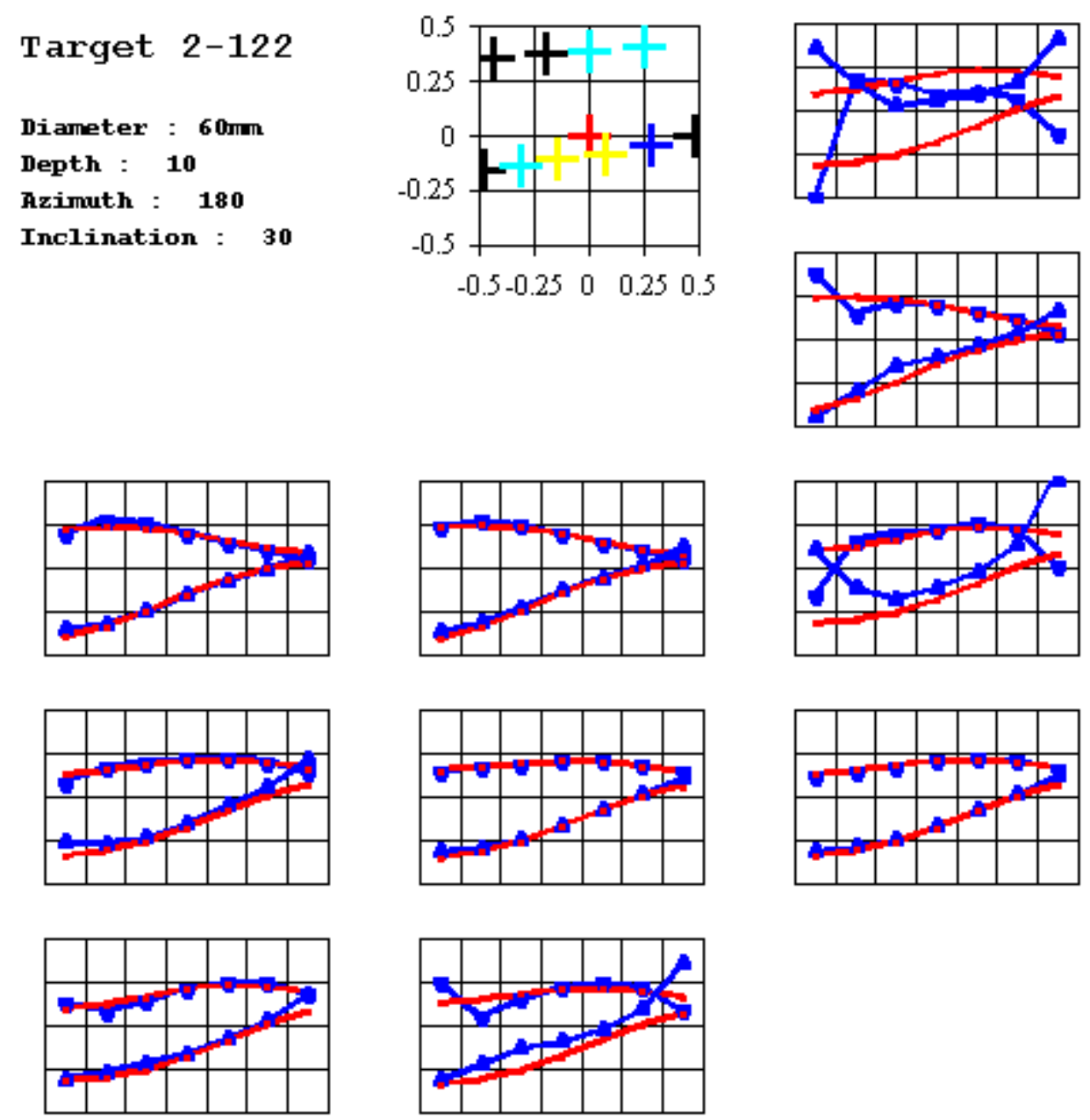
Target 2-124

Diameter : $60 \mathrm{~mm}$

Depth : 20

Azimuth : $\mathbf{3 3 0}$

Inclination : 10
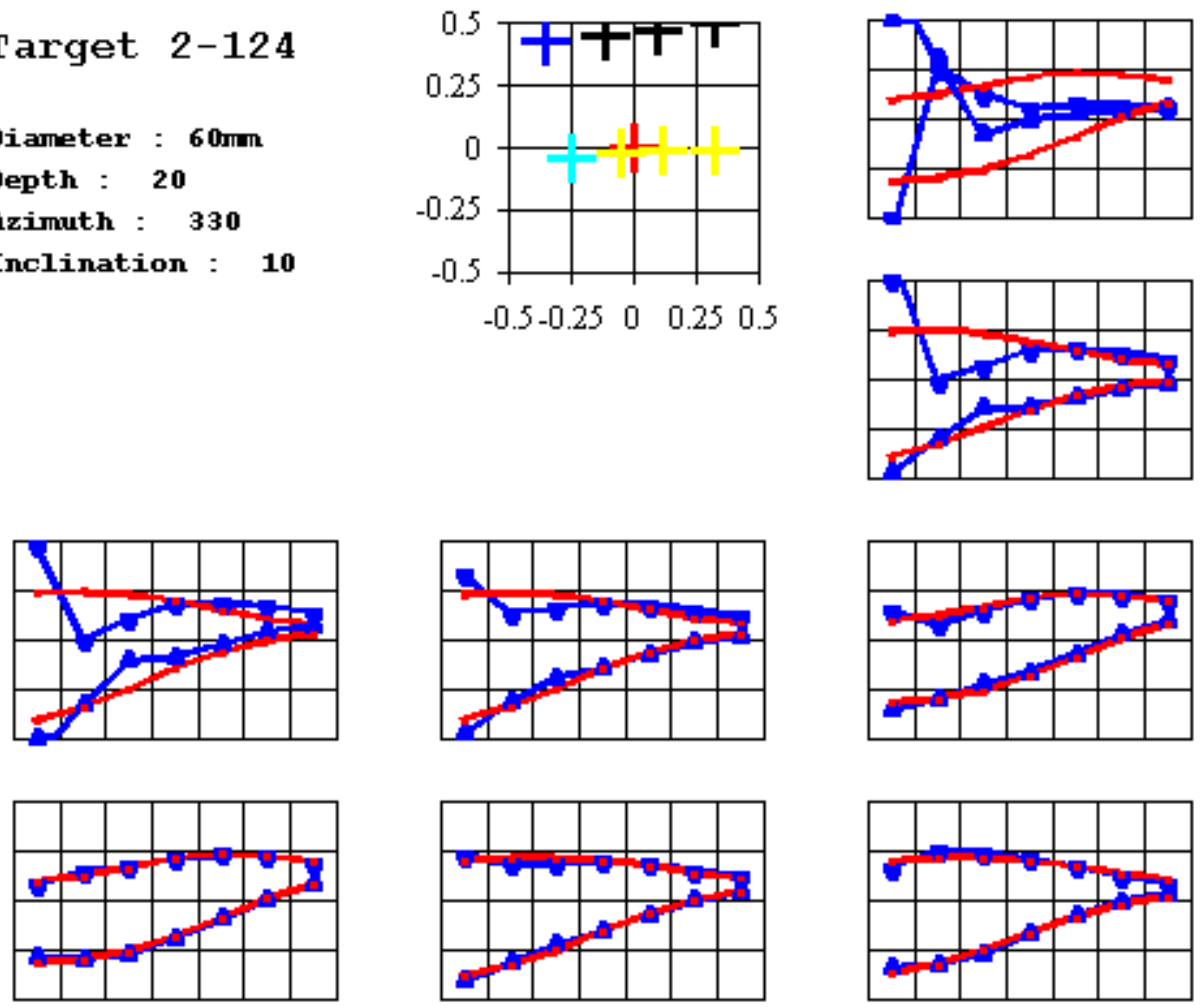

Target 2-126

Diameter : $81 \mathrm{~mm}$

Depth : $\mathbf{3 5}$

Azimuth : 45

Inclination : 45
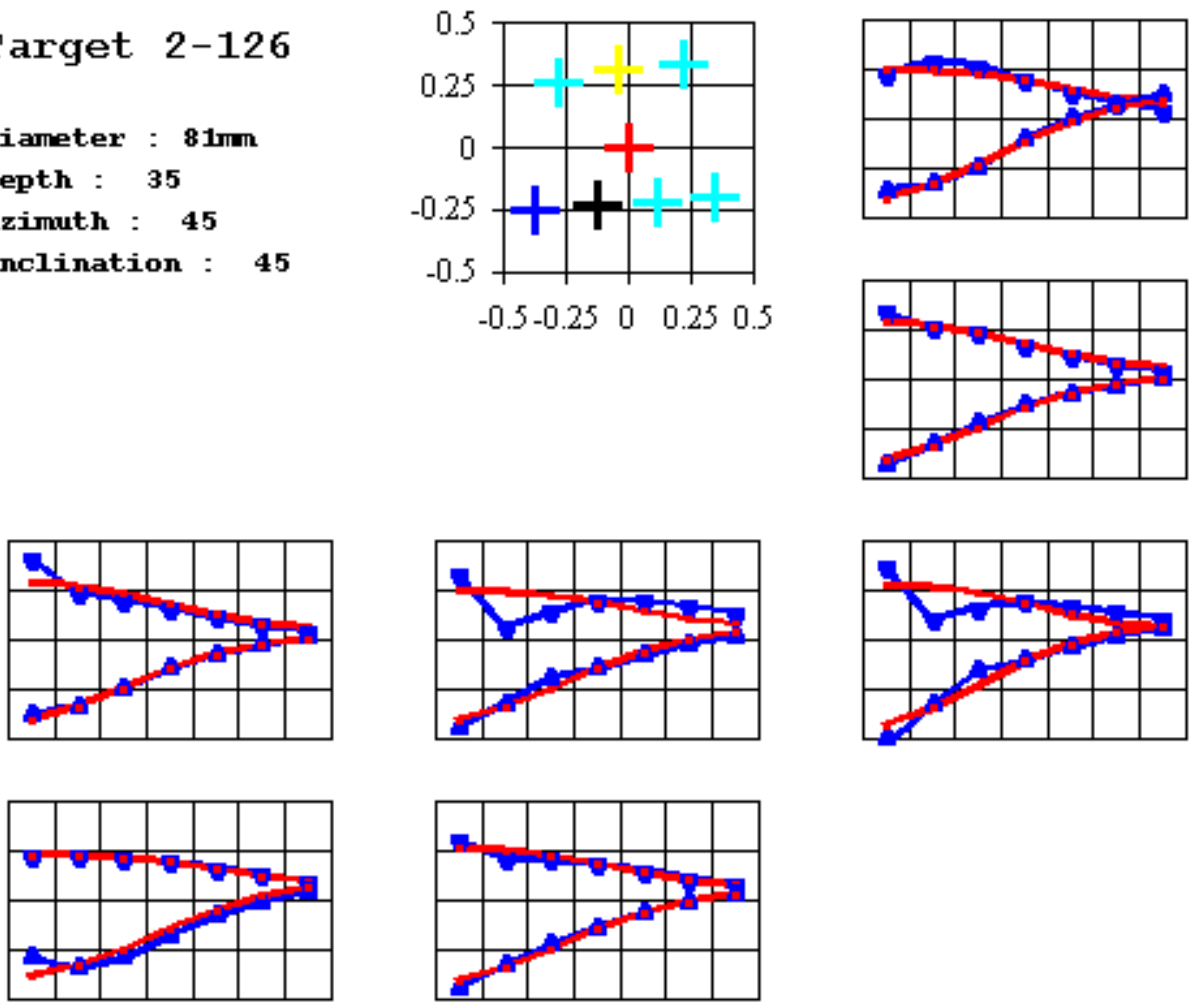
Target $2-128$

Diameter : $60 \mathrm{~mm}$

Depth : 10

Azimuth : 95

Inclination : $\mathbf{2 0}$
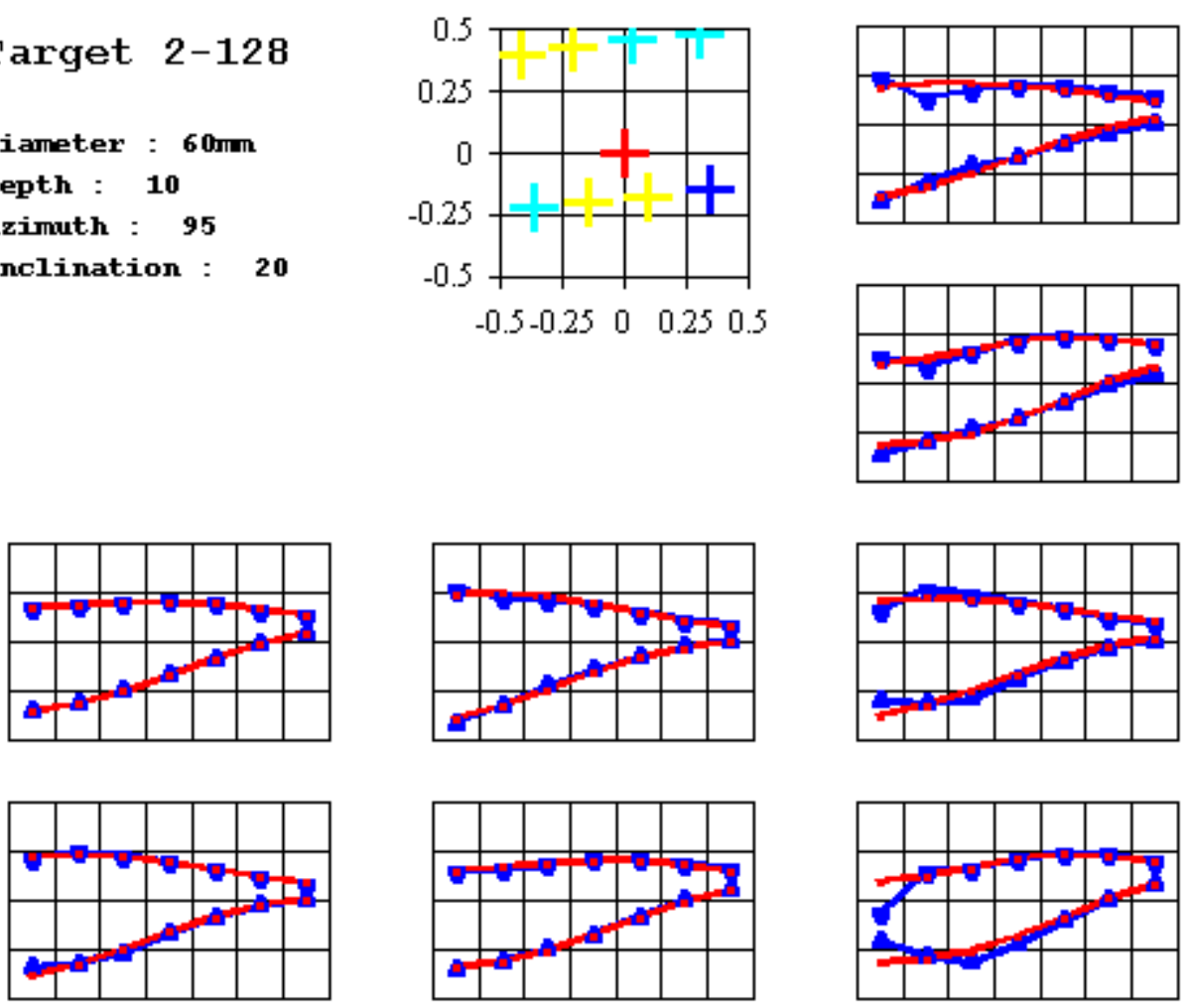
Target $2-130$

Diameter : $81 \mathrm{~mm}$

Depth : 70

Azimuth : 0

Inclination : 0
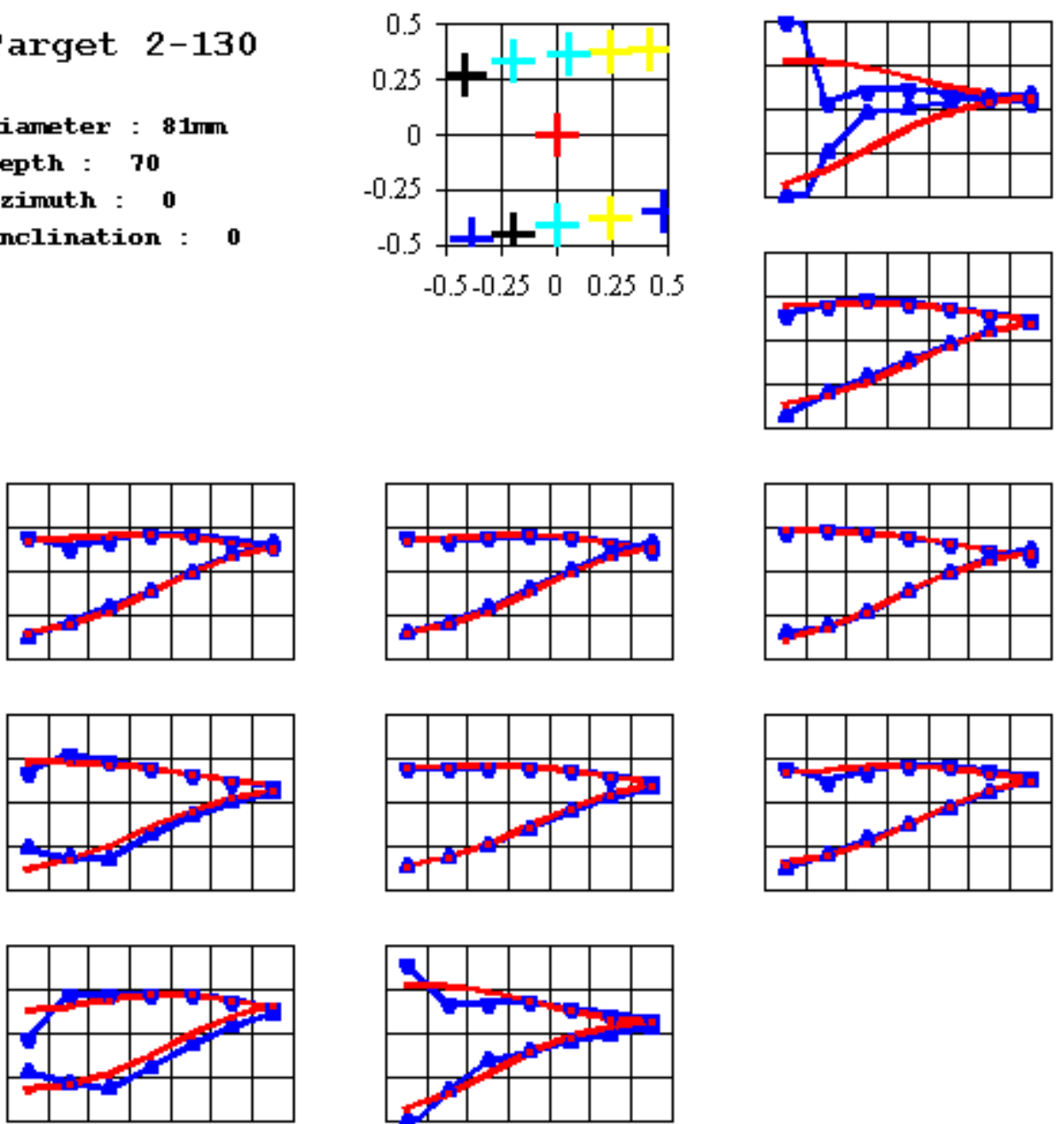
Target $2-131$

Diameter : $\mathbf{8 1 m m}$

Depth : 25

Azimuth : 0

Inclination : 0
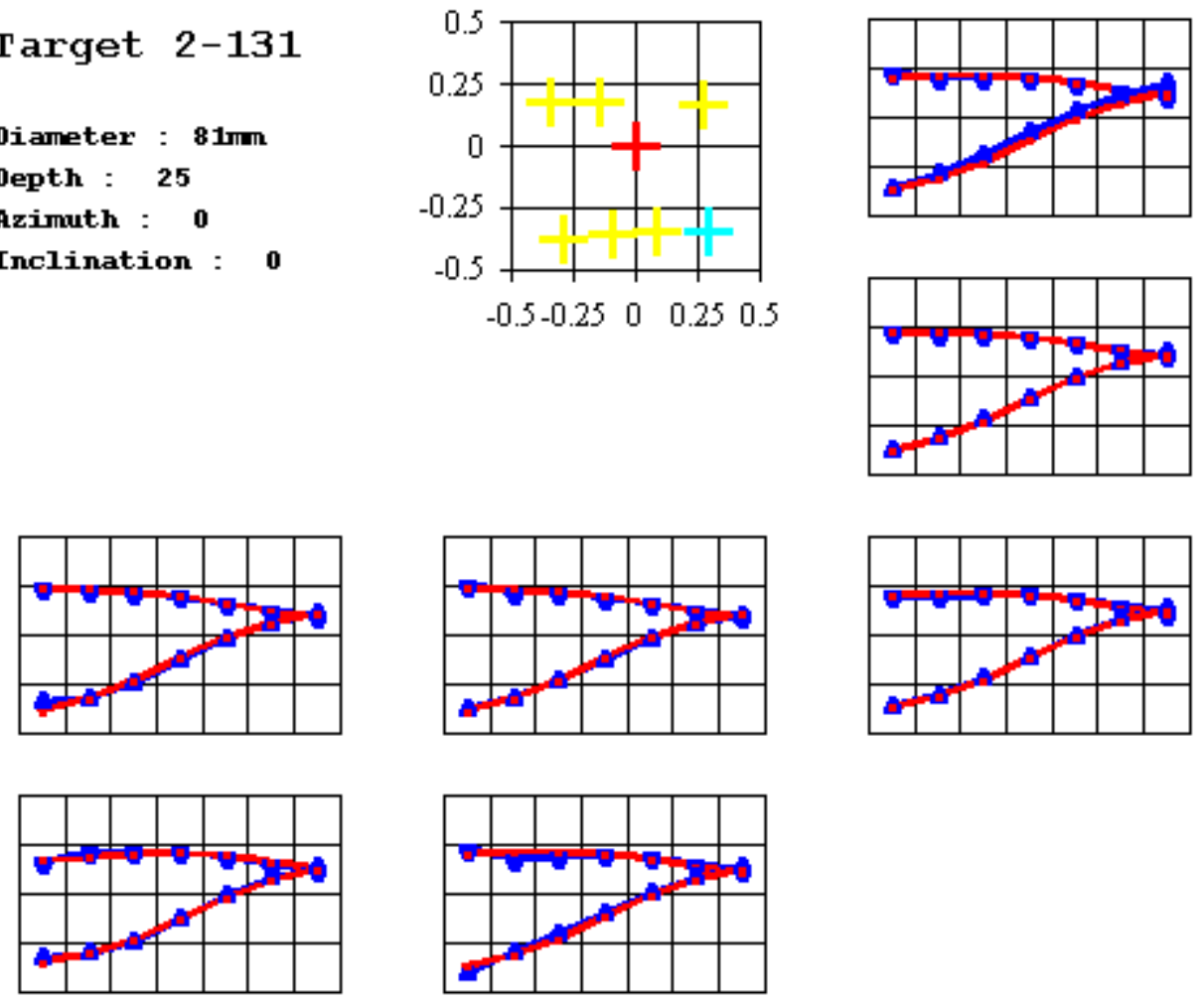

Target 2-132

Diameter : $5.0 i n$

Depth : 91

Azimuth : 90

Inclination : 0
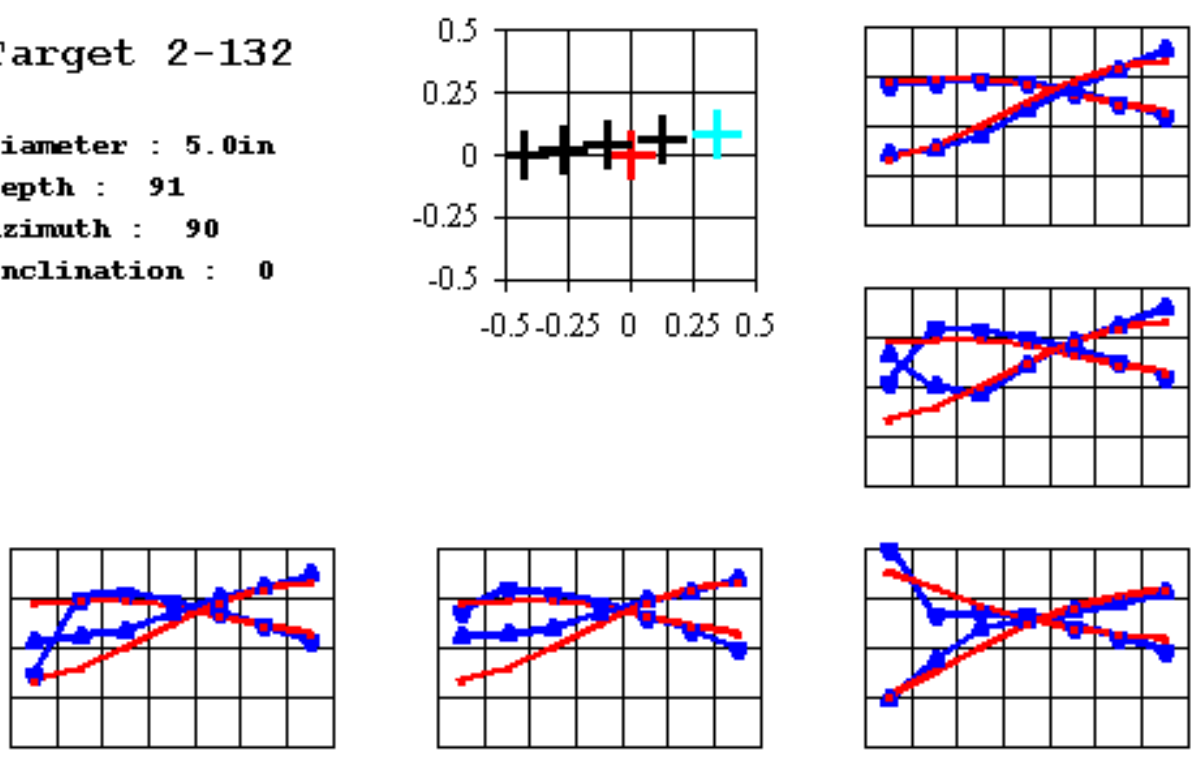
Target 2-134

Diameter : 4.2in

Depth : 40

Azimuth : 0

Inclination : 0
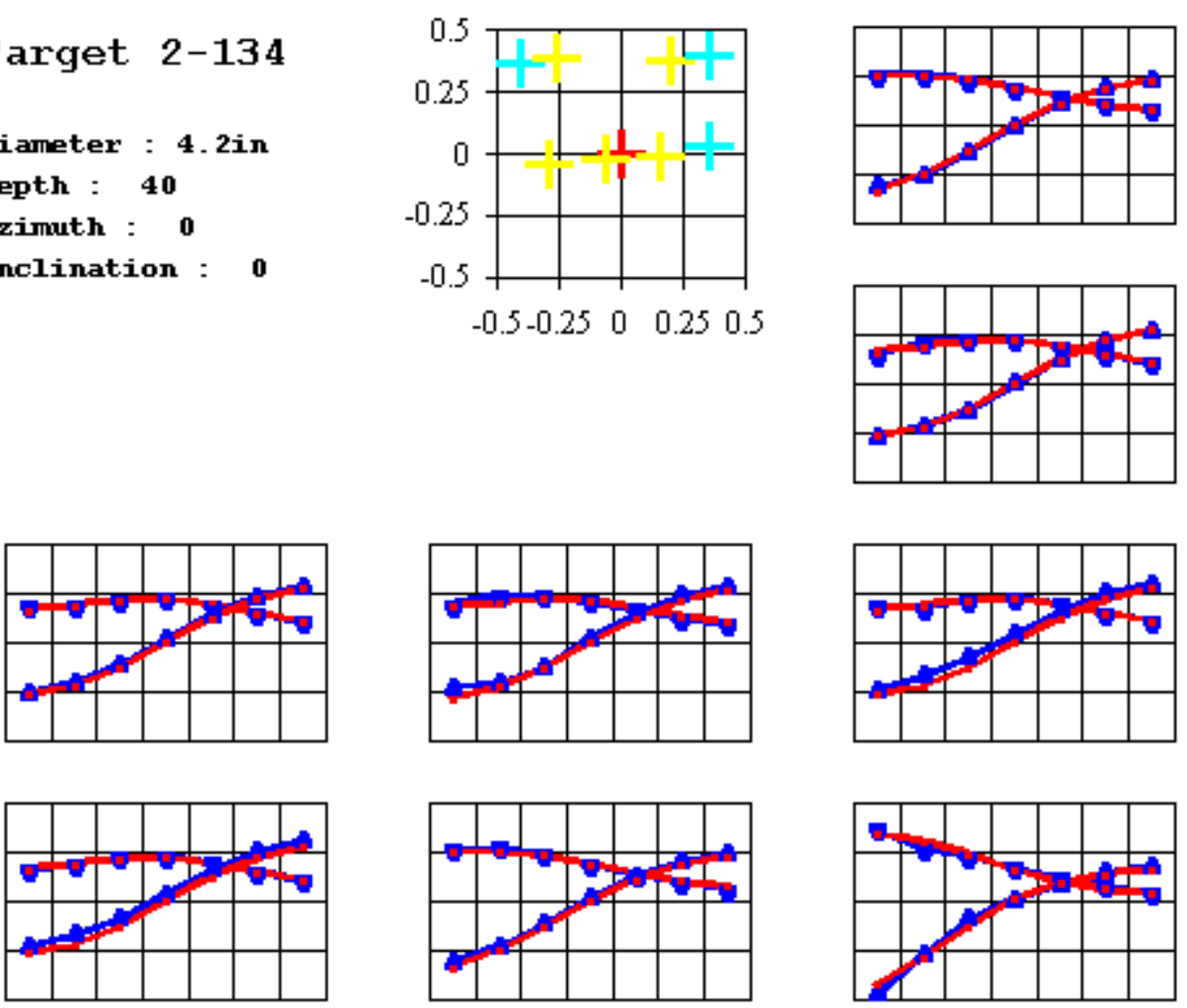

Target 2-136

Diameter : $\mathbf{2 0 m m}$

Depth : 5

Azimuth : 120

Inclination : $\mathbf{1 0}$
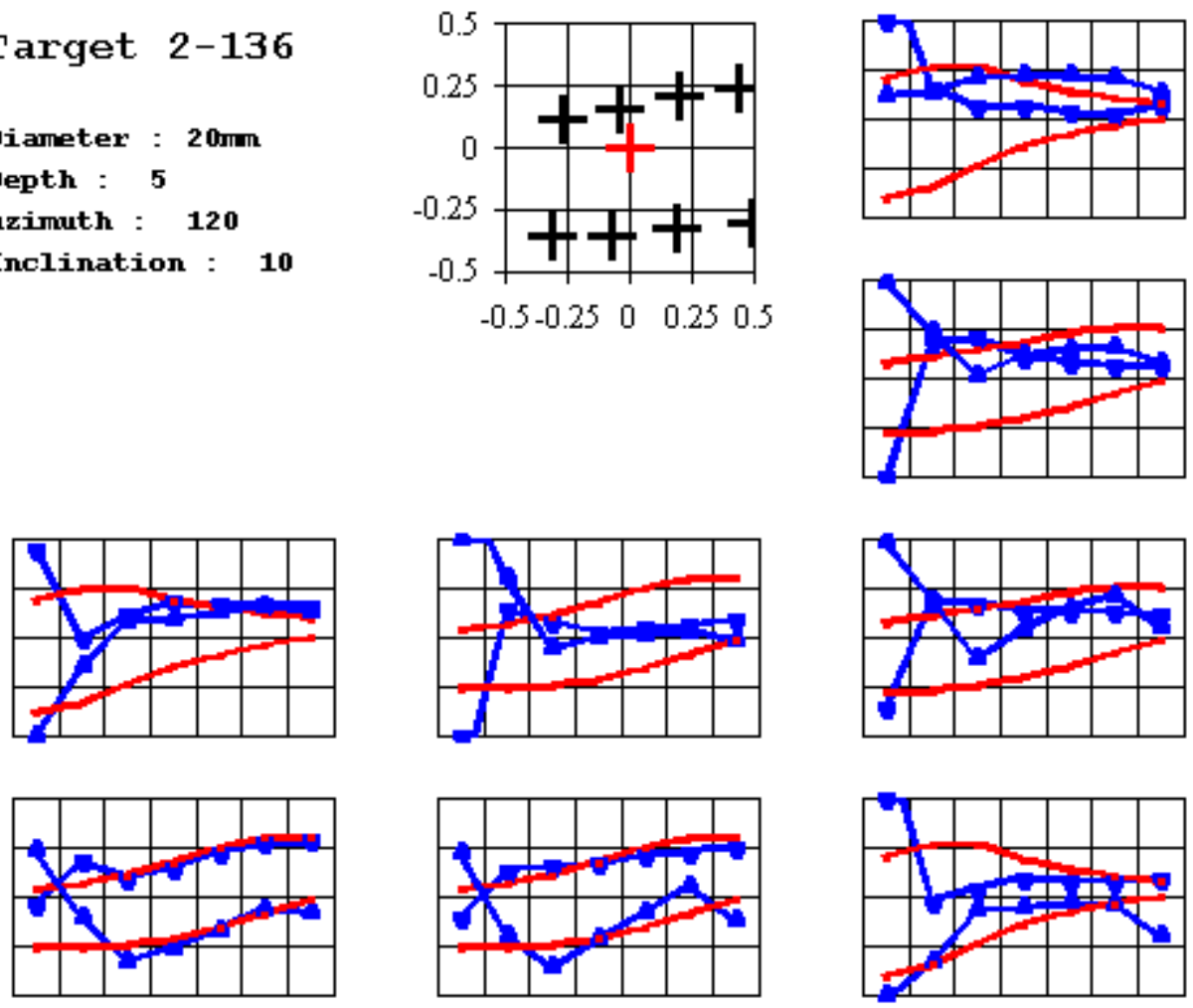
Target $2-138$

Diameter : $20 \mathrm{~mm}$

Depth : 5

Azimuth : 275

Inclination : 15
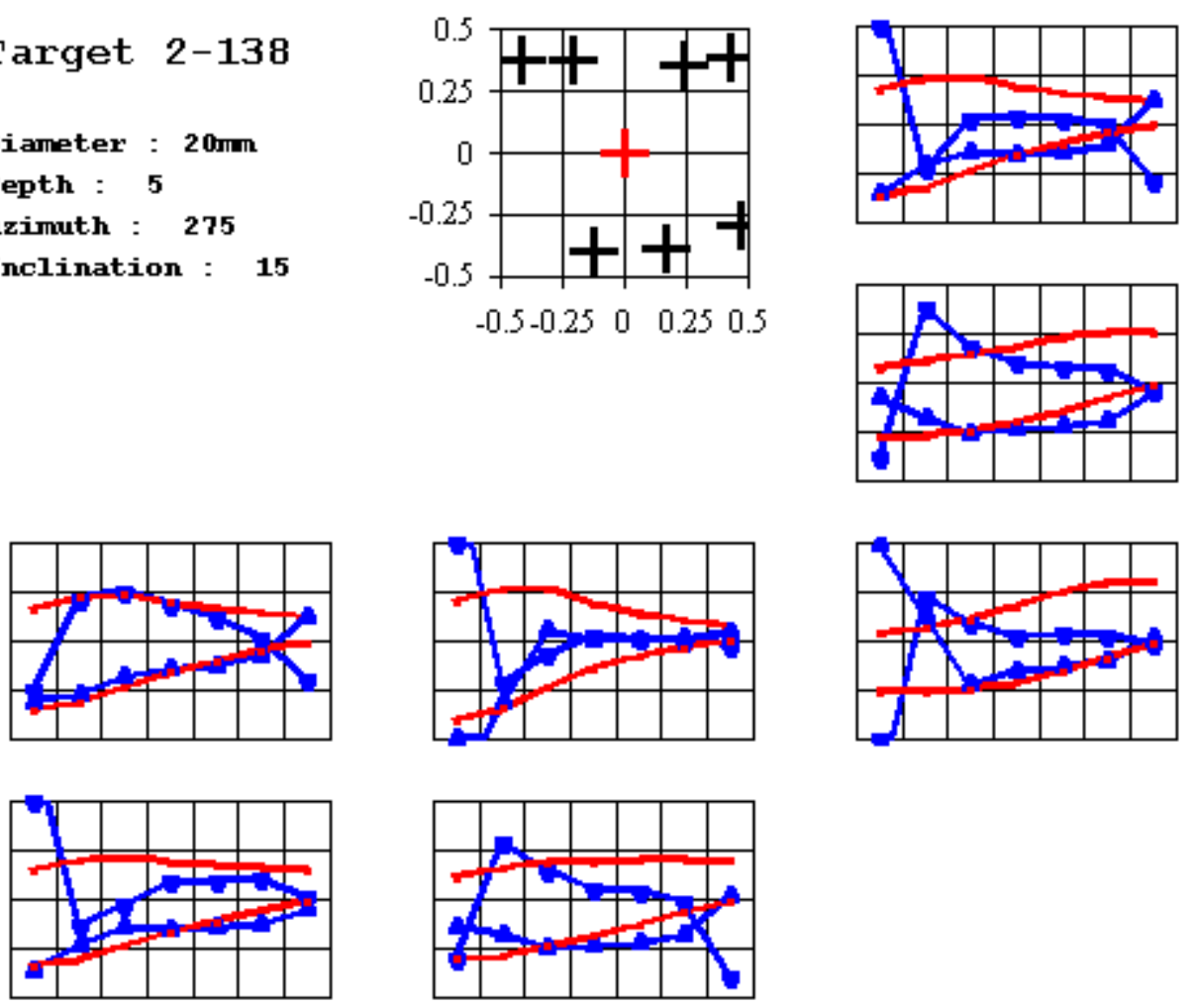

Target $2-140$

Diameter : $105 \mathrm{~mm}$

Depth : 70

Azimuth : $\mathbf{3 3 0}$

Inclination : 10
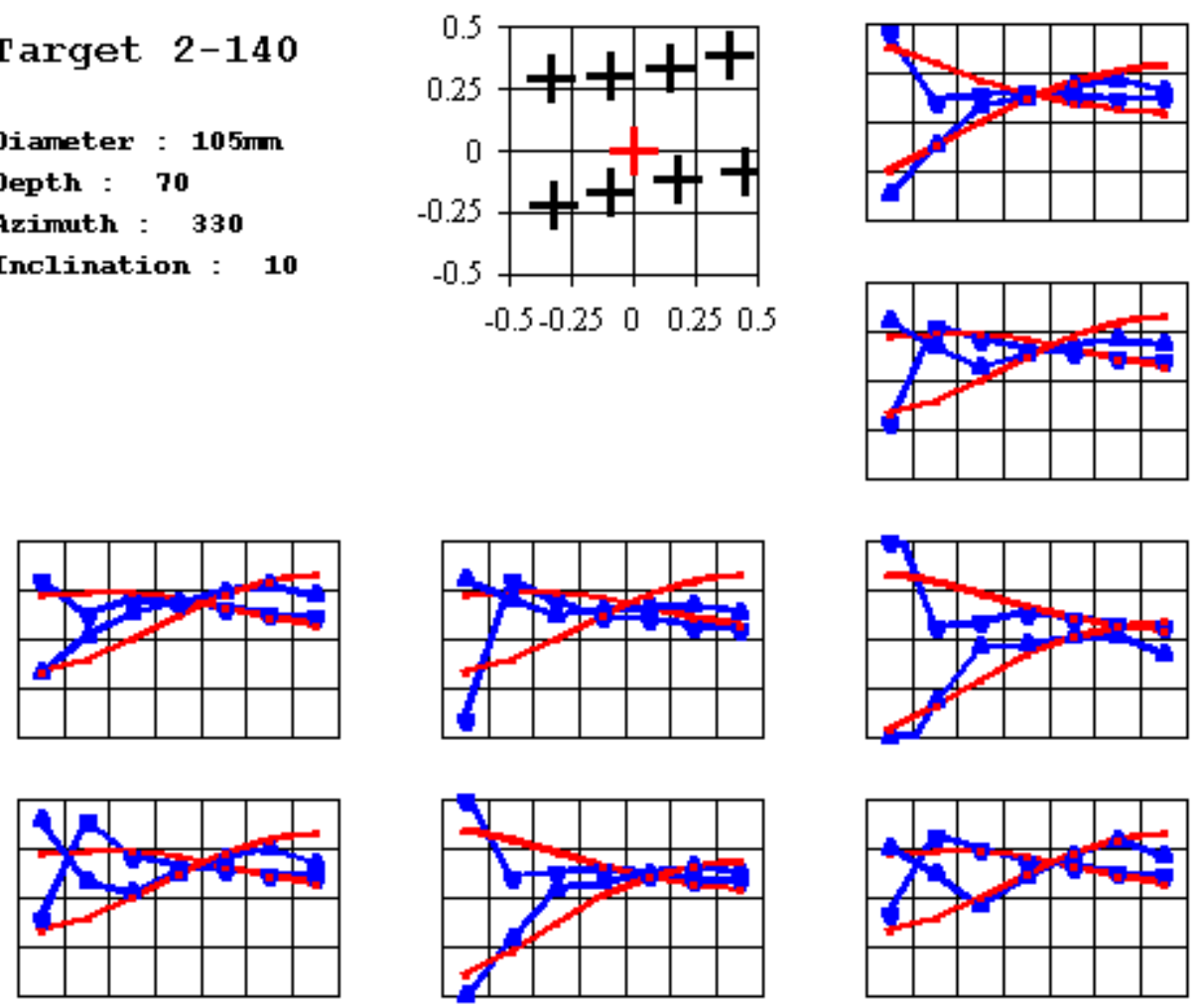
Target $2-142$

Diameter : $152 \mathrm{~mm}$

Depth : 91

Azimuth : $\mathbf{3 0 0}$

Inclination : $\mathbf{4 5}$
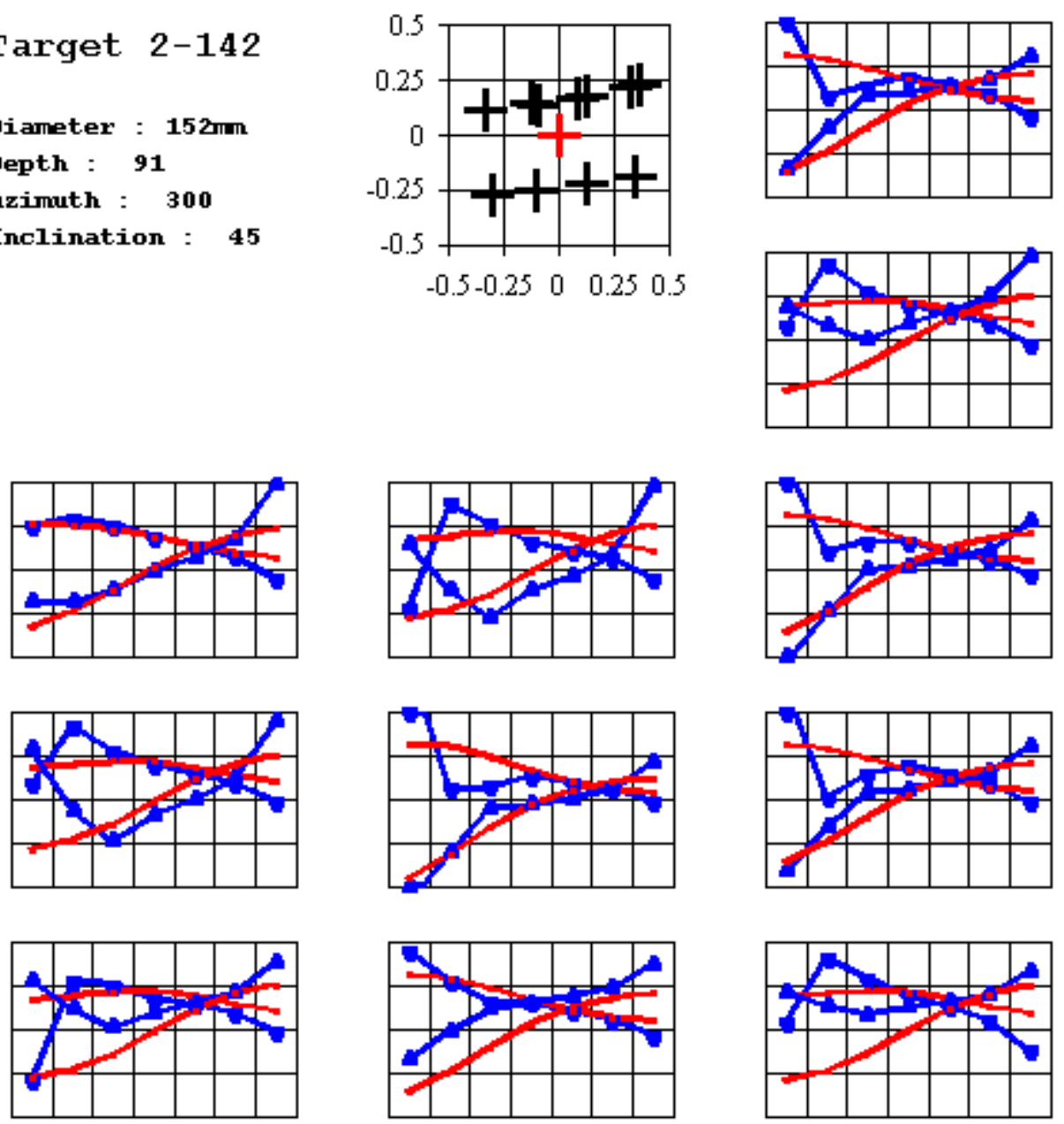
Target 2-144

Diameter : $152 \mathrm{~mm}$

Depth : 45

Azimuth : $\mathbf{5 0}$

Inclination : $\mathbf{3 0}$
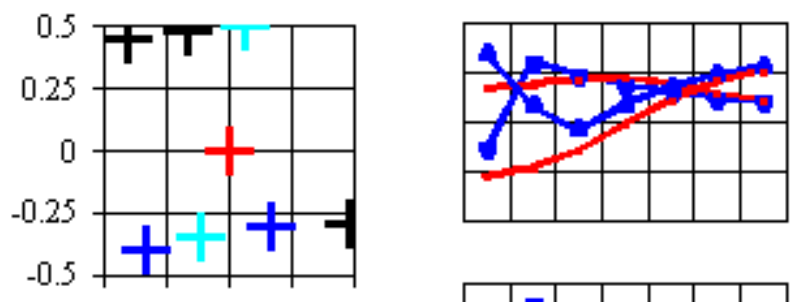

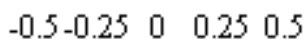
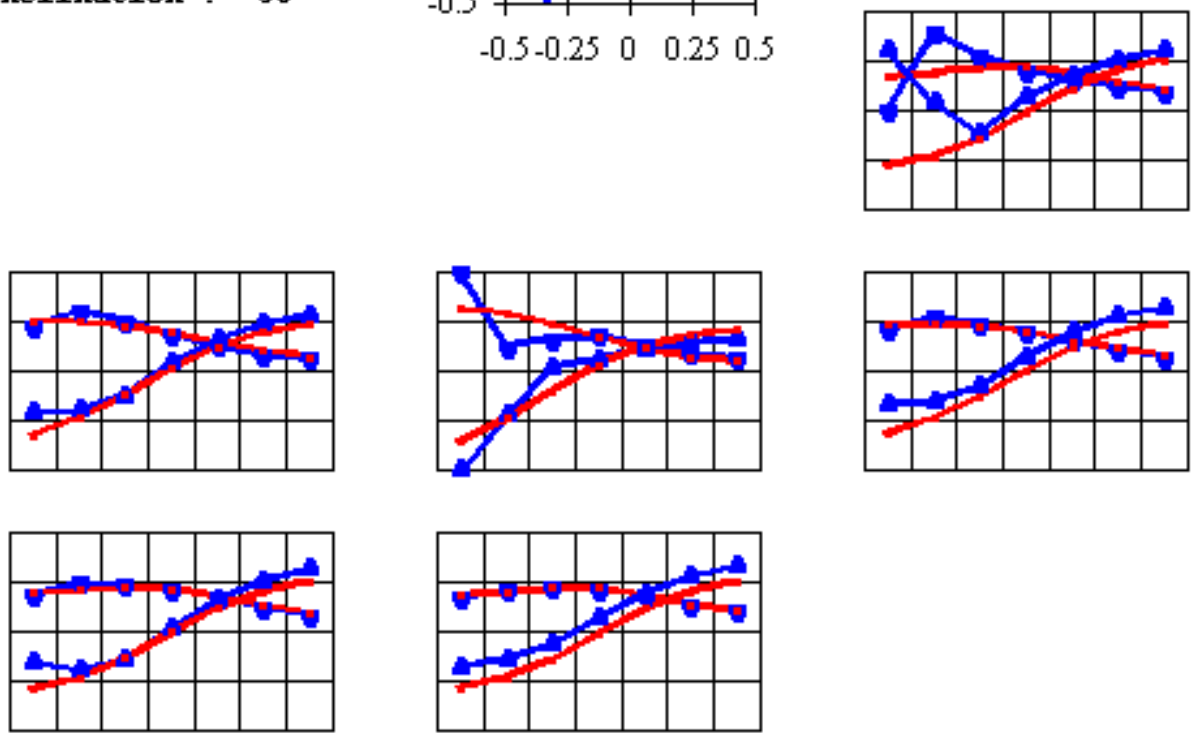
Target 2-146

Diameter : $20 \mathrm{~mm}$

Depth : 0

Azimuth : 0

Inclination : 90
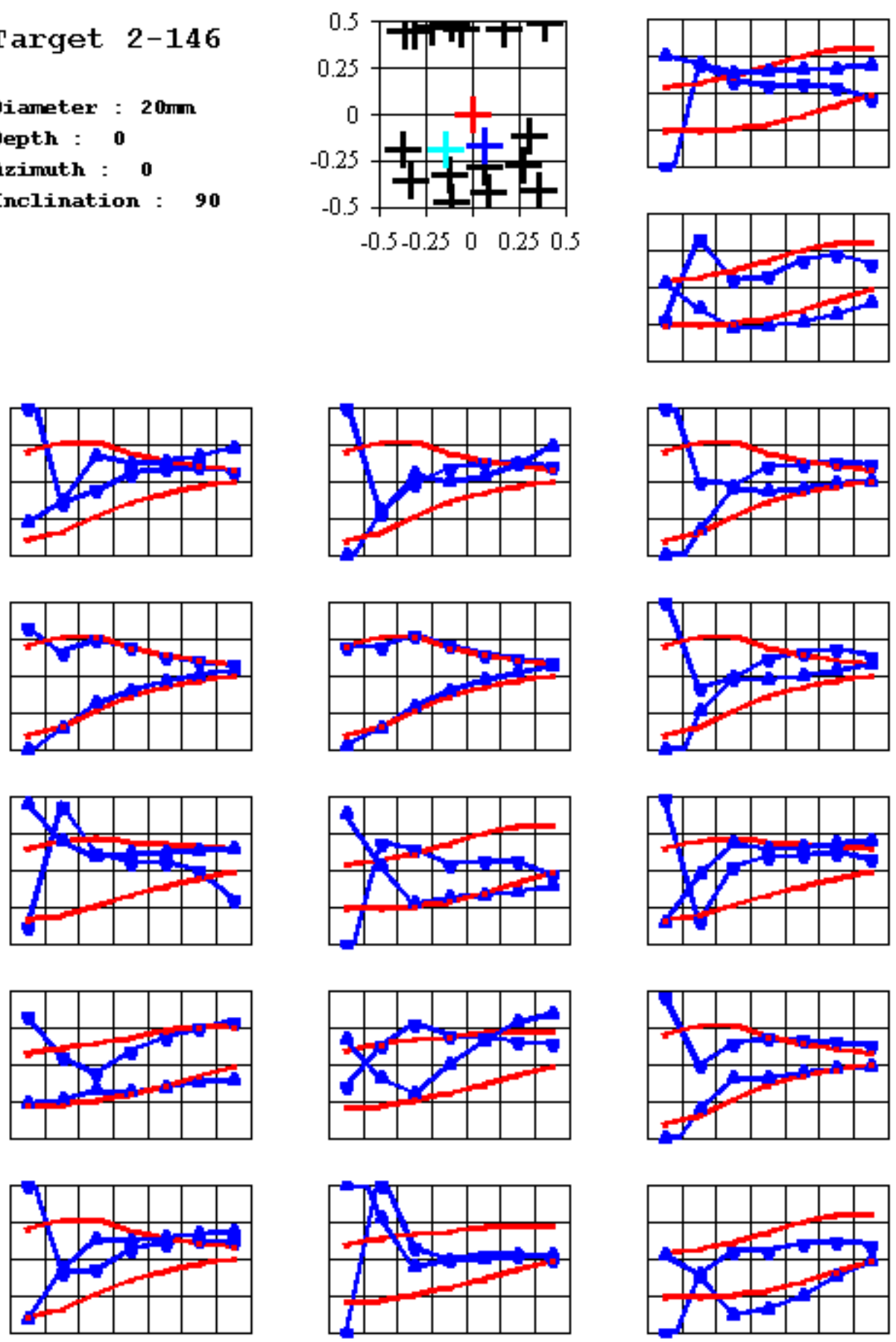
Target 2-148

Diameter : $20 \mathrm{~mm}$

Depth : 0

Azimuth : 0

Inclination : $\mathbf{- 9 0}$
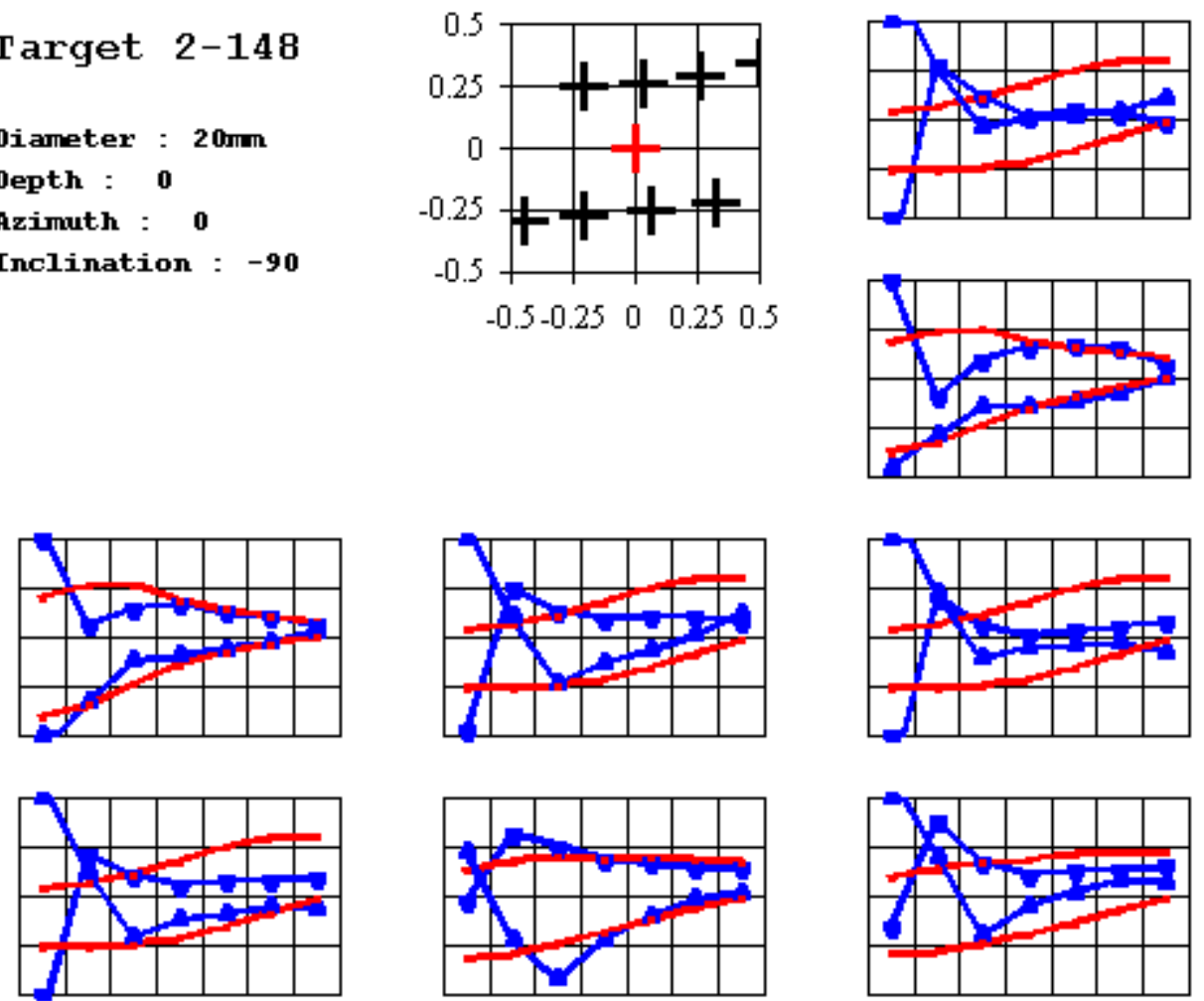
Target 2-150

Diameter : $20 \mathrm{~mm}$

Depth : 10

Azimuth : 0

Inclination : 0
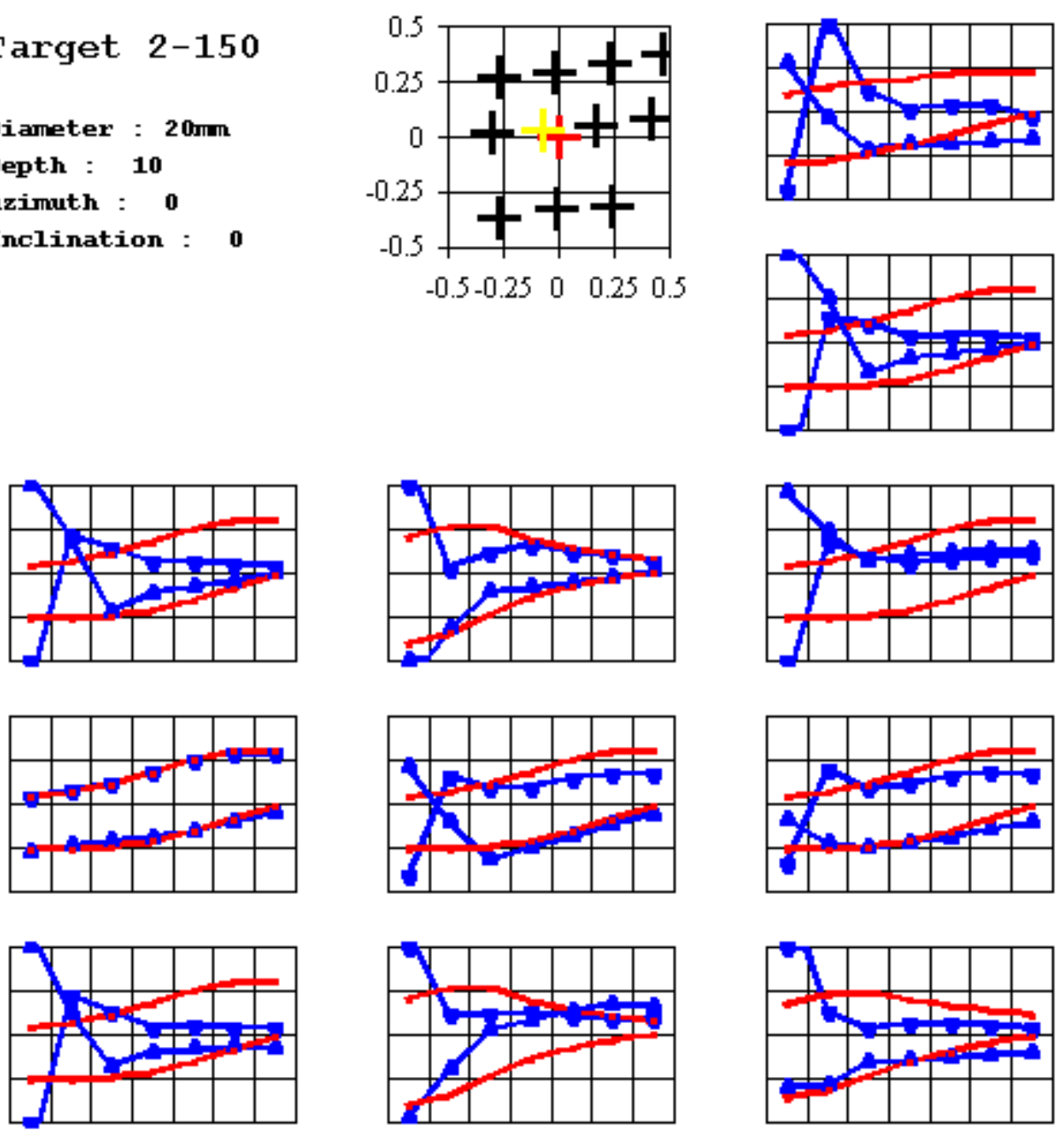
Target 2-152

Diameter : $57 \mathrm{~mm}$

Depth : 25

fzimuth : 90

Inclination : 0
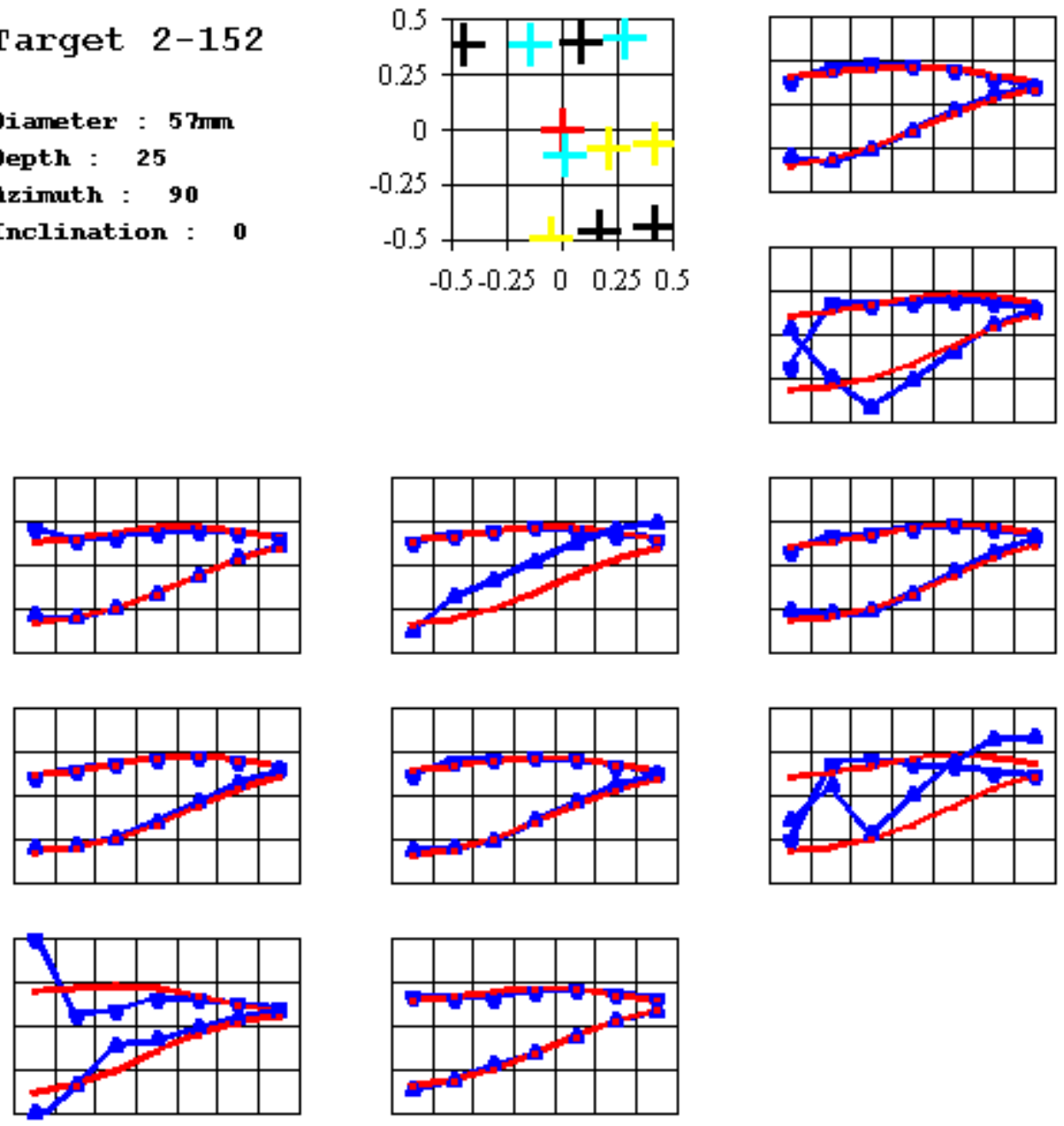
Target 2-154

Diameter : 5 7mm

Depth : 20

Azimuth : 180

Inclination : 45
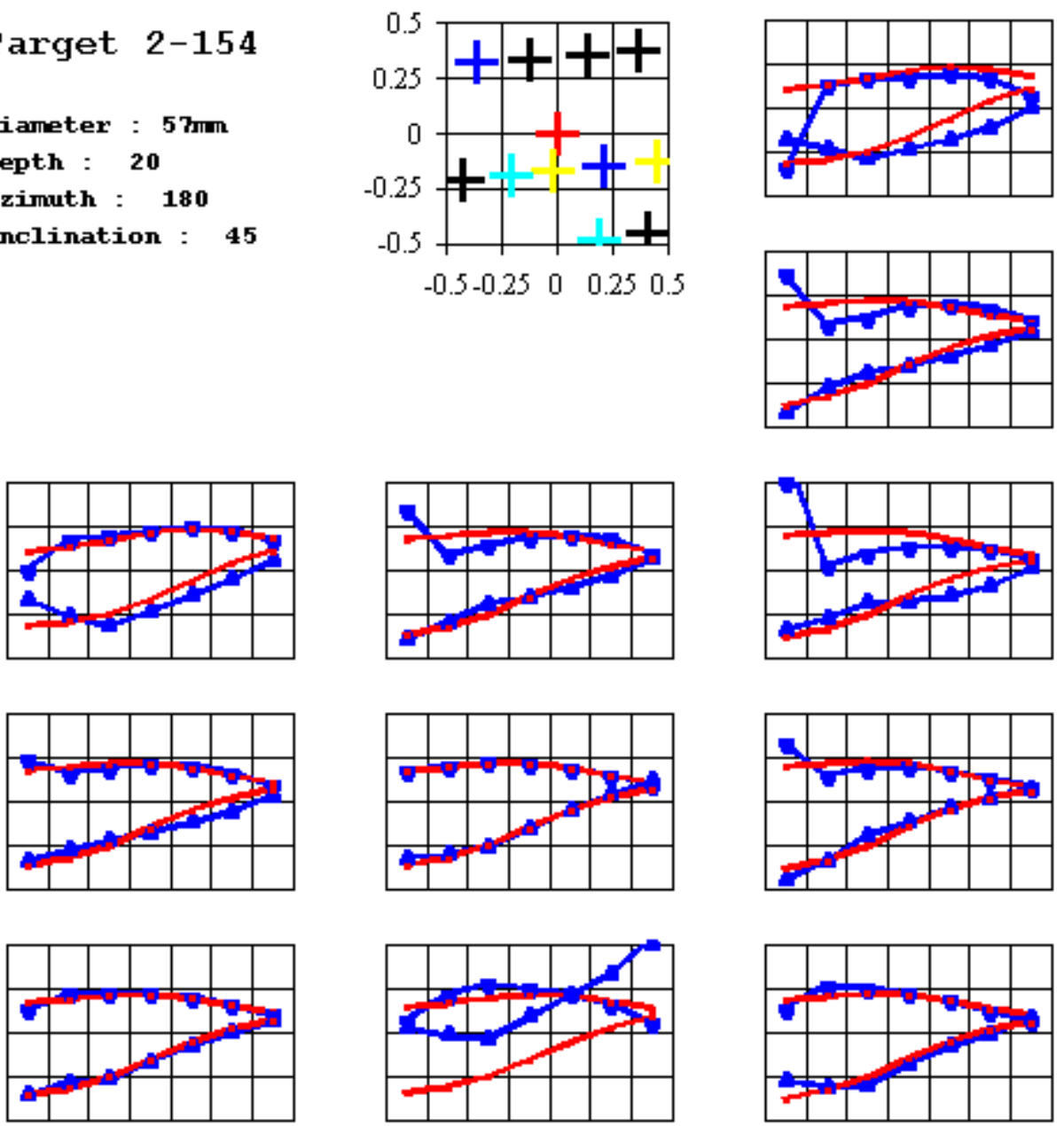
Target $2-156$

Diameter : $155 \mathrm{~mm}$

Depth : 102

Azimuth : 0

Inclination : 90
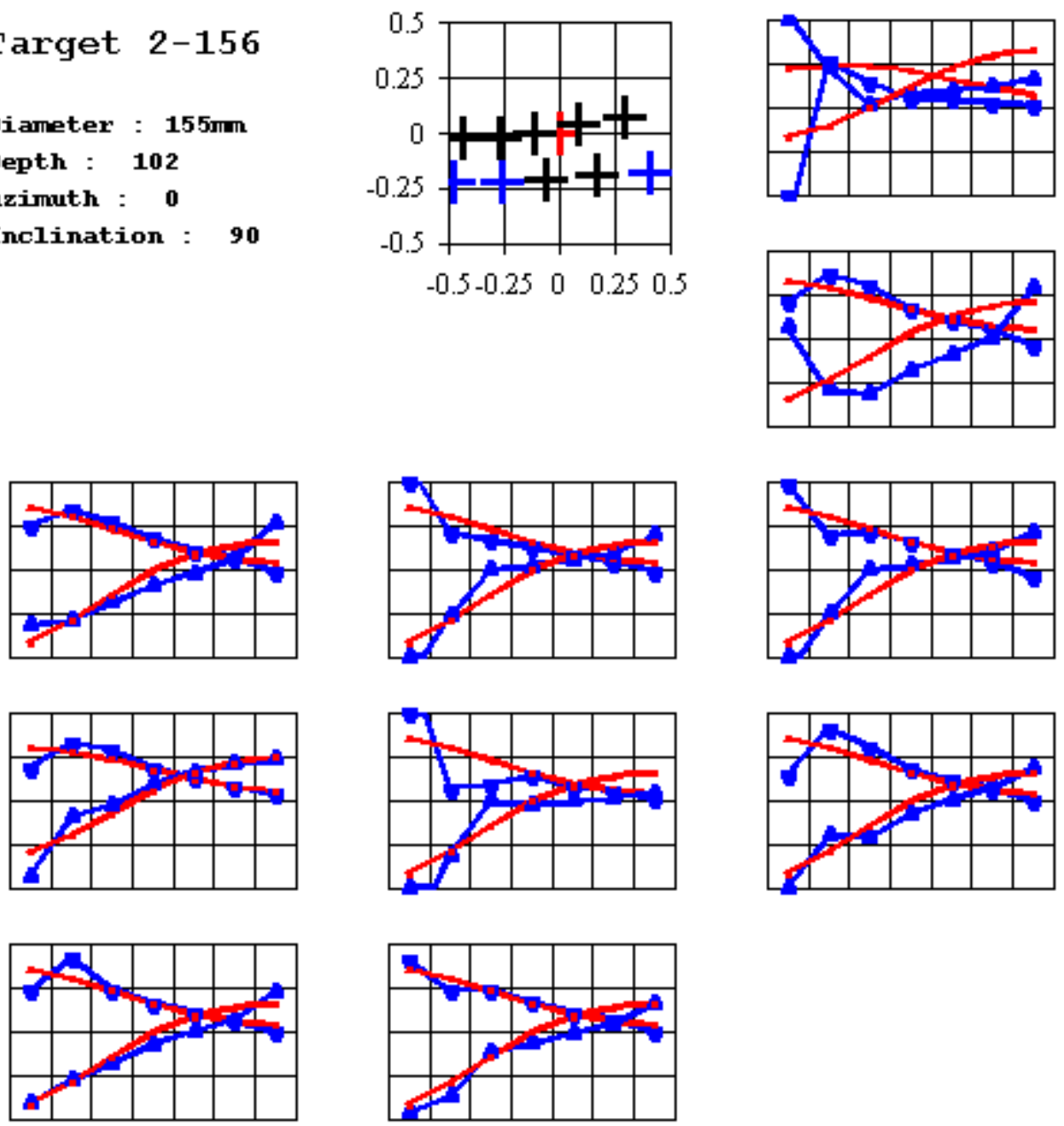
Target $2-158$

Diameter : $20 \mathrm{~mm}$

Depth : 10

Azimuth : 220

Inclination : 20
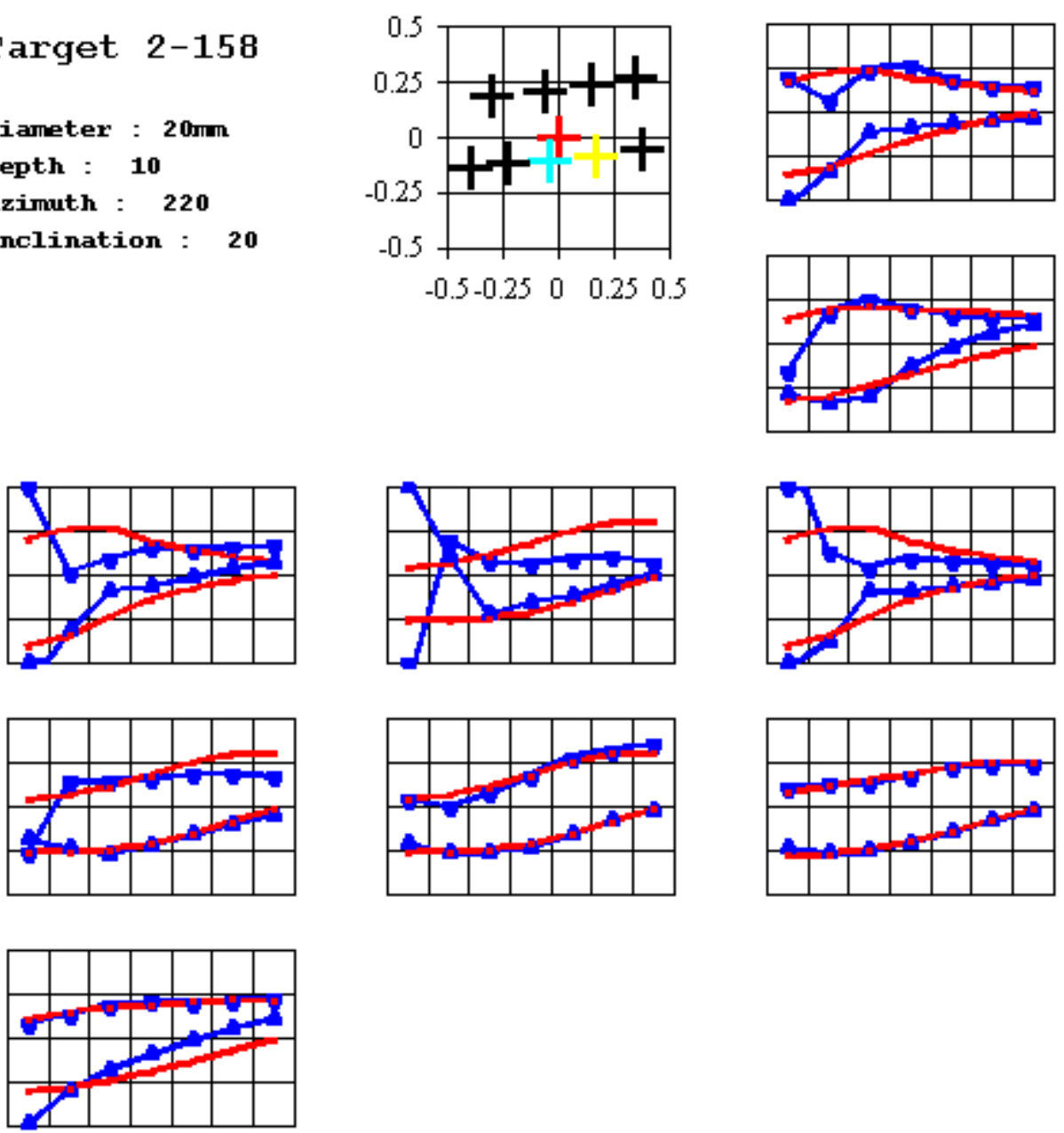
Target $\quad 2-160$

Diameter : $5 \mathrm{hmm}$

Depth : 35

Azimuth : 270

Inclination : 40
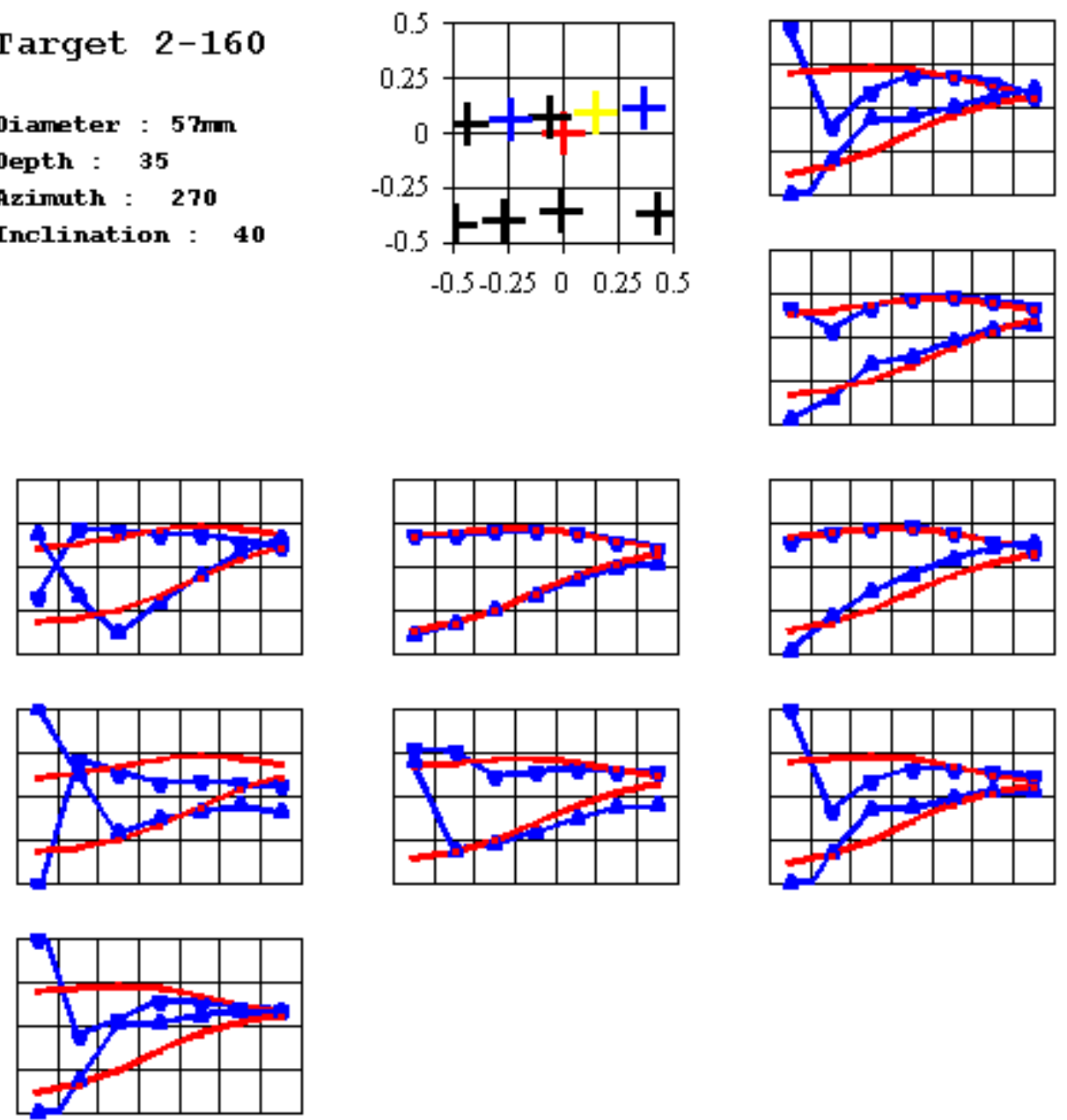
Target $2-162$

Diameter : $76 \mathrm{~mm}$

Depth : 50

Azimuth : 0

Inclination: 0
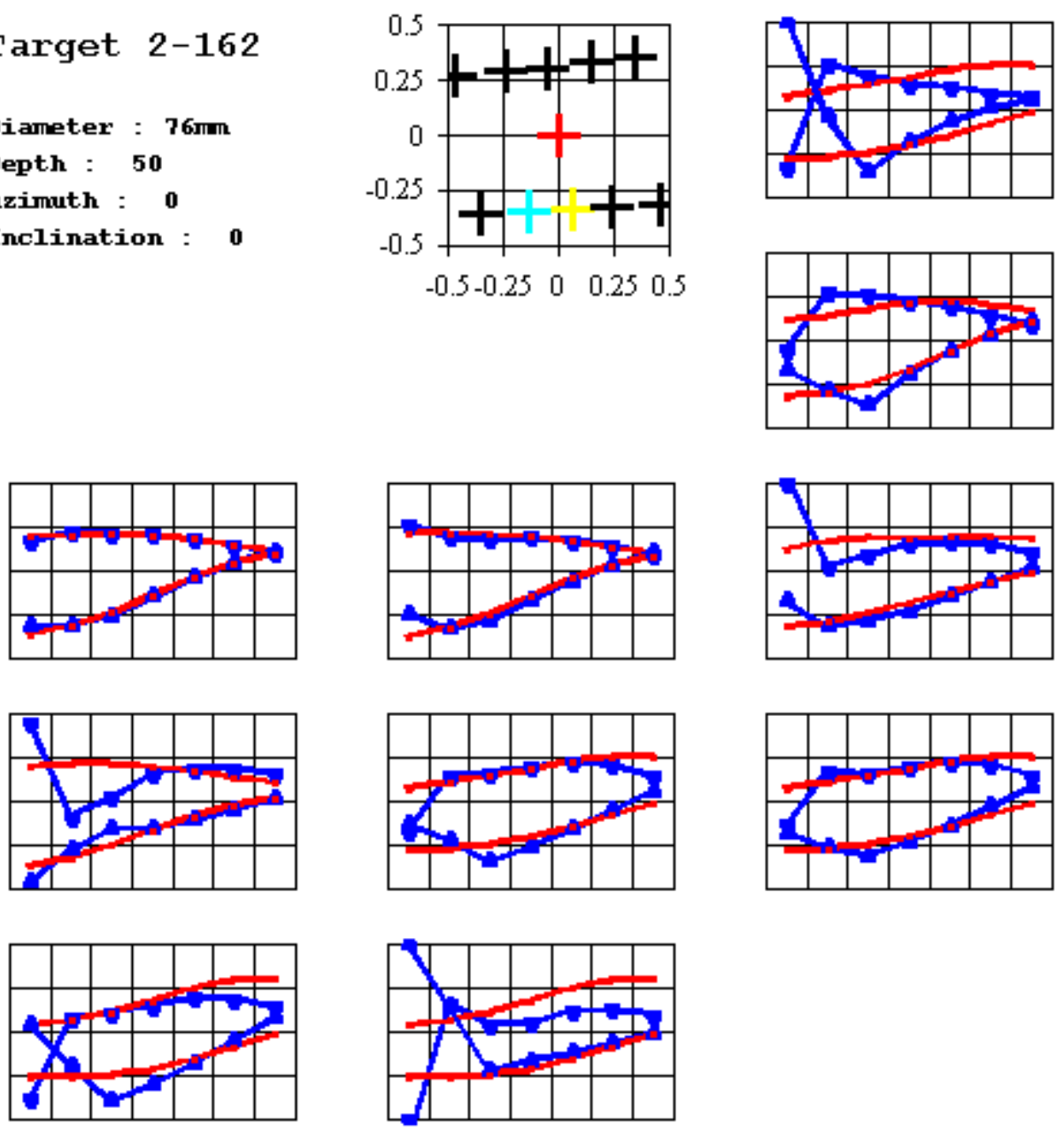

Target 2-164

Diameter : 2.75in

Depth : 60

Azimuth : 90

Inclination : 10
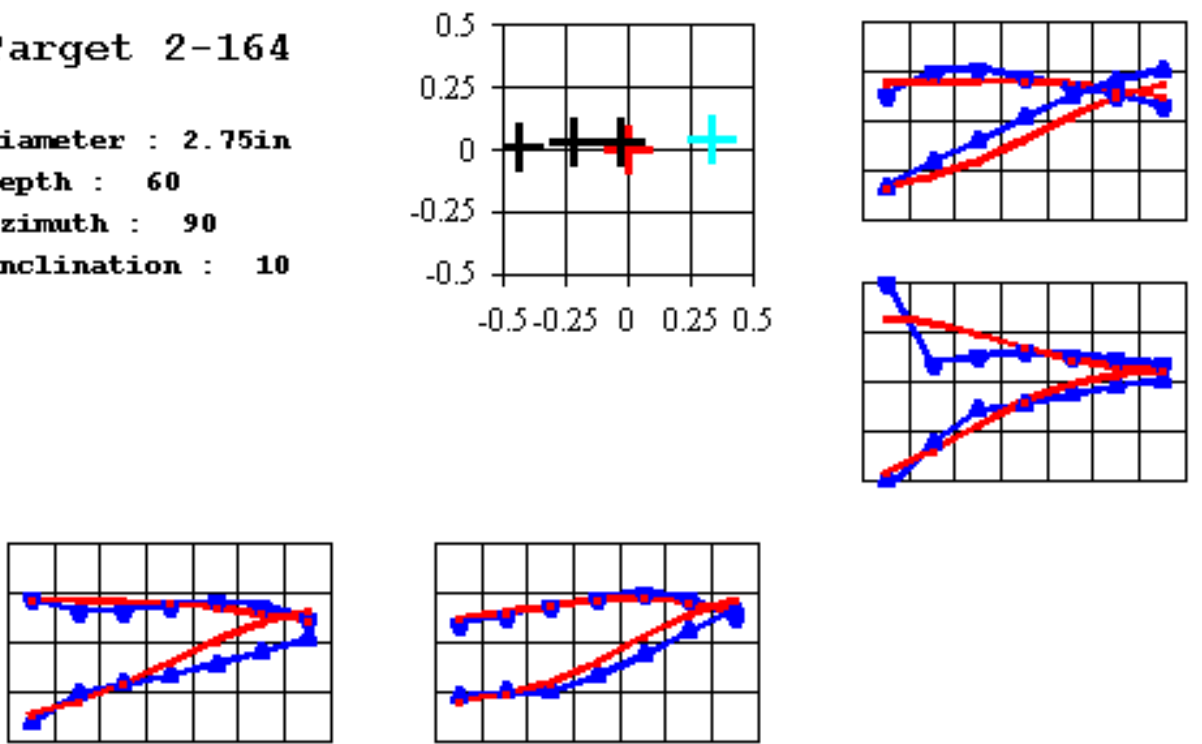
Target 2-166

Diameter : $2.75 i n$

Depth : 75

Azimuth : 180

Inclination : 20

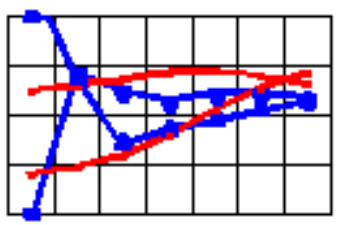

Target $3-68$

Diameter : $60 \mathrm{~mm}$

Depth : $\mathbf{2 0}$

fzimuth : 0

Inclination : 90
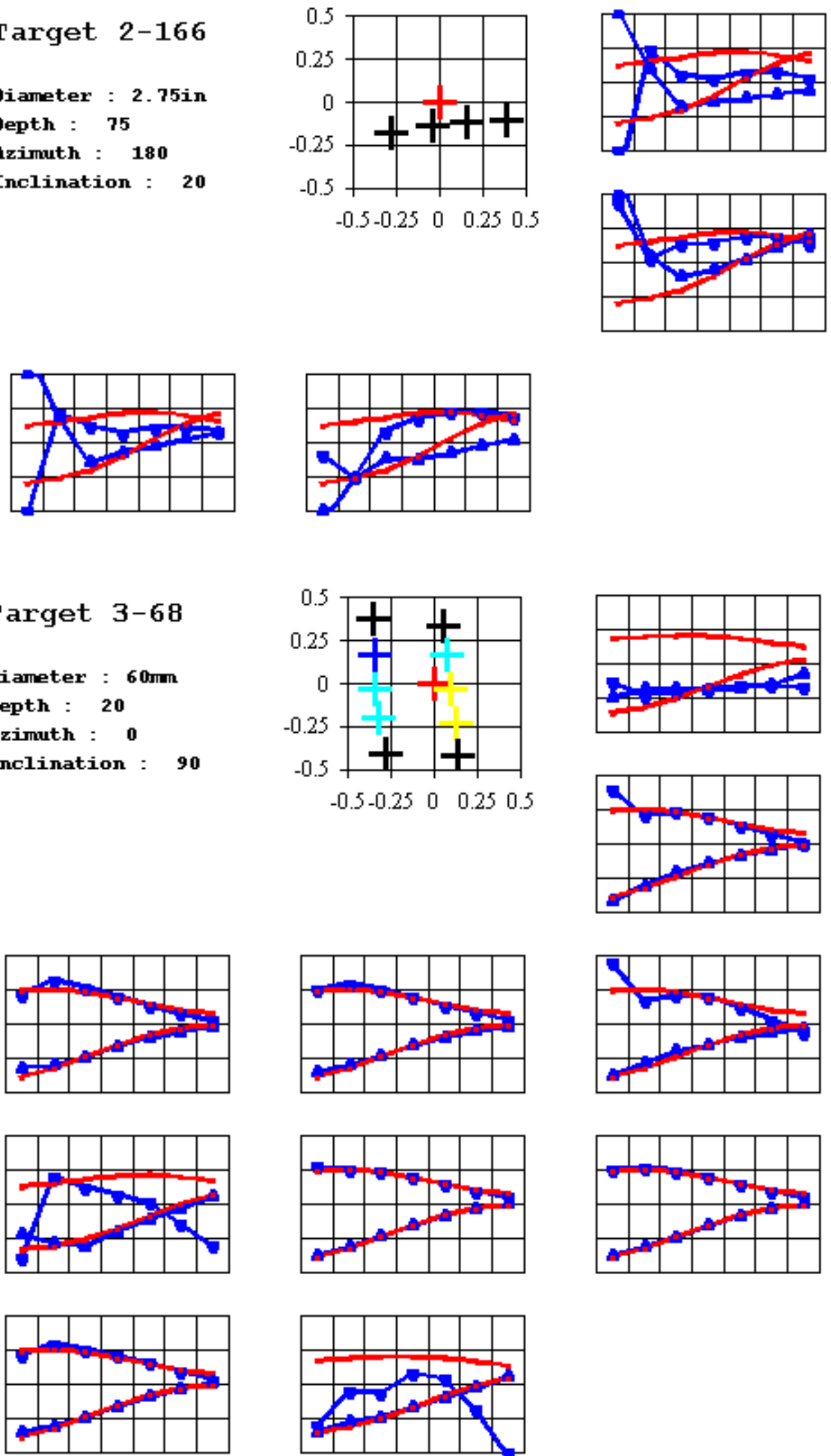

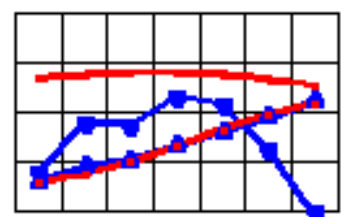


Target $3-70$

Diameter : $81 \mathrm{~mm}$

Depth : $\mathbf{2 5}$

Azimuth : 0

Inclination : 0
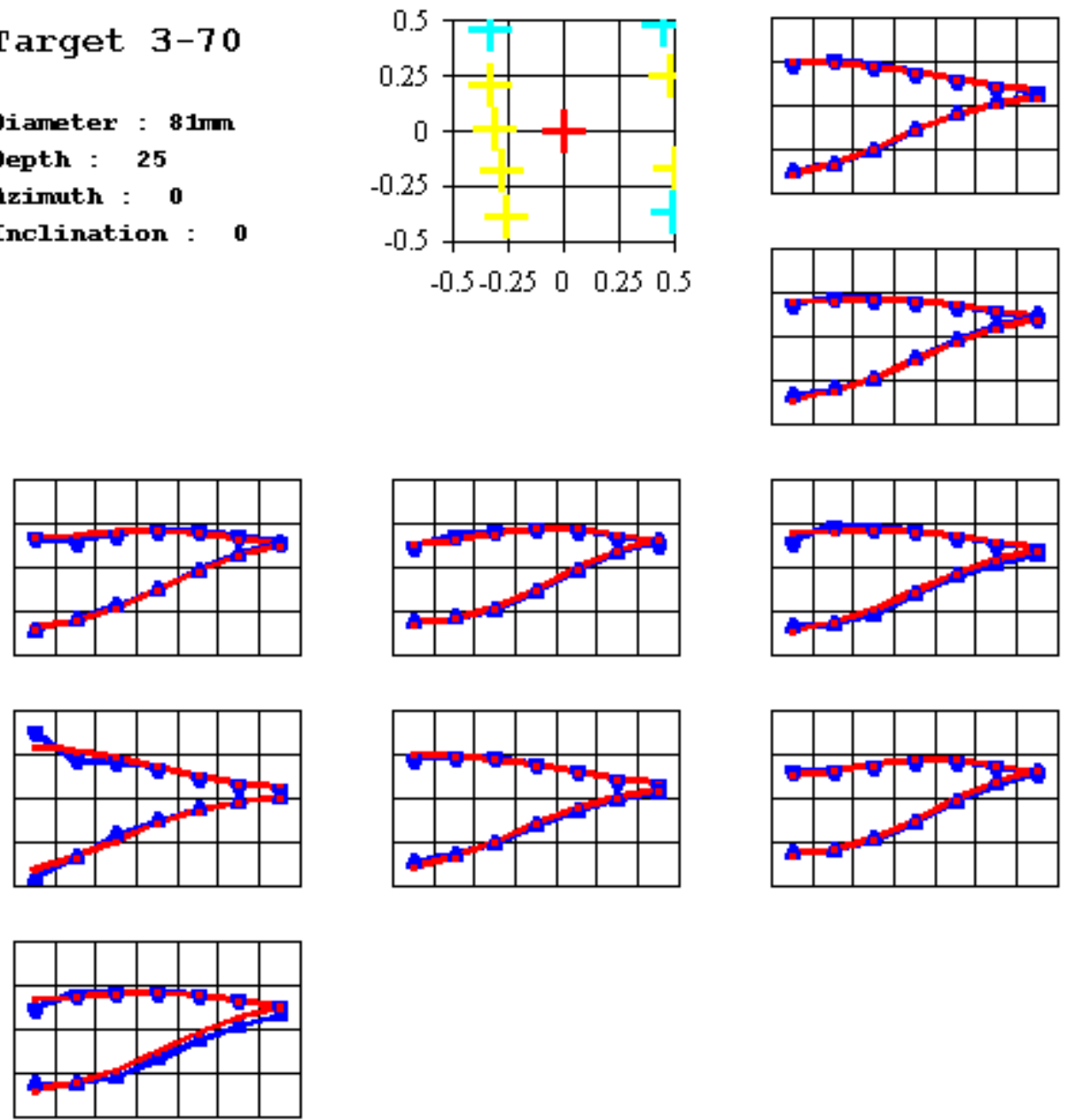
Target $3-72$

Diameter : $60 \mathrm{~mm}$

Depth : 25

Azimuth : 45

Inclination : $\mathbf{3 0}$
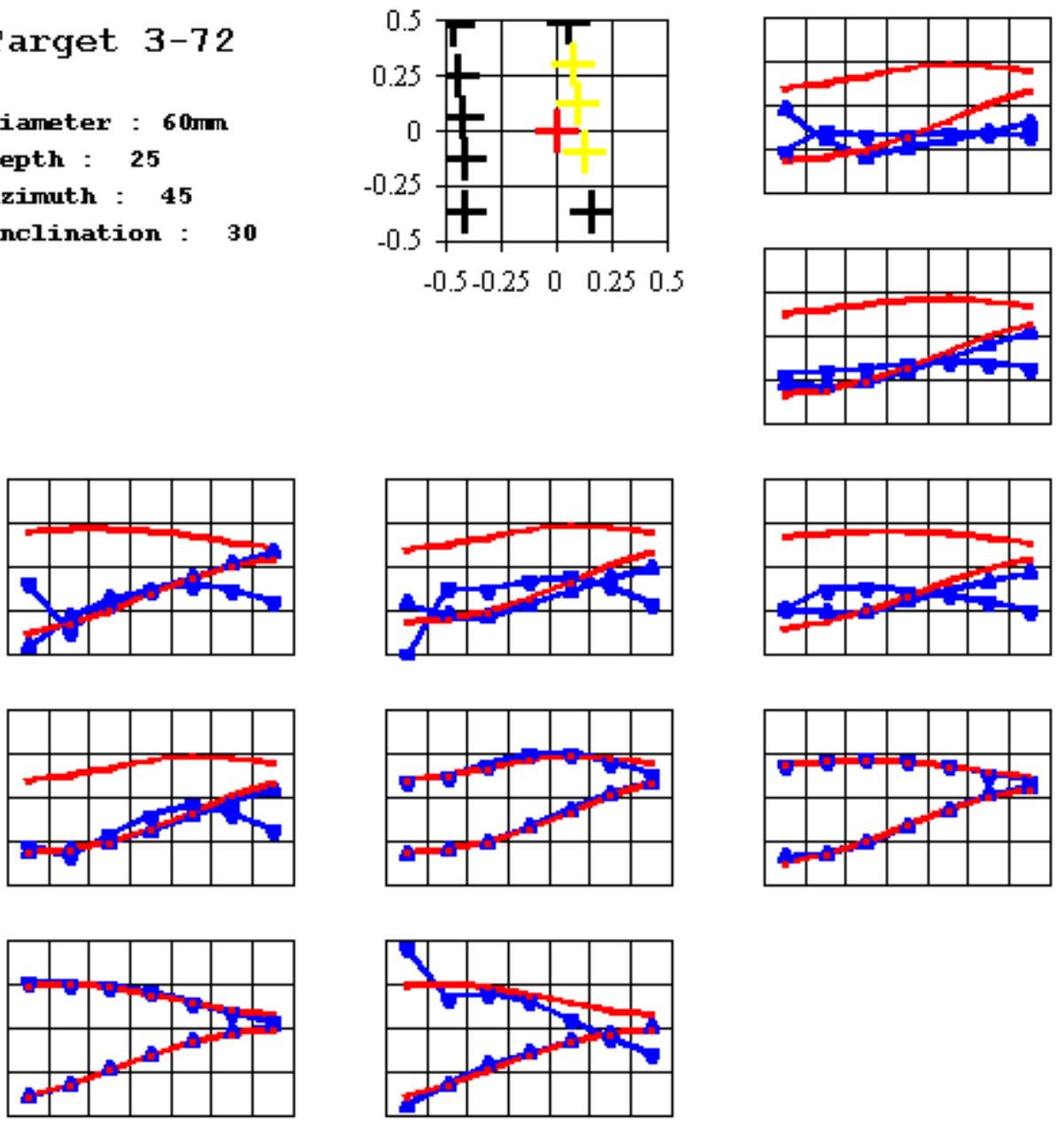
Target $3-74$

Diameter : $60 \mathrm{~mm}$

Depth : $\mathbf{3 0}$

Azimuth : $\mathbf{2 2 5}$

Inclination : 45
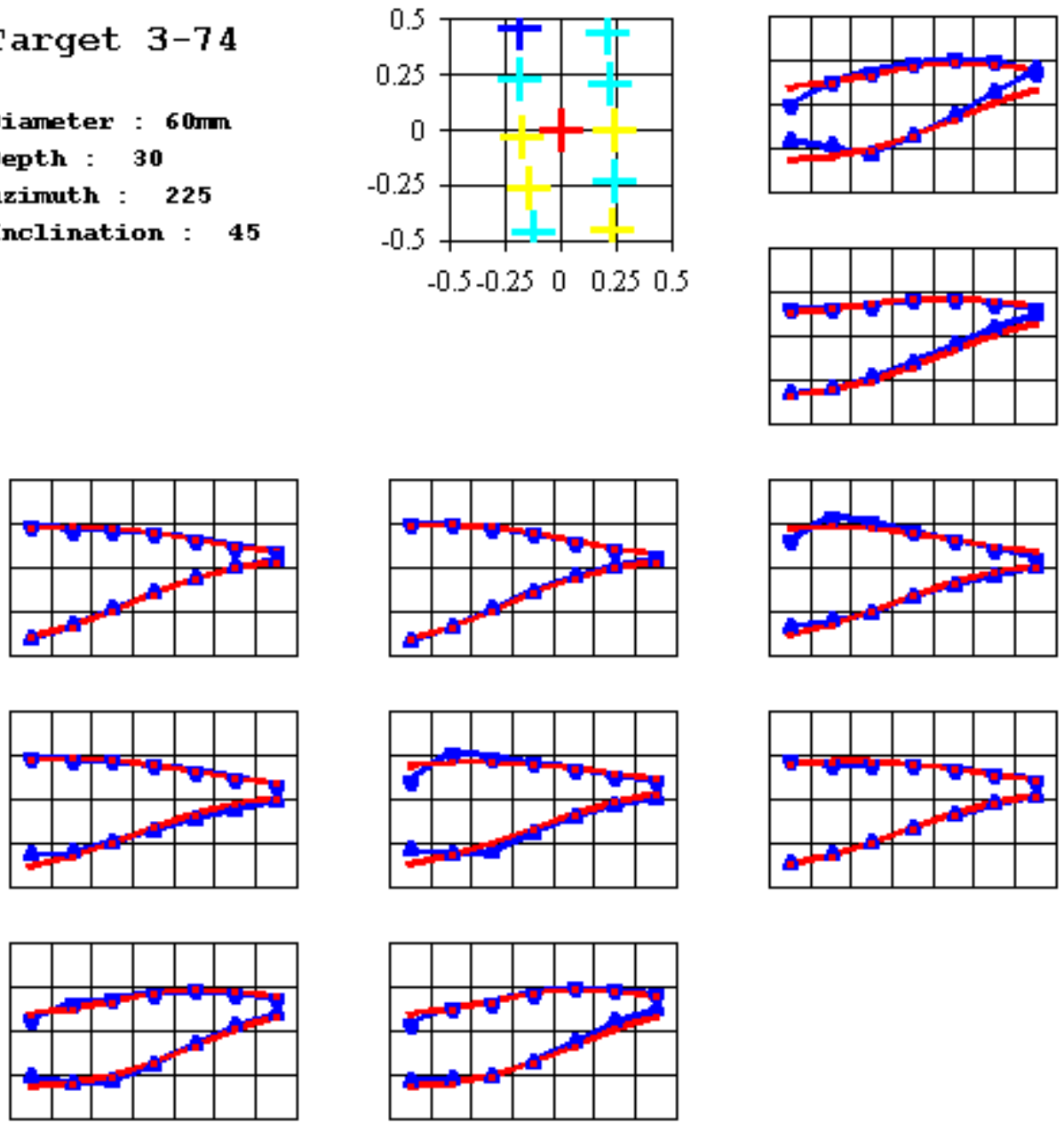

Target $3-76$

Diameter : $81 \mathrm{~mm}$

Depth : $\mathbf{2 0}$

fzimuth : 0

Inclination : $\mathbf{- 9 0}$
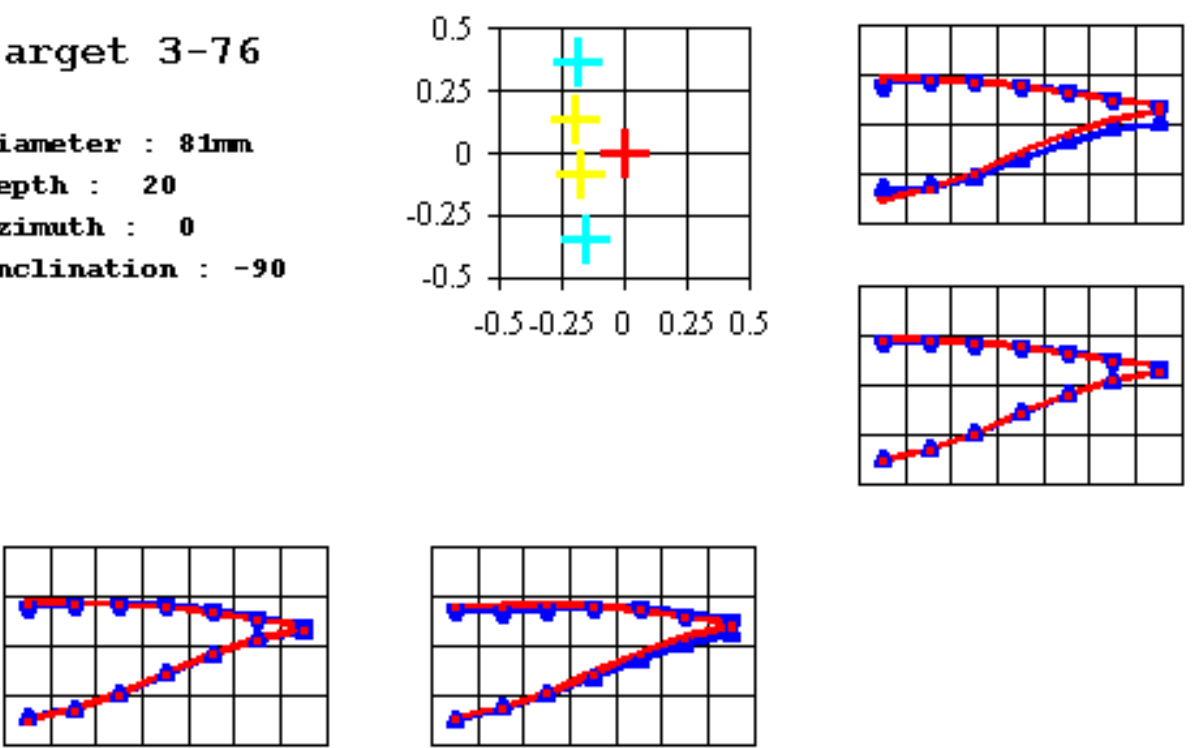
Target $3-78$

Diameter : $60 \mathrm{~mm}$

Depth : $\mathbf{3 5}$

fzimuth : $\mathbf{3 3 0}$

Inclination : 40
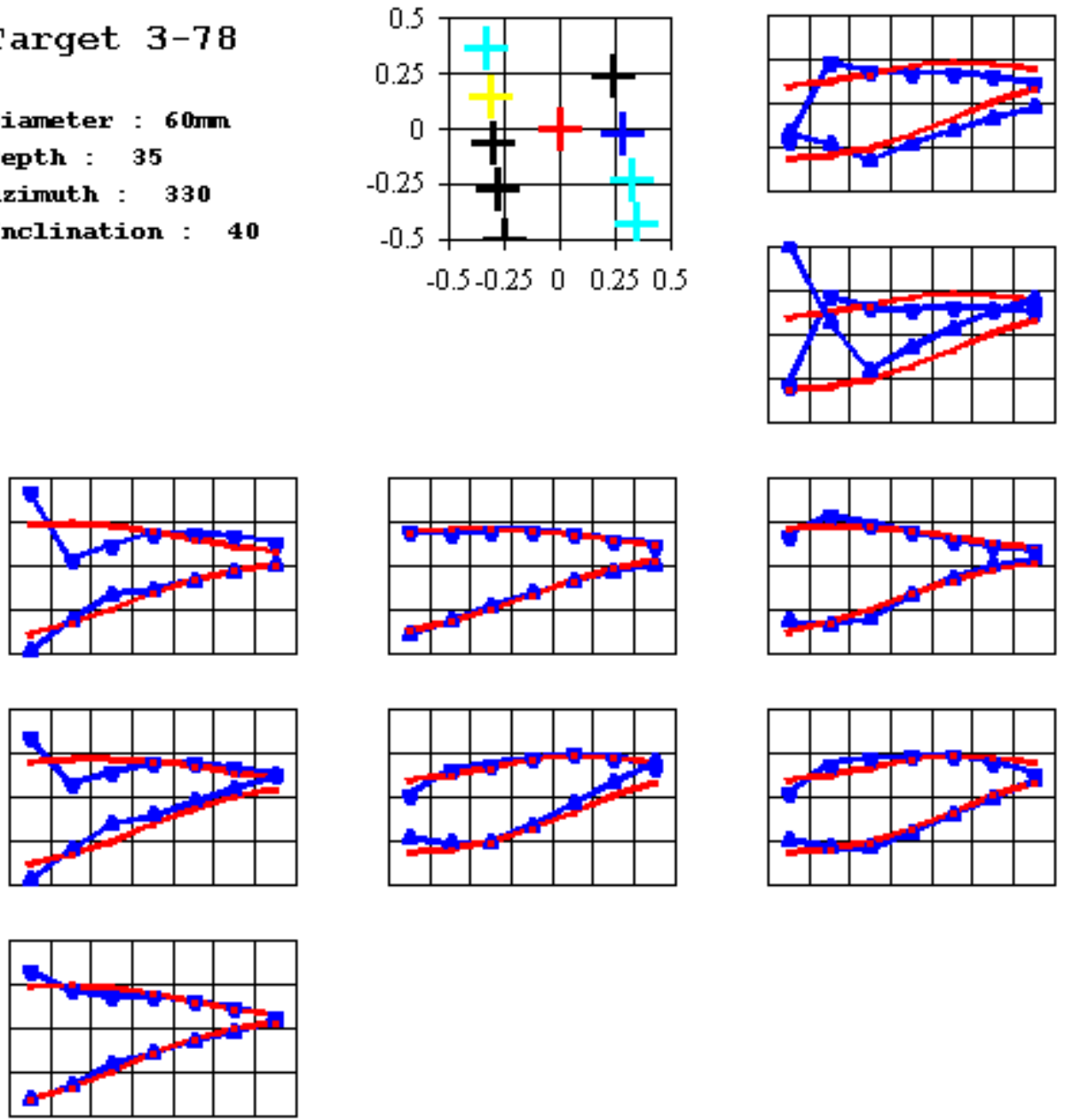
Target $3-80$

Diameter : $81 \mathrm{~mm}$

Depth : 25

Azimuth : 180

Inclination : 0
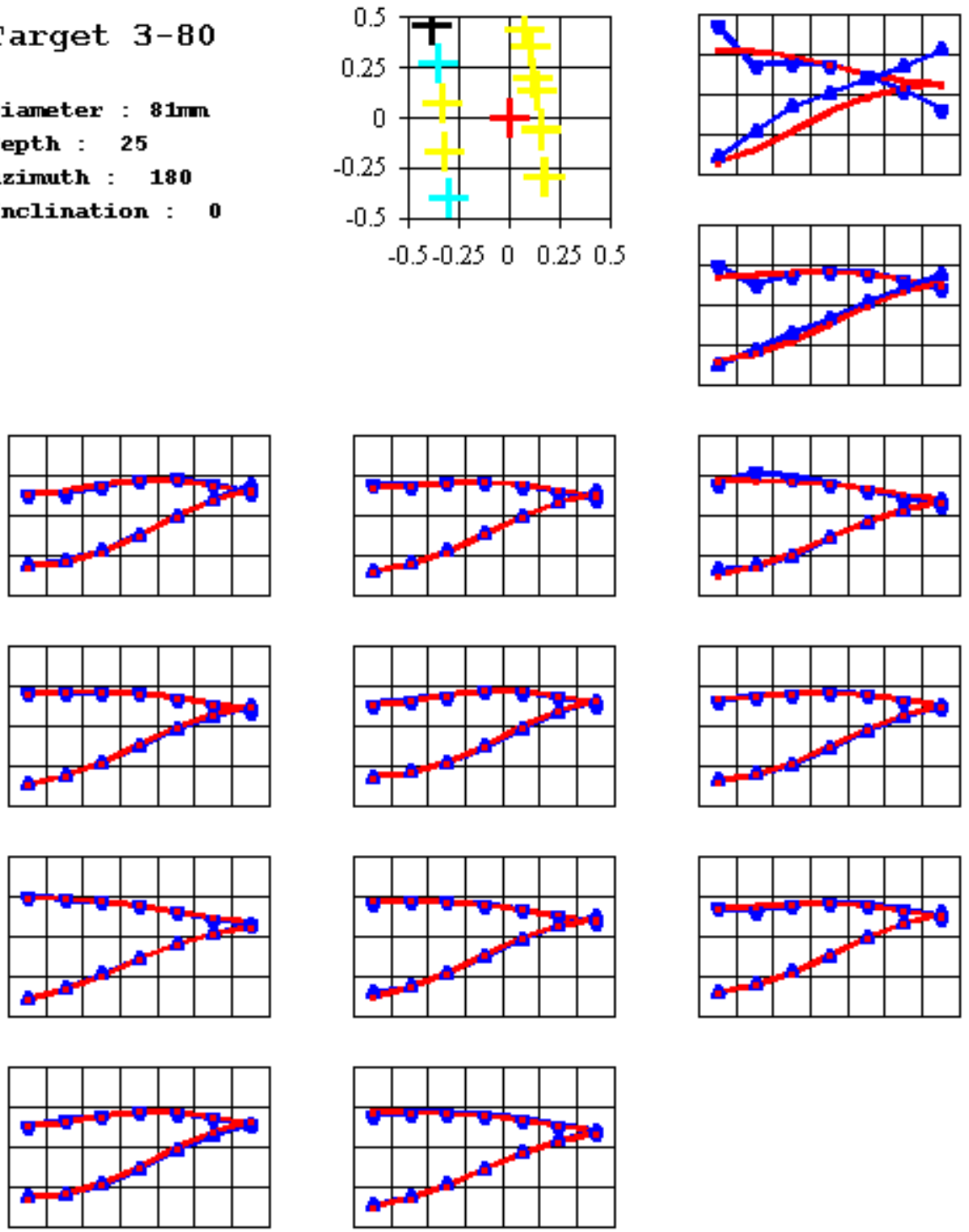
Target $3-82$

Diameter : 60mm

Depth : 20

Azimuth : 270

Inclination : 15
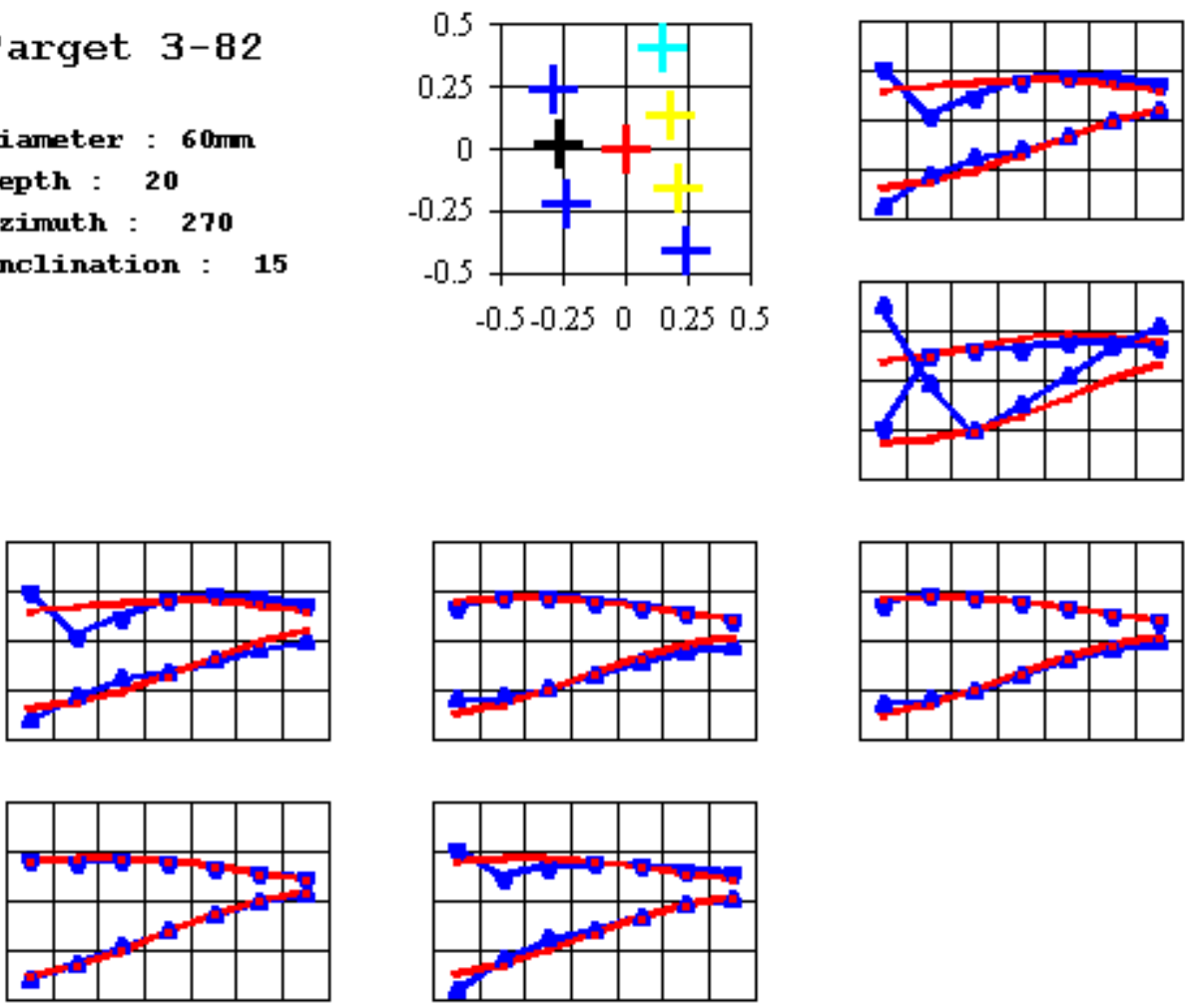
Target $\mathbf{3 - 8 4}$

Diameter : $81 \mathrm{~mm}$

Depth : 25

Azimuth : 0

Inclination : 0
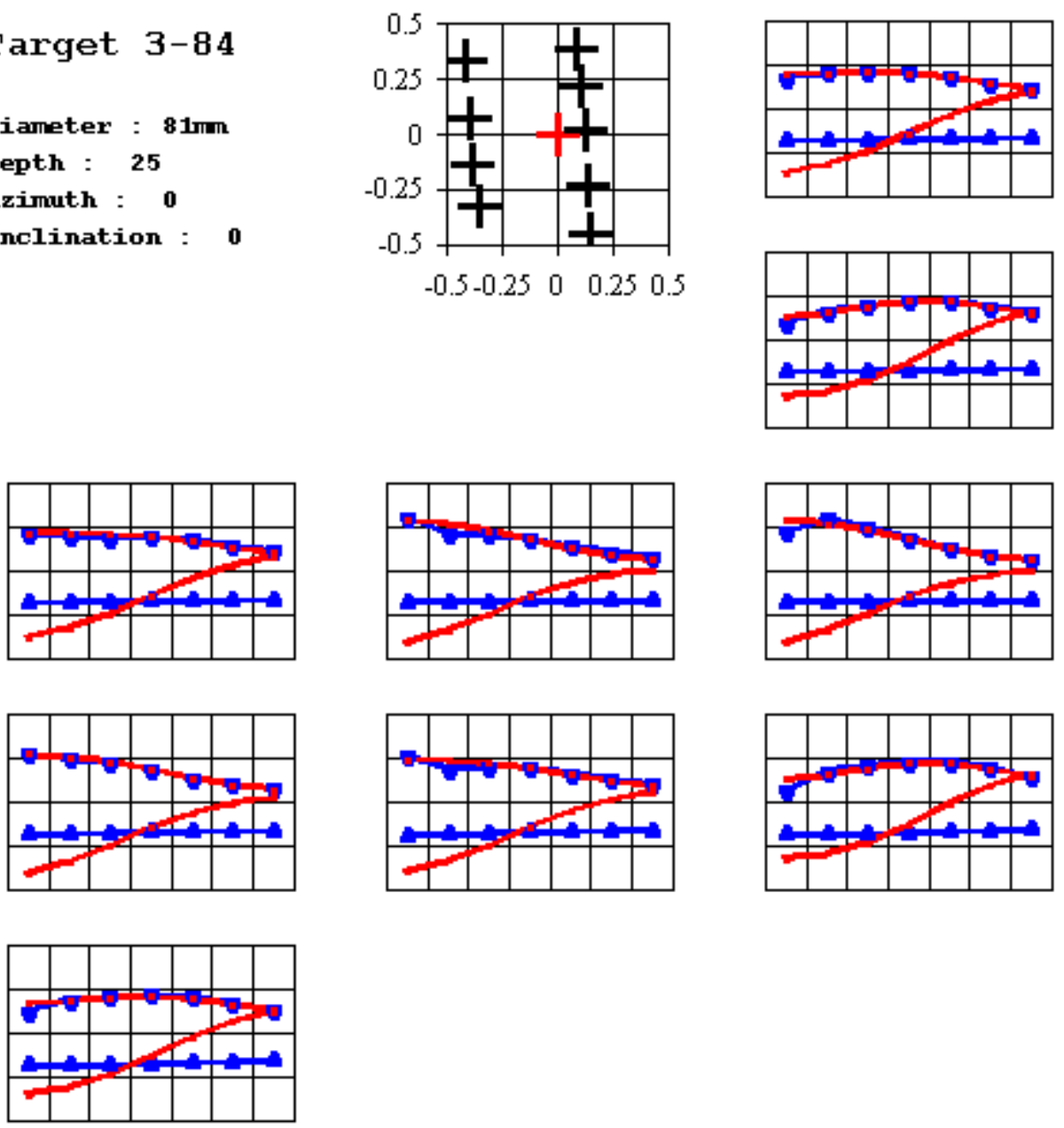
Target $\quad 3-86$

Diameter : $20 \mathrm{~mm}$

Depth : 1

Azimuth : 0

Inclination : 90
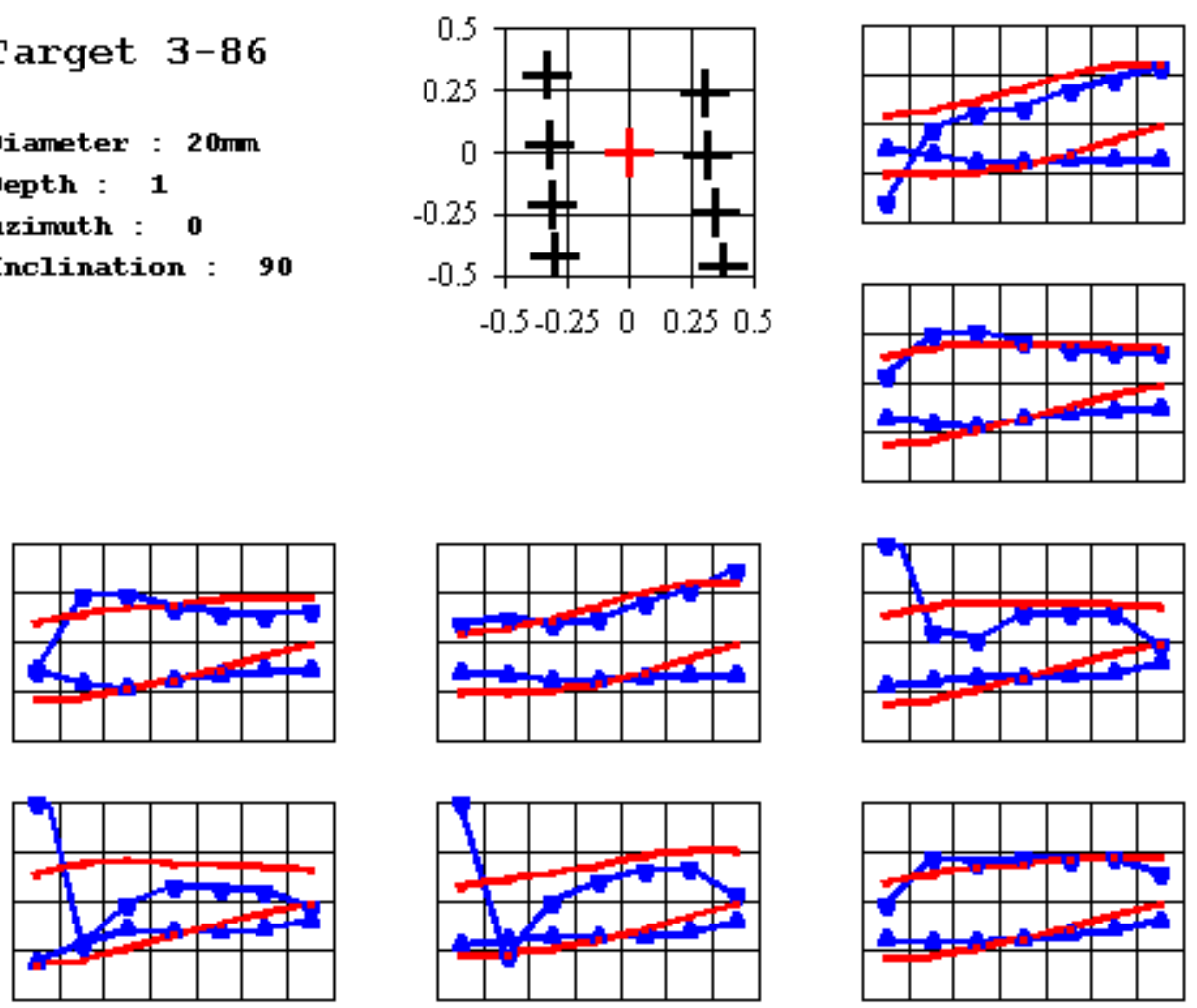

Target $\mathbf{3 - 8 8}$

Diameter : $20 \mathrm{~mm}$

Depth : 1

Azimuth : 0

Inclination : 0
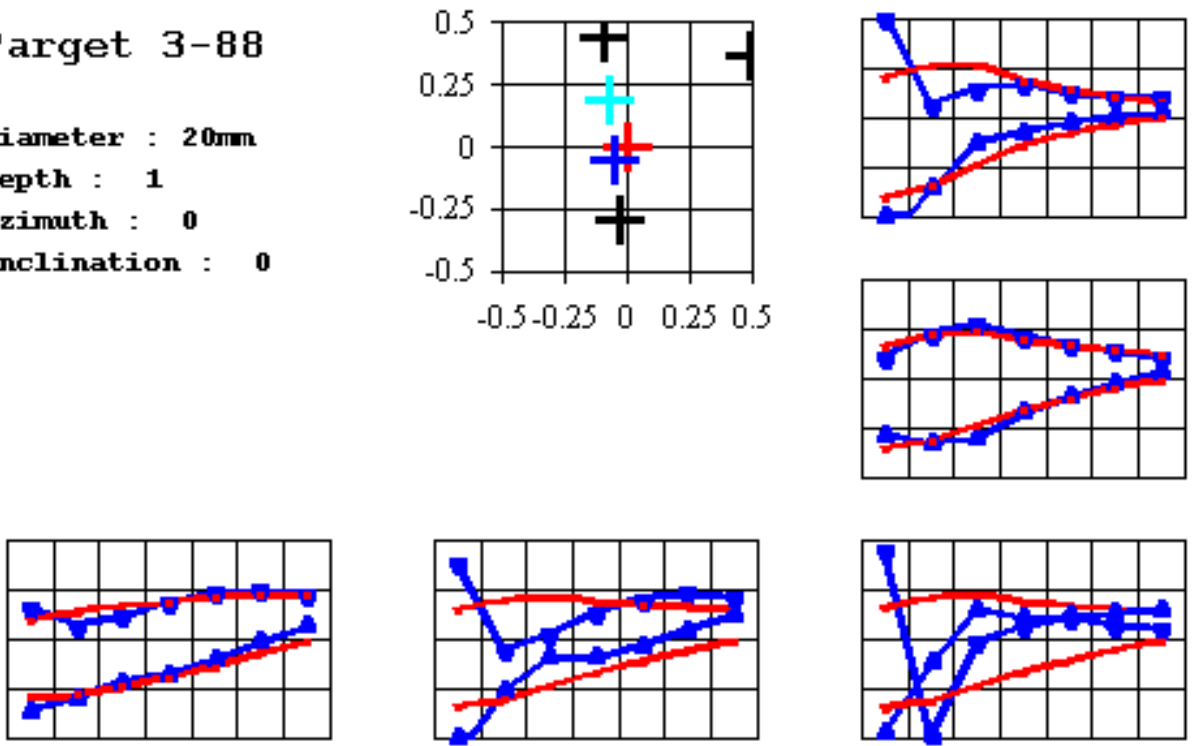
Target $3-90$

Diameter : $20 \mathrm{~mm}$

Depth : $\mathbf{1 5}$

Azimuth : 90

Inclination : 0
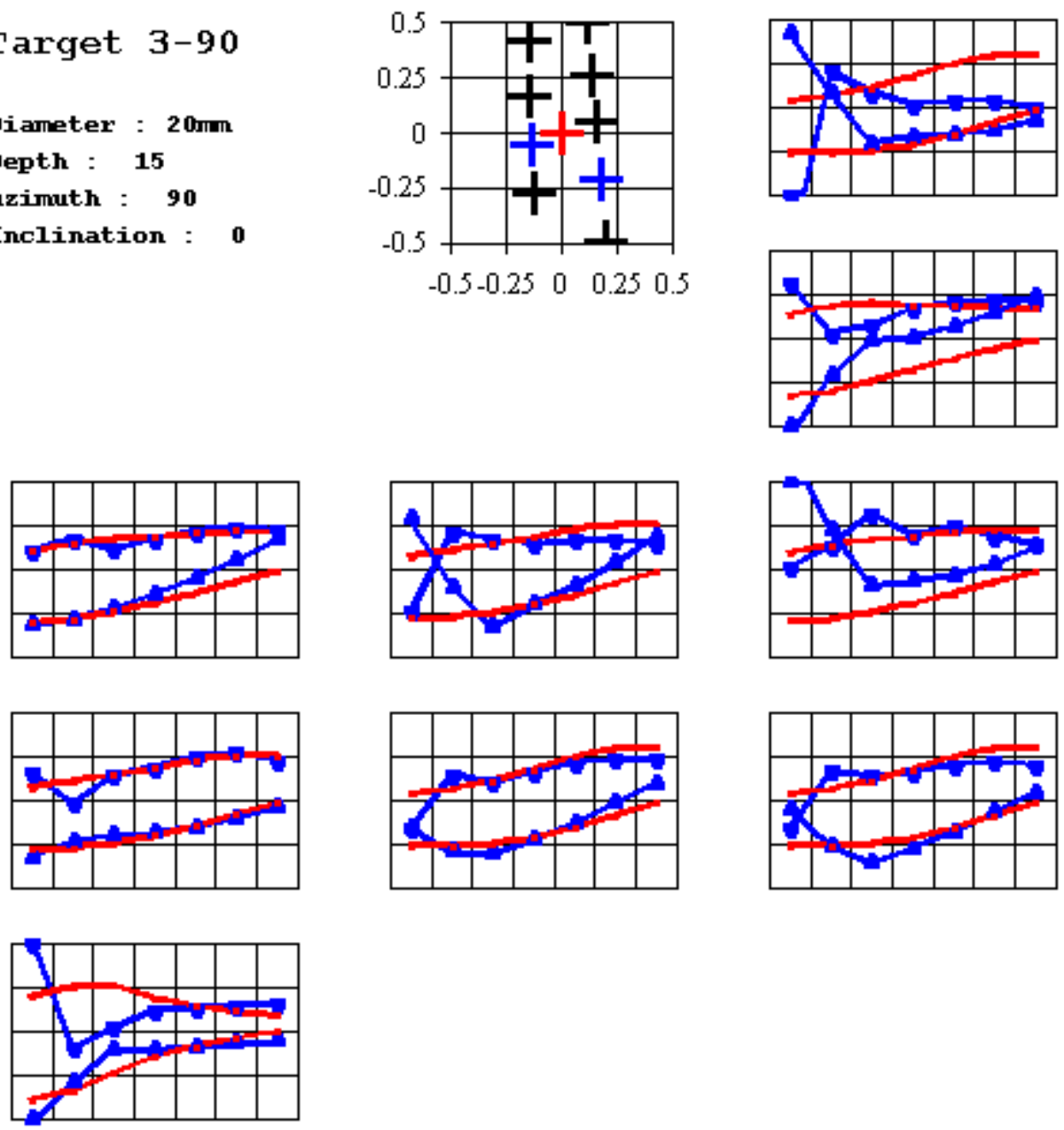
Target 3-92

Diameter : $20 \mathrm{~mm}$

Depth : 15

Azimuth : 120

Inclination : $\mathbf{3 0}$
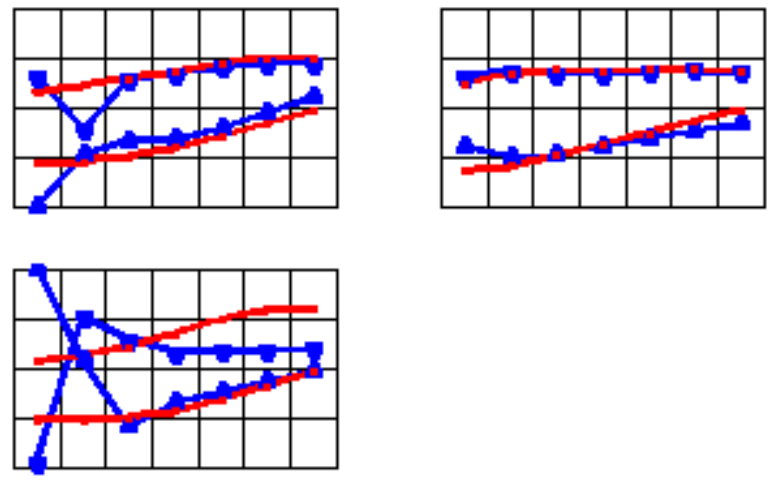

Target 3-94

Diameter : $57 \mathrm{~mm}$

Depth : 35

Azimuth : $\mathbf{3 3 0}$

Inclination : 0
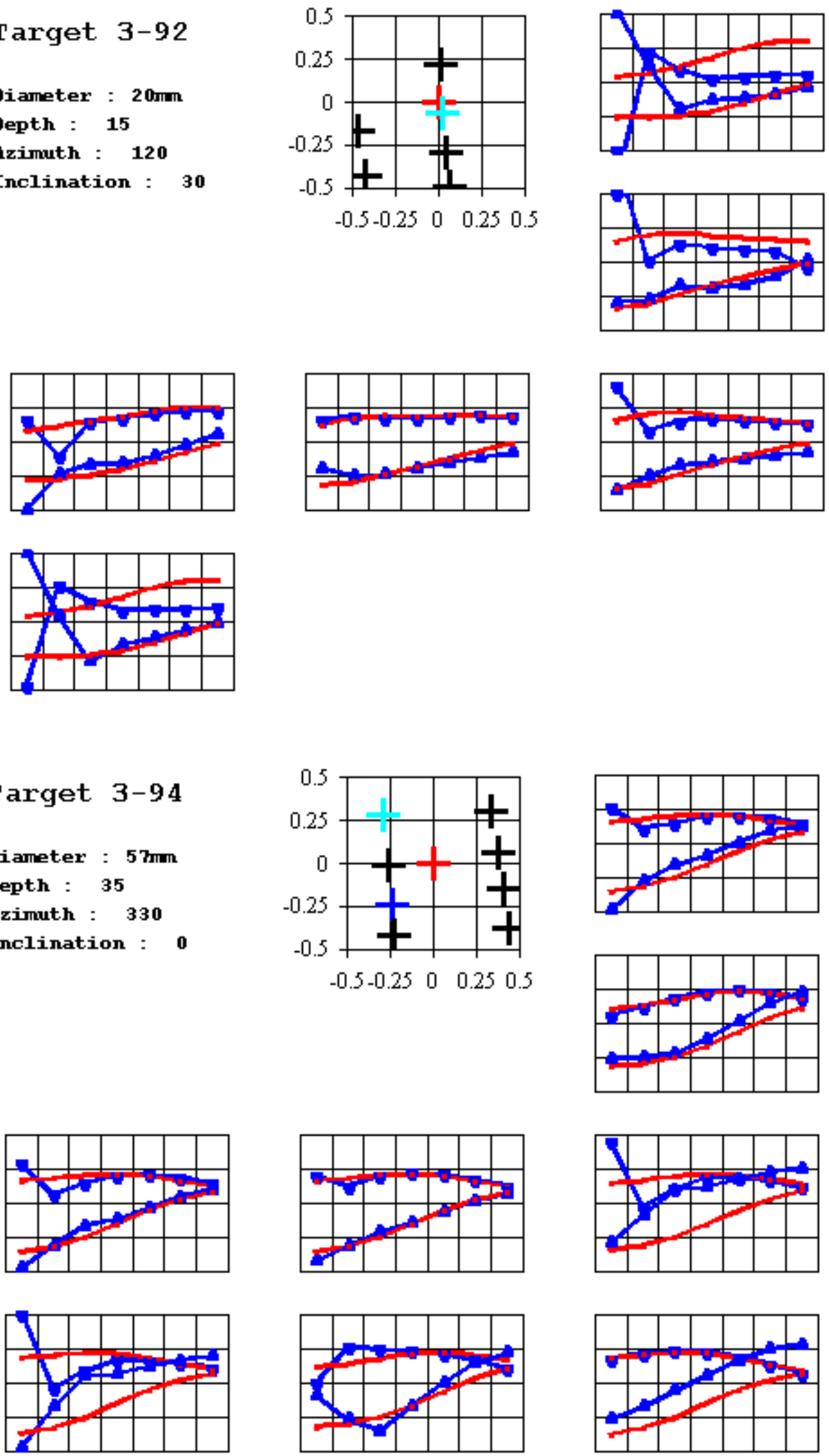
Target $3-98$

Diameter : $105 \mathrm{~mm}$

Depth : $\mathbf{5 0}$

Azimuth : 0

Inclination : -45
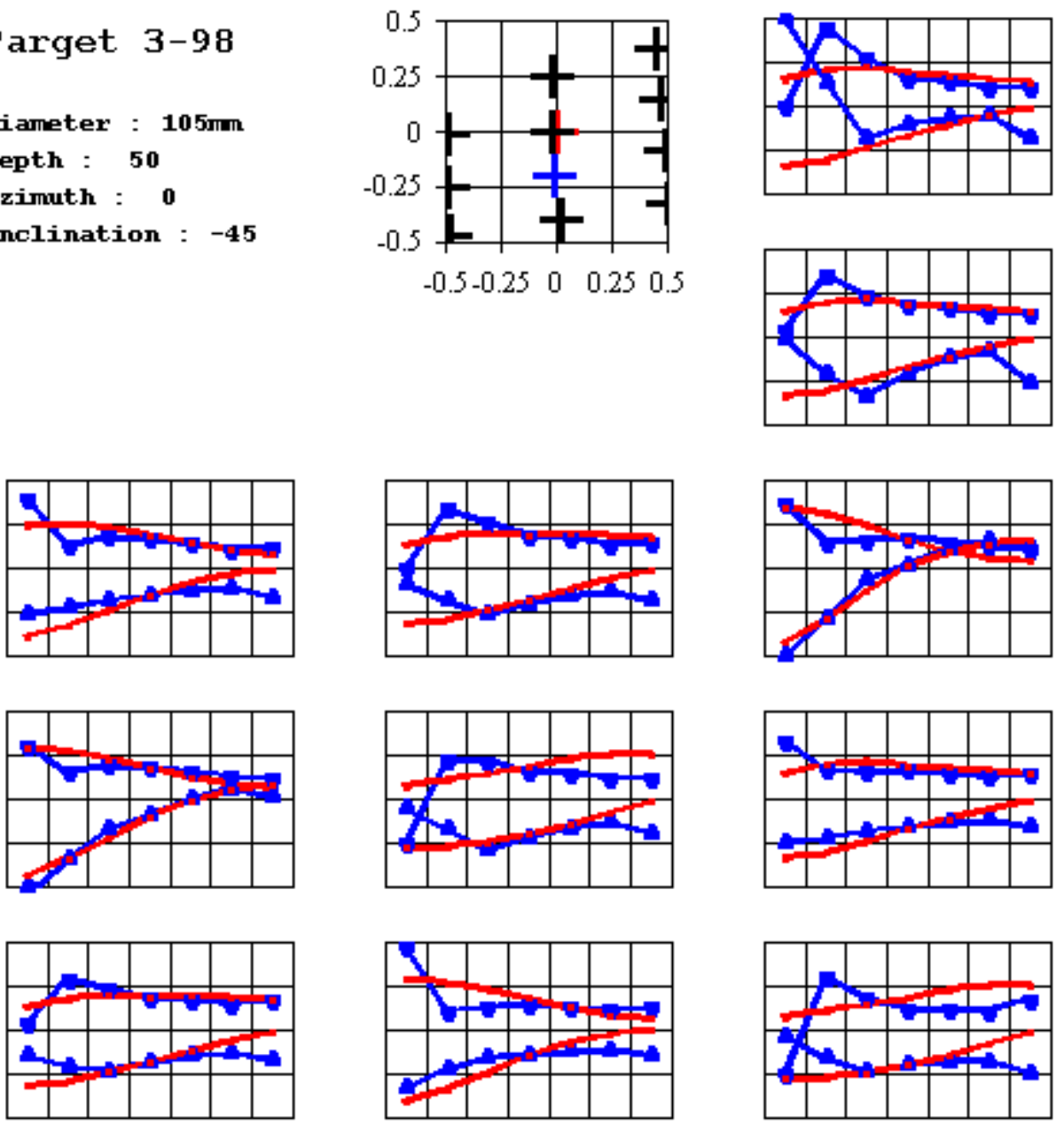

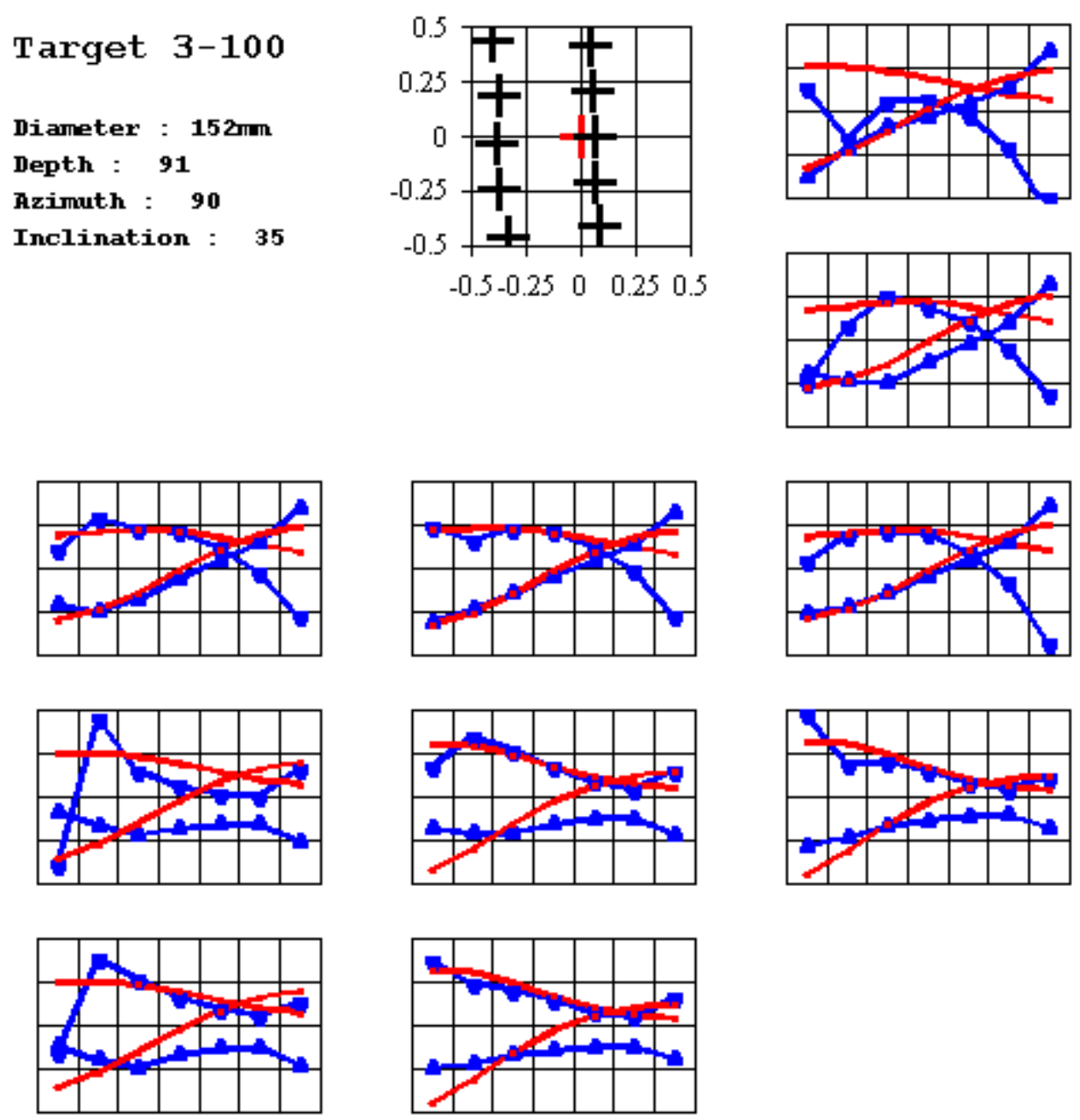
Target $3-102$

Diameter : $155 \mathrm{~mm}$

Depth : 120

Azimuth : $\mathbf{3 0}$

Inclination : $\mathbf{2 0}$
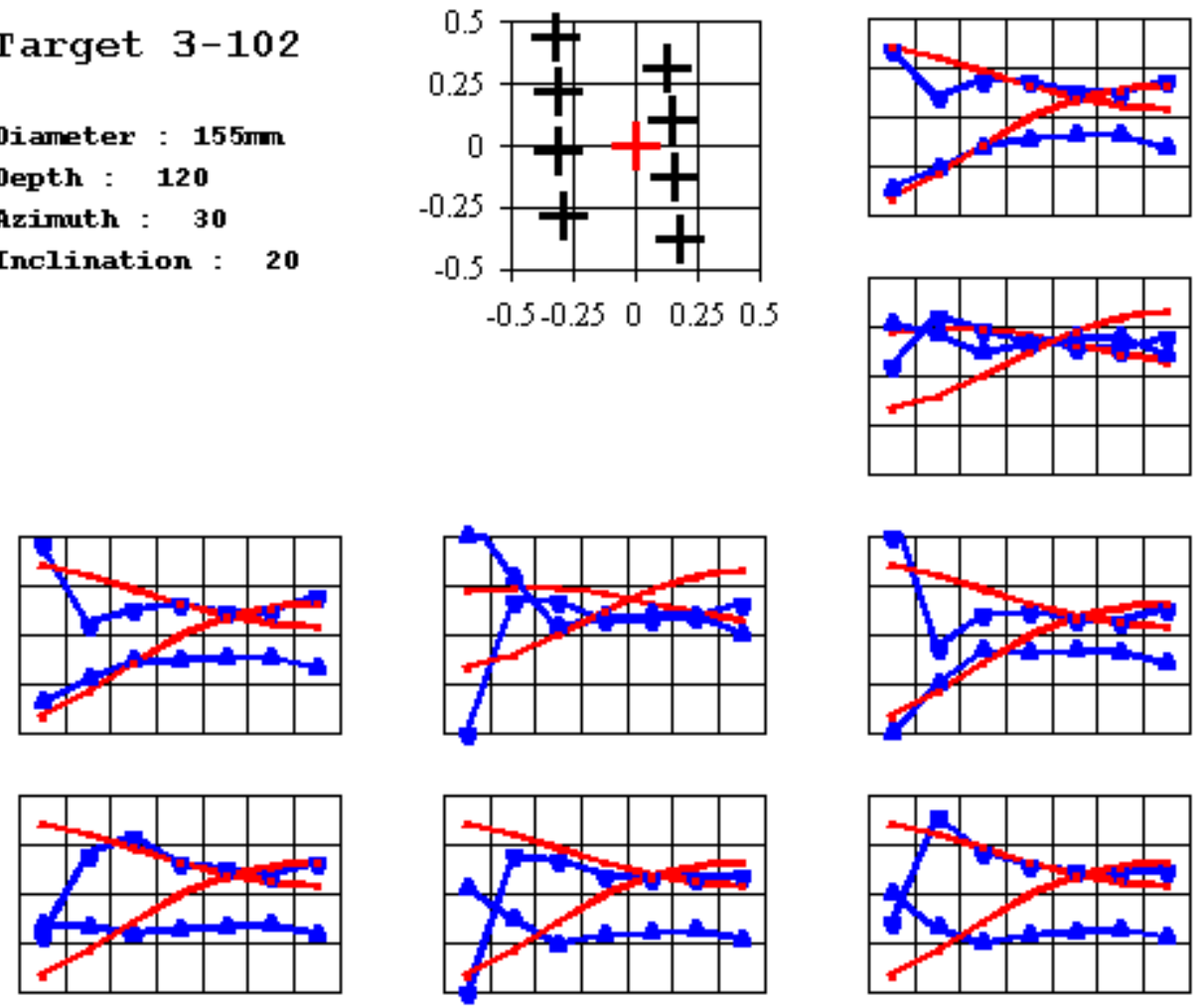

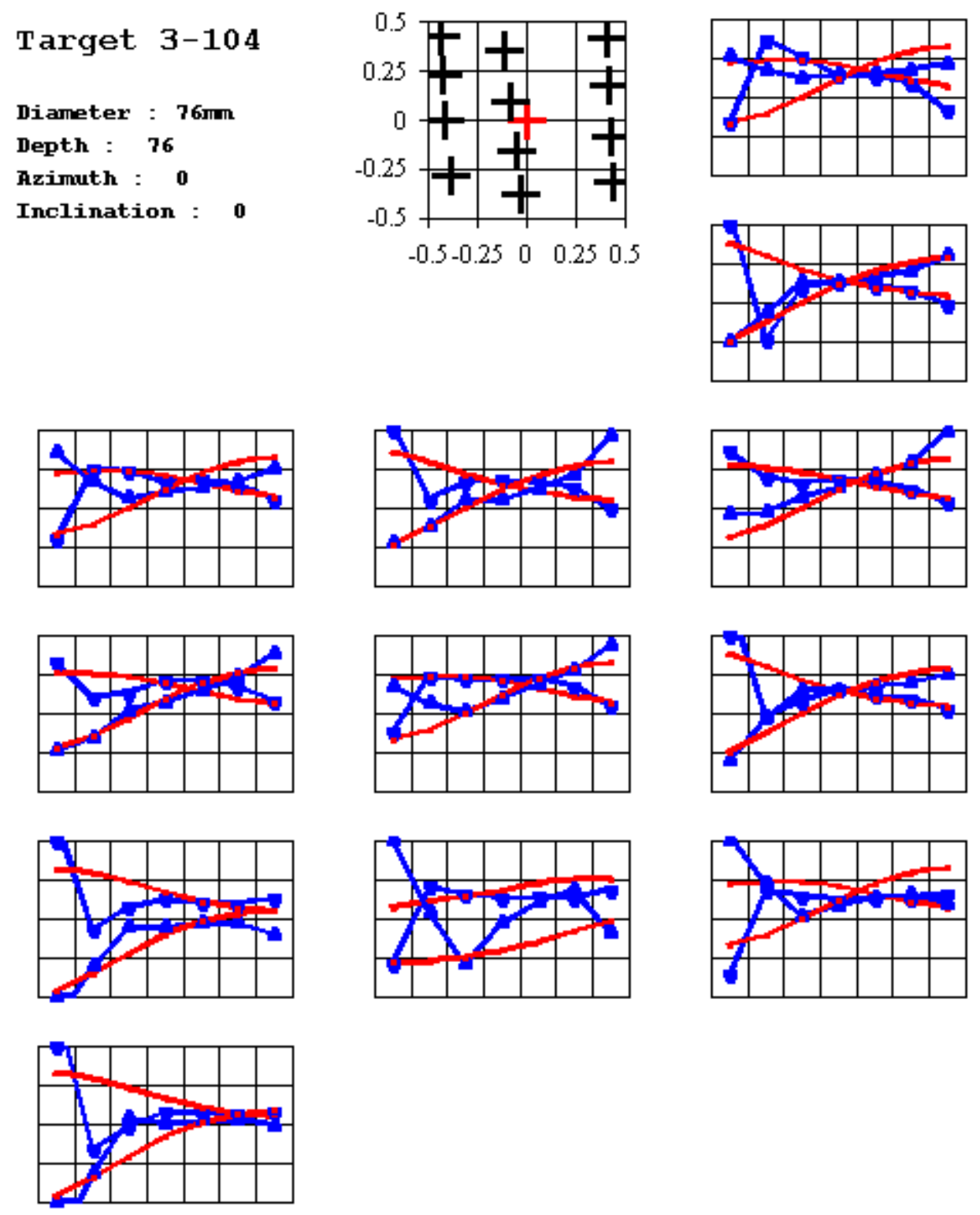
Target $3-106$

Diameter : 2.75in

Depth : 50

Azimuth : 120

Inclination : $\mathbf{3 0}$
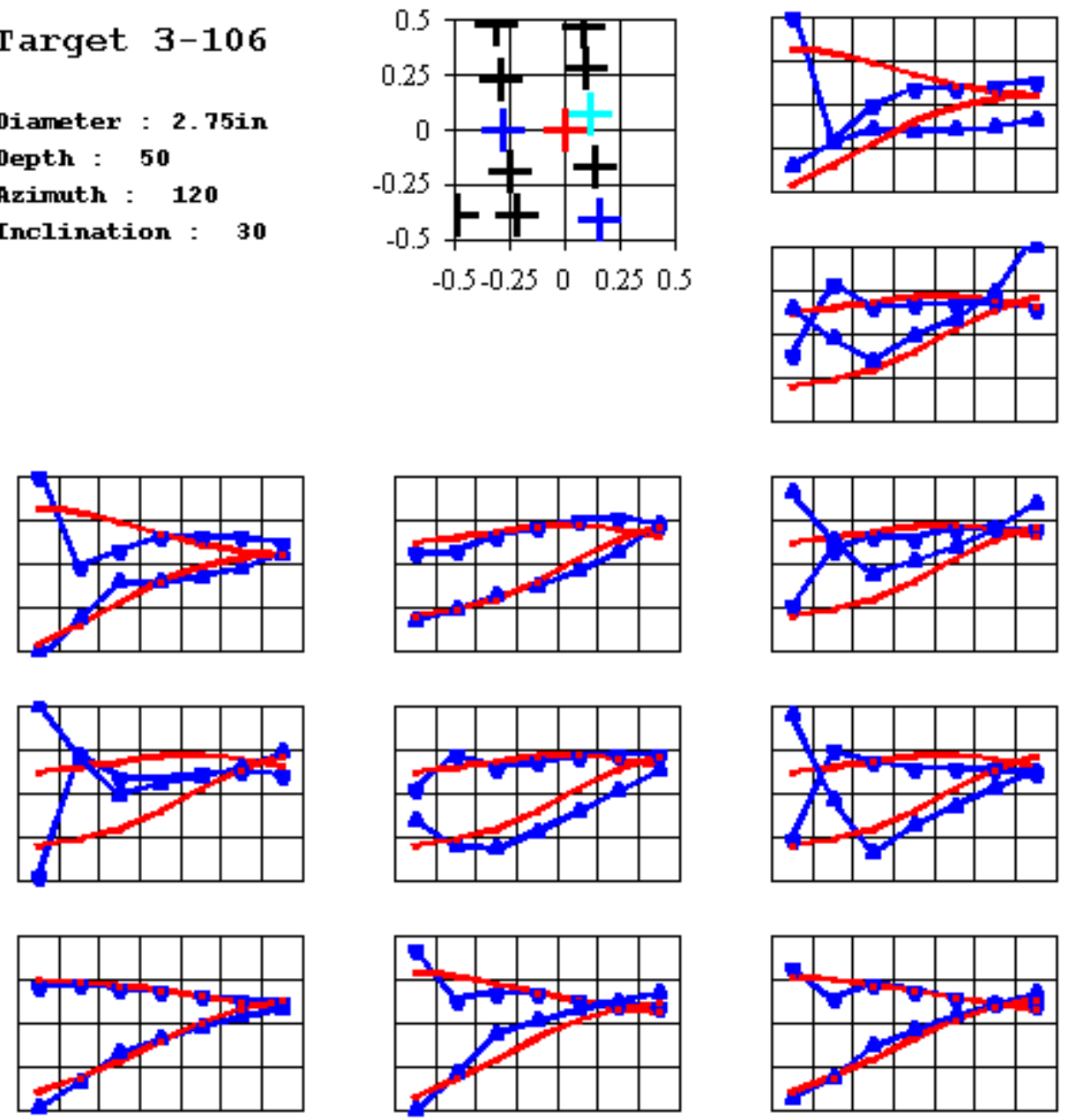


\section{Appendix C Code Listings}

\section{Gem-3 Analysis Program}

\section{Main.frm}

Private Sub Check1_Click(Index As Integer)

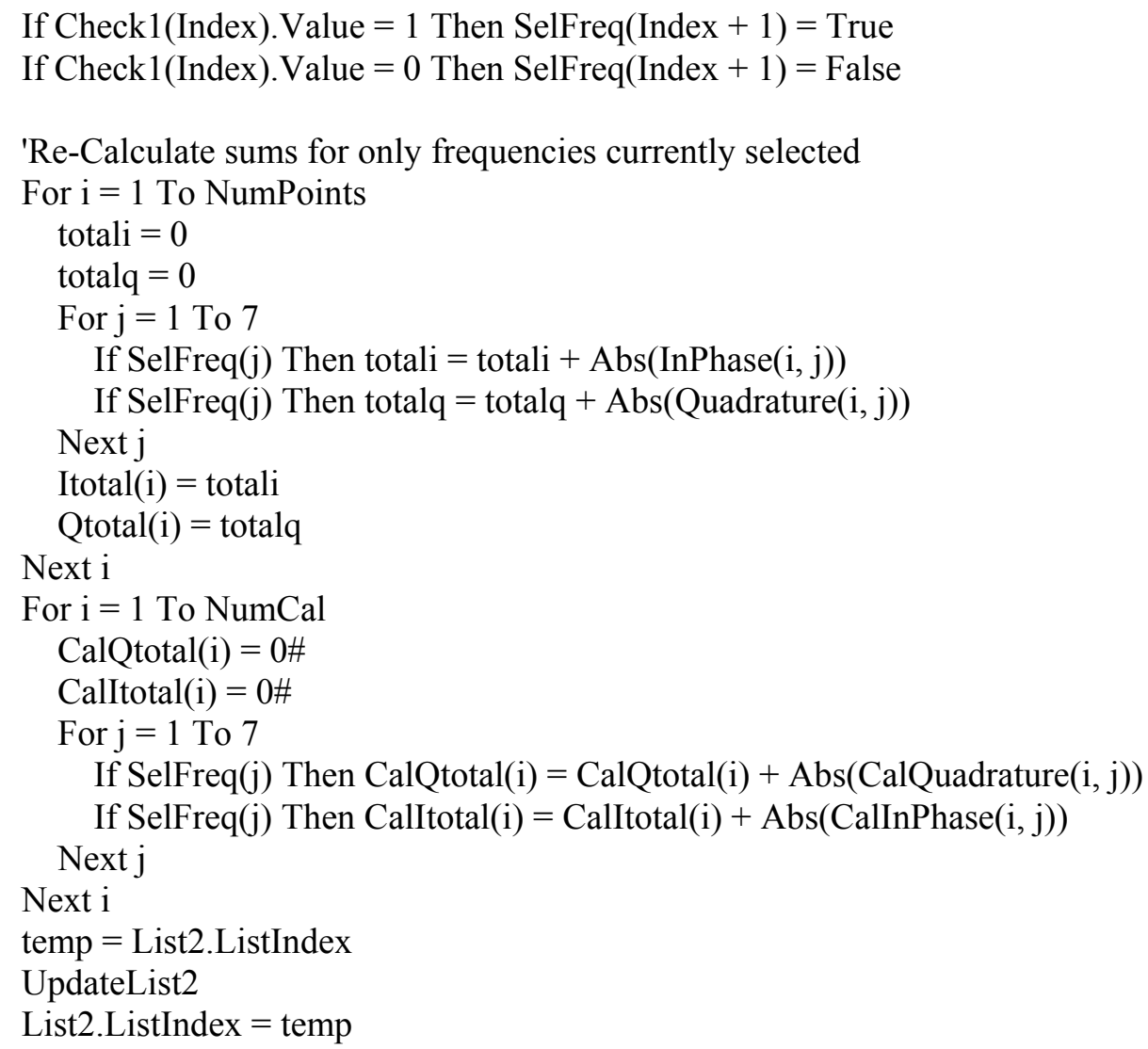


List2_Click

End Sub

Private Sub Check2_Click()

ShowBest $=$ Not ShowBest

temp $=$ List 2. ListIndex

UpdateList2

List2.ListIndex $=$ temp

List2_Click

End Sub

Private Sub Classify_Click()

Open "matches.txt" For Output As \#2

Threshold $=$ Thresh_text.Text

For $\mathrm{j}=1$ To 12

For $\mathrm{k}=1$ To 12

Confusion $(\mathrm{j}, \mathrm{k})=0$

Next k

Next j

For $m=1$ To NumItems

$\operatorname{Max}=0$

ItemMinDist $(m)=999999 \#$

For $\mathrm{j}=\operatorname{ItemStart}(\mathrm{m})$ To ItemEnd(m)

If $($ Qtotal $(j)+\operatorname{Itotal}(j)>$ Max $)$ Then

$\operatorname{Max}=\operatorname{Qtotal}(\mathrm{j})+\operatorname{Itotal}(\mathrm{j})$

$\mathrm{i}=\mathrm{j}$

End If

If $(\operatorname{Tdist}(\mathrm{j})<\operatorname{ItemMinDist}(\mathrm{m}))$ Then $\operatorname{ItemMinDist}(\mathrm{m})=\operatorname{Tdist}(\mathrm{j})$

Next j

FindCombinedMatches (i)

Print \#2, Format(TargetId(i), "@@@@@ ), Format(Qtotal(i)+ Itotal(i), "00000 ") +

Format(Tdist(i), "0.000")

For $\mathrm{j}=1$ To 8

Print \#2, CmatchUxo(j) \& Space(25 - Len(CmatchUxo(j))) \&

Format(CmatchError(j), "0.0000 ")

Next j

found $=0$

For $\mathrm{k}=1$ To TgtNum

If TargetId(i) $=\operatorname{TgtId}(\mathrm{k})$ Then

For $\mathrm{j}=1$ To 11

If TgtType $(k)=\operatorname{UXO}(j)$ Then Row $=\mathrm{j}$

If $\operatorname{InStr}($ CmatchUxo(1), UXO(j)) $>0$ Then 


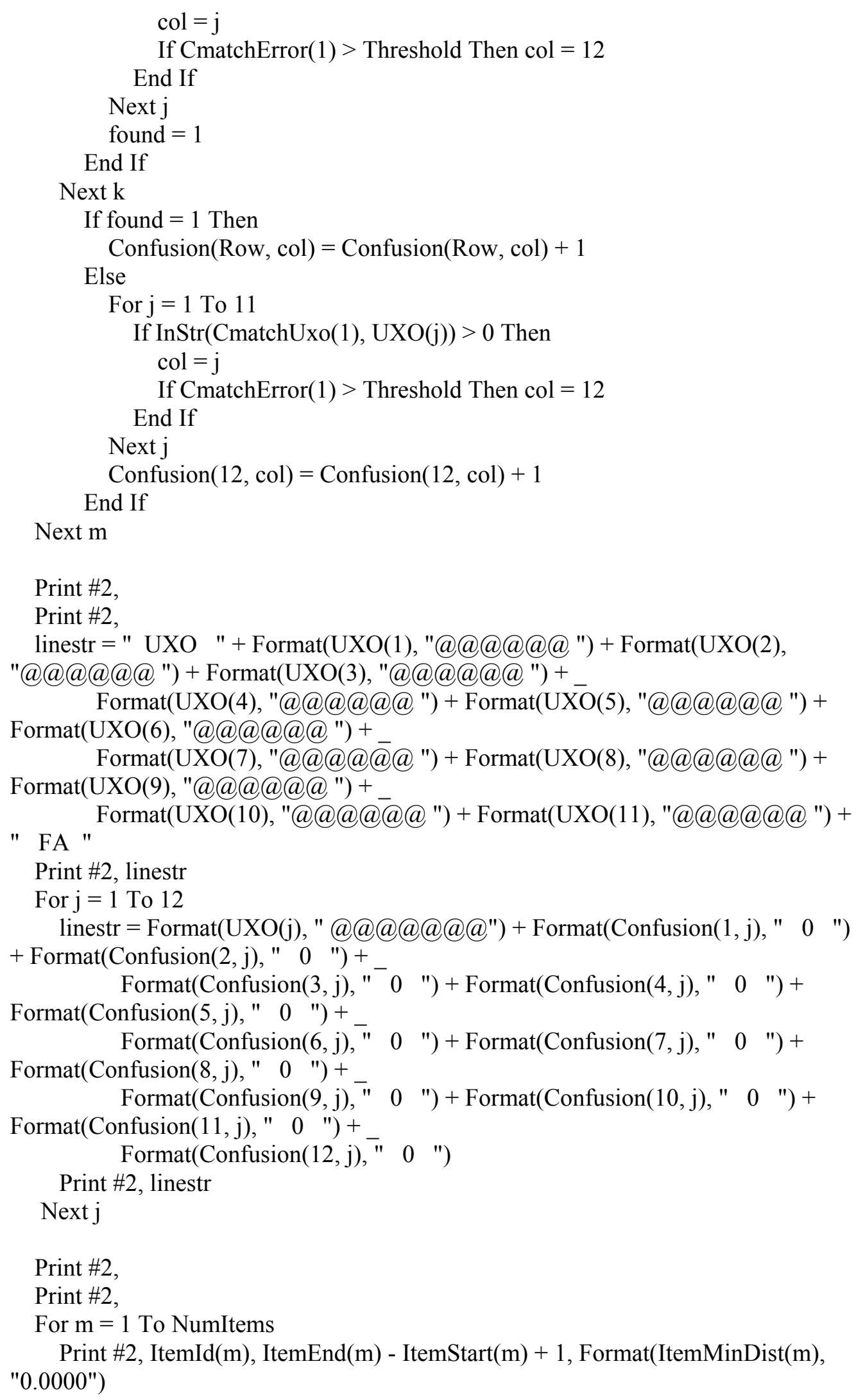


Next $m$

Close \#2

ans = MsgBox("Done", vbOKOnly)

End Sub

Private Sub List3_Click()

$\mathrm{j}=$ List3.ListIndex +1

If $\mathrm{j}<=0$ Then Exit Sub

For $\mathrm{i}=1$ To NumItems

If TargetId(ItemStart(i)) $=$ TgtId(j) Then Exit For

Next $i$

If $\mathrm{i}<=$ NumItems Then

$\mathrm{k}=\mathrm{i}$

List1.ListIndex $=\mathrm{k}-1$

Else

ans $=$ MsgBox("Target not found", vbOKOnly)

Exit Sub

End If

List1_Click

End Sub

Private Sub MatchAll_old_Click()

Open "allmatches.txt" For Output As \#2

For $\mathrm{i}=1$ To NumPoints

FindCombinedMatches (i)

Print \#2, Format(TargetId(i), "@@@@@ ), Format(Qtotal(i)+ Itotal(i), "00000 ") +

Format(Tdist(i), "0.000")

For $\mathrm{j}=1$ To 8

Print \#2, CmatchUxo(j) \& Space(25 - Len(CmatchUxo(j))) \&

Format(CmatchError(j), "0.0000 ")

Next j

Next i

Close \#2

ans = MsgBox("Done", vbOKOnly)

End Sub

Private Sub List1_Click()

Dim info As String 


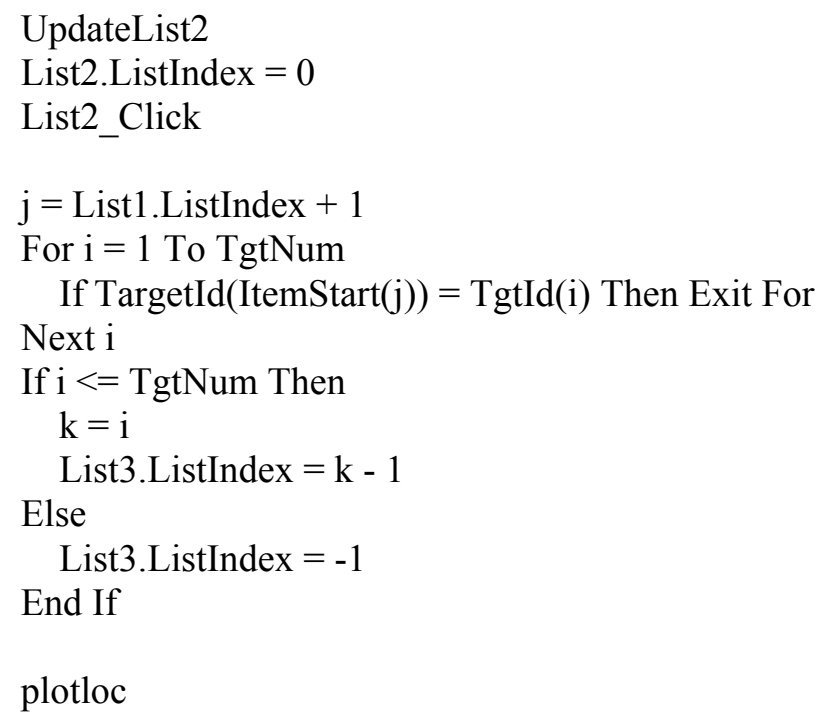

End Sub

Private Sub List2_Click()

NextPlot $=$ ItemStart $($ NextItem $)+$ List2.ListIndex plotloc

plotiq

End Sub

Private Sub UpdateList2()

List2.Clear

$\mathrm{k}=$ List1.ListIndex +1

NextItem $=\mathrm{k}$

For $\mathrm{i}=\operatorname{ItemStart}(\mathrm{k})$ To ItemEnd(k)

FindCombinedMatches (i)

BestMatchUxo(i) $=$ CmatchUxo(1)

BestMatchError(i) = CmatchError(1)

info=Format(TargetId(i), "@@@@@ )+Format(Qtotal(i)+ Itotal(i), "00000 ") +

Format(Tdist(i), "0.000 ")

For $\mathrm{j}=1$ To 8

info $=$ info + CmatchUxo(j) \& Space $(19-\operatorname{Len}($ CmatchUxo(j) $)) \&$

Format(CmatchError(j), "0.0000 ")

If (Not ShowBest) And List3.ListIndex $>=0$ Then

If $\operatorname{InStr}($ CmatchUxo(j), TgtType(List3.ListIndex +1$))>0$ Then

BestMatchUxo(i) $=$ CmatchUxo(j)

BestMatchError(i) $=$ CmatchError(j)

End If

End If

Next j 


\section{List2.AddItem info Next i}

End Sub

Private Sub Exit_Click()

Unload Me

End Sub

Private Sub Form_Load()

Dim linestr As String

$$
\begin{aligned}
& \mathrm{UXO}(1)=" 20 \mathrm{~mm} " \\
& \mathrm{UXO}(2)=" 57 \mathrm{~mm} " \\
& \mathrm{UXO}(3)=" 60 \mathrm{~mm} " \\
& \mathrm{UXO}(4)=" 2.75 \mathrm{in} " \\
& \mathrm{UXO}(5)=" 76 \mathrm{~mm} " \\
& \mathrm{UXO}(6)=" 81 \mathrm{~mm} " \\
& \mathrm{UXO}(7)=" 105 \mathrm{~mm} " \\
& \mathrm{UXO}(8)=\text { "4.2in" } \\
& \mathrm{UXO}(9)=\text { "5.0in" } \\
& \operatorname{UXO}(10)=" 152 \mathrm{~mm} " \\
& \mathrm{UXO}(11)=" 155 \mathrm{~mm} " \\
& \mathrm{UXO}(12)=\text { "Non-Ord" } \\
& \text { NextPlot }=0 \\
& \text { NextTgt }=0 \\
& \text { For } \mathrm{i}=1 \text { To } 7 \\
& \text { SelFreq(i) }=\text { True } \\
& \text { Next } \mathrm{i} \\
& \text { Threshold }=0.1 \\
& \text { Thresh text. Text }=\text { Threshold } \\
& \text { ShowBest }=\text { True }
\end{aligned}
$$

End Sub

Public Sub NewArea(area As Integer)

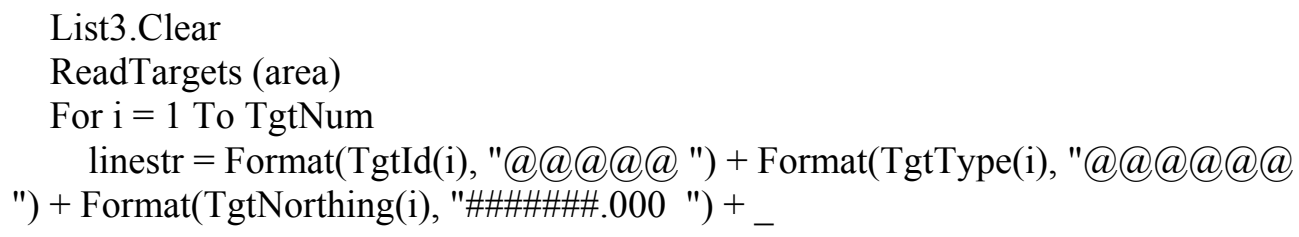


Format(TgtEasting(i), "\#\#\#\#\#.000 ") + Format(TgtDepth(i), "000 ")

+ Format(TgtAzimuth(i), "000 ") +

Format(TgtInclination(i), "000")

List3.AddItem linestr

Next i

List1.Clear

ReadArea (area)

For $\mathrm{i}=1$ To NumItems

List1.AddItem ItemId(i)

Next i

List1.ListIndex $=0$

List1_Click

PrintComparison

End Sub

Public Sub CalStats()

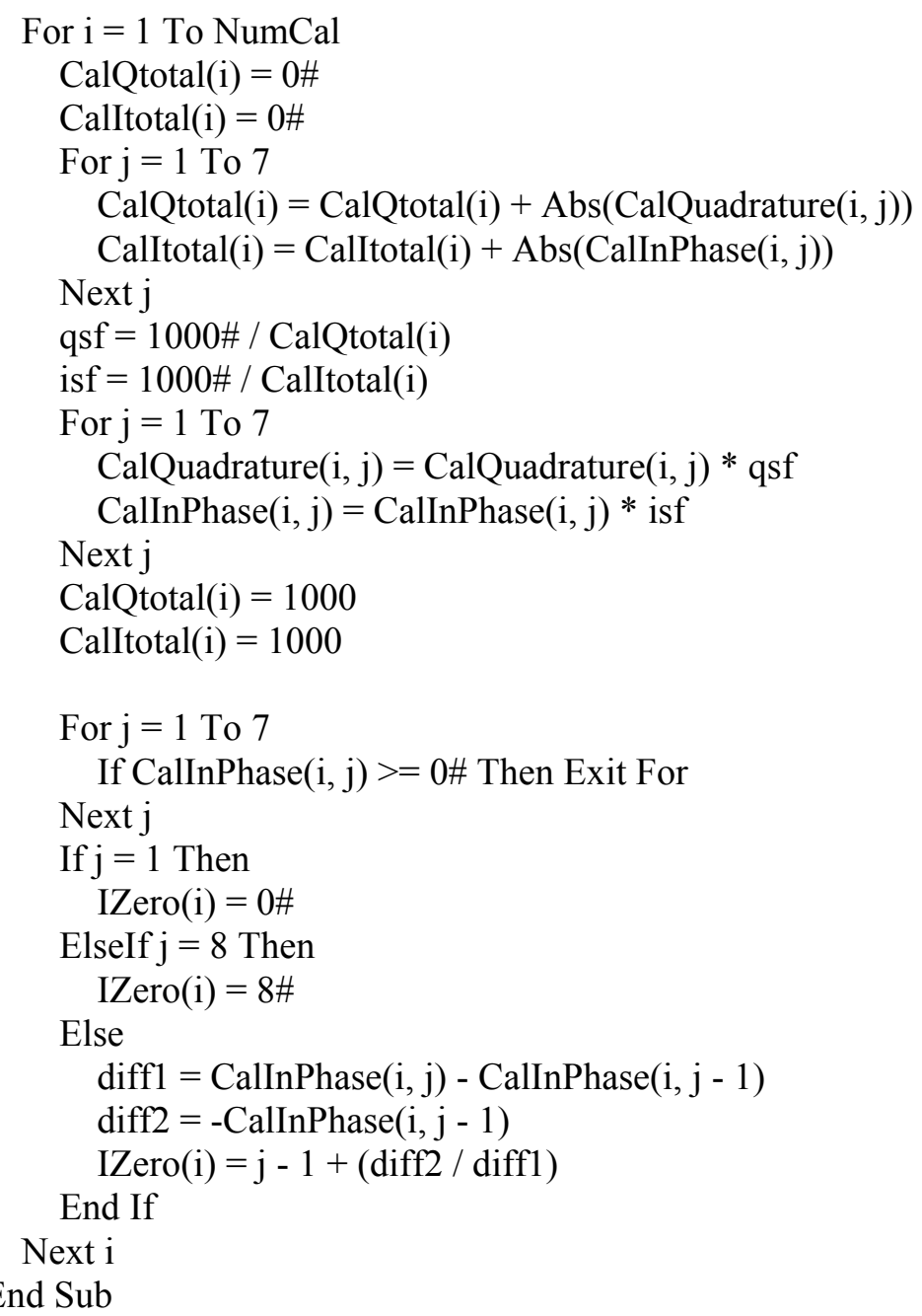


Public Sub FindCombinedMatches(i As Integer)

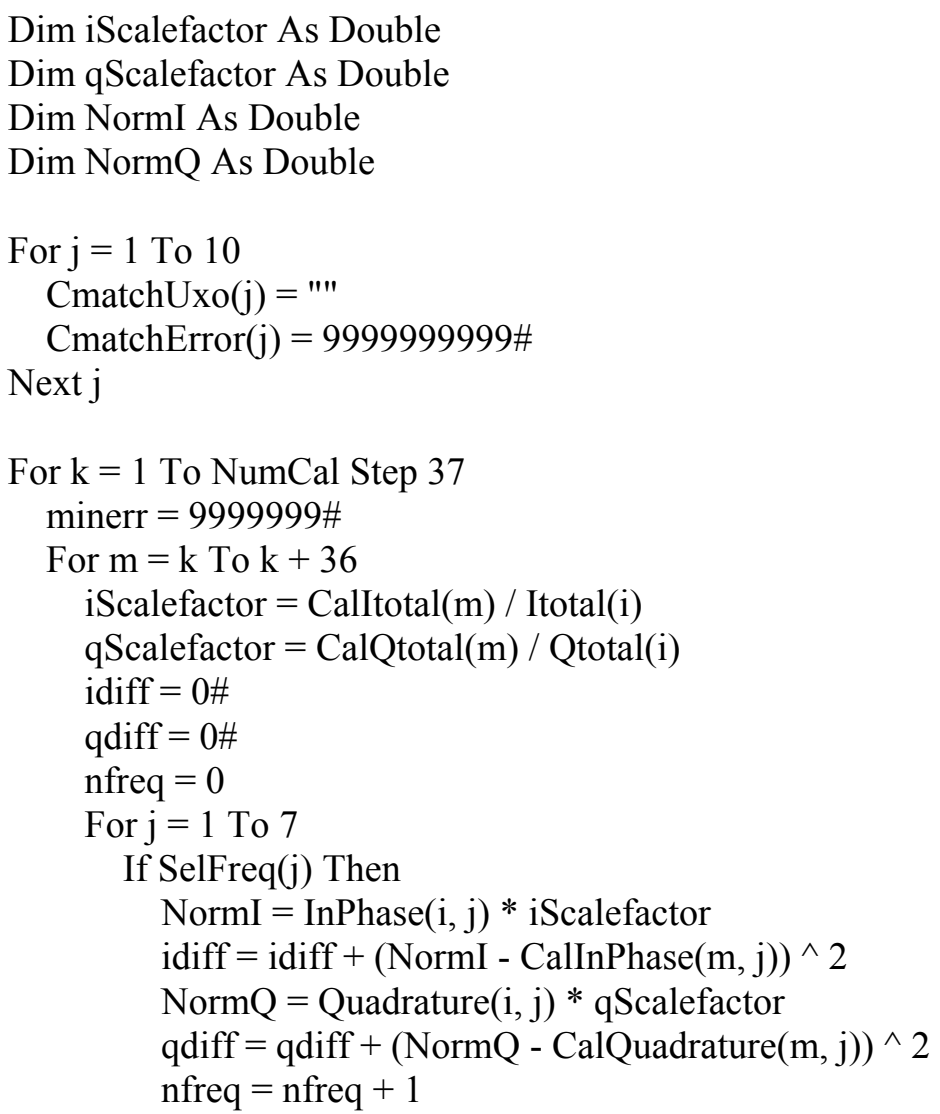

\section{End If}

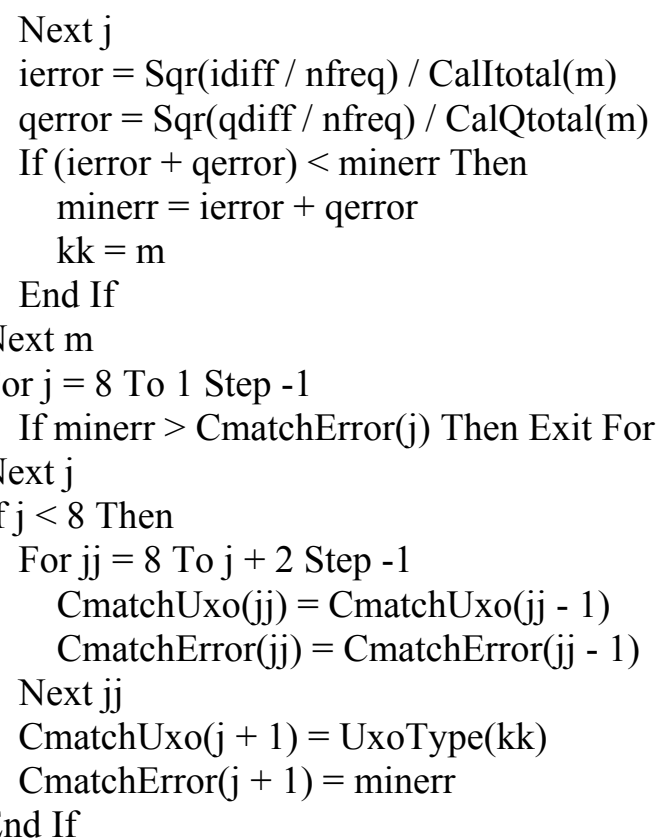

End If 


\section{Next k}

\section{End Sub}

Private Sub MatchAll_Click()

Open "allmatches.txt" For Output As \#2

For $\mathrm{i}=1$ To TgtNum

prtstr1=Format(TgtId(i), "@@@@ ")+Format(TgtType(i), "@@@@@@ )

")

prtstr1 $=$ prtstr1 + Format(TgtDepth(i), "\#\#0 ") + Format(TgtInclination(i), "\#\#0

For ii $=$ ItemStart(i) To ItemEnd(i)

FindCombinedMatches (ii)

For $\mathrm{j}=1$ To 8

If $\operatorname{Mid}($ CmatchUxo(j), 1, InStr(CmatchUxo(j), ",") - 1) = TgtType(i) Then prtstr1 $=$ prtstr $1+$ Format $($ CmatchError(j), "0.0000 ")

\section{End If}

Next j

Next ii

Print \#2, prtstr1

Next i

Close \#2

ans $=$ MsgBox("Done", vbOKOnly)

End Sub

Sub PrintComparison()

Dim NormI(1 To 7) As Double

Dim NormQ(1 To 7) As Double

Open "tgt_err.txt" For Output As \#2

' Open "tgt_mag.txt" For Output As \#3

For $\mathrm{i}=1$ To TgtNum

prtstr1=Format(TgtId(i),"@@@@ ")+Format(TgtType(i), "@@@@@@ ")

prtstr1 = prtstr1 + Format(TgtDepth(i), "\#\#0") + Format(TgtInclination(i), "\#\#0

")

prtstr2=Format(TgtId(i),"@@@@ )+Format(TgtType(i), "@@@@@@

prtstr2 = prtstr2 + Format(TgtDepth(i), "\#\#0 ") + Format(TgtInclination(i), "\#\#0

")

calmatch $=0$

For $\mathrm{j}=19$ To NumCal Step 37

If TgtType(i) $=$ CalType $(j)$ Then 


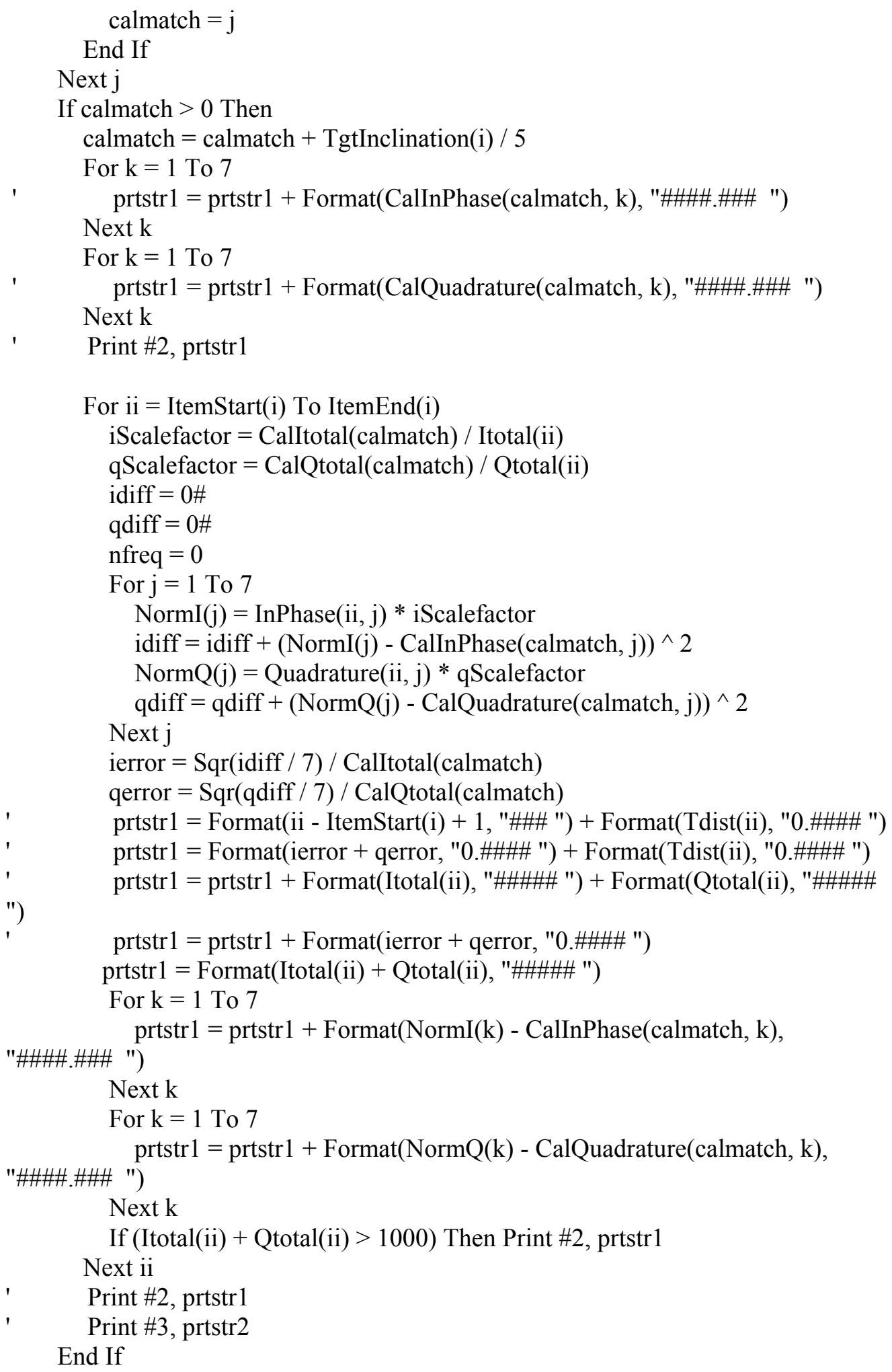


Next i

Close \#2

- Close \#3

End Sub

Sub findmatches()

$$
\begin{aligned}
& \mathrm{UXO}(1)=" 20 \mathrm{~mm} " \\
& \mathrm{UXO}(2)=" 57 \mathrm{~mm} " \\
& \mathrm{UXO}(3)=" 60 \mathrm{~mm} " \\
& \mathrm{UXO}(4)=" 2.75 \mathrm{in} " \\
& \mathrm{UXO}(5)=" 81 \mathrm{~mm} " \\
& \mathrm{UXO}(6)=" 4.2 \mathrm{in} " \\
& \mathrm{UXO}(7)=" 152 \mathrm{~mm} " \\
& \mathrm{UXO}(8)=" 155 \mathrm{~mm} " \\
& \mathrm{UXO}(9)=" 76 \mathrm{~mm} " \\
& \mathrm{UXO}(10)=" 105 \mathrm{~mm} " \\
& \mathrm{UXO}(11)=" 5.0 \mathrm{in} "
\end{aligned}
$$

1 ReadArea

Open "allmatches.txt" For Output As \#2

For $\mathrm{j}=1$ To 11

Score $(j)=0$

Next j

For $\mathrm{j}=1$ To 11

For $\mathrm{k}=1$ To 11

Confusion $(\mathrm{j}, \mathrm{k})=0$

Next k

Next j

For $\mathrm{k}=1$ To TgtNum

If BestFit(k) $>0$ Then

$\mathrm{i}=$ BestFit(k)

$\mathrm{i}=\mathrm{k}$

FindQMatches (i)

FindIMatches (i)

FindCombinedMatches (i)

Print \#2, Format(TargetId(i), "@@@@@"),Format(Iz, "0.00 ")

Print \#2, " InPhase Matches Quadrature Matches"

For $\mathrm{j}=1$ To 8

Print \#2, ImatchUxo(j) \& Space(25 - Len(ImatchUxo(j))) \&

Format(ImatchError(j), "0.0000 "),

QmatchUxo(j) \& Space(25 - Len(QmatchUxo(j))) \&

Format(QmatchError(j), "0.0000 "),

CmatchUxo(j) \& Space $(25$ - Len(CmatchUxo(j))) \&

Format(CmatchError(j), "0.0000 ") 


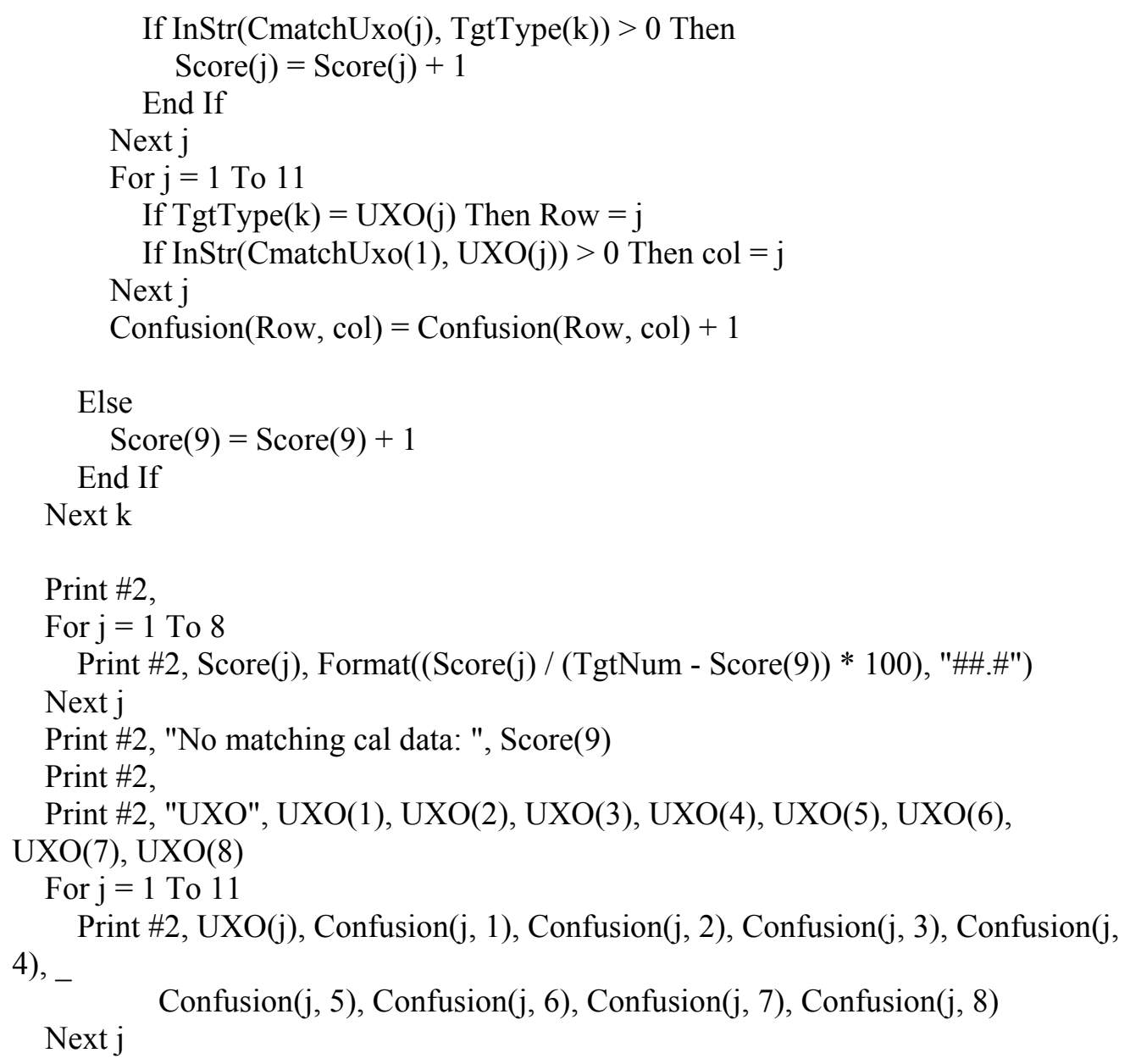

End Sub

Private Sub Option1_Click(Index As Integer)

NewArea $(\operatorname{Index}+1)$

End Sub

Private Sub Plot_Click()

plotUXO.Show 1

CurTarget $=$ List 3 .ListIndex +1

End Sub

Sub plotiq() 
$\mathrm{j}=$ NextPlot

calmatch $=0$

For $\mathrm{jj}=1$ To NumCal

If BestMatchUxo $(\mathrm{j})=\mathrm{UxoType}(\mathrm{jj})$ Then calmatch $=\mathrm{jj}$

Next jj

MSChart1.RowCount $=7$

MSChart1. ColumnCount $=4$

1 $\quad$ MSChart2. RowCount $=7$

MSChart1.Plot.SeriesCollection(1).SeriesMarker.Auto $=$ False

MSChart1.Plot.SeriesCollection(2).SeriesMarker.Auto $=$ False

MSChart1.Plot.SeriesCollection(3).SeriesMarker.Auto $=$ False

MSChart1.Plot.SeriesCollection(4).SeriesMarker.Auto $=$ False

MSChart1.Plot.SeriesCollection.Item(1).DataPoints(-1).Marker.Size $=100$

MSChart1.Plot.SeriesCollection.Item(1).DataPoints(-1).Marker.Style =

VtMarkerStyleFilledSquare

MSChart1.Plot.SeriesCollection.Item(2).DataPoints(-1).Marker.Size $=100$

MSChart1.Plot.SeriesCollection.Item(2).DataPoints(-1).Marker.Style =

VtMarkerStyleFilledSquare

MSChart1.Plot.SeriesCollection.Item(3).DataPoints(-1).Marker.Size $=100$

MSChart1.Plot.SeriesCollection.Item(3).DataPoints(-1).Marker.Style =

VtMarkerStylePlus

MSChart1.Plot.SeriesCollection.Item(4).DataPoints(-1).Marker.Size $=100$

MSChart1.Plot.SeriesCollection.Item(4).DataPoints(-1).Marker.Style = VtMarkerStylePlus

If calmatch $>0$ Then

Scalefactorq $=$ CalQtotal $($ calmatch $) /$ Qtotal $(\mathrm{j})$

Scalefactori $=$ CalItotal $($ calmatch $) /$ Itotal $(\mathrm{j})$

With MSChart1

For $\mathrm{k}=1$ To 7

NormI $=$ InPhase $(\mathrm{j}, \mathrm{k}) *$ Scalefactori

.Row $=\mathrm{k}$

.Column $=1$

.Data $=$ CalInPhase $($ calmatch, $\mathrm{k})$

. Column $=2$

. Data $=$ NormI

Next k

.Title.Text $=$ "Target " + TargetId(j) + " In Phase"

End With

With MSChart2

For $\mathrm{k}=1$ To 7

NormQ $=$ Quadrature $(\mathrm{j}, \mathrm{k}) *$ Scalefactorq

Row $=\mathrm{k}$

.Column $=3$

.Data $=$ CalQuadrature (calmatch, $\mathrm{k})$

. Column $=4$

. Data $=$ NormQ 


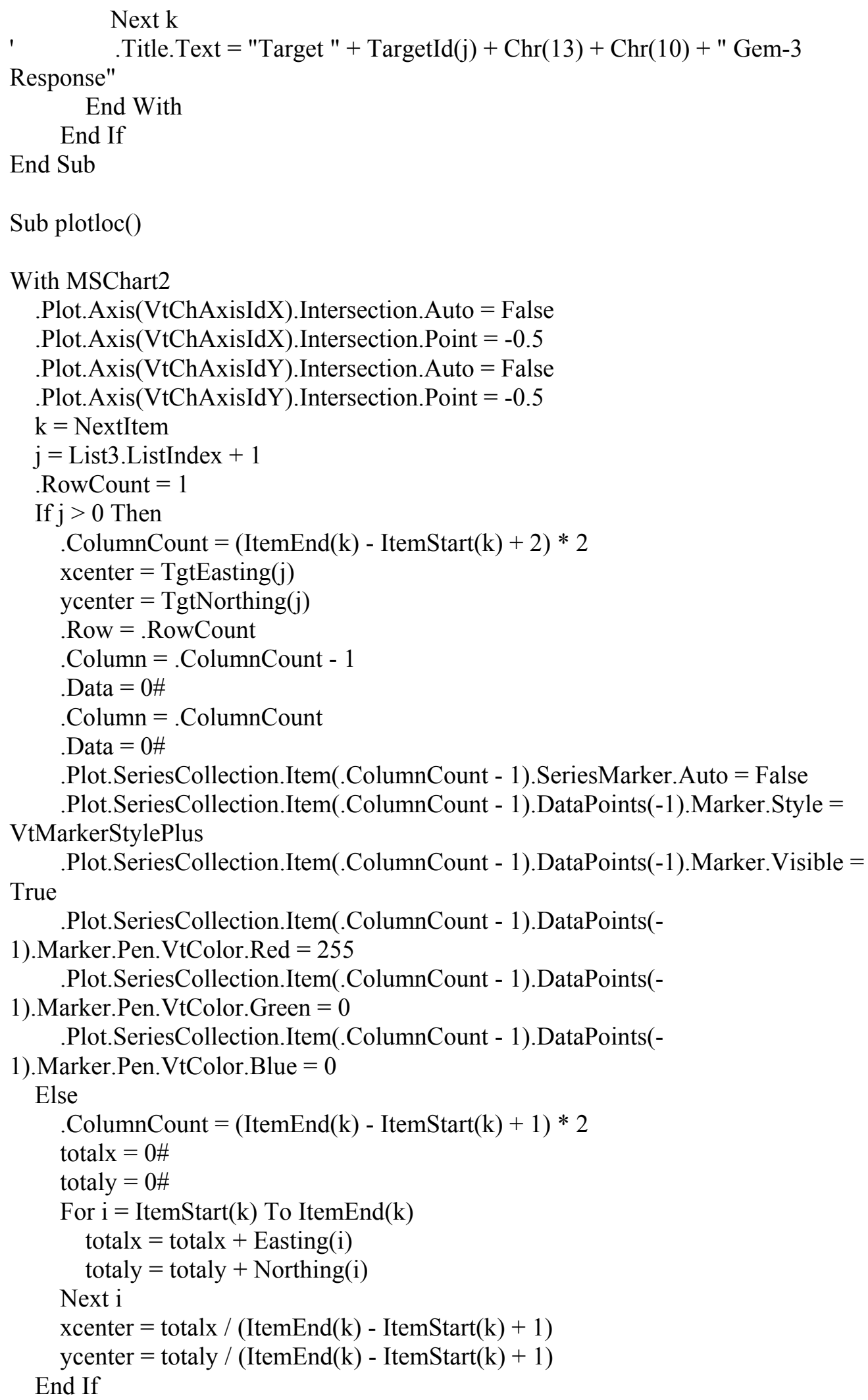


For $\mathrm{i}=\operatorname{ItemStart}(\mathrm{k})$ To ItemEnd(k)

.Plot.SeriesCollection.Item((i - ItemStart(k)) $* 2+1)$.SeriesMarker.Auto $=$ False

.Plot.SeriesCollection.Item((i - ItemStart(k)) * 2 + 1).DataPoints(-

1).Marker.Style = VtMarkerStylePlus

.Plot.SeriesCollection.Item((i - ItemStart(k)) * 2 + 1).DataPoints(-

1).Marker.Visible $=$ True

.Plot.SeriesCollection.Item((i - ItemStart(k)) * 2 + 1).DataPoints(-

1).Marker.Pen.VtColor.Red $=0$

.Plot.SeriesCollection.Item((i - ItemStart(k)) * 2 + 1).DataPoints(-

1).Marker.Pen.VtColor.Green $=0$

.Plot.SeriesCollection.Item((i - ItemStart $(\mathrm{k})) * 2+1)$.DataPoints(-

1).Marker.Pen.VtColor.Blue $=0$

If BestMatchError(i) < Threshold Then

.Plot.SeriesCollection.Item((i - ItemStart(k)) *2+1).DataPoints(-

1).Marker.Pen.VtColor.Red $=0$

'.Plot.SeriesCollection.Item((i - ItemStart(k)) * 2+ 1).DataPoints(-

1).Marker.Pen.VtColor.Green $=($ Threshold - BestMatchError(i) $) /$ Threshold $* 255$

.Plot.SeriesCollection.Item((i - ItemStart(k)) *2+1).DataPoints(-

1).Marker.Pen.VtColor.Green $=0$

.Plot.SeriesCollection.Item((i - ItemStart(k)) *2+1).DataPoints(-

1).Marker.Pen.VtColor.Blue $=255$

End If

If BestMatchError(i) < Threshold / $3 * 2$ Then

.Plot.SeriesCollection.Item((i - ItemStart(k)) *2+1).DataPoints(-

1).Marker.Pen.VtColor.Red $=0$

.Plot.SeriesCollection.Item((i - ItemStart(k)) *2+1).DataPoints(-

1).Marker.Pen.VtColor.Green $=255$

.Plot.SeriesCollection.Item((i - ItemStart(k)) $* 2+1)$.DataPoints(-

1).Marker.Pen.VtColor.Blue $=255$

End If

If BestMatchError(i) < Threshold / 3 Then

.Plot.SeriesCollection.Item((i - ItemStart(k)) *2+1).DataPoints(-

1).Marker.Pen.VtColor.Red $=255$

.Plot.SeriesCollection.Item((i - ItemStart(k)) *2+1).DataPoints(-

1).Marker.Pen.VtColor.Green $=255$

.Plot.SeriesCollection.Item((i - ItemStart(k)) *2+1).DataPoints(-

1).Marker.Pen.VtColor.Blue $=0$

End If

If $(\mathrm{i}$ - ItemStart $(\mathrm{k})=$ List2.ListIndex $)$ Then

.Plot.SeriesCollection.Item((i - ItemStart(k)) *2+1).DataPoints(-

1).Marker.Style $=$ VtMarkerStyleDiamond

' .Plot.SeriesCollection.Item((i - ItemStart(k)) * $2+1)$.DataPoints(-

1).Marker.Pen.VtColor.Red $=255$

'.Plot.SeriesCollection.Item((i - ItemStart(k)) * 2 + 1).DataPoints(-

1).Marker.Pen.VtColor.Green $=255$

1.Plot.SeriesCollection.Item((i - ItemStart(k)) * 2+ 1).DataPoints(-

1).Marker.Pen.VtColor.Blue $=0$

End If 


$$
\begin{aligned}
& . \text { Row }=1 \\
& . \text { Column }=(\mathrm{i}-\text { ItemStart }(\mathrm{k})) * 2+1 \\
& . \text { Data }=\text { Easting }(\mathrm{i})-\text { xcenter } \\
& . \text { Column }=(\mathrm{i}-\text { ItemStart }(\mathrm{k})) * 2+2 \\
& . \text { Data }=\text { Northing }(\mathrm{i})-\text { ycenter }
\end{aligned}
$$

Next i

End With

End Sub

Private Sub Thresh_text_LostFocus()

Threshold $=$ Thresh_text.Text

End Sub

lo.bas

Public Sub ReadCal()

Open "c:ljpg \cal.txt" For Input As \#1

$\mathrm{i}=1$

While Not EOF(1)

For $\mathrm{k}=\mathrm{i}$ To $\mathrm{i}+36$ Step 18

Input \#1, UxoType (k)

CalType(k) = Mid(UxoType(k), 1, InStr(UxoType(k), ",") - 1)

CalDepth(k) = Val(Mid(UxoType(k), InStr(UxoType(k), ",") + 1))

CalInclination(k) = Val(Mid(UxoType(k), InStr(UxoType(k), "cm,") + 3))

For $\mathrm{j}=1$ To 7

Input \#1, CalInPhase $(\mathrm{k}, \mathrm{j})$

Next j

Input \#1, UxoType(k)

For $\mathrm{j}=1$ To 7

Input \#1, CalQuadrature $(\mathrm{k}, \mathrm{j})$

Next j

Next k

ichar $=\operatorname{InStr}($ UxoType(i), "90")

For $\mathrm{k}=\mathrm{i}+1$ To $\mathrm{i}+17$

UxoType $(\mathrm{k})=$ UxoType $(\mathrm{i})$

CalType $(\mathrm{k})=$ CalType $(\mathrm{i})$

CalDepth $(\mathrm{k})=$ CalDepth $(\mathrm{i})$

CalInclination $(\mathrm{k})=90-(\mathrm{k}-\mathrm{i}) * 5$

Mid(UxoType(k), ichar, 2)=Format(90 - (k - i)*5, "@@")

For $\mathrm{j}=1$ To 7

CalInPhase $(k, j)=$ CalInPhase $(i, j)+(k-i) / 18 \# *($ CalInPhase $(i+18, j)-$ CalInPhase (i, j)) 


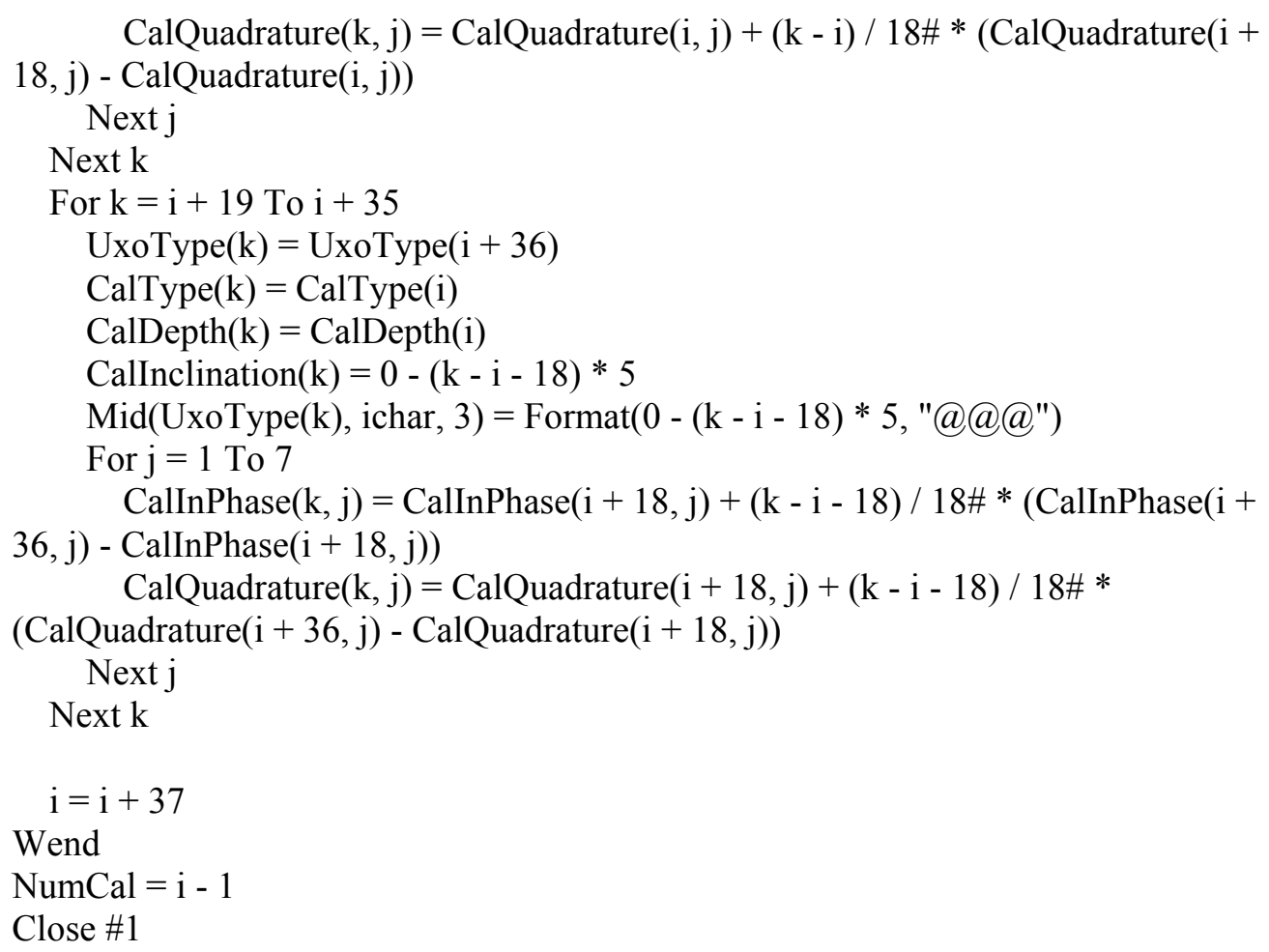

End Sub

Public Sub ReadArea(area As Integer)

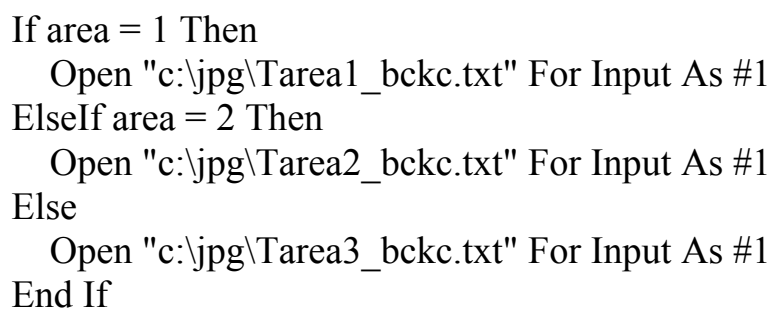


'Decode target id

If area $>1$ Then

' $\quad$ tempstr $=\operatorname{Left}($ TargetId(i), 1) + Mid(TargetId(i), 4)

' $\quad$ TargetId(i) $=$ tempstr

' End If

cpos = InStr(TargetId(i), "-")

If Mid(TargetId(i), cpos, 2) = "-0" Then

tempstr $=\operatorname{Mid}(\operatorname{TargetId}(\mathrm{i}), 1, \operatorname{cpos}-1)$

Else

tempstr $=\operatorname{Str}($ area $)+\operatorname{Mid}(\operatorname{TargetId}(\mathrm{i})$, cpos $)$

End If

TargetId(i) $=$ Trim(tempstr)

'Calculate sum of all frequencies

totali $=0$

totalq $=0$

For $\mathrm{j}=1$ To 7

totali $=$ totali $+\operatorname{Abs}(\operatorname{InPhase}(i, j))$

totalq $=$ totalq $+\operatorname{Abs}($ Quadrature $(i, j))$

Next j

Itotal(i) $=$ totali

Qtotal(i) = totalq

'Calculate distance to actual target

Tdist $(i)=-1$

For $\mathrm{j}=1$ To TgtNum

If TargetId(i) $=\operatorname{TgtId}(\mathrm{j})$ Then

$\mathrm{dx}=$ TgtEasting(j) - Easting(i)

$\mathrm{dy}=$ TgtNorthing(j) - Northing(i)

$\operatorname{Tdist}(\mathrm{i})=\operatorname{Sqr}\left((\operatorname{TgtNorthing}(\mathrm{j})-\operatorname{Northing}(\mathrm{i}))^{\wedge} 2+(\operatorname{TgtEasting}(\mathrm{j})-\right.$ Easting(i) $)$

$\wedge 2)$

\section{Exit For}

End If

Next j

'Keep track of each separate item If (ii $>0$ ) Then

If TargetId(i) $\diamond$ ItemId(ii) Then

$\mathrm{ii}=\mathrm{ii}+1$

ItemEnd(ii - 1) $=\mathrm{i}-1$

ItemStart(ii) $=\mathrm{i}$

ItemId(ii) $=$ TargetId(i)

End If

Else

ii $=1$

ItemId(ii) $=$ TargetId(i)

ItemStart(ii) $=\mathrm{i}$

End If 


$$
\begin{aligned}
& \quad \mathrm{i}=\mathrm{i}+1 \\
& \text { Wend } \\
& \text { NumPoints }=\mathrm{i}-1 \\
& \text { NumItems }=\mathrm{ii} \\
& \text { ItemEnd(ii) }=\mathrm{i}-1
\end{aligned}
$$

Close \#1

End Sub

Public Sub ReadTargets(area As Integer)

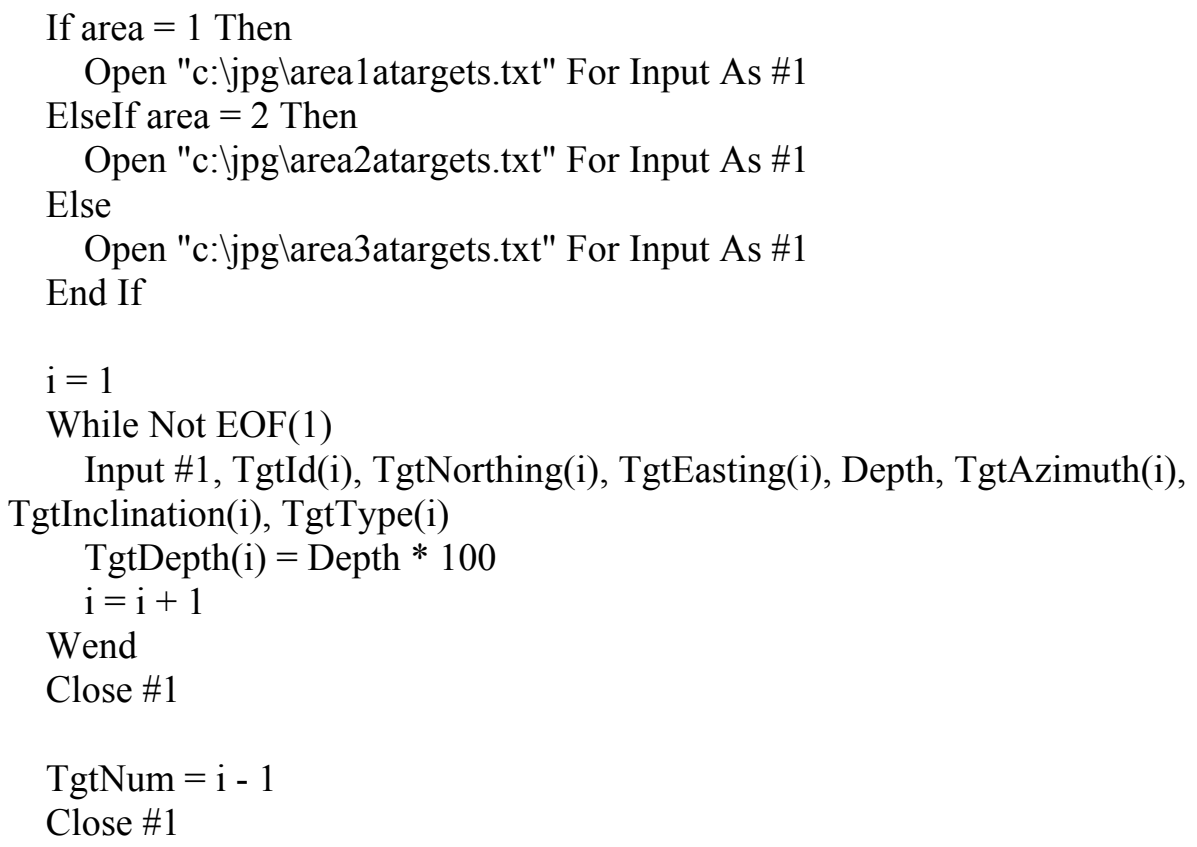

End Sub

Public Sub ReadClutter()

Open "c:ljpg \area123Clutter.txt" For Input As \#1

$\mathrm{i}=1$

While Not EOF(1)

Input \#1, TgtNorthing(i), TgtEasting(i), TgtId(i)

$$
\mathrm{i}=\mathrm{i}+1
$$

Wend

TgtNum $=\mathrm{i}-1$

Close \#1

End Sub 


\section{Globals.bas}

Global UxoType(1 To 10000) As String Global CalInPhase(1 To 10000, 1 To 7) As Double Global CalQuadrature(1 To 10000, 1 To 7) As Double Global CalType(1 To 10000) As String Global CalDepth(1 To 10000) As Integer Global CalInclination(1 To 10000) As Integer

Global CalQtotal(1 To 10000) As Double

Global CalItotal(1 To 10000) As Double

Global NumCal As Integer

Global InPhase(1 To 10000, 1 To 7) As Double

Global Quadrature(1 To 10000, 1 To 7) As Double

Global Northing(1 To 10000) As Double

Global Easting(1 To 10000) As Double

Global TargetId(1 To 10000) As String

Global Qtotal(1 To 10000) As Double

Global Itotal(1 To 10000) As Double

Global Tdist(1 To 10000) As Double

Global NumPoints As Integer

Global IZero(1 To 10000) As Double

Global CmatchUxo(1 To 10) As String

Global BestMatchUxo(1 To 10000) As String

Global CmatchError(1 To 10) As Double

Global BestMatchError(1 To 10000) As Double

Global Iz As Double

Global TgtId(1 To 500) As String

Global TgtEasting(1 To 500) As Double

Global TgtNorthing(1 To 500) As Double

Global TgtDepth(1 To 500) As Integer

Global TgtAzimuth(1 To 500) As Integer

Global TgtInclination(1 To 500) As Integer

Global TgtType(1 To 500) As String

Global TgtNum As Integer

Global ItemId(1 To 10000) As String

Global ItemStart(1 To 10000) As Integer

Global ItemEnd(1 To 10000) As Integer

Global ItemMinDist(1 To 10000) As Double

Global NumItems As Integer

Global NextPlot As Integer

Global NextItem As Integer

Global BestFit(1 To 10000) As Integer

Global BestCal(1 To 10000) As Integer

Global Score(1 To 11) As Integer

Global Confusion(1 To 12, 1 To 12) As Integer

Global UXO(1 To 12) As String 
Global SelFreq(1 To 7) As Boolean

Global Threshold As Double

Global ShowBest As Boolean

Global CurTarget As Integer

\title{
PlotUXO.frm
}

\author{
Private Sub Cancel_Click() \\ NextPlot $=$ NextPlot +1 \\ Unload Me \\ plotUXO.Show
}

End Sub

Private Sub Form_Load()

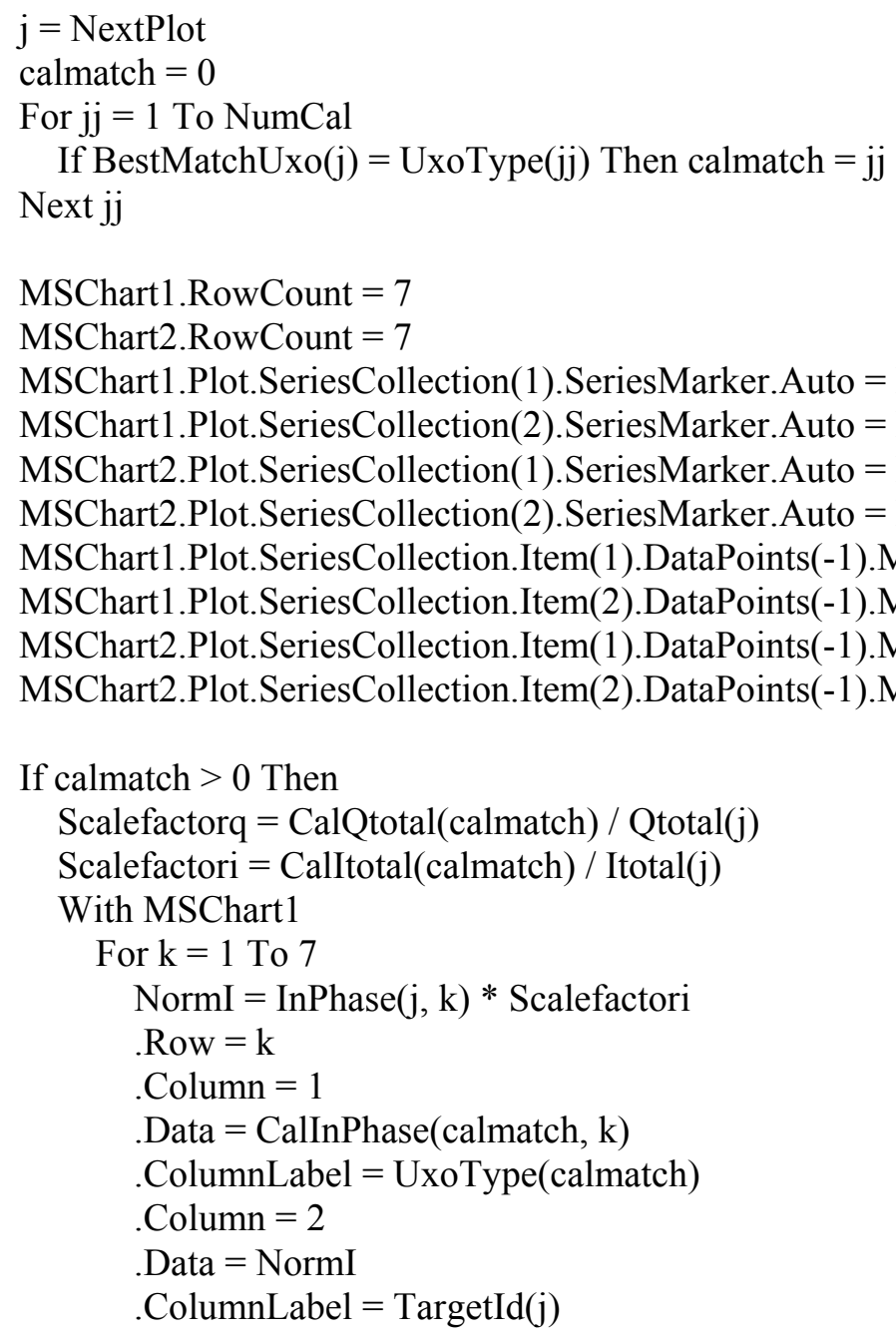




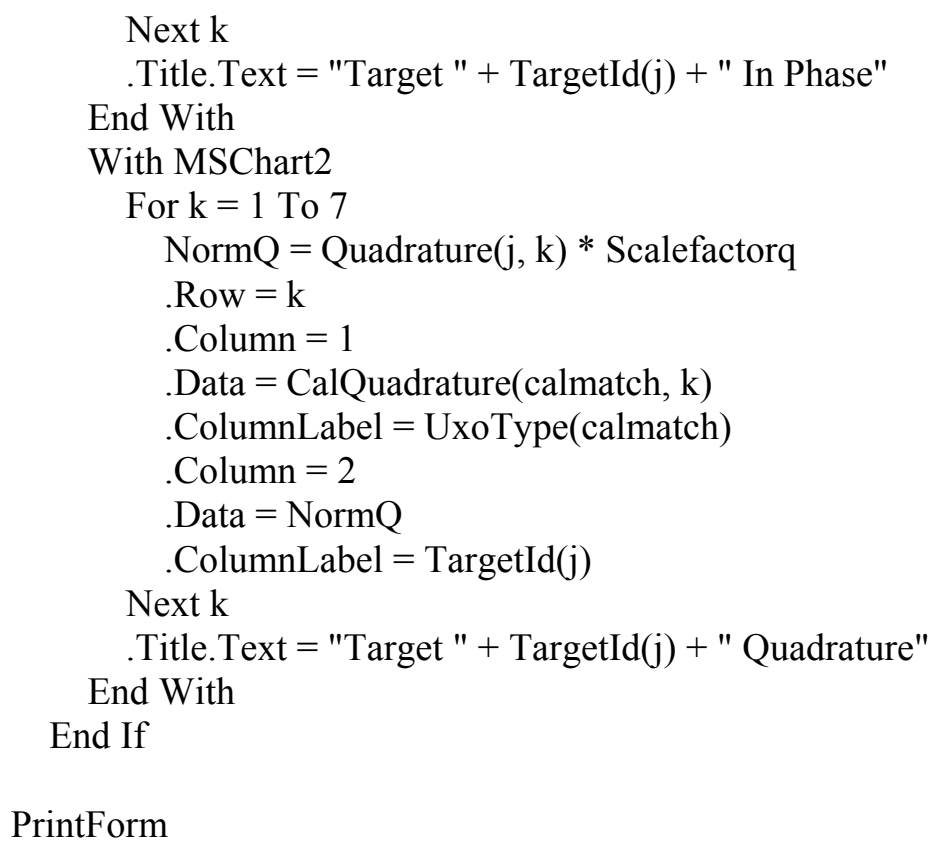

Next k

.Title. Text $=$ "Target " + TargetId(j) + " In Phase"

End With

With MSChart2

For $\mathrm{k}=1$ To 7

NormQ $=$ Quadrature $(\mathrm{j}, \mathrm{k}) *$ Scalefactorq

Row $=\mathrm{k}$

.Column $=1$

.Data $=$ CalQuadrature $($ calmatch, $\mathrm{k})$

.ColumnLabel $=$ UxoType $($ calmatch $)$

.Column $=2$

Data $=$ NormQ

.ColumnLabel $=$ TargetId $(\mathrm{j})$

Next k

.Title.Text $=$ "Target " + TargetId $(j)+$ " Quadrature"

End With

End If

PrintForm

End Sub 


\section{GridScan Program}

Sub dpos_maxima()

Rem this subprogram locates the maxima in the dpos box and outputs them Rem it also writes out the data as inphase for the area and quad for the area Rem the case of Tag_this true is the global histogram of the entire data set Rem false is the local target case

Open wheredata\$ For Input As \#1

Open dposq \$ For Output As \#2

Open dposi\$ For Output As \#3

Do While $\operatorname{EOF}(1)=$ False

icount $=$ icount +1

Rem read in the data for the selected data segment( global and partial)

For $\mathrm{j}=1$ To 14

Input \#1, Rawdata( $0, j$, icount $)$, Rawdata( $1, j$, icount $)$

Next j

Input \#1, posdata( 0 , icount $)$, posdata( 1 , icount $)$

Input $\# 1$, dummy\$

For $\mathrm{j}=8$ To 14

Print \#2, Rawdata( $0, j$, icount $) ;$ " , ";

Print \#3, Rawdata( $1, \mathrm{j}$, icount); " , ";

Next j

Print \#2, posdata( 0 , icount); " , "; posdata(1, icount); " , "; dummy\$

Print \#3, posdata( 0 , icount); " , "; posdata( 1 , icount); " , "; dummy\$

Loop

Close \#1

Close \#2

Close \#3

End Sub

Sub lochist_this()

Rem the case of Tag_this true is the global histogram of the entire data set

Rem false is the local target case

Floop $=\mathrm{n}$

For $\mathrm{m}=1$ To Floop

Rem inserting code for Call initalizelocal and get

$\operatorname{Rem} * * * * * * * * * * * * * * * * * * * * * * * * * * * * * * * * * * * * * * * * * * * * * * * * * * * * * * * * * * * * * * * *$

$\operatorname{Rem} * * * * * * * * * * * * * * * * * * * * * * * * * * * * * * * * * * * * * * * * * * * * * * * * * * * * * * * * * * * * * * *$

Rem this file does not contain the targets

Rem if you try to locate target maxima from here you get junk

$\mathrm{fl}=$ "C:\WINDOWS $\backslash$ Desktop $\backslash$ Hist_temp $\backslash "$ + TVal + "hist_where_" + id $\$(\mathrm{~m})+$

".txt"

Rem initalize the position extrema 


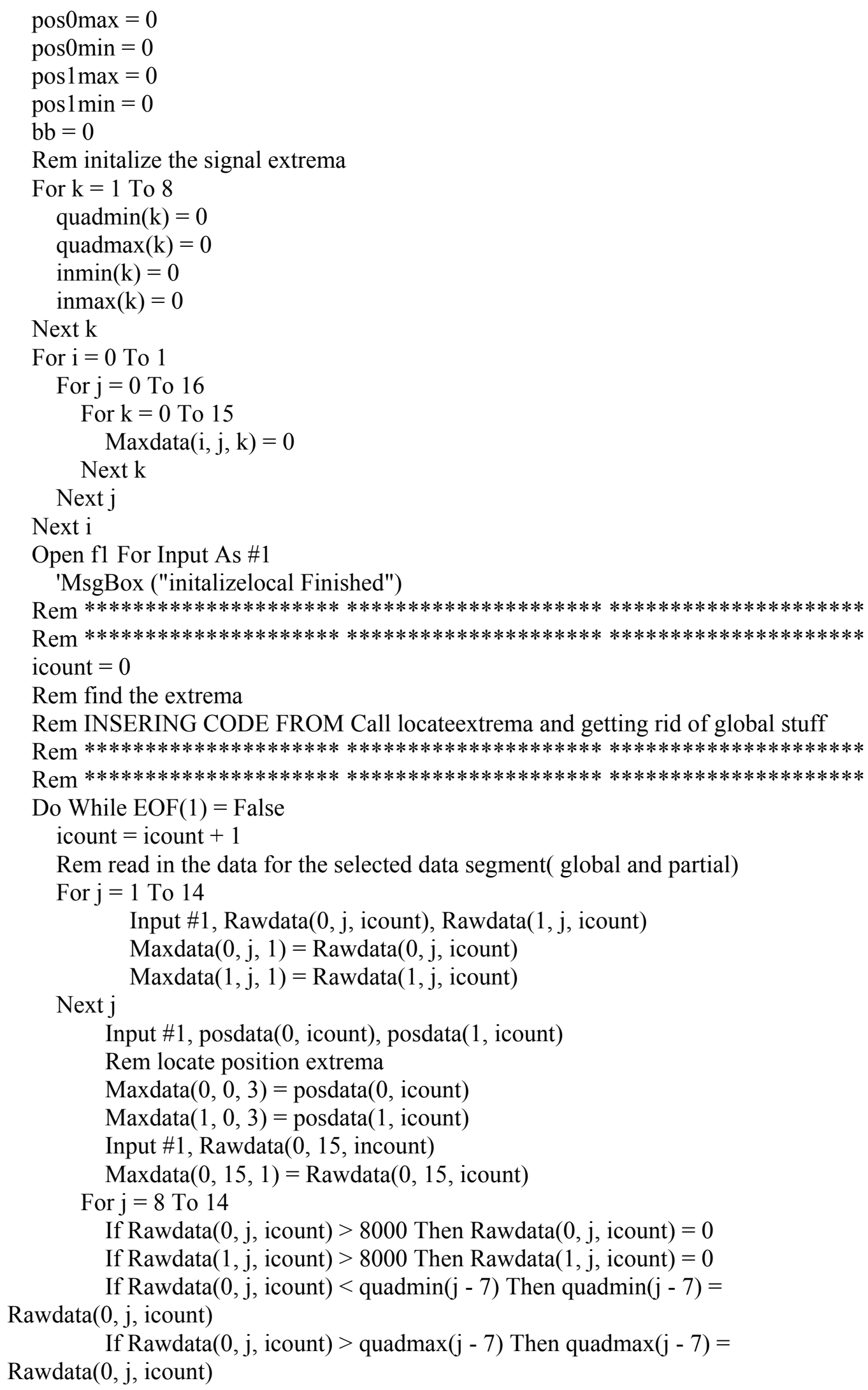




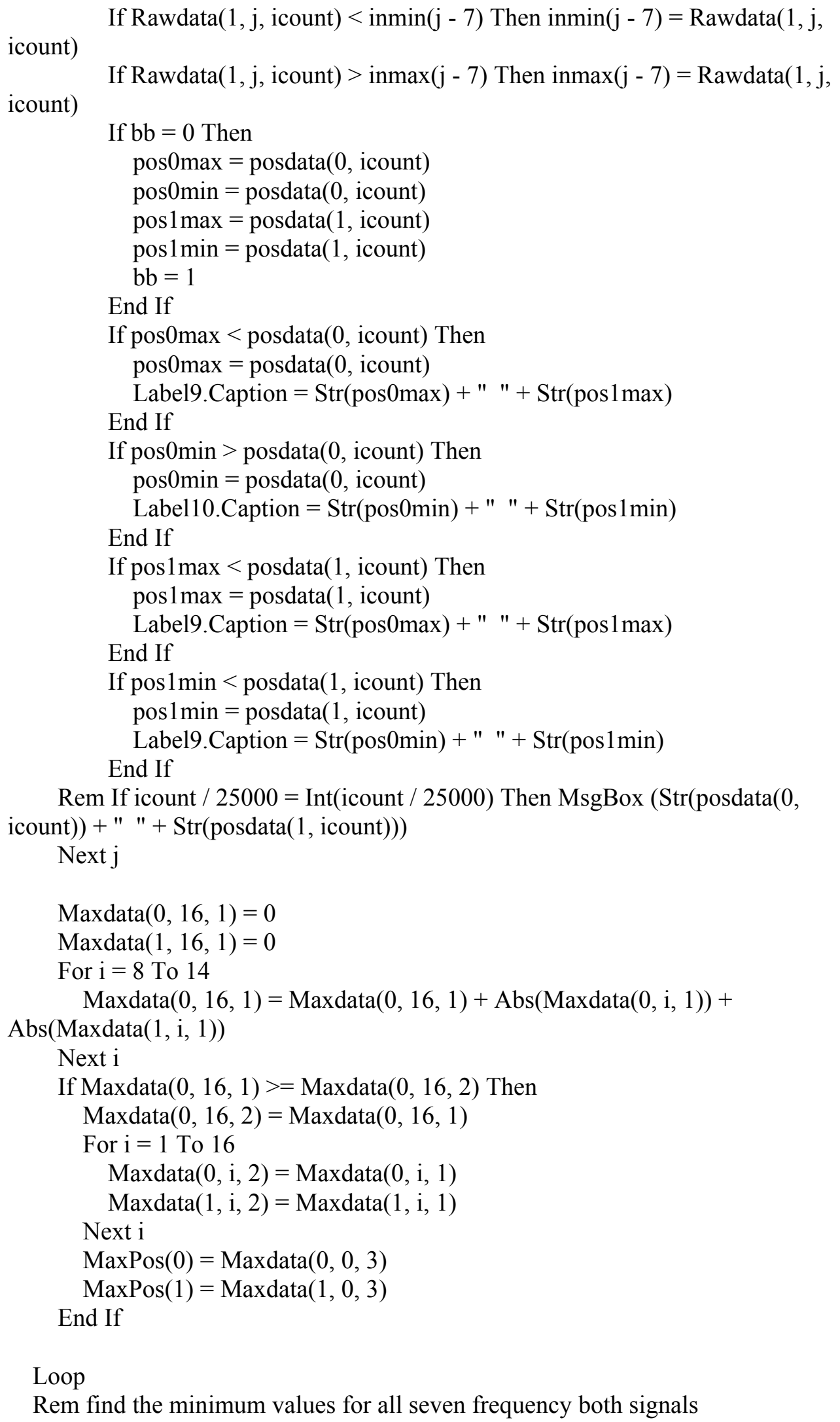


gquadmin $=$ quadmin $(1)$

ginmin $=$ inmin $(1)$

For $\mathrm{i}=2$ To 7

If gquadmin $>$ quadmin(i) Then gquadmin = quadmin(i)

If ginmin $>$ inmin(i) Then ginmin $=$ inmin(i)

Next i

'MsgBox ("localextrema Finished")

$\operatorname{Rem} * * * * * * * * * * * * * * * * * * * * * * * * * * * * * * * * * * * * * * * * * * * * * * * * * * * * * * * * * * * * * * * *$

$\operatorname{Rem} * * * * * * * * * * * * * * * * * * * * * * * * * * * * * * * * * * * * * * * * * * * * * * * * * * * * * * * * * * * * * * * *$

Rem output maxima data for the local case

Rem this is where the problem was

Rem localout is putting out the maxima in the dbk - dpos box

Rem not the dpos box

Rem I have gotten rid of Call localout

jcount $=$ icount

Close \#1

Rem histogram in amplitude for each frequnecy and phase

Call dohist

$\operatorname{Rem} * * * * * * * * * * * * * * * * * * * * *$

Rem put out histogram for each

Rem filenames are quadf1, inf1, ...

Rem get these names

Rem for the global file

Call localhistout

Next m

End Sub

Sub new_hist_this()

Rem note that tag_this hasbeen removed and this code will only deal wiht the

Rem global data set

Rem this subprogram gets the data read in and generates a histogram for the

Rem entire area under investigation as found in $\mathrm{fl}$

Open rawdataa\$ For Input As \#1

For $\mathrm{i}=1$ To 30

Input \#1, head\$(i)

Next i

icount $=0$

Rem find the extrema

Rem insering instead of calling Call locateextrema

$\operatorname{Rem} * * * * * * * * * * * * * * * * * * * * * * * * * * * * * * * * * * * * * * * * * * * * * * * * * * * * * * * * * * * * * * *$

$\operatorname{Rem} * * * * * * * * * * * * * * * * * * * * * * * * * * * * * * * * * * * * * * * * * * * * * * * * * * * * * * * * * * * * * * *$

Do While $\operatorname{EOF}(1)=$ False

icount $=$ icount +1

Rem read in the data for the selected data segment(global and partial)

For $\mathrm{j}=1$ To 14

Input $\# 1$, Rawdata( $0, j$, icount $)$, Rawdata( $1, j$, icount $)$

Next $j$

Input \#1, posdata( 0 , icount), posdata(1, icount)

For $\mathrm{j}=8$ To 14 
If $\operatorname{Rawdata}(0, \mathrm{j}, \mathrm{icount})>8000$ Then $\operatorname{Rawdata}(0, \mathrm{j}, \mathrm{icount})=0$

If $\operatorname{Rawdata}(1, j$, icount $)>8000$ Then $\operatorname{Rawdata}(1, j$, icount $)=0$ $\mathrm{j}$, icount)

If $\operatorname{Rawdata}(0, j$, icount $)<\operatorname{quadmin}(j-7)$ Then quadmin $(j-7)=\operatorname{Rawdata}(0$, j, icount)

If $\operatorname{Rawdata}(0, j$, icount $)>$ quadmax $(j-7)$ Then quadmax $(j-7)=\operatorname{Rawdata}(0$, icount)

If $\operatorname{Rawdata}(1, j$, icount $)<\operatorname{inmin}(j-7) \operatorname{Then} \operatorname{inmin}(j-7)=\operatorname{Rawdata}(1, j$, icount)

If $\operatorname{Rawdata}(1, j$, icount $)>\operatorname{inmax}(j-7)$ Then $\operatorname{inmax}(j-7)=\operatorname{Rawdata}(1, j$,

\section{If $b b=0$ Then}

$\operatorname{pos} 0 \max =\operatorname{posdata}(0$, icount $)$

$\operatorname{pos} 0 \min =\operatorname{posdata}(0$, icount $)$

$\operatorname{pos} 1 \max =\operatorname{posdata}(1$, icount $)$

pos $1 \min =\operatorname{posdata}(1$, icount $)$

$\mathrm{bb}=1$

End If

If $\operatorname{pos} 0 \max <\operatorname{posdata}(0$, icount $)$ Then

$\operatorname{pos} 0 \max =\operatorname{posdata}(0$, icount $)$

Labe19.Caption $=\operatorname{Str}(\operatorname{pos} 0 \max )+" \quad "+\operatorname{Str}(\operatorname{pos} 1 \max )$

End If

If $\operatorname{pos} 0 \mathrm{~min}>\operatorname{posdata}(0$, icount $)$ Then

$\operatorname{pos} 0 \mathrm{~min}=\operatorname{posdata}(0$, icount $)$

Label10.Caption $=\operatorname{Str}(\operatorname{pos} 0 \mathrm{~min})+"$ " $+\operatorname{Str}(\operatorname{pos} 1 \mathrm{~min})$

End If

If $\operatorname{pos} 1 \max <\operatorname{posdata}(1$, icount $)$ Then

$\operatorname{pos} 1 \max =\operatorname{posdata}(1$, icount $)$

Labe19.Caption $=\operatorname{Str}(\operatorname{pos} 0 \max )+"$ " + Str(pos1max $)$

End If

If $\operatorname{pos} 1 \mathrm{~min}<\operatorname{posdata}(1$, icount $)$ Then

pos1 $\min =\operatorname{posdata}(1$, icount $)$

Labe19.Caption $=\operatorname{Str}(\operatorname{pos} 0 \mathrm{~min})+" \quad "+\operatorname{Str}(\operatorname{pos} 1 \mathrm{~min})$

End If

Next j

Loop

Rem find the minimum values for all seven frequency both signals

gquadmin $=$ quadmin $(1)$

ginmin $=$ inmin $(1)$

For $\mathrm{i}=2$ To 7

If gquadmin $>$ quadmin(i) Then gquadmin $=$ quadmin(i)

If ginmin $>$ inmin(i) Then ginmin $=$ inmin(i)

Next i

'MsgBox ("localextrema Finished")

$\operatorname{Rem} * * * * * * * * * * * * * * * * * * * * * * * * * * * * * * * * * * * * * * * * * * * * * * * * * * * * * * * * * * * * * * *$

$\operatorname{Rem} * * * * * * * * * * * * * * * * * * * * * * * * * * * * * * * * * * * * * * * * * * * * * * * * * * * * * * * * * * * * * * *$

numtosearch $=$ icount

Rem I cahanged the variable name to numtosearch in here

Rem I left the one below because I am not sure where it is used again

jcount $=$ icount 
Close \#1

Rem histogram in amplitude for each frequnecy and phase

$\operatorname{Rem} * * * * * * * * * * * * * * * * * * * * * * * * * * * * * * * * * * * * * * * * * * * * * * * * * * * * * * * * * * * * * * *$

$\operatorname{Rem} * * * * * * * * * * * * * * * * * * * * * * * * * * * * * * * * * * * * * * * * * * * * * * * * * * * * * * * * * * * * * * *$

Rem replacing Call dohist wiht code found there

Rem init array space

For $\mathrm{j}=1$ To 8000

For $\mathrm{i}=1$ To 7

$\operatorname{histo}(0, \mathrm{i}, \mathrm{j})=0$

$\operatorname{histo}(1, \mathrm{i}, \mathrm{j})=0$

Next i

Next j

$\mathrm{n}=1$

Rem calculates histgrams for all cases

For icount $=1$ To numtosearch

For $\mathrm{k}=8$ To 14

$\mathrm{j}=\mathrm{k}-7$

histo0index $=\operatorname{Int}(\operatorname{Rawdata}(0, \mathrm{k}$, icount $)$ - gquadmin $)$

histolindex $=\operatorname{Int}(\operatorname{Rawdata}(1, \mathrm{k}$, icount $)-$ ginmin $)$

$\operatorname{histo}(0, \mathrm{j}$, histo0index $)=\operatorname{histo}(0, \mathrm{j}$, histo0index $)+1$

$\operatorname{histo}(1, \mathrm{j}$, histolindex $)=\operatorname{histo}(1, \mathrm{j}$, histolindex $)+1$

Next k

Next icount

Rem find the mean value for the histogram

Rem put it at the end of the histogram

Rem sum up the number of items at each location

For $\mathrm{i}=1$ To 7

$\operatorname{histsum}(0, i)=0$

$\operatorname{histsum}(1, i)=0$

Next $\mathrm{i}$

For $\mathrm{i}=1$ To 7

num $0=0$

num $1=0$

For $\mathrm{j}=1$ To 8000

num0 $=$ num0 $+\operatorname{Abs}(\operatorname{histo}(0, i, j))$

num1 $=$ num1 $+\operatorname{Abs}(\operatorname{histo}(1, \mathrm{i}, \mathrm{j}))$

$\operatorname{histsum}(0, i)=\operatorname{histsum}(0, i)+j * \operatorname{Abs}(\operatorname{histo}(0, i, j))$

$\operatorname{histsum}(1, \mathrm{i})=\operatorname{histsum}(1, \mathrm{i})+\mathrm{j} * \operatorname{Abs}(\operatorname{histo}(1, \mathrm{i}, \mathrm{j}))$

Next j

histmean $(0, \mathrm{i})=\operatorname{histsum}(0, \mathrm{i}) / \operatorname{num} 0$

$\operatorname{histmean}(1, \mathrm{i})=\operatorname{histsum}(1, \mathrm{i}) /$ num 1

Next $\mathrm{i}$

$\operatorname{Rem} * * * * * * * * * * * * * * * * * * * * * * * * * * * * * * * * * * * * * * * * * * * *$

'MsgBox ("Dohist Finished")

$\operatorname{Rem} * * * * * * * * * * * * * * * * * * * * * * * * * * * * * * * * * * * * * * * * * * * * * * * * * * * * * * * * * * * * * * *$

$\operatorname{Rem} * * * * * * * * * * * * * * * * * * * * * * * * * * * * * * * * * * * * * * * * * * * * * * * * * * * * * * * * * * * * * * *$ 


\section{$\operatorname{Rem} * * * * * * * * * * * * * * * * * * * * *$}

Rem put out histogram for each

Rem filenames are quadf1, inf1, ...

Rem get these names

Rem for the global file

Rem

Rem

Rem i am replacing Call globalhistout wiht the code

Rem this produces a the files found in $\mathrm{f} 2$

Rem I dont know why we still need them

Rem I think this should all be one file

Open histonames\$ For Input As \#1

For $\mathrm{i}=1$ To 7

Input \#1, histoname $(0, \mathrm{i})$

Input \#1, histoname $(1, \mathrm{i})$

'Msgbox (histoname $(0, \mathrm{i})+$ " " + histoname $(1, \mathrm{i})$ )

Next i

Close \#1

For $\mathrm{i}=1$ To 7

Open histoname $(0$, i) For Output As \#2

Open histoname(1, i) For Output As \#3

Print \#2, 8000; " qmin, "; quadmin(i); ", qmax "; quadmax(i)

Print \#3, 8000; " imin, "; inmin(i); ", imax "; inmax(i)

For $\mathrm{j}=1$ To 8000

Print \#2, histo $(0, i, j)$

Print \#3, histo( $1, i, j)$

Next $\mathrm{j}$

Close \#2

Close \#3

Next i

'MsgBox ("globalhistout Finished")

End Sub

Sub dohist()

Rem init array

For $\mathrm{j}=1$ To 8000

For $\mathrm{i}=1$ To 7

$\operatorname{histo}(0, i, j)=0$

$\operatorname{histo}(1, \mathrm{i}, \mathrm{j})=0$

Next i

Next $\mathrm{j}$

$\mathrm{n}=1$

Rem calculates histgrams for all cases

For icount $=1$ To jcount

For $\mathrm{k}=8$ To 14

$\mathrm{j}=\mathrm{k}-7$

histo0index $=\operatorname{Int}(\operatorname{Rawdata}(0, \mathrm{k}$, icount $)-$ gquadmin $)$

histolindex $=\operatorname{Int}(\operatorname{Rawdata}(1, \mathrm{k}$, icount $)-$ ginmin $)$

$\operatorname{histo}(0, \mathrm{j}$, histo0index $)=\operatorname{histo}(0, \mathrm{j}$, histo0index $)+1$ 
$\operatorname{histo}(1, \mathrm{j}$, histo1 index $)=\operatorname{histo}(1, \mathrm{j}$, histolindex $)+1$

Next k

Next icount

Rem find the mean value for the histogram

Rem put it at the end of the histogram

Rem sum up the number of items at each location

For $i=1$ To 7

$\operatorname{histsum}(0, i)=0$

$\operatorname{histsum}(1, i)=0$

Next i

For $\mathrm{i}=1$ To 7

'MsgBox ("start")

num $0=0$

num $1=0$

For $\mathrm{j}=1$ To 8000

num0 $=$ num0 $+\operatorname{Abs}($ histo $(0, i, j))$

num $1=$ num $1+\operatorname{Abs}($ histo $(1, i, j))$

$\operatorname{histsum}(0, i)=\operatorname{histsum}(0, i)+j * \operatorname{Abs}(\operatorname{histo}(0, i, j))$

$\operatorname{histsum}(1, i)=\operatorname{histsum}(1, i)+j * \operatorname{Abs}(\operatorname{histo}(1, i, j))$

Next j

$\operatorname{histmean}(0, \mathrm{i})=\operatorname{histsum}(0, \mathrm{i}) /$ num0

$\operatorname{histmean}(1, \mathrm{i})=\operatorname{histsum}(1, \mathrm{i}) /$ num 1

Next $\mathrm{i}$

$\operatorname{Rem} * * * * * * * * * * * * * * * * * * * * * * * * * * * * * * * * * * * * * * * * * * * *$

'MsgBox ("Dohist Finished")

End Sub

Sub globalhistout()

Open histonames\$ For Input As \#1

For $\mathrm{i}=1$ To 7

Input $\# 1$, histoname $(0, i)$

Input \#1, histoname $(1, \mathrm{i})$

'Msgbox (histoname $(0, \mathrm{i})+$ " " + histoname $(1, \mathrm{i})$ )

Next i

Close \#1

For $\mathrm{i}=1$ To 7

Open histoname $(0$, i) For Output As \#2

Open histoname $(1$, i) For Output As \#3

Print \#2, 8000; " qmin, "; quadmin(i); ", qmax "; quadmax(i); ", pos0min ";

Str\$(pos0min); ", pos0max "; Str\$(pos0max); ", pos1min "; Str\$(pos1min); ", pos1max "; Str\$(pos 1 max)

Print \#3, 8000; " imin, "; inmin(i); ", imax "; inmax(i); ", pos0min ";

Str\$(pos0min); ", pos0max "; Str\$(pos0max); ", pos1min "; Str\$(pos1min); ", pos1max "; Str\$(pos1max)

For $\mathrm{j}=1$ To 8000

Print \#2, histo $(0, i, j)$

Print \#3, histo $(1, i, j)$

Next j

Close \#2

Close \#3 
Next $\mathrm{i}$

'MsgBox ("globalhistout Finished")

End Sub

Sub localhistout()

$\mathrm{f} 2=$ "C: $\backslash$ WINDOWS $\backslash$ Desktop $\backslash$ Hist_temp $\backslash "+$ TVal + "hist_where_" + id $\$(\mathrm{~m})+$ "_Q_" + ".txt"

$\mathrm{f} 3=$ "C: $\backslash$ WINDOWS $\backslash$ Desktop $\backslash$ Hist_temp $\backslash "$ + TVal + "hist_where_" + id $\$(\mathrm{~m})+$

"I

$\mathrm{f6}=$ "C: $\backslash$ WINDOWS $\backslash$ Desktop $\backslash$ Hist_temp $\backslash "+$ TVal + "hist_mean_" + AreaNum +

"_Q.txt"

$\mathrm{f} 7$ = "C: $\backslash$ WINDOWS $\backslash$ Desktop $\backslash$ Hist_temp $\backslash "$ + TVal + "hist_mean_" + AreaNum + "I.txt"

Open 12 For Output As \#2

Open f3 For Output As \#3

Open f6 For Append As \#6

Open $\mathrm{f} 7$ For Append As \#7

Print \#2, 8000; " , "

gquadmin $=$ quadmin $(1)$

gquadmax $=$ quadmax $(1)$

ginmin $=$ inmin $(1)$

$\operatorname{ginmax}=\operatorname{inmax}(1)$

For $\mathrm{i}=1$ To 7

Print \#2, quadmin(i); " , "; quadmax(i); " , ";

Print \#3, inmin(i); ", "; inmax(i); " , ";

Next $\mathrm{i}$

Rem output section for target specific file

Print \#2, gquadmin; ", pos0min, "; Str\$(pos0min);

Print \#3, ginmin; ", pos0min, "; Str\$(pos0min);

For $\mathrm{j}=1$ To 8000

Hgrp0 = ""

Hgrp 1 = ""

For $\mathrm{i}=1$ To 7

Hgrp0 $=\operatorname{Hgrp} 0+\operatorname{Str} \$($ histo $(0, i, j))+", "$

Hgrp1 = Hgrp1 + Str\$(histo $(1, \mathrm{i}, \mathrm{j}))+$ ", "

Next $\mathrm{i}$

Print \#2, Hgrp0

Print \#3, Hgrp1

Next j

Rem print a blank line and then the histogram offset and mean

Print \#2, "'"

Print \#3, "'

Print \#2, "mean values"

Print \#3, "mean values"

Print \#6, gquadmin; " , ";

Print \#7, ginmin; " , ";

For $\mathrm{i}=1$ To 7

Print \#2, histmean $(0, i)$; " , ";

Print \#3, histmean(1, i); " , ";

Print \#6, histmean $(0, \mathrm{i})$; " , "; 


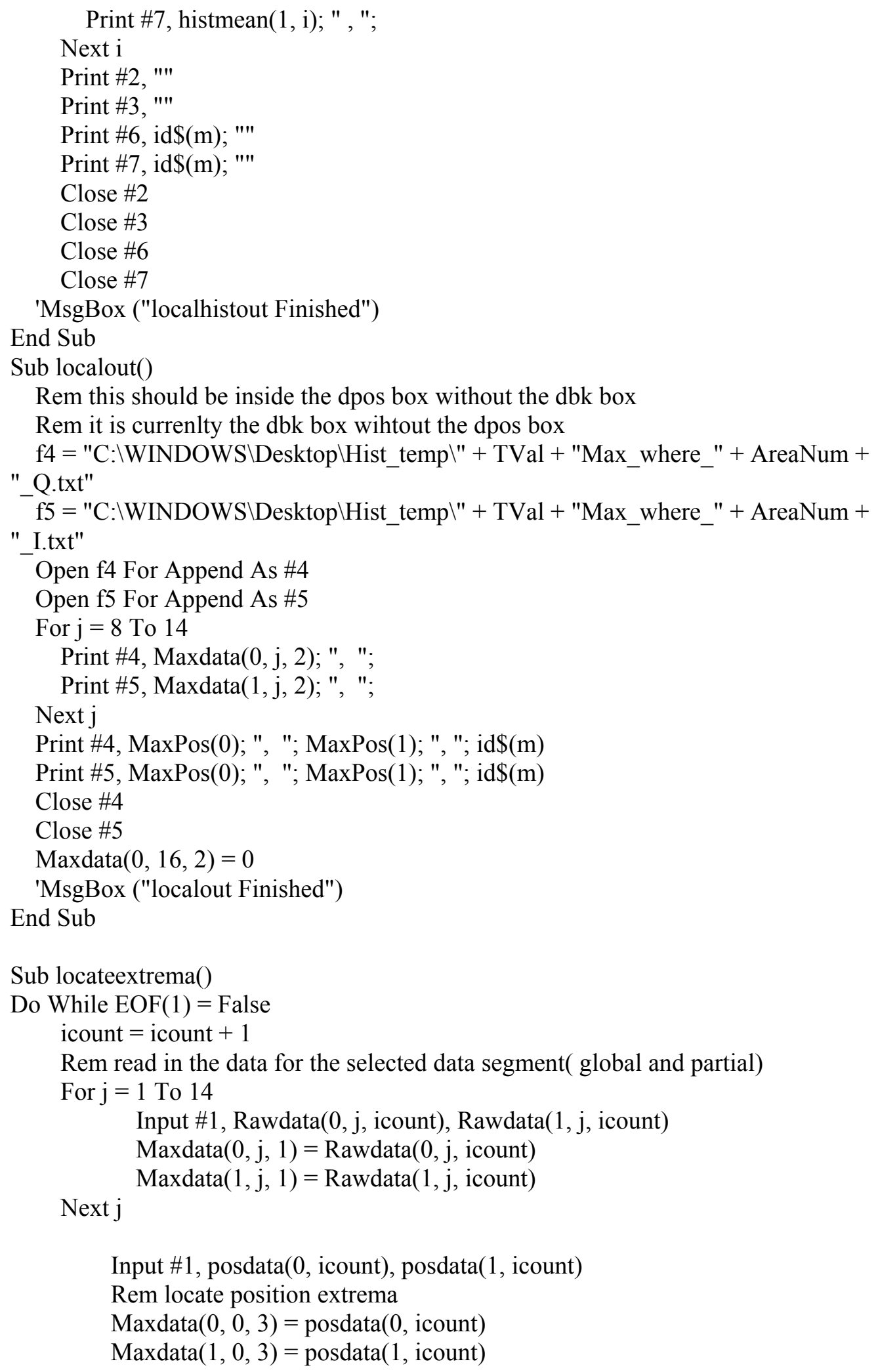


If Tag_This $=$ False Then

Input $\# 1$, Rawdata( 0,15 , incount)

$\operatorname{Maxdata}(0,15,1)=\operatorname{Rawdata}(0,15$, icount $)$

End If

For $\mathrm{j}=8$ To 14

If $\operatorname{Rawdata}(0, j$, icount $)>8000$ Then $\operatorname{Rawdata}(0, j$, icount $)=0$

If $\operatorname{Rawdata}(1, \mathrm{j}$, icount $)>8000$ Then $\operatorname{Rawdata}(1, \mathrm{j}$, icount $)=0$

icount)

If $\operatorname{Rawdata}(0, j$, icount $)<$ quadmin $(j-7)$ Then quadmin $(j-7)=\operatorname{Rawdata}(0, j$,

icount)

If $\operatorname{Rawdata}(0, j$, icount $)>\operatorname{quadmax}(j-7) \operatorname{Then} q u a d m a x(j-7)=\operatorname{Rawdata}(0, j$,

If $\operatorname{Rawdata}(1, \mathrm{j}$, icount $)<\operatorname{inmin}(j-7)$ Then $\operatorname{inmin}(j-7)=\operatorname{Rawdata}(1, j$, icount $)$

If $\operatorname{Rawdata}(1, j$, icount $)>\operatorname{inmax}(j-7) \operatorname{Then} \operatorname{inmax}(j-7)=\operatorname{Rawdata}(1, j$,

icount)

If $b b=0$ Then

$\operatorname{pos} 0 \mathrm{max}=\operatorname{posdata}(0$, icount $)$

$\operatorname{pos} 0 \mathrm{~min}=\operatorname{posdata}(0$, icount $)$

$\operatorname{pos} 1 \mathrm{max}=\operatorname{posdata}(1$, icount $)$

$\operatorname{pos} 1 \mathrm{~min}=\operatorname{posdata}(1$, icount $)$

$\mathrm{bb}=1$

End If

If $\operatorname{pos} 0 \max <\operatorname{posdata}(0$, icount $)$ Then

$\operatorname{pos} 0 \mathrm{max}=\operatorname{posdata}(0$, icount $)$

Labe19.Caption $=\operatorname{Str}(\operatorname{pos} 0 \max )+"$ " $+\operatorname{Str}(\operatorname{pos} 1 \max )$

End If

If $\operatorname{pos} 0 \mathrm{~min}>\operatorname{posdata}(0$, icount $)$ Then

$\operatorname{pos} 0 \mathrm{~min}=\operatorname{posdata}(0$, icount $)$

Label10.Caption $=\operatorname{Str}(\operatorname{pos} 0 \mathrm{~min})+"$ " + Str(pos1min $)$

End If

If pos1max $<\operatorname{posdata}(1$, icount $)$ Then

$\operatorname{pos} 1 \max =\operatorname{posdata}(1$, icount $)$

Labe19.Caption $=\operatorname{Str}(\operatorname{pos} 0 \max )+" ~ "+\operatorname{Str}(\operatorname{pos} 1 \max )$

End If

If pos1min $<\operatorname{posdata}(1$, icount $)$ Then

pos $1 \mathrm{~min}=\operatorname{posdata}(1$, icount $)$

Labe19.Caption $=\operatorname{Str}(\operatorname{pos} 0 \mathrm{~min})+"$ " + Str(pos1min $)$

End If

Rem If icount $/ 25000=\operatorname{Int}($ icount $/ 25000)$ Then MsgBox $(\operatorname{Str}(\operatorname{posdata}(0$, icount $))+$

" " + Str(posdata $(1$, icount $)))$

Next j

$\operatorname{Maxdata}(0,16,1)=0$

$\operatorname{Maxdata}(1,16,1)=0$

For $\mathrm{i}=8$ To 14

$\operatorname{Maxdata}(0,16,1)=\operatorname{Maxdata}(0,16,1)+\operatorname{Abs}(\operatorname{Maxdata}(0, i, 1))+$ $\operatorname{Abs}(\operatorname{Maxdata}(1, \mathrm{i}, 1))$ 


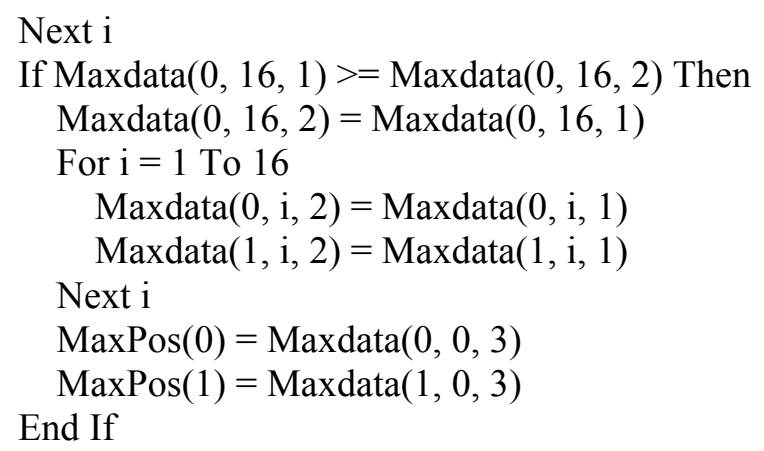

Next i

If $\operatorname{Maxdata}(0,16,1)>=\operatorname{Maxdata}(0,16,2)$ Then

$\operatorname{Maxdata}(0,16,2)=\operatorname{Maxdata}(0,16,1)$

For $\mathrm{i}=1$ To 16

$\operatorname{Maxdata}(0, i, 2)=\operatorname{Maxdata}(0, i, 1)$

$\operatorname{Maxdata}(1, i, 2)=\operatorname{Maxdata}(1, i, 1)$

Next i

$\operatorname{MaxPos}(0)=\operatorname{Maxdata}(0,0,3)$

$\operatorname{MaxPos}(1)=\operatorname{Maxdata}(1,0,3)$

End If

\section{Loop}

Rem find the minimum values for all seven frequency both signals

gquadmin $=$ quadmin $(1)$

ginmin $=$ inmin $(1)$

For $\mathrm{i}=2$ To 7

If gquadmin $>$ quadmin(i) Then gquadmin $=$ quadmin(i)

If ginmin $>$ inmin(i) Then ginmin $=$ inmin(i)

Next i

'MsgBox ("localextrema Finished")

End Sub

Sub initalizelocal()

$\mathrm{f} 1=$ "C: $\backslash$ WINDOWS $\backslash$ Desktop $\backslash$ Hist_temp $\backslash "$ + TVal + "hist_where_" + id $\$(\mathrm{~m})+$ ".txt"

If Dir(f1) = "" Then

Open f1 For Output As \#9

Print \#9, "1, 1, 1, 1, 1, 1, 1, 1, 1, 1, 1, 1, 1, 1, 1, 1, 1, 1, 1, 1, 1, 1, 1, 1, 1, 1, 1, 1, 1, 1, $"+\mathrm{id} \$(\mathrm{~m})$

Close \#9

\section{End If}

Rem initalize the position extrema

$\operatorname{pos} 0 \max =0$

$\operatorname{pos} 0 \min =0$

$\operatorname{pos} 1 \max =0$

$\operatorname{pos} 1 \min =0$

$\mathrm{bb}=0$

Rem initalize the signal extrema

For $\mathrm{k}=1$ To 8

quadmin $(\mathrm{k})=0$

quadmax $(\mathrm{k})=0$

$\operatorname{inmin}(\mathrm{k})=0$

$\operatorname{inmax}(\mathrm{k})=0$

Next k

For $\mathrm{i}=0$ To 1

For $\mathrm{j}=0$ To 16

For $\mathrm{k}=0$ To 15

$\operatorname{Maxdata}(i, j, k)=0$

Next k

Next j 
Next $i$

Open f1 For Input As \#1

'MsgBox ("initalizelocal Finished")

End $\mathrm{Sub}$

\author{
Private Sub AllIt_Click() \\ If AllIt.Checked $=$ False Then \\ TarIt .Checked $=$ False \\ FragIt. Checked $=$ False \\ AllIt.Checked $=$ True \\ GphxIt.Checked $=$ False
}

End If

End Sub

Private Sub AOneIt_Click()

If AOneIt. Checked $=$ False Then

AOneIt.Checked $=$ True

ATwoIt.Checked $=$ False

AThreeIt.Checked $=$ False

End If

End Sub

Private Sub AThreeIt_Click()

If AThreeIt.Checked $=$ False Then

AOneIt.Checked $=$ False

ATwoIt. Checked $=$ False

AThreeIt.Checked $=$ True

End If

End Sub

Private Sub ATwoIt_Click()

If ATwoIt.Checked $=$ False Then

AOneIt.Checked $=$ False

ATwoIt.Checked $=$ True

AThreeIt.Checked $=$ False

End If

End Sub 
Private Sub Command2_Click()

Rem determine which area to get data from

If AOneIt.Checked $=$ True Then

AreaNum $=" 1 "$

End If

If ATwoIt.Checked $=$ True Then

AreaNum = "2"

End If

If AThreeIt.Checked = True Then

AreaNum = "3"

End If

If TarIt.Checked $=1$ Then

$\mathrm{TVal}=$ "T"

End If

If FragIt.Checked $=1$ Then

TVal $=$ "F"

End If

If AllIt.Checked $=1$ Then

TVal = "A"

End If

If GphxIt.Checked = 1 Then

TVal $=$ "GT"

End If

Form1. MousePointer $=$ vbHourglass

'f1 = wheredata $\$$

$\mathrm{fl}=$ "C: $\backslash$ WINDOWS $\backslash$ Desktop $\backslash "$ + TVal + "where_" + AreaNum + "a_data.txt"

$\mathrm{f} 2=$ "C: $\backslash$ WINDOWS $\backslash$ Desktop $\backslash$ Hist_temp $\backslash "+$ TVal + "hist_mean_" + AreaNum +

"Q.txt"

$\mathrm{f} \overline{3}=$ "C: $\backslash$ WINDOWS $\backslash$ Desktop $\backslash$ Hist_temp $\backslash "$ + TVal + "hist_mean_" + AreaNum + "_I.txt"

$\mathrm{f} 4 \$=$ "c: $\backslash$ windows $\backslash$ desktop $\backslash$ Hist_temp $\backslash "$ + TVal + "Area" + AreaNum + "a_data_q.txt"

$\mathrm{f} 5 \$=$ "c: $\backslash$ windows $\backslash$ desktop $\backslash$ Hist temp $\backslash "$ + TVal + "Area" + AreaNum + "a data i.txt"

f6\$ = "c:|windows \desktop \Hist_temp $\backslash "$ + TVal + "Area" + AreaNum +

"a_data_q_bckc.txt"

$\mathrm{f} 7 \overline{\$}=$ "c: $\backslash$ windows $\backslash$ desktop $\backslash$ Hist temp $\backslash "$ + TVal + "Area" + AreaNum +

"a_data_i_bckc.txt"

Open f1 For Input As \#1

Open $\mathrm{f} 2$ For Input As \#2

Open f3 For Input As \#3

icount $=0$

Do While $\operatorname{EOF}(1)=$ False

icount $=$ icount +1

Rem read in the data for the selected data segment( global and partial) 


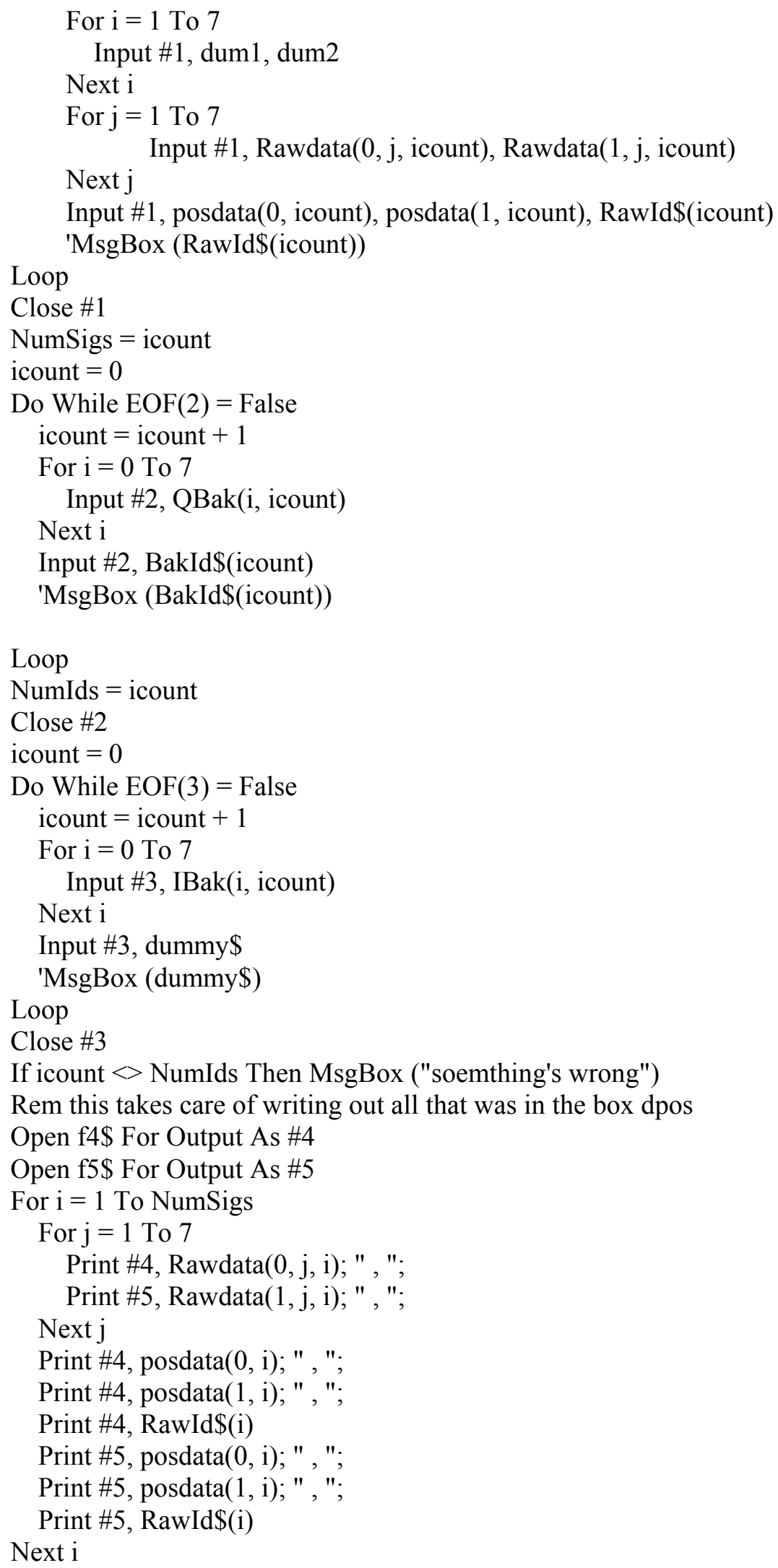


Close \#4

Close \#5

Rem now we need to do the background subtraction from rawdata and do it again

For $\mathrm{i}=1$ To NumSigs

For $\mathrm{j}=1$ To NumIds

If $\operatorname{RawId} \$(\mathrm{i})=\operatorname{BakId} \$(\mathrm{j})$ Then

For $\mathrm{k}=1$ To 7

$\operatorname{Rawdata}(0, \mathrm{k}, \mathrm{i})=\operatorname{Rawdata}(0, \mathrm{k}, \mathrm{i})-\mathrm{QBak}(\mathrm{k}, \mathrm{j})-\mathrm{QBak}(0, \mathrm{j})$

$\operatorname{Rawdata}(1, \mathrm{k}, \mathrm{i})=\operatorname{Rawdata}(1, \mathrm{k}, \mathrm{i})-\operatorname{IBak}(\mathrm{k}, \mathrm{j})-\operatorname{IBak}(0, \mathrm{j})$

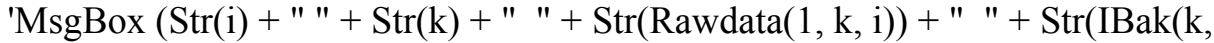

$\mathrm{j}))+"$ " + Str( $\operatorname{IBak}(0, \mathrm{j}))+"$ " + $\operatorname{BakId} \$(\mathrm{j}))$

Next k

End If

Next $j$

Next $\mathrm{i}$

Rem write out the new files

Open f6\$ For Output As \#6

Open f7\$ For Output As \#7

For $\mathrm{i}=1$ To NumSigs

For $\mathrm{j}=1$ To 7

Print \#6, Rawdata(0, j, i); " , ";

Print \#7, Rawdata(1, j, i); " , ";

Next j

Print \#6, posdata $(0,1)$; " , ";

Print \#6, posdata(1, i); " , ";

Print \#6, RawId\$(i)

Print \#7, posdata( 0,1$)$; " , ";

Print \#7, posdata(1, i); " , ";

Print \#7, RawId\$(i)

Next i

Close \#6

Close \#7

Form1. MousePointer $=$ vbDefault

End Sub

Private Sub importit_Click()

End Sub

Private Sub Form_Load()

Rem this sets the area that is being processed.

'AreaNum = "1" 


\section{End Sub}

Private Sub FragIt_Click()

If FragIt.Checked $=$ False Then

TarIt.Checked $=$ False

FragIt.Checked $=$ True

AllIt. Checked $=$ False

GphxIt.Checked $=$ False

End If

End Sub

Private Sub GphxIt_Click()

If GphxIt.Checked = False Then

TarIt. Checked $=$ False

FragIt . Checked $=$ False

Allit. Checked $=$ False

GphxIt.Checked $=$ True

End If

End Sub

Private Sub ingemittest_Click()

If AOneIt.Checked $=$ True Then AreaNum = "1"

End If

If ATwoIt.Checked $=$ True Then AreaNum = "2"

End If

If AThreeIt.Checked = True Then AreaNum = "3"

End If

If TarIt.Checked $=$ True Then $\mathrm{TVal}=$ "T"

End If

If FragIt.Checked $=$ True Then $\mathrm{TVal}=$ "F"

End If

If AllIt.Checked $=$ True Then TVal = "A"

End If

If GphxIt.Checked = True Then

TVal = "GT"

End If

$\mathrm{cc}=1$ 


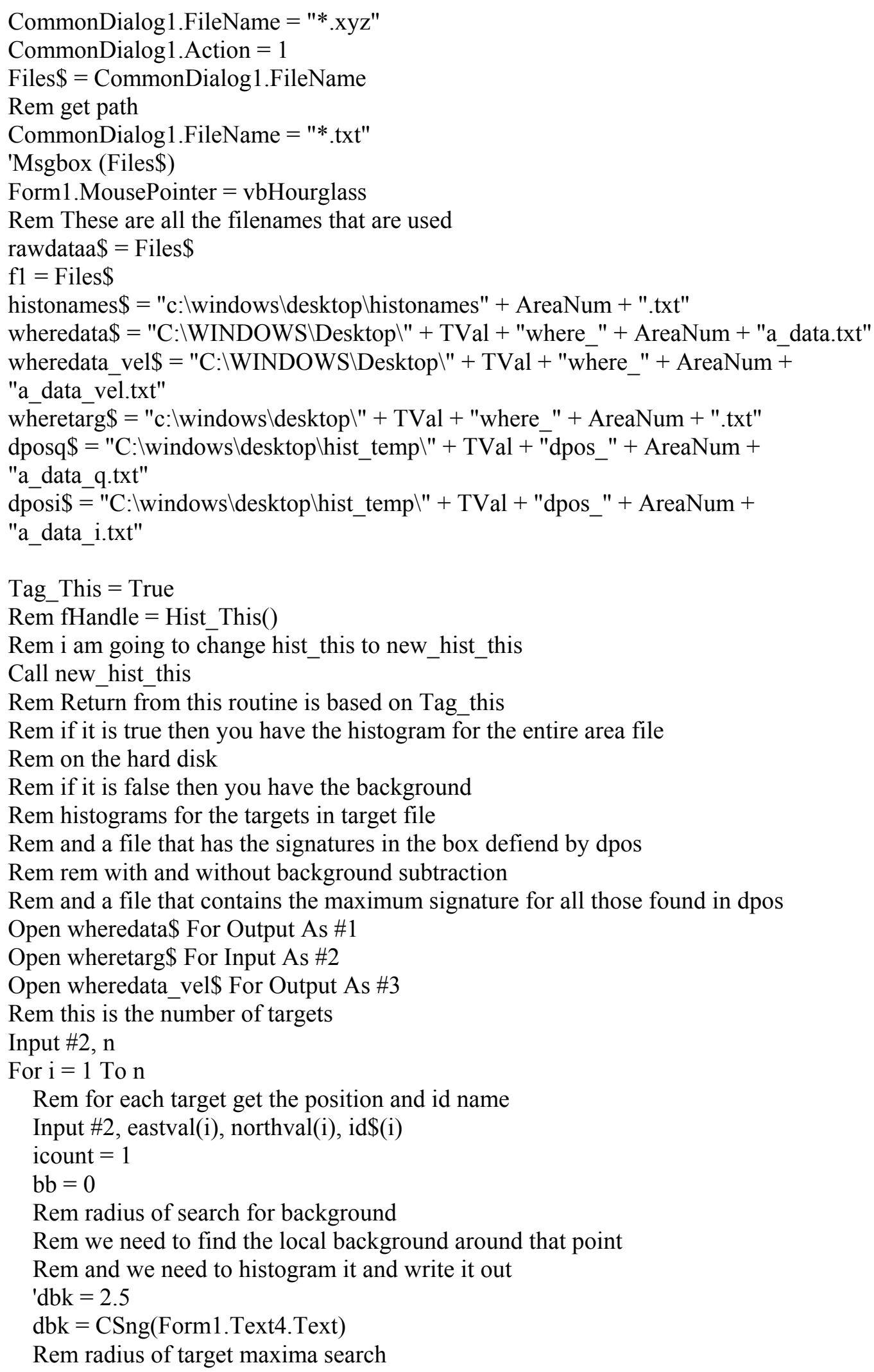




$$
\begin{aligned}
& \text { 'dpos }=0.25 \\
& \text { dpos }=\text { CSng(Form1.Text5.Text) } \\
& \text { 'dbox }=0.5 \\
& \text { dbox }=\text { CSng(Form1.Text6.Text) } \\
& \text { Rem init postion holders } \\
& \text { nd }=0 \\
& \text { ed }=0
\end{aligned}
$$

loopto:

Rem $\mathrm{p}^{*} \mathrm{~d}$ was the last position

pnd $=$ nd

ped $=$ ed

Rem radial of last position

$\mathrm{pr}=\left(\mathrm{pnd}^{\wedge} 2+\mathrm{ped}^{\wedge} 2\right)^{\wedge} 0.5$

Rem posiition of item

nd $=\operatorname{posdata}(0$, icount $)$

ed $=\operatorname{posdata}(1$, icount $)$

Rem for $\mathrm{dx}=$ zero make $\mathrm{dth}=0$ to avoid an error.

$\mathrm{dx}=$ pnd $-\mathrm{nd}$

If $\mathrm{dx}=0$ Then

$\mathrm{dth}=0$

Else

$\mathrm{dth}=\operatorname{Atn}(\mathrm{dy} / \mathrm{dx})$

End If

dy $=$ ped - ed

$\mathrm{ddr}=\left(\mathrm{dx}^{\wedge} 2+\mathrm{dy}^{\wedge} 2\right)^{\wedge} 0.5$

rnow $=\left(\mathrm{nd}^{\wedge} 2+\mathrm{ed}^{\wedge} 2\right)^{\wedge} 0.5$

If $\mathrm{Abs}(\mathrm{pr}-\mathrm{rnow})>0.025$ Then

'If nd $<$ (northval(i) + dpos) And nd $>$ (northval(i) - dpos) And ed $<$ (eastval(i) +

dpos) And ed $>$ (eastval(i) - dpos) Then

' For $\mathrm{j}=1$ To 14

' Print \#1, Rawdata(0, j, icount); ", "; Rawdata(1, j, icount); ", ";

' Next j

' Print \#1, ed; ", "; nd; ", "; id\$(i)

'End If

Rem this is selecting the area to look at

Rem the problem has been that this area is where we are

Rem trying to generate the maxima from and we have

Rem cut out the target values

Rem so the maxima is not there anymore

'MsgBox ("first test")

If nd $<$ (northval(i) + dpos) And nd $>$ (northval(i) - dpos) And ed $<$ (eastval(i) + dpos) And ed $>$ (eastval(i) - dpos) Then

'MsgBox (Str(northval(i) + dpos) + " " + Str(nd) + " " + Str(northval(i) - dpos)

+ " " + Str(eastval(i) + dpos) + " " + Str(ed) + " " + Str(eastval(i) - dpos))

Rem this data goes to the maxima file and is a canidate for a valid signal

For $\mathrm{j}=1$ To 14

Print \#1, Rawdata(0, j, icount); ", "; Rawdata(1, j, icount); ", "; 
Next j

Print \#1, ed; ", "; nd; ", "; id\$(i)

Print \#3, ed; ", "; nd; ", "; id\$(i); " , "; dx; " , "; dy

End If

If nd $<$ (northval(i) + dbk) And nd $>$ (northval(i) - dbk) And ed $<$ (eastval(i) + dbk) And ed $>$ (eastval(i) - dbk) And Not (nd $<$ (northval(i) + dbox) And nd $>$

(northval(i) - dbox) And ed $<$ (eastval(i) + dbox) And ed $>$ (eastval(i) - dbox)) Then

+ ".txt"

f5 = "C: \WINDOWS \Desktop \Hist_temp $\backslash "+$ TVal + "hist_where_" + id\$(i)

'MsgBox (f5 + " opened")

Open f5 For Append As \#5

For $\mathrm{j}=1$ To 14

Print \#5, Rawdata( $0, j$, icount); ", "; Rawdata( $1, \mathrm{j}$, icount); ", ";

Next j

Print \#5, ed; ", "; nd; ", "; id\$(i)

Close \#5

End If

End If

icount $=$ icount +1

Next i

If icount $<=$ jcount Then GoTo loopto

Close \#3

Close \#2

Close \#1

'commenting out this part to speed up the output

'this is the part that does the individual histograming

Rem this is going to go away

Rem hist this is going to become

Rem lochist this and no global stuff will be there

Rem it will only deal with target items

'Tag_This $=$ False

'fHandle $=$ Hist_This ()

Rem call lochist_this

Call lochist_this

Rem the histograms now exist

Rem the problem was that the maxima file had been written without the dpos box in the data set

Rem I am now going to call a subprogram to create them

Rem it will be a variation of Hist this

Rem but it will be called dpos_maxima

Call dpos_maxima 
Form1.MousePointer $=$ vbDefault

MsgBox ("search complete" + Chr(13) + "icount = " + CStr(icount $)+\operatorname{Chr}(13)+$ "jocunt $="+\operatorname{CStr}($ jcount $))$

End Sub

Public Function Hist_This()

Rem the case of Tag_this true is the global histogram of the entire data set

Rem false is the local target case

If Tag_This $=$ True Then

Rem read off the header

Open rawdataa\$ For Input As \#1

For $\mathrm{i}=1$ To 30

Input $\# 1$, head\$(i)

Next i

Floop $=1$

Else

Floop $=n$

End If

For $\mathrm{m}=1$ To Floop

If Tag_This $=$ False Then

Rem initalize variables for the local case

Call initalizelocal

End If

icount $=0$

Rem find the extrema

Call locateextrema

If Tag_This $=$ False Then

Rem output maxima data for the local case

Call localout

End If

jcount $=$ icount

Close \#1

Rem histogram in amplitude for each frequnecy and phase

Call dohist

$\operatorname{Rem} * * * * * * * * * * * * * * * * * * * * *$

Rem put out histogram for each

Rem filenames are quadf 1, infl, ...

Rem get these names

Rem for the global file

If Tag_This $=$ True Then

Call globalhistout

Else

Call localhistout 


\section{End If}

Next m

End Function

Private Sub TarIt_Click()

If TarIt. Checked $=$ False Then

TarIt.Checked $=$ True

FragIt. Checked $=$ False

AllIt. Checked $=$ False

GphxIt.Checked $=$ False

End If

End Sub

Private Sub toolit_Click()

End Sub 


\title{
GridTool Program
}

\author{
Sub setscroll() \\ LLIMIT $=$ Form 1. HScroll1. Value \\ ULIMIT = Form1.HScroll2.Value \\ ULIMIT $=$ TX $*$ LLIMIT / Form1.HScroll3.Max \\ LLIMIT $=$ TX $*$ ULIMIT $/$ Form1.HScroll2.Max \\ If ULIMIT $<$ LLIMIT Then \\ dummy $=$ LLIMIT \\ LLIMIT $=$ ULIMIT \\ ULIMIT $=$ dummy \\ End If \\ For $\mathrm{i}=$ LLIMIT To ULIMIT \\ $\operatorname{map}(i)=\operatorname{RGB}(75,75,75)$ \\ Next $i$ \\ End Sub
}

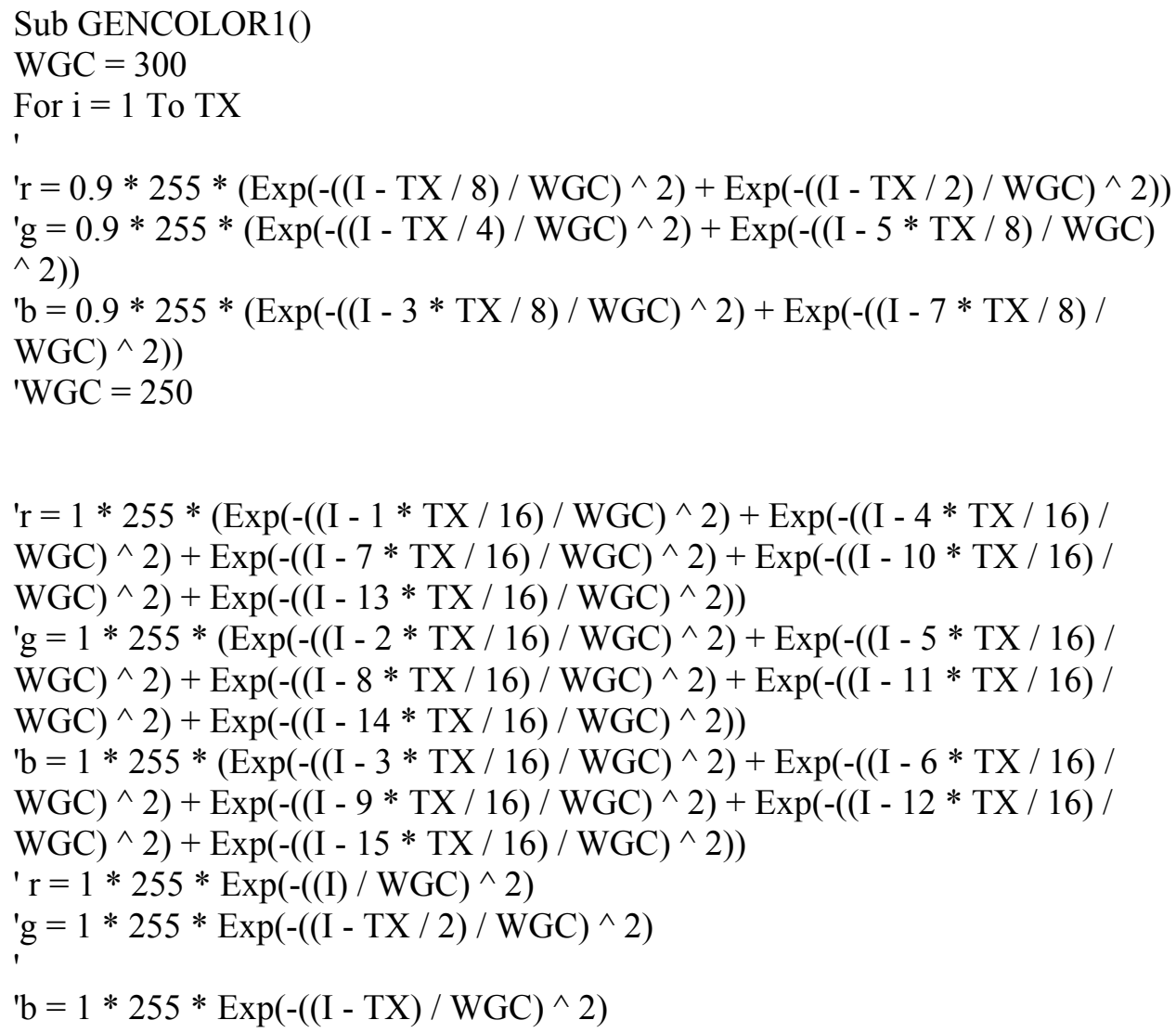




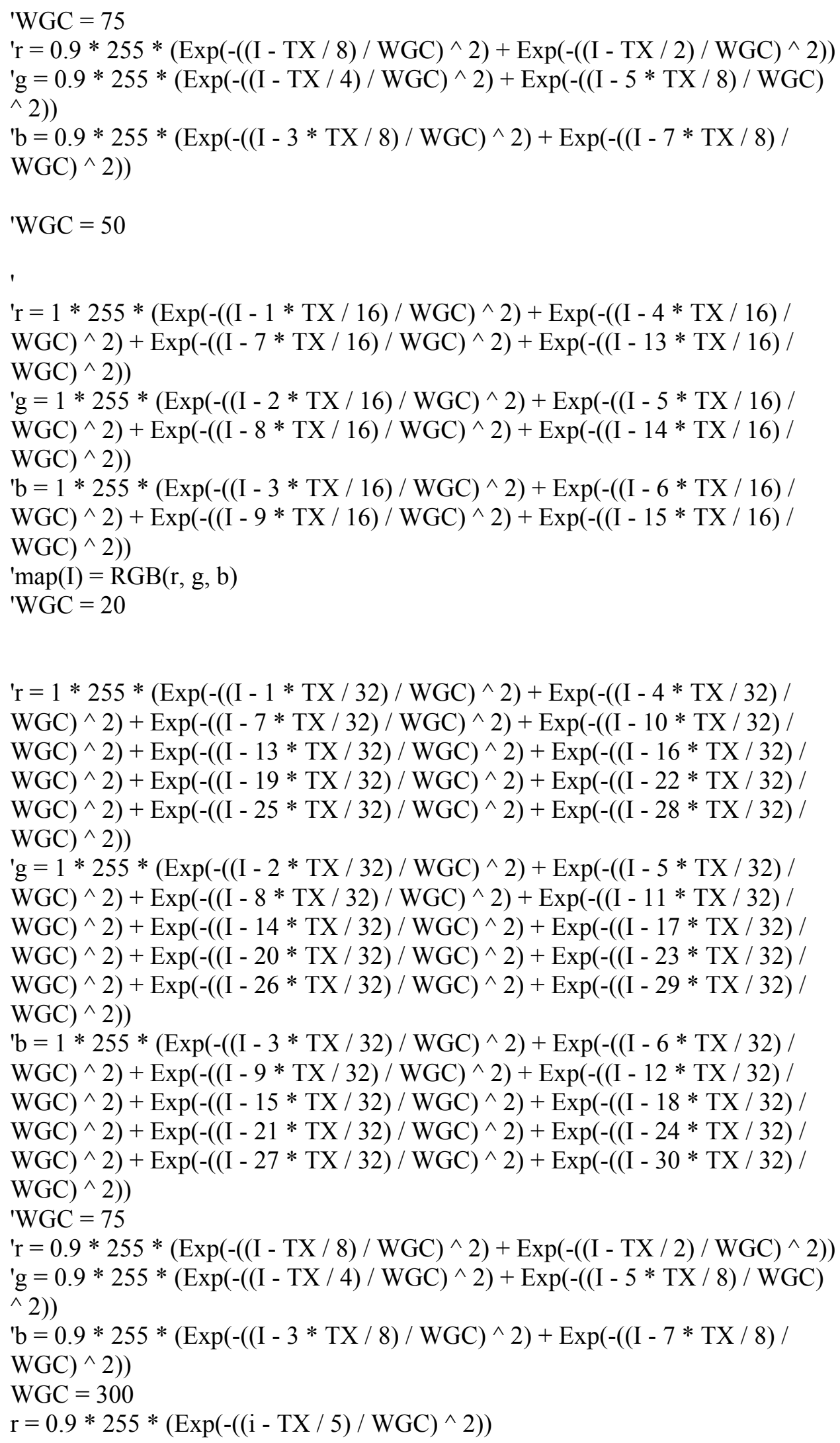




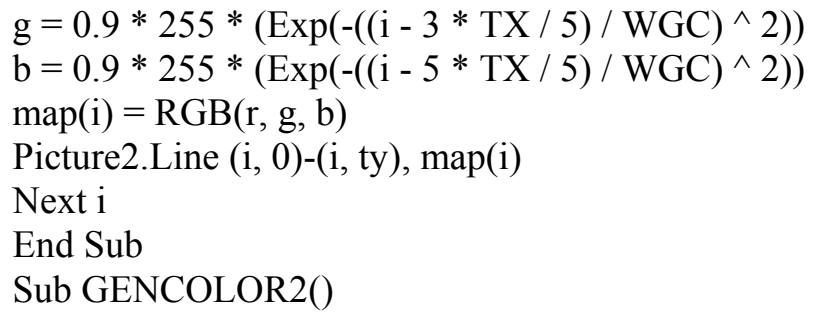

End Sub

Private Sub Command3_Click()

End Sub

Private Sub Command4_Click()

End Sub

Private Sub Command5_Click()

End Sub

Private Sub importit_Click()

End Sub

Private Sub Form_Load()

End Sub

Private Sub ingemittest_Click()

stuff $=0$

$\mathrm{cc}=1$

CommonDialog1.FileName $=$ "*.xyz"

maxspeed $=10000$

CommonDialog1.Action $=1$

Files $\$=$ CommonDialog1.FileName

Rem get path

CommonDialog 1.FileName $=$ "*.txt"

Rem MsgBox (Files\$)

f1 $\$=$ Files $\$$

Text1.Text $=$ Files $\$$

TESTSET $=\operatorname{Val}($ Text5.Text $)-\operatorname{Val}($ Text4.Text $)$

Skip $=$ True

skipto $=\operatorname{Val}($ Text4.Text $)$ 


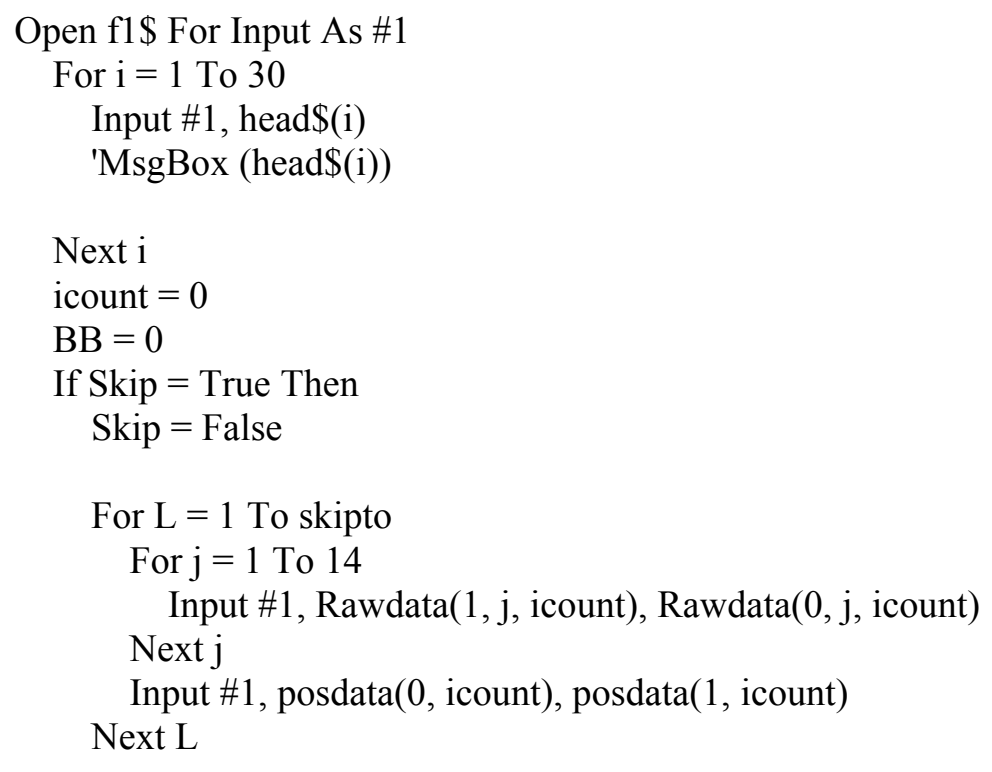

End If

Do While $\operatorname{EOF}(1)=$ False

icount $=$ icount +1

If icount $>$ TESTSET Then GoTo getout

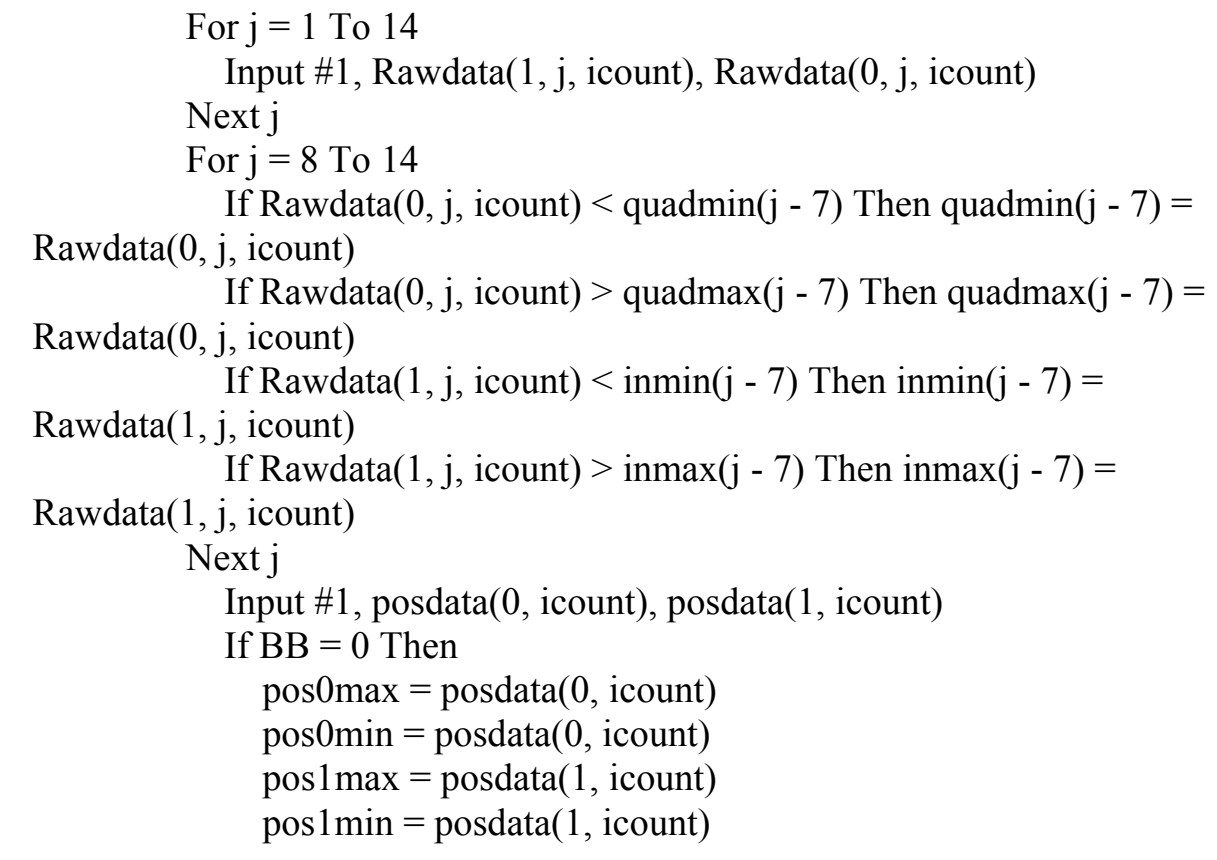


'Print \#3, "'"
Next j

Picture2. ScaleMode $=3$ ' Set ScaleMode to

Picture2.DrawWidth $=1$ ' Set DrawWidth.

Picture2.ForeColor $=$ QBColor(4) ' Set background to red.

Picture2.Cls ' Clear form.

$\mathrm{TX}=$ Picture2.ScaleWidth

ty $=$ Picture2.ScaleHeight

$\mathrm{BB}=0$

For $\mathrm{LL}=1$ To 7

Call GENCOLOR1

DOITAGAIN:

Label5.Caption $=$ "FREQ " + Str\$(LL)

Picture1.BackColor $=\mathrm{QBColor}(0)$

Picture1.ScaleMode $=3$ ' Set ScaleMode to

Picture1.DrawWidth $=1$ ' Set DrawWidth.

Picture1.ForeColor $=$ QBColor(4) ' Set background to red.

Picture1.Cls ' Clear form.

$\mathrm{dx}=\operatorname{pos} 0 \mathrm{max}-\operatorname{pos} 0 \mathrm{~min}$

$\mathrm{dy}=$ pos $1 \mathrm{max}-\operatorname{pos} 1 \mathrm{~min}$

$\mathrm{sx}=$ Picture1.ScaleWidth

sy $=$ Picture1.ScaleHeight

$\mathrm{dz}=$ quadmax $(\mathrm{LL})-$ quadmin $(\mathrm{LL})$

Label6.Caption $=$ Str $\$($ quadmin $($ LL $))$

Label7.Caption $=$ Str $\$($ quadmax $(L L))$

Rem CLEAR HISVAL

Picture3.ScaleMode $=3$ ' Set ScaleMode to

Picture3.DrawWidth $=1$ ' Set DrawWidth.

Picture3.ForeColor $=$ QBColor(4) ' Set background to red.

Picture3.Cls ' Clear form.

TX3 = Picture3.ScaleWidth

TY3 $=$ Picture3.ScaleHeight

Rem ZEROHIST

For $\mathrm{i}=1$ To TX3

$\operatorname{HISVAL}(\mathrm{i})=1$

Next $\mathrm{i}$

For $\mathrm{i}=1$ To TESTSET

$\mathrm{xx}=\mathrm{sx} *(\operatorname{posdata}(0, \mathrm{i})-\operatorname{pos} 0 \mathrm{~min}) / \mathrm{dx}$

yy $=$ sy - sy $*(\operatorname{posdata}(1, i)-\operatorname{pos} 1 \mathrm{~min}) / \mathrm{dy}$

$\mathrm{ZZ}=\mathrm{TX} *(\operatorname{Rawdata}(0,7+\mathrm{LL}, \mathrm{i})-$ quadmin(LL) $) / \mathrm{dz}$

If $\mathrm{ZZ}<0$ Then $\mathrm{ZZ}=1$

HISVAL $(Z Z)=$ HISVAL $(Z Z)+1$

Picture1.PSet (xx, yy), map(ZZ)

If $\mathrm{i} / 1000=\operatorname{Int}(\mathrm{i} / \mathrm{1000})$ Then DoEvents

Next $i$ 


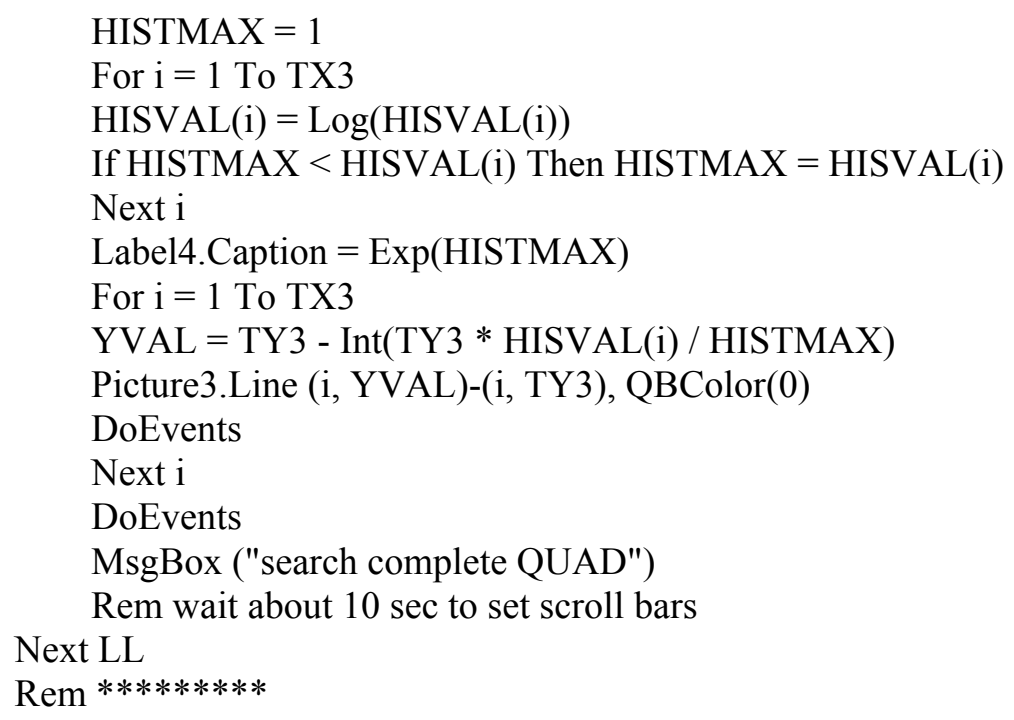




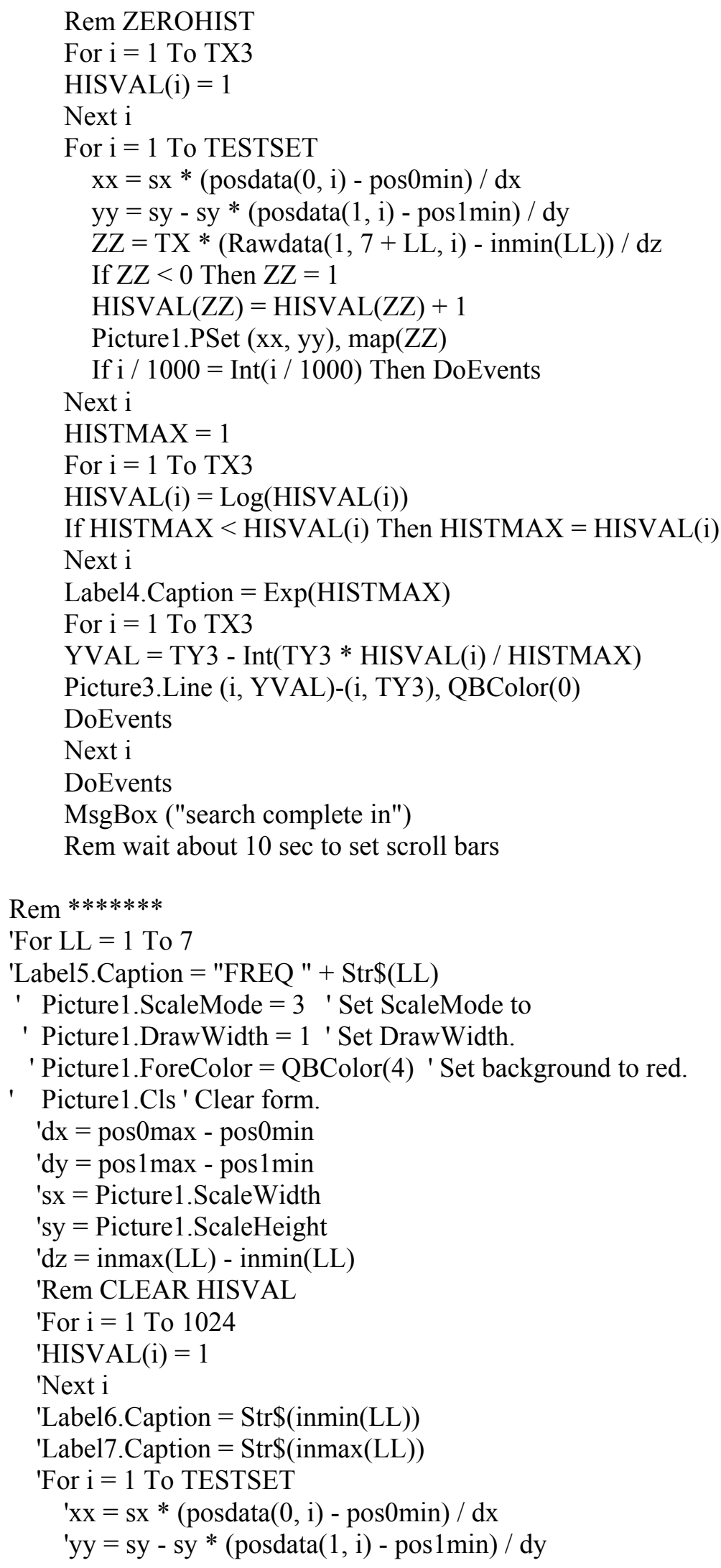




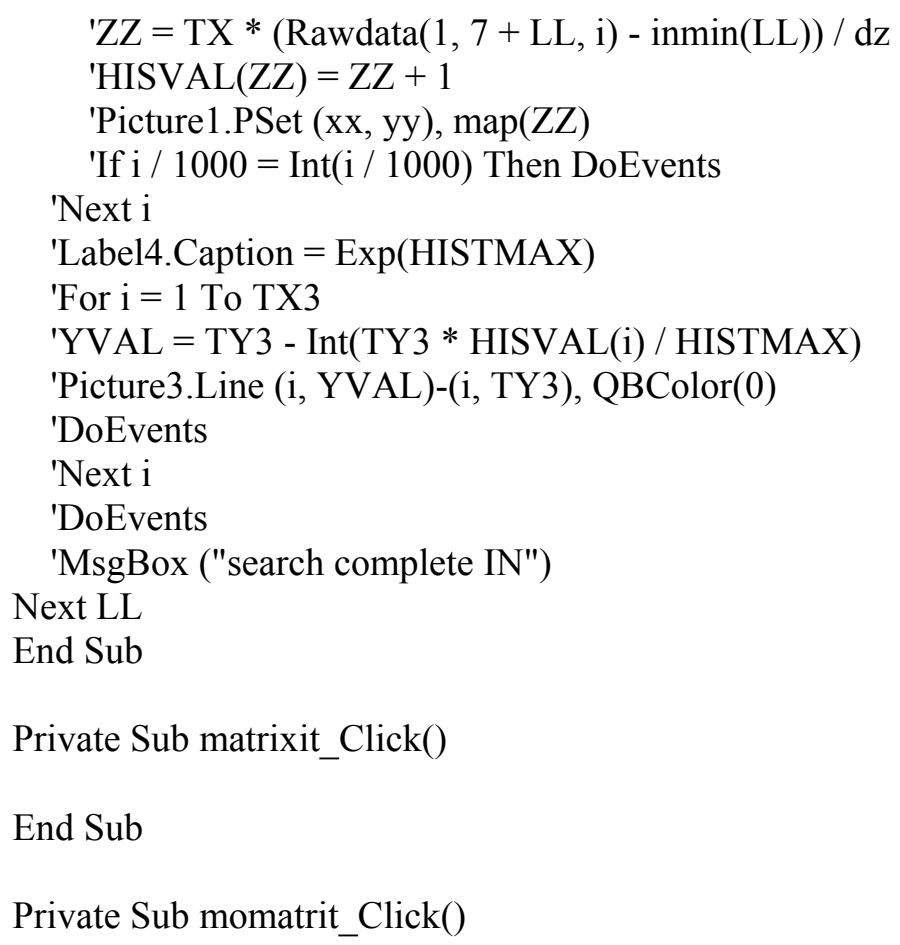

End Sub

Private Sub penit_Click()

End Sub

Private Sub rmatrit_Click()

\section{End Sub}

Private Sub toolit Click()

Picture2. ScaleMode $=3$ ' Set ScaleMode to

Picture2.DrawWidth $=1$ ' Set DrawWidth.

Picture2.ForeColor $=$ QBColor(4) ' Set background to red.

Picture2.Cls ' Clear form.

$\mathrm{TX}=$ Picture2.ScaleWidth

ty $=$ Picture2.ScaleHeight 


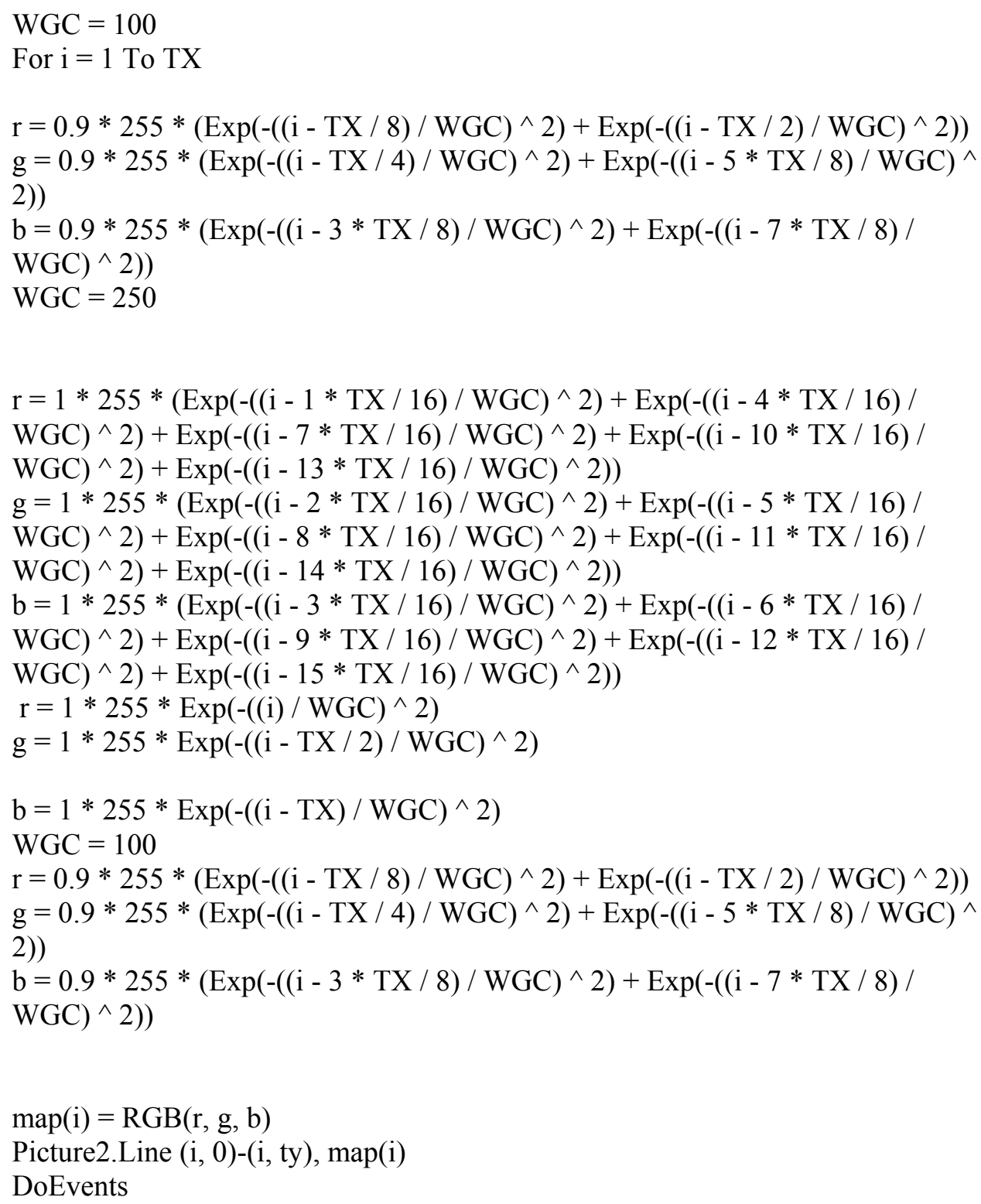

Next i

End Sub 


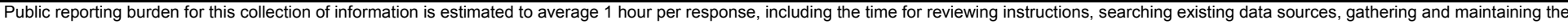

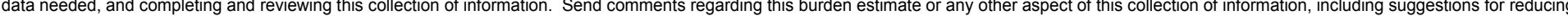

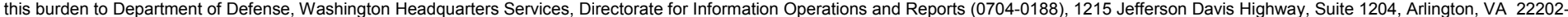

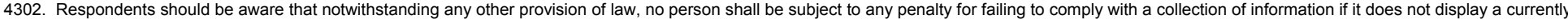
valid OMB control number. PLEASE DO NOT RETURN YOUR FORM TO THE ABOVE ADDRESS.

\begin{tabular}{l|l|l} 
1. REPORT DATE (DD-MM-YYYY) & 2. REPORT TYPE & 3. DATES COVERED (FrOm - TO)
\end{tabular}

September 2002

Final report

4. TITLE AND SUBTITLE

Analysis of GEM-3 Data from the Advanced UXO Detection/Discrimination Technology

Demonstration - U.S. Army Jefferson Proving Ground, Madison, Indiana

5a. CONTRACT NUMBER

\section{AUTHOR(S)}

Ricky A. Goodson, Hollis H. (Jay) Bennett, Jr., Tere' A. DeMoss,

Diane M. Cargile, John C. Morgan, Morris P. Fields

\section{PERFORMING ORGANIZATION NAME(S) AND ADDRESS(ES)}

U.S. Army Engineer Research and Development Center

Environmental Laboratory

3909 Halls Ferry Road

Vicksburg, MS 39180-6199;

Illinois Institute of Technology Research Institute

10 W. $35^{\text {th }}$ Street

Chicago, IL 60616-3717

\section{SPONSORING / MONITORING AGENCY NAME(S) AND ADDRESS(ES)}

10. SPONSOR/MONITOR'S ACRONYM(S)

Headquarters, U.S. Army Corps of Engineers

ERDC/EL TR-02-25

Washington, DC 20314-1000

11. SPONSOR/MONITOR'S REPORT NUMBER(S)

\section{DISTRIBUTION / AVAILABILITY STATEMENT}

Approved for public release; distribution is unlimited.

\section{SUPPLEMENTARY NOTES}

\section{ABSTRACT}

This report documents the analysis of the GEM-3 data collected for the Advanced UXO Detection/Discrimination Technology Demonstration at the U.S. Army Jefferson Proving Ground (JPG), Madison, IN. The analysis is funded through the 62720A/AF25 Program “301U-Subsurface UXO Detection and Discrimination” for Project "UXO03-Advance Sensor Data Analysis Techniques for Improved Buried Target Detection." The approach taken in the U.S. Army Engineer Research and Development Center's analysis of the performance of the GEM-3 at JPG was to extract data points collected near each of the actual target locations and compare them to the calibration data acquired with known targets at the beginning of the test. This was done to determine how well the data collected near each actual target matched the calibration signatures for the same ordnance type and the extent to which the data could be differentiated from other ordnance types of nonordnance clutter. Classification of the targets was performed using a simple template-matching algorithm. This procedure resulted in an exact classification match for nearly half of the targets for which calibration data were available and a match to a similarly sized target for more than two-thirds of the medium and large targets. The statistical variability of the measurements and the effect of ordnance depth on classification performance were also examined.

\section{SUBJECT TERMS}

Anomalies

Calibration

Histogram

Multifrequency

Electromagnetic induction spectroscopy Signatures

16. SECURITY CLASSIFICATION OF:

a. REPORT

UNCLASSIFIED b. ABSTRACT

UNCLASSIFIED c. THIS PAGE

UNCLASSIFIED
Spectroscopy

Target discrimination

UXO

17. LIMITATION OF ABSTRACT

\begin{tabular}{c|l}
$\begin{array}{c}\text { 18. NUMBER } \\
\text { OF PAGES }\end{array}$ & \begin{tabular}{l} 
19a. NAME OF RESPONSIBLE PERSON \\
\cline { 2 - 2 } 219
\end{tabular} \\
$\begin{array}{l}\text { 19b. TELEPHONE NUMBER (include area } \\
\text { code) }\end{array}$ \\
\hline
\end{tabular}

